\title{
ROTORDYNAMIC FORCE COEFFICIENTS OF \\ POCKET DAMPER SEALS
}

\author{
A Dissertation \\ by \\ BUGRA H. ERTAS \\ Submitted to the Office of Graduate Studies of \\ Texas A\&M University \\ in partial fulfillment of the requirements for the degree of \\ DOCTOR OF PHILOSOPHY
}

August 2005

Major Subject: Mechanical Engineering 


\title{
ROTORDYNAMIC FORCE COEFFICIENTS OF POCKET DAMPER SEALS
}

\author{
A Dissertation \\ by \\ BUGRA H. ERTAS \\ Submitted to the Office of Graduate Studies of \\ Texas A\&M University \\ in partial fulfillment of the requirements for the degree of \\ DOCTOR OF PHILOSOPHY
}

\begin{abstract}
Approved by:
Chair of Committee, John M. Vance

Committee Members, Dara Childs

Gerald Morrison

Charles Lessard

Head of Department, Dennis O'Neal
\end{abstract}

August 2005

Major Subject: Mechanical Engineering 


\author{
ABSTRACT \\ Rotordynamic Force Coefficients of Pocket Damper Seals. \\ (August 2005) \\ Bugra H. Ertas, B.S., Texas Tech University; \\ M.S., Texas A\&M University \\ Chair of Advisory Committee: Dr. John M. Vance
}

The present work describes experiments conducted on several pocket damper seal (PDS) designs using a high pressure annular gas seal test rig. Both rotating and non-rotating tests were conducted for a 12, 8, and 6 bladed PDS. The objective of the tests was to determine the rotordynamic force coefficients and leakage for the different PDS while varying parameters such as: (1) clearance ratio, (2) rotor surface speed, (3) PDS pressure differential, and (4) excitation frequency. Two different methods were used to determine frequency dependent force coefficients: (1) the impedance method, which involved using a baseline subtraction and (2) the dynamic pressure response method, which comprised of measuring seal cavity dynamic pressure and phase relationship to vibration. Both methods were used to determine coefficients, but the dynamic pressure response method revealed insights to the dynamics of the PDS that were the first of its kind and allowed the comparison to the damper seal theory at the most fundamental of levels. The results indicated that the conventional PDS possessed high positive damping, negative and positive stiffness, and same sign cross-coupled coefficients. Another objective of the work is to investigate a new fully partitioned PDS design and accompany experimental results with the development of a modified damper seal theory. The new fully partitioned PDS design was shown to give twice as much damping as the conventional design and revealed the ability to modify direct stiffness without degradation in direct damping. Finally, both the conventional theory and the newly proposed theory predictions are compared to experimentally determined force coefficients. The last objective was to evaluate the leakage characteristics of the different designs and to investigate the effect of blade profile on seal leakage. Results showed that beveled tooth blade profiles yield higher mass flow leakage compared to rectangular blade profiles. 


\section{DEDICATION}

The following work is dedicated to my advisor Dr. John Vance, who encouraged me to pursue a doctorate degree at the Turbomachinery Laboratory. I am truly grateful for the knowledge and opportunities he has given to me in graduate school. 


\section{ACKNOWLEDGEMENTS}

I would like to thank Dr. Vance for taking me on this project and for providing guidance when I needed it. He has been an invaluable source of knowledge through this research. Sincere appreciation is extended to Dr. Childs for his guidance and support. Also, great thanks for allowing me to use his test facility. Thanks to Dr. Morrison and Dr. Lessard for their comments and suggestions. I would also like to thank Arthur Picardo, John Wade, Bradley Kerr, and Ahmed Gamal for their help with test rig assembly and operation. 


\section{TABLE OF CONTENTS}

Page

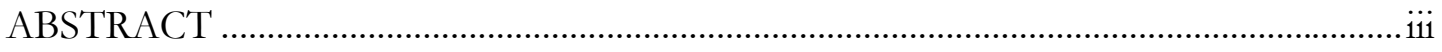

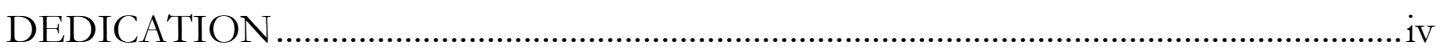

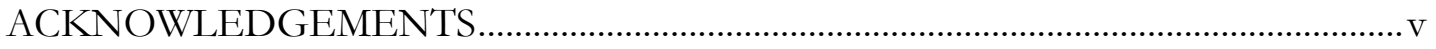

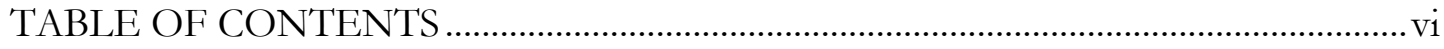

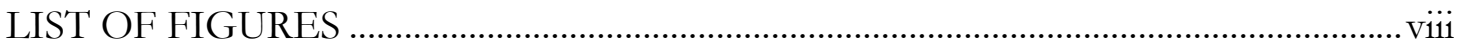

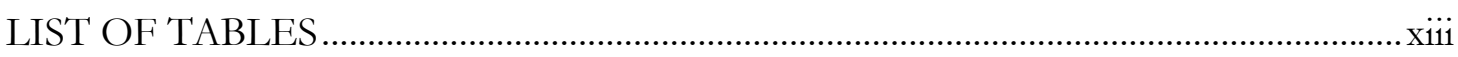

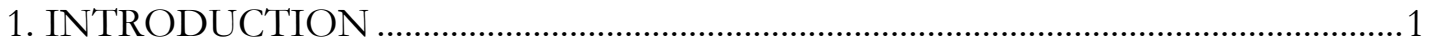

1.1 Annular Gas Seals in Turbomachinery ..................................................................

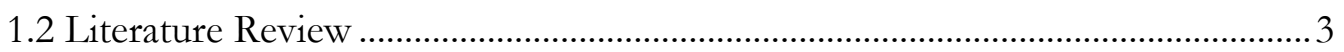

1.3 Research Objectives........................................................................................... 9

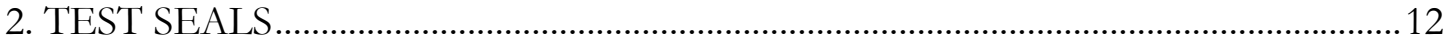

2.1 Twelve Bladed Pocket Damper Seal .........................................................................12

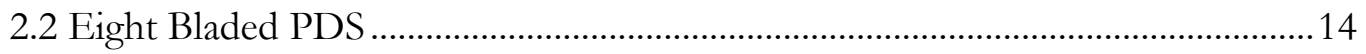

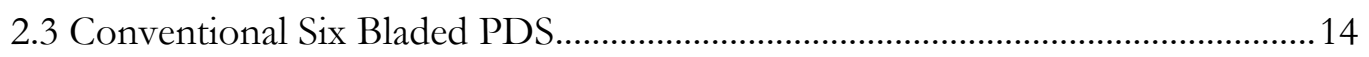

2.4 Fully Partitioned Six Bladed PDS .............................................................................14

2.5 Conventional Six Bladed PDS Blade Profiles .............................................................. 16

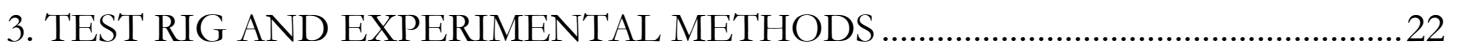

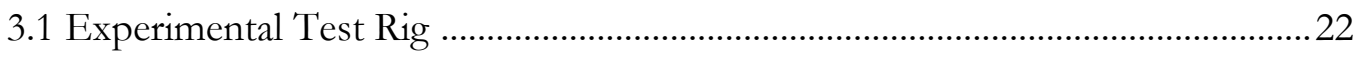

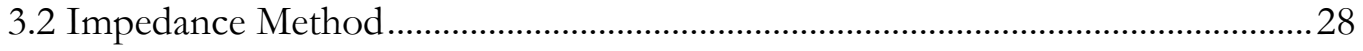

3.3 Rotordynamic Force Coefficients ……………..................................................... 33

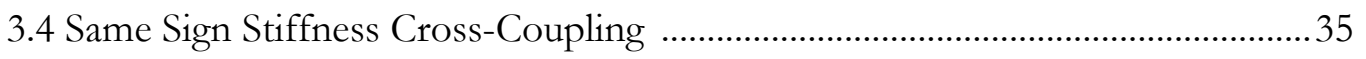

3.5 Dynamic Pressure Response Method .......................................................................4

4. IMPEDANCE METHOD EXPERIMENTAL RESULTS...........................................50

4.1 Baseline Force Coefficients ……………................................................................. 50

4.2 Twelve Bladed PDS Force Coefficients ...............................................................56

4.3 Eight Bladed PDS Force Coefficients ....................................................................5

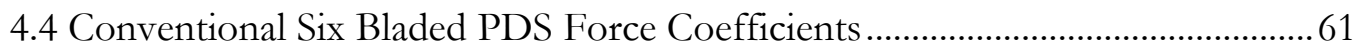

4.5 Fully Partitioned Six Bladed PDS Force Coefficients .............................................62

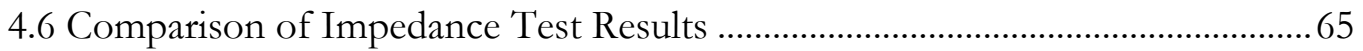




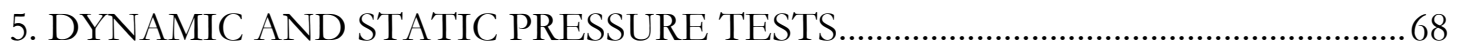

5.1 Cavity Coefficients for Eight Bladed PDS ................................................................68

5.2 Cavity Coefficients for Conventional Six Bladed PDS........................................... 82

5.3 Cavity Coefficients for Fully Partitioned Six Bladed PDS ..................................... 98

5.4 Six Bladed PDS- Static Pressure Tests..................................................................107

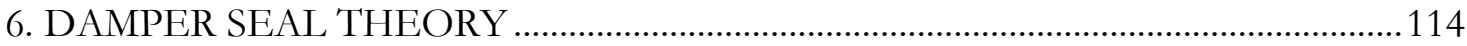

6.1 Mass Flow Derivation and Static Analysis ........................................................... 115

6.2 Control Volume Analysis for Compressible Flow ……......................................... 120

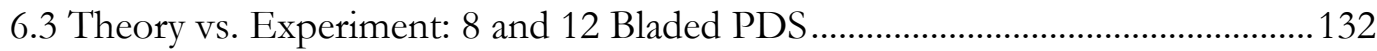

7. FULLY PARTITIONED DAMPER SEAL VS CONVENTIONAL PDS ................134

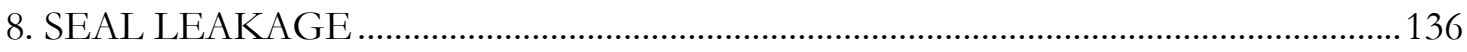

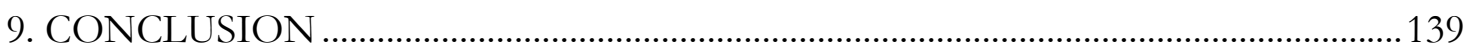

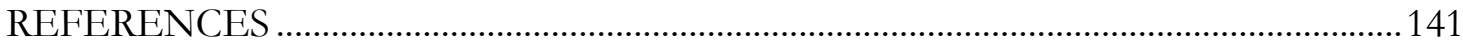

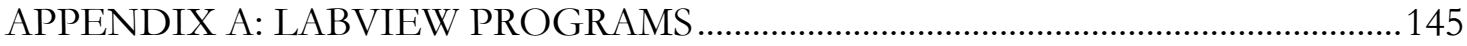

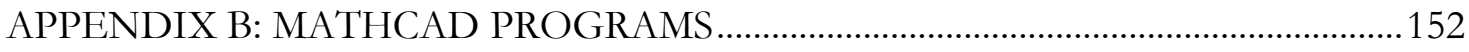

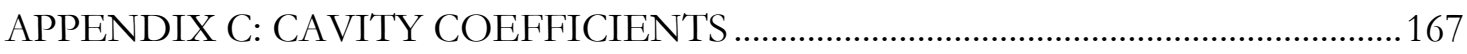

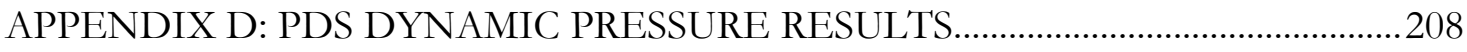

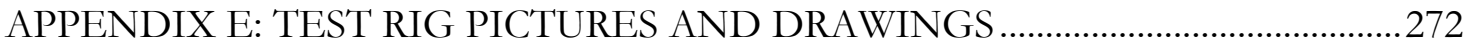

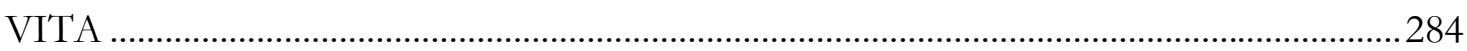




\section{LIST OF FIGURES}

Page

Figure 1. Annular Gas Seals in a Straight-Through Compressor .......................................... 2

Figure 2. Labyrinth Seal vs. Pocket Damper Seal ................................................................... 3

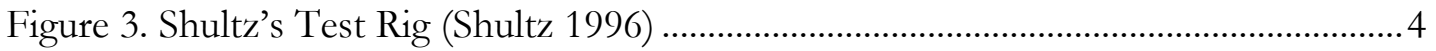

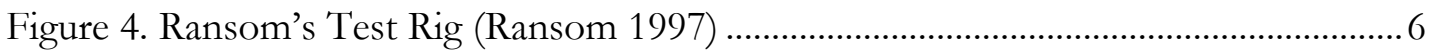

Figure 5. Rotating Test Rig (Laos 1999) .............................................................................. 7

Figure 6. Ball Bearing Test Rig (Ertas and Vance 2004).................................................... 8

Figure 7. Twelve Bladed PDS Configurations ....................................................................... 13

Figure 8. Eight Bladed PDS Configurations ...................................................................... 15

Figure 9. Conventional Six Bladed PDS Configuration.......................................................17

Figure 10. New Fully Partitioned PDS Design .................................................................... 18

Figure 11. Pressure Transducer Detail for Six Bladed Seals ..................................................19

Figure 12. Conventional Six Bladed PDS Blade Modification .............................................20

Figure 13. High Pressure Annular Gas Seal Test Rig............................................................22

Figure 14. High Pressure Test Rig Cross Section (Dawson, 2000) ....................................23

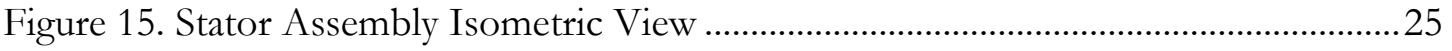

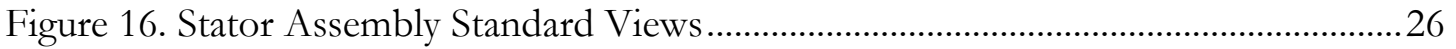

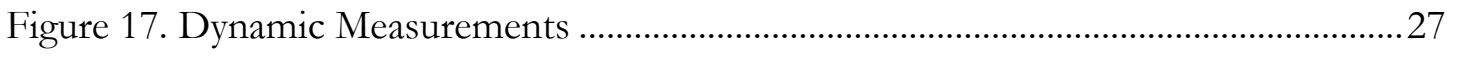

Figure 18. Pseudorandom Force Excitation: 6 Bladed PDS Configurations ........................ 30

Figure 19. Experimental Measurements: Impedance Method .................................................. 31

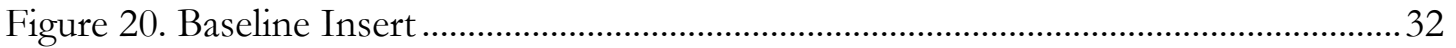

Figure 21. Force Coefficients Effects on Flexible Rotor ....................................................... 34

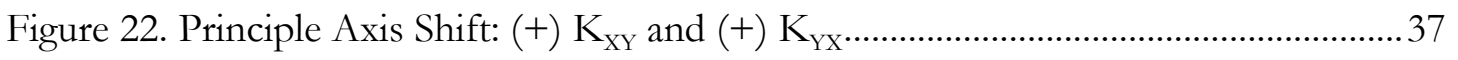

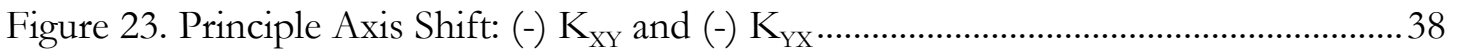

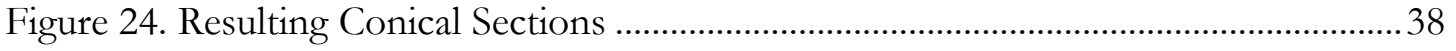

Figure 25. Same Sign Stiffness Cross-Coupling Coefficient Forces ..................................... 39

Figure 26. Same Sign Stiffness Cross-Coupling Force Components ...................................... 40

Figure 27. Orbit Distortion from Same Sign Stiffness Cross-Coupling.................................. 40

Figure 28. Dynamic Pressure Response to Vibratory Motion .................................................. 47

Figure 29. Experimental Measurements: Pressure Response Method................................... 49 


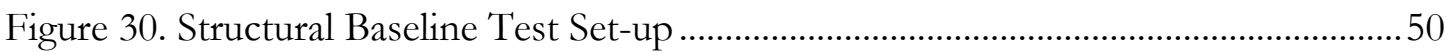

Figure 31. Structural Compliance of Test Rig Stator Assembly ...........................................52

Figure 32. Real and Imaginary Components of Dynamic Stiffness .....................................52

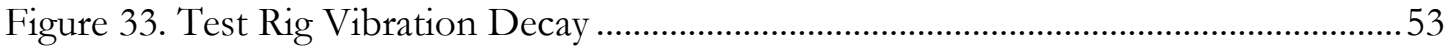

Figure 34. Baseline Direct Coefficients Y Direction-Impedance Method........................... 55

Figure 35. Baseline Cross-coupled Coefficients-Impedance Method..................................... 55

Figure 36. Twelve Bladed PDS 10,200 rpm: Direct Coefficients Impedance Method ..... 56

Figure 37. Twelve Bladed PDS 15,200 rpm: Direct Coefficients Impedance Method ..... 57

Figure 38. Twelve Bladed PDS: Cross-Coupled Stiffness Coefficients Impedance

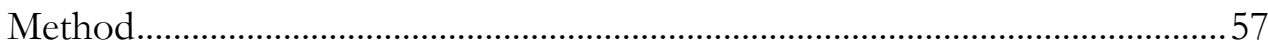

Figure 39. Twelve Bladed PDS- Cross-coupled Damping Coefficients Impedance

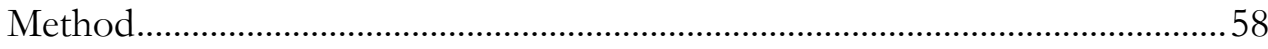

Figure 40. Eight Bladed PDS-Direct Coefficients 10,200 rpm Impedance Method ..........60

Figure 41. Eight Bladed PDS-Direct Coefficients 15,200 rpm Impedance Method ..........60

Figure 42. Eight Bladed PDS-Direct Coefficients 20,200 rpm Impedance Method..........61

Figure 43. Eight Bladed PDS-Cross-Coupled Stiffness Coefficients Impedance

Method 61

Figure 44. Conventional Six Bladed PDS-Direct Coefficients Impedance Method ........62

Figure 45. Conventional Six Bladed PDS-Cross-Coupled Coefficients Impedance

Method

Figure 46. FP Six Bladed PDS-Direct Coefficients 10,200 rpm Impedance Method ........64

Figure 47. FP Six Bladed PDS-Direct Coefficients 15,200 rpm Impedance Method ........64

Figure 48. FP Six Bladed PDS-Cross-Coupled Coefficients................................................... 65

Figure 49. Direct Damping Coefficients: Impedance Method ..............................................66

Figure 50. Direct Stiffness Coefficients: Impedance Method................................................67

Figure 51. Stator Assembly Section View C-C: Eight Bladed Seal Cavity Pressures......... 70

Figure 52. Front Control Panel: NI 4472-16 Channel Simultaneous DAQ Board ............ 71

Figure 53. LABVIEW Front Panel-8 Bladed PDS ................................................................. 72

Figure 54. Phasor Plot for Diverging and Straight-Through Cavities .................................. 72

Figure 55. Static Seal Cavity Pressure Measurements ............................................................. 73 
Figure 56. Peak Pressures and Vibration Amplitudes 8 Bladed PDS 1:1 Clr Ratio............75

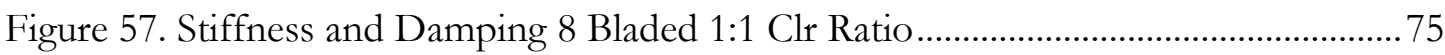

Figure 58. Pressure Phase and Force Density: 8 Bladed PDS 1:1 Clr Ratio.........................75

Figure 59. Asynchronous Pressure Frequencies 8 Bladed PDS 1:1 Clr Ratio......................76

Figure 60. Stiffness and Damping 8 Bladed 1:1.5 Clr Ratio 1.2 mils pk-pk .....................77

Figure 61. Stiffness and Damping 8 Bladed PDS 1:1.5 Clr Ratio 1.8 mils pk-pk ........... 78

Figure 62. Peak Pressures and Vibration 8 Bladed 1:1.5 Clr Ratio 1.2 mils pk-pk ......... 79

Figure 63. Phase and Force Density 8 Bladed PDS 1:1.5 Clr Ratio 1.2 mils pk-pk ....... 79

Figure 64. Phase and Force Density 8 Bladed PDS 1:1.5 Clr Ratio 1.8 mils pk-pk ....... 79

Figure 65. Pressure Spectrum for Diverging 8 Bladed PDS $(200 \mathrm{~Hz})$................................. 80

Figure 66. Conventional Six Bladed PDS Single Row of Cavities: Transducer Installation 82

Figure 67. Front Panel Dynamic Measurements Display: 0 Degree Angle

X Direction Excitation $40 \mathrm{~Hz}$ .84

Figure 68. Front Panel: Data Reduction Conventional Six Bladed PDS ............................. 85

Figure 69. Dynamic Pressure Phase: 45 Degree Location......................................................... 86

Figure 70. Direct Stiffness and Damping: Cavity Coefficients Conventional Six

Bladed PDS .86

Figure 71. Cavity Coefficients: 0 Degree Position Cross-Coupled Stiffness and

Damping 88

Figure 72. Row Coefficients: 45 Degree Position Cross-Coupled and Direct Stiffness ... 88 Figure 73. Row Coefficients: 45 Degree Position Cross-Coupled and Direct Damping . 88

Figure 74. Conventional 6 Bladed PDS Y Dir. Direct Coefficients- Pressure Method .... 89

Figure 75. Conventional 6 Bladed PDS X Dir. Direct Coefficients- Pressure Method.... 89

Figure 76. Conventional 6 Bladed PDS Y Dir. Cross-Coupled Coefficients-

Pressure Method

Figure 77. Conventional 6 Bladed PDS X Dir. Cross-Coupled Coefficients-

Pressure Method 90

Figure 78. Local Pressure Modulation-Inactive Plenums 2 and 4, 90 and 0 Degrees ....... 90

Figure 79. Local Pressure Modulation-Inactive Plenums 2 and 4, 270 Degrees. .90 
Figure 80. X Direction Excitation- 0 Degree Position $100 \mathrm{~Hz}$............................................. 91

Figure 81. X Direction Excitation- 0 Degree Position 200 Hz ............................................92

Figure 82. X Direction Excitation- 45 Degree Position $100 \mathrm{~Hz}$.......................................... 93

Figure 83. X Direction Excitation- 45 Degree Position 200 Hz .......................................... 94

Figure 84. X Direction Excitation- 90 Degree Position $100 \mathrm{~Hz}$........................................... 95

Figure 85. X Direction Excitation- 90 Degree Position 200 Hz ........................................... 96

Figure 86. Single Row of Pockets FP 6 Bladed PDS: Pressure Transducer

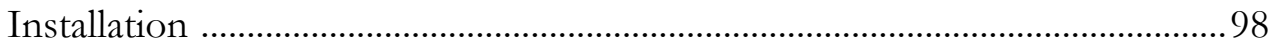

Figure 87. Fully Partitioned Seal: Y Direction Excitation 100 Hz -90 Degree

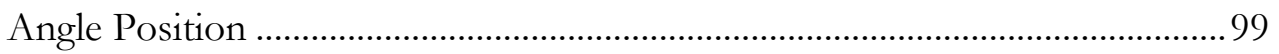

Figure 88. Front Panel: Data Reduction Fully Partitioned Six Bladed PDS .......................100

Figure 89. Stand Alone Cavities- Diverging and Converging Configurations..................... 101

Figure 90. Phasor Diagram- Stand Alone Cavities ................................................................101

Figure 91. Dynamic Cavity Pressure Phase- FP Design vs. Conventional Design............102

Figure 92. Direct Damping-Cavity Coefficients Fully Partitioned Seal 0 Degree

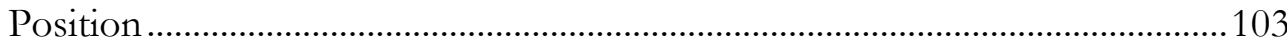

Figure 93. Direct Stiffness-Cavity Coefficients Fully Partitioned Seal 0 Degree

Position 103

Figure 94. Direct Force Coefficients Y Dir: FP PDS vs. Conventional PDS ..................... 104

Figure 95. Direct Force Coefficients X Dir: FP PDS vs. Conventional PDS .....................104

Figure 96. Cross-Coupled Force Coefficients Y Dir: FP PDS vs. Conventional PDS ..... 104

Figure 97. Cross-Coupled Force Coefficients X Dir: FP PDS vs. Conventional PDS.....105

Figure 98. High Frequency Spikes in Dynamic Cavity Pressure Measurements................ 106

Figure 99. Benckert and Wachter's Test Rig and Experimental Results (1980).................109

Figure 100. Static Pressure Measurements Displacement Pattern: 15,200 rpm ................. 110

Figure 101. Static Pressure Measurements: Rotor Offset Displacement Tests...................112

Figure 102. Negative Stiffness Effect in Diverging Seals .................................................112

Figure 103. Stand-Alone Cavities vs. Sequential Cavities .....................................................114

Figure 104. Single Row of Three Pressurized Pockets ….....................................................115

Figure 105. Mass Flow through a Single Constriction...........................................................116 
Figure 106. Flow Coefficient for Various Gases ................................................................118

Figure 107. PDS Cavity Clearance Area for Arbitrary Rotor Displacement .......................123

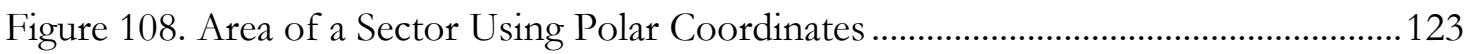

Figure 109. PDS Clearance Area for Cavity-Centered Rotor Displacement .......................125

Figure 110. Dynamic Cavity Pressures-Theoretical Predications Fully

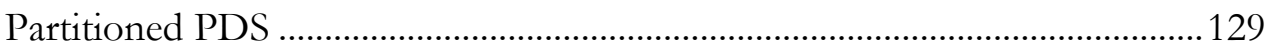

Figure 111. Direct Damping Coefficients for Varying Pitch Ratios .................................... 130

Figure 112. Direct Stiffness Coefficients for Varying Pitch Ratios.......................................131

Figure 113. Theory vs. Impedance Method: 12 Bladed PDS Direct Damping ................. 132

Figure 114. Theory vs. Impedance Method: 12 Bladed PDS Direct Stiffness ....................132

Figure 115. Theory vs. Impedance Method: 8 Bladed PDS Stiffness and Damping ........133

Figure 116. Theory vs. Experiments: Conventional 6 Bladed PDS Stiffness and

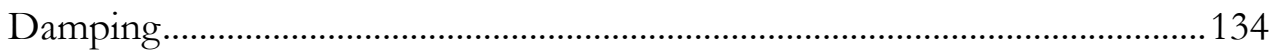

Figure 117. Theory vs. Experiments: FP 6 Bladed PDS Stiffness and Damping..............135

Figure 118. Six Bladed PDS Experimental Results ...............................................................135

Figure 119. Static Cavity Pressure Measurements vs Theory: FP PDS ...............................137 


\section{LIST OF TABLES}

Page

Table 1. Summarized PDS Geometry and Parameters ..........................................................21

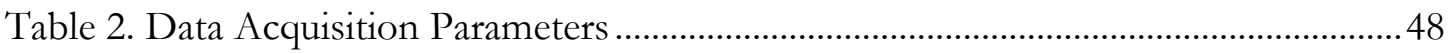

Table 3. Baseline Test Matrix - Impact Hammer Tests .............................................................. 51

Table 4. Structural Baseline Coefficients-Impact Hammer Tests .........................................53

Table 5. Baseline Impedance Test Matrix ...................................................................................5

Table 6. Test Matrix-12 Bladed PDS Impedance Method .....................................................56

Table 7. Test Matrix-8 Bladed PDS Impedance Method ........................................................... 59

Table 8. Test Matrix-Conventional 6 Bladed PDS Impedance Method ................................ 62

Table 9. Test Matrix-Fully Partitioned 6 Bladed PDS Impedance Method..........................64

Table 10. Configurations Tested Using the Impedance Method .........................................66

Table 11. Test Matrix- Eight Bladed Dynamic Pressure Tests ...............................................71

Table 12. Cavity Flow Parameters: 8 Bladed 1:1 ...................................................................76

Table 13. Inactive Plenum Pressure Magnitudes .....................................................................97

Table 14. Gamal's Results for Static Direct and Cross-coupled Stiffness (2003) .............. 107

Table 15. Cavity Pressure Measurements: Rotating Displacement Test Results ................111

Table 16. Cases for Fully Partitioned PDS-Variable Pitch Ratio ...........................................130

Table 17. Leakage Results for 6 Bladed PDS Configurations.............................................138

Table 18. Inlet Blade and Exit Blade Discharge Coefficients .............................................138 


\section{INTRODUCTION}

\subsection{Annular Gas Seals in Turbomachinery}

Annular gas seals in turbomachinery are necessary components that are used to seal interstage pressures in turbines and compressors. Gas seals are primarily non-contact annular seals that are comprised of multiple axially spaced blades or surface patterns that provide a restrictive path for the working fluid that impedes the leakage between machine stages. Figure 1 shows a typical centrifugal compressor with annular gas seals in various locations around the rotor. The primary aim of annular gas seals is to prevent fluid leakage from high pressure regions to low pressure regions, which directly affects the thermodynamic efficiency of the machine. A secondary function or effect of gas seals is the contribution to the mechanical dynamics of the rotor-bearing system, which is generated from fluid-structure interaction forces between the rotor and stator components. When analytically modeling rotor-bearing systems to evaluate dynamic stability and response, engineers consider seals to have dynamic coefficients similar to hydrodynamic bearings. Like bearings in a rotor assembly, gas seals exhibit force coefficients that can either improve or degrade the dynamic stability of the rotor-bearing system and also can influence the vibration response of the machine. Gas annular seals, such as labyrinth seals, possess negligible added mass terms and can generally be expressed by the reaction force model (Childs 1993) as shown in Equation 1.1. This equation relates the seal forces due to rotor motion and velocity through direct $\left(\mathrm{K}_{\mathrm{XX}}, \mathrm{K}_{\mathrm{YY}}\right.$, $\left.\mathrm{C}_{\mathrm{XX}}, \mathrm{C}_{\mathrm{YY}}\right)$ and cross-coupled $\left(\mathrm{K}_{\mathrm{XY}}, \mathrm{K}_{\mathrm{YX}}, \mathrm{C}_{\mathrm{XY}}, \mathrm{C}_{\mathrm{YX}}\right)$ linear force coefficients.

$$
-\left[\begin{array}{c}
F_{X} \\
F_{Y}
\end{array}\right]=\left[\begin{array}{cc}
K_{X X} & K_{X Y} \\
K_{Y X} & K_{Y Y}
\end{array}\right] \cdot\left[\begin{array}{c}
X \\
Y
\end{array}\right]+\left[\begin{array}{cc}
C_{X X} & C_{X Y} \\
C_{Y X} & C_{Y Y}
\end{array}\right] \cdot\left[\begin{array}{c}
\dot{X} \\
\dot{Y}
\end{array}\right]
$$

The most common type of annular gas seal used in present day machines is the labyrinth seal (Figure 2). Although labyrinth seals are easy to manufacture and provide adequate sealing of internal fluid pressures, they possess characteristics that degrade the dynamic stability of rotor-bearing systems. The undesirable effect on dynamic stability can mainly be attributed to destabilizing stiffness cross-coupling $\left(\mathrm{K}_{\mathrm{XY}}=-\mathrm{K}_{\mathrm{YX}}\right)$ that arises from fluid rotation in the annular plenums within the seal that are in the direction of rotor whirl.

This dissertation follows the style of ASME Journal of Tribology. 
The rotation of the fluid amplifies rotor vibration by feeding energy into the whirl orbit and can result in rotordynamic instability.

Pocket damper seals have been used in several centrifugal compressor applications, primarily at the center seal location on back to back machines, and also on balance pistons, in efforts to attenuate rotor vibration response and increase rotordynamic stability. Unlike the labyrinth seal, the pocket damper seal (PDS) as shown in Figure 2 is fabricated using baffle walls between paired blades to restrict circumferential fluid flow developed by rotor rotation and impeller stage pre-swirl. In addition to restricting circumferential flow, incorporating baffle walls within paired blades gives rise to larger valued dynamic pressure oscillations in the cavities during machine operation, which yield significant direct damping coefficients. Pocket damper seals also exhibit other force coefficients as described in Equation 1. Knowing these force coefficients is necessary for performing an accurate analysis simulating the dynamic response and stability of a rotor-bearing system. Therefore, experimental tests are required to measure force coefficients and are also needed to compare with existing theories so the dynamic behavior of machines can be predicted.
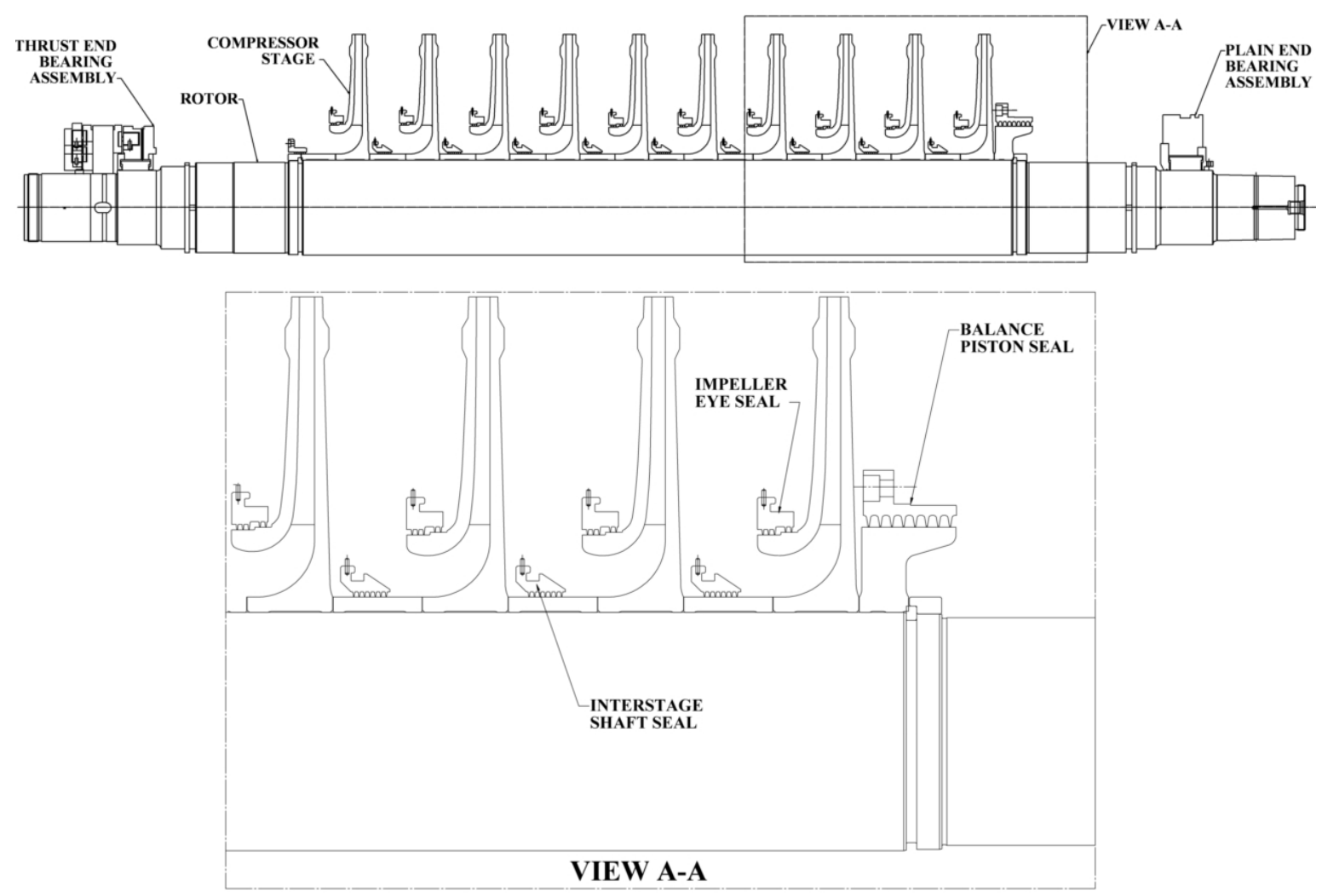

Figure 1. Annular gas Seals in a Straight-Through Compressor 


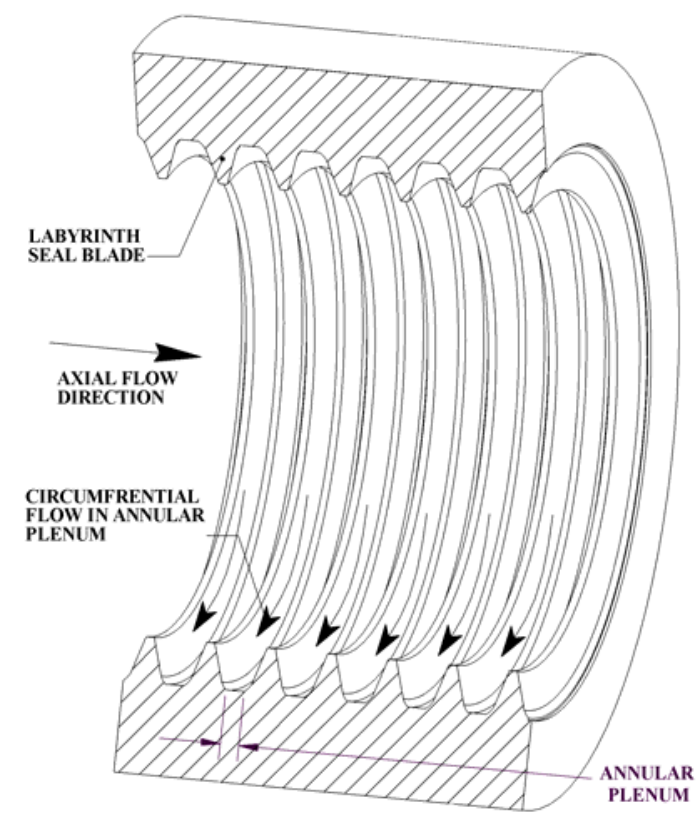

(a) Labyrinth seal

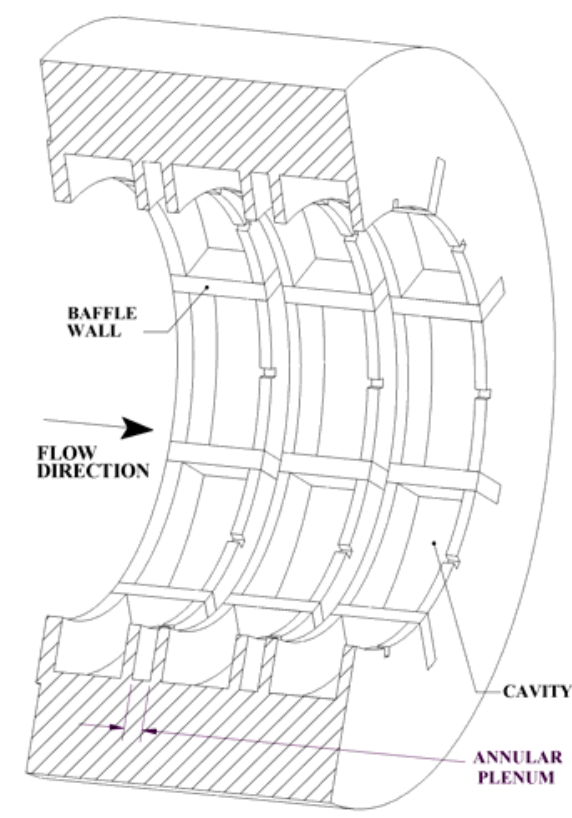

(b) Six bladed pocket damper seal

Figure 2. Labyrinth Seal vs. Pocket Damper Seal

\subsection{Literature Review}

Alford (1965) developed a theory focused on explaining the destabilizing forces produced in axial flow turbomachinery. Considering the labyrinth seals, he used a onedimensional axial bulk flow analysis that showed the difference in the axial flow modulation across the inlet and exit blades of a seal from rotor vibration. This produced an unbalanced pressure distribution around the rotor surface; giving rise to destabilizing forces or stabilizing forces dependent on the seal geometry. Although Alford's 1965 work was a significant development in the fundamental understanding of flow-induced forces in gas seals, he failed to consider circumferential flow in the analysis. In fact, Alford's analysis would have been more accurate if he had incorporated baffle walls between the blades of his labyrinth seal restricting circumferential flow; similar to the PDS design. Due to this fact, the conventional PDS analysis is fundamentally the same as Alford's 1965 analysis on labyrinth seals.

Vance et al. in 1993 developed a model for a gas damper actuator for application in aircraft engines. The premise of the design was to use a series of actuators and the rotor vibration to produce a pressure-induced reaction force that would oppose the rotor vibration, therefore yielding positive damping. Using a bulk flow approach the analysis showed that the damping produced was positive if the inlet area was modulated while the exit area was kept 
fixed. This work resulted in the development of the fundamental control volume analysis used for the present day PDS theory. Closely following the actuator analysis, Sundararajan and Vance (1993a) improved the model by incorporating choked flow. The result was that damping increased a lesser amount with increased inlet pressures when considering choked flow.

Initial experimental testing of the conventional PDS design was first conducted by Vance and Shultz in 1996. Vance and Shultz measured cavity pressures, leakage, and direct damping coefficients for a 2-bladed four pocket damper seal with inlet pressures up to 70 psig leaking to atmosphere (14.7 psig) and also developed a refined analytical model based on the bulk flow equations generated by Sundararajan and Vance (1993b). A cross sectional view of Shultz's test rig is shown in Figure 3. The cantilevered shaft-journal assembly (No. 5) was non-rotating. The two bladed diverging clearance (1:2 clearance ratio) PDS (No. 4) was mounted on top of test rig base (No. 1). Pressurized air entered the chamber and exited from the top in between the last blade of the PDS and the journal.

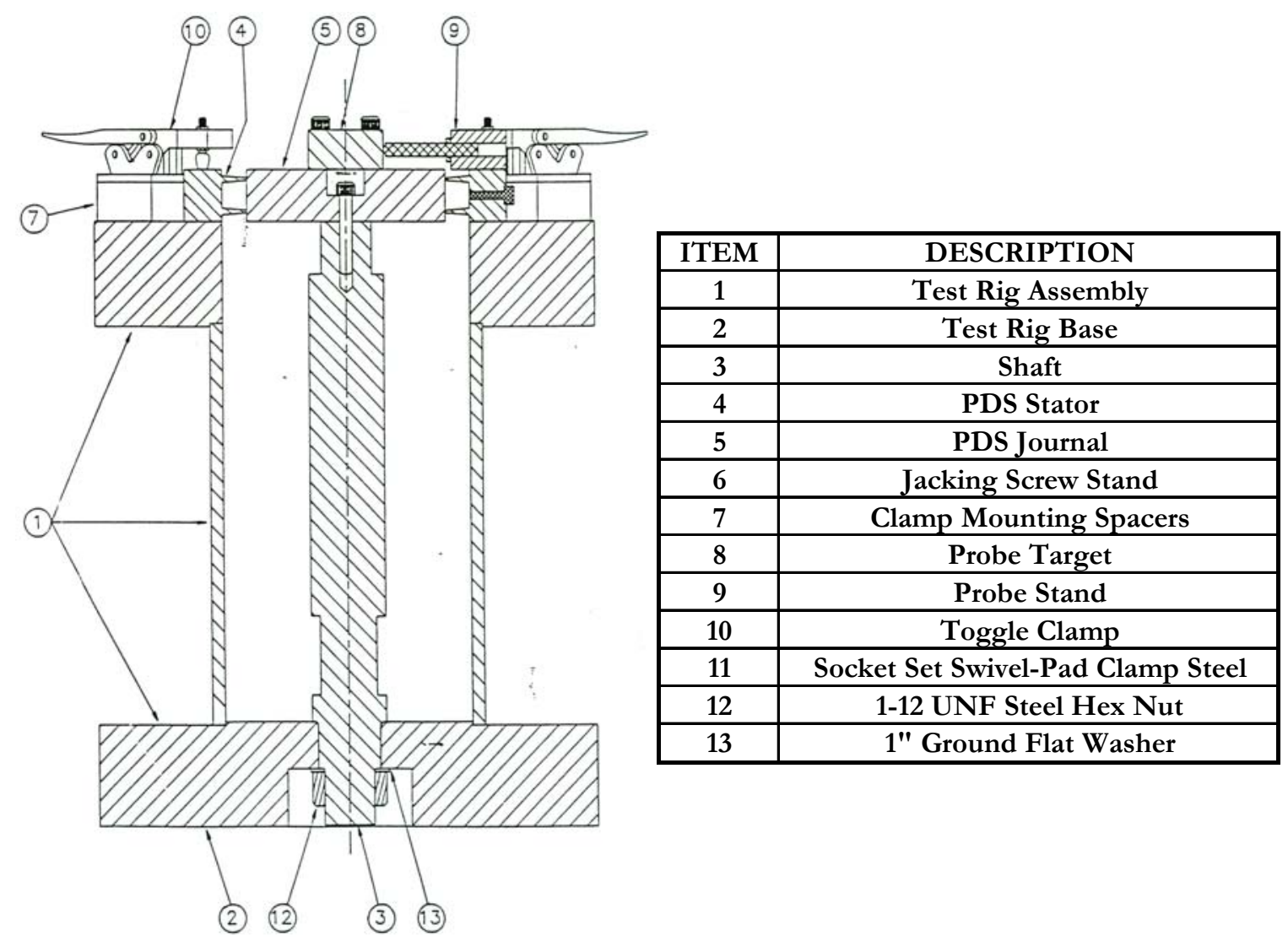

Figure 3. Shultz's Test Rig (Shultz 1996) 
The motion was measured with a non-contact eddy current proximity probe and the system was excited using an impact hammer. Damping was measured using log decrement free vibration tests on the non-rotating test rig, which had a test frequency of $200 \mathrm{~Hz}$. Additionally, Vance and Schultz measured cavity pressures as a function of inlet pressure and compared the pressure magnitude and phase with the analytical results at $200 \mathrm{~Hz}$. This study only investigated the effect of inlet pressure on damping, dynamic pressures peaks, and leakage. All parameters where shown to linearly increase with increasing inlet pressure. Vance and Schultz also conducted tests on the same PDS design with the seal reversed so that the clearance was a converging, which yielded a highly unstable system due to the negative damping.

In $1995 \mathrm{Li}$ and Vance presented the first rotating tests with the PDS. They tested PDS with two and three blades on a test rig rotor mounted on ball bearings up to 70 psig (4.83 bar) inlet pressure. Several coast-down measurements were taken for the PDS and compared to a conventional labyrinth seal. In addition to the coast-down tests, impact hammer tests were performed for determining the damping and stiffness of the seal. The coast down tests were used to observe the effects of the PDS on the critical speed amplitude; whereas, the impact hammer tests were focused on extracting force coefficients. These tests were conducted for many inlet pressures and the results showed that PDS had significantly more damping then the conventional labyrinth seal design. A follow on project by Droste (1995) and Li and Vance (1995) performed experiments on three and four bladed PDS. These seals were different than the previously tested seals because all the blades possessed the same inside bore diameter, but the downstream blades had notches machined into them. This configuration resulted in twice as much damping compared to the initial PDS design.

In 1997 Ransom tested a two bladed PDS design that yielded a full set of rotordynamic coefficients. The test rig used for the experiments is shown in Figure 4 and comprised of a seal housing that was vertically supported by flexible rods with a vertical rotor bearing system. This test rig was used to extract bearing coefficients, but Ransom used it to perform impact hammer tests in the $\mathrm{X}$ and $\mathrm{Y}$ directions at different inlet pressures (up to 45 psig or 4.06 bar) and rotor speeds. The impact tests were used to generate transfer functions in both orthogonal directions and indicated very little cross-coupling stiffness and almost no cross-coupled damping. The tests also showed the PDS to have negative direct stiffness and positive direct damping which increased in magnitude with increasing inlet pressures. Follow 
up tests were conducted in 1998 by Ransom et al. on the same test rig for a four bladed PDS design. The tests demonstrated the insensitivity of stiffness cross-coupling for different journal speeds, indicating the benefits of low destabilizing forces in the PDS.

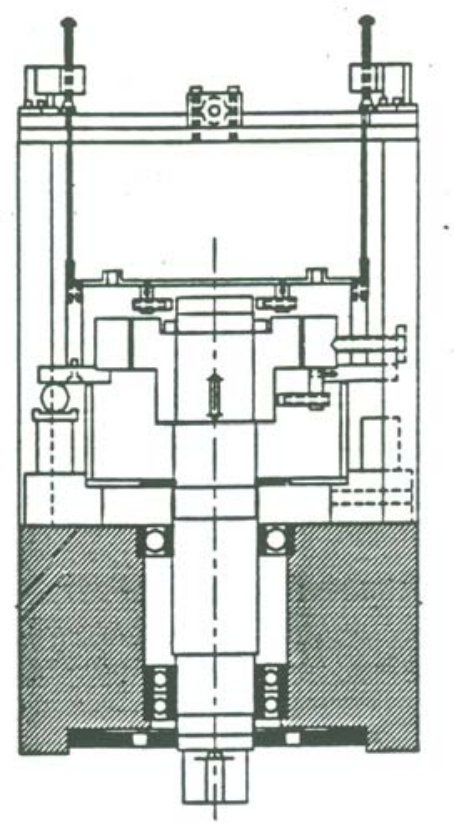

Figure 4. Ransom’s Test rig (Ransom 1998)

Further tests were conducted by Laos (1999), which involved both non-rotating and rotating tests on four bladed damper seals with eight circumferential pockets at pressures up to 80 psig. The test rig used for his experiments is shown in Figure 5. The rotor is supported by two self aligning ball bearings one of which is mounted on a compliant squirrel cage support. The journal is located at the left outboard end of the rotor assembly and is inserted into the two back-to-back test seals. Inlet pressure is supplied into the center plenum of the seal housing. The air flow exits at both ends of the housing. The first type of tests that Laos conducted were impact hammer rap tests aimed at determining direct force coefficients. $\mathrm{He}$ showed that the PDS exhibited positive damping and negative stiffness force coefficients that increased in magnitude as the inlet pressure was increased. This result agreed with past experiments. Also, Laos performed rotating coast-down tests at 7000-3000 rpm to observe the effects of the PDS on critical speed amplitudes. Tests were conducted with and without the PDS assembled into the rotor-bearing system. The rotating tests showed an extraordinary ability to suppress critical speed amplitudes. In addition to his experimental work, Laos also 
developed a theory for off-centered operation and predicted negative cross-coupled stiffness coefficients that possessed the same sign.

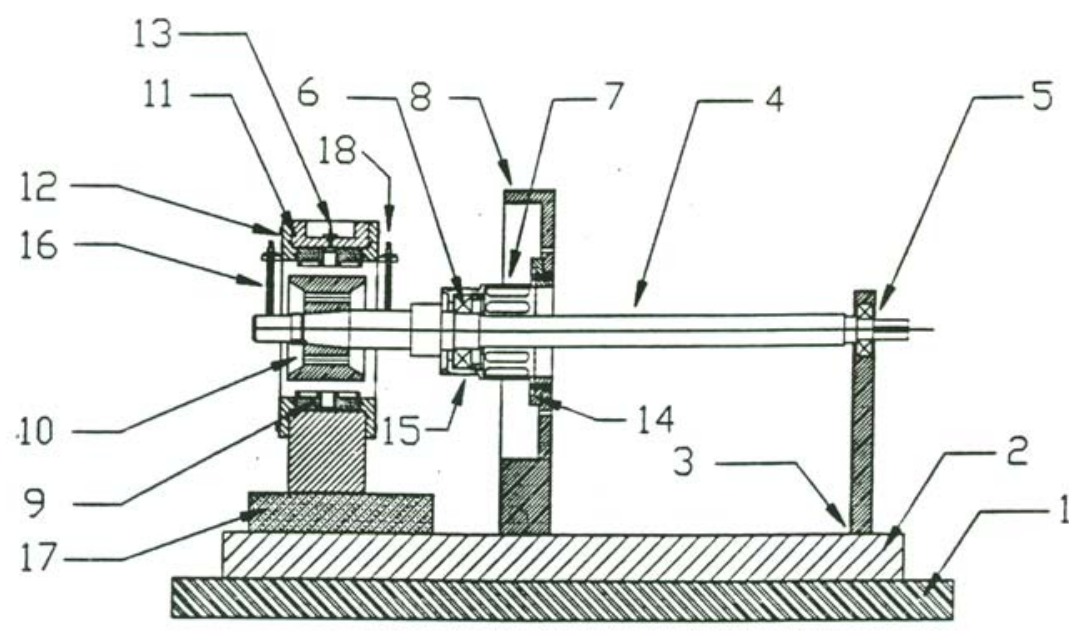

\begin{tabular}{|c|c|}
\hline ITEM & DESCRIPTION \\
\hline 1 & Steel Plate \\
\hline 2 & Base Plate \\
\hline 3 & Alignment Channel \\
\hline 4 & Rotor Shaft \\
\hline 5 & Self-Aligning Bearing \\
\hline 6 & Self-Aligning Bearing \\
\hline 7 & Squirrel Cage Ribs \\
\hline 8 & Damper Housing \\
\hline 9 & Test Seal \\
\hline 10 & Seal Journal \\
\hline 11 & O'rings \\
\hline 12 & Seal Housing \\
\hline 13 & Pressure Transducer \\
\hline 14 & Centering Plate \\
\hline 15 & Squirrel Cage \\
\hline 16 & Outboard End Prox Probe \\
\hline 17 & Leveling Plate \\
\hline 18 & Inboard End Prox Probe \\
\hline
\end{tabular}

Figure 5. Rotating Test Rig (Laos 1999)

Li et al. (2000) tested a 4 bladed PDS with 4 circumferential pockets on the test rig shown in Figure 4. The tests conducted were similar to the previous tests in that both impact hammer tests and rotating tests were administered at inlet pressure up to 36 psig. The conclusions to the tests were interesting because they resulted in negative same sign crosscoupled coefficients even though their theoretical predications showed opposite sign and same magnitude stiffness cross-coupling. This result (same sign cross-coupling) was rejected in its authenticity and was believed to be related with the stiffness asymmetry of the test rig. 
Although the same sign stiffness cross-coupled coefficients were labeled as a test rig phenomena and not the inherent behavior of the PDS, these experimental results for crosscoupled stiffness correlated with Laos' (1999) findings and would also agree with future findings by several researchers.

The next set of experimental tests was conducted by Vance et al. (2002) and they focused on investigating the effects of high frequency excitation and $\Pi$ groups associated with the PDS. The test rig used for these experiments is shown in Figure 6 and was originally used to test for ball bearing radial stiffness. It proved to be a good test rig to test PDS because of the low baseline damping and the high frequency rotor eigenvalues. The first type of test performed was the impact hammer free vibration tests for inlet seal pressures from 1.7-6.0 bar. Experiments showed that damping increased with increasing inlet pressure at higher frequencies of vibration up to $300 \mathrm{~Hz}$. In addition to the free vibration tests, critical speed coast down tests were conducted with and without the damper seal pressurized. The damper seal dramatically decreased the critical speed vibration response by one-third the response without the PDS.

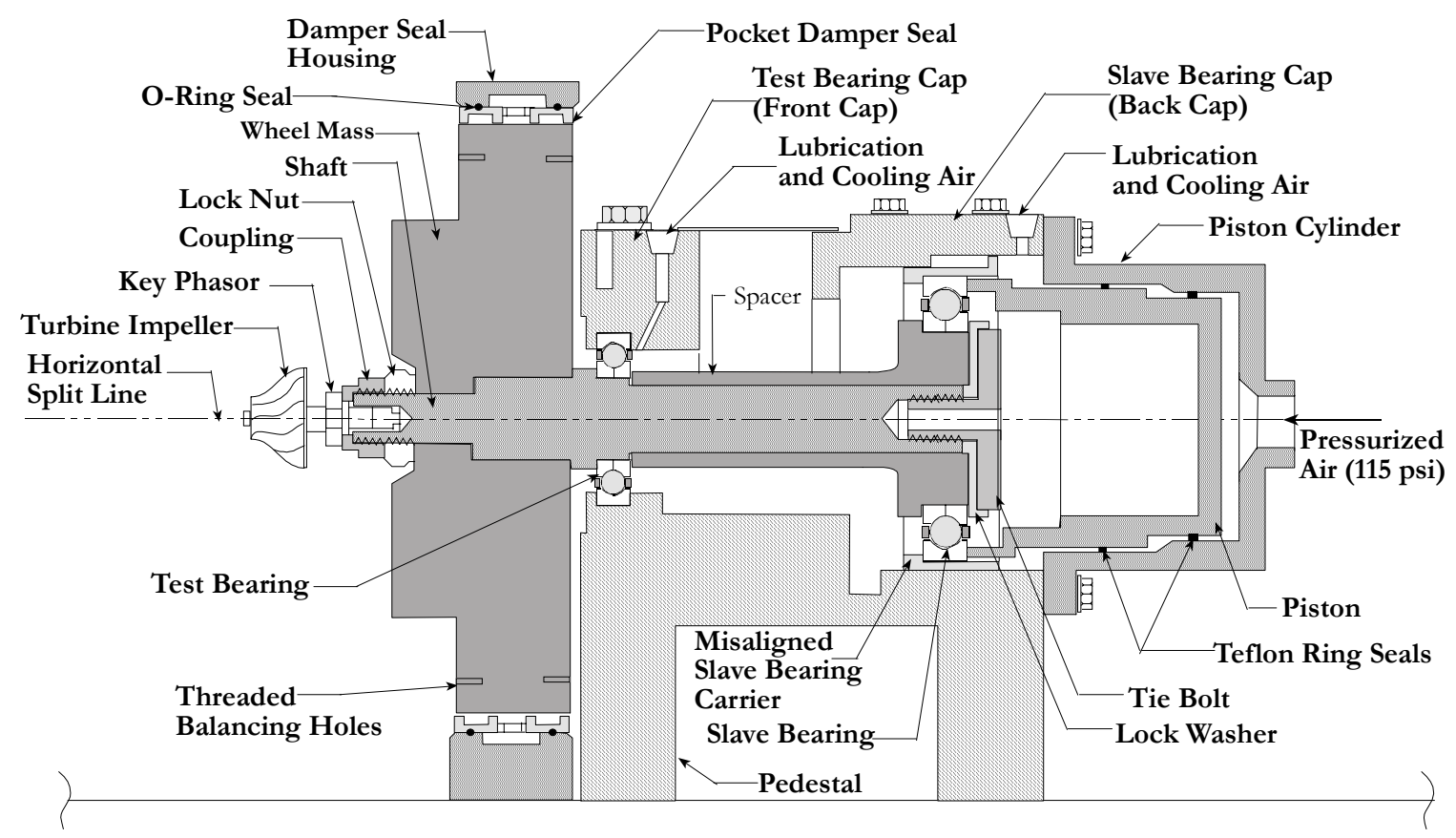

Figure 6. Ball Bearing Test Rig (Ertas and Vance 2004) 
The most recent analytical work was presented by Aguilar (2002) on the topic of nonlinear vibration amplitude effects on PDS coefficients. This analysis considered both axial and circumferential continuity equations and also used a second order nonlinear Taylor series expansion for expressing the control volume partial differential equation terms. His analysis was generated for a 2-bladed 4-pocket diverging PDS. The results showed that vibration amplitude has a weak effect on PDS force coefficients. Also, he predicted same sign stiffness cross-coupling coefficients (same as Laos did in his 1999 work). This was contradictory to the analytical work by Li et al. (2000).

The latest experiments were carried out by Gamal (2003). Gamal investigated the leakage characteristics for the 12 bladed and 8 bladed 1:1 clearance ratio PDS. He discovered that the 8 bladed PDS leaked less than the 12 bladed PDS, which was contradictory to the analytical simulations. This raised questions on the effects of blade profile on leakage, because the 12 bladed seal and the 8 bladed PDS possess different blade profile shapes. The leakage findings by Gamal for these two seals acted as the motivation for the presently conducted research on blade profiles of the six bladed PDS. Gamal also measured the direct stiffness and static cross-coupled stiffness of the 12 and 8 bladed seals. His experiments showed same sign stiffness cross-coupling coefficients, which agree with most (with one analytical exception) earlier results as previously discussed. His tests were conducted on the same high pressure gas seal test rig as the present work.

\subsection{Research Objectives}

There are several objectives of the present research, that when combined, define a broader research objective, which is to provide the potential to improve the rotordynamic and thermodynamic performance of turbomachinery by the use of damper seals. Improving rotordynamic performance can be accomplished by providing engineers with experimental results of PDS that are applicable to the operating conditions observed in the industry. Therefore, unlike previous tests conducted on PDS, the present tests are performed at high inlet pressures (1,000 psi), adequate pressure differentials (400-900 psi), sufficient rotor speeds (10,200-20,200 rpm), and a test frequency range between 20-300 Hz, which encompasses a large range of operating conditions in the field. Since past tests with PDS were limited to low inlet pressures, verification of the damper seal theory for high pressures was nonexistent. The experiments conducted in this work will not only provide comparisons 
to the damper seal theory, but will also validate the theory at the cavity level with the use of the dynamic pressure response method.

Conventional pocket damper seals are designed to possess a diverging clearance geometry in efforts to maximize damping capacity that results in enhanced rotor-bearing stability. Another consequence of diverging clearance seals is the inherent direct negative stiffness. The presence of negative stiffness is not a contributor to dynamic instability, but can moderately lower the locations of critical speeds. In some cases where the operating speed is lower and relatively close to the critical speed, negative stiffness can pose a synchronous response problem, which restricts the use of a diverging clearance PDS even though the seal suppresses the asynchronous instability. To remedy the negative stiffness problem associated with synchronous response, another objective of this research is to present and verify a new PDS design that can have significantly more positive direct stiffness without degrading the damping capacity. The new fully partitioned design is a modification from the conventional 6 bladed PDS and the comparison of the force coefficients and the seal leakage between the two seals is presented. In addition, the experimental results are accompanied by a modified theory that accounts for the new seal design.

The final objective of the present research was to determine the influence of bladed profile on seal leakage. Turbomachinery stage leakage is an important characteristic when considering the application of a particular seal in a machine. The seal leakage contributes to the overall efficiency of a machine and based on previous experiments by Gamal, it seems that the blade profile plays an important role with the leakage amount for a PDS. Therefore, the work presented here looks at two different blade profiles: (1) a rectangular profile and (2) a beveled or chamfered profile. Unlike Gamal's tests where there was little experimental control because of the varying parameters between the 12 and 8 bladed PDS, the leakage tests conducted for this work are conducted on the same seal with identical geometric parameters. The only parameter which differs from the two tests is the shape of the blade seal, which eliminates the effect of all other parameters on leakage differences such as cavity depth, number of blades, axial pitch, inside bore diameters, and notch geometry. 
Some important questions that will be answered in the present work are:

(1) What characteristics do PDS have that affects the dynamics of rotating equipment?

(2) How does blade profile affect seal leakage?

(3) How does experiments compare with theory for high inlet pressures?

(4) Do the $X$ and Y cross-coupled stiffness coefficients have the same sign?

(5) What is the effect of same sign cross-coupling coefficients on rotordynamics?

(6) Do the inactive plenums with no partition walls contribute to the overall seal coefficients? Is there pressure modulation in these plenums?

(7) What is the effect of clearance ratio on cavity pressure phase?

(8) How does the new fully partitioned design compare with the conventional design? Can the direct stiffness be changed to be more positive without degrading damping capacity?

(9) How does the modified theory prediction of coefficients compare with the experimental results from the new design?

(10) What is the effect of axial pitch ratio on predictions of direct stiffness and damping for the new design? 


\section{TEST SEALS}

Experimental tests were performed using 7 different damper seal designs, one of which is a new design. The test seals are comprised of a 12 bladed seal, an 8 bladed seal, and a 6 bladed seal. All the damper seals were machined as pairs (two of each type) from high strength 4140 steel, due to the high inlet pressures. The seals were machined in pairs because of the back-to-back assembly in the test rig. Also, the seals were fabricated using two different manufacturing techniques. The rotor diameter was manufactured to be 4.500 inches $(114.3 \mathrm{~mm})$, and all test seals possessed a bore diameter of 4.510 inches (114.554 $\mathrm{mm})$, which yields a radial clearance of 0.005 inches $(0.127 \mathrm{~mm})$ from the rotor.

\subsection{Twelve Bladed Pocket Damper Seal}

The twelve bladed PDS was the first to be tested and is shown in Figure 7. This seal was manufactured by inserting bar stock into 8 angular slots machined into the seal body where they were brazed in an oven under high temperature, and then the inactive plenums were machined. The twelve bladed seal was fabricated using this method due to the small balde-to-blade-spacing of the cavities in combination with the large cavity depth of 1.400 inches $(35.56 \mathrm{~mm})$. Two different configurations were tested with the twelve bladed PDS: (1) 1:1 clearance ratio and (2) 1:2 clearance ratio. The 1:1 clearance ratio represents the ratio of the inlet blade clearance area from the rotor surface to the exit blade clearance area from the rotor surface. For the 1:1 case both the inlet and exit areas are equivalent therefore labeling the configuration as a "straight through" seal with no notches. The second configuration, the 1:2 clearance ratio) utilizes curvilinear notches (as shown in Figure 7) on the exit blade making the exit clearance area from the rotor equal 2 times the inlet clearance, which is referred to as a diverging clearance configuration. One important feature worth noting for the twelve bladed PDS is the blade profile. The blade profile, shown in Figure 7 Detail E, illustrates a double chamfer profile resulting in 6 blades with a bevel on the upstream flow side of a blade and 6 blades with a bevel on the downstream flow side. Bevels are usually machined into seal blades to minimize damage in anticipation of rotor to stator interaction during operation and also to further reduce leakage. Typically bevels are machined on the downstream side of bladed profiles, but for the 12 bladed PDS, that was not feasible due to the small active pitch $(0.208 \mathrm{in} / 5.283 \mathrm{~mm})$ in combination with the existence of baffle walls in the plenum, making it impossible to machine with a lathe. 


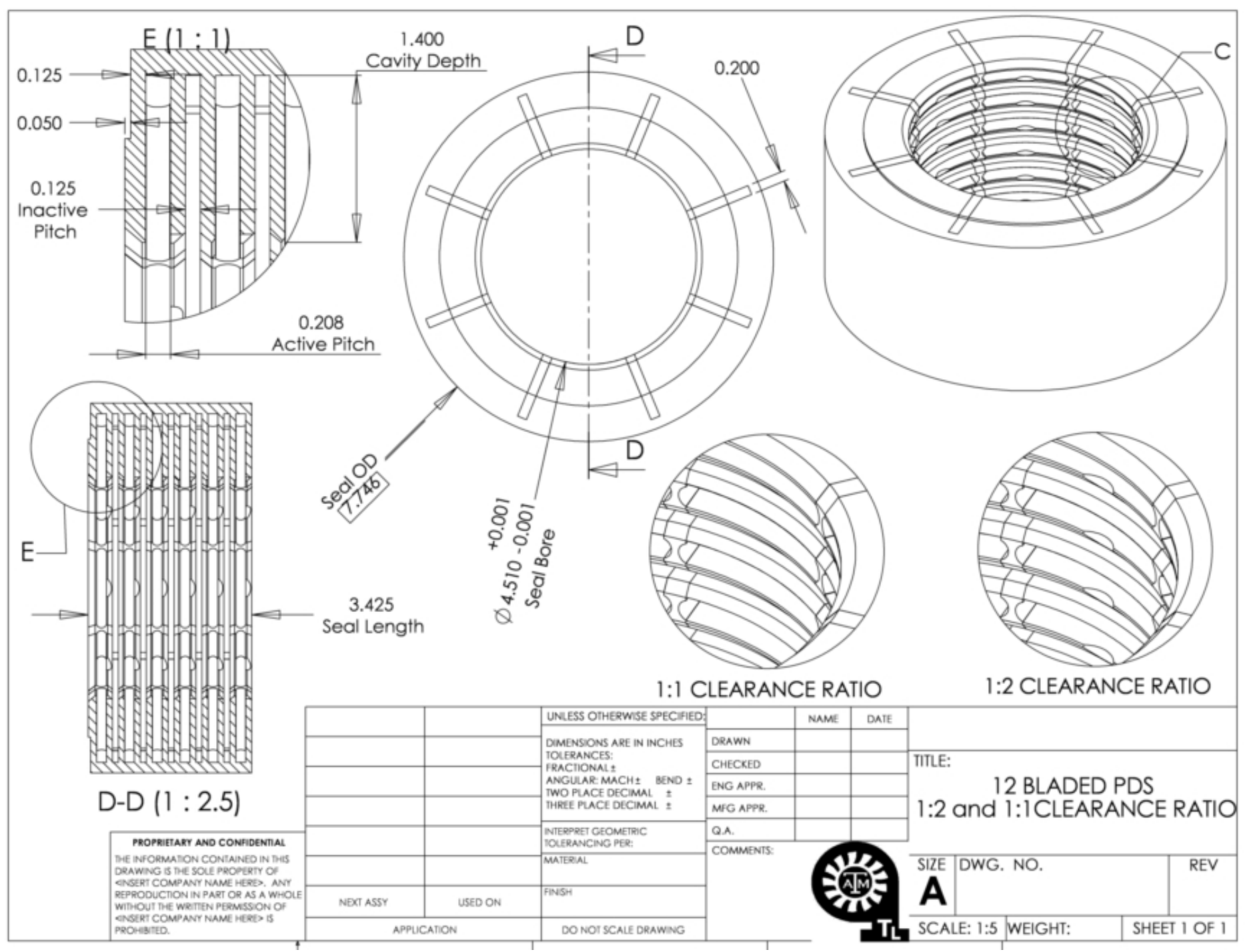

Figure 7. Twelve Bladed PDS Configurations 


\subsection{Eight Bladed PDS}

The next seal tested was the eight bladed PDS, shown in Figure 8. Unlike the 12 bladed seal the eight bladed seal was manufactured from a single piece of round stock, therefore a brazing process was not required. Also, the eight bladed seal was fabricated with different shaped cavities, rectangular notches rather than curvilinear notches, and rectangular blade profiles. The eight bladed seal was tested for two clearance ratios, 1:1 clearance ratio (straight through configuration) and a 1:1.5 clearance ratio (diverging clearance). This seal is composed of 4 circumferential rows of 8 equally spaced cavities separated by three inactive plenums (no cavities). Four pressure taps were machined into the two middle cavities for pressure transducers, which will be discussed later. This seal was expected to exhibit larger force coefficients because of the larger axial pitch of the active cavities.

\subsection{Conventional Six Bladed PDS}

The six bladed seal, shown in Figure 9, possessed the largest axial pitch for the active cavities giving it the potential for the largest valued force coefficients. This seal was manufactured like the twelve bladed PDS, using separate bar stock to make up the partition walls and also required a brazing process after inserting the barriers into the machined slots in the body of the seal. Only one clearance ratio of 1:2 was tested for the six bladed seal, and the notches were rectangular in shape. In addition to testing the rectangular profile blades, the six bladed seal was modified to incorporate beveled profile blades to investigate the effect of blade profile on leakage. To accommodate the dynamic pressure method for determining frequency dependent force coefficients the six bladed PDS was equipped with pressure taps in each cavity for measuring dynamic and static pressure levels. Note that the conventional six bladed PDS has a total of 24 diverging clearance cavities. These 24 cavities are called active cavities because they possess a dynamic pressure component in response to system vibration. The two inactive plenums, that separate the active cavities, are theorized to have a constant pressure with no dynamic pressure component.

\subsection{Fully Partitioned Six Bladed PDS}

A 2002 paper presented by Li et al. discusses the testing of a new type of PDS and compares the results of the experiments with previous experiments using a conventional PDS with the same geometry except the configuration of the partition walls between the paired blades. Although the test did not yield force coefficients, critical speed tests suggested that the new design exhibited higher positive stiffness and more damping. 


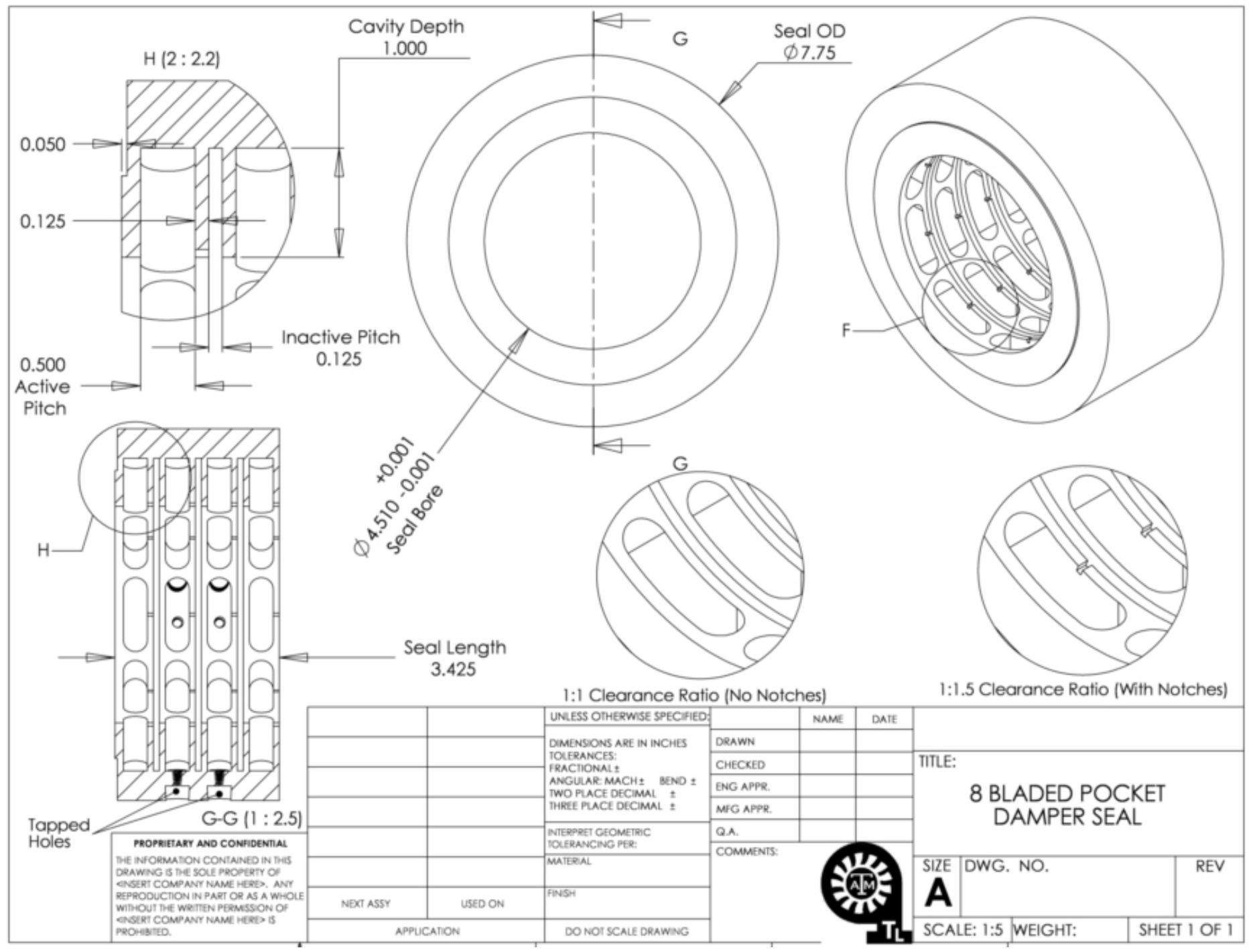

Figure 8. Eight Bladed PDS Configurations 
Figure 10 shows the new fully partitioned PDS design and how the design differs from the conventional PDS. The fully partitioned design incorporates baffle walls throughout the entire axial length of the seal creating sequentially placed cavities that have diverging and converging clearance geometries. Unlike the conventional PDS design, the fully partitioned design has 24 diverging cavities and 16 converging clearance cavities, yielding a total of 40 active cavities in the seal. With this new configuration the 3 circumferential rows of diverging cavities cease to be bounded by constant pressures of the 2 inactive plenums as shown in the conventional design (Figure 9). The boundaries to the cavities within the seal are now bordered by other cavities which possess a dynamic pressure modulated by the rotor vibration. This implies that the converging cavities, which were previously inactive annular plenums with no dynamic pressure oscillations, will contribute to the force coefficients of the overall PDS, resulting in a fully active cavity seal. The fully partitioned design also has tapped holes in every cavity for measuring static and dynamic pressures. The detail of the tapped holes for the conventional and fully partitioned 6 bladed PDS are shown in Figure 11.

\subsection{Conventional Six Bladed PDS Blade Profiles}

The last seal configuration tested was the six bladed conventional PDS with modified blade profiles. To determine the effect of beveled geometry on leakage the conventional 6 bladed PDS was modified to have beveled chamfers machined on the downstream side of each of the blades. Figure 12 shows the modifications incorporated to the six bladed PDS.

Table 1 summarizes the geometry for all 7 test configurations. The cavity depth values for all the seals were optimized at $70 \mathrm{~Hz}$ for maximum damping, which is the typical location of the first natural frequency for industrial centrifugal compressors. The inactive pitch for the 12 and 8 bladed seals was designed to be 0.125 in $(3.175 \mathrm{~mm})$, but the inactive

pitch for the six bladed configurations was required to be 0.200 in $(5.08 \mathrm{~mm})$ to accommodate the pressure transducers. 


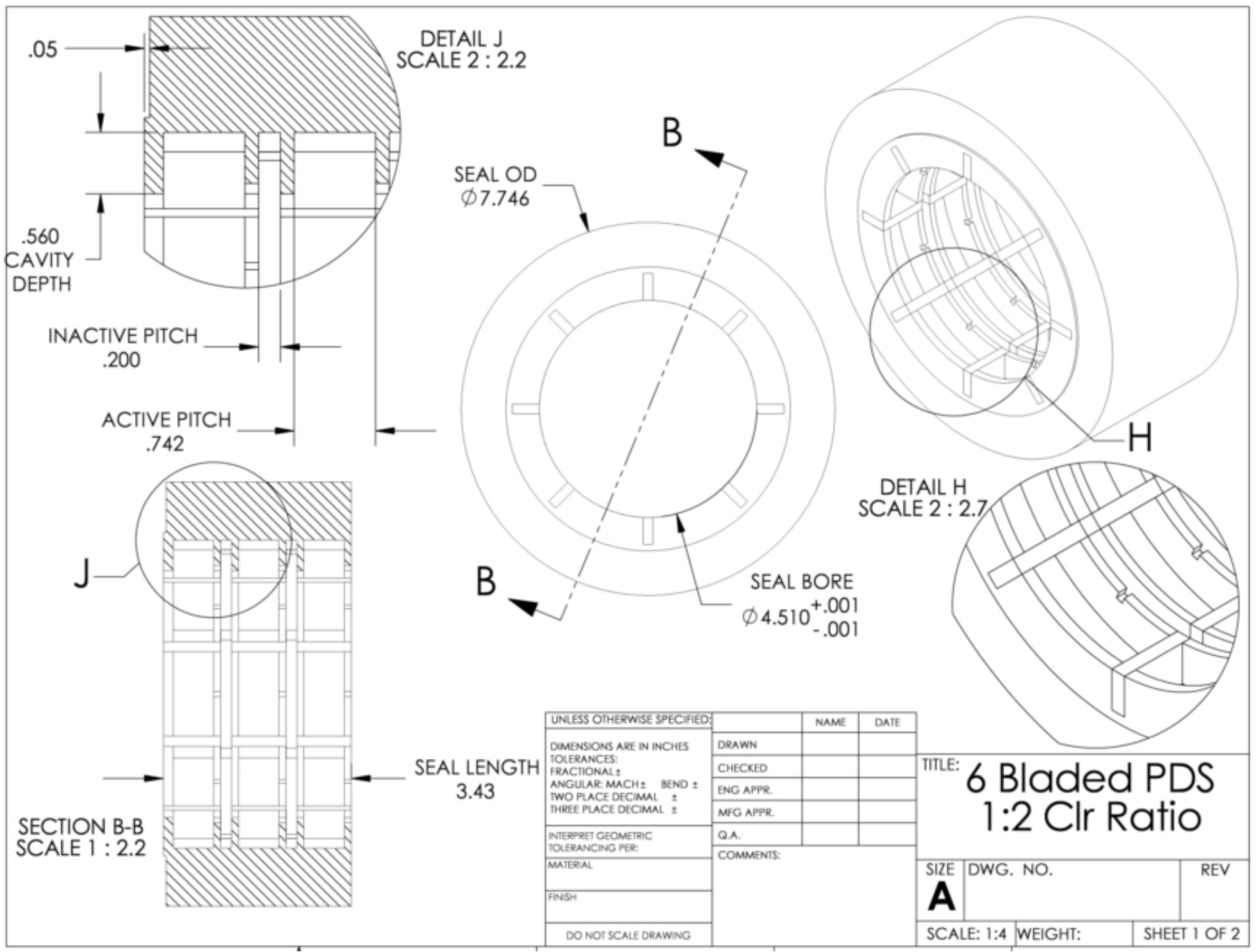

Figure 9. Conventional Six Bladed PDS Configuration 


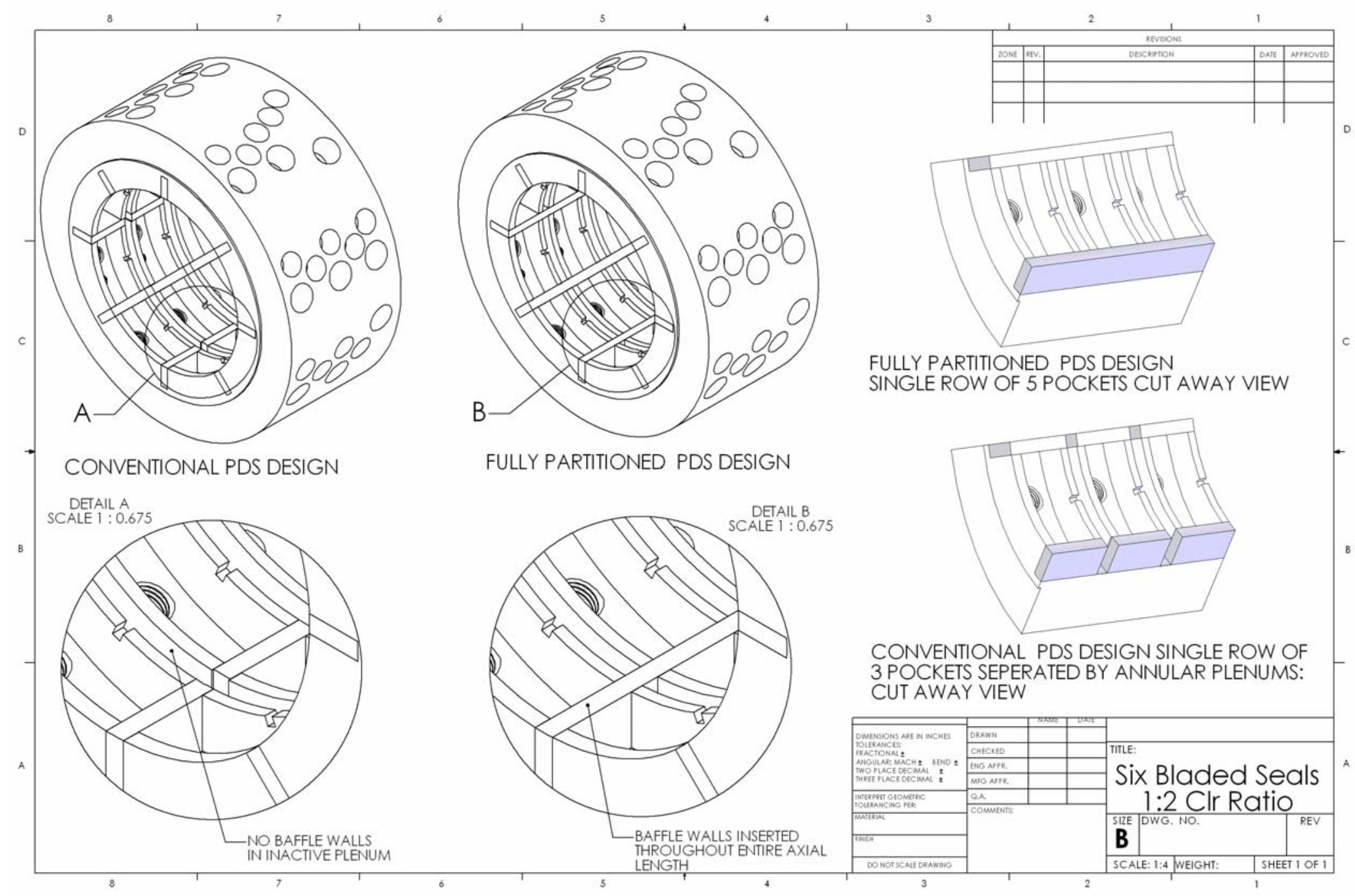

Figure 10. New Fully Partitioned PDS Design 


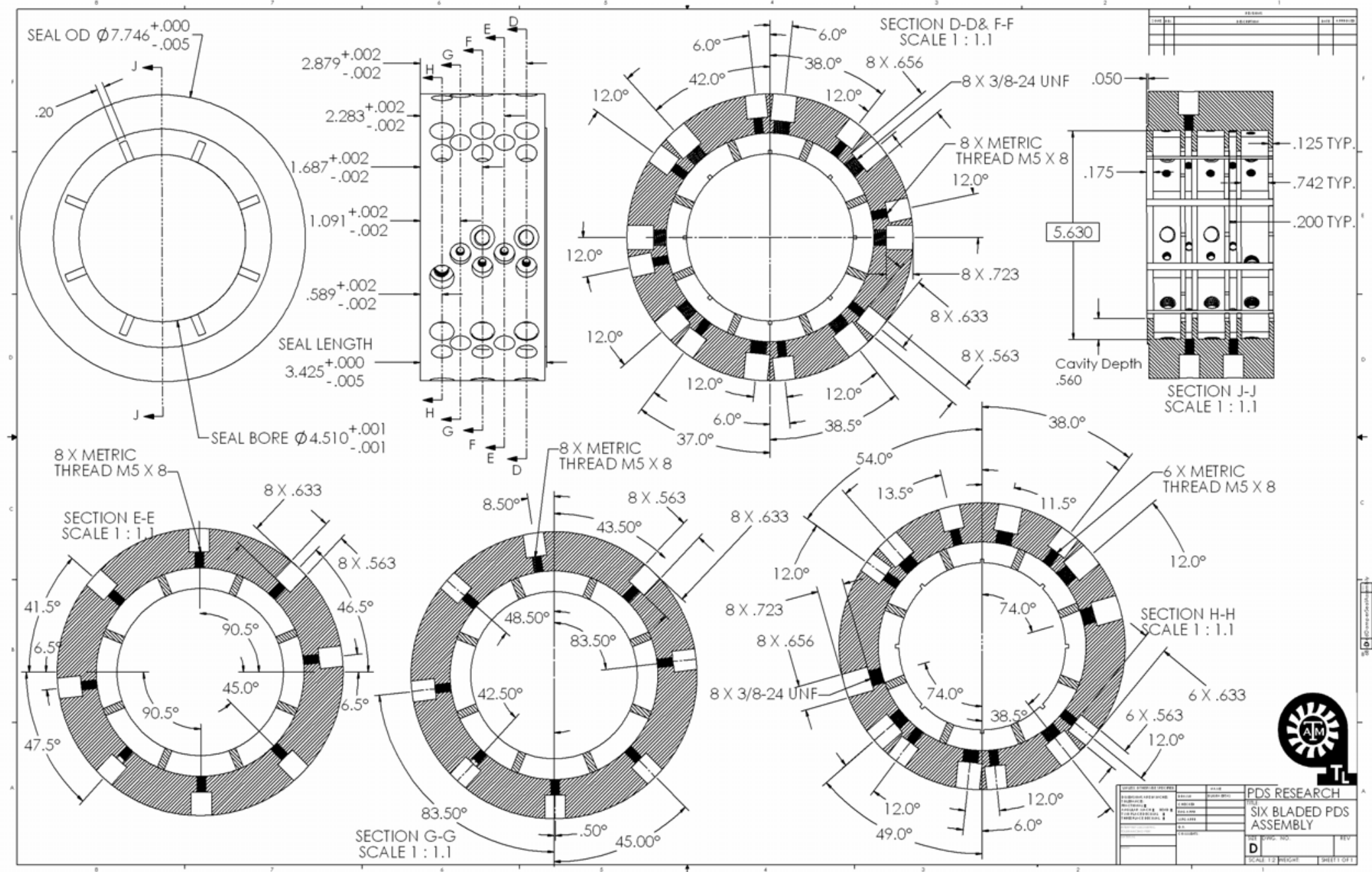

Figure 11. Pressure Transducer Detail for Six Bladed Seals 


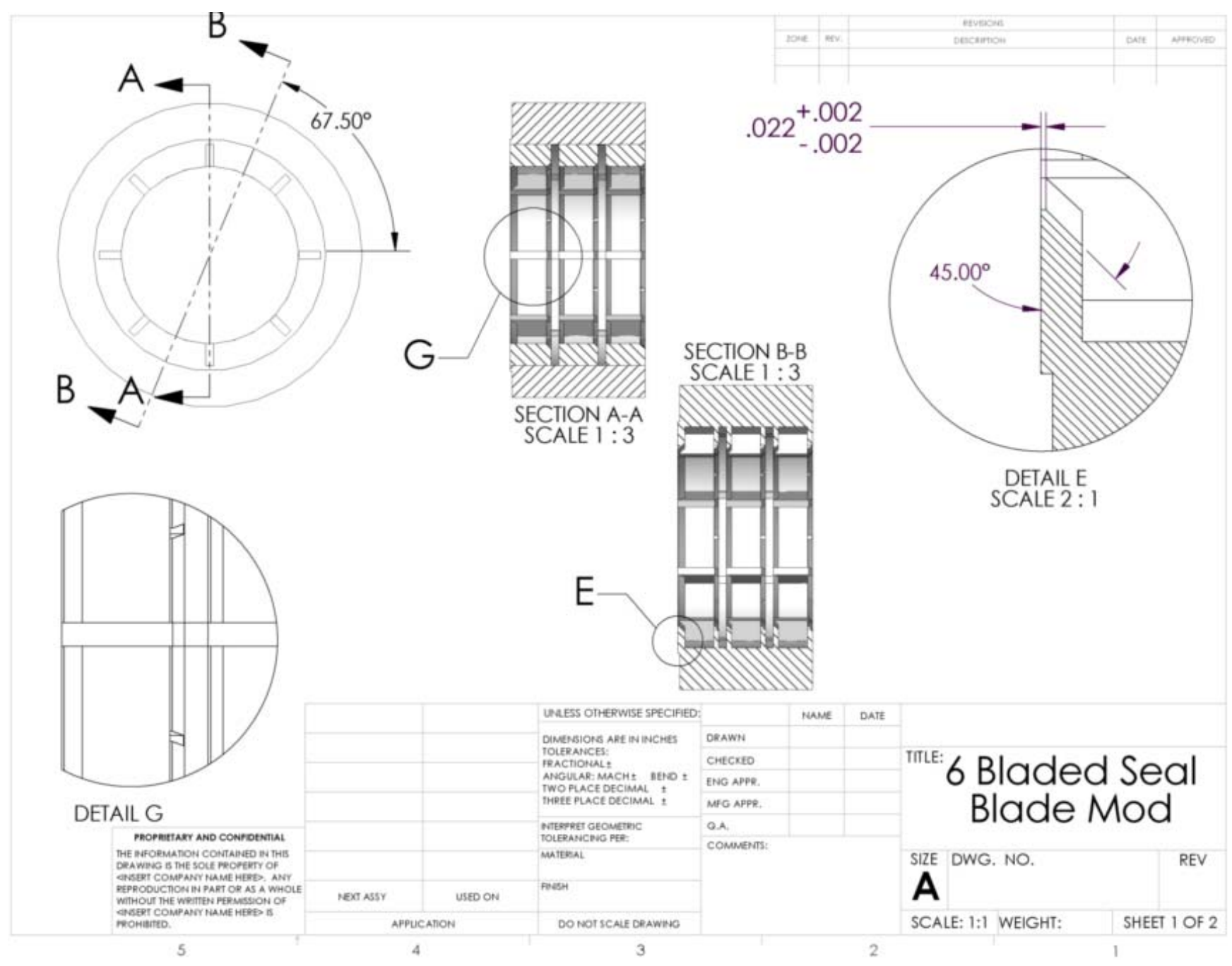

Figure 12. Conventional Six Bladed PDS Blade Modification 
Table 1. Summarized PDS Geometry and Parameters

\begin{tabular}{|c|c|c|c|c|c|c|c|c|c|c|}
\hline $\begin{array}{c}\text { SEAL } \\
\text { CONFIG }\end{array}$ & DESIGN & $\begin{array}{c}\text { NO. } \\
\text { BLADES }\end{array}$ & $\begin{array}{c}\text { CLEARANCE } \\
\text { RATIO }\end{array}$ & $\begin{array}{l}\text { CAVITY } \\
\text { DEPTH }\end{array}$ & $\begin{array}{l}\text { INACTIVE } \\
\text { PITCH }\end{array}$ & $\begin{array}{c}\text { ACTIVE } \\
\text { PITCH }\end{array}$ & $\begin{array}{c}\text { SEAL } \\
\text { LENGTH }\end{array}$ & $\begin{array}{c}\text { SEAL } \\
\text { OD }\end{array}$ & $\begin{array}{c}\text { SEAL } \\
\text { BORE DIA. }\end{array}$ & $\begin{array}{c}\text { BLADE } \\
\text { PROFILE }\end{array}$ \\
\hline 1 & $\begin{array}{c}\text { CONVENTIONAL } \\
\text { PDS }\end{array}$ & 12 & 1 TO 1 & $\begin{array}{c}1.400 \mathrm{in} \\
35.56 \mathrm{~mm}\end{array}$ & $\begin{array}{l}0.125 \mathrm{in} \\
3.175 \mathrm{~mm}\end{array}$ & $\begin{array}{c}0.208 \mathrm{in} \\
5.283 \mathrm{~mm}\end{array}$ & $\begin{array}{c}3.425 \text { in } \\
87.00 \mathrm{~mm}\end{array}$ & $\begin{array}{c}7.75 \mathrm{in} \\
196.85 \mathrm{~mm}\end{array}$ & $\begin{array}{c}4.510 \mathrm{in} \\
114.55 \mathrm{~mm}\end{array}$ & $\begin{array}{c}\text { DOUBLE } \\
\text { CHAMFER }\end{array}$ \\
\hline 2 & $\begin{array}{c}\text { CONVENTIONAL } \\
\text { PDS }\end{array}$ & 12 & 1 TO 2 & $\begin{array}{c}1.400 \mathrm{in} \\
35.56 \mathrm{~mm}\end{array}$ & $\begin{array}{c}0.125 \mathrm{in} \\
3.175 \mathrm{~mm}\end{array}$ & $\begin{array}{c}0.208 \mathrm{in} \\
5.283 \mathrm{~mm}\end{array}$ & $\begin{array}{c}3.425 \mathrm{in} \\
87.00 \mathrm{~mm}\end{array}$ & $\begin{array}{c}7.75 \mathrm{in} \\
196.85 \mathrm{~mm}\end{array}$ & $\begin{array}{c}4.510 \mathrm{in} \\
114.55 \mathrm{~mm}\end{array}$ & $\begin{array}{c}\text { DOUBLE } \\
\text { CHAMFER }\end{array}$ \\
\hline 3 & $\begin{array}{c}\text { CONVENTIONAL } \\
\text { PDS }\end{array}$ & 8 & 1 TO 1 & $\begin{array}{c}1.00 \mathrm{in} \\
25.4 \mathrm{~mm}\end{array}$ & $\begin{array}{c}0.125 \mathrm{in} \\
3.175 \mathrm{~mm}\end{array}$ & $\begin{array}{c}0.50 \mathrm{in} \\
12.7 \mathrm{~mm}\end{array}$ & $\begin{array}{c}3.425 \text { in } \\
87.00 \mathrm{~mm}\end{array}$ & $\begin{array}{c}7.75 \mathrm{in} \\
196.85 \mathrm{~mm}\end{array}$ & $\begin{array}{c}4.510 \mathrm{in} \\
114.55 \mathrm{~mm}\end{array}$ & RECTANGULAR \\
\hline 4 & $\begin{array}{c}\text { CONVENTIONAL } \\
\text { PDS }\end{array}$ & 8 & 1 TO 1.5 & $\begin{array}{c}1.00 \mathrm{in} \\
25.4 \mathrm{~mm}\end{array}$ & $\begin{array}{c}0.125 \text { in } \\
3.175 \mathrm{~mm}\end{array}$ & $\begin{array}{c}0.50 \mathrm{in} \\
12.7 \mathrm{~mm}\end{array}$ & $\begin{array}{c}3.425 \text { in } \\
87.00 \mathrm{~mm}\end{array}$ & $\begin{array}{c}7.75 \mathrm{in} \\
196.85 \mathrm{~mm}\end{array}$ & $\begin{array}{c}4.510 \mathrm{in} \\
114.55 \mathrm{~mm}\end{array}$ & RECTANGULAR \\
\hline 5 & $\begin{array}{c}\text { CONVENTIONAL } \\
\text { PDS }\end{array}$ & 6 & 1 TO 2 & $\begin{array}{c}0.56 \mathrm{in} \\
14.224 \mathrm{~mm}\end{array}$ & $\begin{array}{c}0.200 \mathrm{in} \\
5.08 \mathrm{~mm}\end{array}$ & $\begin{array}{c}0.742 \mathrm{in} \\
18.847 \mathrm{~mm}\end{array}$ & $\begin{array}{c}3.425 \text { in } \\
87.00 \mathrm{~mm}\end{array}$ & $\begin{array}{c}7.75 \mathrm{in} \\
196.85 \mathrm{~mm}\end{array}$ & $\begin{array}{c}4.510 \mathrm{in} \\
114.55 \mathrm{~mm}\end{array}$ & RECTANGULAR \\
\hline 6 & $\begin{array}{c}\text { CONVENTIONAL } \\
\text { PDS }\end{array}$ & 6 & 1 TO 2 & $\begin{array}{c}0.56 \text { in } \\
14.224 \mathrm{~mm}\end{array}$ & $\begin{array}{l}0.200 \mathrm{in} \\
5.08 \mathrm{~mm}\end{array}$ & $\begin{array}{c}0.742 \text { in } \\
18.847 \mathrm{~mm}\end{array}$ & $\begin{array}{c}3.425 \text { in } \\
87.00 \mathrm{~mm}\end{array}$ & $\begin{array}{c}7.75 \mathrm{in} \\
196.85 \mathrm{~mm}\end{array}$ & $\begin{array}{c}4.510 \mathrm{in} \\
114.55 \mathrm{~mm}\end{array}$ & $\begin{array}{c}\text { BEVELED ON } \\
\text { DOWNSTREAM } \\
\text { SIDE }\end{array}$ \\
\hline 7 & $\begin{array}{c}\text { FULLY } \\
\text { PARTITIONED } \\
\text { PDS }\end{array}$ & 6 & 1 TO 2 & $\begin{array}{c}0.56 \text { in } \\
14.224 \mathrm{~mm}\end{array}$ & $\begin{array}{l}0.200 \mathrm{in} \\
5.08 \mathrm{~mm}\end{array}$ & $\begin{array}{c}0.742 \text { in } \\
18.847 \mathrm{~mm}\end{array}$ & $\begin{array}{c}3.425 \text { in } \\
87.00 \mathrm{~mm}\end{array}$ & $\begin{array}{c}7.75 \mathrm{in} \\
196.85 \mathrm{~mm}\end{array}$ & $\begin{array}{c}4.510 \mathrm{in} \\
114.55 \mathrm{~mm}\end{array}$ & RECTANGULAR \\
\hline
\end{tabular}




\section{TEST RIG AND EXPERIMENTAL METHODS}

The following section discusses the test rig, the experimental methods used to determine frequency dependent force coefficients and the testing procedures.

\subsection{Experimental Test Rig}

The main requirements for the testing were: (1) the necessity of the test facility to support experiments at 1,000 psig (68.95 bar), (2) the ability to dynamically excite the system at variable frequencies up to $300 \mathrm{~Hz}$ in two orthogonal directions, and (3) different rotor surface speeds needed to be employed. A picture of the high pressure testing facility used for the PDS experiments is shown in Figure 13. Item 1 is the variable frequency drive which interfaces with the test rotor through a Lufkin Gear box. Also shown in Figure 13 are the two bearing supports, the $\mathrm{Y}$ direction hydraulic shaker, the support bracket to the shaker, and the stator housing assembly where the test seals are located. The high pressure test rig was originally developed (Childs, D. and Hale, K., 1994) to test hydrostatic bearings, but was later modified (Dawson, M., 2000) to support testing of annular gas seals. A detailed cross section of the high pressure test rig is shown in Figure 14 (Dawson, 2000). The stator housing is a floating (suspended around rotor) assembly that interfaces the test rig at the bearing supports by the use of six pitch stabilizers (three on each end). To limit the axial thrust in the test rig the test seals are mounted in a back-to-back configuration where the inlet air is supplied at the center plenum (between the test seals) of the stator assembly.

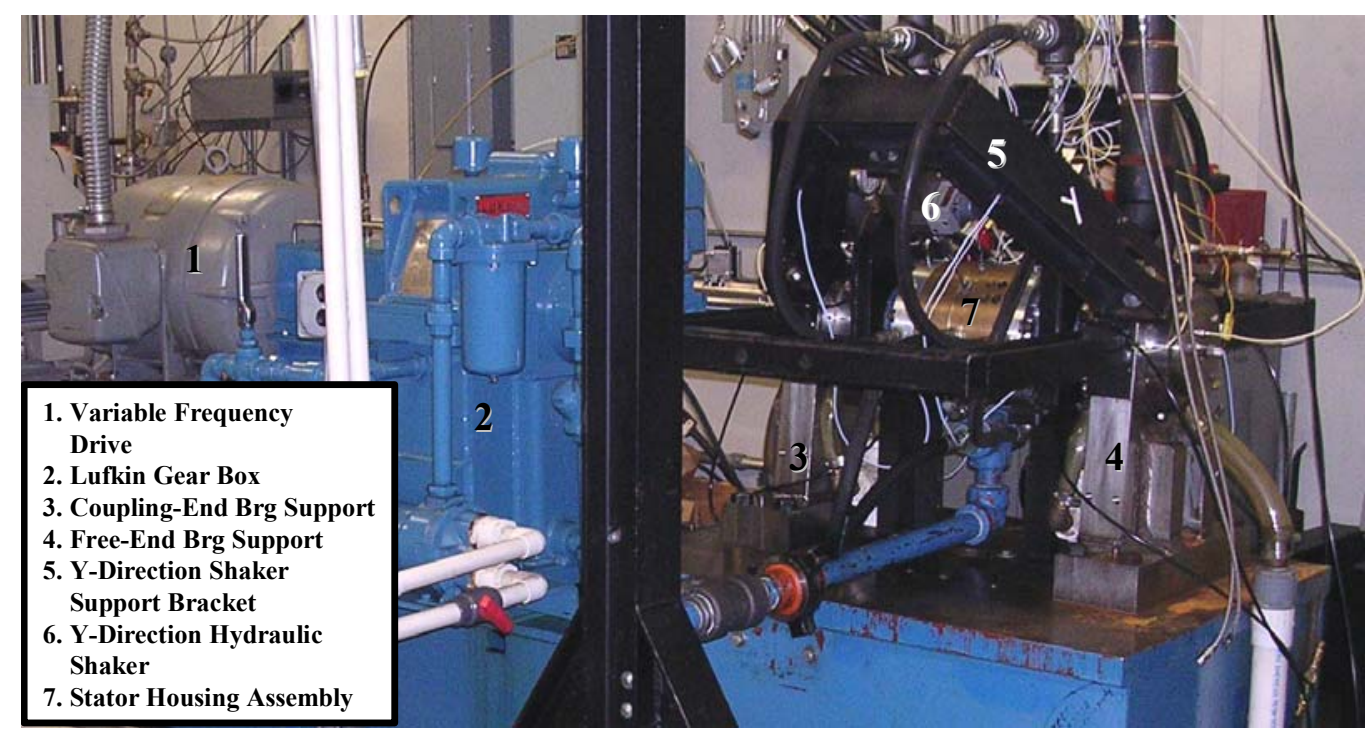

Figure 13. High Pressure Annular Gas Seal Test Rig 


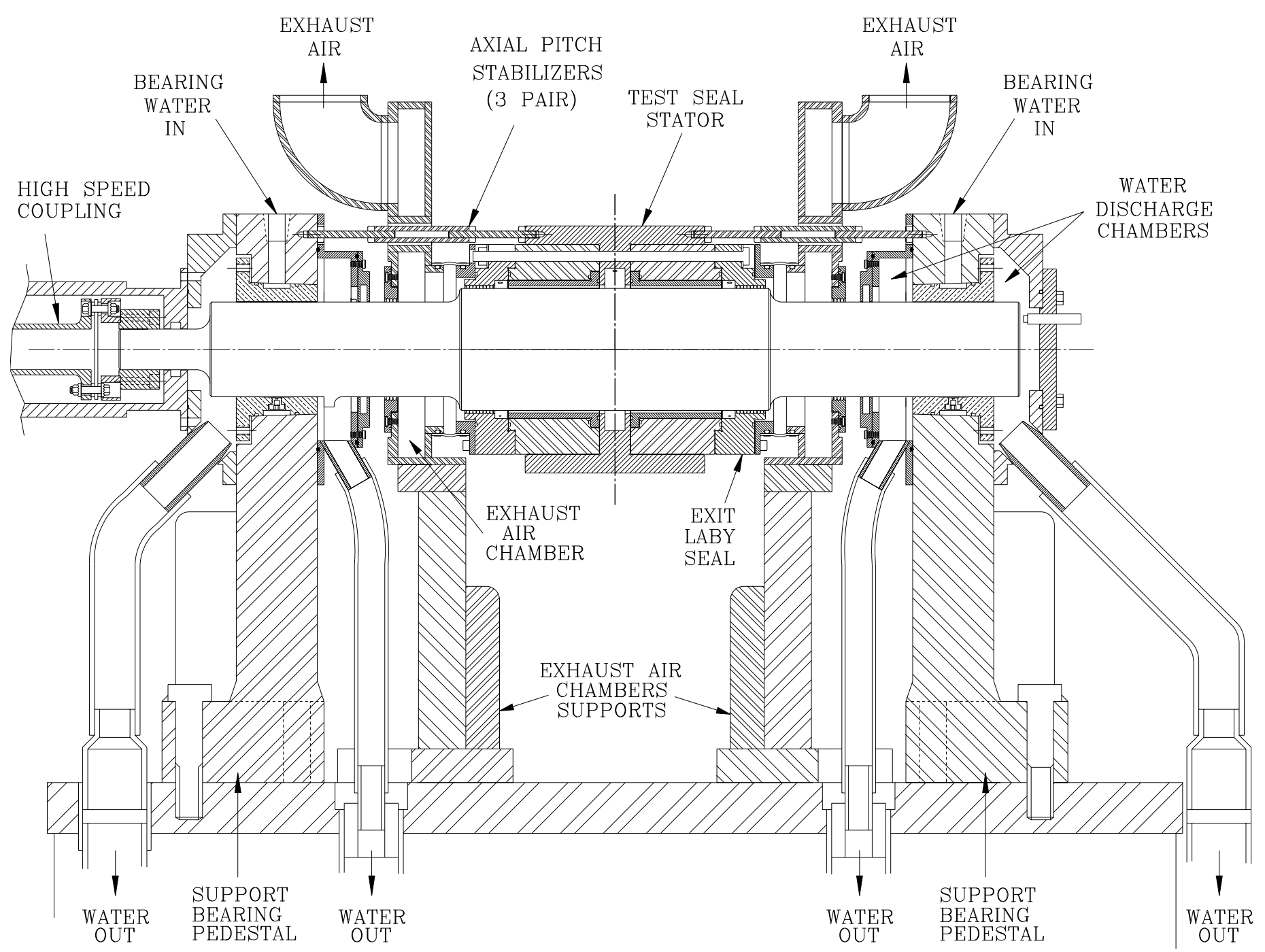

Figure 14. High Pressure Test Rig Cross Section (Dawson, 2000) 
The rotor is supported by two hydrostatic water bearings and it has a first natural frequency above the test frequency range of 20-300 Hz.

Several static and dynamic measurements were required during experiments. Figure 15 shows an isometric view of the stator assembly. The static measurements included static inlet pressure taken at the center inlet plenum of the stator housing, temperature at the inlet plenum, static back pressures at the exit of the test seals, and finally the exit gas temperature at the test seal exit. The back pressure to the test seals was controlled by a valve that varies the flow through the three ports on each labyrinth seal. Each labyrinth seal possessed a radial clearance of 0.004 inches $(0.1016 \mathrm{~mm})$ from the rotor surface and was required to hold the back pressure during testing. An extra addition made to the stator assembly was the use of two radial squirrel cages that were bolted to the axial face of the labyrinth seals and interfaced the test rig at the flange of the exhaust chamber housing. Diverging clearance seals exhibit direct negative stiffness and if the negative stiffness surpasses the magnitude of the baseline direct stiffness the stator assembly becomes statically unstable making it impossible to perform dynamic testing. This phenomenon was observed for the diverging eight bladed seal, and the six bladed seal, therefore two squirrel cage radial stiffeners were designed to increase the baseline radial stiffness and allow for static stability while testing at 1,000 psig inlet pressure.

To determine rotordynamic force coefficients, for two different testing methods, several dynamic measurements were taken. Figure 16 shows the standard views of the stator housing assembly and Figure 17 represents the A-A cross sectional front view revealing all the dynamic measurements taken during the experiments. The first method of testing performed in the experiments was the system impedance method, which required the measurement of the external system dynamic forces, stator displacement motion, and stator acceleration. Four proximity probes constrained at the labyrinth seal, two in line with the X direction and two in line with the $\mathrm{Y}$ direction, were used to measure the relative motion between the stator housing and the rotor.

The second experimental method implemented was the dynamic pressure response method for determining frequency dependent force coefficients. This method required the measurement of PDS cavity dynamic pressures in combination with relative vibratory displacement between the stator housing assembly and the test rig rotor. The static cavity pressure was also measured for optimizing discharge coefficients. 


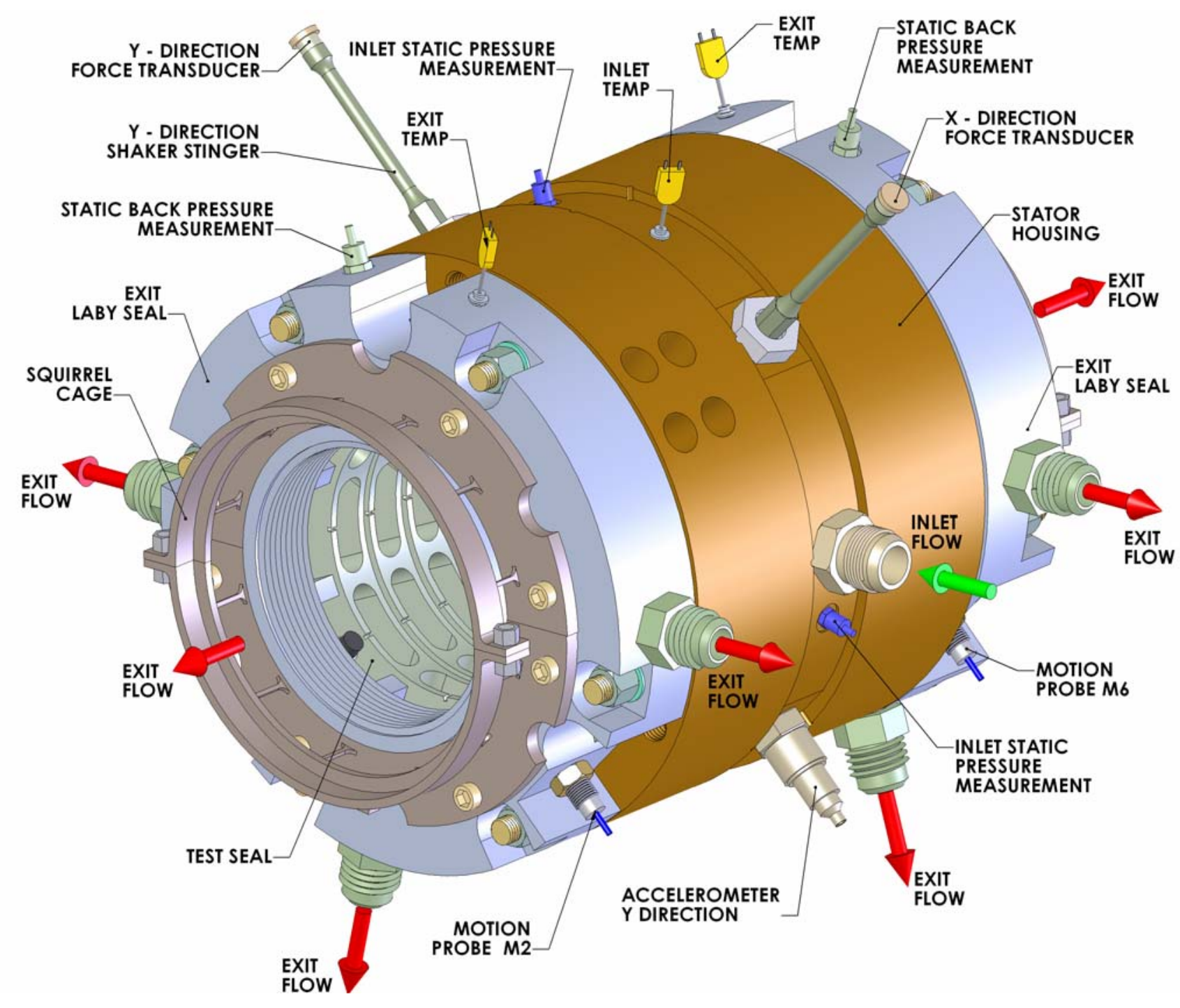

Figure 15. Stator Assembly Isometric View 

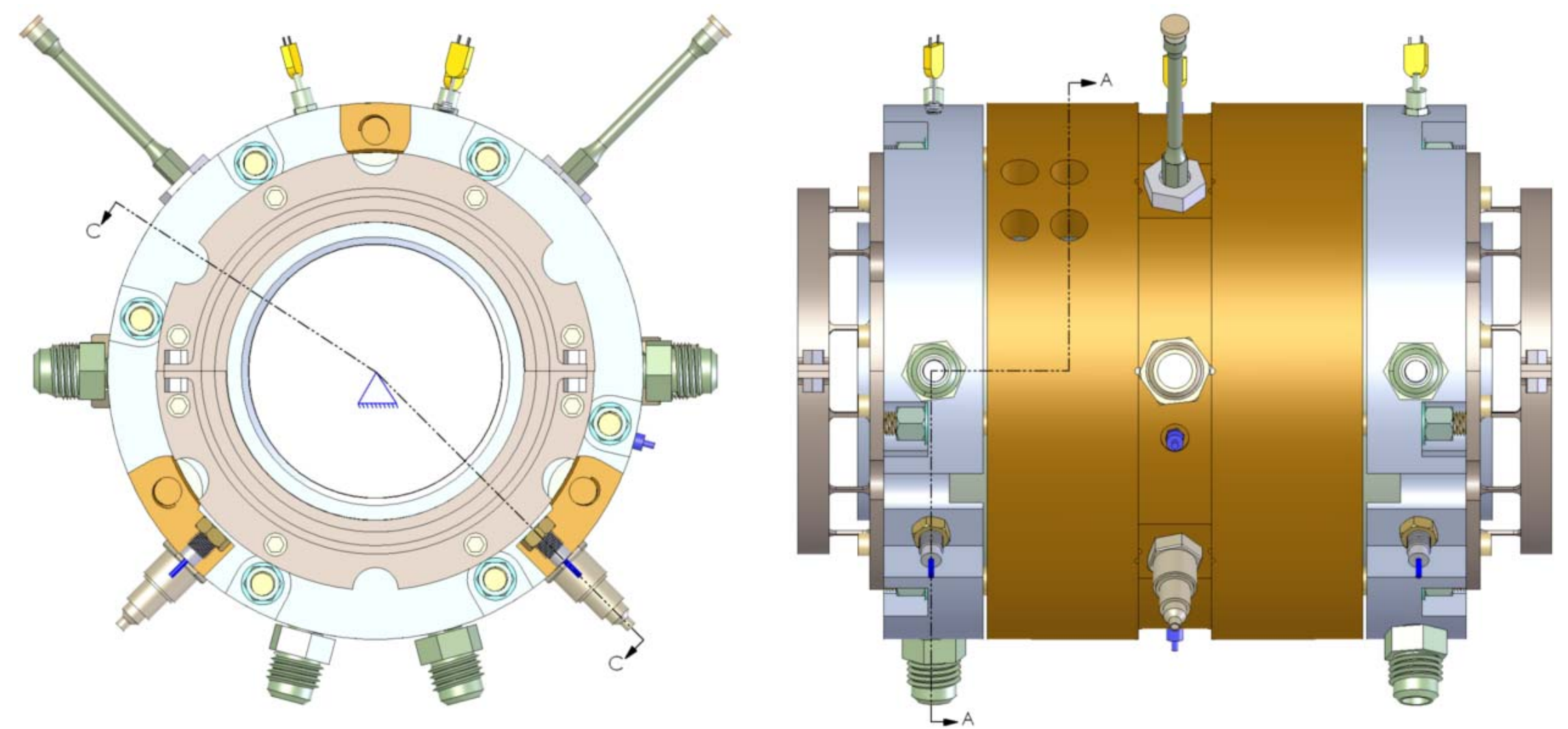

Figure 16. Stator Assembly Standard Views 


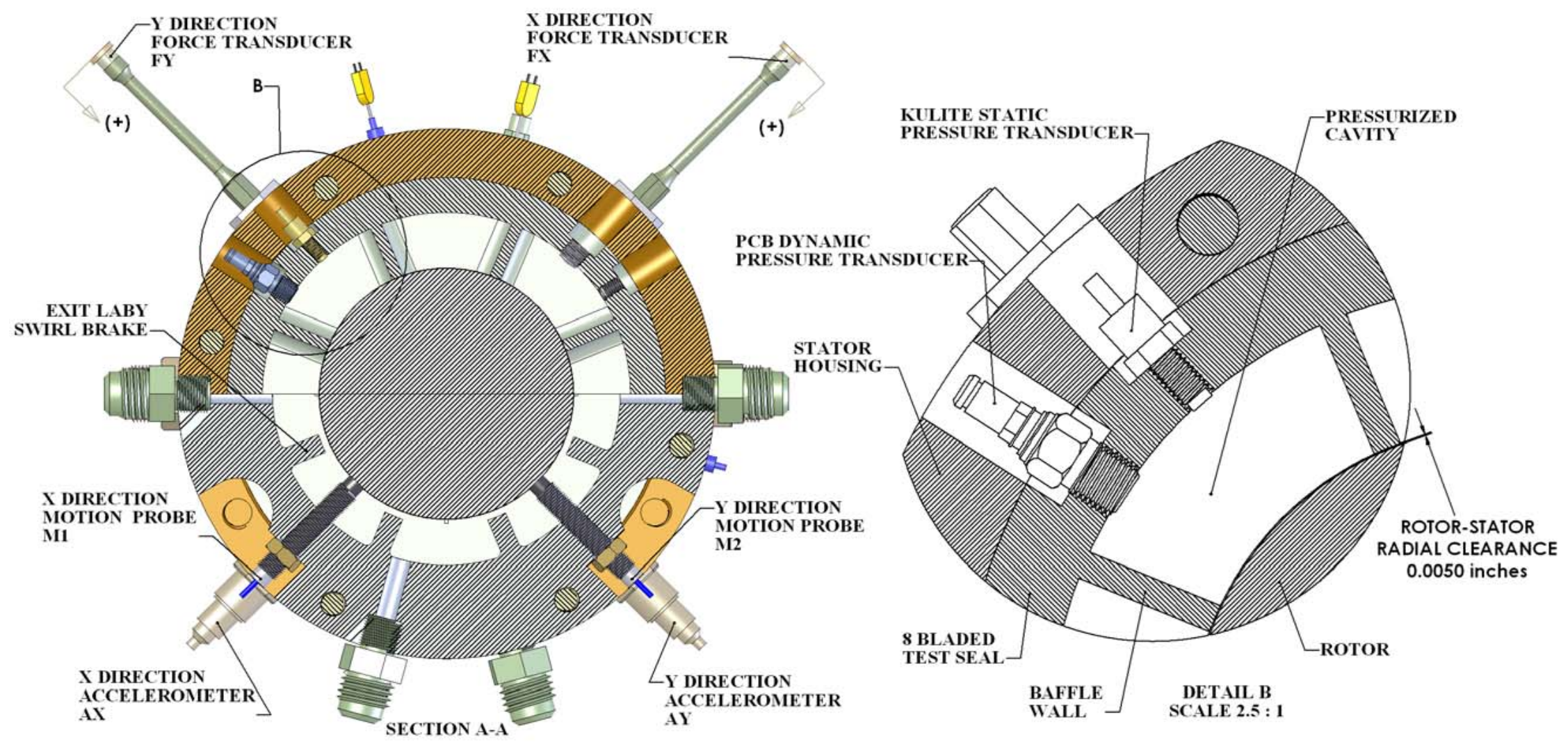

Figure 17. Dynamic Measurements 


\subsection{Impedance Method}

The first method used to determine frequency dependent force coefficients was the system impedance method (Childs, D. and Hale, K., 1994). It is labeled as an impedance method because the method measures the total system impedance (actually dynamic stiffness), which is comprised of the pitch stabilizers, exit labyrinth seals, squirrel cage radial stiffeners, and the test seals. The first step in the derivation was to develop the equations of motion for the dynamic system shown in Figure 17. Note that the complex equations of motion are derived in the frequency domain, and that each equation possesses a real component and an imaginary component. Equations 3.1 and 3.2 show the X direction force equations, where Equation 3.1 represents the direct equation of motion and Equation 3.2 represents the cross-coupled equation of motion. Equations 3.3 and 3.4 show the $\mathrm{Y}$ direction equations of motion. The left hand side of Equations 3.1-3.4 is the effective force components (Equations 3.5-3.8), which are defined as the dynamic shaker forces minus the stator inertia forces from the system, where $M$ is the stator assembly mass and $A_{i j}$ is the measured system acceleration from the accelerometers.

$X$ Direction Equations of Motion:

$$
\begin{aligned}
& F_{x x}-M A_{x x}=\left(K_{x x}-m_{x x} \omega^{2}+j \omega C_{x x}\right) \cdot D_{x x}+\left(K_{x y}-m_{x y} \omega^{2}+j \omega C_{x y}\right) \cdot D_{x y} \\
& F_{y x}-M A_{y x}=\left(K_{x x}-m_{x x} \omega^{2}+j \omega C_{x x}\right) \cdot D_{y x}+\left(K_{x y}-m_{x y} \omega^{2}+j \omega C_{x y}\right) \cdot D_{y y}
\end{aligned}
$$

Y Direction Equations of Motion:

$$
\begin{aligned}
& F_{y y}-M A_{y y}=\left(K_{y y}-m_{y y} \omega^{2}+j \omega C_{y y}\right) \cdot D_{y y}+\left(K_{y x}-m_{y x} \omega^{2}+j \omega C_{y x}\right) \cdot D_{y x} \\
& F_{x y}-M A_{x y}=\left(K_{y y}-m_{y y} \omega^{2}+j \omega C_{y y}\right) \cdot D_{x y}+\left(K_{y x}-m_{y x} \omega^{2}+j \omega C_{y x}\right) \cdot D_{x x}
\end{aligned}
$$

Effective Forces in X Direction:

$$
\begin{aligned}
& f_{x x}=F_{x x}-M A_{x x} \\
& f_{y x}=F_{y x}-M A_{y x}
\end{aligned}
$$

Effective Forces in Y Direction:

$$
f_{y y}=F_{y y}-M A_{y y}
$$

$$
f_{x y}=F_{x y}-M A_{x y}
$$

Also note that each force equation has a contribution of direct coefficients and crosscoupled coefficients. Since we are working in the complex domain each equation has a real part (determines stiffness) and an imaginary part (determines damping). 
The next step is to define direct and cross-coupled impedances (Equations 3.9-3.12). Substituting Equations 3.5-3.12 into Equations 3.1-3.4 yields the final equation of motion in matrix form (Equation 3.13). The force matrix of Equation 3.13 and the displacement matrix are terms that are measured during testing. Once these measurements are made one can solve for the impedances (Equation 3.14-3.17), which are composed of the stiffness and damping terms.

Direct and Cross-Coupled Impedance For X Direction:

$H_{x x}=K_{x x}+j \omega C_{x x}$

$H_{x y}=K_{x y}+j \omega C_{x y}$

Direct and Cross-Coupled Impedance For Y Direction:

$$
\begin{aligned}
& H_{y y}=K_{y y}+j \omega C_{y y} \\
& H_{y x}=K_{y x}+j \omega C_{y x}
\end{aligned}
$$

Final Equation of Motion In Matrix Form:

$\left[\begin{array}{ll}f_{x x} & f_{y x} \\ f_{x y} & f_{y y}\end{array}\right]=\left[\begin{array}{ll}H_{x x} & H_{x y} \\ H_{y x} & H_{y y}\end{array}\right] \cdot\left[\begin{array}{ll}D_{x x} & D_{y x} \\ D_{x y} & D_{y y}\end{array}\right]$

Direct Impedance in X Direction:

$$
H_{x x}=\frac{f_{x x} \cdot D_{y y}-f_{y x} \cdot D_{x y}}{D_{x x} \cdot D_{y y}-D_{y x} \cdot D_{x y}}
$$

Cross-Coupled Impedance in X Direction:

$$
H_{x y}=\frac{f_{x x} \cdot D_{y x}-f_{y x} \cdot D_{x x}}{D_{x y} \cdot D_{y x}-D_{y y} \cdot D_{x x}}
$$

Direct Impedance in Y Direction:

$$
H_{y y}=\frac{f_{y y} \cdot D_{x x}-f_{x y} \cdot D_{y x}}{D_{y y} \cdot D_{x x}-D_{y x} \cdot D_{x y}}
$$

Cross-Coupled Impedance in Y Direction:

$$
H_{y x}=\frac{f_{y y} \cdot D_{x y}-f_{x y} \cdot D_{y y}}{D_{x y} \cdot D_{y x}-D_{y y} \cdot D_{x x}}
$$

The force excitation used in the tests is a pseudo-random complex frequency waveform tailored to contain several frequencies in the signal that acts as the reference input signal from which all other signals are phased from. A waveform was constructed for each 
of the three sets of PDS by performing single frequency amplitude tests for $\mathrm{X}$ and $\mathrm{Y}$ directions to determine the amplitude ratio between the different frequency components. The amplitude-ratio plots for building the excitation waveforms are listed in the Appendix. Unlike single frequency excitation tests, which require a separate test for each frequency, performing a test using a multi-tone excitation yields results for multiple frequencies, therefore requiring only one shake test. Figure 18 illustrates the excitation waveform for the six-bladed PDS. Each test contained thirty-two 0.1 second long excitations resulting in 3.2 seconds of data collection in each direction. The excitations for the 12 and eight bladed PDS tests are shown in the Appendix.

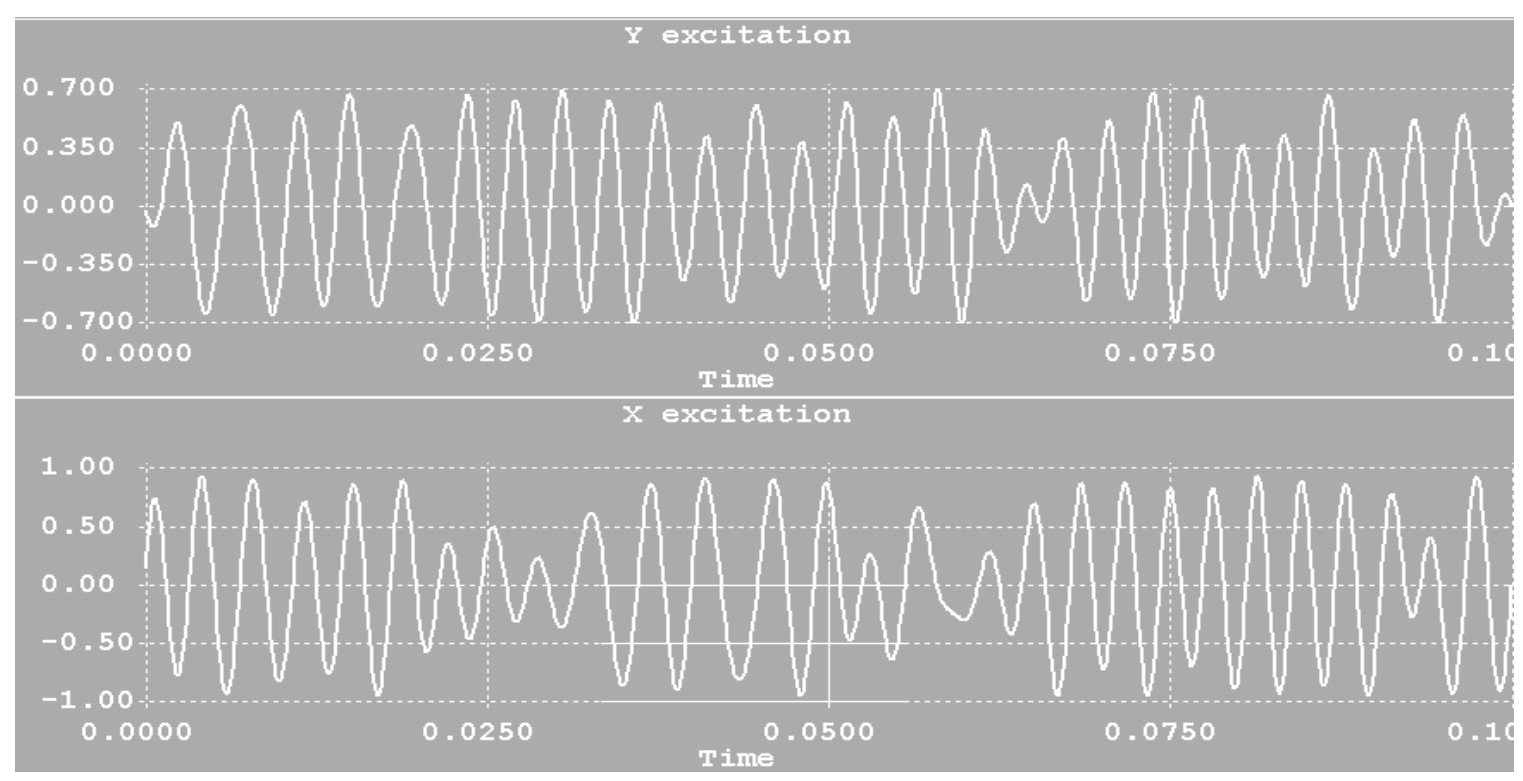

Figure 18. Pseudorandom Force Excitation: 6 Bladed PDS Configurations

Figure 19 shows the dynamic measurements recorded for a $\mathrm{Y}$ direction excitation of the 6 bladed PDS stator assembly. Measurements shown in this figure include forces in the $\mathrm{X}$ and $\mathrm{Y}$ directions ( $\mathrm{FX}$ and $\mathrm{FY}$ ), stator accelerations ( $\mathrm{AX}$ and $\mathrm{AY}$ ), and stator motion relative to the rotor surface (M1, M5, M2, and M6). The black time trace represents the Y direction shaker force input, and the red trace shows the vibration response of the stator assembly in the $\mathrm{Y}$ direction. Also shown in Figure 19 is the stator acceleration measurement used to subtract the inertial forces. To determine the four unknown impedance functions one must generate four independent equations. These equations are generated from two independent excitations. First, the stator assembly is excited in the $\mathrm{X}$ direction, which yields 
two equations, a direct equation of motion and a cross-coupled equation of motion. Then the system is excited in the $\mathrm{Y}$ direction, which also yields a direct equation of motion and a cross-coupled equation of motion. The direct and cross-coupled impedance values for the $\mathrm{X}$ and $\mathrm{Y}$ directions are calculated at each test frequency from the four independent equations.

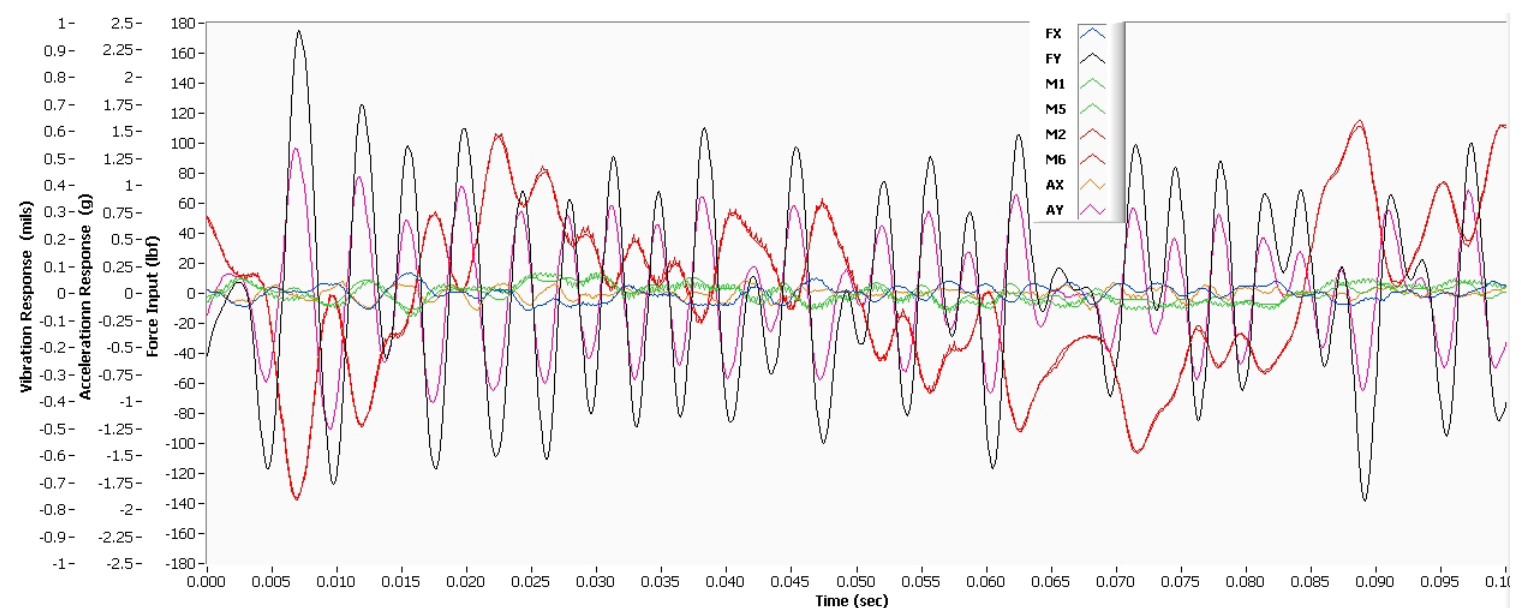

Figure 19. Experimental Measurements: Impedance Method

Before determining the test seal coefficients, one must realize that the impedance measured in the procedure described above is the system impedance, which is composed of contributions from the entire mechanical system such as the pitch stabilizers, exit labyrinth seals, and the radial squirrel cage stiffeners. Extracting the PDS force coefficients will require a separate assembly of the rig and a baseline test using baseline or blank inserts in place of the test seals. The objective of the baseline test is to measure the contribution of all other components in the system other than the test seals. This is achieved by replacing the test seals by smooth baseline seals (Figure 20) with large clearances from the rotor resulting in negligible effects from the inserts. During the baseline test it was observed that the pressure drop at the baseline inserts was $\sim 6$ psig (0.41 bar) with an inlet pressure of $\sim 700$ psi (48.26 bar). After measuring the system impedance with the baseline inserts $\left[H_{i j}\right]_{B L}$ it is subtracted from the system impedance with the test seals $\left[H_{i j}\right]_{T S}$ yielding the resultant test seal impedance $\left[H_{i j}\right]_{R}$ (Equation 18). 
$\left[\begin{array}{ll}H_{x x} & H_{x y} \\ H_{y x} & H_{y y}\end{array}\right]_{R}=\left[\begin{array}{ll}H_{x x} & H_{x y} \\ H_{y x} & H_{y y}\end{array}\right]_{T S}-\left[\begin{array}{ll}H_{x x} & H_{x y} \\ H_{y x} & H_{y y}\end{array}\right]_{B L}$
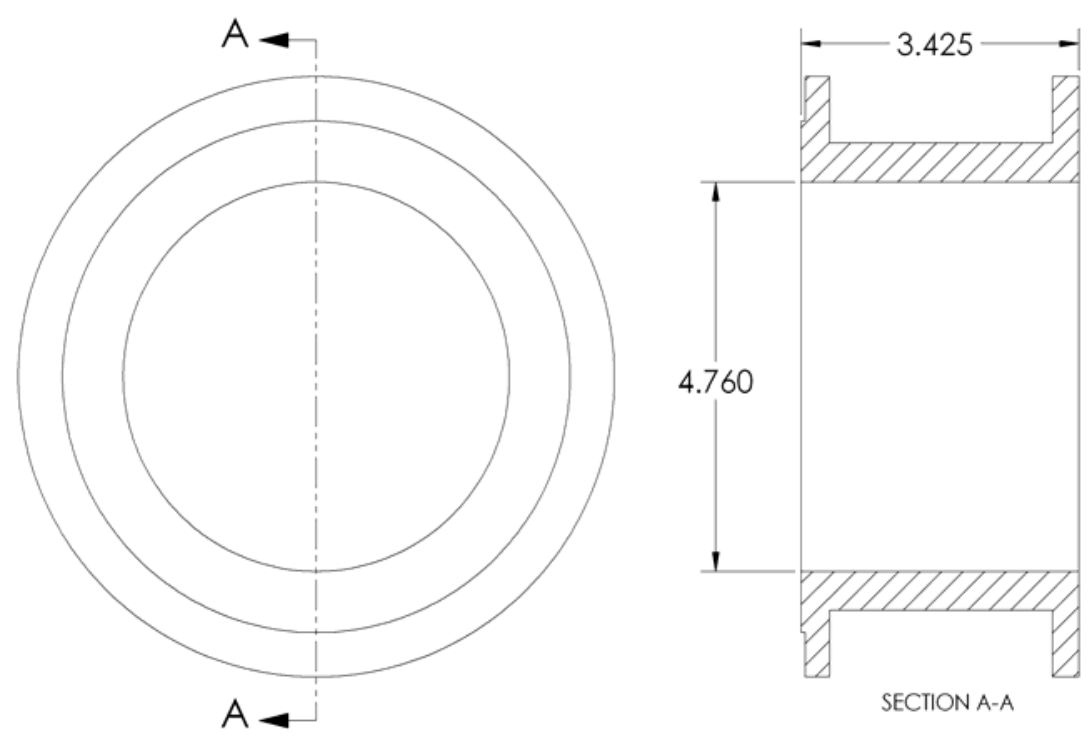

Figure 20. Baseline Insert 


\subsection{Rotordynamic Force Coefficients}

Figure 21 shows a flexible symmetric rotor with a centered disk that illustrates the different type of linearized rotordynamic force coefficients acting on the rotor bearing system from a PDS. Point $\mathrm{O}$ is located on the bearing center line, point $\mathrm{P}$ is the center of the rotor disk, and $\mathrm{M}$ is the location of the mass imbalance. Let us assume that the rotor spin $\omega$ and the whirl frequency $\dot{\phi}$ are the same resulting in synchronous circular rotor whirl. The dynamic response of point $\mathrm{P}$ from the bearing centerline point $\mathrm{O}$, which is defined by the $\mathrm{Y}_{0}$ and $\mathrm{X}_{0}$ coordinates, is dictated by various force coefficients depending on the proximity of $\omega$ relative to the rotor- bearing system natural frequency $\left(\omega_{\mathrm{n}}\right)$. Part $\mathrm{C}$ of Figure 21 shows two unit directions $\vec{r}$ and $\vec{t}$, where $\vec{r}$ is the displacement vector and $\vec{t}$ represents the velocity vector. At low frequencies $\left(\omega<<\omega_{\mathrm{n}}\right.$ and $\beta$ near 0 degrees) the rotor response $\mathrm{R}$ is dictated by the elastic stiffness force $K \cdot r$, where $K=K_{x x}=K_{y y}$. Direct stiffness values $\left(\mathrm{K}_{\mathrm{xx}}\right.$ and $\mathrm{K}_{\mathrm{YY}}$ ) can be directly determined by observing the real component of the direct impedance functions $H_{X X}$ and $H_{Y Y}$, because the system inertia is subtracted from the shaker force signal; and that PDS exhibit negligible added mass effects. The first subscript represents the direction of the excitation, where in this method the excitation is the forcing function. Therefore, $\mathrm{K}_{\mathrm{XX}}$ is the stiffness coefficient that relates an $\mathrm{X}$ direction force excitation to an $\mathrm{X}$ direction motion and $\mathrm{K}_{\mathrm{XY}}$ is the stiffness coefficient that relates an $\mathrm{X}$ direction force to a $\mathrm{Y}$ direction displacement. In mechanical systems, direct stiffness acts as a restoring force opposing the rotor displacement, but for diverging clearance PDS configurations the seal exhibits a negative stiffness force that acts in the direction of rotor displacement. Also in line with the direct stiffness is the cross-coupled damping term $\mathrm{C}_{\mathrm{C}}$, but cross-coupled damping for PDS is low in magnitude and has little contribution to the rotordynamics. Nevertheless $\mathrm{C}_{\mathrm{C}}$ can be extracted from the imaginary part of the cross-coupled impedance functions $H_{X Y}$ and $H_{Y X}$. When $\omega$ approaches the natural frequency $\omega_{\mathrm{n}}$, the imbalance becomes in phase with the velocity vector $(\beta=90 \mathrm{deg})$ and the rotor response $\mathrm{R}$ is now dictated by the direct damping $\mathrm{C}$ and the cross-coupled or cross-axis stiffness $\mathrm{K}_{\mathrm{D}}$, where $C=C_{X X}=C_{Y Y}$, and the subscript $\mathrm{D}$ stands for destabilizing. The direct damping $\left(\mathrm{C}_{\mathrm{XX}}\right.$ and $\left.\mathrm{C}_{\mathrm{YY}}\right)$ is calculated from the imaginary component of the direct impedance functions by dividing by the excitation frequency. 


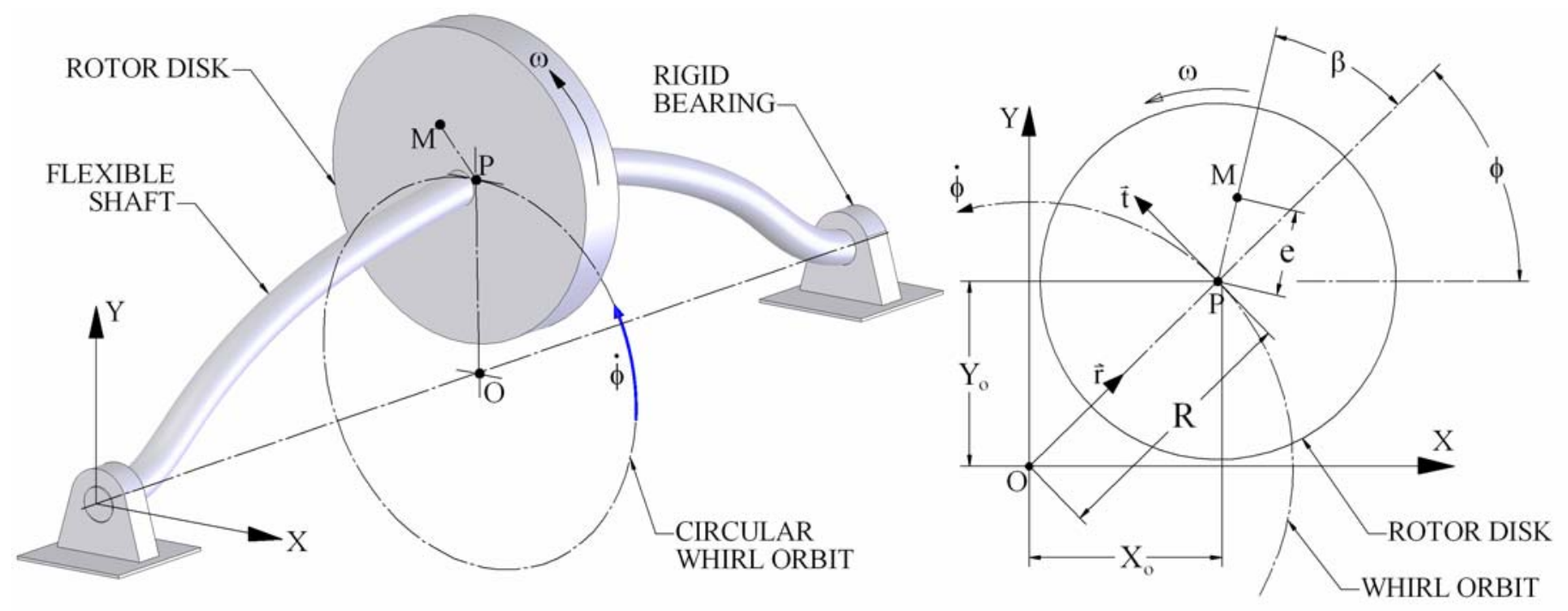

(a) FLEXIBLE ROTOR

(b) ROTOR GEOMETRY
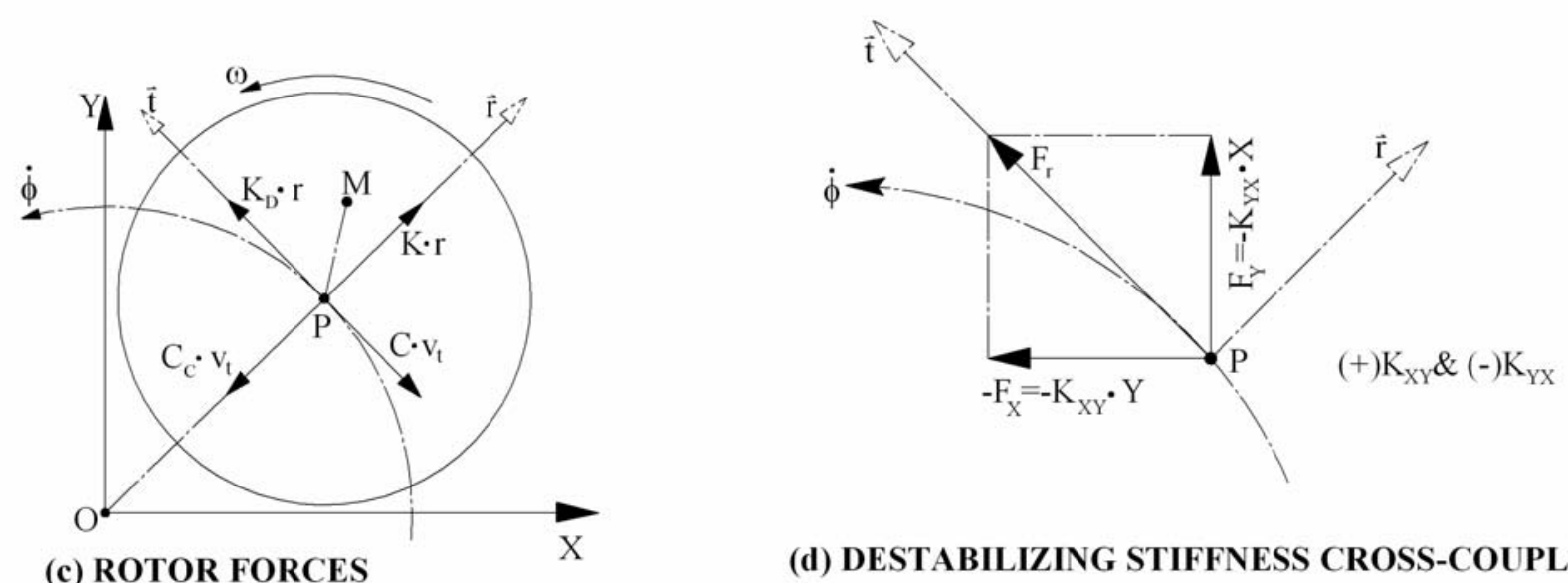

(d) DESTABILIZING STIFFNESS CROSS-COUPLING

Figure 21. Force Coefficients Effects on Flexible Rotor 
For forward whirl, direct damping reduces the rotor response at the critical speed. In addition to rotordynamic response, engineers must also consider the effects of coefficients on dynamic stability. Rotordynamic stability is dictated by two types of forces: (1) direct damping and (2) cross-coupled or cross-axis stiffness. Direct damping in a non-rotating inertial frame of reference is considered to be stabilizing to a rotor-bearing system. This is apparent in Part C of Figure 21 that shows the damping force acting in the opposing direction of the rotor whirl velocity vector. Although large positive values of direct damping is a favorable characteristic for seals, it is not the ultimate indicator to the damping capacity. One must consider the effective damping, which also accounts for the destabilizing stiffness cross-coupling force, when simulating the rotordynamics for a machine. The cross-coupled stiffness force is found through the real part of the cross-coupled impedance values, namely $\mathrm{H}_{\mathrm{XY}}$ and $\mathrm{H}_{\mathrm{YX}}$. Note that the presence of the $\mathrm{K}_{\mathrm{XY}}$ and $\mathrm{K}_{\mathrm{YX}}$ terms is not always destabilizing (opposing direct damping); there are some special requirements. In Figure 21 part $\mathrm{d}$ the stiffness cross-coupling force component $F_{r}$ is tangential to the whirl orbit. This implies that for circular orbits the $\mathrm{K}_{\mathrm{XY}}$ and $\mathrm{K}_{\mathrm{YX}}$ term must be have opposite for stiffness crosscoupling to be destabilizing. The effective damping is defined in Equation 3.19-3.20.

$$
\begin{aligned}
& C_{e f f(X)}=C_{X X}-\frac{K_{X Y}}{\dot{\phi}}=C_{X X}-\frac{K_{D}}{\dot{\phi}} \\
& C_{e f f(Y)}=C_{Y Y}-\frac{K_{Y X}}{\dot{\phi}}=C_{Y Y}-\frac{K_{D}}{\dot{\phi}}
\end{aligned}
$$

\subsection{Same Sign Stiffness Cross-Coupling}

Past research on PDS has shown that the cross-coupled stiffness coefficients in the $\mathrm{X}$ and Y directions possess the same sign. Analytical work by Li et al. (1998), Laos (1999), and Aguilar (2002) have shown same sign cross-coupled stiffness coefficients. Also showing same sign cross-coupled coefficients are experiments from Li et al. (2000) and Gamal (2003). Same sign stiffness cross-coupling can be represented by a symmetric stiffness matrix (Equation 3.21) from a general equation of motion) for a two degree of freedom linear system (Equation 1.1), where $\mathrm{K}$ is the direct stiffness coefficients $\left(\mathrm{K}_{\mathrm{XX}}=\mathrm{K}_{\mathrm{YY}}=\mathrm{K}\right), \mathrm{k}$ is the positive valued cross-coupled stiffness coefficients $\left(+\mathrm{K}_{\mathrm{XY}}=+\mathrm{K}_{\mathrm{YX}}=\mathrm{k}\right)$, and $\bar{K}$ is the stiffness matrix. The characteristic equation of $\bar{K}$ (Equation 3.23 ) is used to solve for the eigenvalues of the stiffness matrix, where $I$ is the identity matrix and $D(\lambda)$ is the 
characteristic determinant; and when expanded the characteristic equation (3.24). The solution of the eigenvalues (Equation 3.25) is then substituted into 3.22, followed by the calculation of the eigenvectors (Equations 3.26-3.29). The shift of the initial axis to the principle axis is shown in Figure 22 and indicates a 45 degree and 135 degree orientation of the principle stiffness axis. Next, the eigenvectors are used to diagonalize the stiffness matrix $(\bar{K})$, which transforms the stiffness matrix to the principle axis (Equations 30-31). Clearly, the transformed stiffness matrix in Equation 31 is not considered to be destabilizing due to the absence of the off-diagonal stiffness cross-coupling terms. A similar analysis can be performed for the case where $\mathrm{K}$ is the direct stiffness coefficients $\mathrm{K}_{\mathrm{XX}}=\mathrm{K}_{\mathrm{YY}}=\mathrm{K}$ and $\mathrm{k}$ is the negative valued cross-coupled stiffness coefficients $-K_{X Y}=-K_{Y X}=-k$ (Equations 3.33-3.44). The principle axis shift is illustrated in Figure 23 and Figure 24 shows the resultant conical sections.

$$
\begin{aligned}
& {\left[\begin{array}{ll}
K & k \\
k & K
\end{array}\right] \cdot\left[\begin{array}{l}
X \\
Y
\end{array}\right]=0} \\
& \bar{K}=\left[\begin{array}{cc}
K-\lambda & k \\
k & K-\lambda
\end{array}\right]
\end{aligned}
$$

$D(\lambda)=\operatorname{det}(\bar{K}-\lambda \mathrm{I})=0$

$K^{2}-2 K \lambda+\lambda^{2}-k^{2}=0$

$\lambda=K \pm k$

For $\lambda=(K+k)$ :

$$
\left[\begin{array}{cc}
K-(K+k) & k \\
k & K-(K+k)
\end{array}\right] \cdot\left[\begin{array}{l}
a_{11} \\
a_{21}
\end{array}\right]=0
$$

$\left[\begin{array}{l}a_{11} \\ a_{21}\end{array}\right]=\left[\begin{array}{l}1 \\ 1\end{array}\right]=a_{1} \quad$ Normalizing the Eigenvector $a_{1}, \quad \hat{a}_{1}=\left[\begin{array}{c}\frac{1}{\sqrt{2}} \\ \frac{1}{\sqrt{2}}\end{array}\right]$

For $\lambda=(K-k)$ :

$$
\left[\begin{array}{cc}
K-(K-k) & k \\
k & K-(K-k)
\end{array}\right] \cdot\left[\begin{array}{l}
a_{11} \\
a_{21}
\end{array}\right]=0
$$


$\left[\begin{array}{l}a_{12} \\ a_{22}\end{array}\right]=\left[\begin{array}{c}1 \\ -1\end{array}\right]=a_{2} \quad$ Normalizing the Eigenvector $a_{2}, \quad \hat{a}_{2}=\left[\begin{array}{c}\frac{1}{\sqrt{2}} \\ -\frac{1}{\sqrt{2}}\end{array}\right]$

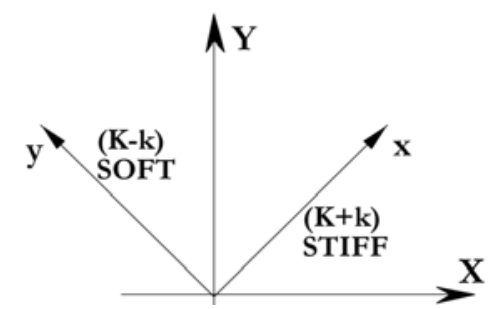

Figure 22. Principle Axis Shift: $(+) K_{\mathrm{XY}}$ and $(+) K_{Y X}$

$$
\begin{aligned}
& A=A^{T}=\left[\begin{array}{ll}
\hat{a}_{11} & \hat{a}_{12} \\
\hat{a}_{21} & \hat{a}_{22}
\end{array}\right]=\frac{1}{\sqrt{2}}\left[\begin{array}{cc}
1 & 1 \\
1 & -1
\end{array}\right] \\
& \frac{1}{\sqrt{2}}\left[\begin{array}{cc}
1 & 1 \\
1 & -1
\end{array}\right] \cdot\left[\begin{array}{ll}
K & k \\
k & K
\end{array}\right] \cdot \frac{1}{\sqrt{2}}\left[\begin{array}{cc}
1 & 1 \\
1 & -1
\end{array}\right]=\left[\begin{array}{cc}
K+k & 0 \\
0 & K-k
\end{array}\right]
\end{aligned}
$$

For $-\mathrm{K}_{\mathrm{XY}}=-\mathrm{K}_{\mathrm{YX}}=-\mathrm{k}$

$\left[\begin{array}{cc}K & -k \\ -k & K\end{array}\right] \cdot\left[\begin{array}{c}X \\ Y\end{array}\right]=0$

$\bar{K}=\left[\begin{array}{cc}K-\lambda & -k \\ -k & K-\lambda\end{array}\right]$

$D(\lambda)=\operatorname{det}(\bar{K}-\lambda \mathrm{I})=0$

$K^{2}-2 K \lambda+\lambda^{2}-k^{2}=0$

$\lambda=K \pm k$

For $\lambda=(K+k)$ :

$\left[\begin{array}{cc}K-(K+k) & -k \\ -k & K-(K+k)\end{array}\right] \cdot\left[\begin{array}{l}a_{11} \\ a_{21}\end{array}\right]=0$

$\left[\begin{array}{l}a_{11} \\ a_{21}\end{array}\right]=\left[\begin{array}{c}1 \\ -1\end{array}\right]=a_{1} \quad$ Normalizing the Eigenvector $a_{1}, \quad \hat{a}_{1}=\left[\begin{array}{c}\frac{1}{\sqrt{2}} \\ -\frac{1}{\sqrt{2}}\end{array}\right]$

$\operatorname{For} \lambda=(K-k)$ : 
$\left[\begin{array}{cc}K-(K-k) & -k \\ -k & K-(K-k)\end{array}\right] \cdot\left[\begin{array}{l}a_{12} \\ a_{22}\end{array}\right]=0$

$\left[\begin{array}{l}a_{12} \\ a_{22}\end{array}\right]=\left[\begin{array}{l}1 \\ 1\end{array}\right]=a_{2} \quad$ Normalizing the Eigenvector $a_{2}, \quad \hat{a}_{2}=\left[\begin{array}{c}\frac{1}{\sqrt{2}} \\ \frac{1}{\sqrt{2}}\end{array}\right]$

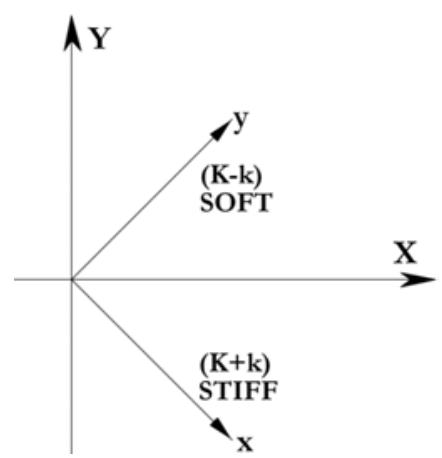

Figure 23. Principle Axis Shift: (-) $K_{X Y}$ and (-) $K_{Y X}$

$$
\begin{aligned}
& A=\left[\begin{array}{ll}
\hat{a}_{11} & \hat{a}_{12} \\
\hat{a}_{21} & \hat{a}_{22}
\end{array}\right]=\frac{1}{\sqrt{2}}\left[\begin{array}{cc}
1 & 1 \\
-1 & 1
\end{array}\right] \\
& A^{T}=\frac{1}{\sqrt{2}}\left[\begin{array}{cc}
1 & -1 \\
1 & 1
\end{array}\right] \\
& \frac{1}{\sqrt{2}}\left[\begin{array}{cc}
1 & -1 \\
1 & 1
\end{array}\right] \cdot\left[\begin{array}{cc}
K & -k \\
-k & K
\end{array}\right] \cdot \frac{1}{\sqrt{2}}\left[\begin{array}{cc}
1 & 1 \\
-1 & 1
\end{array}\right]=\left[\begin{array}{cc}
K+k & 0 \\
0 & K-k
\end{array}\right]
\end{aligned}
$$

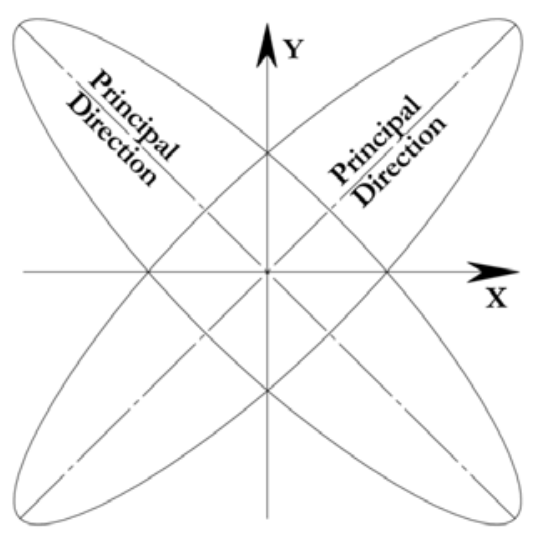

Figure 24. Resulting Conical Sections 
The previous analysis has shown how the symmetric stiffness matrix was diagonalized to produce an asymmetric stiffness matrix at the principal axis where the matrix is diagonal but orthotropic. To better understand this result a more illustrative explanation is presented. Unlike the behavior of equal and opposite valued cross-coupled coefficients, same sign stiffness cross-coupling does not generate a continuous follower force in the direction of rotor whirl velocity. Figure 25 shows two cases: (1) negative $K_{\mathrm{XY}}$ and $\mathrm{K}_{\mathrm{YX}}$ and (2) positive $K_{X Y}$ and $K_{Y X}$. Each case shows a resultant stiffness cross-coupling force $F_{r}$ as it rotates one complete cycle from point 1 to point 8 . Note that points $1,3,5$, and 7 show purely tangential forces and points $2,4,6$, and 8 are purely radial forces. The tangential forces can be considered as forces that affect the energy input into the whirl orbit whereas the radial forces are restoring forces like direct stiffness. Based on the force diagram there are two issues to be considered: (1) radial distortion force on the orbit and (2) tangential forces responsible for rotordynamic stability.

First, consider the radial forces acting on the circular orbit. Figure 26 shows a particular same sign stiffness cross coupling resultant force $F_{r}$ at an angle between points 1 and 2 , which decomposes into a tangential component $F_{t}$ and a radial component $F_{R}$. The radial component $F_{R}$ is a maximum at points $2,4,6$, and 8 and zero at points $1,3,5$, and 7 .

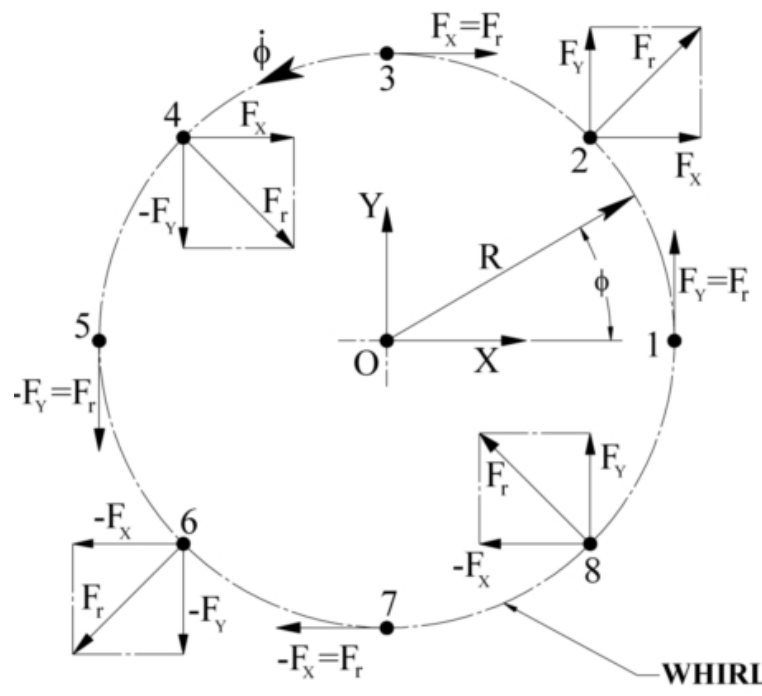

(a) Negative $K_{\mathbf{X Y}}$ and $K_{\mathbf{Y X}}$

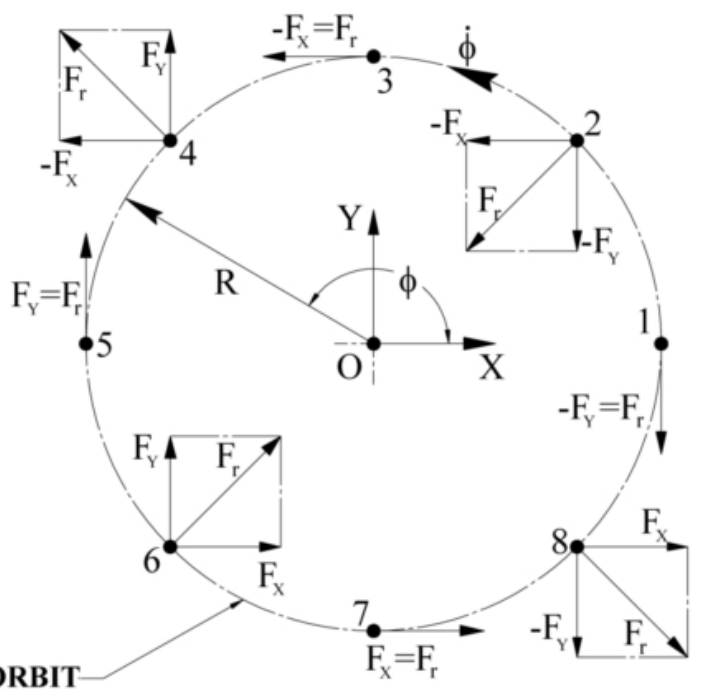

(b) Positive $\mathrm{K}_{\mathrm{xY}}$ and $\mathrm{K}_{\mathrm{yx}}$

Figure 25. Same Sign Stiffness Cross-Coupling Coefficient Forces 


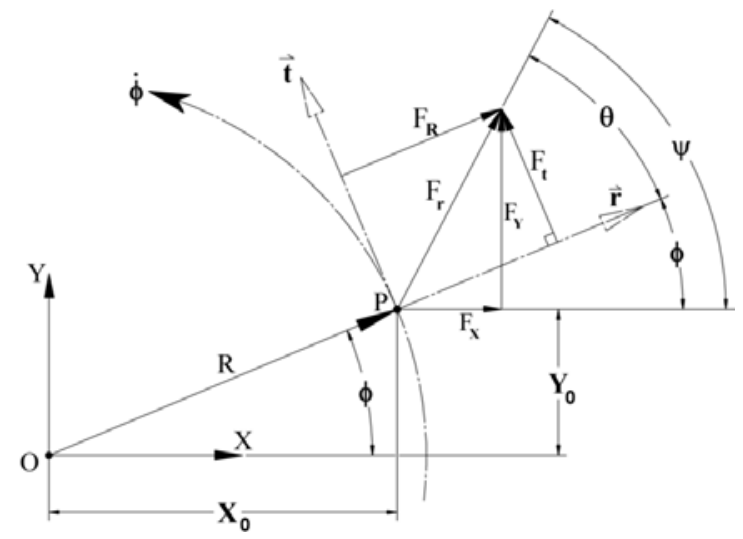

Figure 26. Same Sign Stiffness Cross-Coupling Force Components

Using the radial force components from same sign stiffness cross-coupling coefficients, a force distribution is generated as shown in Figure 27. The dotted line force distribution represents positive valued stiffness cross-coupling coefficients and the solid line force distribution illustrates negative stiffness cross-coupling values. As a result of the radial distortion forces, the orbit shape becomes elliptical with the major axis of the ellipse either at 45 degrees (negative valued stiffness cross-coupling) or 135 degrees (positive valued stiffness cross-coupling).
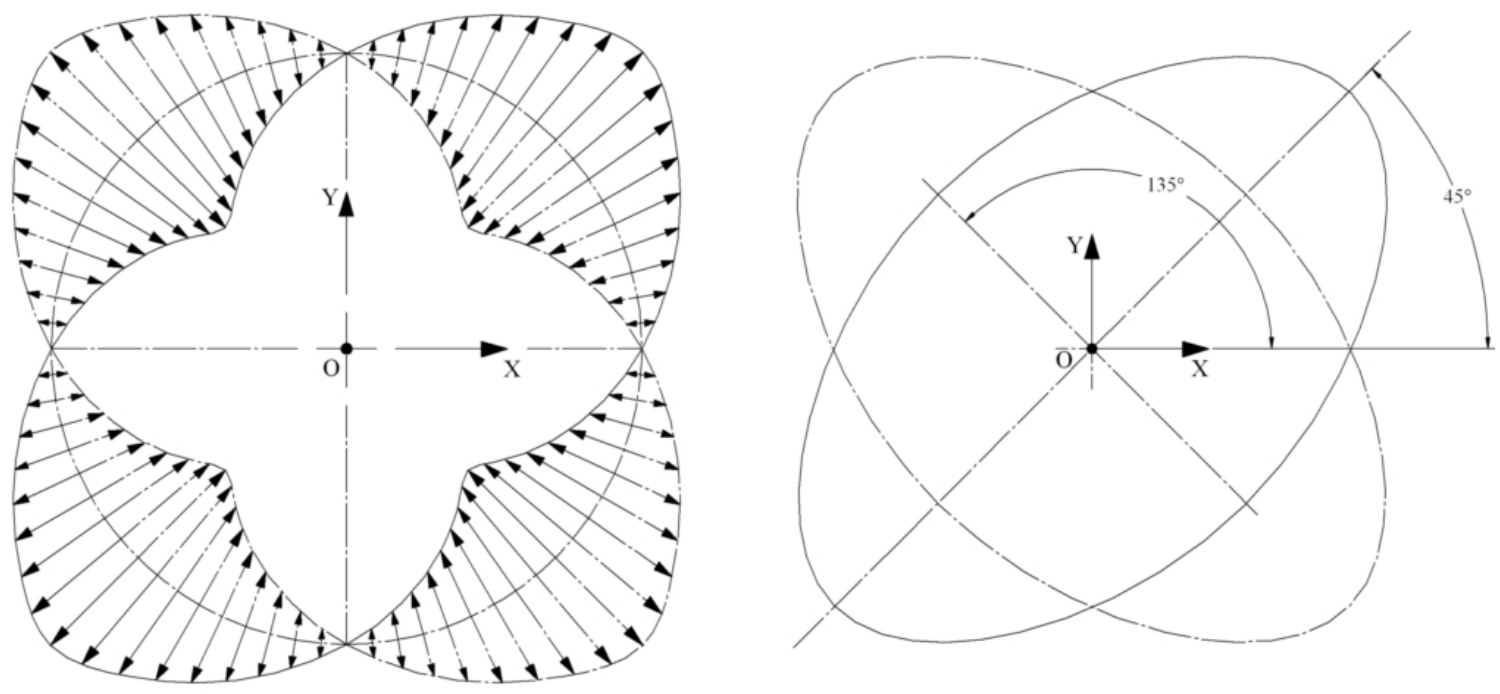

$-(-) \mathrm{K}_{\mathrm{XY}} \& \mathrm{~K}_{\mathrm{YX}}$

$(+) \mathrm{K}_{\mathrm{XY}} \& \mathrm{~K}_{\mathrm{YX}}$

(a) Radial Distortion Forces Imposed on Circular Orbit

(b) Distorted Elliptical Orbits

Figure 27. Orbit Distortion from Same Sign Stiffness Cross-Coupling 
The previous analysis suggests that same sign cross-coupled stiffness is not destabilizing since there appears to be no net energy input per cycle for the special cases considered. The following analysis is more general and more rigorous. The objective is to determine the influence of same sign stiffness cross-coupling on dynamic stability. This is achieved by performing a complex eigenvalue stability analysis on a single point mass with 2 degrees of freedom. The equations of motion for the system in the $\mathrm{X}$ and $\mathrm{Y}$ directions are shown in Equations 3.44-3.45. These equations will address the unstable case first, which is when $K_{X Y}=-K_{Y X}$. Note that the equations only account for direct stiffness and the unstable cross-coupled stiffness terms; there is no damping in the analysis. Assuming exponential functions (Equation 3.46-3.47) for the $\mathrm{x}$ and $\mathrm{y}$ motion yields Equation 3.48-3.49. Expressing the modified equations of motion in matrix form gives 3.50. The equation shown in 3.50 implies that the determinant of the coefficient element matrix is equal to zero, which gives the relationship shown in Equation 3.51 and solving for $\mathrm{S}^{2}$ results in Equation 3.52.

$$
\begin{aligned}
& \text { Unstable Case: } \mathrm{K}_{\mathrm{XY}}=\mathrm{k}, \mathrm{K}_{\mathrm{YX}}=-\mathrm{k} \text {, and } \mathrm{K}_{\mathrm{XX}}=\mathrm{K}_{\mathrm{YY}}=\mathrm{K} \\
& m \ddot{x}+K x+k y=0 \\
& m \ddot{y}+K y-k x=0 \\
& x=\mathrm{X} e^{S T} \text { and } \ddot{x}=S^{2} \mathrm{X} e^{S T} \\
& y=\mathrm{Y} e^{S T} \text { and } \ddot{y}=S^{2} \mathrm{Y} e^{S T} \\
& m\left(S^{2} X e^{S T}\right)+K\left(\mathrm{X} e^{S T}\right)+k\left(\mathrm{Y} e^{S T}\right)=0 \\
& m\left(S^{2} \mathrm{Y} e^{S T}\right)+K\left(\mathrm{Y} e^{S T}\right)-k\left(\mathrm{X} e^{S T}\right)=0 \\
& {\left[\begin{array}{cc}
\left(m S^{2}+K\right) & k \\
-k & \left(m S^{2}+K\right)
\end{array}\right] \cdot\left\{\begin{array}{l}
\mathrm{X} \\
\mathrm{Y}
\end{array}\right\}=\left[\begin{array}{l}
0 \\
0
\end{array}\right]} \\
& \left(m S^{2}+K\right)^{2}+k^{2}=0 \\
& S^{2}=-\frac{K}{m} \pm i \frac{k}{m}
\end{aligned}
$$

Next, $\mathrm{S}$ is defined to have a real and imaginary part where $\omega$ is the frequency (Equation 3.53). The relationship from 3.53 is substituted into 3.52, which gives Equation 3.54. The real part of Equation 3.54 is representative of the frequency and the imaginary part of Equation 3.54 is responsible for the stability.

$S=\lambda \pm i \omega$ where $\lambda$ and $\omega$ are real numbers 
$S^{2}=\left(\lambda^{2} \mp \omega^{2}\right) \pm i(2 \lambda \omega)$

Since the analysis is focused on the system stability and not the frequency, the next step is to equate the imaginary part of Equation 3.54 to the imaginary part of Equation 3.52. Next, we solve for $\lambda$ (Equation 3.56) and note that $\lambda$ is real and positive; clearly an unstable system.

$2 \lambda \omega=\frac{k}{m}$

$\lambda=\frac{k}{2 m \omega}$

So far the analysis has shown that for a system with $K_{X Y}=-K_{Y X}$ the imaginary part of the eigenvalue solution is real and positive yielding an unstable system. For the next part consider stiffness cross-coupling where $\mathrm{K}_{\mathrm{XY}} \neq \mathrm{K}_{\mathrm{YX}}$ and both coefficients are positive. Using this assumption the equations of motion are shown in Equations 3.57-3.58. Unlike the previous analysis that necessitated the square root of a negative valued $-k^{2}$ between Equations 3.51 and 3.52 this case requires the square root of a positive real number $\left(\mathrm{k}_{1} \mathrm{k}_{2}\right)^{1 / 2}= \pm \alpha$ as shown in Equation 3.61. The eigenvalue solution is purely real and does not possess an imaginary part, which suggests that there is no contribution to the vibration; the contribution is purely to static stability. Rearranging Equation 3.61 and solving using the negative sign and positive sign yields Equations 3.63-3.64. The solution of S will depend on the sign of the direct stiffness and the magnitude of $\mathrm{k}$ in relation to $\mathrm{K}$. Therefore, if $\mathrm{S}$ is imaginary than the system is statically stable and if $S$ is a real number then the system is statically unstable. The important result from this analysis is that the eigenvalue solution for positive $\mathrm{K}_{\mathrm{XY}}$ and $\mathrm{K}_{\mathrm{YX}}$ values fails to possess an imaginary component, therefore rendering the effects to the mechanical system to be purely static and not dynamic. The conclusion is that there is no direct influence on rotordynamic stability.

Stable Case: $K_{X Y} \neq K_{Y X}$ and both positive

$$
\begin{aligned}
& m \ddot{x}+K x+k_{1} y=0 \\
& m \ddot{y}+K y+k_{2} x=0 \\
& {\left[\begin{array}{cc}
\left(m S^{2}+K\right) & k_{1} \\
k_{2} & \left(m S^{2}+K\right)
\end{array}\right] \cdot\left\{\begin{array}{l}
\mathrm{X} \\
\mathrm{Y}
\end{array}\right\}=\left[\begin{array}{l}
0 \\
0
\end{array}\right]} \\
& \left(m S^{2}+K\right)^{2}-k_{1} k_{2}=0
\end{aligned}
$$


$\pm\left(m S^{2}+K\right)=\sqrt{k_{1} k_{2}}= \pm \alpha \quad \alpha$ is a real number

$m S^{2}+(K \mp \alpha)=0$

(+) Sign

$S^{2}=\frac{-K-\alpha}{m}$

(-) Sign

$S^{2}=\frac{\alpha-K}{m}$

Another approach to rigorously determining the influence of same sign crosscoupling is to analyze the energy fed into the whirling motion of the rotor by stiffness crosscoupling. Murphy in his 1984 dissertation work shows a detailed analysis in Appendix D where he analyzes the cross-coupled Jeffcott Rotor. In his analysis he discusses and computes the effects of cross-coupled stiffness and cross-coupled damping on stability for several elliptical orbits with different percent asymmetries. In regards to stiffness crosscoupling and stability, he proceeds to develop an expression for the energy input into a generalized whirl orbit with variable eccentricity from cross-coupled springs (Equation 3.65). The energy input is calculated by integrating the force times the displacement around the closed curve of the ellipse, which is essentially a line integral that calculates the net work done on the system (Equation 3.65). Then by the use of Green's Theorem, the line integral is converted to an area integral yielding Equation 3.66. Next, Equation 3.66 is reduced to Equation 3.68, where $\mathrm{A}$ is the area of the ellipse. Note that for same sign stiffness crosscoupling the energy input or net work done on the dynamic system is zero, therefore not affecting the stability. He also explains how same sign stiffness cross-coupling coefficients have the effect of increasing the stiffness asymmetry in the $\mathrm{X}$ and $\mathrm{Y}$ directions.

$$
\begin{aligned}
& E=\int_{C}\left(-K_{X Y} y d x-K_{Y X} x d y\right) \\
& E=\iint_{R}\left(\frac{\partial\left(-K_{Y X} x\right)}{\partial x}-\frac{\partial\left(-K_{X Y} y\right)}{\partial y}\right) d x d y \\
& E=\iint_{R}\left(-K_{Y X}+K_{X Y}\right) d x d y \\
& E=\left(-K_{Y X}+K_{X Y}\right) \cdot A
\end{aligned}
$$




\subsection{Dynamic Pressure Response Method}

The second experimental method used to determine force coefficients was the dynamic pressure response method. The PDS tested in the present work are comprised of several cavities configured by the baffle walls and the seal blades. Each cavity possesses a dynamic pressure during stator vibration, and the pressure in the cavities can be transformed into a force by integrating the pressure over the projected area of the cavity onto the rotor surface. The knowledge of the internal seal forces due to stator vibration can allow one to calculate frequency dependent rotordynamic coefficients. This method differs from the system impedance method from several points of view. First, the dynamic pressure method directly measures the seal forces, and the seal forces do not have to be extracted by subtracting the impedance values from two separate tests, namely the system impedance test with the test seals and the system impedance test with the baseline inserts. This method requires one assembly to determine the seal coefficients, which eliminates the error and uncertainty associated with the separate baseline test necessary for the system impedance method. Second, the dynamic pressure method provides unique information which can be directly compared with the damper seal theory on the most fundamental of levels, such as the frequency dependent cavity pressure phase relationship relative to vibration motion, dynamic pressure peaks, and the calculation of force coefficients for each individual cavity. Knowing the pressure phase relationship to vibration can give insight to the cavities with the strongest frequency dependency and can lead to optimized pitch ratios and clearance ratios to maximize stiffness or damping. Also, the phase measurement is the basis for understanding the internal dynamics of the new fully partitioned PDS design in terms of the seal providing either positive or negative stiffness and either positive or negative damping for the individual cavities. The pressure peak can be compared to the theoretical predications and thereby validate the assumptions for excluding viscous and inertial effects from the analytical model. There are some disadvantages to this method. First, the pressure is only being measured at a single location in the cavity, therefore small pressure variation that may arise near the rotor surface (where there exists a flow velocity) are not measured. Second, the pressure under the circumferential partition walls are not being measured, but these walls account for a small portion of the projected area on the rotor (less than $2 \%$ ).

Although PDS have not been tested before using the dynamic pressure response method, there has been work conducted on different components that used dynamic 
pressure to determine force coefficients. The earliest work found for utilizing dynamic pressures to determine force characteristics involves experiments with squeeze film dampers (SFD). Vance and Kirton (1975) carried out a test which was the first of its kind on SFD. They experimentally investigated the hydrodynamic force response of a SFD using a controlled orbit test rig. This involved measuring the pressure wave around the circumference of the journal while administering circular centered orbits and then integrating the dynamic pressure around the journal surface to determine the force components of the squeeze film. This method for determining force coefficients for SFD was adopted for future tests conducted by numerous researchers such as Hibner and Bansal (1979), San Andres (1985), and Zeidan (1989).

Gas seal testing is another area in which cavity pressures have been used to extract force coefficients. Experimental tests by Kwanka et.al (1993), Millsaps and Sanchez (1993), and Benckert and Wachter (1980) used pressure transducers to determine direct and crosscoupled stiffness coefficients in different configurations of labyrinth seals. The most recent and thorough work has been by Kaneko et.al (2003) who used pressure transducers on the rotor surface to determine a full set (direct and cross-coupled damping and stiffness) of frequency dependent coefficients for a converging hole pattern liquid seal. This work utilized the motion of the rotor in combination with the pressure transducers to integrate the pressure around the surface of the rotor for determining coefficients.

The dynamic pressure response method described in the present work has slight differences from the previous methods that used pressures to determine coefficients. Unlike the SFD experiments which only necessitate one transducer for circular centered orbits, the PDS configuration required multiple pressure transducers to instrument all cavities for a full set of coefficients. Also, the tests comprised of two independent linear motion excitations rather than the controlled circular orbit testing. The dynamic pressure response method is not an impedance method, because an impedance method requires that the system response is measured relative to a known force imposed on the system in the frequency domain. The dynamic pressure method measures only the system motion and the cavity pressures. The seal forces are computed from the measured pressures. This method is valid because dynamic forces generated from pressures are uniquely determined by the stator's vibratory motion. Figure 28 shows the PDS-journal assembly and pressure distribution for the two directions of excitation. To determine direct and cross-coupled force coefficients the system 
was excited in two independent directions $\mathrm{X}$ and $\mathrm{Y}$, therefore yielding four pressure response equations for an individual cavity (Equation 3.69). Unlike the system impedance method which uses the force signal as the input excitation and reference signal, the dynamic pressure method uses the motion $\left(D_{i j}\right)$ as the input excitation and the dynamic cavity pressure as the response to the excitation. Therefore, the motion signal is the reference signal from which all other signals are phased from. Also, the first subscript shown in Equation 3.69 represents the direction of excitation, which is the motion not the force signal as in the system impedance method. The cavity response force can be determined by integrating the dynamic cavity pressure along the circumference of the rotor (Equations 3.70-3.71), where $\bar{F}_{i}$ is the pressure force. It is also important to decompose each pressure generated cavity forces into the $\mathrm{X}$ and $\mathrm{Y}$ directions. Next, the matrix shown in Equation 3.69 can be solved for the seal coefficients in the frequency domain as a function of the pressure induced dynamic cavity forces and the vibratory motion (Equations 3.72-3.75).

$$
\begin{aligned}
& {\left[\begin{array}{ll}
\bar{F}_{x x} & \bar{F}_{y x} \\
\bar{F}_{x y} & \bar{F}_{y y}
\end{array}\right]=\left[\begin{array}{ll}
\left(K_{x x}+i C_{x x} \omega\right) & \left(K_{x y}+i C_{x y} \omega\right) \\
\left(K_{y x}+i C_{y x} \omega\right) & \left(K_{y y}+i C_{y y} \omega\right)
\end{array}\right] \cdot\left[\begin{array}{ll}
D_{x x} & D_{y x} \\
D_{x y} & D_{y y}
\end{array}\right]} \\
& \bar{F}_{X}=\int_{\theta_{1}}^{\theta_{2}}\left(P(i) \cdot \cos \theta_{0}\right) d A \\
& \bar{F}_{Y}=\int_{\theta_{1}}^{\theta_{2}}\left(P(i) \cdot \sin \theta_{0}\right) d A \\
& \left(K_{x x}+i C_{x x} \omega\right)=\frac{\bar{F}_{x x} \cdot D_{y y}-\bar{F}_{y x} \cdot D_{x y}}{D_{x x} \cdot D_{y y}-D_{y x} \cdot D_{x y}} \\
& \left(K_{x y}+i C_{x y} \omega\right)=\frac{\bar{F}_{x x} \cdot D_{y x}-\bar{F}_{y x} \cdot D_{x x}}{D_{x y} \cdot D_{y x}-D_{y y} \cdot D_{x x}} \\
& \left(K_{y y}+i C_{y y} \omega\right)=\frac{\bar{F}_{y y} \cdot D_{x x}-\bar{F}_{x y} \cdot D_{y x}}{D_{y y} \cdot D_{x x}-D_{y x} \cdot D_{x y}} \\
& \left(K_{y x}+i C_{y x} \omega\right)=\frac{\bar{F}_{y y} \cdot D_{x y}-\bar{F}_{x y} \cdot D_{y y}}{D_{x y} \cdot D_{y x}-D_{y y} \cdot D_{x x}}
\end{aligned}
$$

The positive $\mathrm{X}$ and $\mathrm{Y}$ direction motion is illustrated in Figure 28, and also shown is the angular location for the 8 circumferential cavities. Cavities in the $0^{\circ}$ and $180^{\circ}$ positions contributed to the $\mathrm{X}$ direction direct and cross-coupled coefficients but did not contribute to the $\mathrm{Y}$ coefficients. 


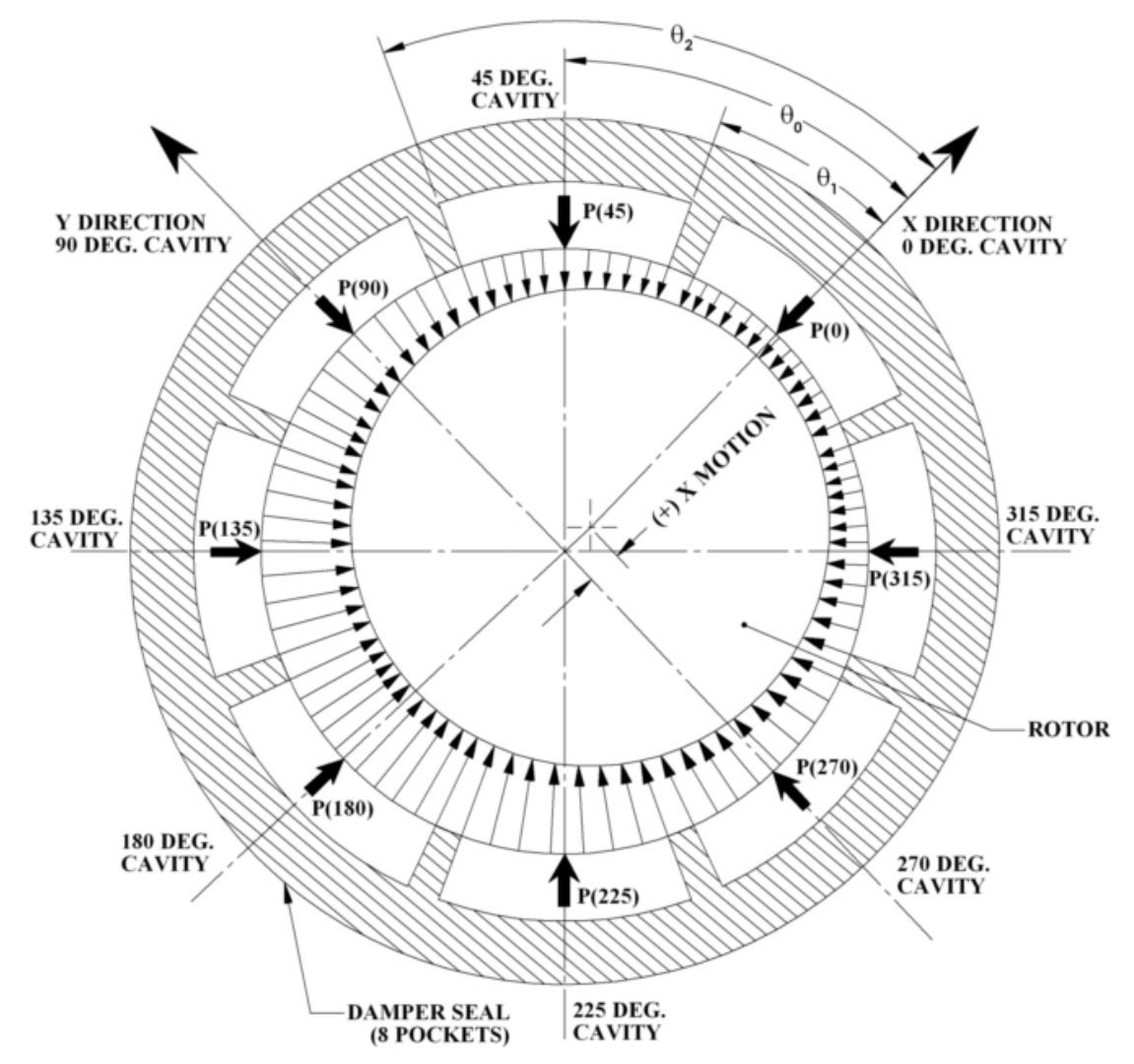

(a) X Direction Excitation

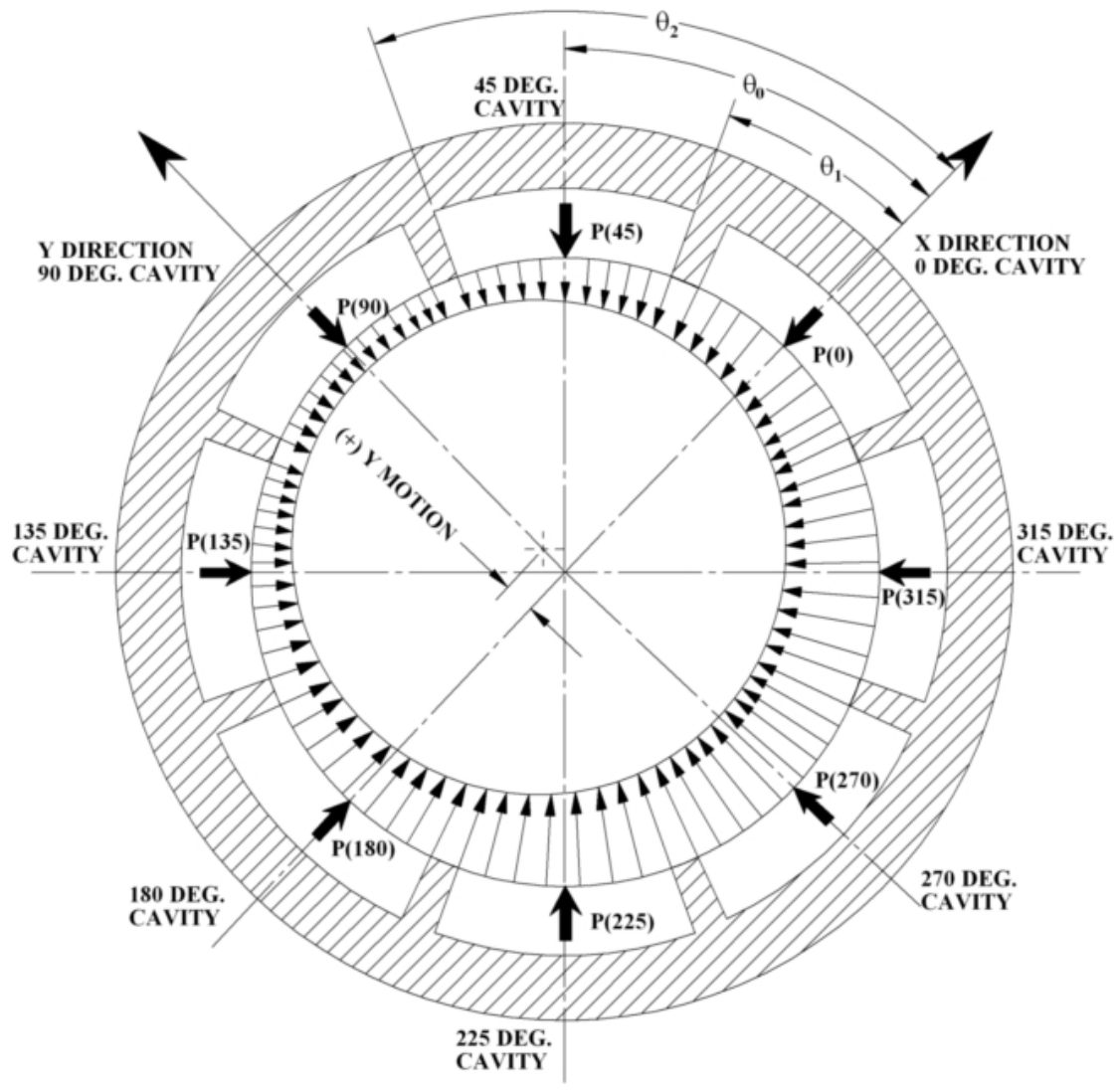

(b) Y Direction Excitation

Figure 28. Dynamic Pressure Response to Vibratory Motion 
In addition, the cavities in the $90^{\circ}$ and $270^{\circ}$ positions only produced coefficients in the $\mathrm{Y}$ direction; they did not generate any direct or cross-coupled coefficients in the X direction. However, the cavities in the $45^{\circ}, 135^{\circ}, 225^{\circ}$, and $315^{\circ}$ positions possessed direct and crosscoupled coefficients in the $\mathrm{X}$ and $\mathrm{Y}$ directions. Also, these cavities in the mid quadrant locations required that the pressure generated dynamic force be decomposed into the $\mathrm{X}$ and Y direction to calculate coefficients. The dynamic pressure method was only administered to the eight bladed PDS and the six bladed PDS, which will be discussed in detail in the next section. The testing scheme involved single frequency excitations in the $\mathrm{X}$ and $\mathrm{Y}$ directions for several test frequencies up to $200 \mathrm{~Hz}$, while measuring the stator motion (M1, M5, M2, M6) and dynamic cavity pressures. The instrument control, data acquisitions, and the data reduction were achieved using LABVIEW software and hardware (hardware specifications shown in Appendix). Table 2 shows the data acquisition parameters used for the dynamic pressure tests. Figure 29 shows a Y direction shake test at $80 \mathrm{~Hz}$ on the fully partitioned six bladed PDS assembly, where P1 through P5 are the dynamic pressures for the row of cavities at the 90 degree location. A shake test at $80 \mathrm{~Hz}$ was also performed in the $\mathrm{X}$ direction.

Table 2. Data Acquisition Parameters

\begin{tabular}{|l|c|}
\hline DAQ PARAMETERS & \\
\hline EXCITATION SIGNAL: & FIXED SINE WAVE \\
\hline SAMPLING RATE: & 8192 SAMPLES/SEC \\
\hline SAMPLE LENGTH: & $0.25 \mathrm{sec}(2048$ samples) \\
\hline SETS OF DATA: & 12 \\
\hline FREQUENCY RANGE: & $0-4096 \mathrm{~Hz}$ \\
\hline FREQUENCY RESOLUTION: & $4 \mathrm{~Hz}$ \\
\hline FREQUENCY DOMAIN POINTS: & 1024 \\
\hline VIBRATION AMPLITUDE: & $1.2-1.8$ mils pk-pk \\
\hline
\end{tabular}



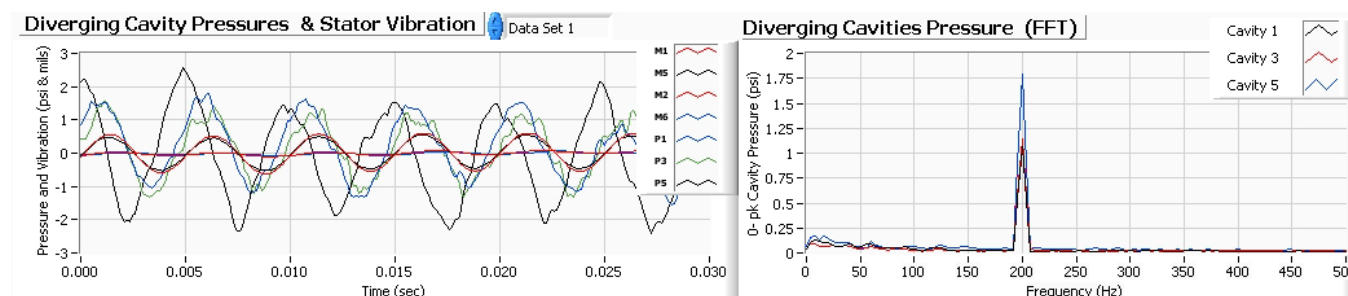

Converging Cavity Pressures \& Stator Vibration
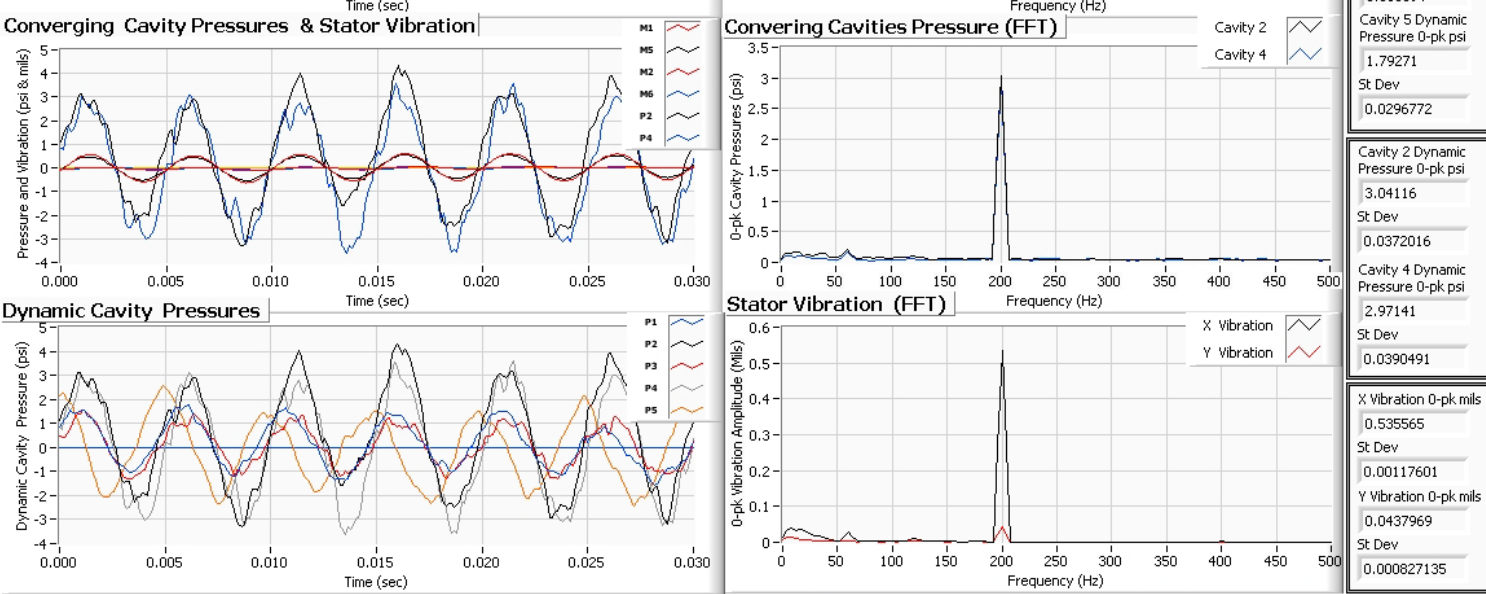

Figure 29. Experimental Measurements: Pressure Response Method 


\section{IMPEDANCE METHOD EXPERIMENTAL RESULTS}

This section presents the experimental results for frequency dependent force coefficients determined using the impedance method. Each PDS configuration was tested for multiple pressure ratios with an approximate inlet pressure of 1,000 psig and different rotor speeds of 10,200, 15,200, and 20,200 rpm. Results are discussed and are compared in terms of pressure ratio, rotor speed, and seal type.

\subsection{Baseline Force Coefficients}

As discussed earlier, the impedance method requires a separate test that uses baseline inserts (Figure 18) in place of the test seals. The first types of tests presented are impact hammer tests performed on the mechanical structure of the test rig for several configurations. The aim of these tests was to extract stiffness and damping coefficients so that they can be compared to the force coefficients generated from the impedance method. Figure 30 shows the test rig mechanical structure, which is composed of the stator housing suspended by the use of the shaker stingers, squirrel cages, and pitch stabilizers. This figure also illustrates the location of the stick-on accelerometer (bottom left) and the force hammer striking location (bottom right); both in line with the Y direction shaker. Impact hammer test were used to measure the inertance and free vibration decay of the test rig structure for the two configurations in Table 3.

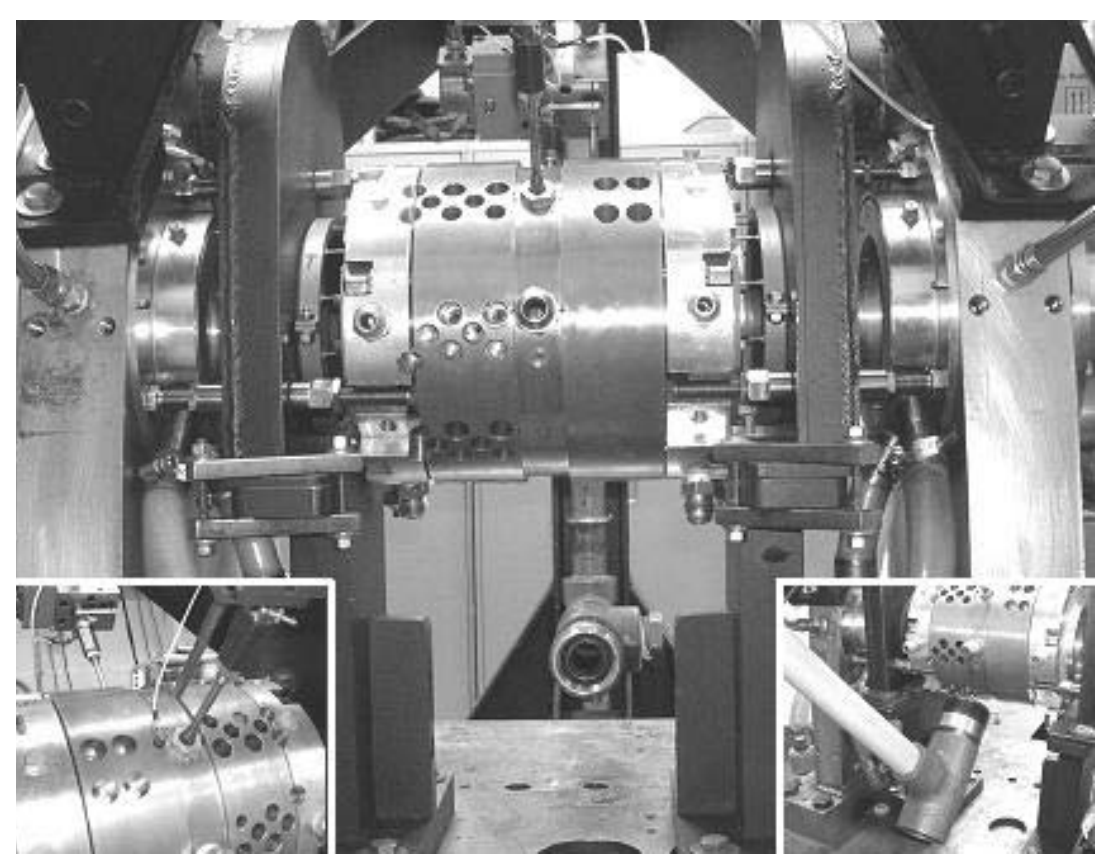

Figure 30. Structural Baseline Test Set-up 
It is important to understand that the mechanical system in question does not include the hydraulic shakers, therefore all tests reported in this section were performed with the shaker stingers not attached. The acceleration and force signal were used to calculate the structural compliance, phase, and coherence with and without the squirrel cage stiffeners. Determining force coefficients was achieved using three different methods. First, the compliance (Figure 31) was curve fit (with LABVIEW software) using a function of the form in Equation 4.1, which assumes linear stiffness and damping coefficients. Both stiffness and mass values were extracted using an iterative scheme, whereas the damping was first found using the 90 degree damping method (Equation 4.2). It is evident from Figure 31 that installing the squirrel cages provided significant radial stiffness and direct damping to the structural system. Based on the curve fit the installation of the squirrel cages resulted in a radial stiffness contribution of $55,155 \mathrm{lb} /$ in $(9.65 \mathrm{MN} / \mathrm{m})$. In addition to yielding 5 times the radial stiffness of the pitch stabilizers, installing the squirrel cage stiffeners also increased the direct damping by a factor more than 4. Although having higher baseline stiffness is advantageous when testing components that exhibit negative direct stiffness, increasing the direct damping of the baseline can pose resolution problems if the damping of the baseline is significantly higher than the damping of the test seals. The results from curve fitting the compliance also indicated that the modal mass of the system increased when the squirrel cages were attached due to the added motion of the exhaust air chambers that interface the stator assembly through the squirrel cages. The second method used to extract stiffness and damping was curve fitting the real and imaginary components (Figure 32) of the dynamic stiffness.

Table 3. Baseline Test Matrix - Impact Hammer Tests

\begin{tabular}{|c|c|c|c|}
\hline TEST NUMBER & SQUIRREL CAGES & SHAKER STINGERS & TEST TYPE \\
\hline \hline 6 & AT'TACHED & NOT AT'TACHED & IMPACT HAMMER-A/F \\
\hline 7 & AT'TACHED & NOT AT'TACHED & LOG DEC TEST \\
\hline 8 & NOT AT'TACHED & NOT AT'TACHED & LOG DEC TEST \\
\hline 9 & NOT AT'TACHED & NOT AT'TACHED & IMPACT HAMMER-A/F \\
\hline
\end{tabular}

$\left|\frac{A}{F}\right| \cdot \frac{1}{\omega^{2}}=\left|\frac{X}{F}\right|=\frac{1}{\sqrt{\left(K-M \omega^{2}\right)^{2}+(C \omega)^{2}}}$

$\left|\frac{X}{F}\right|=\frac{1}{C \omega} \quad$ when the phase $=90^{\circ}$ 
The real part of the dynamic stiffness was easily curve fit, but the imaginary component and damping posed problems due to the erratic fluctuations. The type of damping being measured in this system is structural or hysteretic damping, which is a function of vibration amplitude. Since these tests were conducted with an impact hammer, the 0-pk force excitation amplitude for each frequency component was not constant, resulting in varying values for the damping as shown in Figure 32. The values obtained for the stiffness and mass from Figure 32 agreed well with the results from curve fitting the compliance and so did the damping for certain frequency ranges.

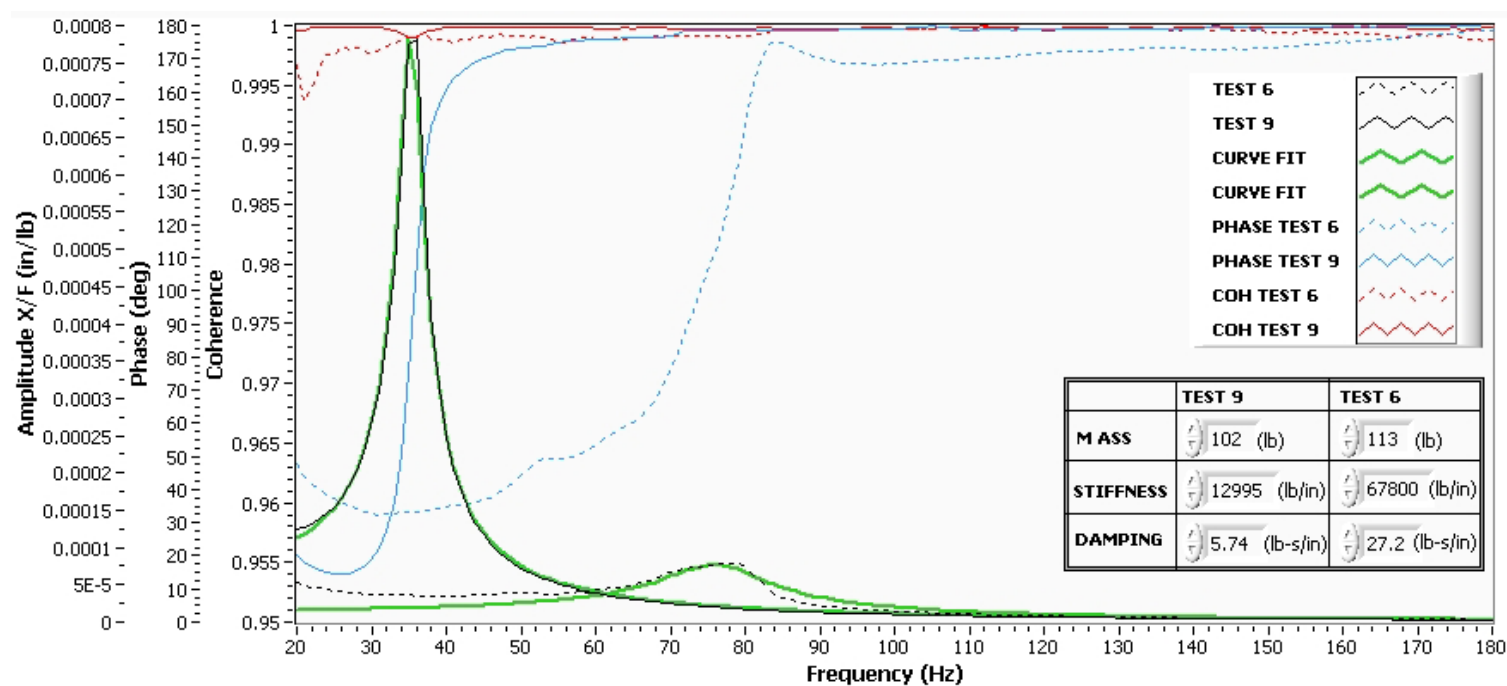

Figure 31. Structural Compliance of Test Rig Stator Assembly

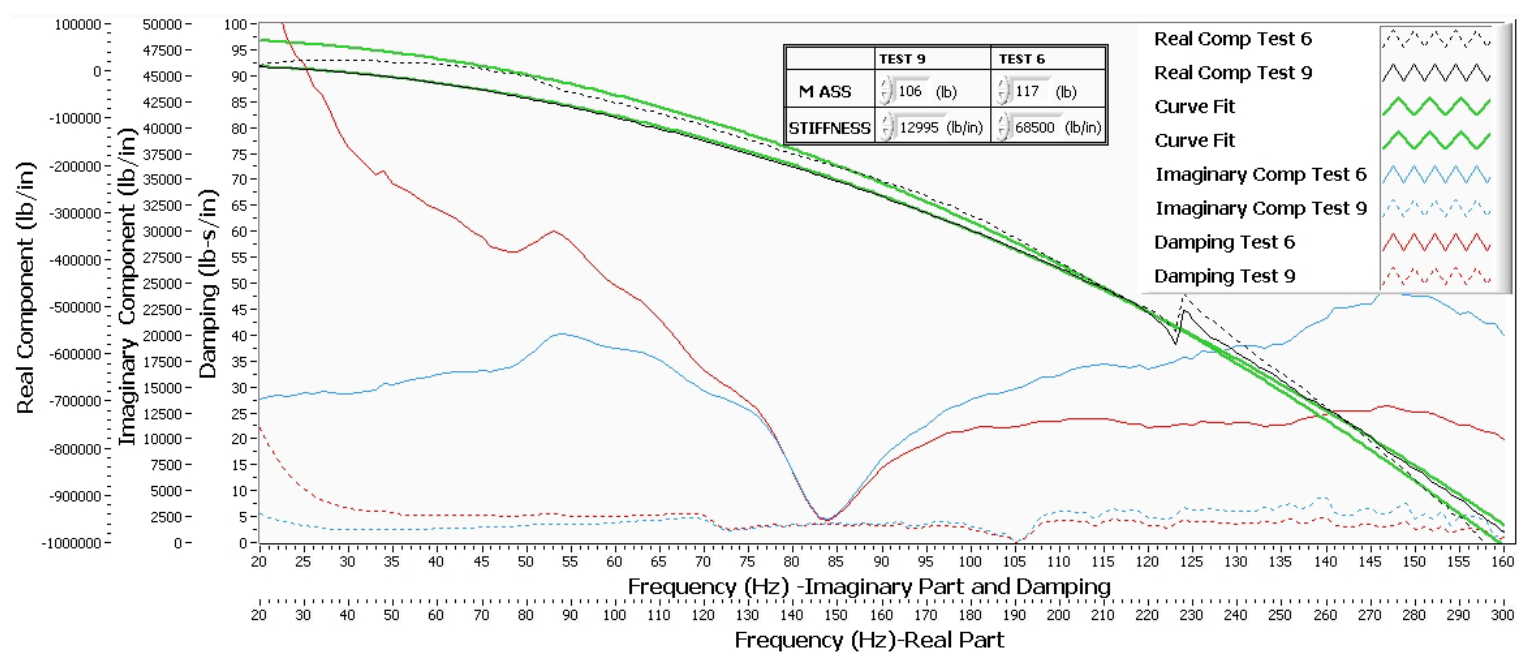

Figure 32. Real and Imaginary Components of Dynamic Stiffness 
Test 6 for 90-170 Hz shows a damping value between 20 to $30 \mathrm{lb}-\mathrm{sec} /$ in and Test 9 shows a damping value of $\sim 5 \mathrm{lb}-\mathrm{sec} /$ in for $30-160 \mathrm{~Hz}$. The last test conducted for the two configurations was a free vibration decay test. The time decay of the acceleration was converted to vibration motion and is shown in Figure 33, where the dotted lines are curve fits described by a function incorporating damping ratio and natural frequency. Using the modal mass and natural frequency, the damping was determined from Equation 4.3. A summary of the results for the impact hammer tests are shown in Table 4.

$\zeta=\frac{C}{C_{c}}=\frac{C}{2 m \omega_{n}}$

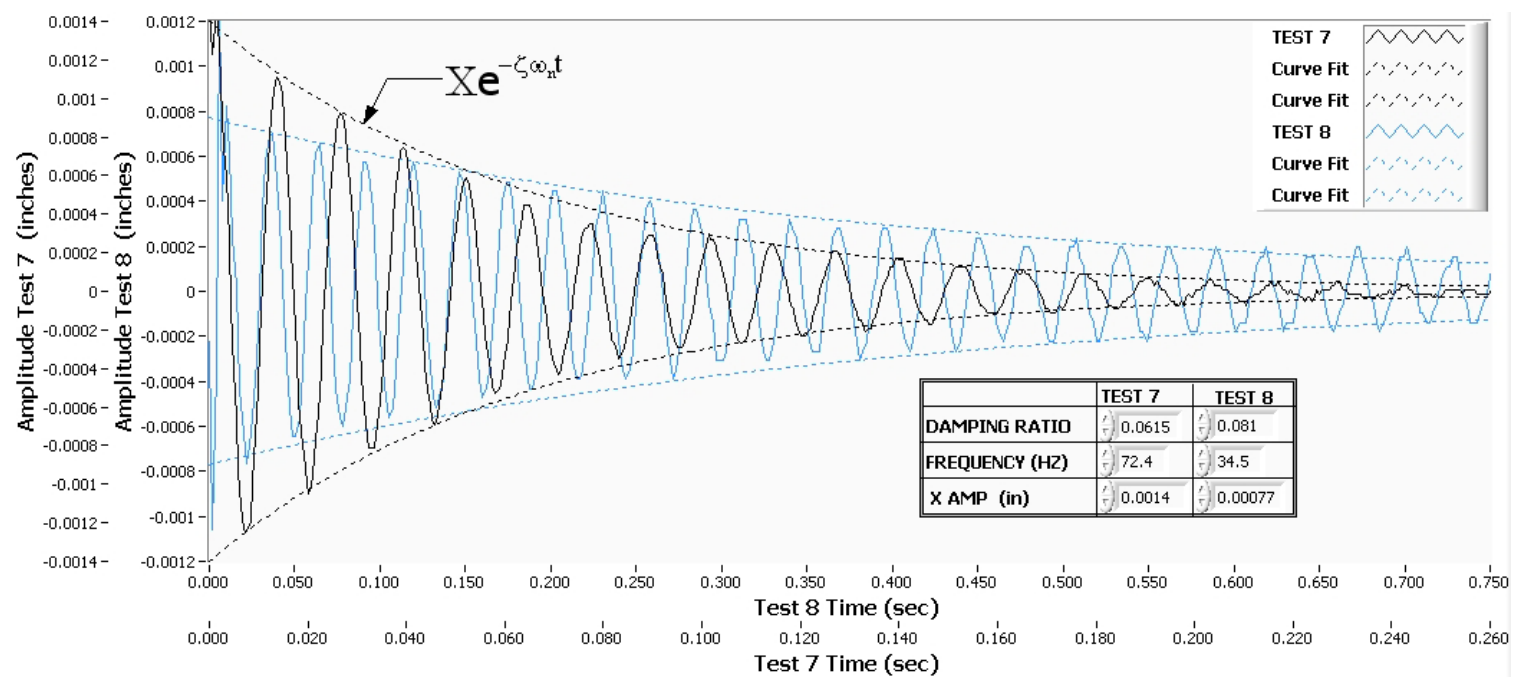

Figure 33. Test Rig Vibration Decay

Table 4. Structural Baseline Coefficients-Impact Hammer Tests

\begin{tabular}{|c|c|c|c|}
\hline \multirow[t]{2}{*}{ CURVE FIT } & \multicolumn{3}{|c|}{ TEST 6} \\
\hline & MASS (lb) & STIFFNESS (lb/in) & DAMPING (lb-s/in) \\
\hline COMPLIANCE & 113 & 67800 & 27.2 \\
\hline REAL PART & 117 & 68500 & - \\
\hline \multirow[t]{3}{*}{\begin{tabular}{|l|} 
IMAGINARY PART \\
\end{tabular}} & - & - & 21.3 \\
\hline & \multicolumn{3}{|c|}{ TEST 9} \\
\hline & MASS (lb) & STIFFNESS (lb/in) & DAMPING (lb-s/in) \\
\hline COMPLIANCE & 102 & 12995 & 5.74 \\
\hline \begin{tabular}{|l} 
REAL PART \\
\end{tabular} & 106 & 12995 & - \\
\hline \multirow[t]{3}{*}{ IMAGINARY PART } & - & - & 6.73 \\
\hline & \multicolumn{3}{|c|}{ TEST 7} \\
\hline & \multicolumn{2}{|c|}{ DAMPING RATIO } & DAMPING (lb-s/in) \\
\hline \multirow[t]{3}{*}{ VIBRATION DECAY } & & 0.0615 & 16.4 \\
\hline & \multicolumn{3}{|c|}{ TEST 8} \\
\hline & \multicolumn{2}{|c|}{ DAMPING RATIO } & DAMPING (lb-s/in) \\
\hline VIBRATION DECAY & & 0.081 & 9.27 \\
\hline
\end{tabular}


The test matrix for the baseline impedance tests is shown in Table 5, from which selected results are presented in Figure 34. The coefficients shown for all the impedance tests, including the baseline, have been divided by two in order to base results on one seal. Baseline tests were conducted for 150-700 psi inlet pressure at increments of 50 psi for both configurations (with and without squirrel cages). It is interesting that the direct damping increase with frequency whereas the direct stiffness decreases with frequency. The linear decrease in direct stiffness with increasing frequency is moderate for the 150 psi and 450 psi cases without the squirrel cage and is not indicative of a mass effect. Also, the fact that the 450 psi case with the squirrel cage possesses a different slope to the trend line of the 450 psi without the squirrel cage is not expected.

Table 5. Baseline Impedance Test Matrix

\begin{tabular}{|c|c|}
\hline \multicolumn{2}{|c|}{ WITHOUT SQUIRREL CAGES } \\
\hline TEST PRESSURE (PSI/BAR) & ROTOR SPEED (RPM) \\
\hline \hline $150 / 10.34$ & $10,200-20,200$ \\
\hline $200 / 13.78$ & $10,200-20,200$ \\
\hline $300 / 20.68$ & $10,200-20,200$ \\
\hline $350 / 24.13$ & $10,200-20,200$ \\
\hline $400 / 27.58$ & $10,200-20,200$ \\
\hline $450 / 31.03$ & $10,200-20,200$ \\
\hline $500 / 34.47$ & $10,200-20,200$ \\
\hline $600 / 41.37$ & $10,200-20,200$ \\
\hline $700 / 48.26$ & $10,200-20,200$ \\
\hline $400 / 27.58$ & Non-Rotating \\
\hline $450 / 31.03$ & Non-Rotating \\
\hline $500 / 34.47$ & Non-Rotating \\
\hline
\end{tabular}

\begin{tabular}{|c|c|}
\hline WITH SQUIRREL CAGES \\
\hline TEST PRESSURE (PSI/BAR) & ROTOR SPEED (RPM) \\
\hline \hline $450 / 31.03$ & $10,200-20,200$ \\
\hline $500 / 34.47$ & $10,200-20,200$ \\
\hline $600 / 41.36$ & $10,200-20,201$ \\
\hline $500 / 34.47$ & Non-Rotating \\
\hline $600 / 41.37$ & Non-Rotating \\
\hline
\end{tabular}

The coefficients for these pressurized impedance tests are not only generated from structural components, but also the fluid pressure forces generated between stator and rotor components. Picardo (2003) also reported direct stiffness coefficients decreasing with frequency for labyrinth seal test on the same test rig. For the low inlet pressure case and at low frequencies the direct stiffness values agree well with the impact hammer test stiffness results. Recall that the baseline results presented here are per seal, therefore need to be multiplied by 2 when compared to the results from the impact hammer tests. The 150 psi case at $20 \mathrm{~Hz}$ has a total direct stiffness value of 12,168 lb/in in comparison to 12,995 lb/in from the impact hammer tests, which results in a $6 \%$ difference that is most likely attributed to the end labyrinth seals at pressure. The results shown in Figure 34 also indicate that the squirrel cages have a stiffness value of $52,354 \mathrm{lb} /$ in at $20 \mathrm{~Hz}$. The radial stiffness of the 
squirrel cages compares very well with results from the impact hammer tests with less than a $1 \%$ deviation between the two methods. The direct damping also agrees well for the 150 psi case with no squirrel cage, which shows a total system damping of $4.92 \mathrm{lb}-\mathrm{sec} /$ in at $70 \mathrm{~Hz}$ compared to the $5.74 \mathrm{lb}-\mathrm{sec} /$ in from the impedance tests. Baseline direct damping results for frequencies below $50 \mathrm{~Hz}$ are not presented due to the large uncertainty. Also measured using the impedance method was the baseline cross-coupled coefficients (Figure 35). The crosscoupled stiffness and damping coefficients for the baseline have small values, which increase as frequency is increased.
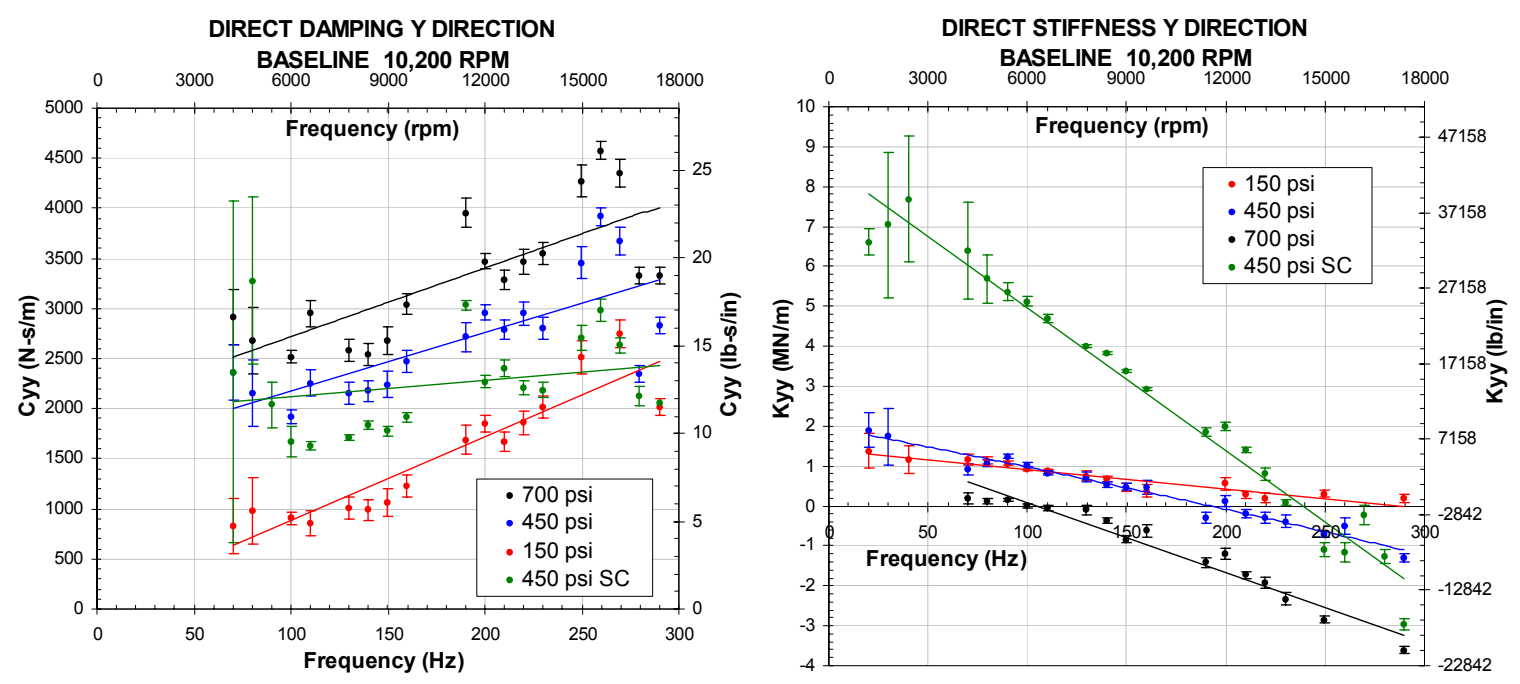

Figure 34. Baseline Direct Coefficients Y Direction-Impedance Method
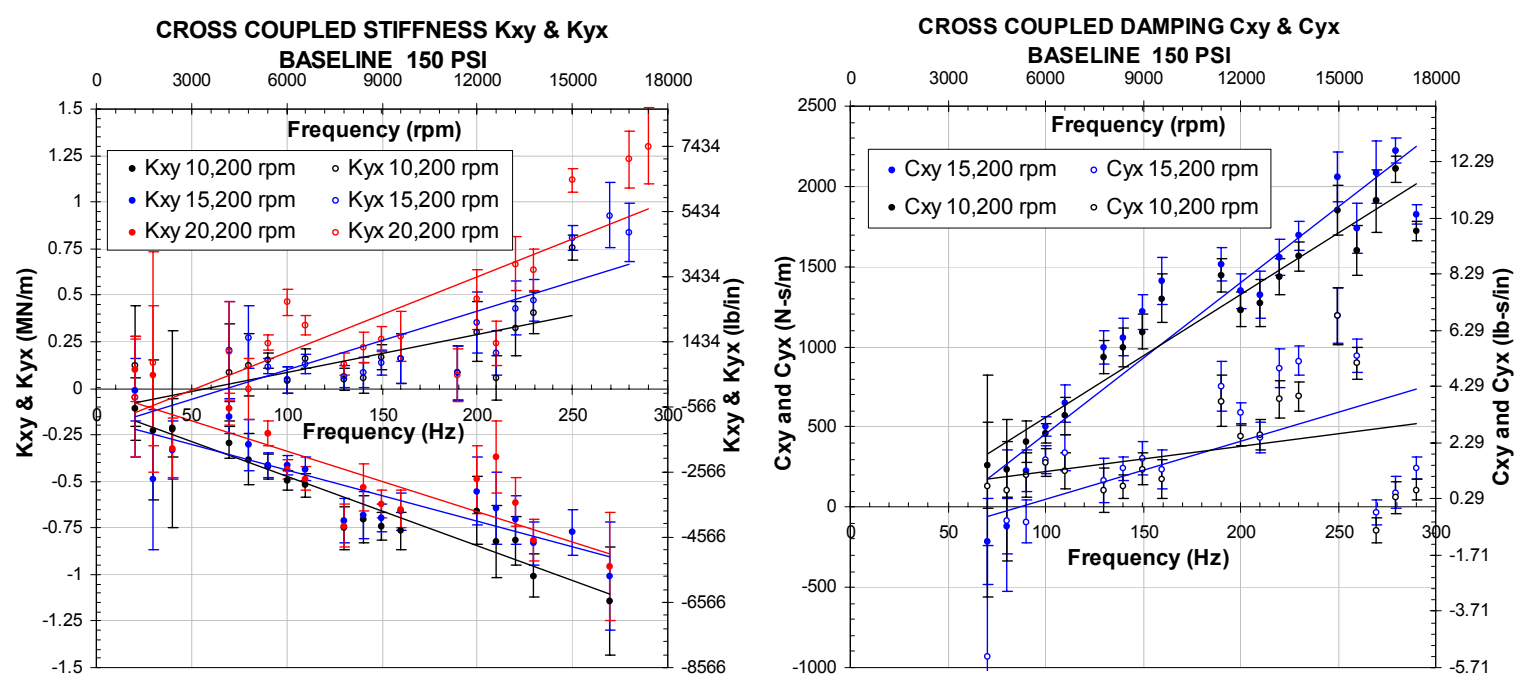

Figure 35. Baseline Cross-coupled Coefficients-Impedance Method 


\subsection{Twelve Bladed PDS Force Coefficients}

The test matrix for the twelve bladed PDS is shown in Table 6. The ideal testing scheme was to test for three pressure ratios and three rotor speeds for each seal. Although the twelve bladed PDS was tested for three pressure ratios only the low pressure ratio is presented, where the inlet pressure was 1,000 psi (68.9 bar). This is due to the significantly large statistical uncertainties associated with the data in combination with the significantly small force coefficients. The direct coefficients are shown in Figures 36-37, from which several conclusions can be made. First, both Figures 36 and 37 shows that the diverging clearance PDS possesses more than twice the direct damping compared to the straight through configuration, and the diverging seal also shows more frequency dependence at the lower frequencies. Another conclusion is that the diverging seal generated significant negative stiffness coefficients that increase with excitation frequency. The straight through configuration yielded direct stiffness coefficients that are small in magnitude, but also increase with excitation frequency.

Table 6. Test Matrix-12 Bladed PDS Impedance Method

\begin{tabular}{|c|c|c|c|}
\hline TEST & ROTOR SPEED & PRESSURE RATIO & CLEARANCE RATIO \\
\hline $\mathbf{1}$ & $10,200 \mathrm{rpm}$ & 0.214 & 1 to 1 \\
\hline $\mathbf{2}$ & $15,200 \mathrm{rpm}$ & 0.211 & 1 to 1 \\
\hline $\mathbf{3}$ & $20,200 \mathrm{rpm}$ & 0.212 & 1 to 1 \\
\hline $\mathbf{4}$ & $10,200 \mathrm{rpm}$ & 0.179 & 1 to 2 \\
\hline $\mathbf{5}$ & $15,200 \mathrm{rpm}$ & 0.179 & 1 to 2 \\
\hline $\mathbf{6}$ & $20,200 \mathrm{rpm}$ & 0.179 & 1 to 2 \\
\hline
\end{tabular}

Inlet Plenum Pressure 1,000 psi (68.9 bar)
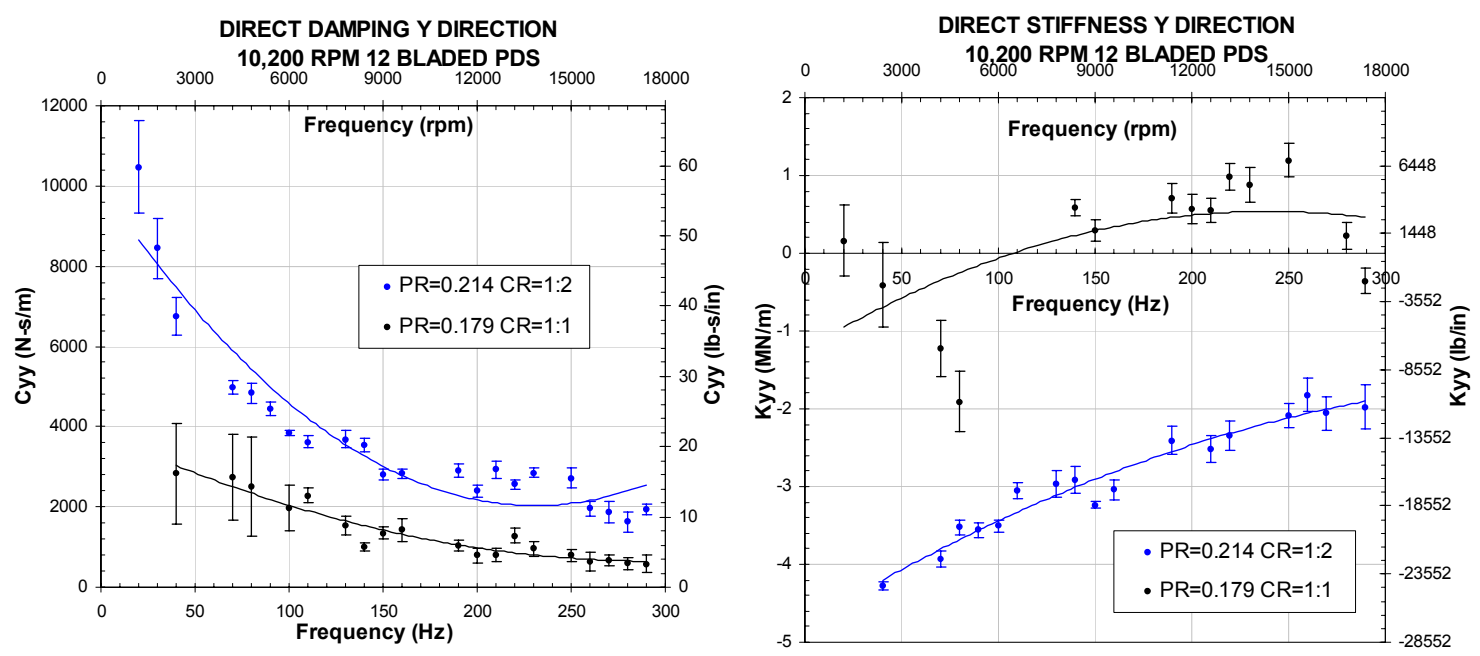

Figure 36. Twelve Bladed PDS 10,200 rpm: Direct Coefficients Impedance Method 
The next set of graphs (Figure 38) show the cross-coupled stiffness for the two configurations at 10,200 rpm and 20,200 rpm. An important conclusion from Figure 38 is that almost all the cross-coupled stiffness data points possess the same sign for a particular frequency. As discussed in Section III, same sign stiffness cross-coupling coefficients do not generate destabilizing forces and therefore do not subtract from the direct damping, but significant values of same sign stiffness cross-coupling can induce stiffness asymmetry into a rotor-bearing system.
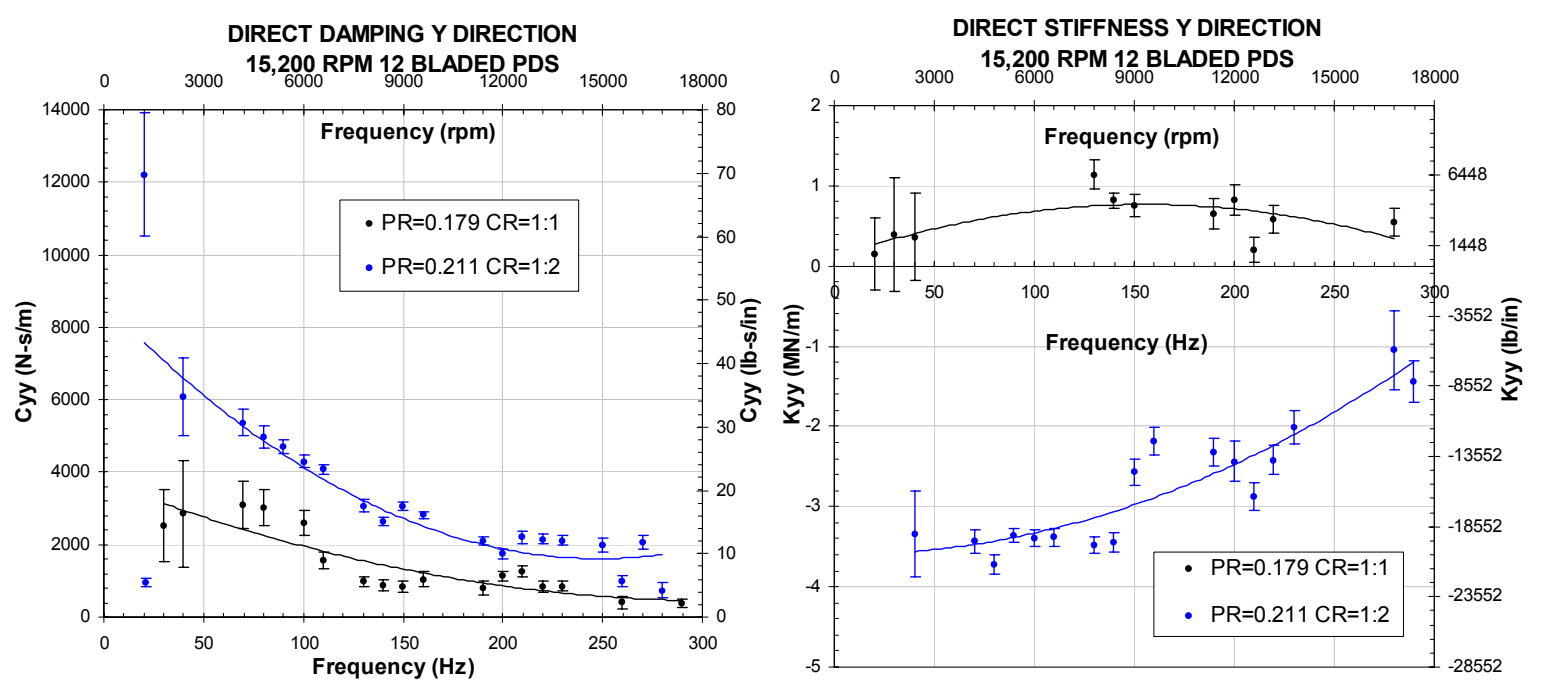

Figure 37. Twelve Bladed PDS 15,200 rpm: Direct Coefficients Impedance Method
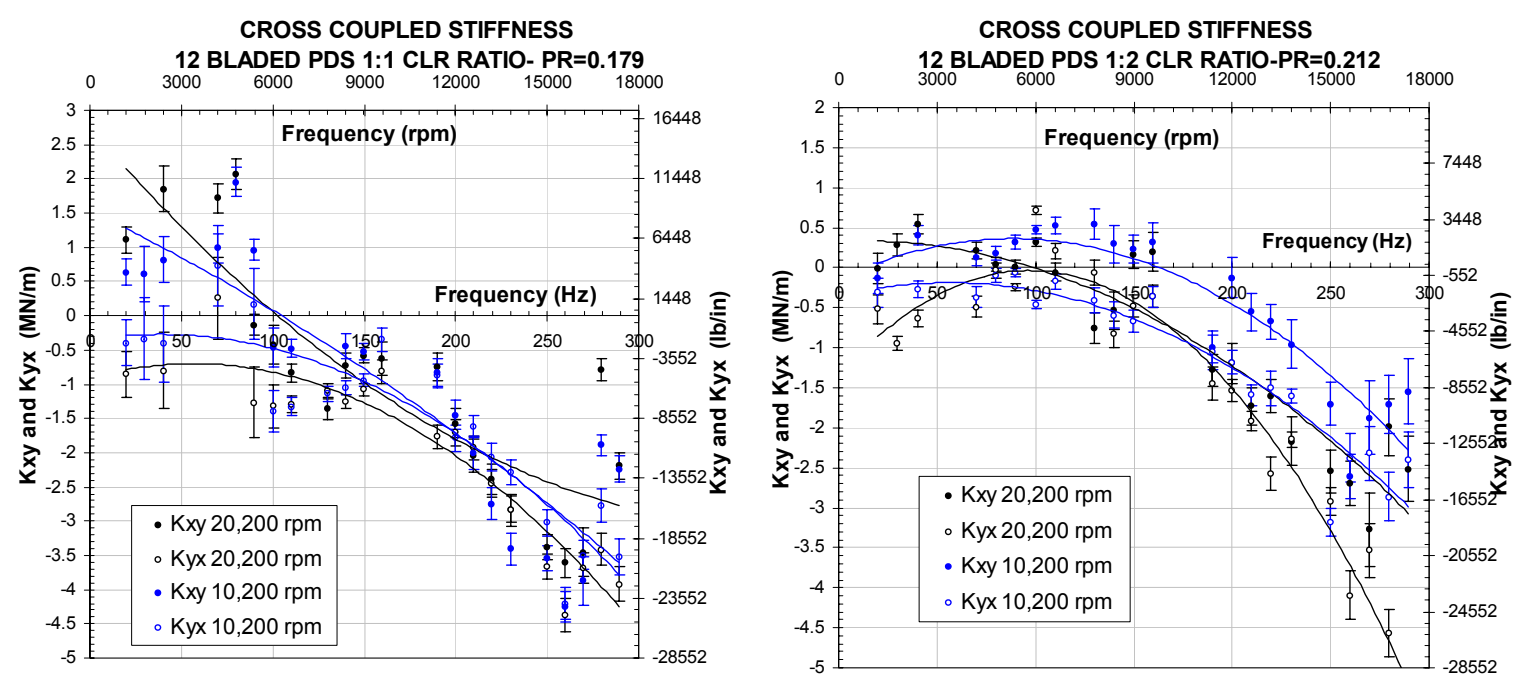

Figure 38. Twelve Bladed PDS: Cross-Coupled Stiffness Coefficients Impedance Method 
The cross-coupled damping coefficients for selected cases are shown in Figure 39. As shown in Figure 21, cross-coupled damping generates a radial force in line with the direct stiffness force. These coefficients are of less concern because of the small magnitudes and the inability of cross-coupled damping to directly influence rotordynamic stability.
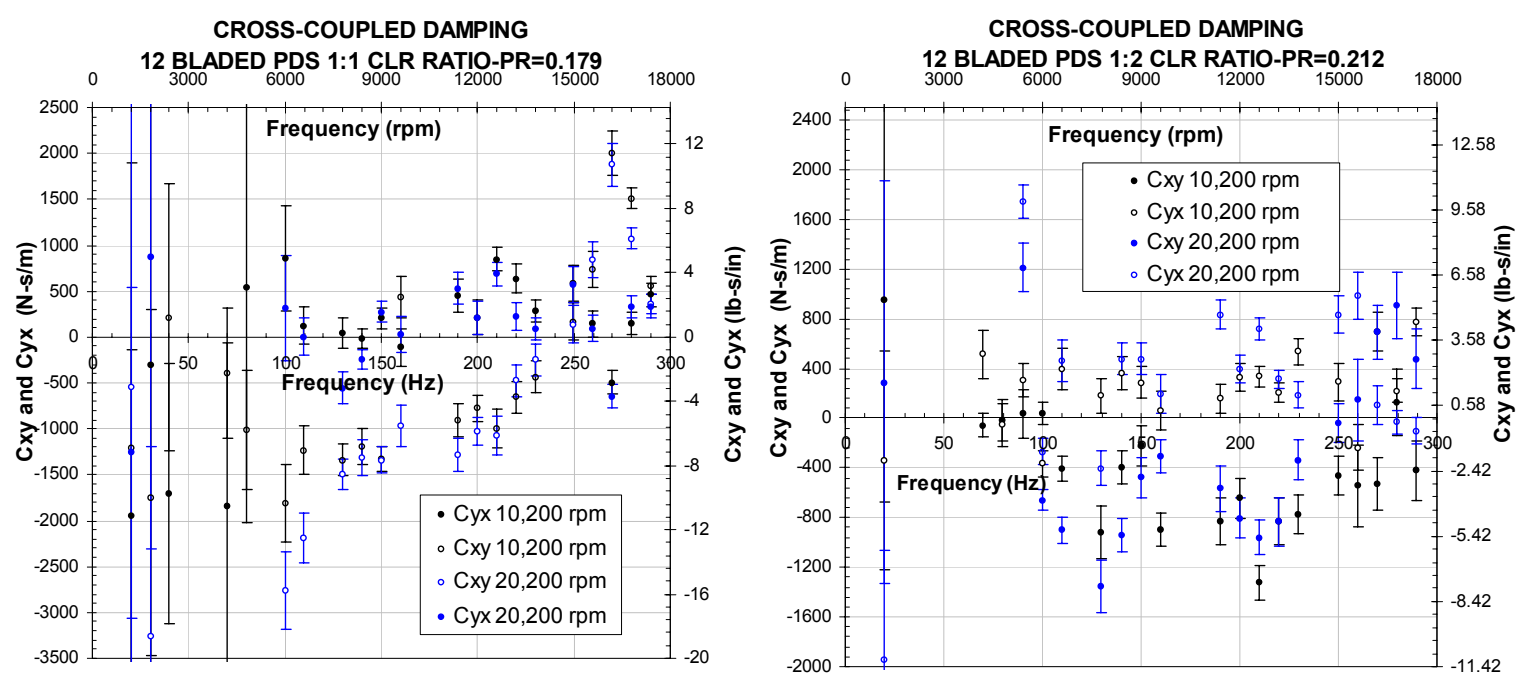

Figure 39. Twelve Bladed PDS- Cross-coupled Damping Coefficients Impedance Method

\subsection{Eight Bladed PDS Force Coefficients}

Experimental results presented in this section are for the eight bladed seal configurations as listed in Table 7. For the 1:1 clearance ratio seal three rotor speeds at three different pressure ratios were tested resulting in 9 tests. After testing the 1:1 clearance ratio seal, the seals were modified by machining notches into the downstream blades at a 1:1.5 clearance ratio, resulting in a diverging seal. The modified diverging clearance eight bladed seals posed static instability problems and restricted dynamic testing due to the negative stiffness, while the test rig was pressurized to the inlet plenum pressure of 1,000 psig (68.9 bar). To accommodate the large negative stiffness of the diverging clearance test seals, a pair of squirrel cage stiffeners was manufactured (as shown in Figures 15-16) to increase the baseline radial stiffness; requiring another round of baseline tests with the baseline inserts and the squirrel cages. Even with the squirrel cages, which added $\sim 56,000 \mathrm{lb} /$ in to the system, the eight bladed 1:1.5 clearance ratio seal could only be tested for one pressure ratio due to the negative stiffness. 
Table 7. Test Matrix-8 Bladed PDS Impedance Method

\begin{tabular}{|c|c|c|c|}
\hline TEST & ROTOR SPEED & PRESSURE RATIO & CLEARANCE RATIO \\
\hline $\mathbf{1}$ & $10,200 \mathrm{rpm}$ & 0.116 & 1 to 1 \\
\hline $\mathbf{2}$ & $15,200 \mathrm{rpm}$ & 0.112 & 1 to 1 \\
\hline $\mathbf{3}$ & $20,200 \mathrm{rpm}$ & 0.110 & 1 to 1 \\
\hline $\mathbf{4}$ & $10,200 \mathrm{rpm}$ & 0.326 & 1 to 1 \\
\hline $\mathbf{5}$ & $15,200 \mathrm{rpm}$ & 0.319 & 1 to 1 \\
\hline $\mathbf{6}$ & $20,200 \mathrm{rpm}$ & 0.308 & 1 to 1 \\
\hline $\mathbf{7}$ & $10,200 \mathrm{rpm}$ & 0.516 & 1 to 1 \\
\hline $\mathbf{8}$ & $15,200 \mathrm{rpm}$ & 0.509 & 1 to 1 \\
\hline $\mathbf{9}$ & $20,200 \mathrm{rpm}$ & 0.490 & 1 to 1 \\
\hline $\mathbf{1 0}$ & $10,200 \mathrm{rpm}$ & 0.531 & 1 to 1.5 \\
\hline $\mathbf{1 1}$ & $15,200 \mathrm{rpm}$ & 0.520 & 1 to 1.5 \\
\hline $\mathbf{1 2}$ & $20,200 \mathrm{rpm}$ & 0.502 & 1 to 1.5 \\
\hline
\end{tabular}

Figures 40-42 display the direct damping and stiffness for all four seals at 10,200 $\mathrm{rpm}, 15,200 \mathrm{rpm}$, and 20,200 rpm. The effect of pressure ratio in the straight through configuration seems to have the most predominant affect at lower frequency ranges of 20-80 $\mathrm{Hz}$, but for higher frequencies the effect of pressure ratio on the direct damping is small. The damping plots in these figures suggests that the parameter that influences the damping capacity the most is the clearance ratio, when comparing the 1:1 clearance ratio cases with the 1:1.5 clearance ratio test at the pressure ratio $(\mathrm{PR})$ of $\sim 0.5$. As observed with the twelve bladed tests, the rotor speed has very little if no effect on the direct damping values of the eight bladed results in Figures 40-42. Also shown in Figure 40-42 are the results for the direct stiffness of the eight bladed PDS configurations. The stiffness results show almost identical values for the high and medium pressure ratio cases, whereas the low pressure case of $\mathrm{PR}=\sim 0.1$ and clearance ratio 1:1 shows lower stiffness even though the delta $\mathrm{P}$ across the seal is larger. The reduction of direct stiffness for increasing pressure differential is contradictory to the conventional theory predictions. Conventional damper seal theory predicts positive direct stiffness for straight through configurations and increasing positive direct stiffness for increasing pressure differentials. Negative direct stiffness for the eight bladed straight through PDS was also measured using the dynamic pressure method, which will be shown in the following section. The diverging 1:1.5 clearance ratio seal possesses negative stiffness throughout the entire frequency range and is significant in magnitude, especially at low frequencies. Next, the cross-coupled stiffness coefficients for the eight bladed PDS configurations are shown in Figure 43. The main conclusion from these results is that the cross-coupling stiffness coefficients are of the same sign and therefore are not destabilizing and do not subtract from the direct damping. 

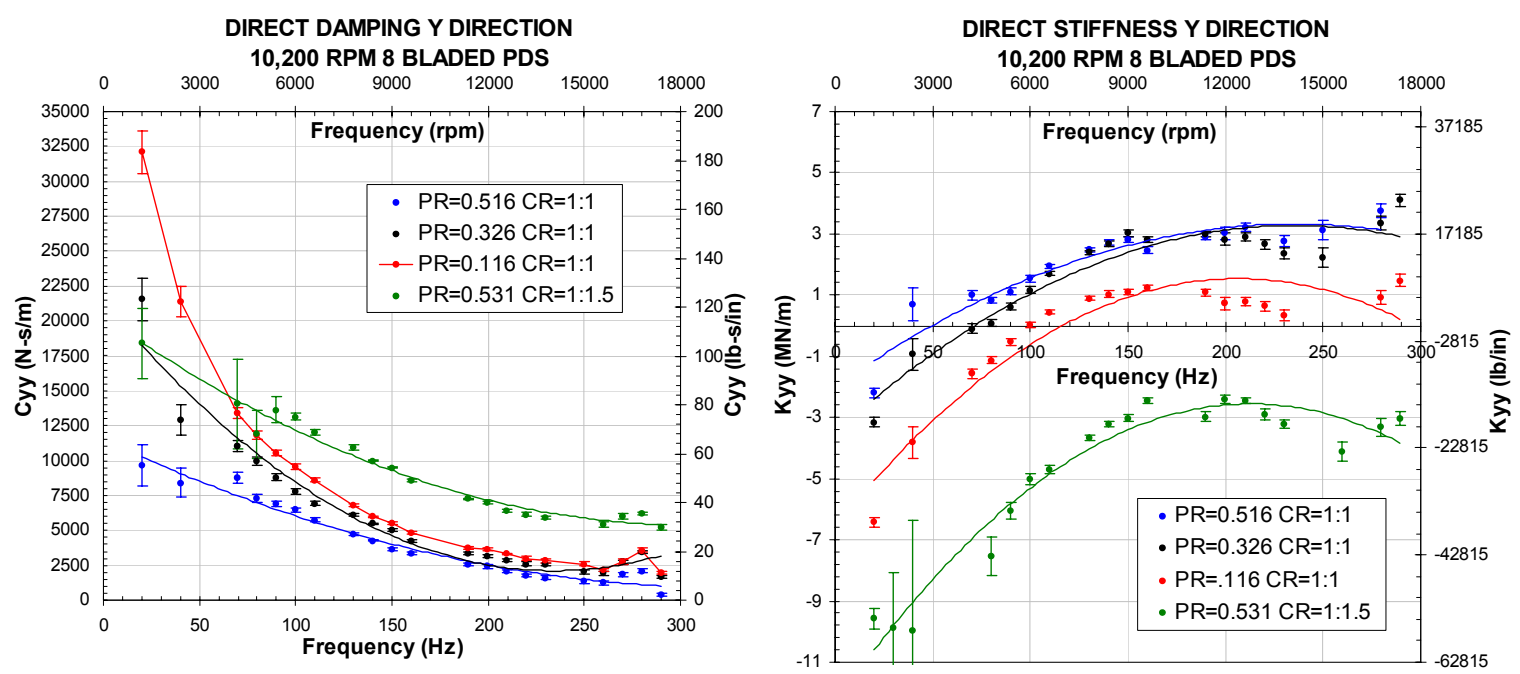

Figure 40. Eight Bladed PDS-Direct Coefficients 10,200 rpm Impedance Method
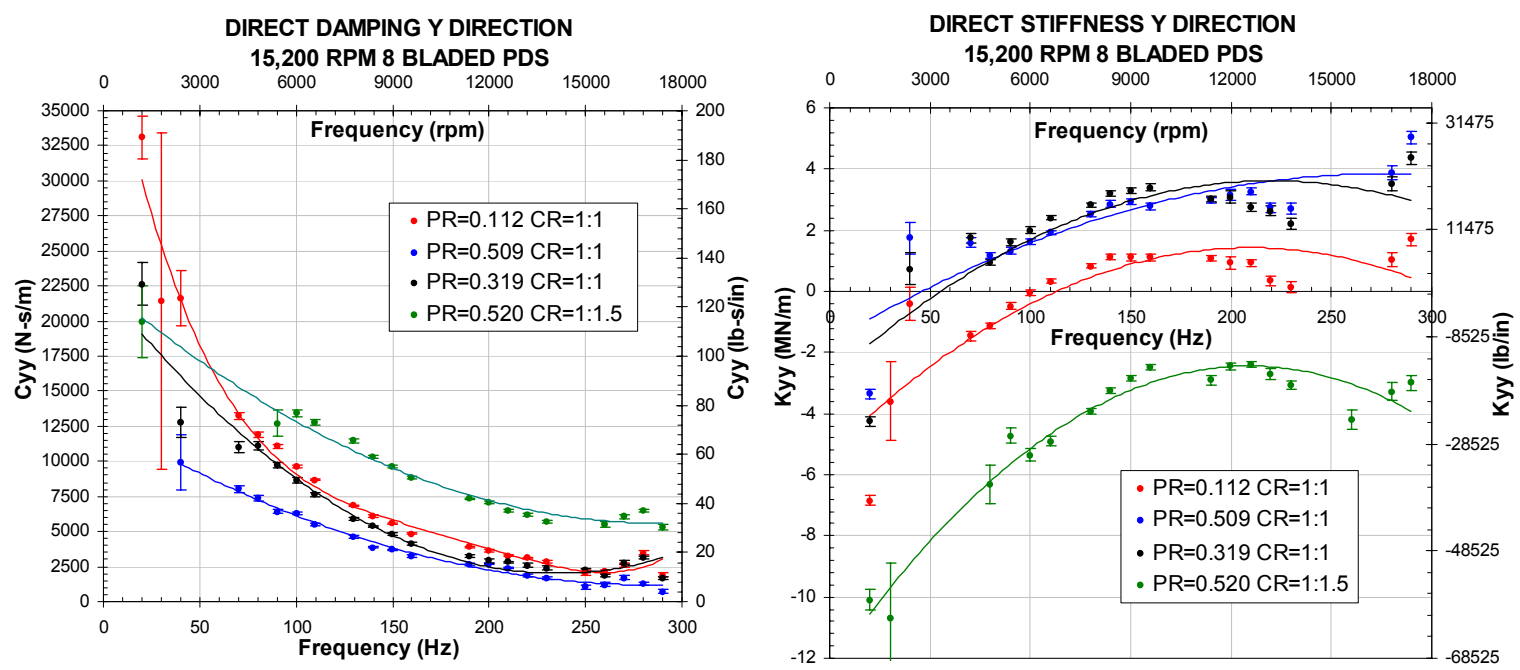

Figure 41. Eight Bladed PDS-Direct Coefficients 15,200 rpm Impedance Method 

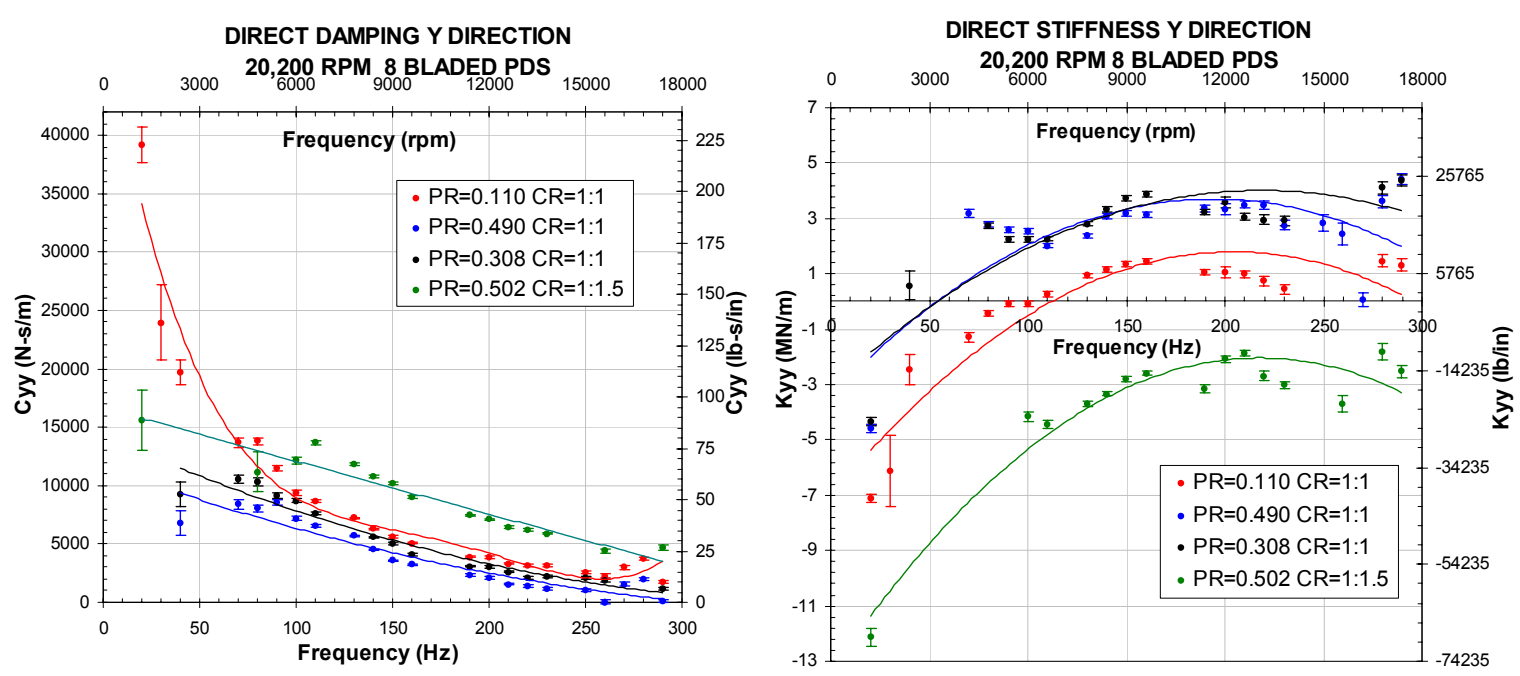

Figure 42. Eight Bladed PDS-Direct Coefficients 20,200 rpm Impedance Method
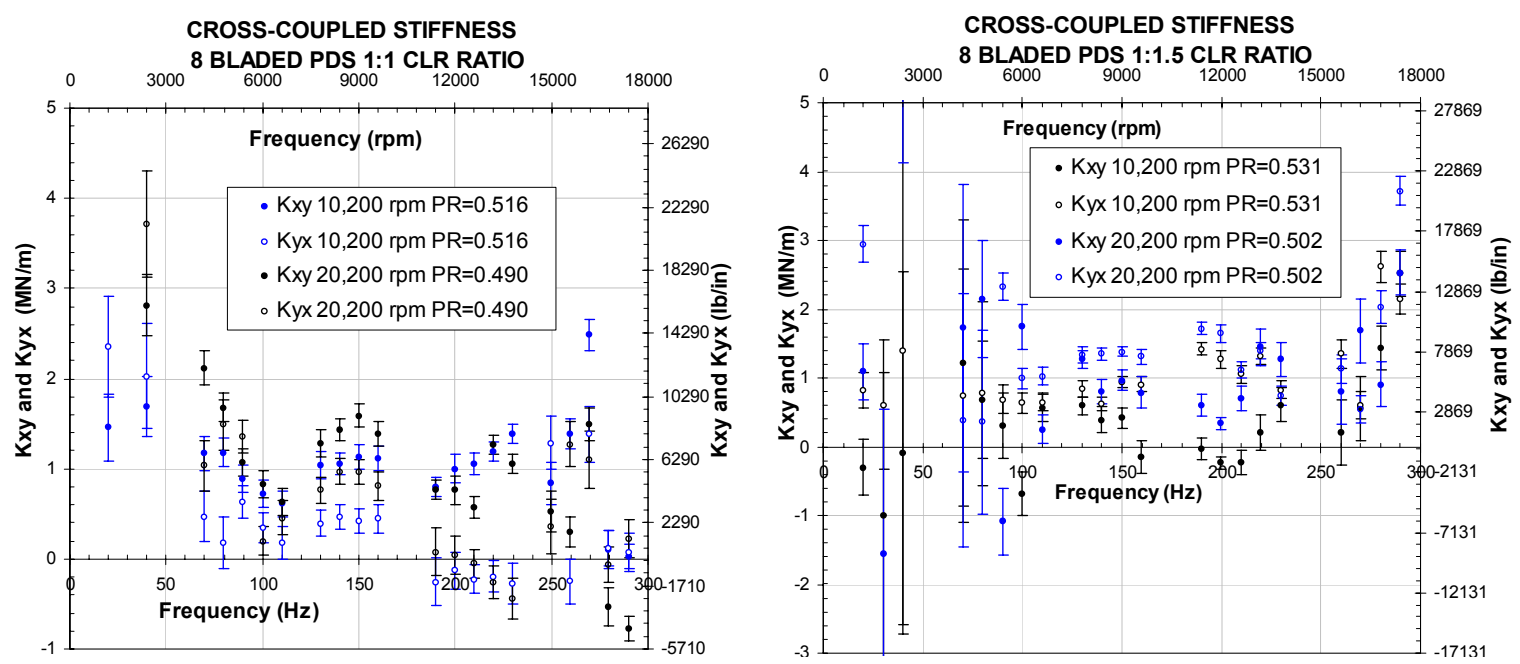

Figure 43. Eight Bladed PDS-Cross-Coupled Stiffness Coefficients Impedance Method

\subsection{Conventional Six Bladed PDS Force Coefficients}

The next two sections in this section will address the two six bladed PDS configurations: (1) conventional six bladed design and (2) fully partitioned (FP) six bladed design. Fist the results for the conventional design are presented for the test matrix shown in Table 8. Only three tests were performed successfully due to the negative stiffness of the test seals. The direct coefficients for 10,200 rpm are shown in Figure 44 at two different pressure ratios. The smaller pressure ratio of 0.496 yielded slightly more direct damping and possessed a less frequency dependent direst stiffness. 
The trend of the cross-coupled stiffness for theses seals (Figure 45) is similar to the previous results. The data points are initially positive at low frequency ranges and decrease in values breaching the zero mark then continuing to decrease with frequency. As shown in the previous results of cross-coupled stiffness, the Kxy and Kyx values possess the same sign, therefore not affecting the effective damping. The cross-coupled damping coefficients are small in magnitude, exhibit large uncertainties compared to the magnitude of the data points, and are also erratic not revealing a distinctive trend.

Table 8. Test Matrix-Conventional 6 Bladed PDS Impedance Method

\begin{tabular}{|c|c|c|c|}
\hline TEST & ROTOR SPEED & PRESSURE RATIO & CLEARANCE RATIO \\
\hline $\mathbf{1}$ & $10,200 \mathrm{rpm}$ & 0.6 & 1 to 2 \\
\hline $\mathbf{2}$ & $15,200 \mathrm{rpm}$ & 0.6 & 1 to 2 \\
\hline $\mathbf{3}$ & $10,200 \mathrm{rpm}$ & 0.498 & 1 to 2 \\
\hline
\end{tabular}

Inlet Plenum Pressure 1,000 psi (68.9 bar)
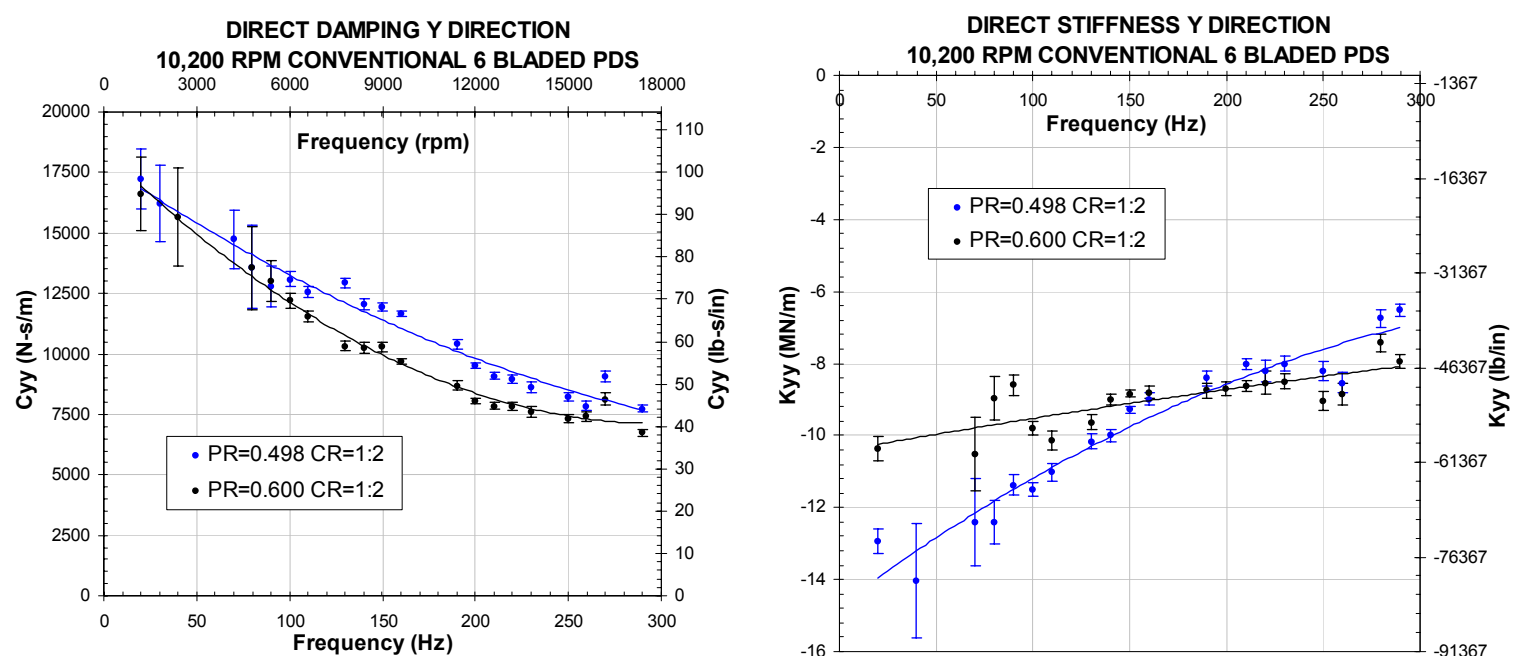

Figure 44. Conventional Six Bladed PDS-Direct Coefficients Impedance Method 

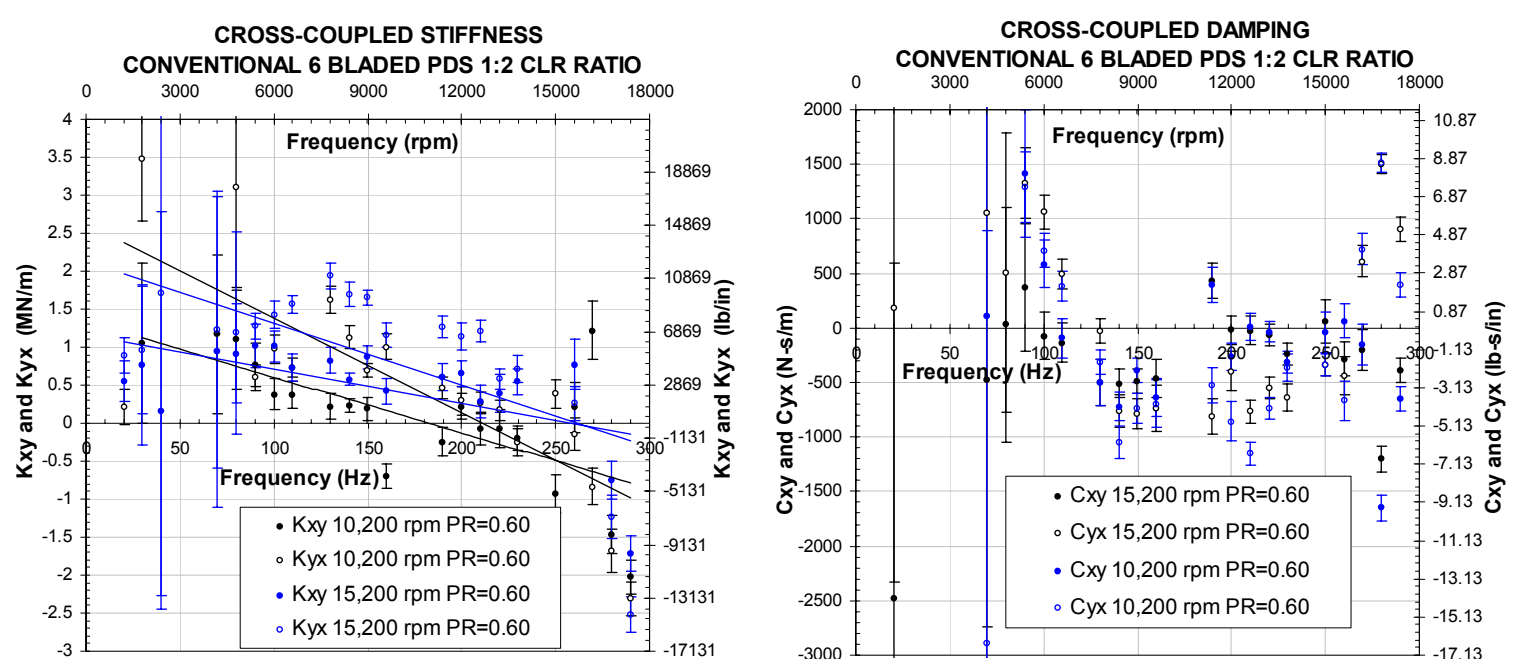

Figure 45. Conventional Six Bladed PDS-Cross-Coupled Coefficients Impedance Method

\subsection{Fully Partitioned Six Bladed PDS Force Coefficients}

The last seal configuration tested using the impedance method was the FP PDS. Recall that the FP PDS was identical to the conventional six bladed seal except for the partition walls in the inactive plenums. Each of the two inactive plenums of the conventional design were transformed into eight equally spaces converging cavities with an axial pitch (0.203 in) more than three time less than the active cavity pitch (0.742 in). Table 9 shows the test matrix and note that this seal was tested successfully for two pressure ratios and three rotor speeds, unlike the conventional PDS that exhibited negative stiffness problems. Figures 46-47 illustrates the direct coefficients at 10,200 rpm and 15,200 rpm. Note that as observed in previous seals the rotor surface speed does not influence the magnitude of the coefficients. The direct damping is the largest measured from all the seals at the low frequencies that peak at a value of $\sim 250 \mathrm{lb}-\mathrm{s} /$ in. At $70-80 \mathrm{~Hz}$, where the first mode eigenvalue is located for most centrifugal compressors, the damping measures $\sim 120 \mathrm{lb}-\mathrm{s} / \mathrm{in}$. The direct stiffness behavior is very interesting because the stiffness breaches the zero mark and becomes positive at around $100 \mathrm{~Hz}$. This behavior is very different than the conventional PDS design where the direct stiffness was measured to be negative throughout the entire frequency range. After becoming positive, the direct stiffness plateaus and reaches a magnitude of $22,000 \mathrm{lb} /$ in. The last figure (Figure 48) shows that the stiffness cross coupling coefficients for this seal also has same signs. 
Table 9. Test Matrix-Fully Partitioned 6 Bladed PDS Impedance Method

\begin{tabular}{|c|c|c|c|}
\hline TEST & ROTOR SPEED & PRESSURE RATIO & CLEARANCE RATIO \\
\hline $\mathbf{1}$ & $10,200 \mathrm{rpm}$ & 0.602 & 1 to 2 \\
\hline $\mathbf{2}$ & $15,200 \mathrm{rpm}$ & 0.588 & 1 to 2 \\
\hline $\mathbf{3}$ & $20,200 \mathrm{rpm}$ & 0.572 & 1 to 2 \\
\hline $\mathbf{4}$ & $10,200 \mathrm{rpm}$ & 0.522 & 1 to 2 \\
\hline $\mathbf{5}$ & $15,200 \mathrm{rpm}$ & 0.52 & 1 to 2 \\
\hline $\mathbf{6}$ & $20,200 \mathrm{rpm}$ & 0.501 & 1 to 2 \\
\hline
\end{tabular}

Inlet Plenum Pressure 1,000 psi (68.9 bar)
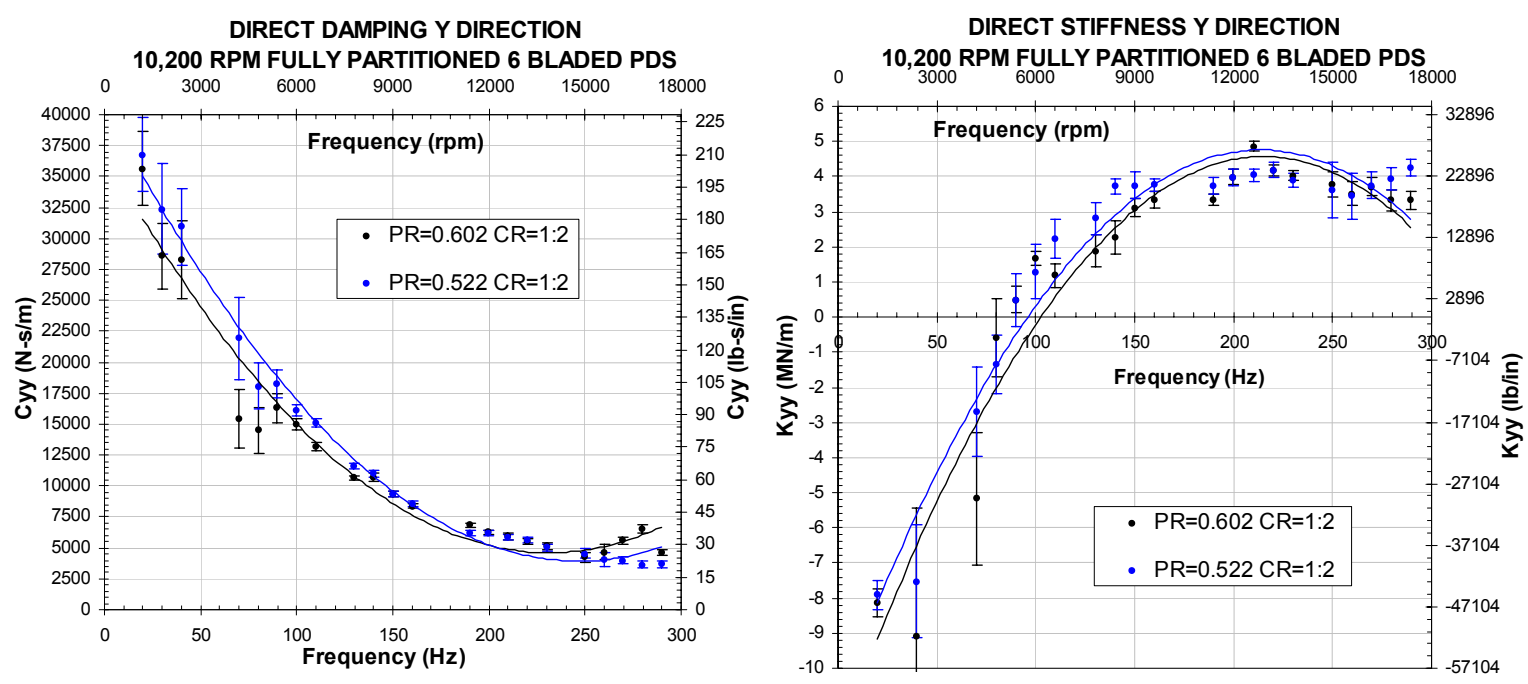

Figure 46. FP Six Bladed PDS-Direct Coefficients 10,200 rpm Impedance Method
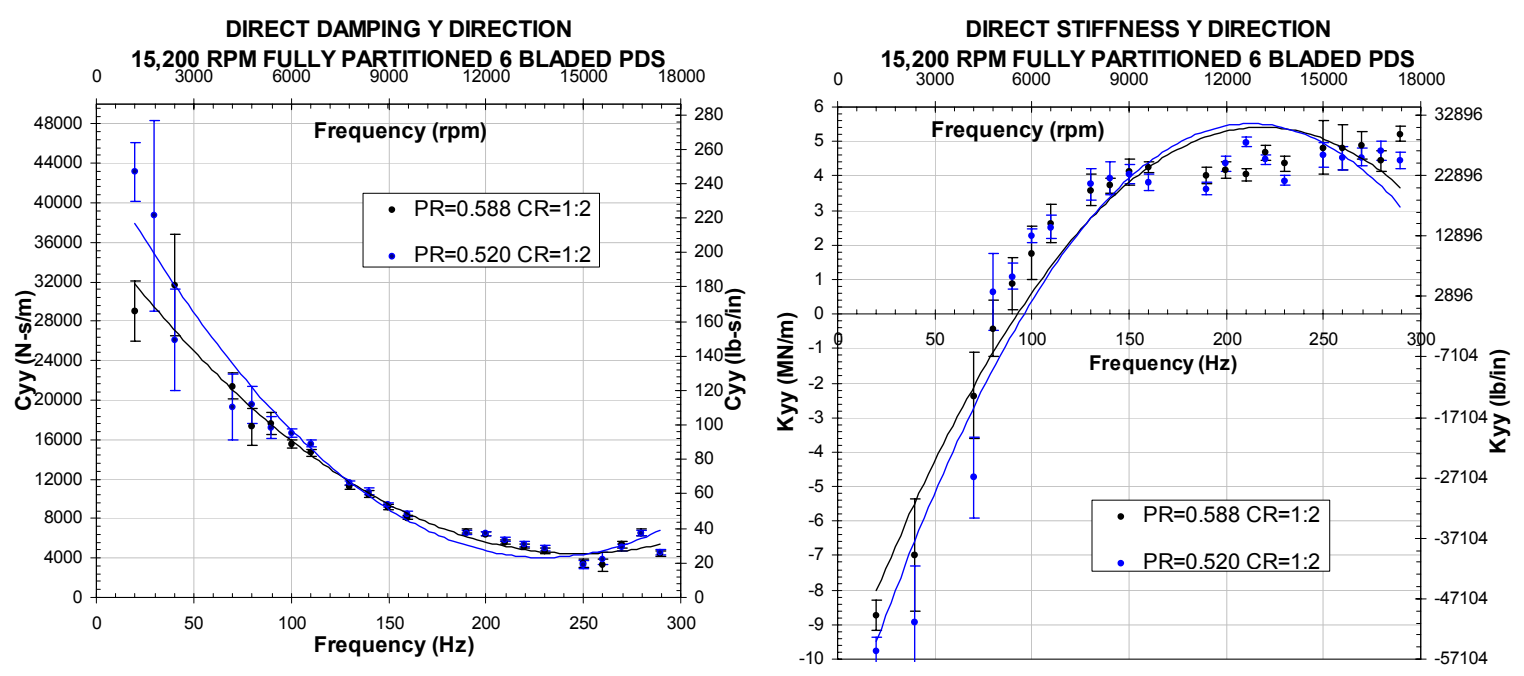

Figure 47. FP Six Bladed PDS-Direct Coefficients 15,200 rpm Impedance Method 

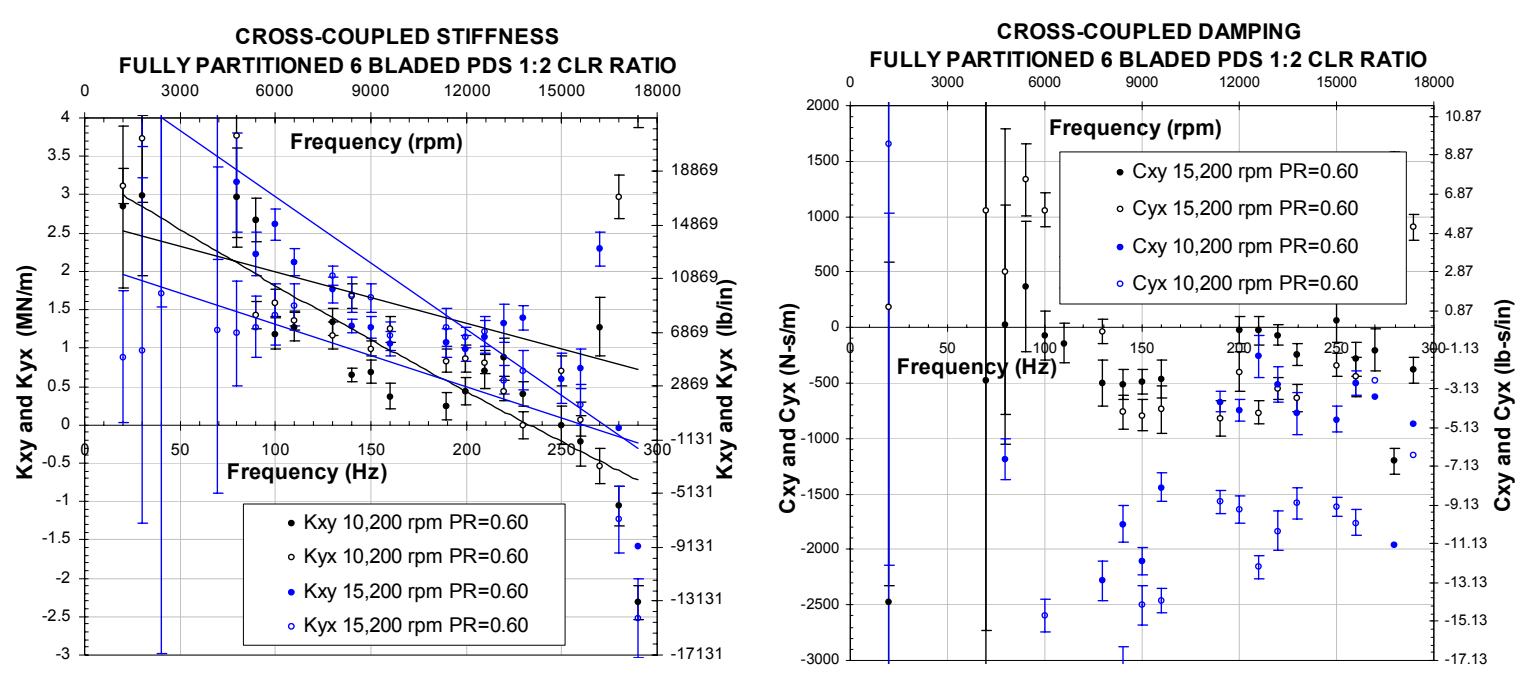

Figure 48. FP Six Bladed PDS-Cross-Coupled Coefficients

\subsection{Comparison of Impedance Test Results}

The focus of this section was the impedance test results of frequency dependent force coefficients for several PDS configurations (Table 10) at different pressure ratios and rotor speeds. Figures 49-50 summarize and compare frequency dependent force coefficients for all the different PDS configurations. Clearly, the magnitude of the direct damping is increased as the number of blades is decreased. Decreasing the number of blades increases the overall axial pitch of the active cavities for the same pitch ratio. The 12 bladed 1:1 clearance ratio PDS possessed the least amount of damping, and the largest amount of damping was produced from the six bladed PDS configurations. It is interesting to note that the damping at the low frequency range $(20-100 \mathrm{~Hz})$ for the FP PDS shows significantly more damping than the conventional design and above $100 \mathrm{~Hz}$ the conventional design retains higher values. The FP design is more frequency dependent compared to the conventional design. Figure 50 shows the direct stiffness for the different PDS. The 12 bladed PDS with the 1:1 clearance ratio was tested to have significantly small values for direct stiffness whereas the six bladed conventional PDS has significant negative stiffness. The eight bladed diverging seal has the second highest negative stiffness values. The trends for the test seals are in accordance with the theory that predicts the highest negative stiffness for diverging PDS, with the least number for blades. The most interesting data from the stiffness is the FP PDS results. Usually in PDS design and optimization, diverging seals that are modified to produce more positive direct damping yield high negative stiffness values. 
For example, the damping can be increased by increasing the clearance ratio, by reducing the number of blades, or increasing the pitch ratio. All of these modifications to the design produce higher damping but also generate higher negative stiffness for conventionally designed PDS. The results here have shown that modifying the conventional design by inserting baffle walls into the inactive plenums yields much higher positive stiffness. The reasoning and dynamics behind this phenomenon with the FP PDS will be discussed in Section V.

Table 10. Configurations Tested Using the Impedance Method

\begin{tabular}{|c|c|c|c|}
\hline CONFIG & SEAL & CLEARANCE RATIO & NUMBER OF TESTS \\
\hline $\mathbf{1}$ & 12 BLADED PDS & to 1 & 3 \\
\hline $\mathbf{2}$ & 12 BLADED PDS & 1 to 2 & 3 \\
\hline $\mathbf{3}$ & 8 BLADED PDS & 1 to 1 & 9 \\
\hline $\mathbf{4}$ & 8 BLADED PDS & 1 to 1.5 & 3 \\
\hline $\mathbf{5}$ & 6 BLADED PDS & 1 to 2 & 3 \\
\hline $\mathbf{6}$ & FP 6 BLADED PDS & 1 to 2 & 6 \\
\hline
\end{tabular}

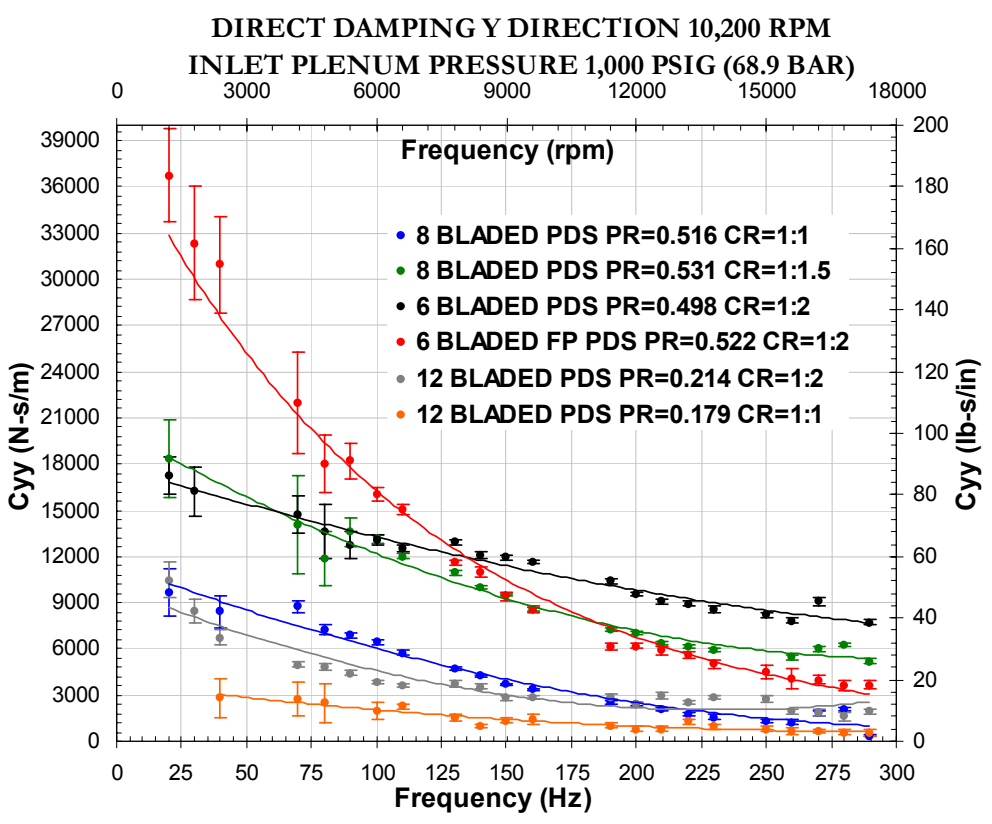

Figure 49. Direct Damping Coefficients: Impedance Method 


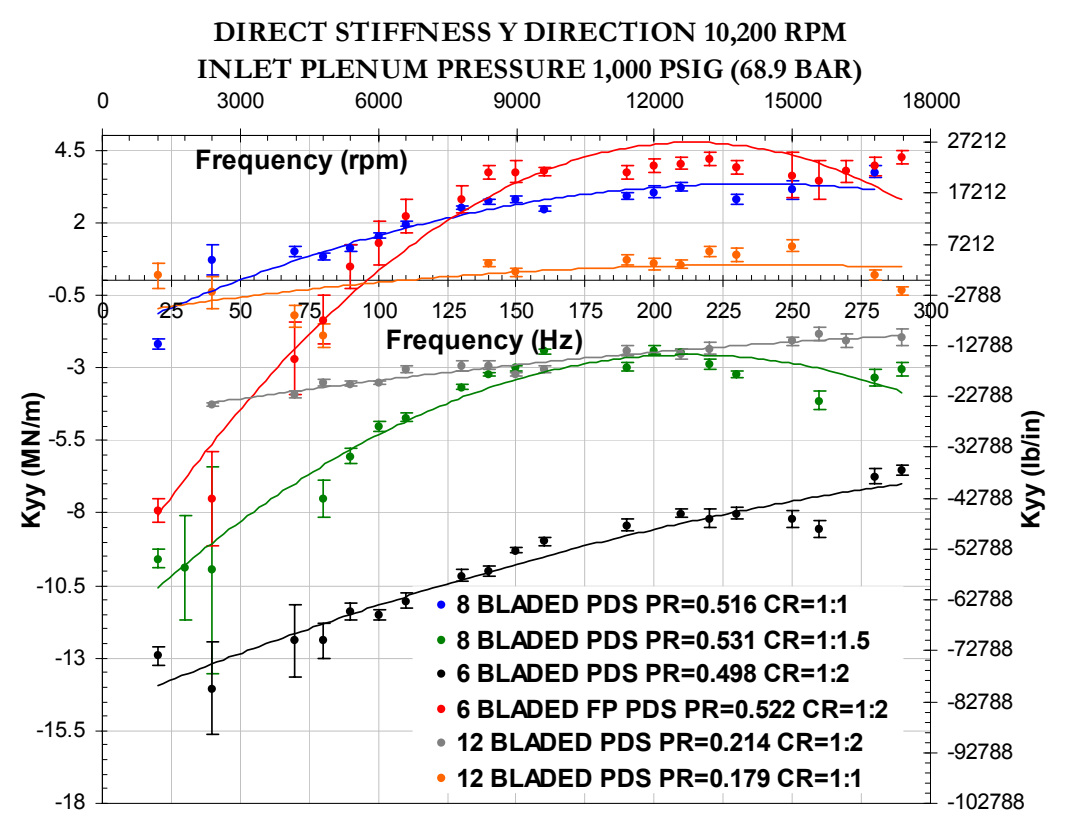

Figure 50. Direct Stiffness Coefficients: Impedance Method 


\section{DYNAMIC AND STATIC PRESSURE TESTS}

This section focuses on experiments performed using dynamic cavity pressures in efforts to determine frequency dependent rotordynamic coefficients. Results shown in this section describe and present testing conducted on 4 different PDS configurations: (1) 8 bladed PDS 1:1 clearance ratio, (2) 8 bladed PDS 1:1.5 clearance ratio, (3) conventional 6 bladed PDS 1:2 clearance ratio, and (4) FP 6 bladed seal 1:2 clearance ratio. Due to space limitations for the 8 bladed PDS design, the 8 bladed force coefficients were only measured for cavities 3 and 5. Also only direct force coefficients are presented for the 8 bladed PDS. Both six bladed PDS designs however show direct and cross-coupled coefficient results for each cavity, therefore allowing the calculation of the overall seal coefficients. This required measurement of dynamic pressures in 40 cavities of the 6 bladed FP PDS design and dynamic pressures in 24 cavities of the conventional design.

\subsection{Cavity Coefficients for Eight Bladed PDS}

The main objectives for the following tests were to use dynamic pressure measurements to calculate the peak pressure magnitude, pressure phase (relative to vibration), force density $(\mathrm{P} / \mathrm{Y})$, and the direct stiffness/damping coefficients of the two cavities. Results from the experiments are compared with theoretical predictions based on the conventional pocket damper seal (PDS) theory by Schultz (1996). Analytical predictions for single pressurized cavities were calculated using a MathCAD program. In addition to the objectives listed above, this section also shows experimental measurements of asynchronous pressure frequencies in the cavities, which have a direct effect on the force density. Two different seal configurations were tested: (a) 8 bladed PDS with 1:1 clearance ratio and (b) 8 bladed PDS with 1:1.5 clearance ratio (notched).

The stator housing configuration used for the 8 bladed cavity pressure tests is shown in Figures 17 and 51, which shows dynamic and static pressure transducers in active cavities 3 and 5 at 90 degrees (in line with the $\mathrm{Y}$ direction). Although this testing configuration is identical to the conventional impedance test set-up shown in Section III, the measurements used for calculating rotordynamic coefficients is different. Conventional impedance testing, for determining dynamic force coefficients, requires the use of a baseline measurement, which accounts for additional forces within the test assembly that are not related to the seal impedance. Unlike the conventional testing method described in previous section, the use of the cavity pressures for determining dynamic force coefficients provides a means to directly 
measure the seal forces without having to account for a baseline subtraction. Not only does this approach eliminate some of the error that accompanies the baseline subtraction, it also allows the direct comparison with the damper seal theory.

The testing scheme involved shaking the stator assembly with respect to the rotor in the $\mathrm{Y}$ direction and measuring vibration motion, static inlet pressure, static back pressure, static cavity pressures, inlet flow temperature, and dynamic cavity pressures. The excitation signal was chosen to be a fixed sine wave for the frequency range of $20 \mathrm{~Hz}-200 \mathrm{~Hz}$ and all tests were conducted with the same pressure differential 450psi-470psi with an inlet plenum pressure of 1,000 psi. The test matrix for these tests is shown in Table 11. Note that the diverging seal was tested for two different vibration amplitudes, whereas the straight-through damper seal was only tested for one vibration amplitude.

The data was acquired using a 16 channel simultaneous sampling NI 4472 DAQ board. The testing method used was a single frequency excitation, not the pseudorandom excitation discussed in Section III. After acquiring the data a LABVIEW virtual instrument was developed for data reduction. Figure 52 shows the front panel DAQ settings and Figure 53 shows the front panel output for the virtual instrument (VI). The VI shown in Figure 53 was developed to display the time trace, the frequency spectra, and also uses 12 averaged sets of data for each excitation frequency to calculate and display the results. The time trace plot possesses all four dynamic signals (M2, M6, P3, P5), but the spectrum plots display the average of M2 and M6 and the two dynamic cavity pressures. Results for the peak signal amplitudes, pressure phase, cavity coefficients, and force density are also displayed in the VI.

The methodology for experimentally determining the direct cavity coefficients was based on two equations (5.1-5.2). These equations define the direct stiffness and damping contributions from a single cavity in a PDS, where $A_{p}$ is the projected area of a single cavity, $\mathrm{y}$ is the 0 -pk vibration amplitude, $\omega$ is the vibration frequency, and $\mathrm{P}_{\mathrm{S}, \mathrm{C}}$ are the pressure components with respect to the displacement and velocity. To better understand the terms in 5.1-5.2 refer to the example vibration signal and pressure signals shown in Figure 54. Consider a sinusoidal motion $Y(\mathrm{t})$ with two different types of cavity pressure signals $\mathrm{P}_{1}(\mathrm{t})$ and $\mathrm{P}_{2}(\mathrm{t})$. The $\mathrm{P}_{2}(\mathrm{t})$ signal is considered to have a phase angle that leads the vibration motion by an angle $90>\phi 2>0$ and the $P_{1}(t)$ signal leads the vibration by an angle $90>\phi 1>180$. These time trace signals can be represented as rotating vectors with the reference zero angle defined at $Y(t)$. 


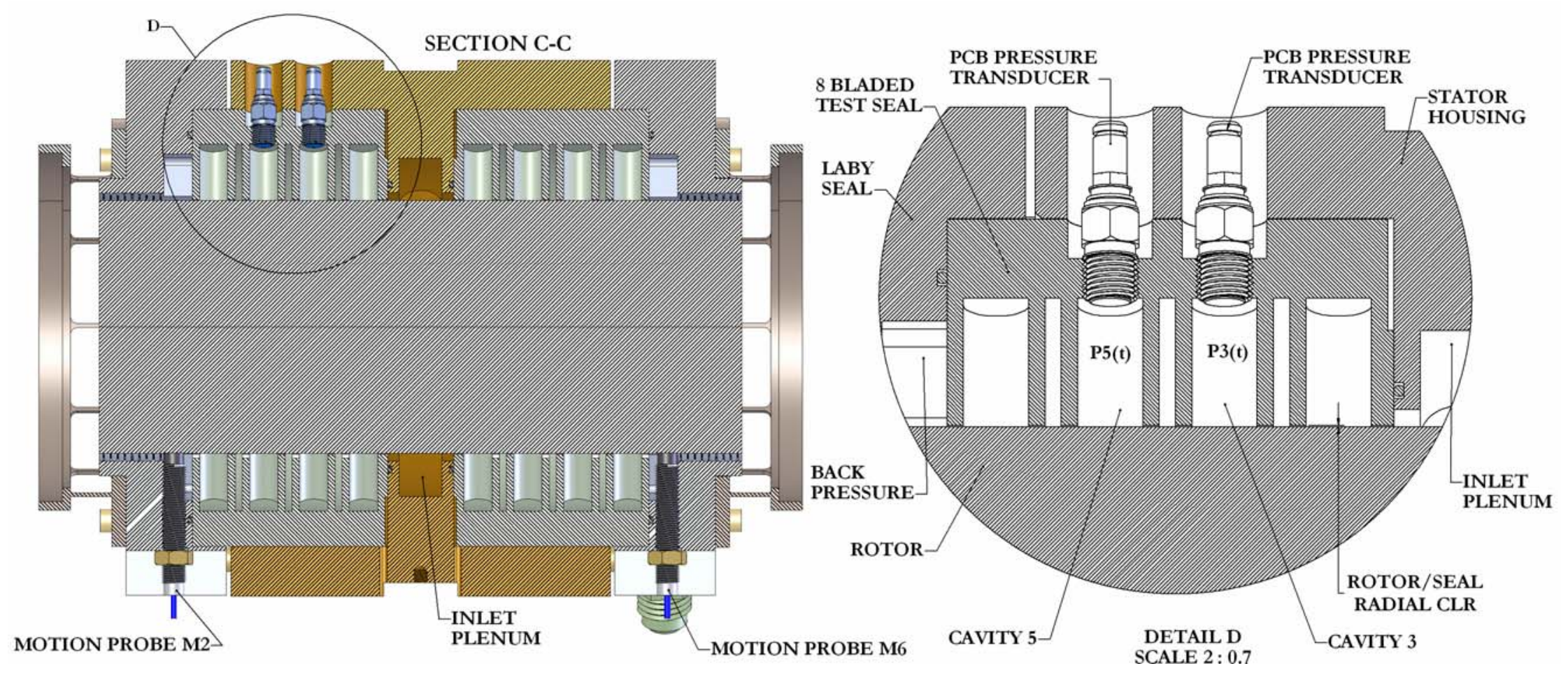

Figure 51. Stator Assembly Section View C-C: Eight Bladed Seal Cavity Pressures 
The $Y$ direction displacement therefore is related to the stiffness coefficient and the $\dot{Y}$ direction represents the velocity, which relates to the damping coefficient. Next, one can decompose the two pressure signals $\mathrm{P}_{1}(\mathrm{t})$ and $\mathrm{P}_{2}(\mathrm{t})$ into the two directions defined by $Y$ and $\dot{Y}$.

Table 11. Test Matrix- Eight Bladed Dynamic Pressure Tests

\begin{tabular}{|c|c|c|c|c|c|}
\hline \multicolumn{2}{|c|}{8 Bladed 1:1 Clearance Ratio } & \multirow{2}{*}{\begin{tabular}{|l|}
$1000-530$ psi Delta $P$ \\
VIBRATION AMPLITUDE (mils) \\
\end{tabular}} & \multicolumn{2}{|c|}{8 Bladed 1:1.5 Clearance Ratio } & \multirow{2}{*}{\begin{tabular}{|l|}
$1000-545$ psi Delta P \\
VIBRATION AMPLITUDE (mils) \\
\end{tabular}} \\
\hline TEST \# & FREQUENCY (Hz) & & TEST \# & FREQUENCY (Hz) & \\
\hline 1 & 20 & $\sim 1.2$ & 11 & 20 & $\sim 1.2$ \\
\hline 2 & 40 & $\sim 1.2$ & 12 & 40 & $\sim 1.2$ \\
\hline 3 & 60 & $\sim 1.2$ & 13 & 60 & $\sim 1.2$ \\
\hline 4 & 80 & $\sim 1.2$ & 14 & 80 & $\sim 1.2$ \\
\hline 5 & 100 & $\sim 1.2$ & 15 & 100 & $\sim 1.2$ \\
\hline 6 & 120 & $\sim 1.2$ & 16 & 120 & $\sim 1.2$ \\
\hline 7 & 140 & $\sim 1.2$ & 17 & 140 & $\sim 1.2$ \\
\hline 8 & 160 & $\sim 1.2$ & 18 & 160 & $\sim 1.2$ \\
\hline 9 & 180 & $\sim 1.2$ & 19 & 180 & $\sim 1.2$ \\
\hline 10 & 200 & $\sim 1.2$ & 20 & 200 & $\sim 1.2$ \\
\hline \multicolumn{2}{|c|}{8 Bladed 1:1.5 Clearance Ratio } & $1000-545$ psi Delta P & & & \\
\hline TEST \# & FREQUENCY (Hz) & VIBRATION AMPLITUDE (mils) & & & \\
\hline 21 & 20 & $\sim 1.8$ & & & \\
\hline 22 & 40 & $\sim 1.8$ & & & \\
\hline 23 & 60 & $\sim 1.8$ & & & \\
\hline 24 & 80 & $\sim 1.8$ & & & \\
\hline 25 & 100 & $\sim 1.8$ & & & \\
\hline 26 & 120 & $\sim 1.8$ & & & \\
\hline 27 & 140 & $\sim 1.8$ & & & \\
\hline 28 & 160 & $\sim 1.8$ & & & \\
\hline 29 & 180 & $\sim 1.8$ & & & \\
\hline 30 & 200 & $\sim 1.8$ & & & \\
\hline
\end{tabular}

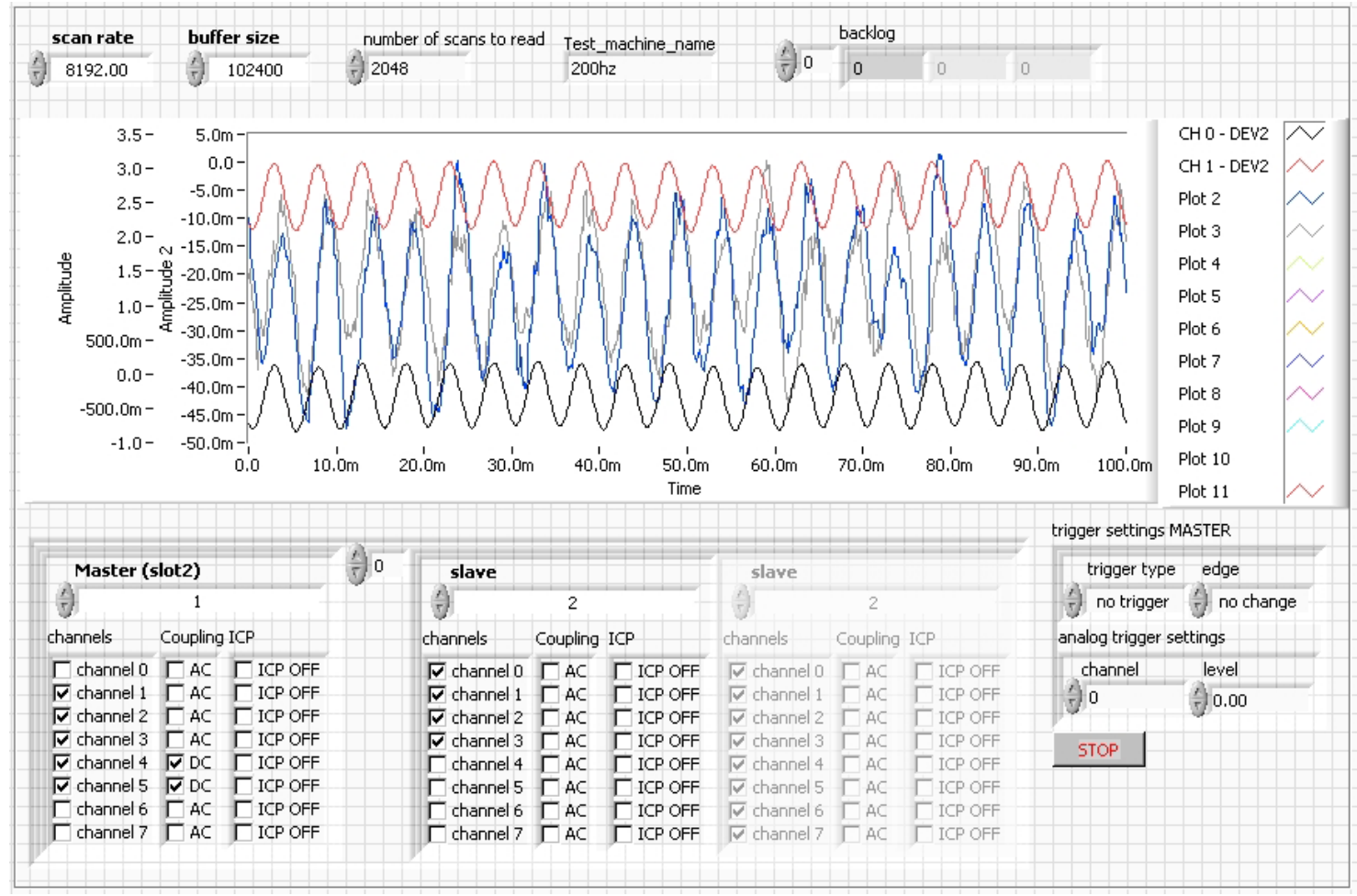

Figure 52. Front Control Panel: NI 4472-16 Channel Simultaneous DAQ Board 


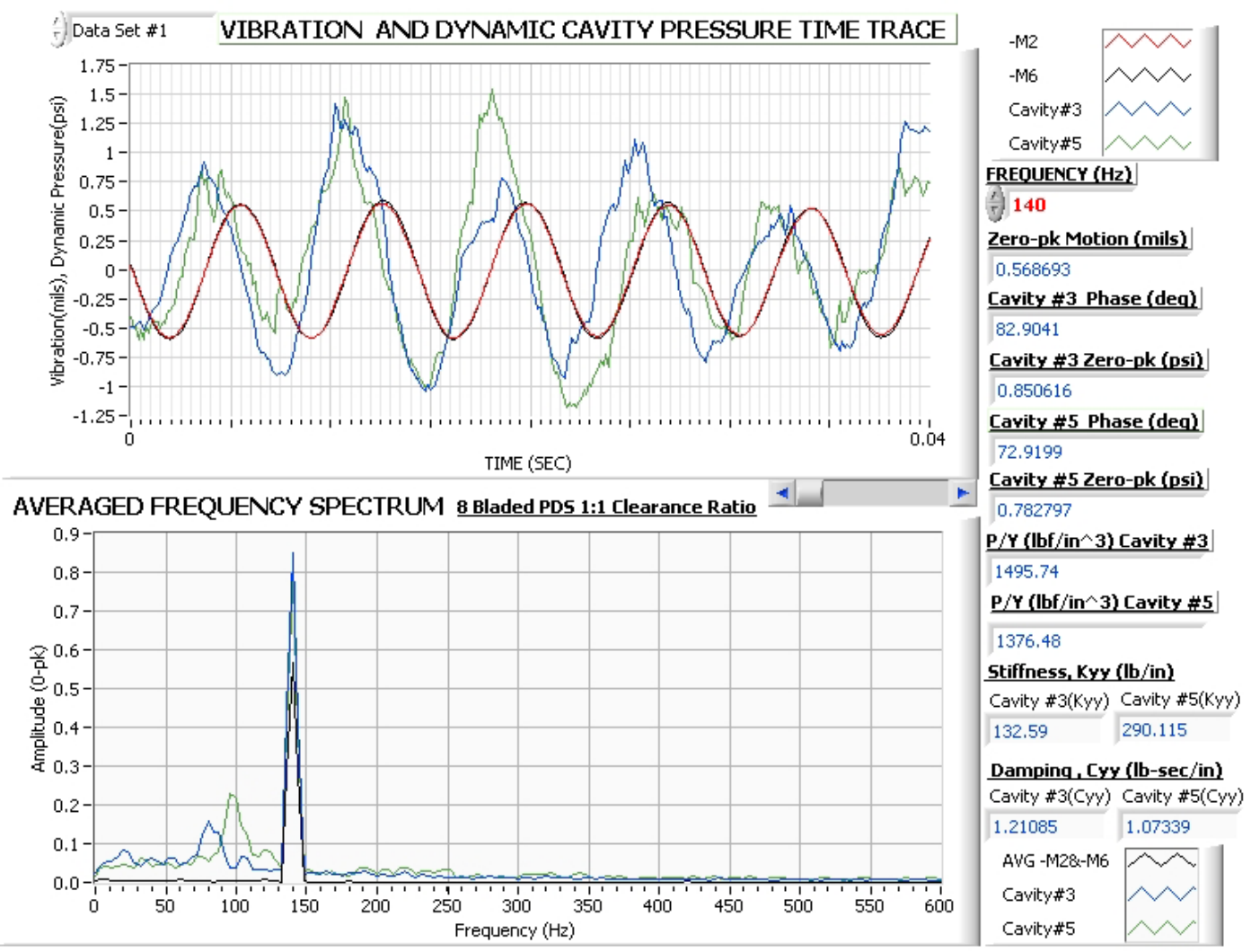

Figure 53. LABVIEW Front Panel-8 Bladed PDS

$\begin{array}{ll}\text { Direct Damping: } & C_{y y}=\frac{P_{S} \cdot A_{P}}{\omega \cdot y} \\ \text { Direct Stiffness: } & K_{y y}=\frac{P_{C} \cdot A_{P}}{y}\end{array}$
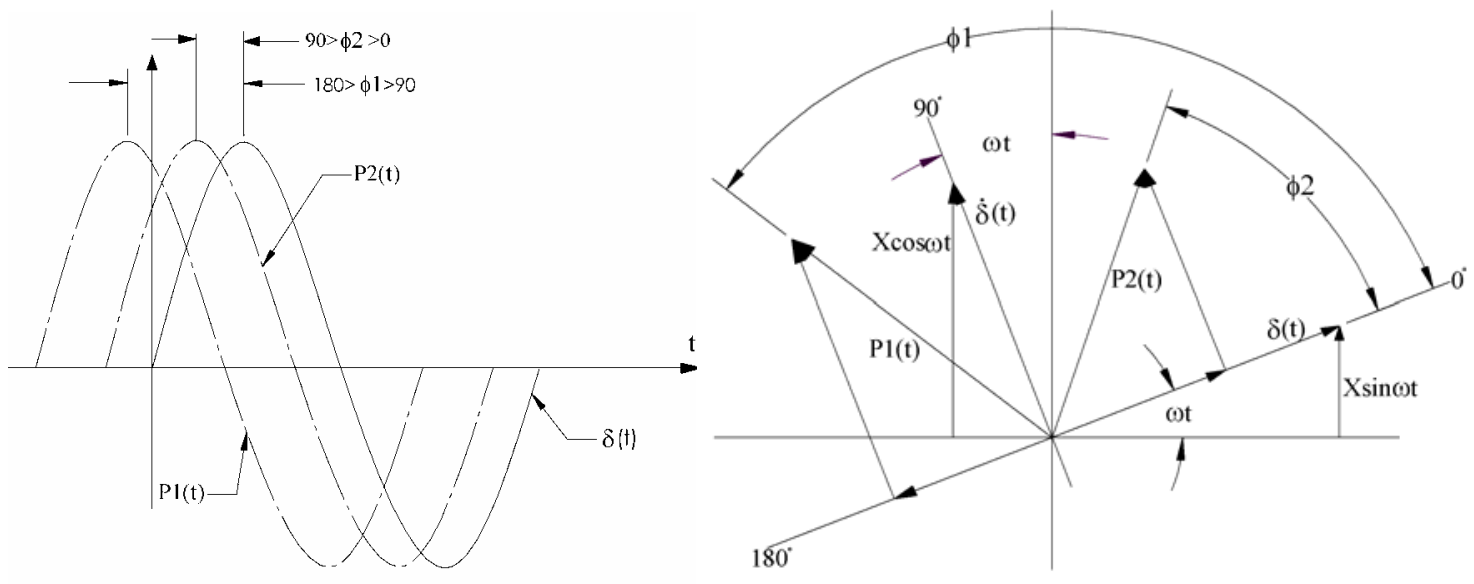

Figure 54. Phasor Plot for Diverging and Straight-Through Cavities 
Note that the $\mathrm{P}_{2}(\mathrm{t})$ has both components in line with the displacement and velocity whereas $\mathrm{P}_{1}(\mathrm{t})$ possesses a component in phase with the velocity but $180^{\circ}$ out of phase with the displacement. When the pressure components are in phase with the displacement and velocity, they produce a positive reaction force against the stator displacement and velocity, hence yielding positive stiffness and damping terms. For phase angles between $180^{\circ}$ and $90^{\circ}$ the cavity pressure $\mathrm{P}_{1}(\mathrm{t})$ resolves into positive damping and negative stiffness terms.

After reducing the experimental data, the PDS theory was used to generate analytical predictions for comparison with experimental results. The first step involved solving for the steady state for the 8 bladed PDS using the incomplete set of measured static pressures (P0, P3, P5, P8) shown in Figure 55. Note that to generate analytical solutions for all the test cases the inactive plenum pressures $\mathrm{P} 2, \mathrm{P} 4$, and $\mathrm{P} 6$ needed to be known. The static pressures P2, P4, and P6 were not measured during testing due to the small axial pitch ( 0.125 in) of the inactive cavities. To match the flow rate and pressures in active cavities 3 and 5 the discharge coefficients were adjusted to 0.75 for the inlet blade and 0.85 for the exit blade. Using the static pressure results for $\mathrm{P} 2, \mathrm{P} 4$, and $\mathrm{P} 6$ the cavity coefficients and parameters were calculated using a MathCad program. Two sample calculations based on the PDS theory can be found in the Appendix, along with the MathCAD code. The experiments conducted for the following results were linear unidirectional vibration tests based on a single degree of freedom impedance model; therefore, only the direct stiffness and direct damping are presented.
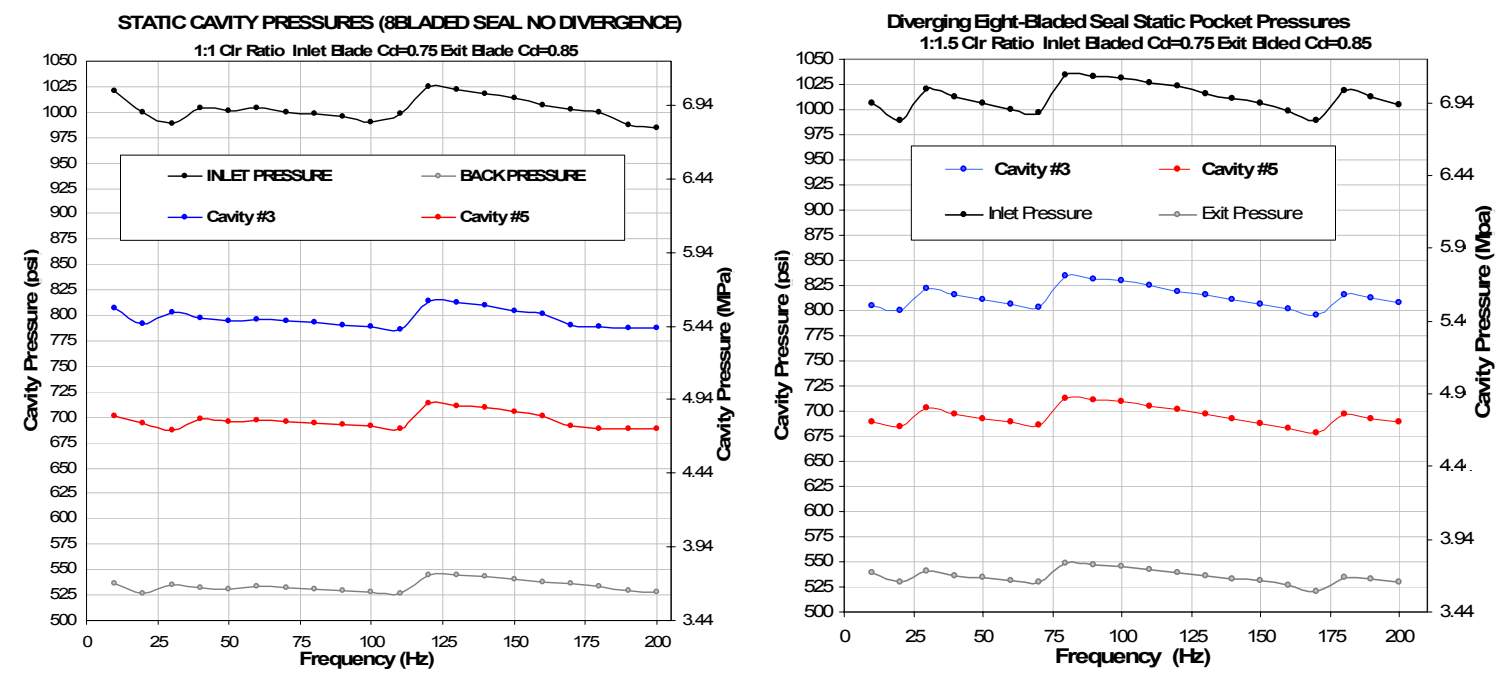

Figure 55. Static Seal Cavity Pressure Measurements 
Vibration amplitudes ranged between 0.5-0.7 mils 0-pk (Figure 56) and peak pressures (Figure 56) are also shown for the experiments and theory. Note that the theoretical predictions for the peak pressures are significantly different from the experimental measurements. The damping and stiffness results for the 8 bladed 1:1 clr ratio seal can be seen in Figure 57. Although the damping results in Figure 57 show similar trends, the theory significantly under predicts the experimental results. For a straight through seal (clr ratio 1:1) the PDS theory predicts positive stiffness values for all frequencies, but the experimental results for stiffness shown in Figure 57 indicate a negative stiffness at low frequencies and a transition to positive stiffness past the frequency range between 100-120 Hz. This is also apparent in Figure 58, which shows the pressure phase as a function of frequency. Figure 58 shows that the phase in cavity 3 crosses the $90^{\circ}$ mark at $\sim 120 \mathrm{~Hz}$ and the pressure phase in cavity 5 crosses $90^{\circ}$ at $\sim 100 \mathrm{~Hz}$.

The second plot shown in Figure 58 displays the force density, which is defined by $\left|\frac{P}{Y}\right|$, where $\mathrm{P}$ is the 0 -pk dynamic pressure and $\mathrm{Y}$ is the 0 -pk vibration amplitude. It is important to note the pressure resonances at $\sim 80 \mathrm{~Hz}$ and $\sim 95 \mathrm{~Hz}$. These are locations where the force density has a local maximum for the $3^{\text {rd }}$ cavity and a local minimum for the $5^{\text {th }}$ cavity. The damping plot in Figure 57 also shows deviations in the data at $80 \mathrm{~Hz}$ and $100 \mathrm{~Hz}$. Figure 57 illustrates that the $3^{\text {rd }}$ cavity increases in damping at $80 \mathrm{~Hz}$ and cavity 5 shows a decrease in damping at $100 \mathrm{~Hz}$. The frequency where the force density shows maximums and minimums is clearly seen in all the frequency spectra of the cavity pressure measurements. Example spectrum plots displaying the resonance frequencies can be seen in Figures 53 and 59. Initially the asynchronous frequencies observed from the pressure probes were thought to be acoustic frequencies, but this hypothesis was discarded because of the difference of the frequencies in the two cavities. Since the cavities possess the exact same geometry and the temperature from cavity 3 to cavity 5 is changing no more than $1^{\circ} \mathrm{F}$, the acoustic frequencies should be very close; given that they are a function of temperature (Lucas et al. 1997). However, the frequencies shown in Figures 53 and 59 display frequencies that with a $\sim 15$ $\mathrm{Hz}$ separation indicating that the resonances in the force density are attributed to another phenomena. 


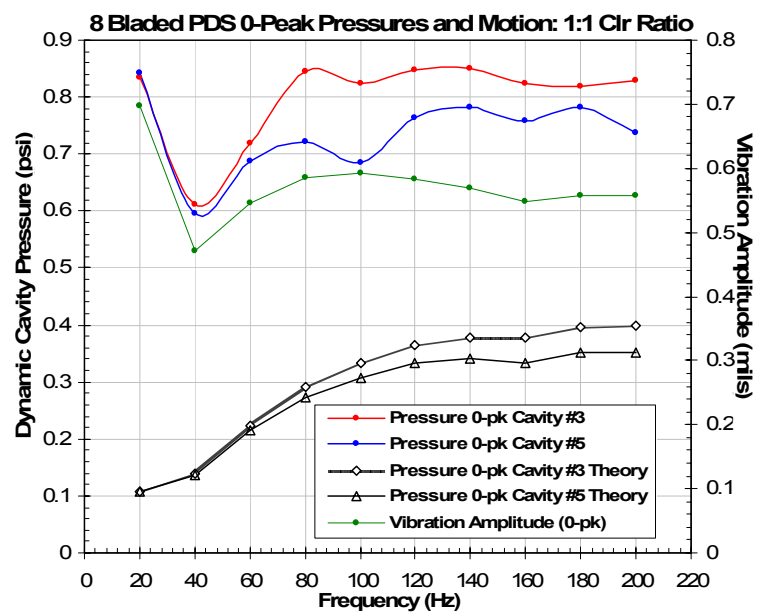

Figure 56. Peak Pressures and Vibration Amplitudes 8 Bladed PDS 1:1 Clr Ratio
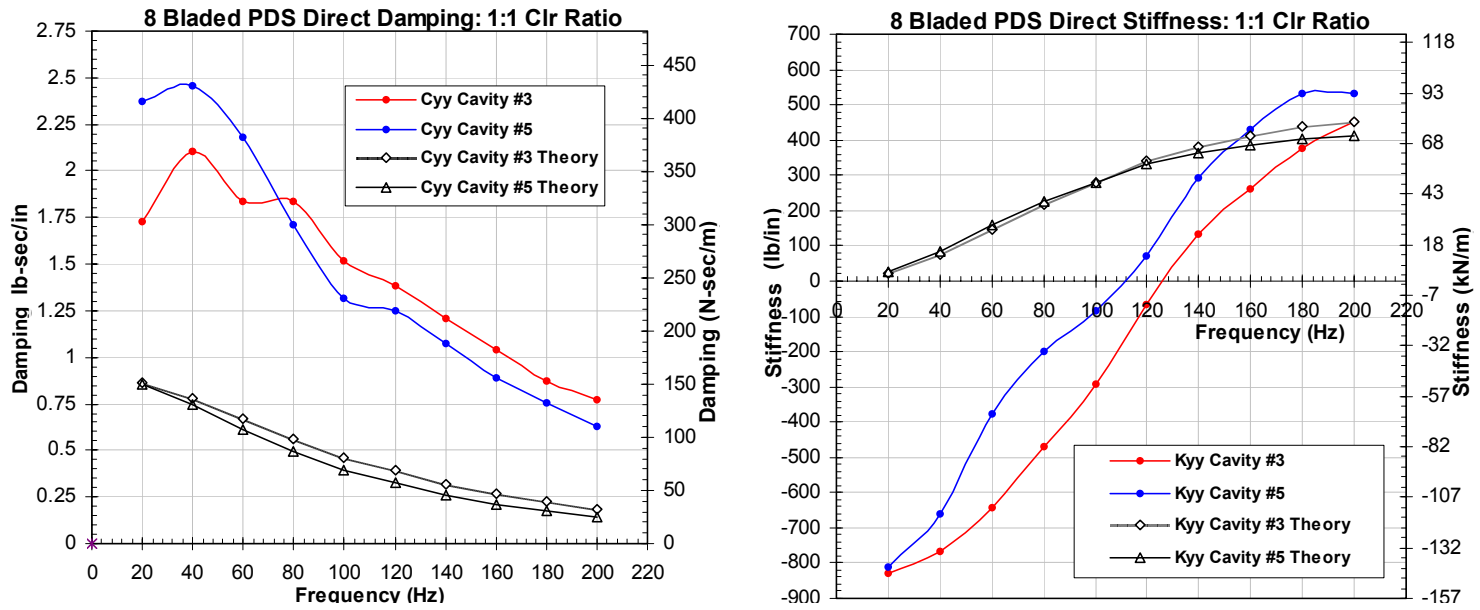

Figure 57. Stiffness and Damping 8 Bladed 1:1 Clr Ratio
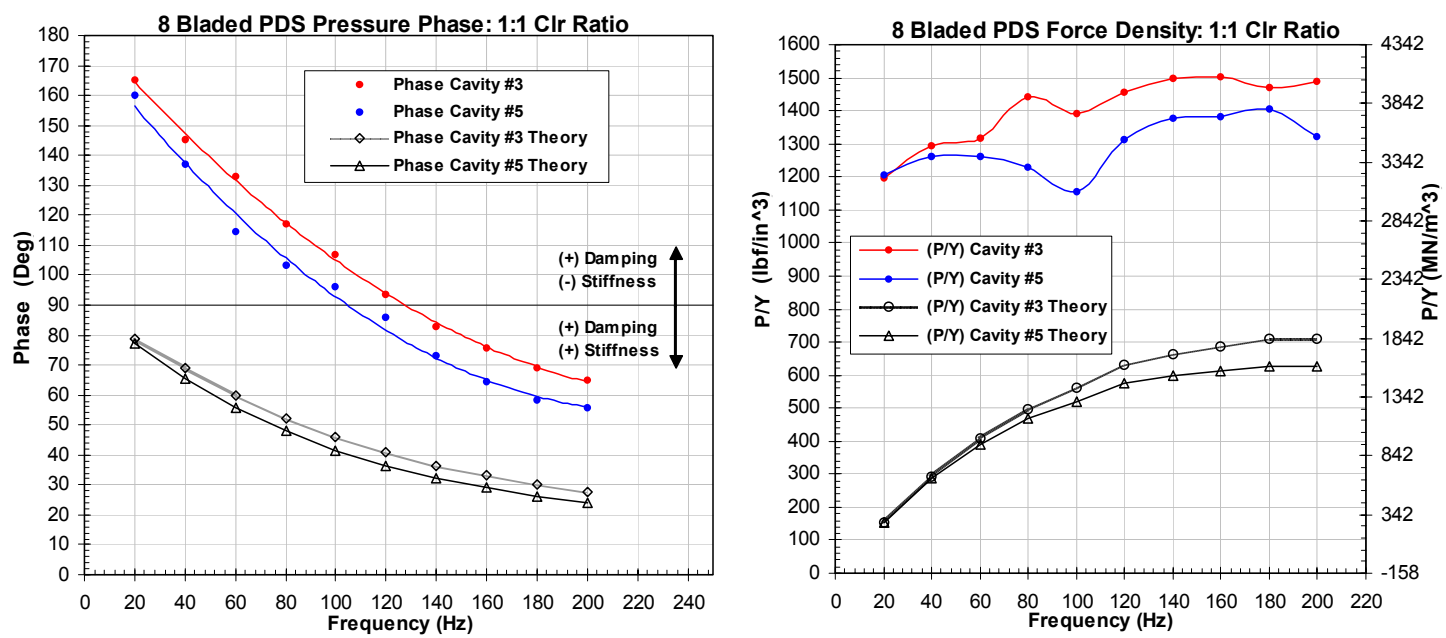

Figure 58. Pressure Phase and Force Density: 8 Bladed PDS 1:1 Clr Ratio 


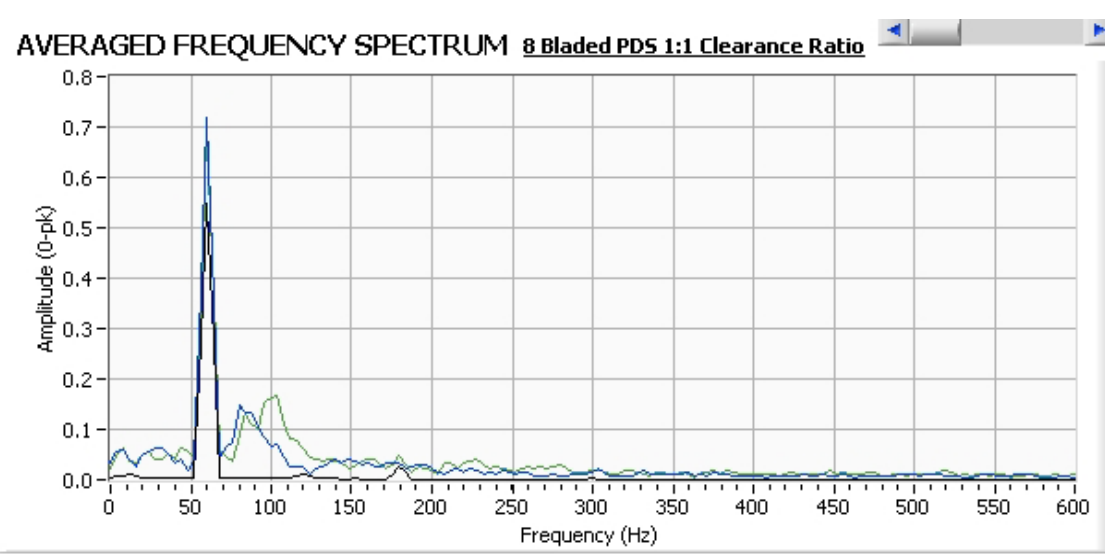

Figure 59. Asynchronous Pressure Frequencies 8 Bladed PDS 1:1 Clr Ratio

One major difference between the two cavities is the static pressure, which also materializes in a difference in gas density. This variation in gas density yields differences in cavity flow velocities, where cavity 3 possesses a lower flow velocity than cavity 5 . Calculating flow velocities was achieved by using the ideal gas equation (Equation 5.3) and the mass flow rate equation (Equation 5.4) and the results are shown in Table 12. It is interesting to note that the $95 \mathrm{~Hz}$ frequency in cavity 5 is $\sim 16 \%$ higher than the asynchronous $80 \mathrm{~Hz}$ frequency of cavity 3 and that the flow velocity in cavity 5 is $\sim 14 \%$ higher than the flow velocity for cavity 3 . The ratio of velocities and frequencies are similar and imply a direct linear relation between the two. The asynchronous frequencies are most likely related to a vortex shedding phenomena, which can be described by a non-dimensional number called the Strouhal number (Equation 5.5). This equation relates the wake shedding frequency $(\mathrm{f})$ to the flow velocity $(\mathrm{V})$ using a characteristic length $\mathrm{L}_{\mathrm{c}}$ and the Strouhal number (St). Using the axial pitch of the cavity for the characteristic length, the Strouhal number becomes $\sim 0.11$.

Table 12. Cavity Flow Parameters: 8 Bladed 1:1

\begin{tabular}{|c|c|c|c|}
\hline INLET RESSURE (PSI) & 1,000 & & \\
\hline EXIT RESSURE (PSI) & 525 & & \\
\hline DELTA P (PSI) & 475 & & \\
\hline INLET TEMP (F) & 56.2 & & \\
\hline EXIT TEMP (F) & 50.9 & & \\
\hline \multirow[t]{2}{*}{ MASS FLOW (KG/S) } & 0.294 & & \\
\hline & CAVTIY 3 & CAVTIY 5 & $\%$ DIFFERENCE \\
\hline PRESSURE (PSI) & 800 & 700 & ------------ \\
\hline FLOW VEOCITY $(\mathrm{m} / \mathrm{s})$ & 94.5 & 109.8 & $14 \%$ \\
\hline FREQUENCY (Hz) & 80 & 95 & $16 \%$ \\
\hline
\end{tabular}


$\rho=\frac{P}{R T}$

$\dot{m}=\rho \cdot A \cdot V$

$S t=\frac{f \cdot L_{c}}{V}$

The next set of results present the diverging clearance cavity results for the 8 bladed PDS. For the diverging seal two different excitation amplitudes were investigated: (1) vibration amplitude of 1.2 mils $(0.0305 \mathrm{~mm})$ pk-pk and (2) vibration amplitude of 1.8 mils pk-pk $(0.0457 \mathrm{~mm})$. Figure 60 shows the results for the stiffness and damping coefficients at 1.2 mils excitation, and Figure 61 shows the coefficient results for the 1.8 mils excitation. The results in Figure 60 indicate that the theory closely matches the experiment for these cases. Figures 60-61 also show that the experimental results between the two excitation amplitudes (1.2 mils and 1.8 mils) are almost identical in magnitude and trend.
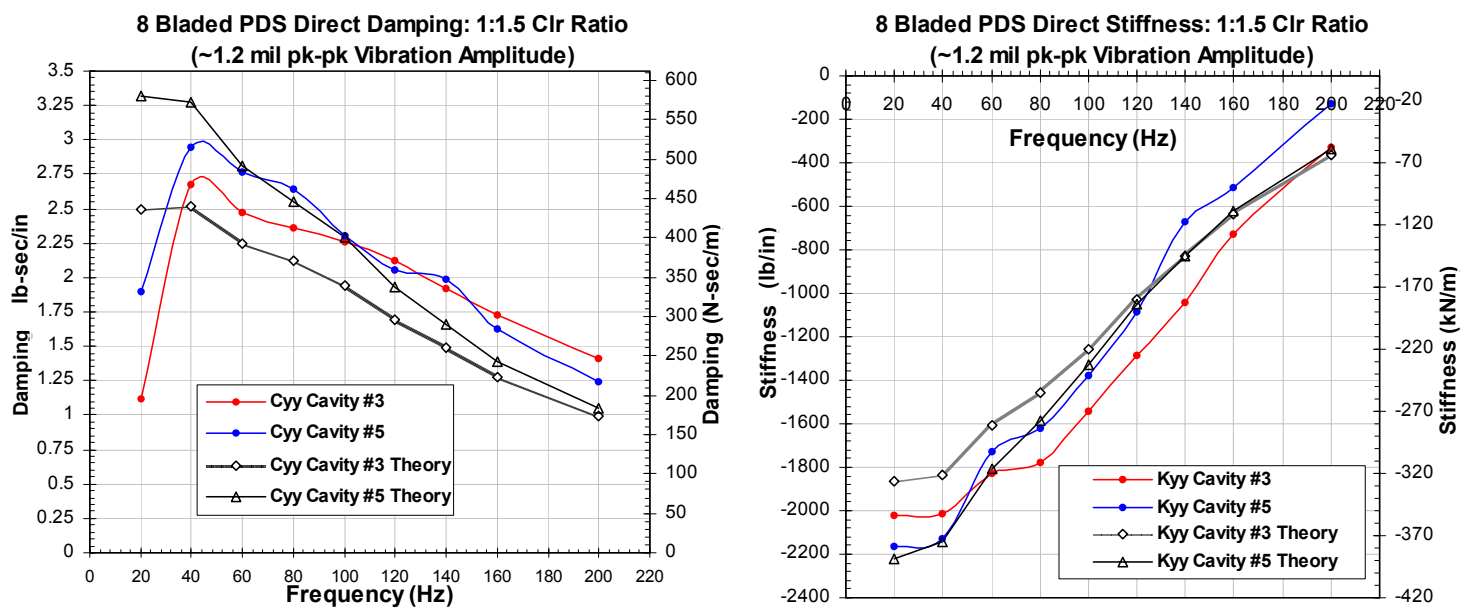

Figure 60. Stiffness and Damping 8 Bladed 1:1.5 Clr Ratio 1.2 mils pk-pk

Vibration and pressure amplitudes for each of the diverging seal test are shown in Figure 62. The final plots in Figures 63-64 display the pressure phase and force density plots for the diverging seal. The effect of the diverging clearance, in the form of the square notches, is evident in all the results compared to the straight through seal results. Comparing the phase plots in Figures 63-64 to the phase plot shown in Figure 58, it is apparent that the phase at $20 \mathrm{~Hz}$ is the same, however, when frequency increases the phase between the two types of seals deviates. The phase shown in Figure 58 indicates a stronger dependence to frequency 
whereas the phase in Figures 63-64 exhibits a weaker dependence on increasing frequency. The stiffness results also show significant change due to the notch.
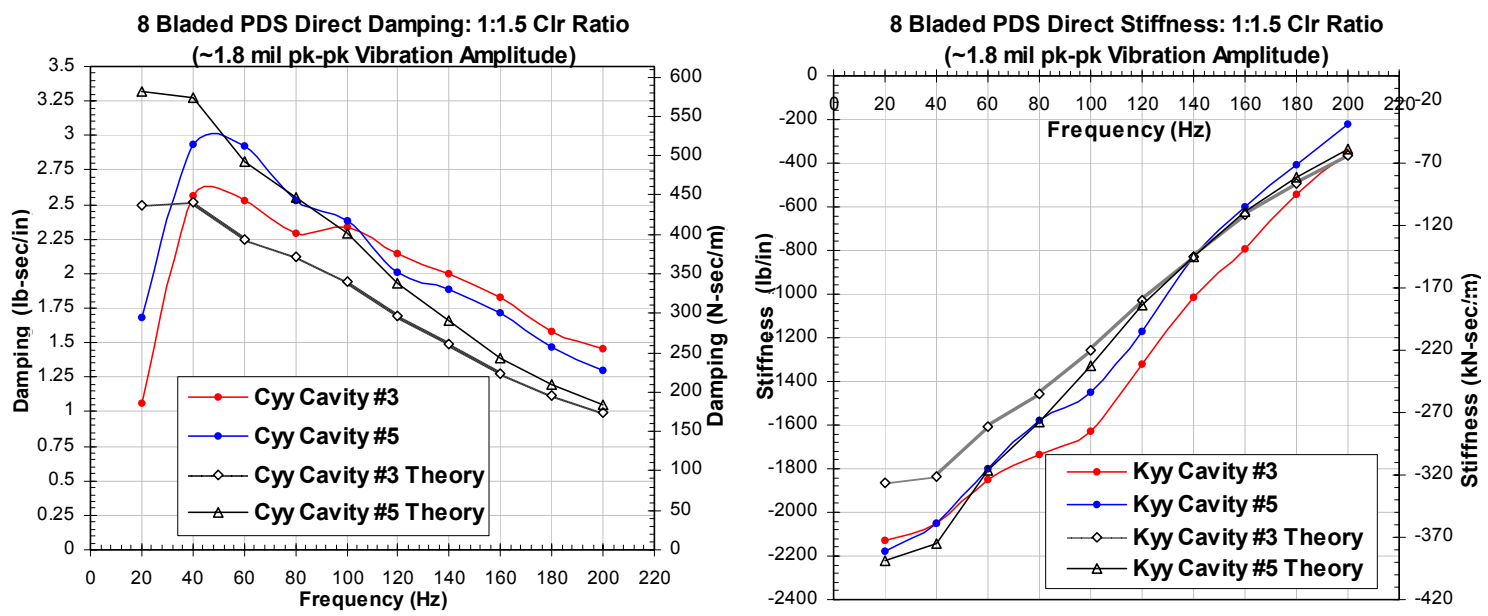

Figure 61. Stiffness and Damping 8 Bladed PDS 1:1.5 Clr Ratio $~ 1.8$ mils pk-pk

The stiffness in Figure 57 starts at $-800 \mathrm{lb} /$ in $(200 \mathrm{~Hz})$ and ends at $500 \mathrm{lb} /$ in $(200$ $\mathrm{Hz})$, but the stiffness plots shown in Figures 60-61 have a range between -2400 lb/in (20 $\mathrm{Hz})$ to $-400 \mathrm{lb} /$ in $(200 \mathrm{~Hz})$. This is a significant change in stiffness, which is due to the increase in phase angle and also an increase in peak cavity pressures. The damping portrayed a moderate increase for the diverging clearance PDS. Damping values ranged from 2.5-0.75 $\mathrm{lb}-\mathrm{sec} /$ in in Figure 57 and 3.0-1.25 lb-sec/in for damping plots in Figures 60-61. The diverging clearance also revealed drastic effects on the force density.

The pressure spectrum plots for the diverging seal also revealed two frequencies other than the forced excitation frequency. Figure 64 shows an example of two frequencies, one at $100 \mathrm{~Hz}$ (cavity 3) and another at $140 \mathrm{~Hz}$ (cavity 5). These values are an increase compared to the cavity frequencies shown in Figures 52 and 58, which were observed to be at $80 \mathrm{~Hz}$ (cavity 3) and $95 \mathrm{~Hz}$ (cavity 5). This increase in shedding frequency makes sense because the leakage for the diverging notched PDS was measured to be larger than the straight through configuration. The diverging seal spectrum plots (shown in the Appendix) differed from the straight through seal due to the increase in signal noise. This can possibly be attributed to the increase in turbulence intensity in the flow caused by the notch. Also the force peak pressure for the diverging seal are considerably larger than the 1:1 clr ratio PDS. 

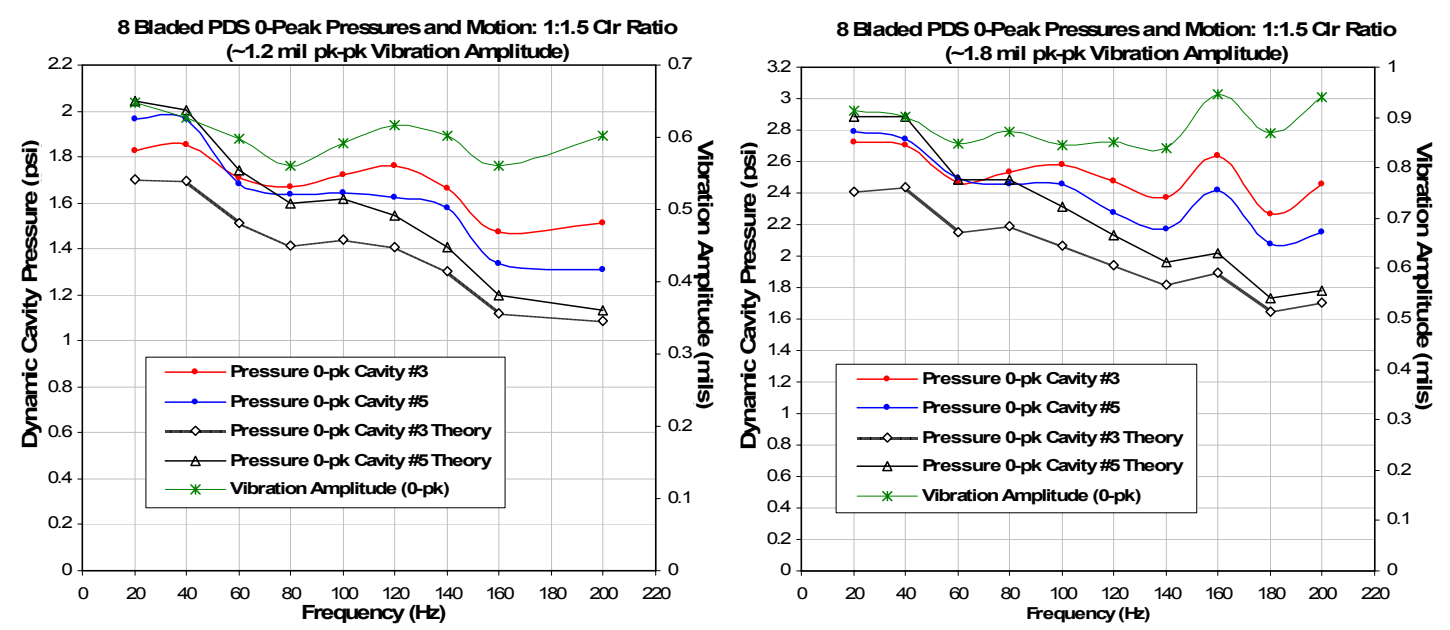

Figure 62. Peak Pressures and Vibration 8 Bladed 1:1.5 Clr Ratio 1.2 mils pk-pk
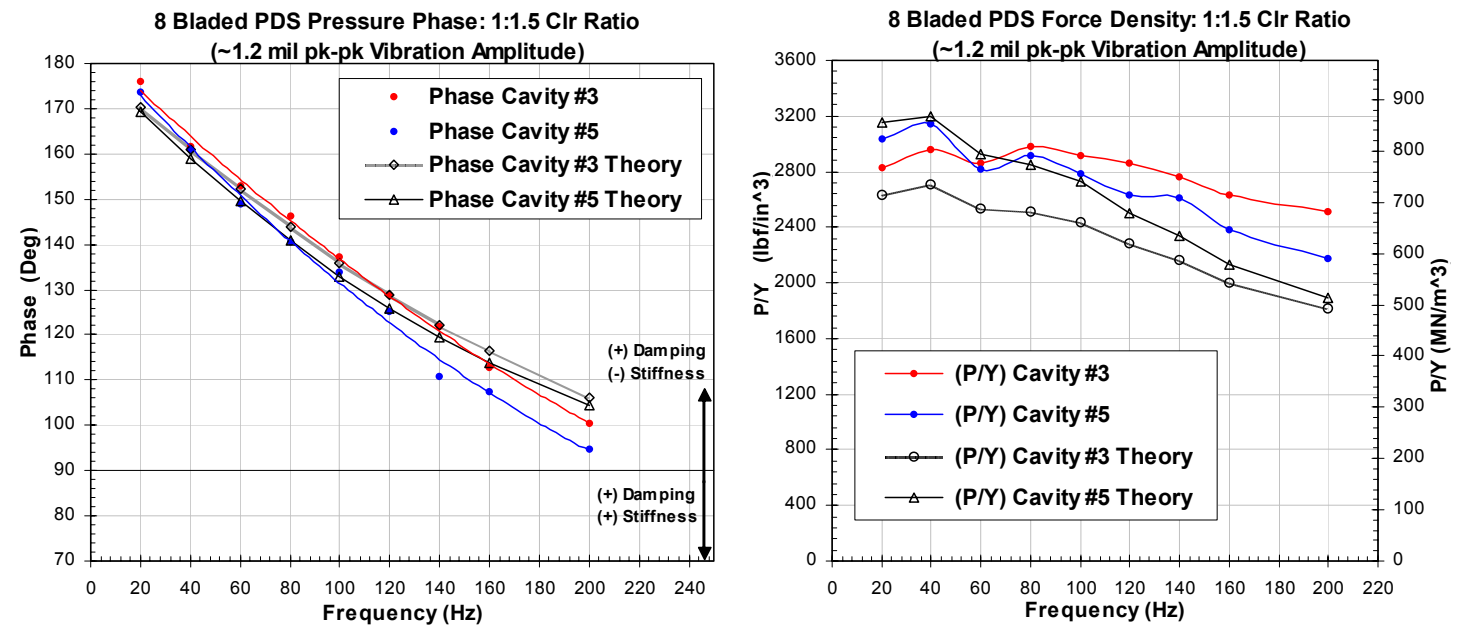

Figure 63. Phase and Force Density 8 Bladed PDS 1:1.5 Clr Ratio $\sim 1.2$ mils pk-pk
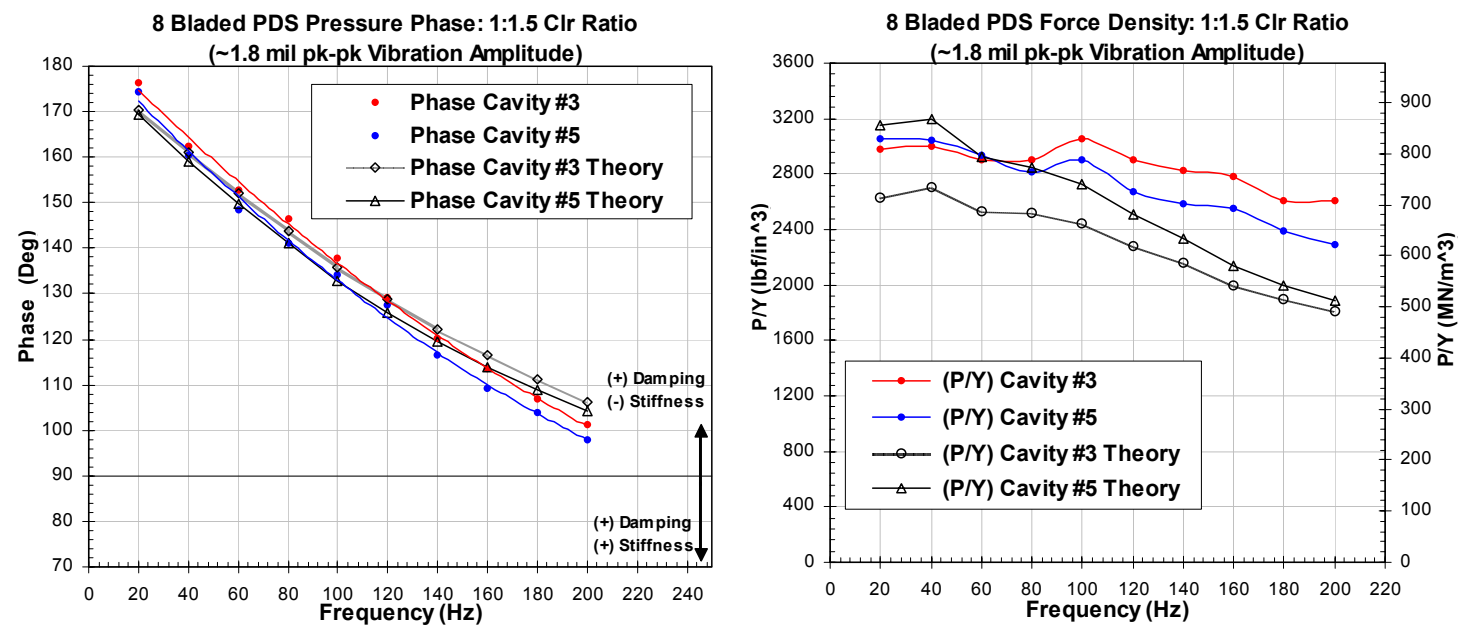

Figure 64. Phase and Force Density 8 Bladed PDS 1:1.5 Clr Ratio $\sim 1.8$ mils pk-pk 


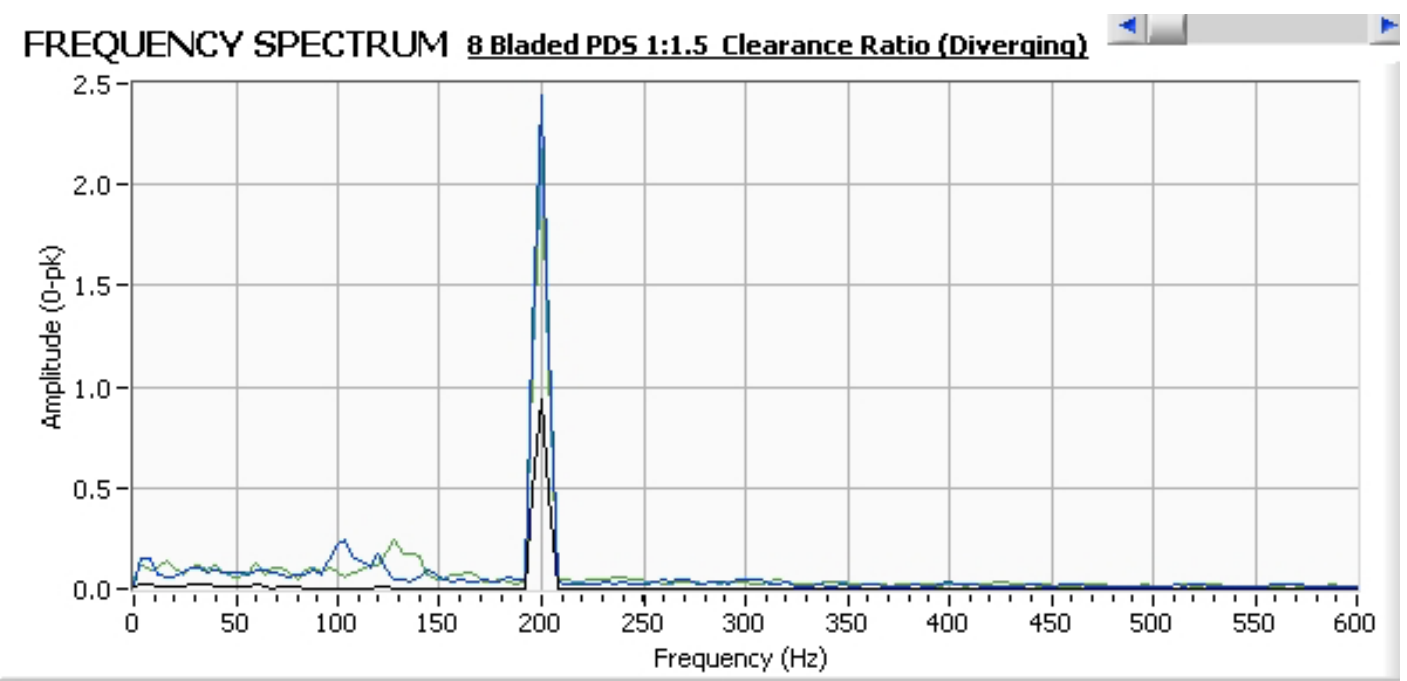

Figure 65. Pressure Spectrum for Diverging 8 Bladed PDS (200 Hz)

The main objective of this section was to compare the conventional damper seal theory for a single cavity with experimental measurements using two different seals. The conclusions to the tests with the 1:1 clearance ratio seal are as follows:

(1) The damping was shown to decrease with frequency

(2) The stiffness was shown to increase with frequency

(3) The phase angle decreases as frequency increases with the range of $20-200 \mathrm{~Hz}$

(4) The theory for the damping, stiffness, and phase is significantly under predicting the values

(5) The theory predicts only positive stiffness, where as the experiments show a cross over from negative stiffness to positive stiffness around $100-120 \mathrm{~Hz}$ 
(6) The force density increases with frequency

(7) Dynamic pressure frequencies away from the forced frequency were observed and are related to a vortex shedding phenomena

The next seal that was tested was the diverging PDS, which has a clearance ratio of 1:1.5.

Conclusions to the tests with this seal are as follows:

(1) The 1:1.5 clearance ratio yielded higher direct damping and more negative direct stiffness. It was also observed that the negative stiffness for the diverging seal increased less with frequency compared to the straight through seal. This behavior is evident in the phase plots as well

(2) The theory closely matches the experimental results for all the plots.

(3) The effect of the different vibration amplitudes (1.2 mils pk-pk and 1.8 mils pk-pk) revealed almost no effect on the results.

(4) Cavity frequencies other than the forced excitation frequency were observed in the pressure spectra at $100 \mathrm{~Hz}$ and $140 \mathrm{~Hz}$. This is an increase compared to the 1:1 clr ratio PDS which makes sense because of the increases flow velocities.

(5) The pressure spectrum for this seal displayed more signal noise; most probably due to the increase in turbulent flow from the rectangular notches. 


\subsection{Cavity Coefficients for Conventional Six Bladed PDS}

Dynamic pressure transducers were used to determine individual cavity coefficients and overall seal coefficients for the conventional six bladed PDS at a back pressure of 620 psi (42.74 bar) and an inlet plenum pressure of 1,000 psi (68.94 bar). The requirement for generating seal coefficients was to measure pressure modulations in 24 active cavities simultaneously with system vibration. In addition to the active cavity pressure measurements, the local pressure modulation in plenums 2 and 4 were also measured. Due to the limitation of 5 dynamic pressure transducers, testing was administered for a single row of cavities in 8 different angular locations as defined in Figure 26. Figure 66 illustrates the pressure transducer installation schematic for a single row of 3 cavities separated by 2 plenums. Note that cavities 1,3 , and 5 consist of both a dynamic transducer and a static transducer, whereas the inactive plenums support a miniature type PCB dynamic transducer. For determining seal coefficients only the dynamic measurements were used; whereas, the static pressure measurements aided in determining discharge coefficients.
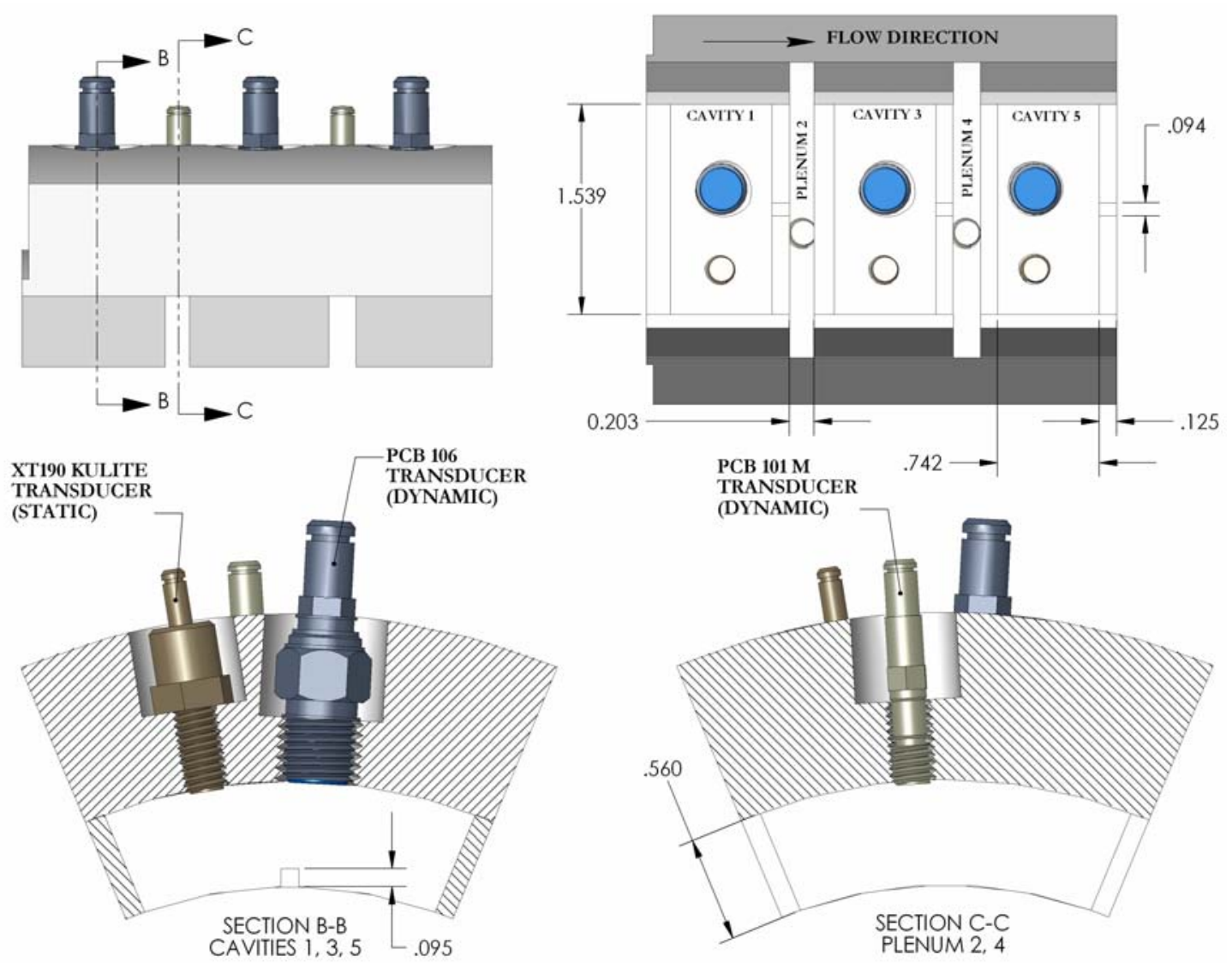

Figure 66. Conventional Six Bladed PDS Single Row of Cavities: Transducer Installation 
Although the dynamic pressure and motion measurements were acquired using the same control panel as shown in Figure 52, the on-line front panel display and data reduction panel were different from the virtual instrument used for the eight bladed seal. Figure 67 shows the display panel that plots the dynamic pressures and vibration motion. The plot on the upper left corner displays the dynamic pressure modulation in the active cavities and also displays the vibration motion. The left middle graph represents the local pressure modulation in inactive plenums 2 and 4 along with vibration motion. The bottom left graph displays only the dynamic pressure measurement at the 3 active cavities and the 2 inactive plenums. This particular example (Figure 67) was for the 0 degree position with an X direction excitation of $40 \mathrm{~Hz}$. The plots shown on the right side represent the FFT of the pressures and the motion, where the top graph is the FFT for the active cavity pressures, the middle graph is the FFT for the local pressure modulation in the inactive plenums, and the bottom right graph displays the FFT of the motion. In addition to the plots, the front panel display calculates the 0 -pk values for all the dynamic signals. The second part of the front panel is the on-line data reduction program output as shown in Figure 68. A virtual instrument was programmed to display the individual cavity phase measurements and the direct/cross-coupled coefficients for the $\mathrm{X}$ and $\mathrm{Y}$ directions. It also calculates the overall row coefficients as shown in the top portion of the output screen.

In the previous section it was shown that diverging clearance cavities possess a dynamic pressure phase relationship to vibration that is between 90 and 180 degrees, resulting in negative stiffness and positive damping. The tests performed for each of the cavity rows of the conventional six bladed PDS confirmed the behavior for frequencies between 20 and $200 \mathrm{~Hz}$, as shown in Figure 69. This figure displays the phase relationship for the row of cavities located at the 45 degree angle position; in relation to the $\mathrm{Y}$ direction and the $\mathrm{X}$ direction. All the phase plots for the other angular locations are shown in the Appendix. An example plot of direct damping and stiffness for individual cavities at the 0 degree angle is shown in Figure 70. The direct damping was measured to be positive with little dependency on frequency, whereas the direct stiffness was negative with more of a frequency dependency. Since the system was excited in two orthogonal directions, crosscoupled coefficients were calculated for each of the cavities. The cross -coupled stiffness and damping for the 0 degree position is shown in Figure 71. 


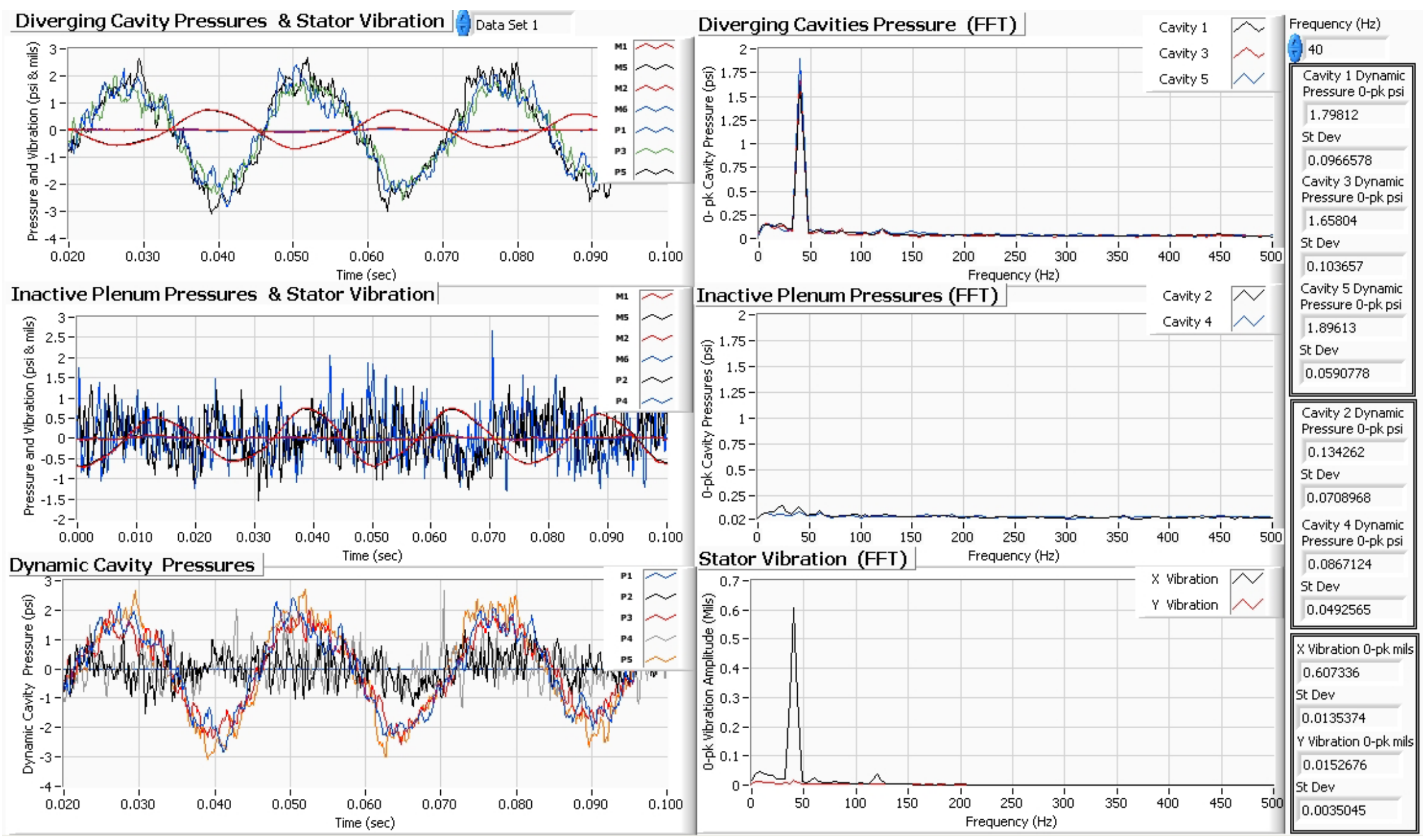

Figure 67. Front Panel Dynamic Measurements Display: 0 Degree Angle X Direction Excitation $40 \mathrm{~Hz}$ 
Rotordynamic Coefficients Row Values X Direction

\begin{tabular}{|c|c|}
\hline \multicolumn{2}{|c|}{ Row Direct Stiffness X Direction (b/b/in) } \\
\hline \multicolumn{2}{|l|}{-4206.47} \\
\hline \multicolumn{2}{|c|}{ Row Direct Stiffness X Direction Standard Dev (lb/in) } \\
\hline \multicolumn{2}{|l|}{92.0555} \\
\hline \multicolumn{2}{|c|}{ Row Direct Damping X Direction (lb-sec/in) } \\
\hline \multicolumn{2}{|l|}{16.3697} \\
\hline \multicolumn{2}{|c|}{ Row Direct Damping $\times$ Direction Standard Dev (lb-seclin } \\
\hline \multicolumn{2}{|c|}{$0.31584 i$} \\
\hline \multirow{2}{*}{\multicolumn{2}{|c|}{ 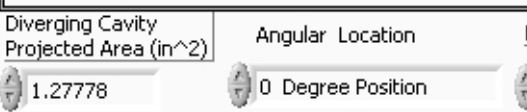 }} \\
\hline & \\
\hline \multicolumn{2}{|c|}{ Cavity \#1 Coefficients } \\
\hline \begin{tabular}{||l|} 
X Direction \\
Direct Stiffness (lbjin)
\end{tabular} & $\begin{array}{l}\text { Y Direction } \\
\text { Direct Stiffness (lb/in) }\end{array}$ \\
\hline-544.409 & 0 \\
\hline Standard Dev & Standard Dev \\
\hline 84.1262 & 0 \\
\hline Direct Damping (b-sec/in) & Direct Damping (lb-sec/in) \\
\hline 5.82497 & 0 \\
\hline Standard Dev & Standard Dev \\
\hline 0.152565 & 0 \\
\hline CC Stiffness (lb/in) & CC Stiffness (b/in) \\
\hline 90.1079 & 0 \\
\hline Standard Dev & Standard Dev \\
\hline 117.977 & 0 \\
\hline CC Damping (lb-seclin) & CC Damping (lb-seclin) \\
\hline 0.802422 & 0 \\
\hline Standard Dev & Standard Dev \\
\hline 0.195132 & 0 \\
\hline X-Shake Phase (Deg) & Y-Shake Phase (Deg) \\
\hline 100.383 & 76.0472 \\
\hline Standard Dev & Standard Dev \\
\hline 1.53205 & 15.4873 \\
\hline
\end{tabular}

Rotordynamic Coefficients Row Values Y Direction

\begin{tabular}{||l||}
\hline Row CC Stiffness X-Direction (blinin) \\
\hline 112.834 \\
\hline Row CC Stiffness X-Direction Standard Dev (bbin) \\
\hline 145.753 \\
\hline Row CC Damping X-Direction (b--seclin) \\
\hline 1.5138 \\
\hline Row CC Damping X-Direction Standard Dev (lb-seclin) \\
\hline 0.528576 \\
\hline
\end{tabular}

\begin{tabular}{||l|}
\hline Row Direct Stiffness Y-Direction (lb/in) \\
\hline 0 \\
Row Direct Stiffness \\
Y-Direction Standard Dev (Ib/in) \\
\hline 0 \\
Row Direct Damping Y-Direction (lb-sec/in) \\
\hline 0 \\
Row Direct Damping Y-Direction Standard Dev (Ib-sec/in) \\
\hline 0 \\
\hline
\end{tabular}

Row CC Stiffness Y-Direction (b/)/in)

Row CC Stiffness Y-Direction Standard Dev (lb/in) 0 Row CC Damping Y-Direction (lb-sec/in) Row CC Damping Y-Direction Standard Dev (lb-sec/in)

\begin{tabular}{|c|c|c|c|}
\hline \multicolumn{2}{|c|}{ Cavity \#3 Coefficients } & \multicolumn{2}{|c|}{ Cavity \#5 Coefficients } \\
\hline \begin{tabular}{|l|l|} 
X Direction \\
Direct Stiffness (lb/in)
\end{tabular} & $\begin{array}{l}\text { Y Direction } \\
\text { Direct Stiffness (lb/in) }\end{array}$ & \begin{tabular}{|l|l|} 
X Direction \\
Direct Stiffness (lb/in) \\
\end{tabular} & $\begin{array}{l}\text { Y Direction } \\
\text { Direct Stiffness (b)/in) }\end{array}$ \\
\hline 835.523 & 0 & -4497.58 & 0 \\
\hline Standard Dev & Standard Dev & Standard Dev & Standard Dev \\
\hline 62.5728 & 0 & 85.2358 & 0 \\
\hline 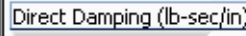 & Direct Damping (lb-sec/in) & Direct Damping (lb-secjin) & Direct Damping (b-sec/in) \\
\hline 4.28185 & 0 & 6.26284 & 0 \\
\hline Standard Dev & Standard Dev & Standard Dev & Standard Dev \\
\hline 0.105729 & 0 & 0.21599 & 0 \\
\hline CC Stiffness (lb)in) & CC Stiffness (b/in) & CC Stiffness (b)/in) & CC Stiffness (Ib/in) \\
\hline-121.351 & 0 & 144.077 & 0 \\
\hline Standard Dev & Standard Dev & Standard Dev & Standard Dev \\
\hline 62.586 & 0 & 67.6417 & 0 \\
\hline CC Damping (b-sec/in) & $\subseteq \subset$ Damping (lb-sec/in) & CC Damping (lb-sec/in) & CC Damping (lb-sec/in) \\
\hline 0.792597 & 0 & -0.0812165 & 0 \\
\hline Standard Dev & Standard Dev & Standard Dev & Standard Dev \\
\hline 0.180445 & 0 & 0.365343 & 0 \\
\hline X-Shake Phase (Deg) & Y-Shake Phase (Deg) & $x$-Shake Phase (Deg) & Y-Shake Phase (Deg) \\
\hline 68.8526 & 110.837 & 145.025 & -18.9819 \\
\hline Standard Dev & Standard Dev & Standard Dev & Standard Dev \\
\hline 1.55473 & 11.5744 & 0.646018 & 42.4315 \\
\hline
\end{tabular}

Figure 68. Front Panel: Data Reduction Conventional Six Bladed PDS 

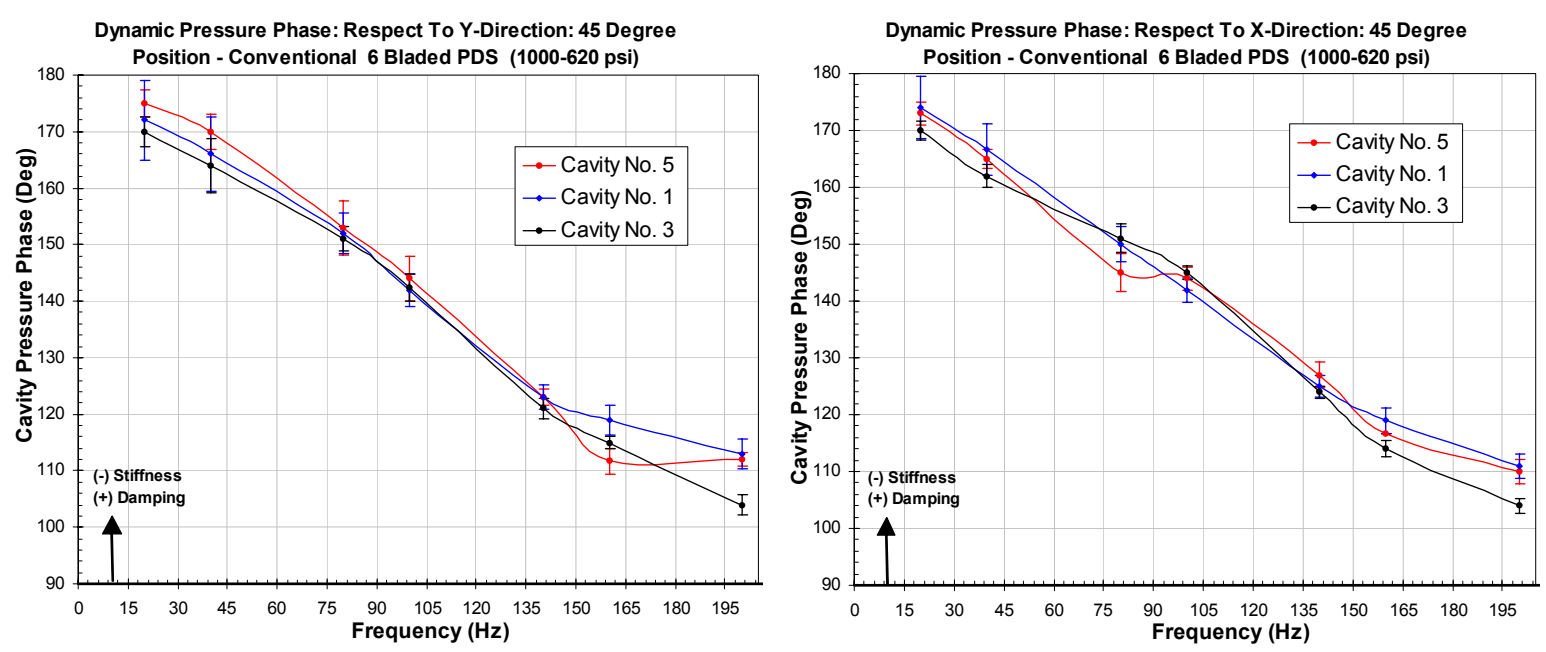

Figure 69. Dynamic Pressure Phase: 45 Degree Location
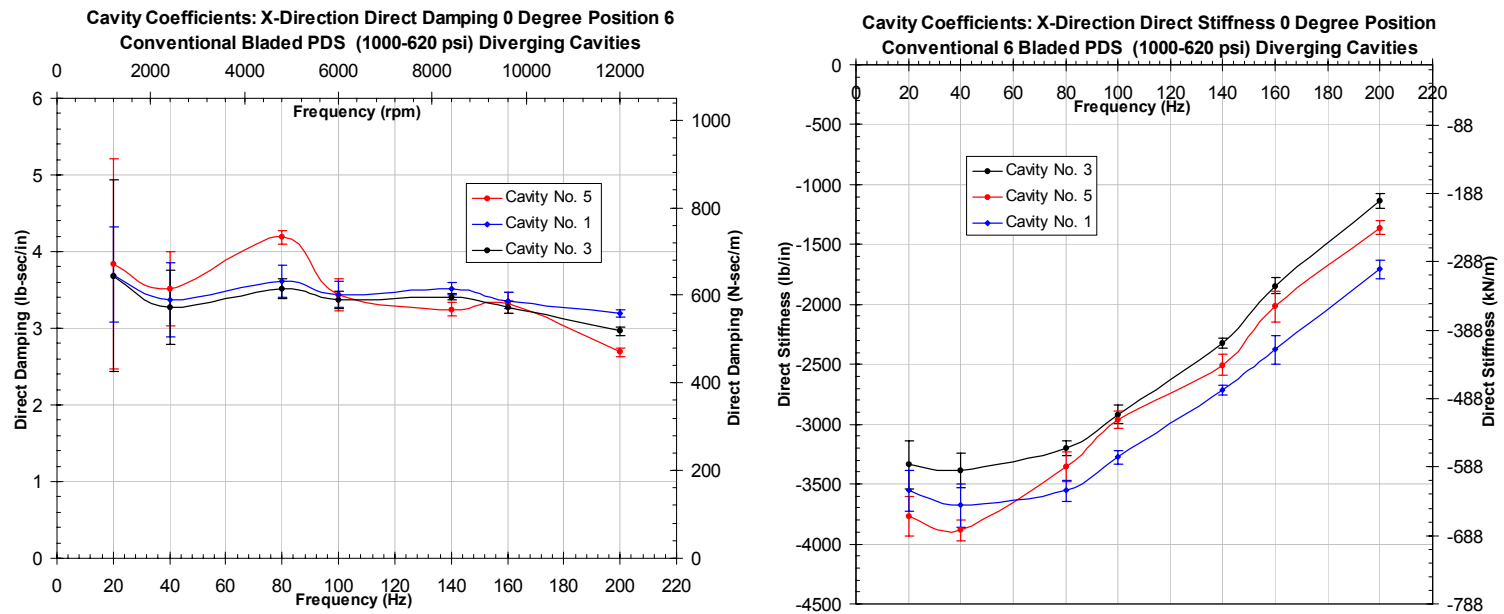

Figure 70. Direct Stiffness and Damping: Cavity Coefficients Conventional Six Bladed PDS

The cross-coupled coefficients for the 0 degree position are small in magnitude and are not the dominating cross-coupled coefficients. The total seal cross-coupled stiffness and damping is mostly determined by the cavities at the 45, 135, 225, and 315 degree positions. Figure 72 shows the cross-coupled and direct stiffness row values for the $\mathrm{X}$ and $\mathrm{Y}$ directions in the 45 degree position and Figure 73 shows the cross-coupled and direct damping. An important observation from Figure 72 is that the cross-coupled stiffness coefficients in the $\mathrm{X}$ and $\mathrm{Y}$ directions possess the same sign and become more positive as frequency increases.

After calculating the row coefficients for each angular position (shown in Appendix) all the row coefficients are added resulting in a full set of rotordynamic coefficients for the $\mathrm{X}$ and Y directions. These graphs are shown in Figures 74-77. The experiments show that the 
damping is positive throughout the entire frequency range while the stiffness is negative.

Also, the cross-coupled stiffness and damping coefficients are of the same sign.

One of the research objectives was to determine if the inactive plenums possess significant pressure modulations and if they contribute to the overall seal coefficients. The level of contribution from the inactive cavities was investigated by measuring two things: (1) dynamic pressure phase relation to vibration, and (2) the local 0-pk plenum pressure magnitudes. First consider the pressure phase for the 0, 90, and 270 degree angular locations shown in Figures 78-79. These figure show that the pressures phase is incoherent until the excitation frequency reaches $100 \mathrm{~Hz}$, which is when the phase converges to 120-140 degrees. The second factors investigated for the inactive plenums measurements was the pressure magnitude, which is shown for six tests performed at the 0, 45, and 90 degree locations (Figures 80-85). The first two tests conducted at 100 and $200 \mathrm{~Hz}$ (Figures 80-81) show local pressure modulations at the 0 degree position for an $\mathrm{X}$ direction excitation, which are $\sim 17 \%$ the magnitude of the dynamic pressures for the active cavities. For the $200 \mathrm{~Hz}$ test, Figure 81 shows an increase in the pressure magnitudes for the inactive plenums at $\sim 40 \%$ the active cavity pressure magnitudes. The tests conducted at the 45 degree angle position for an $\mathrm{X}$ direction excitation are shown in Figures 82-83. The $100 \mathrm{~Hz}$ case shows the presence of significant pressure modulation for the inactive plenum 2, whereas the inactive plenum 4 pressure is significantly smaller. At $200 \mathrm{~Hz}$, the results for the 45 degree angle position are similar to the 0 degree angle position $200 \mathrm{~Hz}$ test; yielding an average inactive plenum pressure that is $46 \%$ of the average active cavity pressure. The last sets of results (Figures $84-$ 85) presented are tests at the 90 degree position for an $\mathrm{X}$ direction excitation at $100 \mathrm{~Hz}$ and $200 \mathrm{~Hz}$. The local pressure modulations at the 90 degree location for an $\mathrm{X}$ direction excitation are significantly smaller than the values for the 0 degree and 45 degree positions. The results for the 4 cases shown in Figures 80-85 are summarized in Table 13. To determine the percent contribution of the inactive plenums, Equations 1 and 2 can be utilized for each plenum in combination with the phase data and the results shown in Table 18. Using these equations the contribution of the two plenums in the X direction at $100 \mathrm{~Hz}$ is $(1.06 \mathrm{lb}-\mathrm{s} /$ in) $2.5 \%$ of the seal direct damping and $(1,847.6 \mathrm{lb} / \mathrm{in}) 5 \%$ of the seal direct stiffness. At $200 \mathrm{~Hz}$ the inactive plenum damping is $(2.62 \mathrm{lb}-\mathrm{s} / \mathrm{in}) 7.1 \%$ of the total seal damping and (0.18188) 18\% of the seal direct stiffness. 

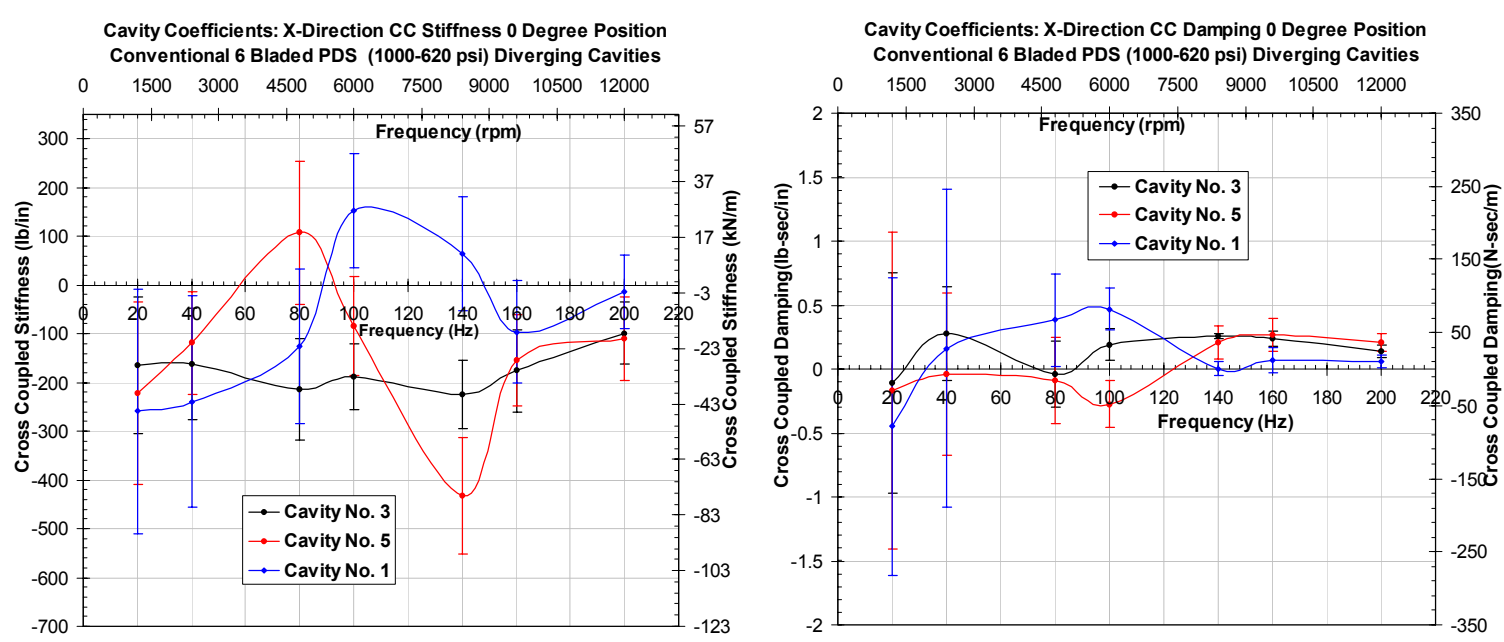

Figure 71. Cavity Coefficients: 0 Degree Position Cross-Coupled Stiffness and Damping
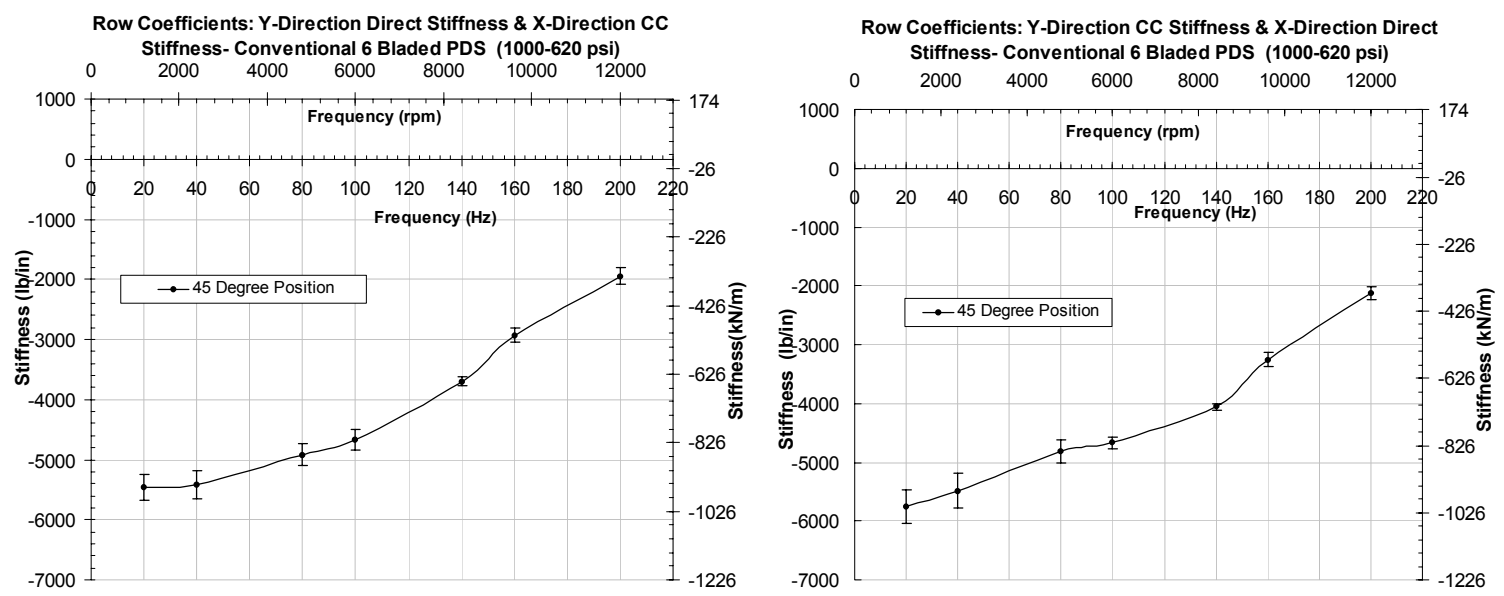

Figure 72. Row Coefficients: 45 Degree Position Cross-Coupled and Direct Stiffness
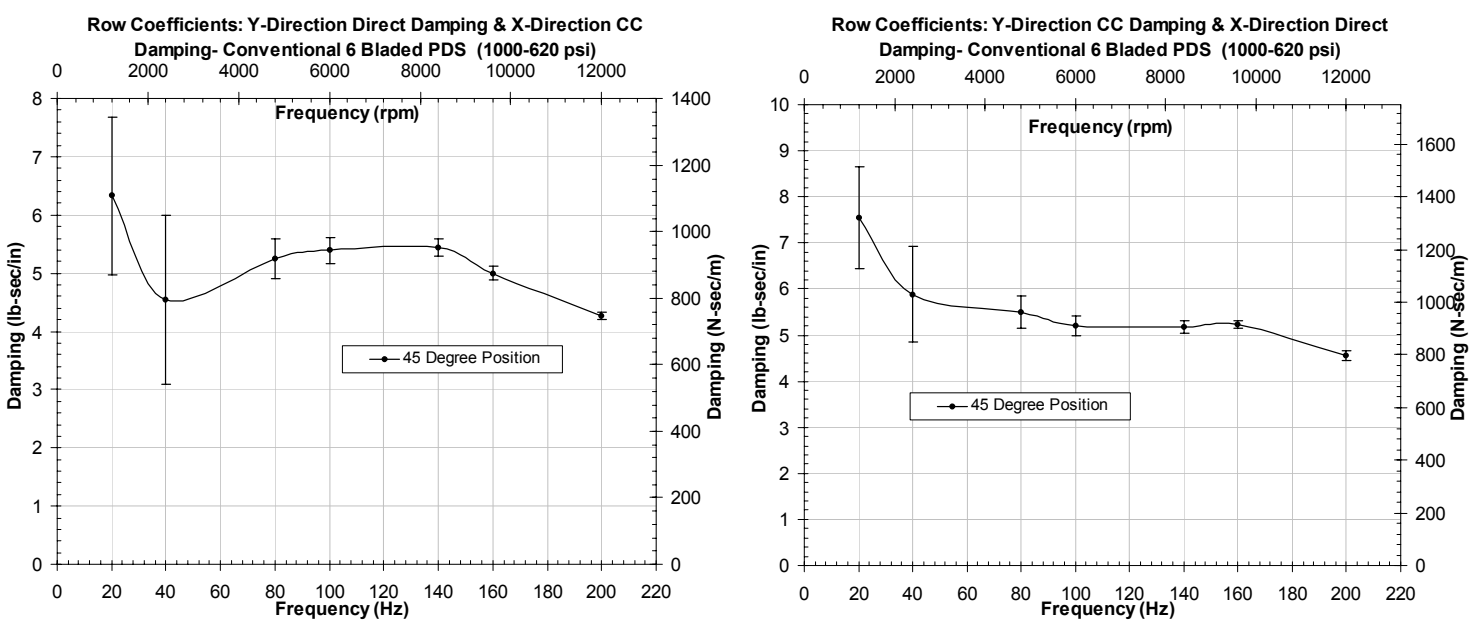

Figure 73. Row Coefficients: 45 Degree Position Cross-Coupled and Direct Damping 

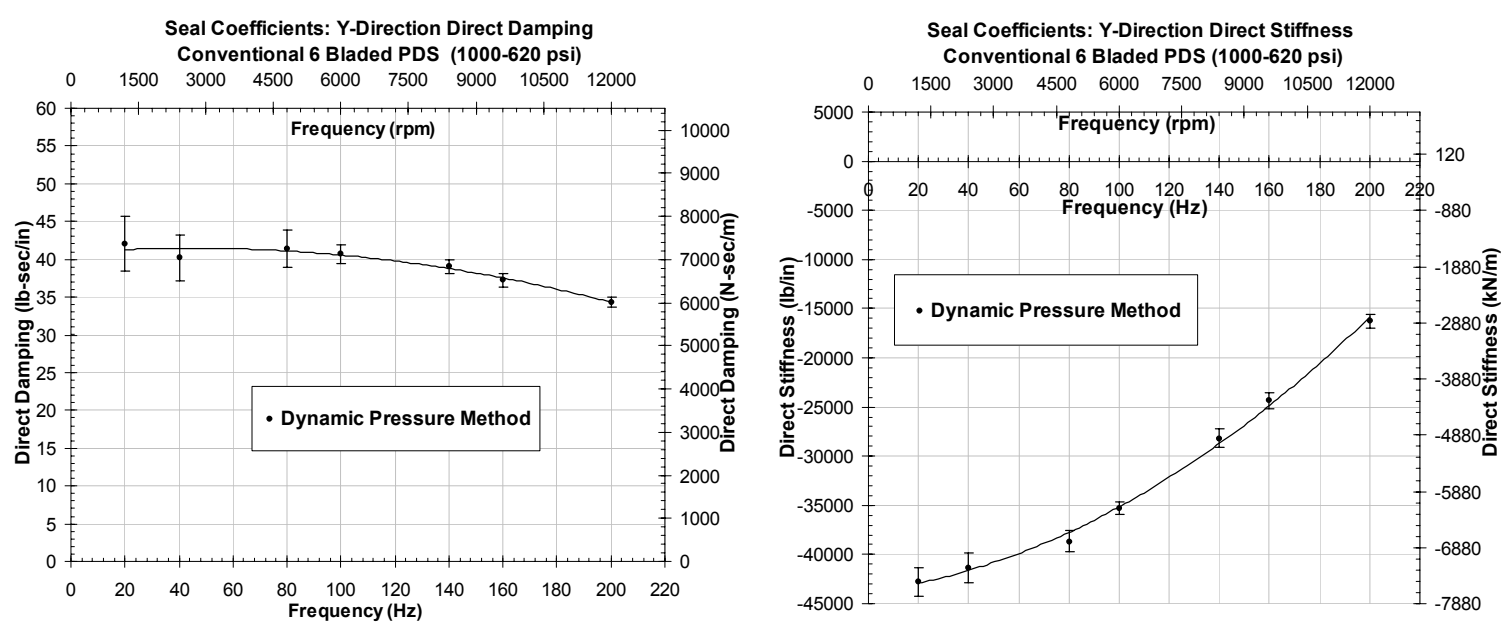

Figure 74. Conventional 6 Bladed PDS Y Dir. Direct Coefficients- Pressure Method
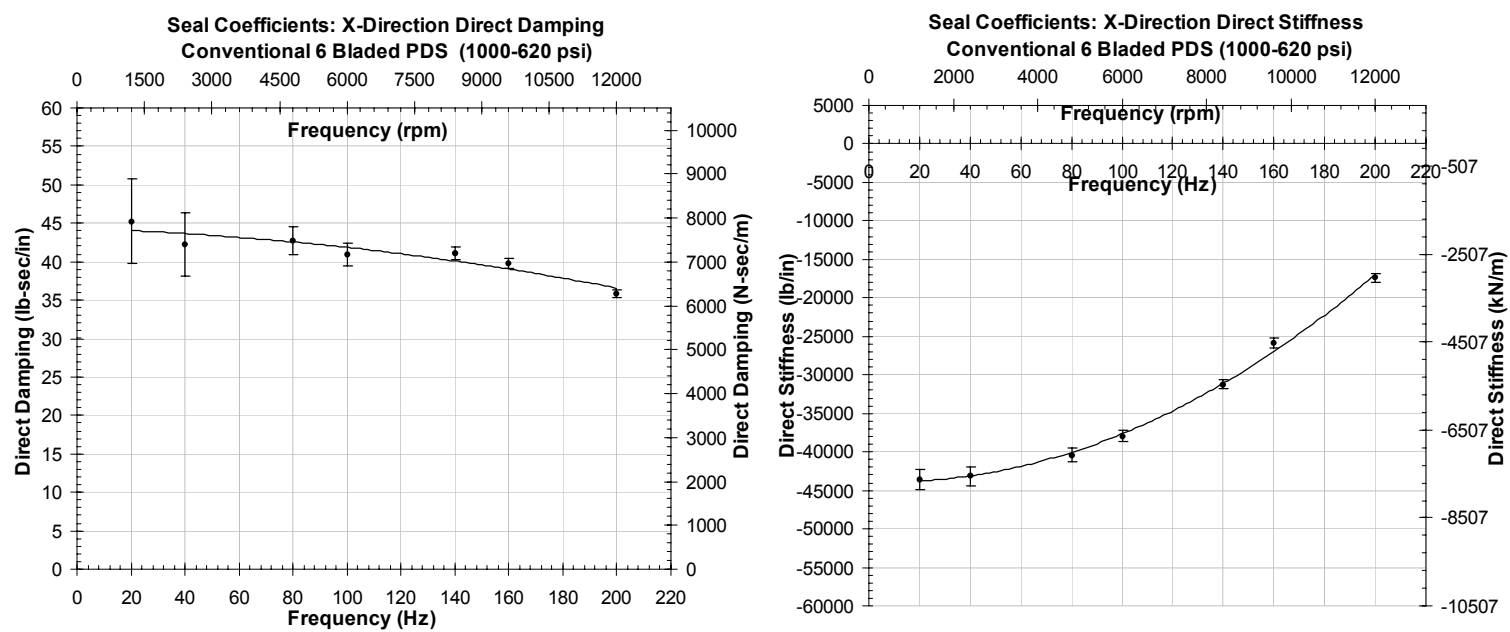

Figure 75. Conventional 6 Bladed PDS X Dir. Direct Coefficients- Pressure Method
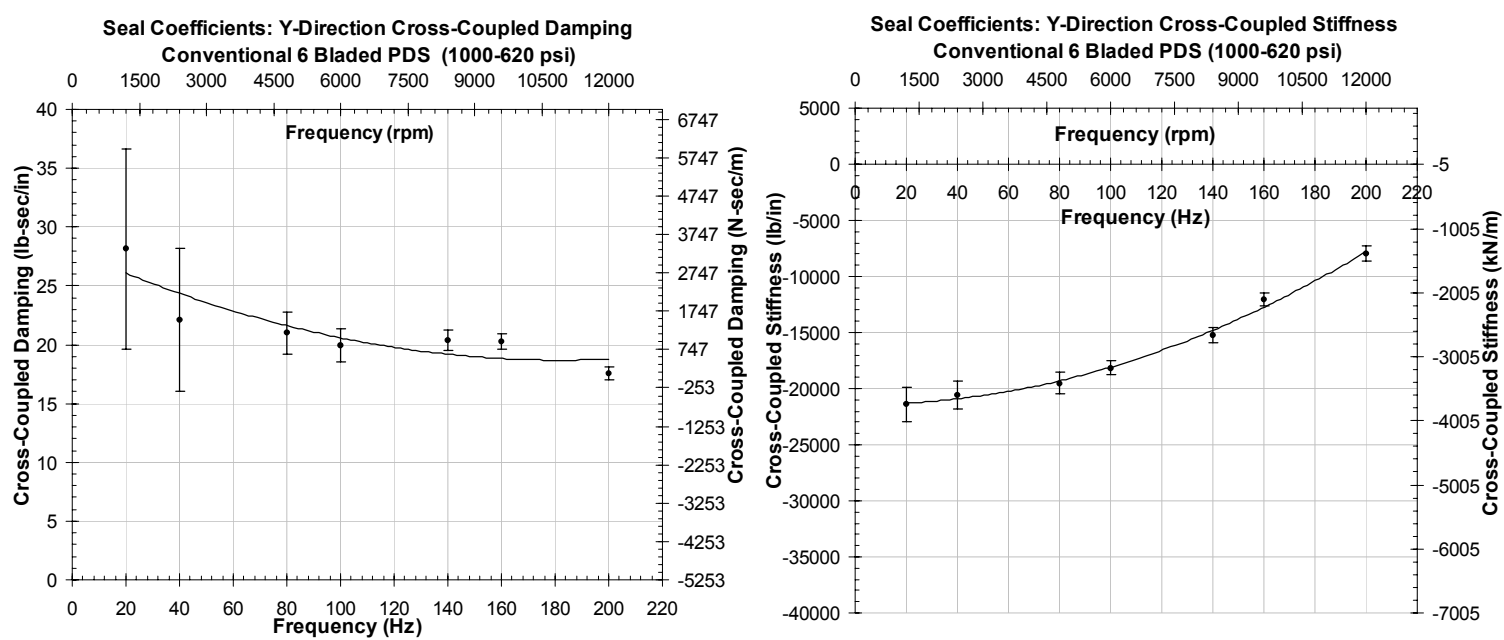

Figure 76. Conventional 6 Bladed PDS Y Dir. Cross-Coupled Coefficients-Pressure Method 

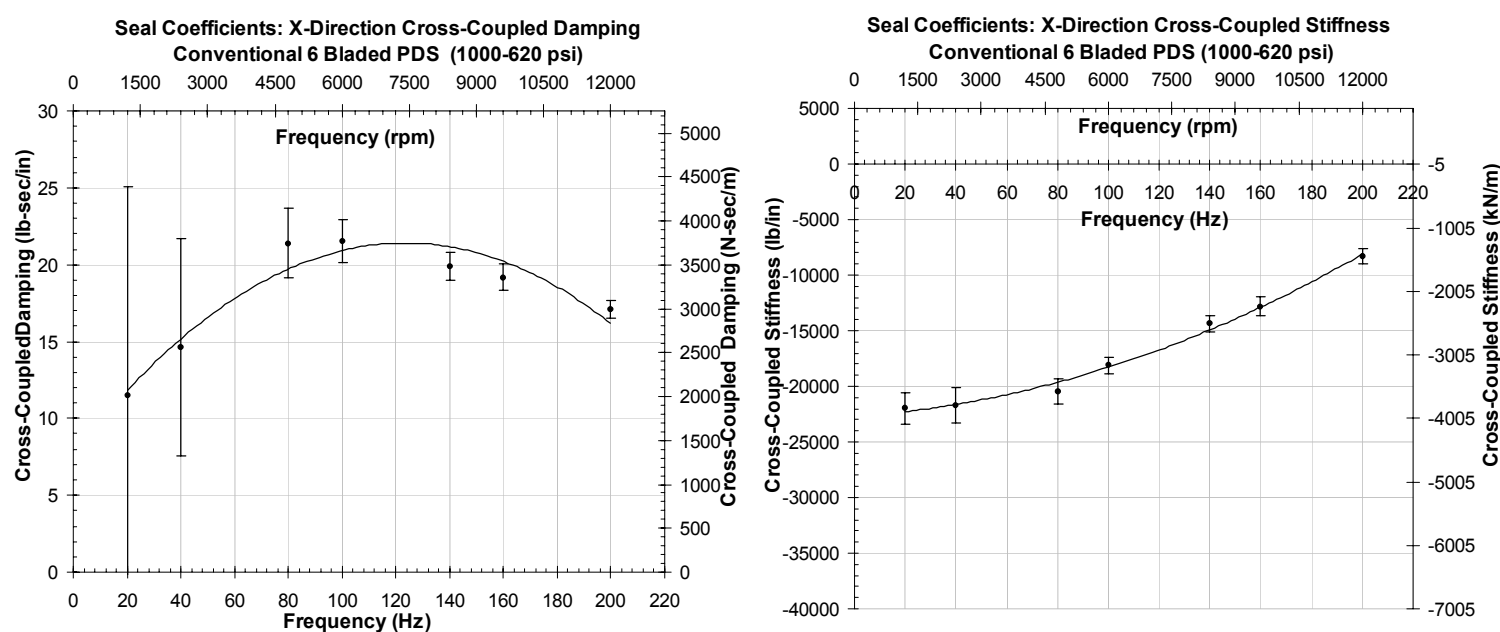

Figure 77. Conventional 6 Bladed PDS X Dir. Cross-Coupled Coefficients-Pressure Method
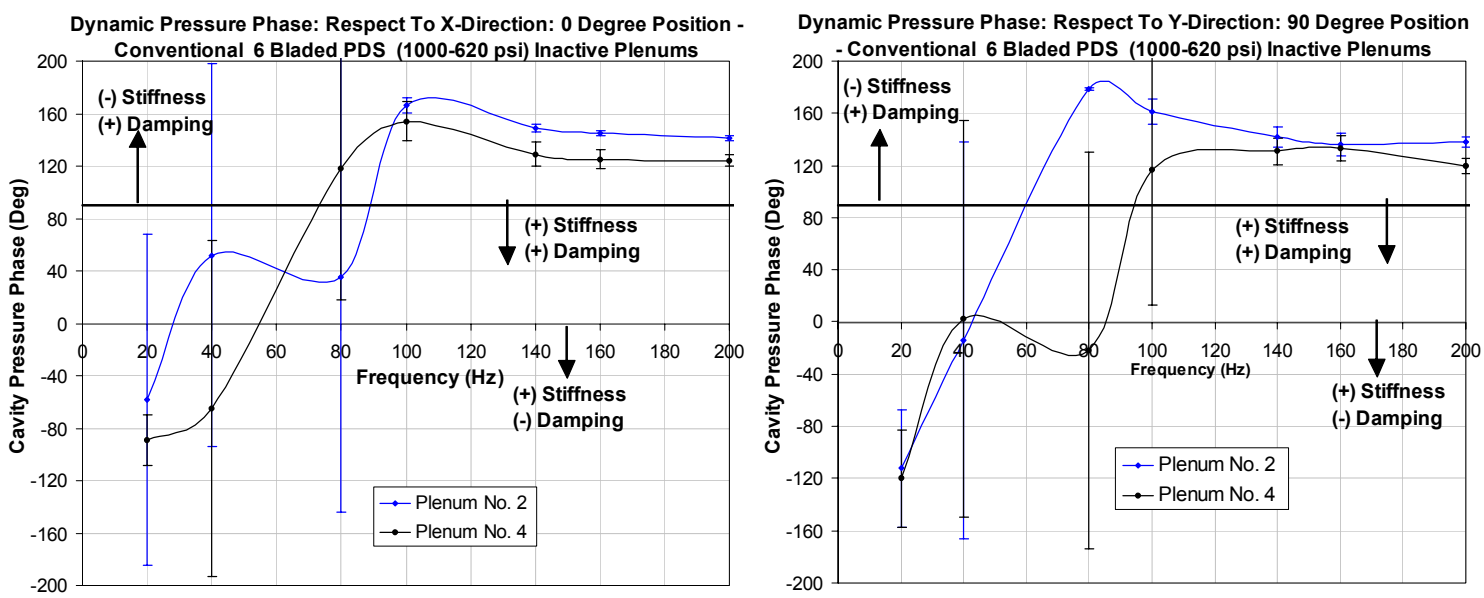

Figure 78. Local Pressure Modulation-Inactive Plenums 2 and 4, 90 and 0 Degrees

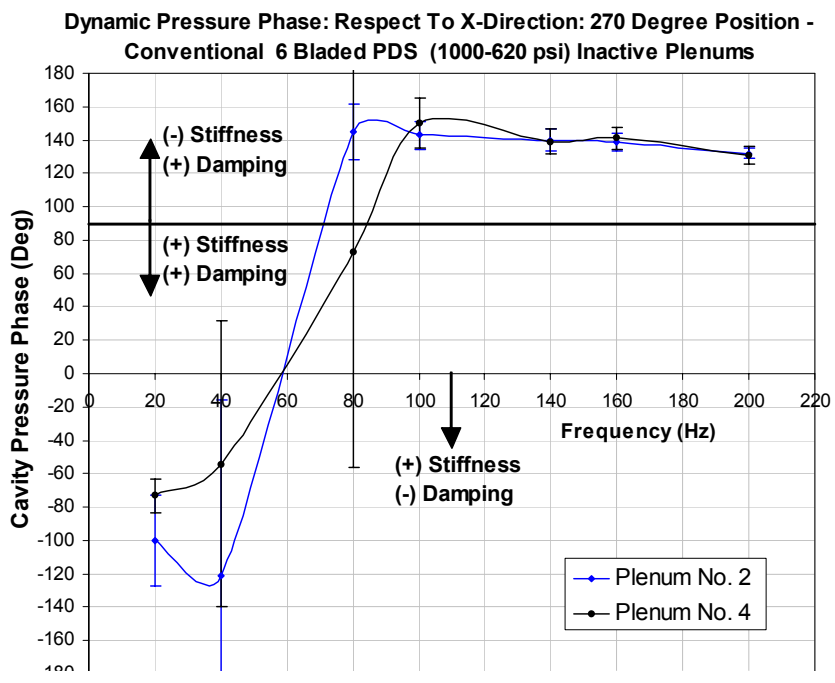

Figure 79. Local Pressure Modulation-Inactive Plenums 2 and 4, 270 Degrees 
Diverging Cavity Pressures \& Stator Vibration

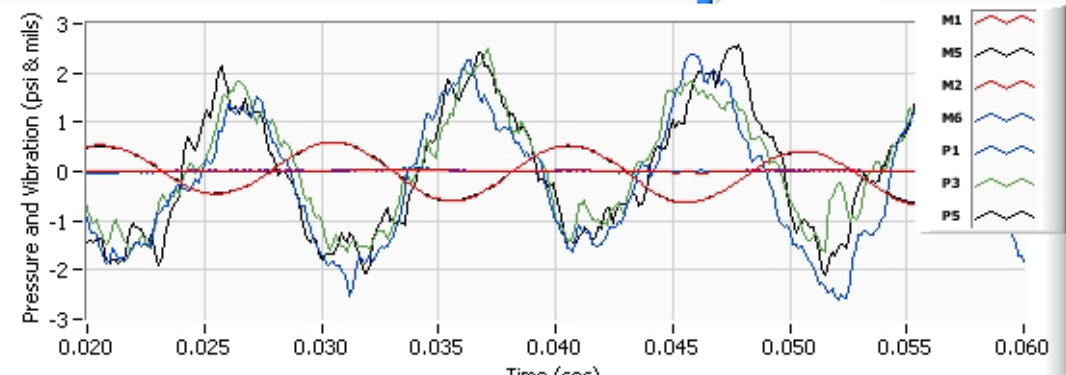

Inactive Plenum Pressures \& Stator Vibration

宸 $2.5-$

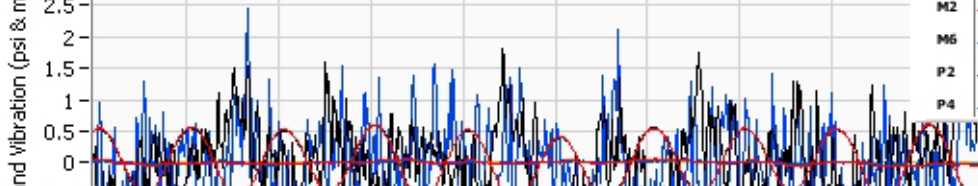

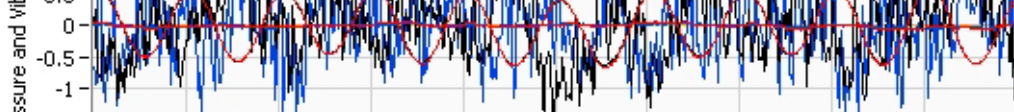

资 $-1-1$

$-1.5-$

$\begin{array}{lllllllllll}0.000 & 0.010 & 0.020 & 0.030 & 0.010 & 0.050 & 0.060 & 0.010 & 0.0180 & 0.090 & 0.100\end{array}$

Dynamic Cavity Pressures $\quad$ Time (sec)

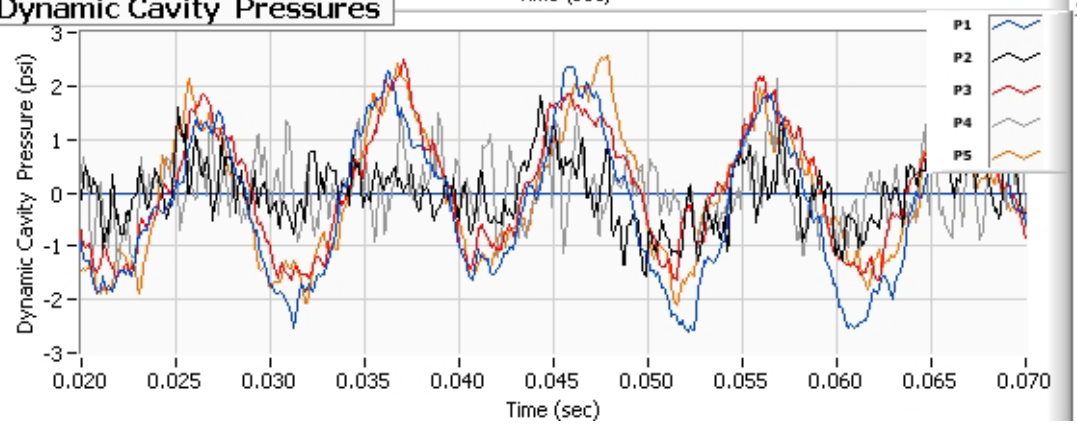

Diverging Cavities Pressure (FFT)
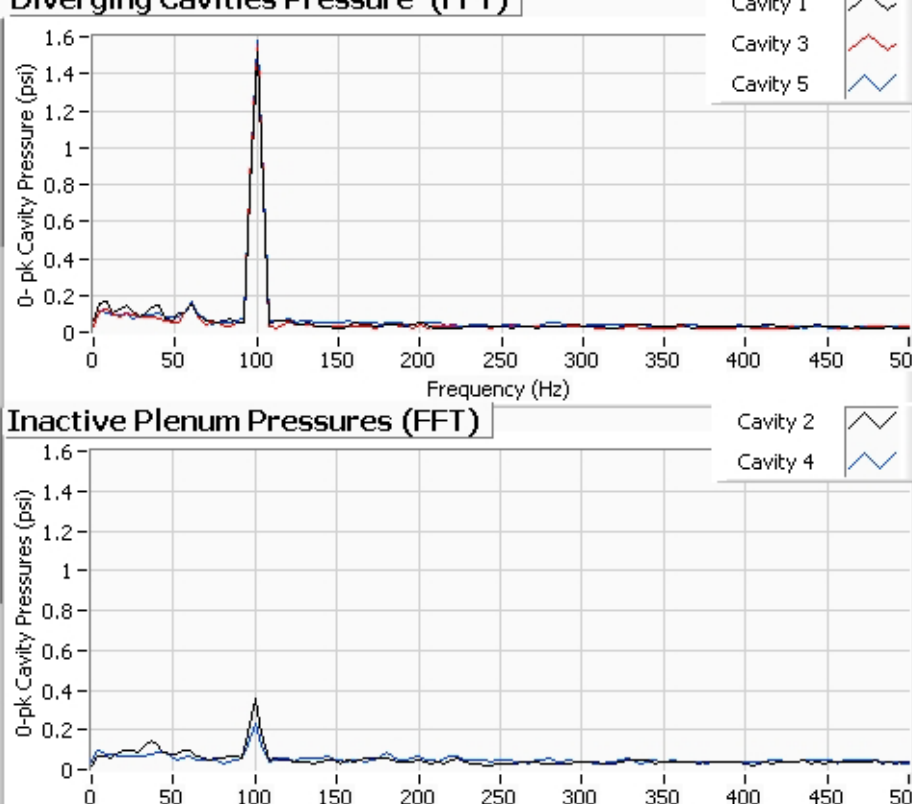

Frequency $(\mathrm{Hz})$

Cavity $2 \longdiv { \sim }$

Cavity $4 \sim$

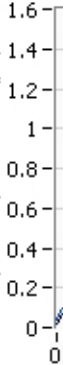

Stator

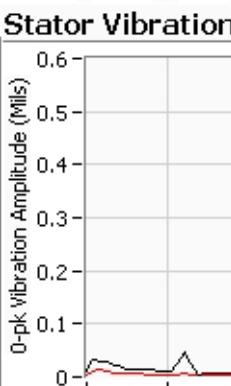

$\begin{array}{cccccccc}\prime & 1 & 1 & 1 & 1 & 1 & 1 & 1 \\ 150 & 200 & 250 & 300 & 350 & 400 & 450 & 500\end{array}$ (FFT) Frequency $(\mathrm{Hz})$

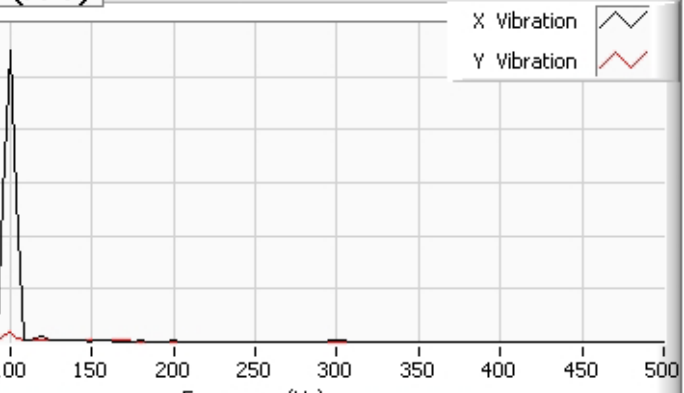

Frequency $(\mathrm{Hz})$

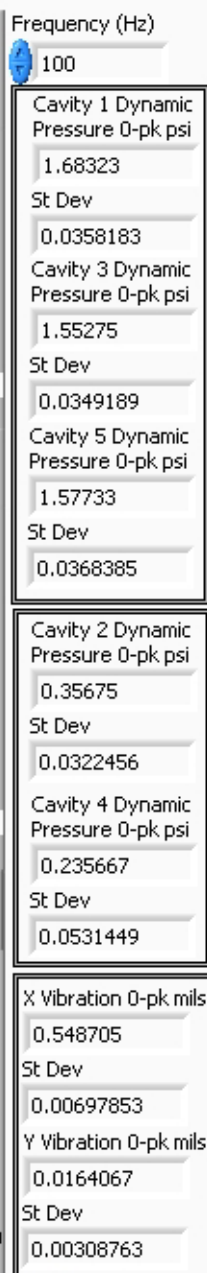

Figure 80. X Direction Excitation- 0 Degree Position $100 \mathrm{~Hz}$ 
Diverging Cavity Pressures \& Stator Vibration 1 슬 Data Set 1

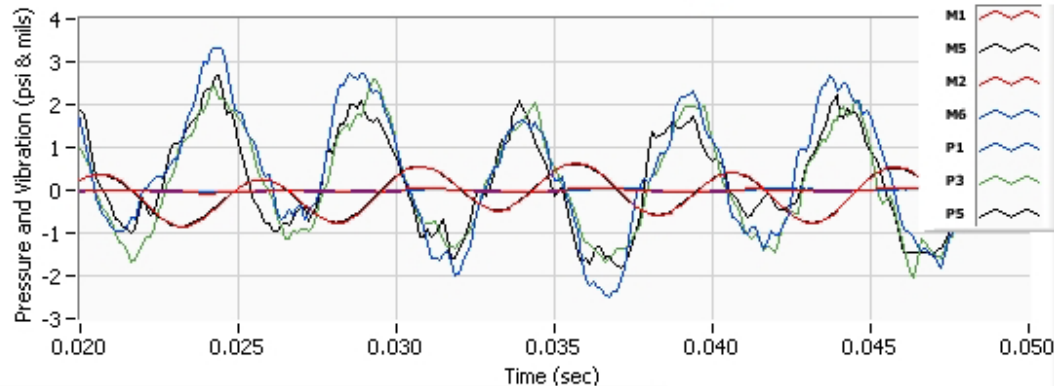

nactive Plenum Pressures \& Stator Vibration

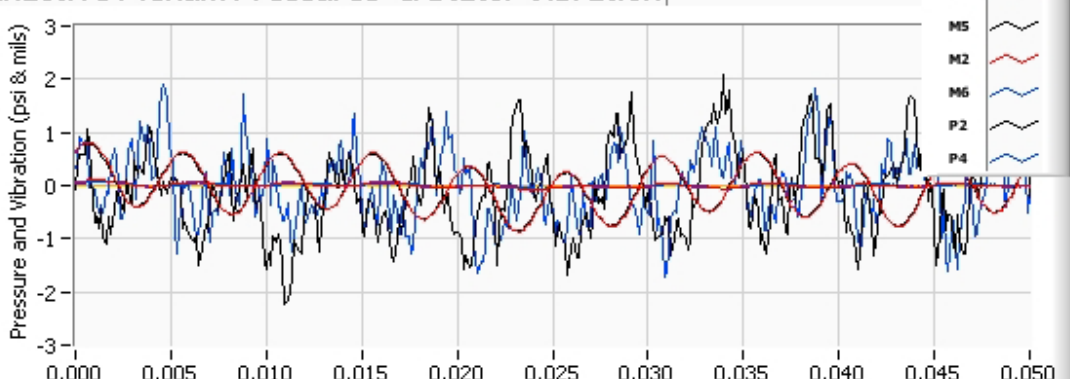

$\begin{array}{lllllllllll}0.000 & 0.005 & 0.010 & 0.015 & 0.020 & 0.025 & 0.030 & 0.035 & 0.040 & 0.045 & 0.050\end{array}$ Dynamic Cavity Pressures 1 Time (sec)

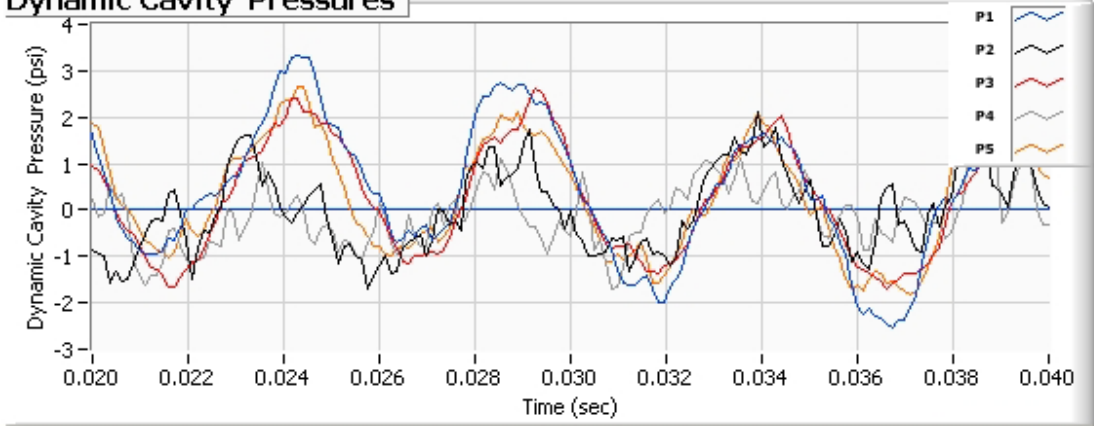

\section{Diverging Cavities Pressure (FFT)}
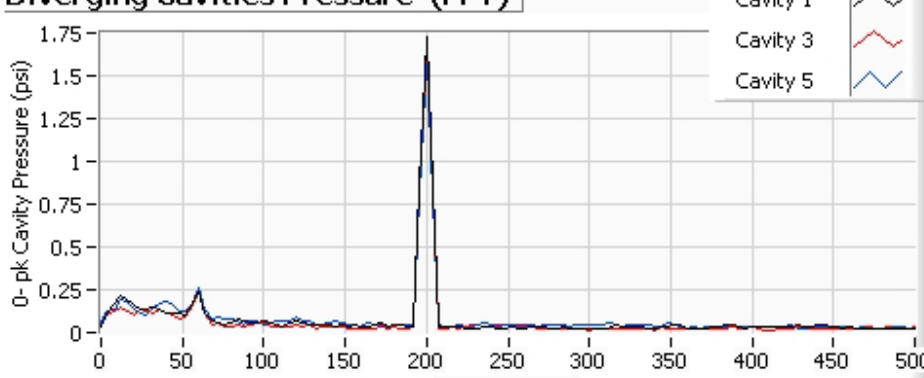

Inactiv

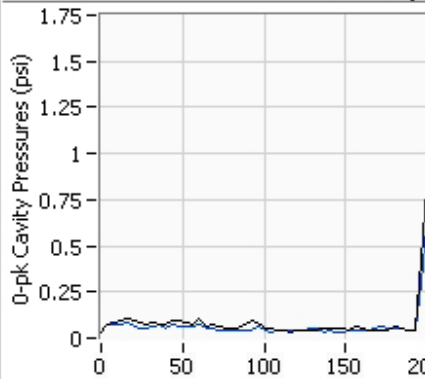

(FFT)

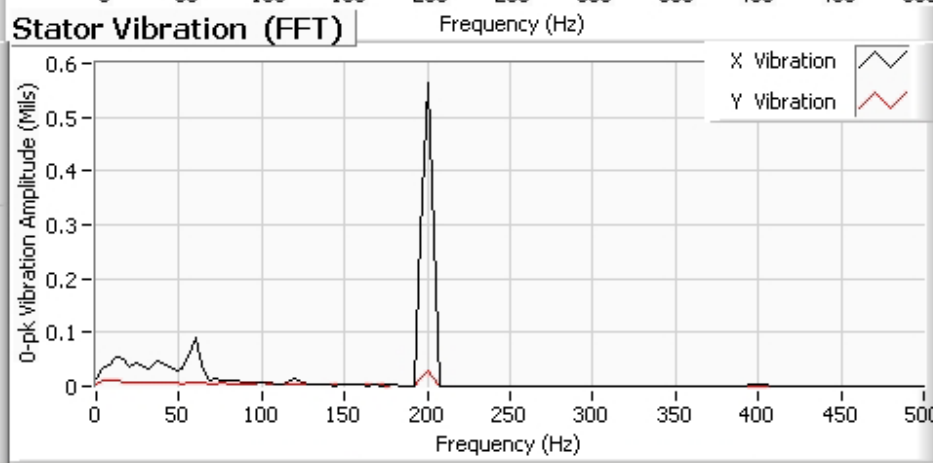
Frequency $(\mathrm{Hz})$

Cavity $2 \longdiv { }$

Cavity $4 \curvearrowright$

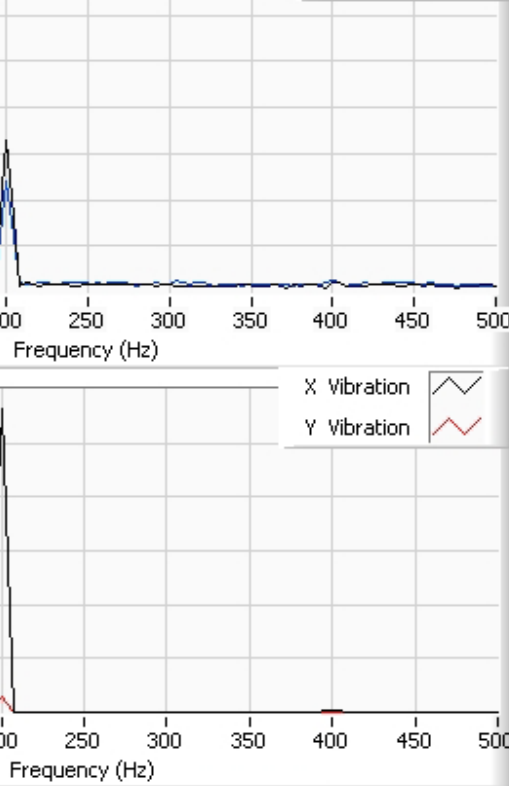

Figure 81. X Direction Excitation- 0 Degree Position $200 \mathrm{~Hz}$ 
Diverging Cavity Pressures \& Stator Vibration 1 Data Set 1

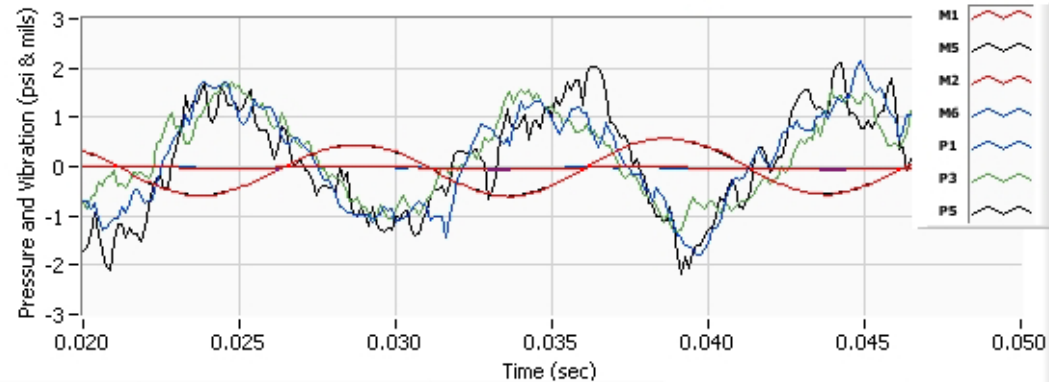

Inactive Plenum Pressures \& Stator Vibration

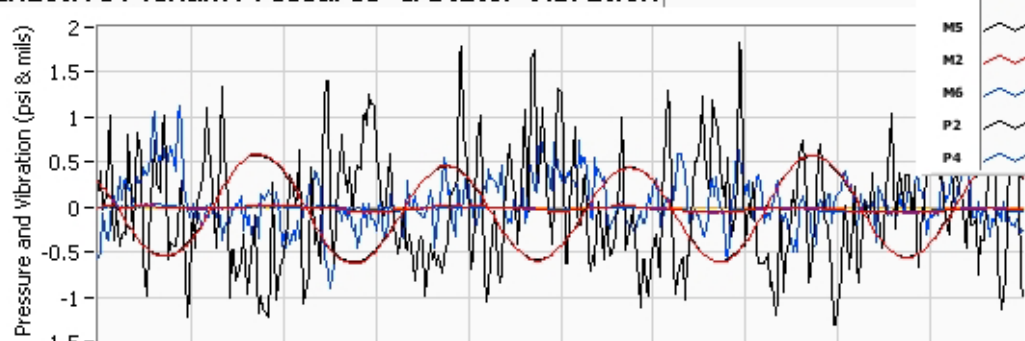

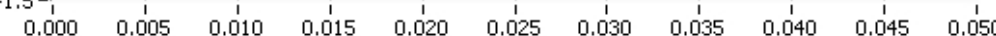

Dynamic Cavity Pressures $\quad$ Time (sec)

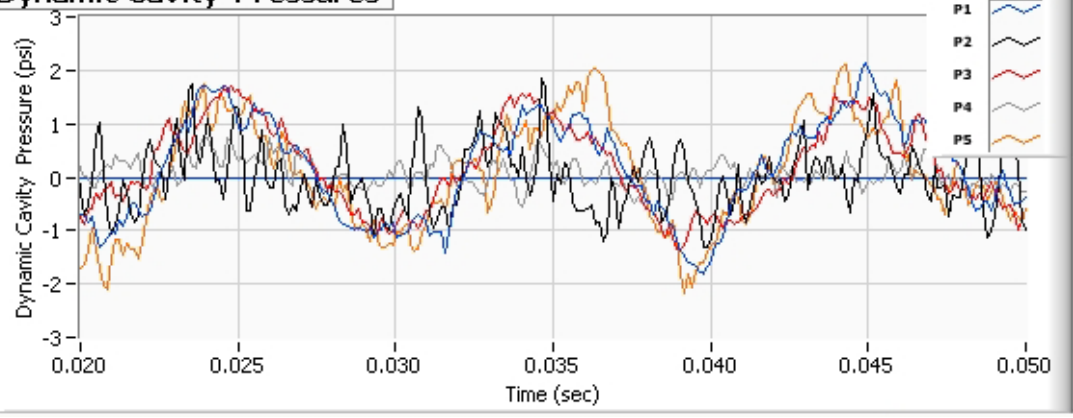

Diverging Cavities Pressure (FFT)
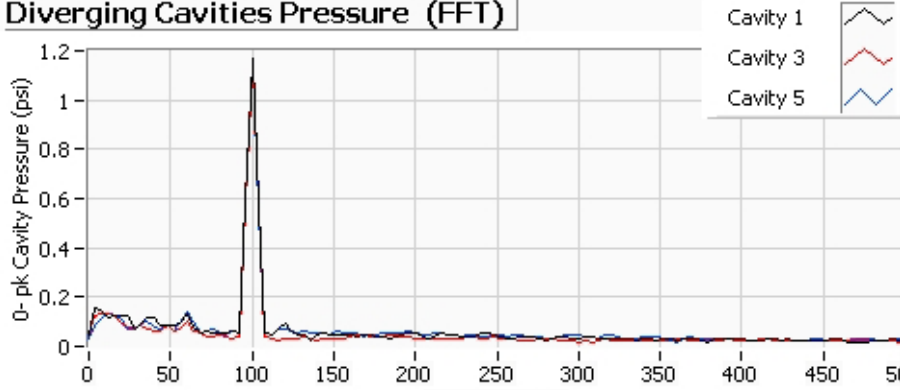

Cavity $2 \longdiv { }$
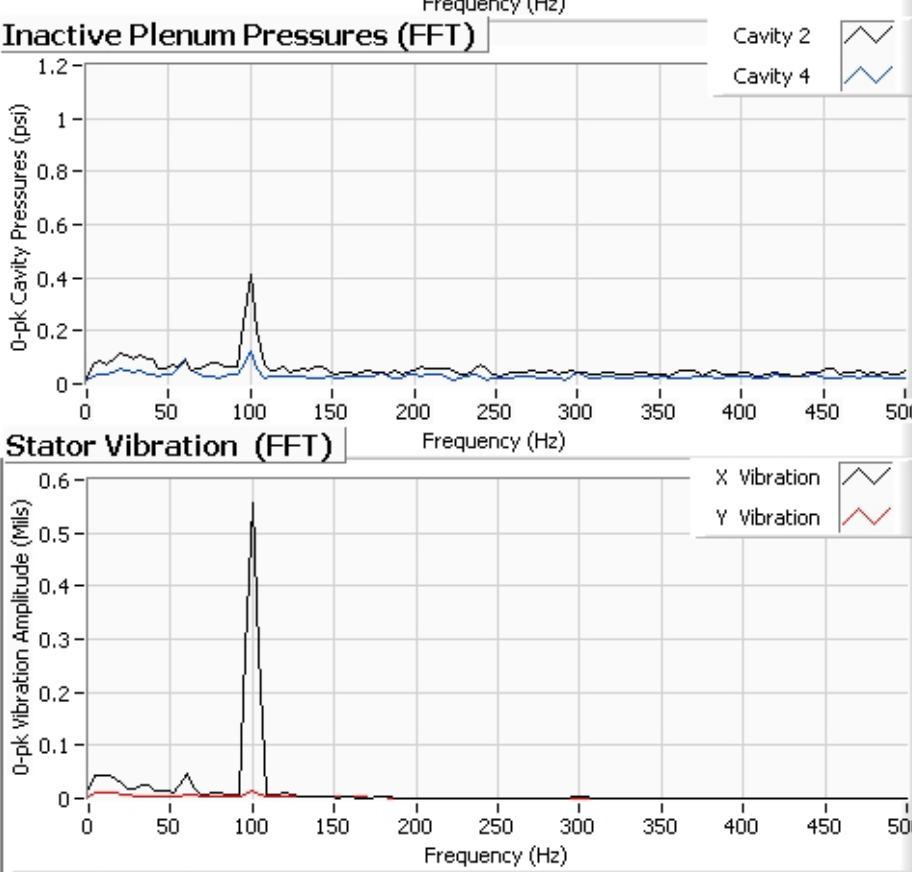

Frequency $(\mathrm{Hz})$

100

Cavity 1 Dynamic Pressure 0-pk psi 1.28953

0.0536725

Cavity 3 Dynamic

Pressure 0-pk psi

1.14896

0.028309

Cavity 5 Dynamic

Pressure 0-pk psi

0.045634

\section{Cavity 2 Dynamic}

0.41042 p

0.41042

St Dev

0.0516259

Cavity 4 Dynamic

Pressure 0-pk psi

0.121873

St Dev

0.0179025

X Vibration 0-pk mils

0.557022

St Dev

0.00347209

Y Vibration 0-pk mils

0.0140627

St Dev

0.00284449

Figure 82. X Direction Excitation- 45 Degree Position $100 \mathrm{~Hz}$ 
Diverging Cavity Pressures \& Stator Vibration 1 Data Set 1

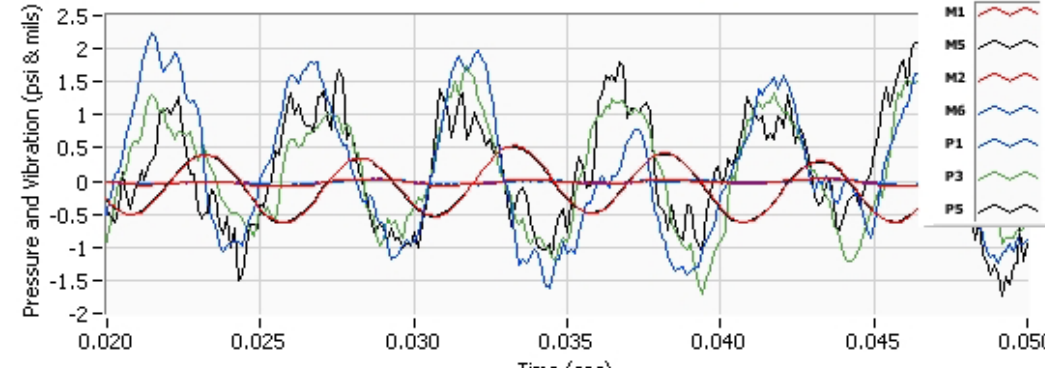

Inactive Plenum Pressures \& Stator Vibration

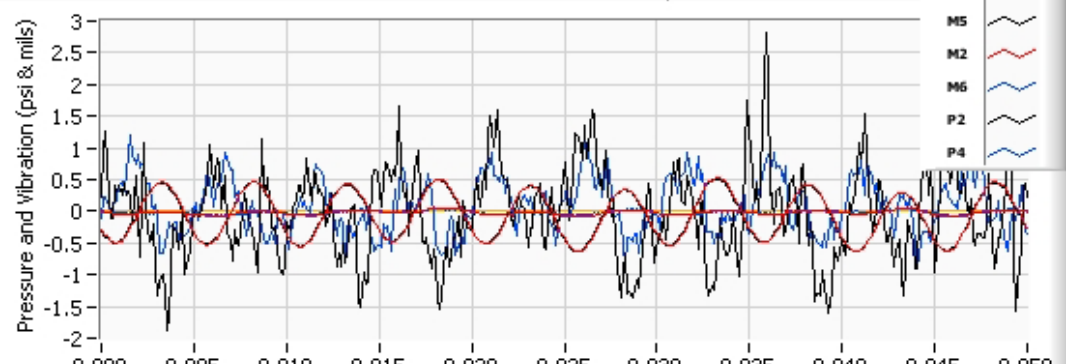

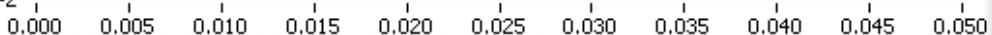

Dynamic Cavity Pressures $\_$Time (sec)

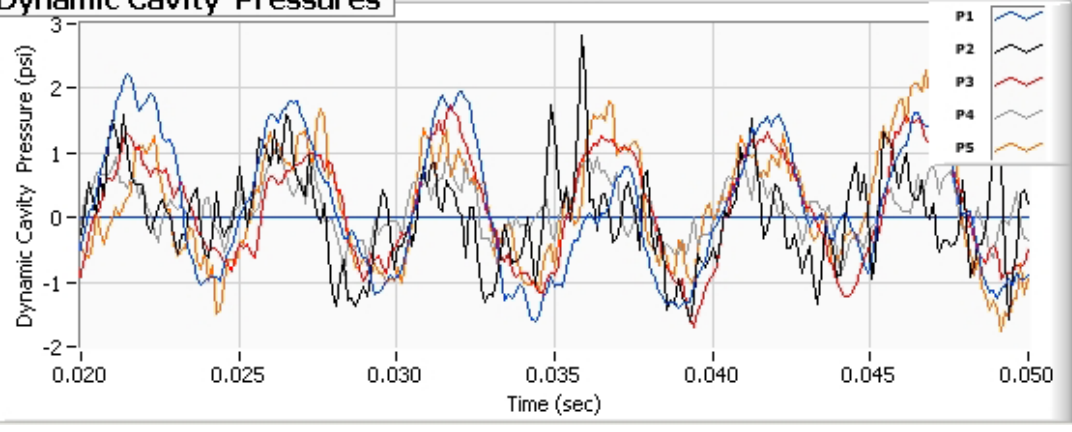

Diverging Cavities Pressure (FFT)
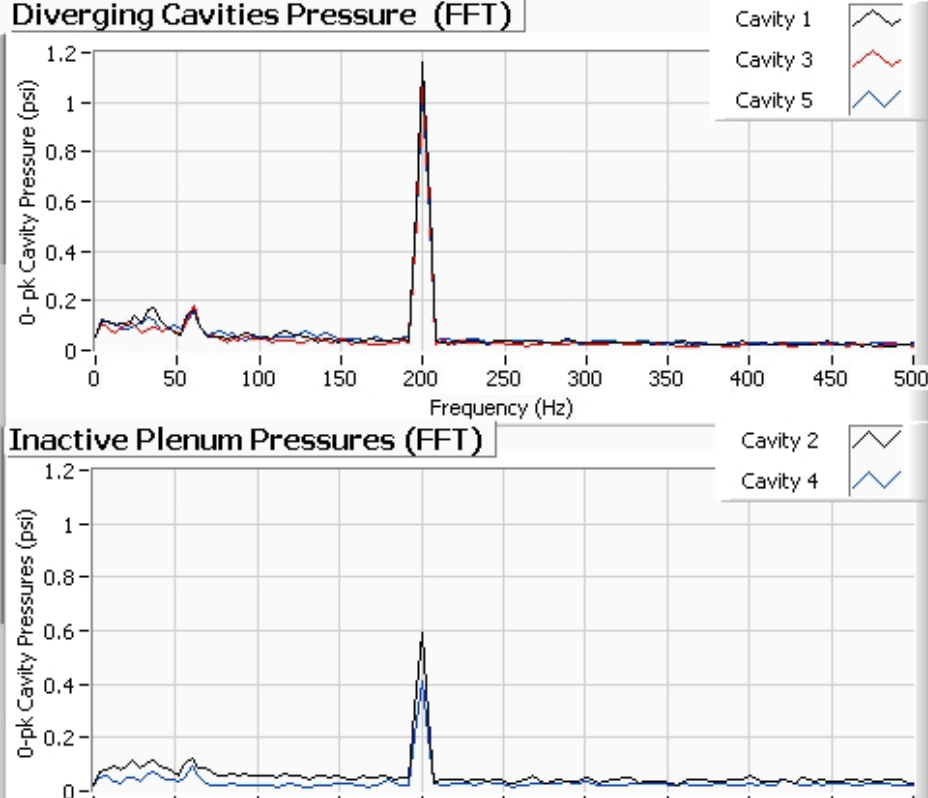

FrT)

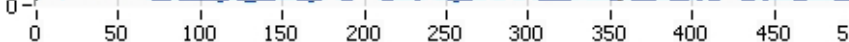
Stator Vibration (FFT) $\quad$ Frequency $(\mathrm{Hz})$

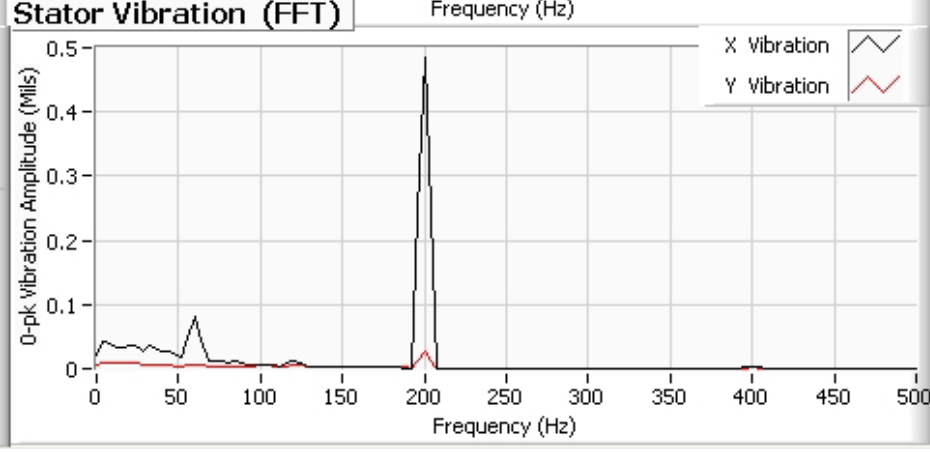

Figure 83. X Direction Excitation- 45 Degree Position $200 \mathrm{~Hz}$ 
Diverging Cavity Pressures \& Stator Vibration

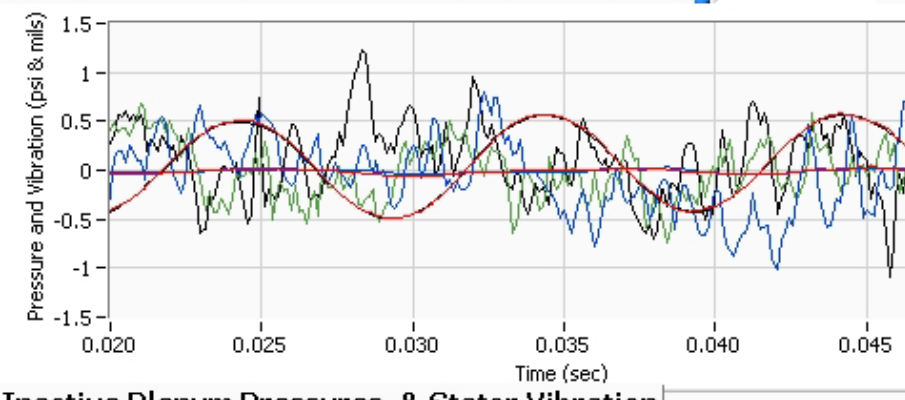

Inactive Plenum Pressures \& Stator Vibration

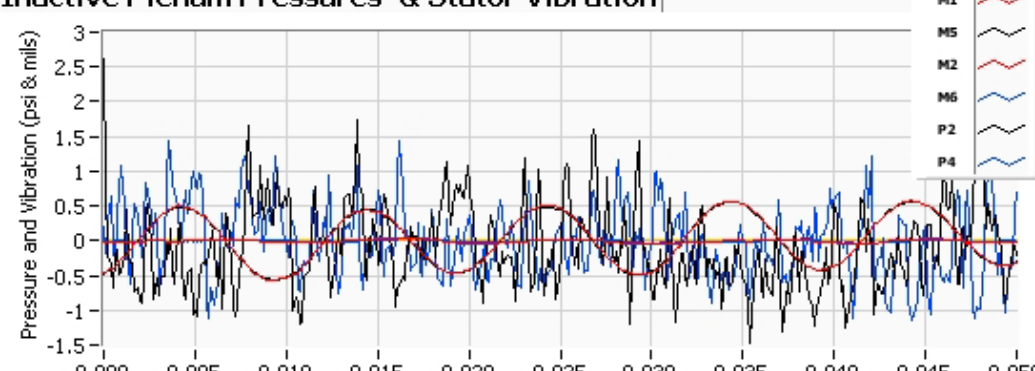

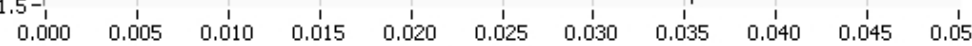

Dynamic Cavity Pressures

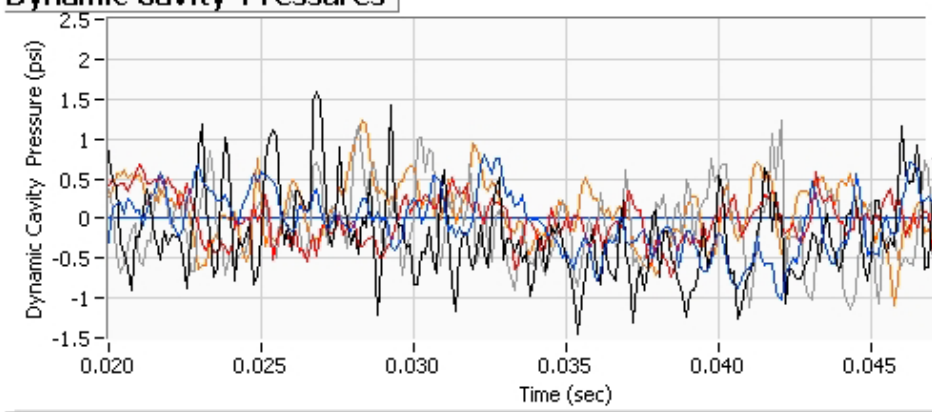

Diverging Cavities Pressure (FFT)

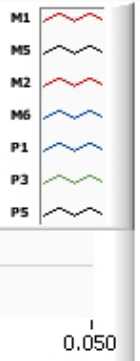

$\mathrm{P1}_{\mathrm{W}}$

$\mathrm{P1}_{\mathbf{P} 2} \widetilde{\sim}$

舟

P4

P5

4 W

ind

1.

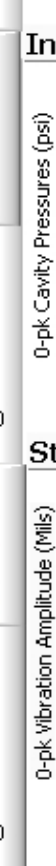

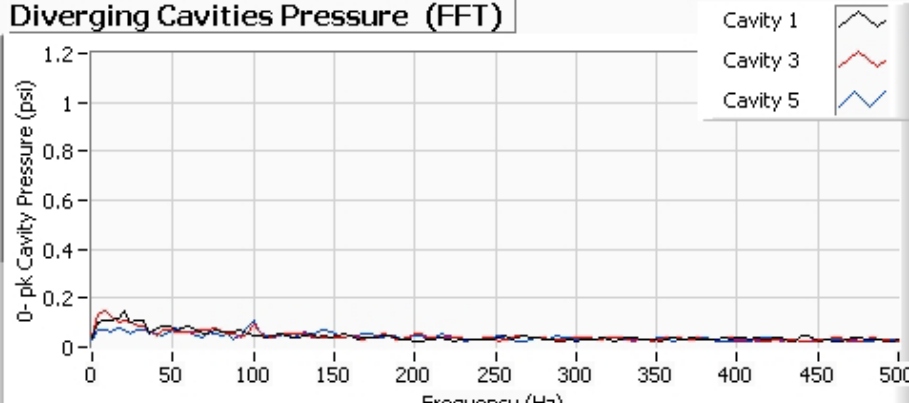

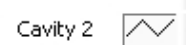

Cavity $4 \sim$

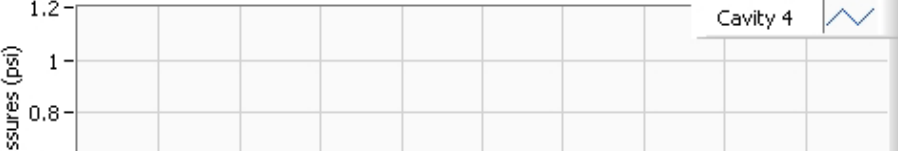

高

空 0.4

高0.2-<smiles>C1C[SiH2]C1</smiles>

5
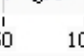

100

Stator Vibration (FFT)

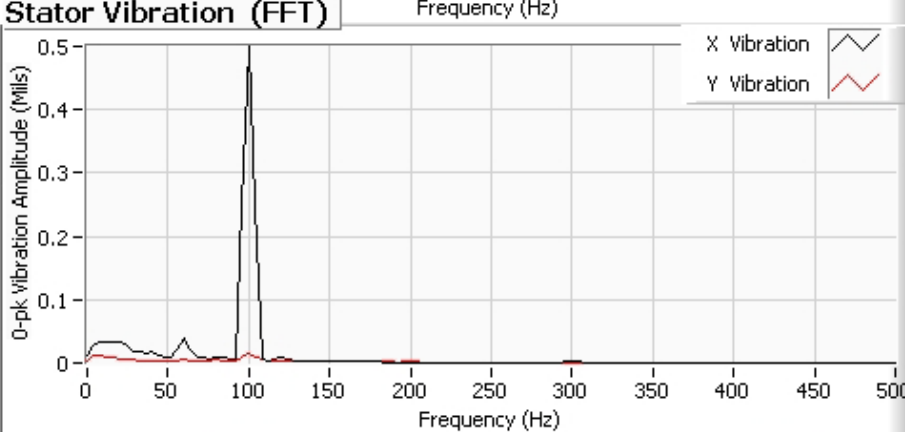

Figure 84. X Direction Excitation- 90 Degree Position 100 Hz 
Diverging Cavity Pressures \& Stator Vibration $1 / 7$ Data Set 1
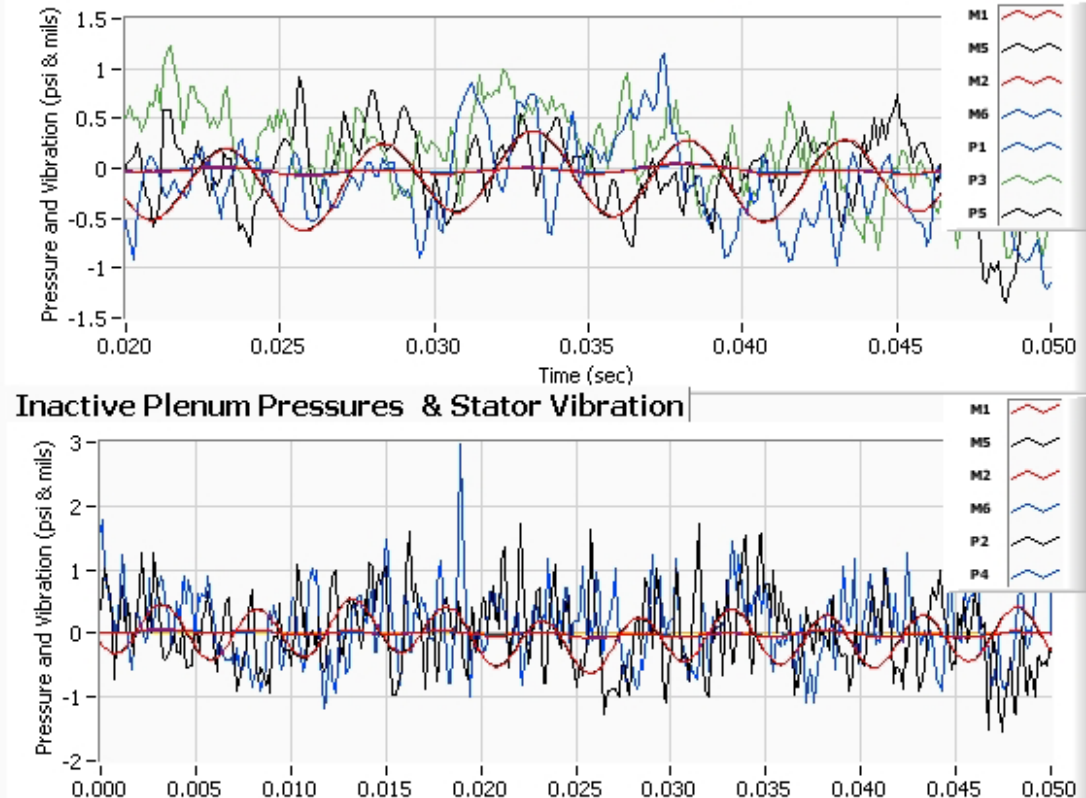

$\begin{array}{lllllllllll}0.000 & 0.005 & 0.010 & 0.015 & 0.020 & 0.025 & 0.030 & 0.035 & 0.040 & 0.045 & 0.050\end{array}$

\section{Dynamic Cavity Pressures}

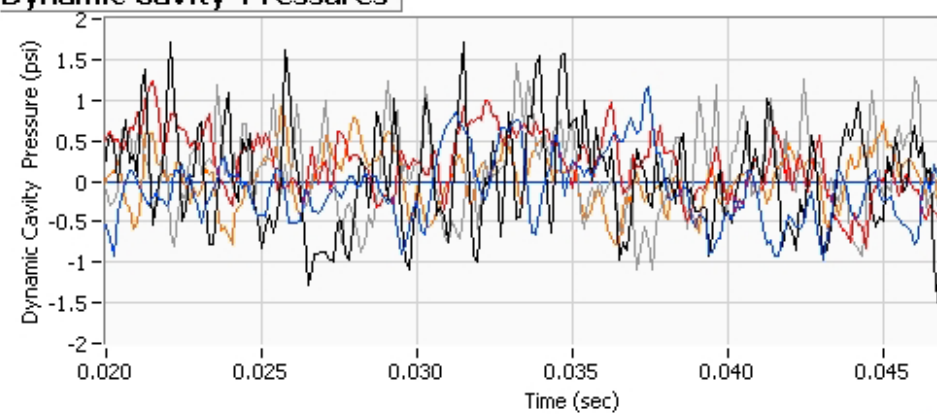

Diverging Cavities Pressure (FFT)

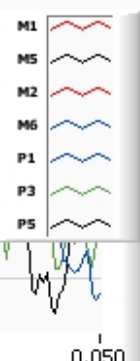

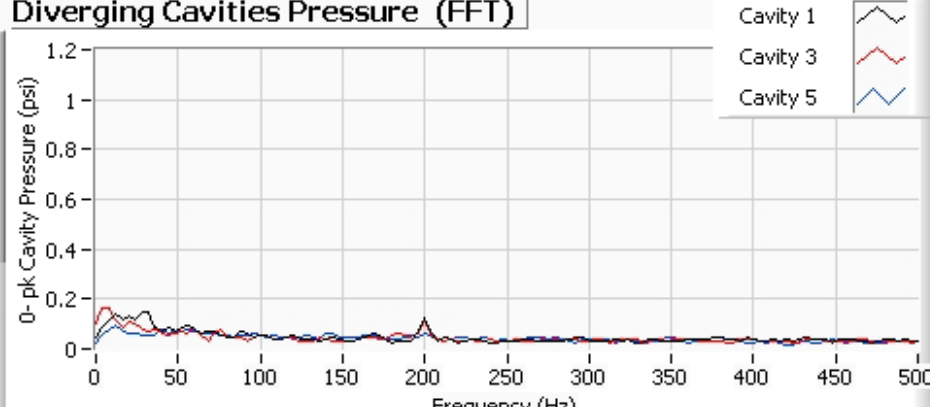
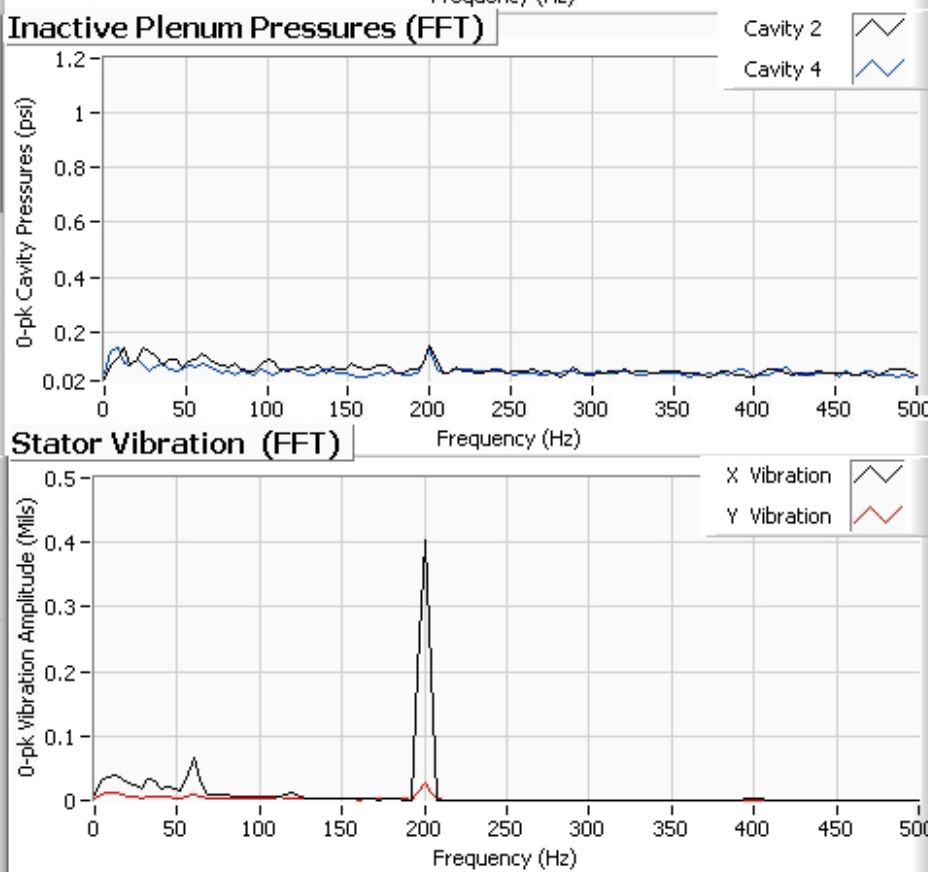
200 Cavity 1 Dynamic Pressure 0-pk psi 0.131971

5t Dev 0.0326018 Cavity 3 Dynamic Pressure 0-pk psi 0.108792

5t Dev

0.0195585

Cavity 5 Dynamic Pressure 0-pk psi 0.0599656

St Dev 0.0189797

Cavity 2 Dynamic Pressure 0-pk psi 0.150727

St Dev 0.0317834 Cavity 4 Dynamic Pressure 0-pk psi 0.141666 St Dev 0.0335754 X vibration 0-pk mils 0.403842 th Dev 0.000996611 $\gamma$ vibration 0-pk mils 0.0260606 5t Dev 0.000950575

Figure 85. X Direction Excitation- 90 Degree Position $200 \mathrm{~Hz}$ 
Table 13. Inactive Plenum Pressure Magnitudes

\begin{tabular}{|c|c|c|c|c|}
\hline FREQ. & $\begin{array}{l}\text { ANGULAR } \\
\text { LOCATION }\end{array}$ & $\begin{array}{l}\text { EXCITATION 0-PK } \\
\text { X AMP. (MILS) }\end{array}$ & $\begin{array}{l}\text { PLENUM } 2 \text { PRESSURE } \\
\text { 0-PK MAGNITUDE PSI }\end{array}$ & $\begin{array}{l}\text { PLENUM } 4 \text { PRESSURE } \\
\text { 0-PK MAGNITUDE PSI }\end{array}$ \\
\hline $100 \mathrm{HZ}$ & 0 DEG & 0.5487 & 0.356 & 0.235 \\
\hline $200 \mathrm{HZ}$ & 0 DEG & 0.5627 & 0.825 & 0.604 \\
\hline $100 \mathrm{HZ}$ & 45 DEG & 0.557 & 0.41 & 0.122 \\
\hline $200 \mathrm{HZ}$ & 45 DEG & 0.485 & 0.591 & 0.413 \\
\hline $100 \mathrm{HZ}$ & 90 DEG & 0.499 & 0.187 & 0.116 \\
\hline $200 \mathrm{HZ}$ & 90 DEG & 0.404 & 0.151 & 0.142 \\
\hline$\overline{\text { FREQ. }}$ & $\begin{array}{l}\text { ANGULAR } \\
\text { LOCATION }\end{array}$ & $\begin{array}{c}\text { FORCE DENSITY }\left(\mathrm{LBF} / \mathrm{IN}^{\wedge} 3\right) \\
(\mathrm{P} / \mathrm{Y}) \text {-PLENUMM } 2\end{array}$ & $\begin{array}{c}\text { FORCE DENSITY }\left(\mathrm{LBF} / \mathrm{IN}^{\wedge} 3\right) \\
(\mathrm{P} / \mathrm{Y}) \text {-PLENUMM } 4\end{array}$ & \\
\hline $100 \mathrm{HZ}$ & 0 DEG & 648.81 & 428.29 & \\
\hline $200 \mathrm{HZ}$ & 0 DEG & 1466.15 & 1073.40 & \\
\hline $100 \mathrm{HZ}$ & 45 DEG & 736.09 & 219.03 & \\
\hline $200 \mathrm{HZ}$ & 45 DEG & 1218.56 & 851.55 & \\
\hline $100 \mathrm{HZ}$ & 90 DEG & 374.75 & 232.46 & \\
\hline $200 \mathrm{HZ}$ & 90 DEG & 373.76 & 351.49 & \\
\hline
\end{tabular}




\subsection{Cavity Coefficients for Fully Partitioned Six Bladed PDS}

The fully partitioned seal used for the following test results possessed the same cavity depth, pitch ratio, clearance, clearance ratio, seal length, pressure ratio, and testing scheme as the conventional six bladed PDS. As discussed in Section II, the fully partitioned design incorporates baffle walls in the two inactive plenums; resulting in the creation of 2 converging cavities. Figure 86 shows the fully partitioned seal and the cavity numbers that are used. Note that cavity 1 is the upstream cavity whereas cavity 5 is the down stream cavity. An example front panel display for a $100 \mathrm{~Hz}$ Y direction excitation test at the 90 degree angular location is shown in Figure 87. The data processing program (Figure 88) for the FP PDS was modified to incorporate two extra cavities (2 and 4) into the overall seal coefficient calculations. The addition of the baffle walls in the inactive plenums resulted in higher pressure modulations that are approximately 10 times larger, when comparing results from Figure 80 to Figure 87.
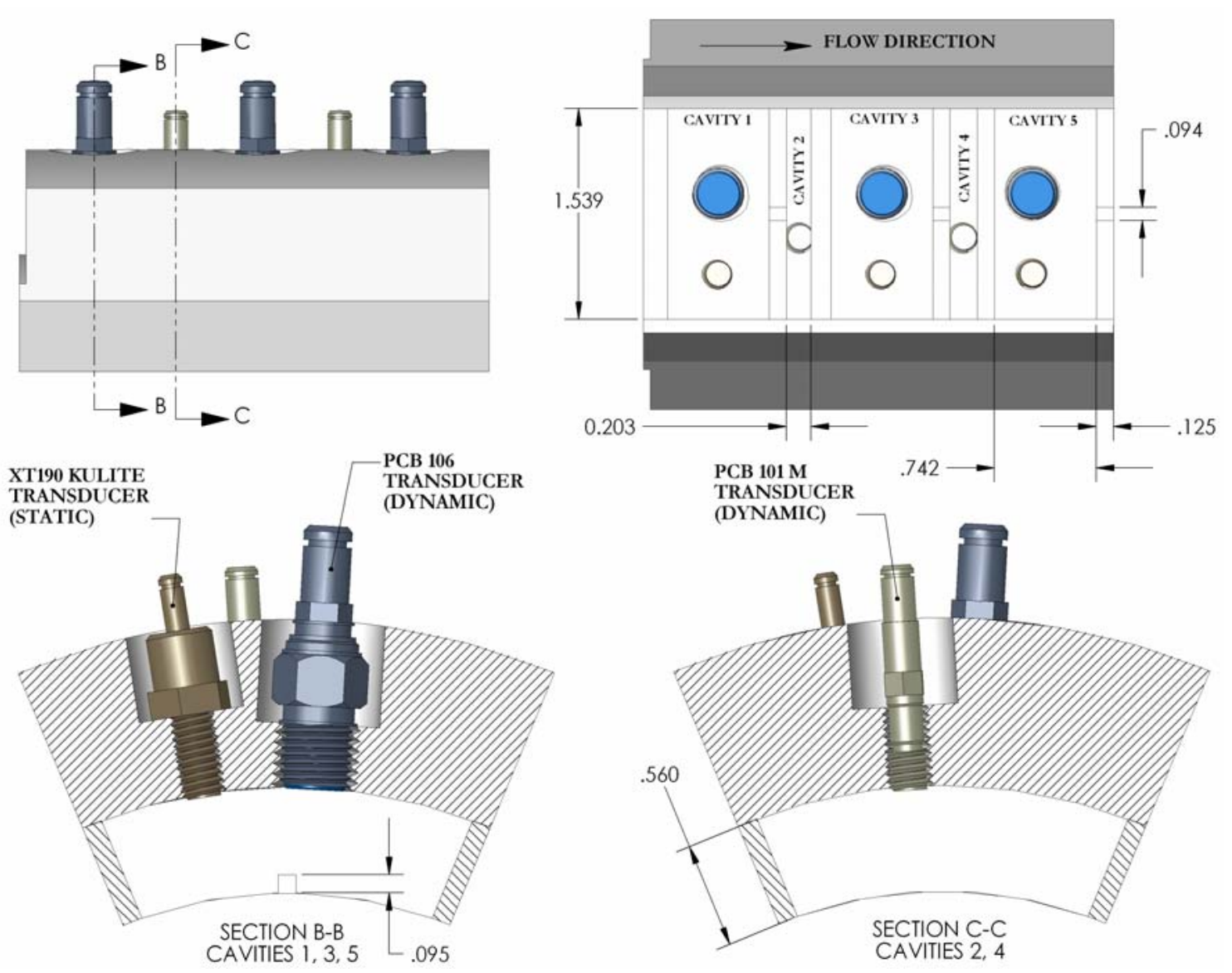

Figure 86. Single Row of Pockets FP 6 Bladed PDS: Pressure Transducer Installation 
Diverging Cavity Pressures \& Stator Vibration 1 Data Set 1

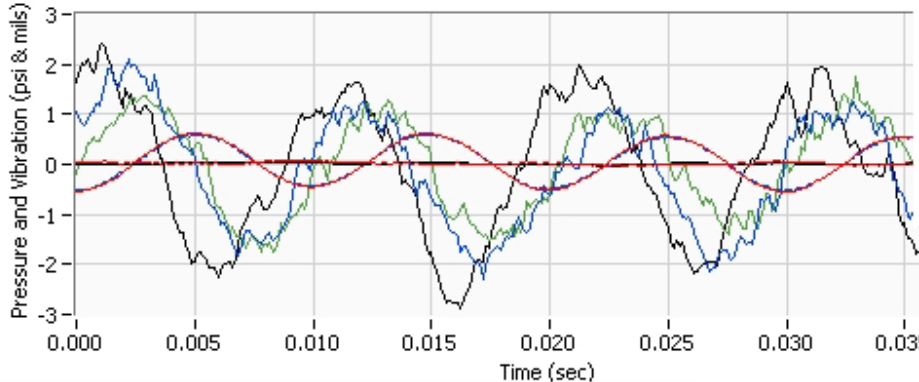

Converging Cavity Pressures \& Stator Vibration

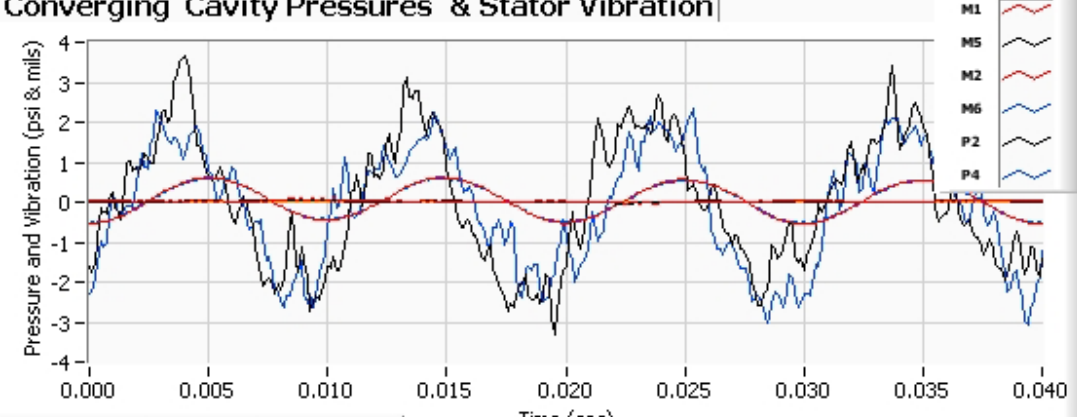

$\begin{array}{lllllllll}0.000 & 0.005 & 0.010 & 0.015 & 0.020 & 0.025 & 0.030 & 0.035 & 0.040\end{array}$

\section{Dynamic Cavity Pressures}

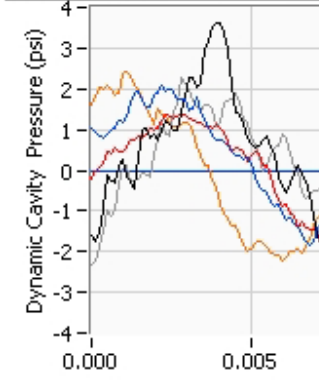

0.005
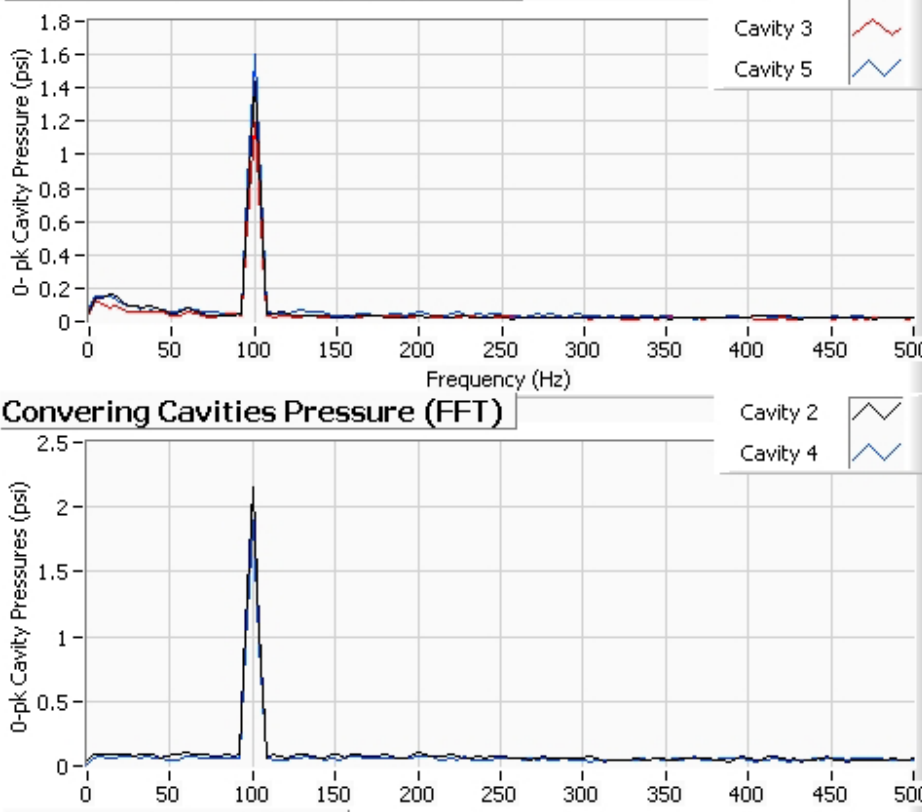
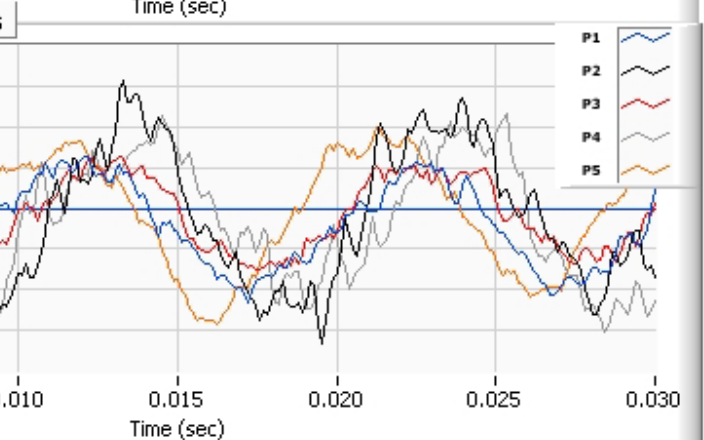

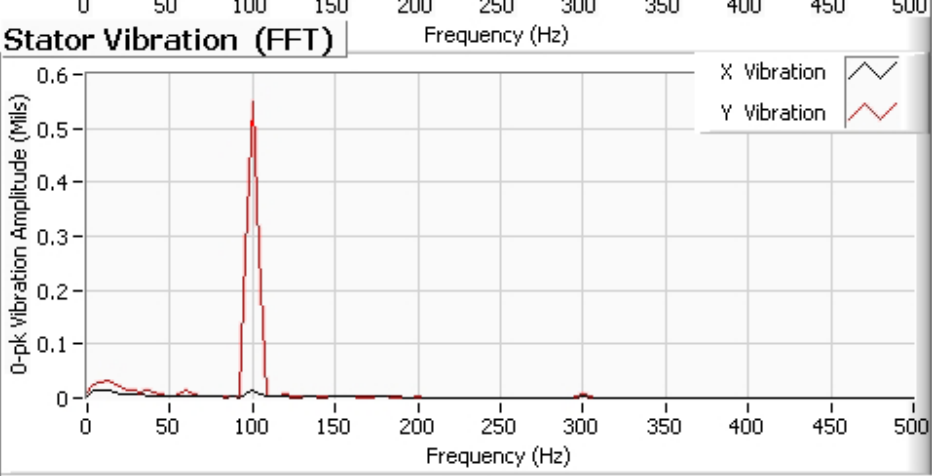

Figure 87. Fully Partitioned Seal: Y Direction Excitation 100 Hz -90 Degree Angle Position 


\begin{tabular}{|c|c|c|c|c|c|c|c|c|c|c|c|}
\hline \multicolumn{4}{|c|}{ Rotordynamic Coefficients Row Values Y Direction } & \multicolumn{6}{|c|}{ Rotordynamic Coefficients Row Values X Direction } & \multicolumn{2}{|c|}{$\begin{array}{l}\text { Diverging Cavity } \\
\text { Projected Area (iin 2) }\end{array}$} \\
\hline \multicolumn{2}{|c|}{ 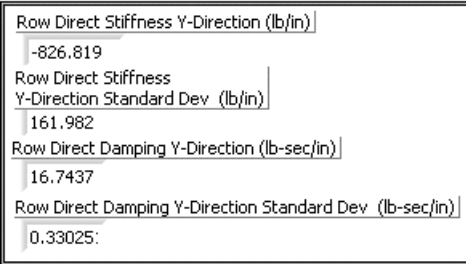 } & \multicolumn{2}{|c|}{ 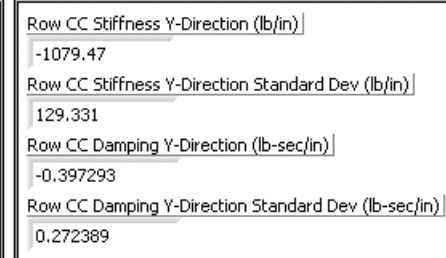 } & \multicolumn{3}{|c|}{ 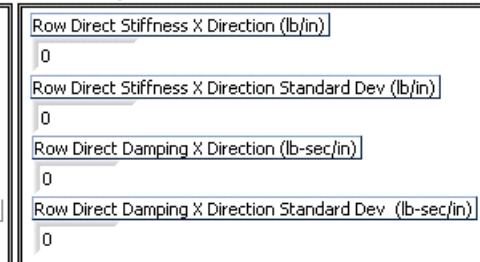 } & \multicolumn{3}{|c|}{ 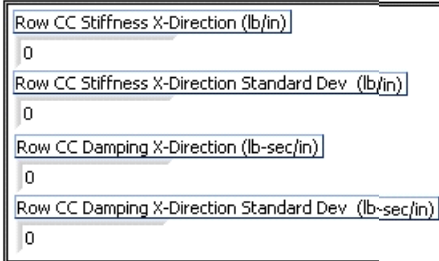 } & $\begin{array}{l}1.27778 \\
\text { Converging Cavity } \\
\text { Projected Área } 2 \text { (it } \\
0.344416 \\
\text { Angular Location } \\
90 \text { Degree } \\
\text { Frequency (Hz) } \\
100\end{array}$ & \\
\hline \multicolumn{2}{|c|}{ Cavity \#1 Coefficients } & \multicolumn{2}{|c|}{ Cavity \#2 Coefficients } & \multicolumn{2}{|c|}{ Cavity \#3 Coefficients } & \multicolumn{3}{|c|}{ Cavity \#4 Coefficients } & \multicolumn{3}{|c|}{ Cavity \#5 Coefficients } \\
\hline $\begin{array}{l}\text { X Direction } \\
\text { Direct Stiffness (lb/in) } \\
0 \\
\text { Standard Dev } \\
\end{array}$ & \begin{tabular}{||l|} 
Y Direction \\
Direct Stiffness (lb/in) \\
-394.366 \\
Standard Dev
\end{tabular} & \begin{tabular}{|l} 
X Direction \\
Direct Stiffness (lbjin) \\
0 \\
Standard Dev \\
\end{tabular} & \begin{tabular}{||l|} 
Y Direction \\
Direct Stiffness (lbjin) \\
812.633 \\
Standard Dev \\
Sand
\end{tabular} & \begin{tabular}{|l|} 
X Direction \\
Direct Stiffness (lb/in) \\
0 \\
Standard Dev \\
\end{tabular} & \begin{tabular}{|l|} 
Y Direction \\
Direct Stiffness (lb)inin) \\
671.425 \\
Standard Dev \\
tand
\end{tabular} & \begin{tabular}{|c|}
$\mathrm{XD}$ \\
Direc \\
0 \\
Stan \\
\end{tabular} & $\begin{array}{l}\text { ection } \\
\text { iffness (b/in) } \\
\text { DDev }\end{array}$ & \begin{tabular}{||l|} 
Y Direction \\
Direct Stiffness (lb/in) \\
974.718 \\
Standard Dev|
\end{tabular} & & $\begin{array}{l}\text { irection } \\
\text { t Stiffness (b/in) } \\
\\
\text { Jard Dev }\end{array}$ & \begin{tabular}{|l|} 
Y Direction \\
Direct Stiffness (lblin) \\
-2891.23 \\
Standard Dev \\
\end{tabular} \\
\hline 0 & $\mid 68.7548$ & 0 & 23. & & $\mid 65.3972$ & 0 & & 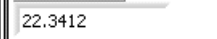 & 0 & & 83.4261 \\
\hline birect Damping & | Direct Damping $($ (b-seclin $) \|$ & Direct Damping (lb-seclin) || & Direct Dam & Direct Damping (lb-sec(jin) & | Direct Damping (b-seclin) & & amping (b-seclin) & | Direct Damping (b-seclin) & Direct & amping (b-seclin) & Direct Damping (b-sec/in) \\
\hline 0 & $\mid \begin{array}{ll}5.86881 \\
65 \text { tandart }\end{array}$ & 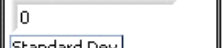 & 1.7207 & & 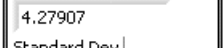 & & & 1.08897 & 0 & & 3.78618 \\
\hline $\begin{array}{l}\text { Standard Dev } \\
0\end{array}$ & $\mid$\begin{tabular}{||l|} 
Standard Dev \\
0.192518
\end{tabular} & $\mid$\begin{tabular}{|l} 
Standard Dev \\
0
\end{tabular} & $\mid \begin{array}{ll}\text { Standard Dev } \\
0.0681029\end{array}$ & $\mid \begin{array}{l}\text { Standard Dev } \\
0\end{array}$ & $\mid$\begin{tabular}{||l|l|} 
Standard Dev \\
0.0761245
\end{tabular} & & $\mathrm{ev}$ & 0.050115 & & a & 0.186659 \\
\hline CC Stiffness (b,jin) & ๔C Stiffness (blifin) & ๔C Stiffness (lb/in) & 〔C Stiffness (blin) & CC 5tiffness (lbin) & ||CC Stiffnes & & $s(1 \mathrm{~b}$ & cc stiffne & & fness (l) & ccsti \\
\hline 0 & -135.562 & 0 & -24.2194 & 0 & $\mid-537.73$ & 0 & & -133.025 & 0 & & -258.045 \\
\hline Standard Dev & Standard Dev & Standard Dev & |Standard Dev & Standard Dev & |Standard Dev & & $\mathrm{ev}$ & Standard Dev & & ard Dev & Standard Dev \\
\hline $\begin{array}{l}0 \\
\text { CC Damping (lb-sec }\end{array}$ & 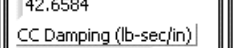 & $\mid \begin{array}{l}0 \\
\text { CC Damping (lb-seclin) }\end{array}$ & $\mid$\begin{tabular}{||l}
48.0878 \\
CC Damping (lb-seclin)
\end{tabular} & $\mid \begin{array}{l}0 \\
\operatorname{Cc} \text { Damping }(\text { b-secjin })\end{array}$ & $\left|\begin{array}{|ll}61.8882 \\
\text { CC Damping (lb-seclin) } \mid\end{array}\right|$ & & mping (lb & $\mid$\begin{tabular}{||l}
23.3939 \\
cc Damping
\end{tabular} & $\frac{0}{C C D a}$ & Samping (lb-seclin)] & $\begin{array}{l}121.605 \\
\text { CCDampit }\end{array}$ \\
\hline 0 & -0.0831431 & & & & -0.216665 & 0 & & -0.0719552 & 0 & & \\
\hline Standarc & Standard Dev & Standard Dev & Standard Dev & Standar & | Standard De: & & & |Standard Dev & & of & 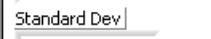 \\
\hline & 0.121183 & $\mid 0$ & & 0 & 0.079287 & 0 & & 0.04768 & 0 & & 0.192405 \\
\hline x-Shake Phase (Deg) & Y-Shake Phase (Deg) & X-shake Phase (Deg) & Y-Shake Phase (Deg)] & x-shake Phase & Y-Shake Phase (Deg) & & ase & Y-Shake : & & hase (De & $\gamma-5 h$ \\
\hline & & $\mid-32.5$ & & & 75.9 & & & & & & 140.4 \\
\hline & & $\mid$ Stand & & & & & & & & & \\
\hline 145.266 & 0.968684 & 120.282 & & & & & & & & & 1.21807 \\
\hline
\end{tabular}

Figure 88. Front Panel: Data Reduction Fully Partitioned Six Bladed PDS 
Alford's work in 1965 showed that for converging clearances the seal cavity produced positive stiffness and negative damping; and diverging clearances yielded positive damping and negative stiffness. His analysis also is valid for the PDS and has been verified by researchers such as Shultz (1996). Figures 89-90 show two examples of PDS cavities and the representative phasor plot for the different clearance geometries. Note that the cavities shown in Figure 89 are stand-alone cavities or cavities which are bounded by a constant pressure. It would be incorrect to assume that the converging geometry cavities 2 and 4 in the FP PDS behave as converging stand-alone cavities as shown in Figure 89. All the internal cavities in the FP PDS design are bounded by time dependent pressures, therefore coupling the dynamics between the 5 active cavities, which results in a favorable result in terms of direct damping for the converging cavities. Figure 91(a) displays the dynamic pressure phase of the five cavities in the 0 degree location.

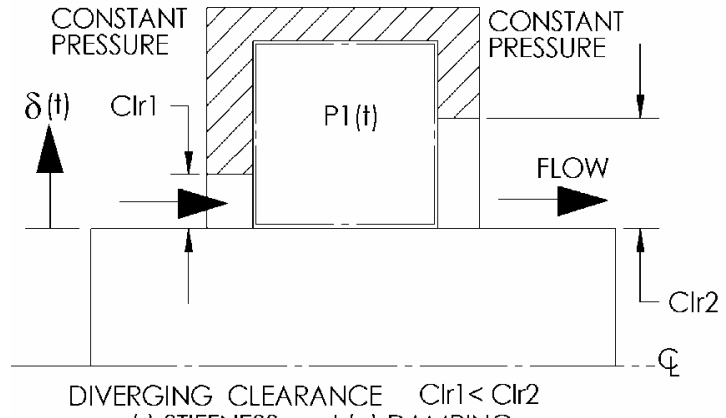

(-) STIFFNESS and (+) DAMPING

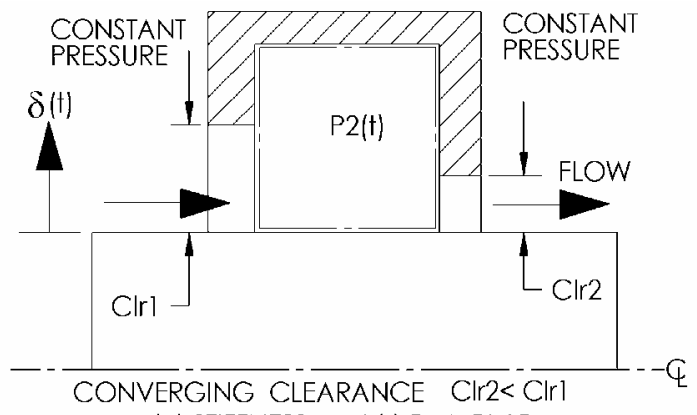

$(+)$ STIFFNESS and (-) DAMPING

Figure 89. Stand Alone Cavities- Diverging and Converging Configurations

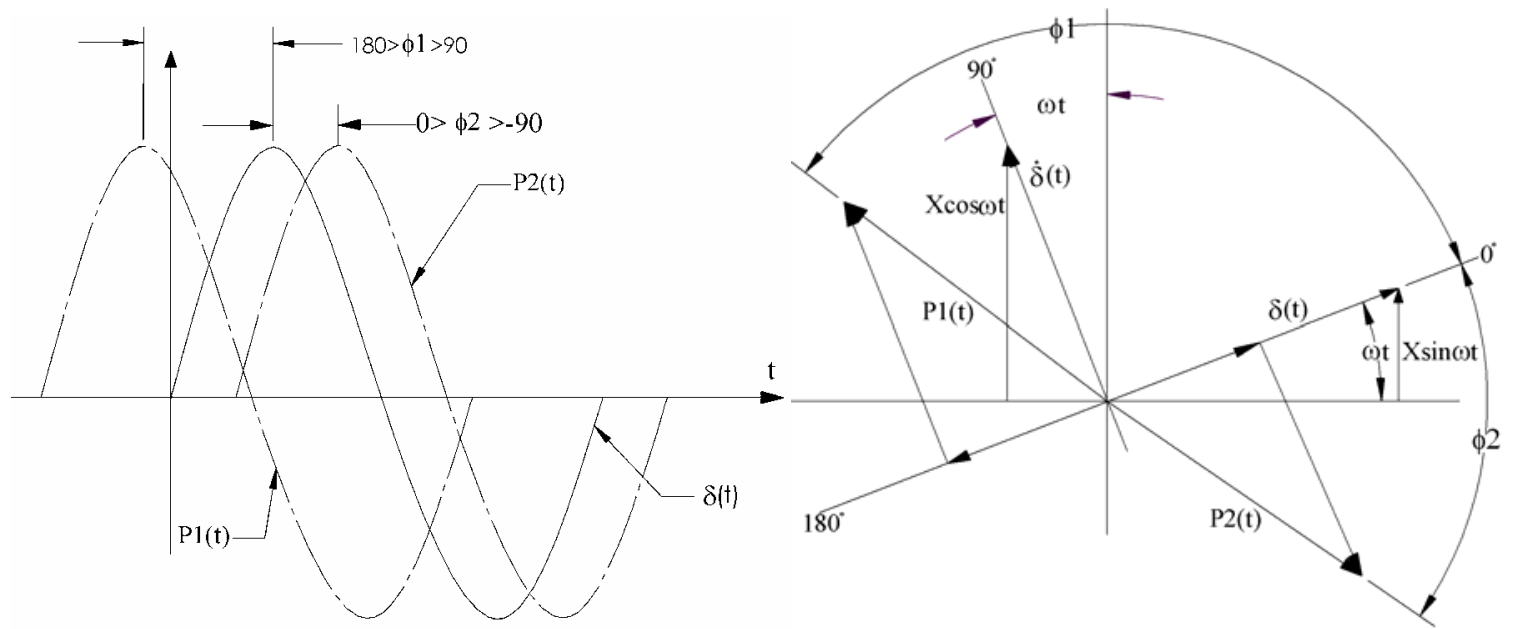

Figure 90. Phasor Diagram- Stand Alone Cavities 
Converging cavities 2 and 4 show a pressure phase between 0 and 90 degree, indicating that these cavities possess positive stiffness and positive damping. Another consequence from the FP design is the change in pressure phase of the diverging active cavities compared to the conventional design pressure phase values. This change is shown in Figure 91, where the pressure phase values for the diverging active cavities of the conventional design in part (b) of the figure are almost identical and indicate negative stiffness and positive damping throughout the test frequency range. The FP design results in pressure modulations between the cavities that are now coupled, resulting in drastically different values for pressure phase in the diverging cavities 1,3 , and 5 . In fact, cavities 1 and 3 cross over to positive stiffness and are more frequency dependent when compared to the pressure phases from the conventional seal tests. Figure 92 displays the direct damping coefficients for the 0 degree position where all cavities possess positive direct damping. The direct stiffness for the same angular location is shown in Figure 93 and indicates that the converging cavities possess positive direct stiffness whereas cavities 1 and 3 have negative direct stiffness at low frequencies and positive direct stiffness for frequencies above $80 \mathrm{~Hz}$.

The cavity coefficients were used to determine row coefficients, which were then added to give the overall seal coefficients. Figures 94-97 show the $\mathrm{Y}$ direction and $\mathrm{X}$ direction force coefficients for both the FP design and the conventional design.

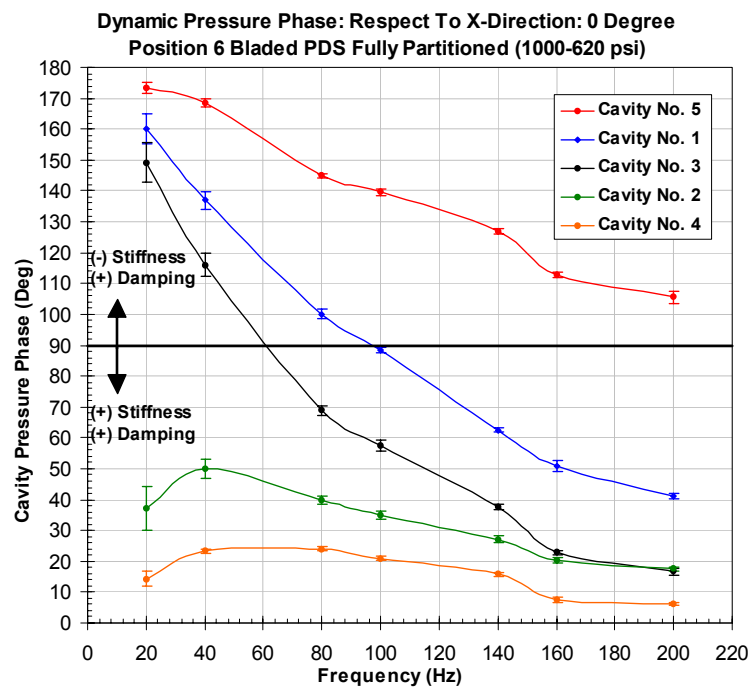

(a) Fully Partitioned Design

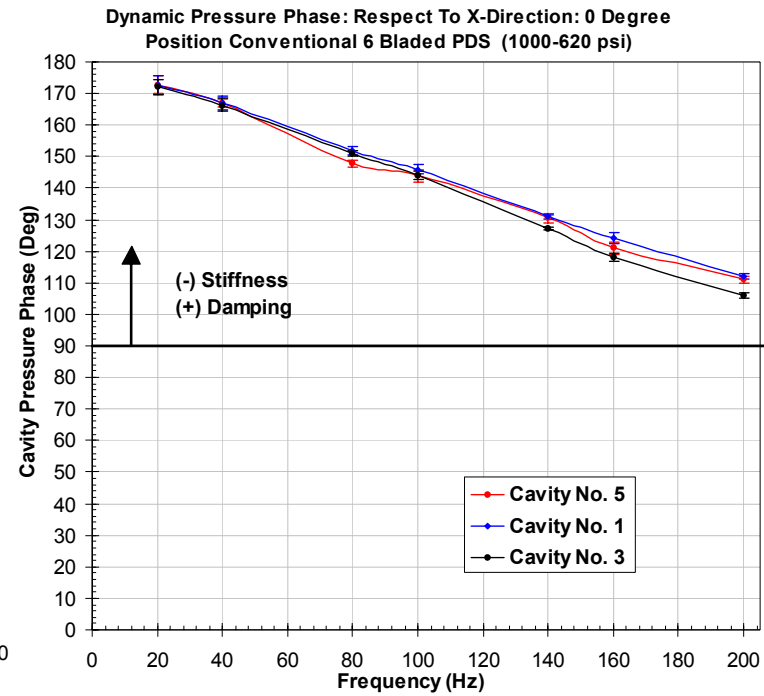

(b) Conventional PDS Design

Figure 91. Dynamic Cavity Pressure Phase- FP Design vs. Conventional Design 
Figures 94-95 show that the FP PDS direct damping between 20-100 Hz is significantly larger than the conventional PDS. The increase in direct damping is attributed to the added damping from the converging cavities and the change in pressure phase, which shifted the phase closer to 90 degrees. For frequencies above $120 \mathrm{~Hz}$ the direct damping of the FP PDS is less than the conventional design. This trend in the FP PDS direct damping was also observed with the impedance tests, where the direct damping crosses over the conventional PDS damping curve around $125 \mathrm{~Hz}$ (Figure 49). The direct stiffness of the FP PDS is $\sim 25 \%$ of the conventional PDS at low frequencies 20 and $40 \mathrm{~Hz}$. For higher frequencies the direct stiffness of the FP design is significantly higher, breaching the 0 stiffness mark at $100 \mathrm{~Hz}$. The cross-coupled coefficients are shown in Figures 96-97.
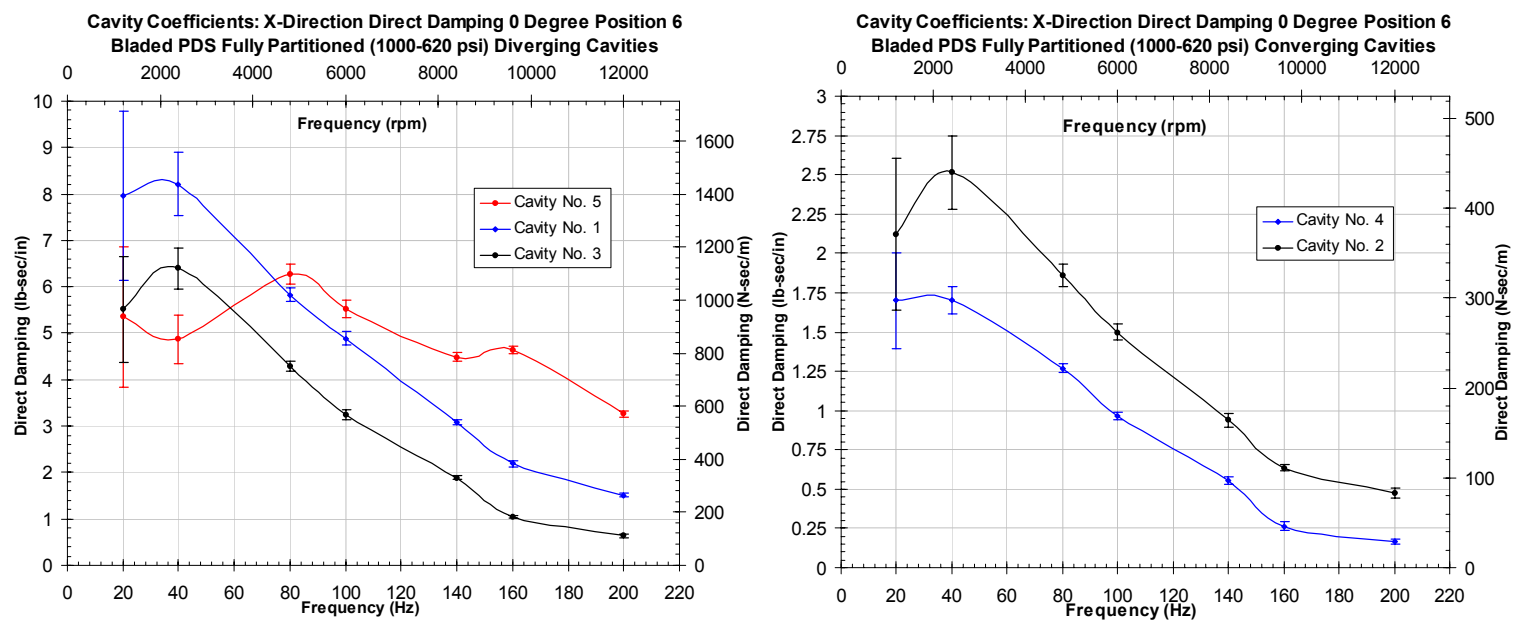

Figure 92. Direct Damping-Cavity Coefficients Fully Partitioned Seal 0 Degree Position
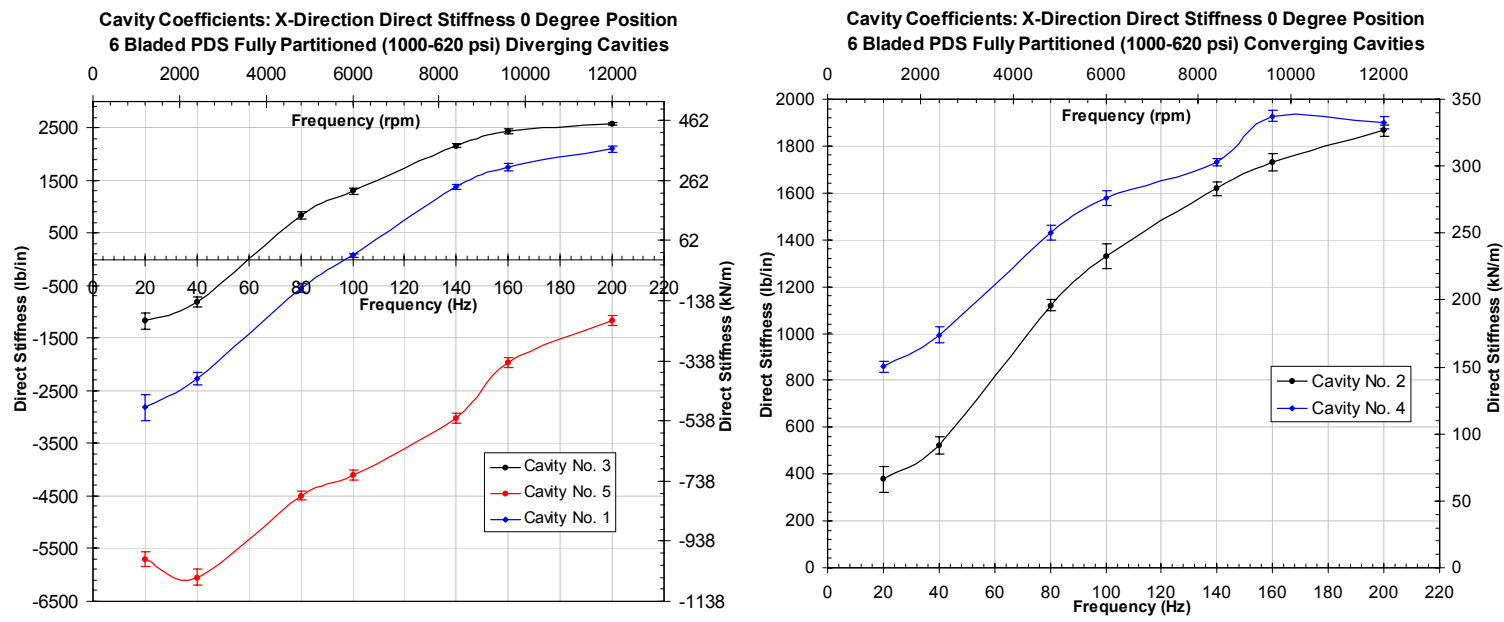

Figure 93. Direct Stiffness-Cavity Coefficients Fully Partitioned Seal 0 Degree Position 

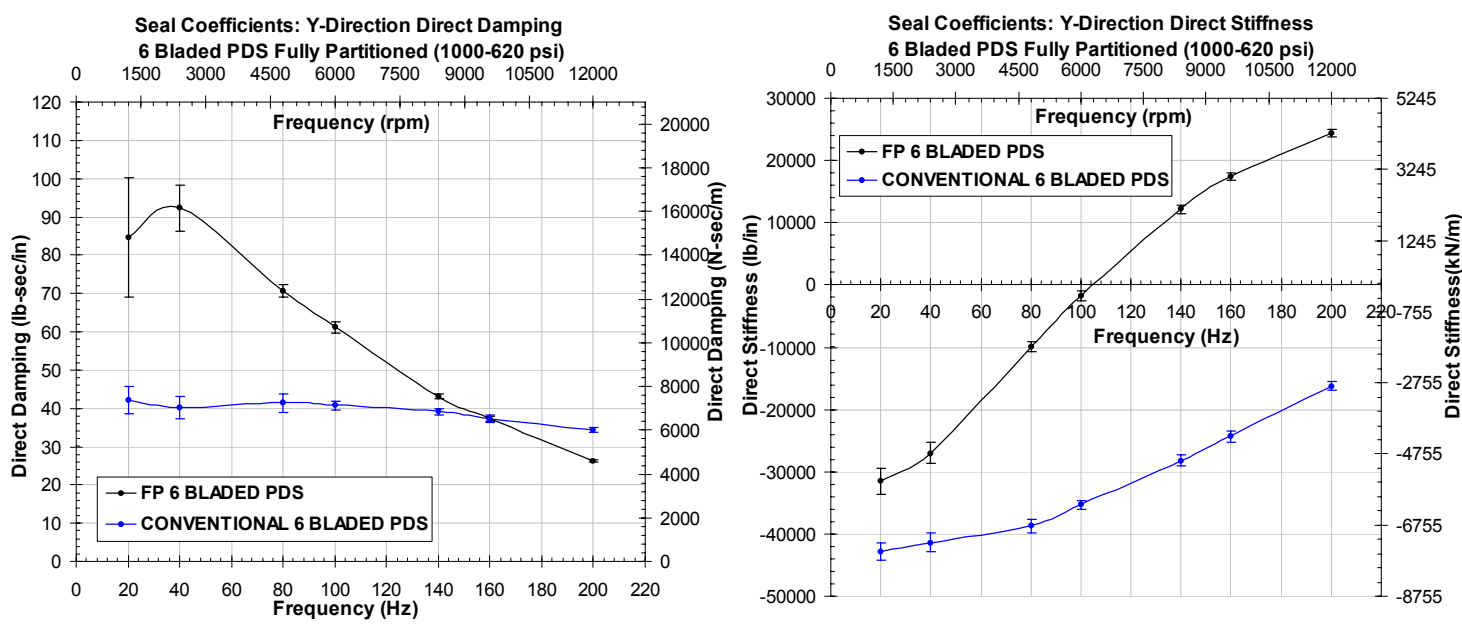

Figure 94. Direct Force Coefficients Y Dir: FP PDS vs. Conventional PDS
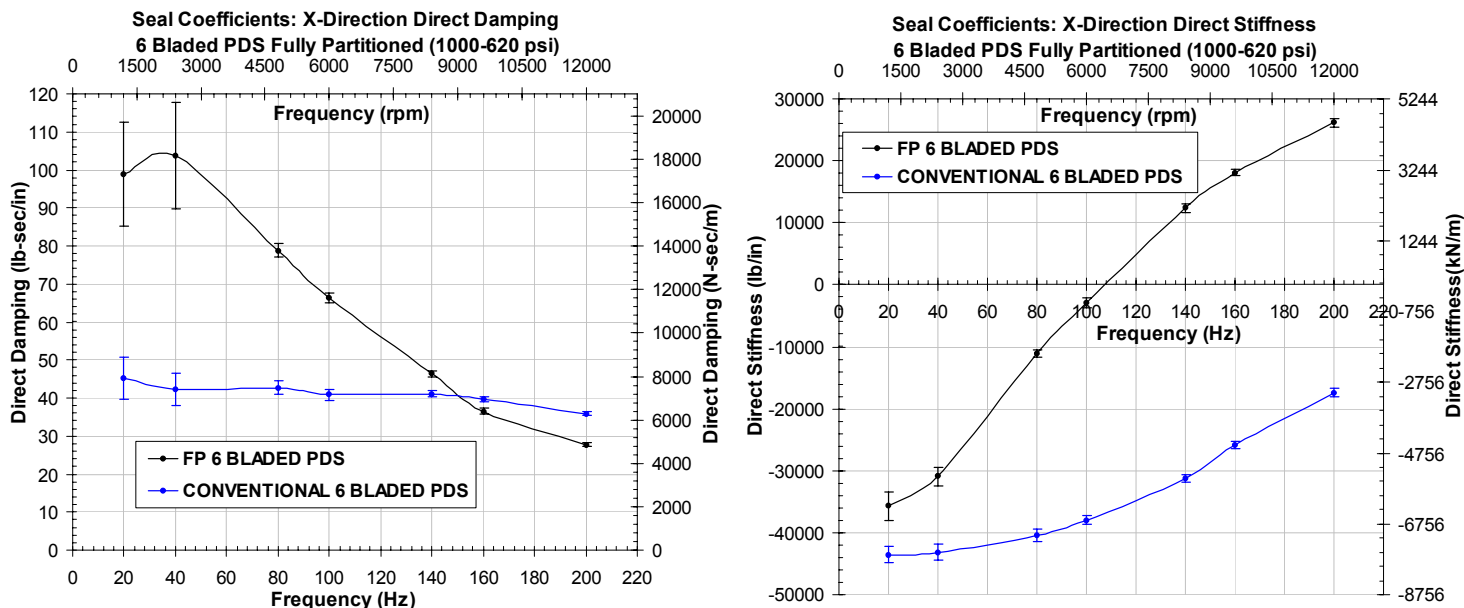

Figure 95. Direct Force Coefficients X Dir: FP PDS vs. Conventional PDS
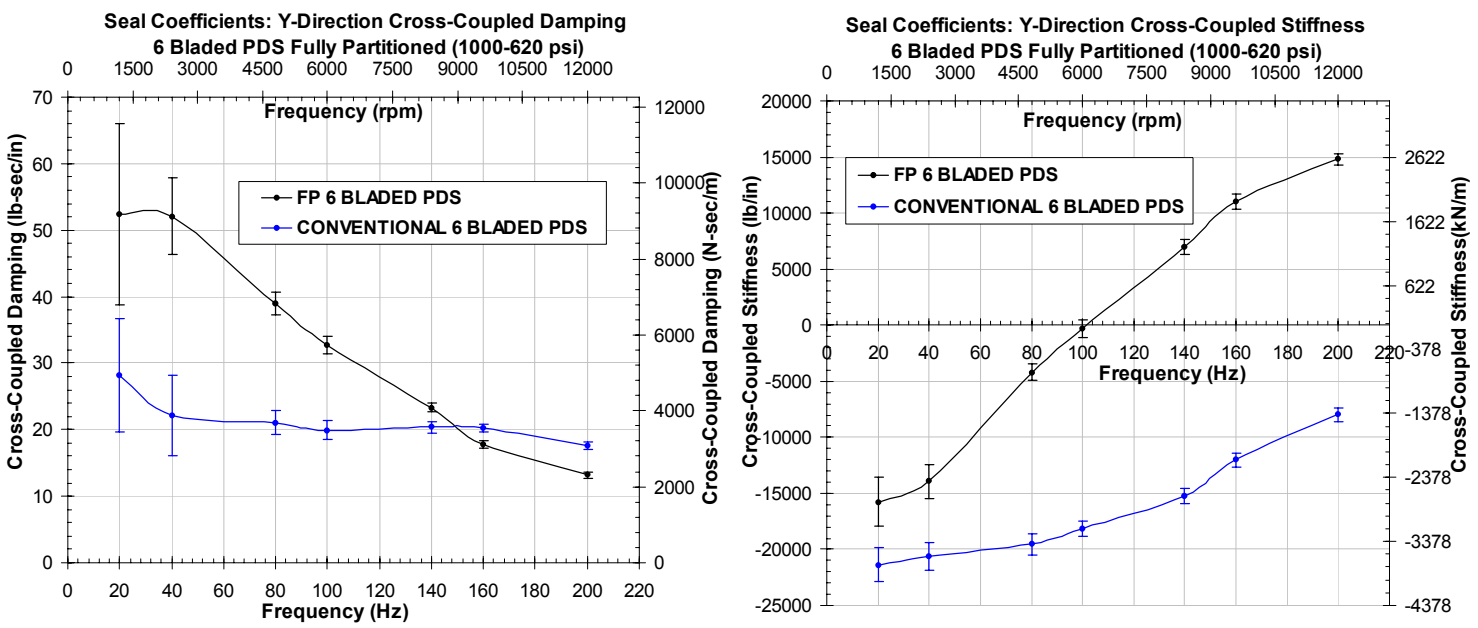

Figure 96. Cross-Coupled Force Coefficients Y Dir: FP PDS vs. Conventional PDS 
The cross-coupled stiffness for the FP seal yielded the same sign, and at low frequencies both are negative and for frequencies above $100 \mathrm{~Hz}$ the cross-coupled stiffness coefficients become positive. Cross-coupled damping coefficients are positive and also possess the same sign.
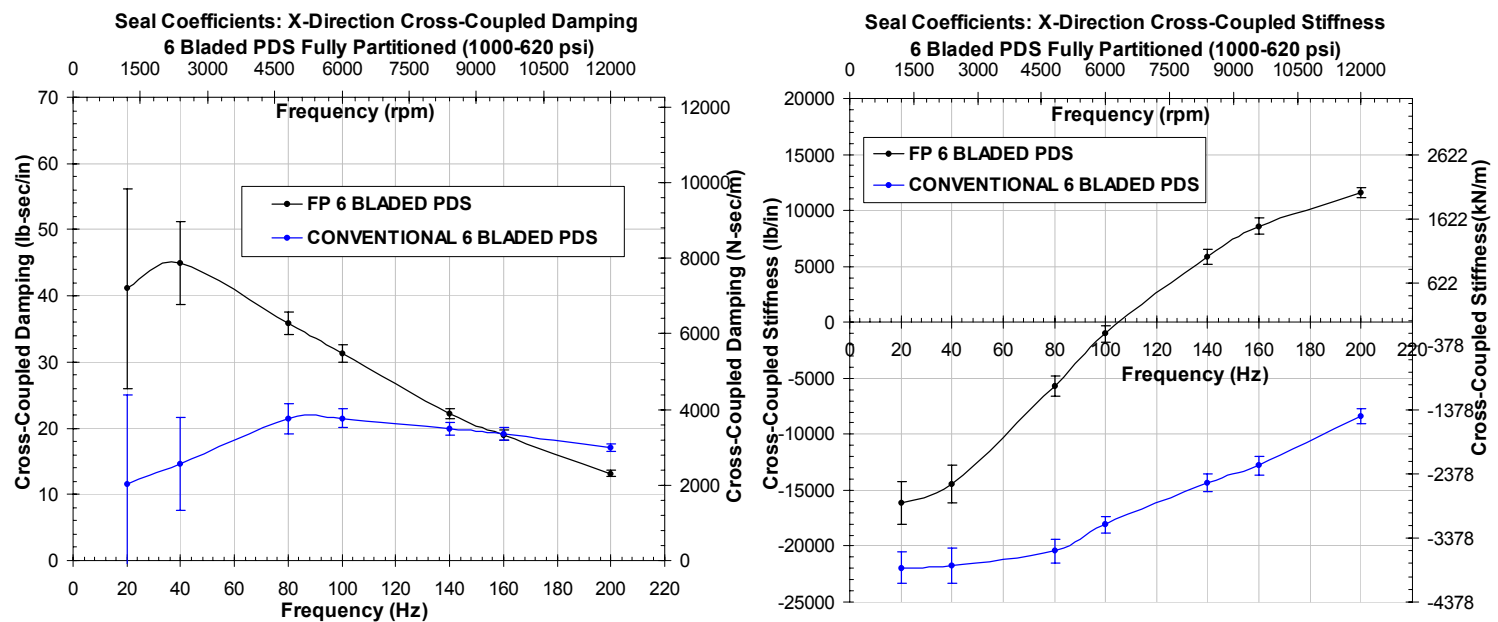

Figure 97. Cross-Coupled Force Coefficients X Dir: FP PDS vs. Conventional PDS

In the previous sections showing the pressure signals in the time domain, there exists a high frequency component. This high frequency component was not easily detectable when averaging was performed for all 12 sets of data. However, when the frequency spectrum was displayed without averaging, frequency spikes where revealed at high frequencies. Figure 98 shows one example of this high frequency component residing at $\sim 3,750 \mathrm{~Hz}$. This test was conducted for the 45 degree angle location at $20 \mathrm{~Hz}$ excitation frequency for an $\mathrm{X}$ direction excitation. These high frequency components were observed in almost all of the tests. Note that the high frequency energy is not observed in the motion signal, as shown in Figure 98, and that it exists in all three pressure signals for the three cavities 1,3 , and 5 . The frequency spikes are not related to the resonance frequency of the pressure transducers, because there natural frequencies are located far above $4 \mathrm{kHz}$. The frequency excitation at $3,750 \mathrm{~Hz}$ is most predominant for the last cavity (cavity 5) and seems to be at a slightly lower location than the frequency spikes for cavities 3 and 1 . Several possible sources were investigated (Lucas et al. 1997) including the acoustic Helmholtz frequency and edge tone flow instabilities as shown with the eight bladed PDS, but no conclusion was reached. The source for the high frequency content is unknown. 

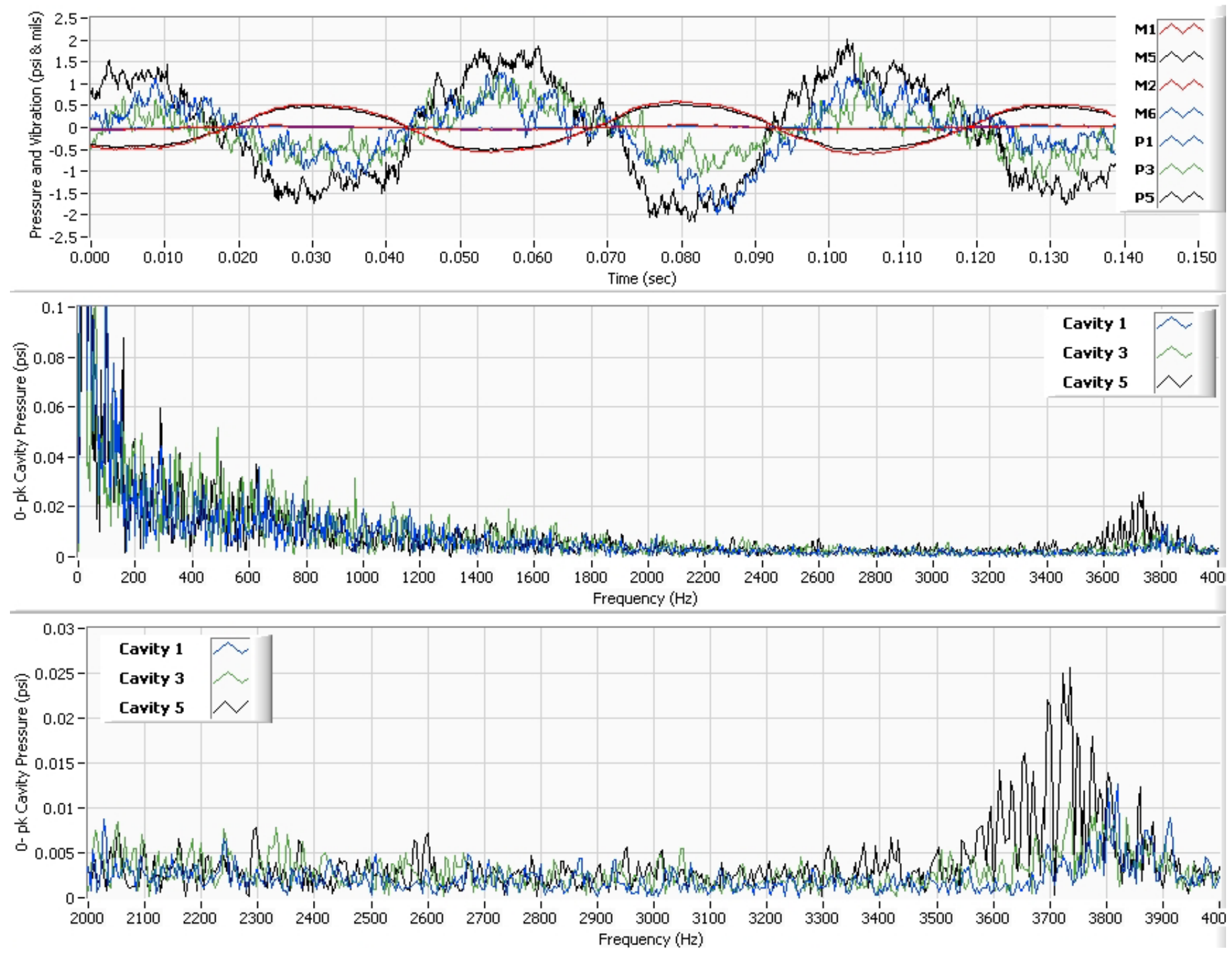

Figure 98. High Frequency Spikes in Dynamic Cavity Pressure Measurements 


\subsection{Six Bladed PDS- Static Pressure Tests}

Past research completed on stiffness cross-coupling coefficients of PDS have shown contradictory results. The main point of contradiction is the signs of the stiffness crosscoupled coefficients Kxy and Kyx. Gamal (2003) performed non-rotating static displacement experiments on the same test rig shown in Figure 14 for the 12 bladed and 8 bladed PDS, but using motion probes and the force transducer readings on the shaker stingers. The objective of Gamal's test was to measure direct and cross-coupled stiffness coefficients for the pressurized 8 and 12 bladed PDS by measuring the displacement of the stator in combination with the static force imposed on the system. To account for and subtract the stiffness effects from the mechanical structure of the test rig, a baseline test without the test assembly pressurized was required. The baseline test for this case did not require a separate assembly but only a test with no air-flow. His tests were conducted in line with the $\mathrm{X}$ and $\mathrm{Y}$ axis, indicating that the stator was not purposely offset before displacing in the $\mathrm{X}$ or $\mathrm{Y}$ directions. Table 14 summarizes his results.

Table 14. Gamal's Results for Static Direct and Cross-coupled Stiffness (2003)

\begin{tabular}{|c|c|c|c|c|}
\hline Test & $\mathrm{K}_{\mathbf{x x}}(\mathrm{Ib} / \mathbf{i n})$ & $\mathrm{K}_{\mathbf{x y}}(\mathrm{Ib} / \mathbf{i n})$ & $\mathrm{K}_{\mathbf{y x}}(\mathrm{Ib} / \mathrm{in})$ & $\mathrm{K}_{\mathbf{y y}}(\mathrm{Ib} / \mathbf{i n})$ \\
\hline 8-Bladed Test 1 & $-46,373.84$ & 767.51 & $8,719.28$ & $-41,075.65$ \\
\hline 8-Bladed Test 2 & $-64,304.27$ & 912.30 & $3,074.13$ & $-60,803.19$ \\
\hline 12-Bladed Test 1 & $-11,094.20$ & -10.01 & -751.51 & $-9,932.93$ \\
\hline 12-Bladed Test 2 & $-39,212.94$ & $-3,330.31$ & $-1,879.96$ & $-36,302.19$ \\
\hline
\end{tabular}

Benchert and Wachter (1980) studied static pressure forces in several labyrinth seal configurations for offset rotor displacements. One of the tests used a new configuration that comprised of 4 circumferentially spaced swirl webs between the labyrinth seal blades. This modification to the straight through labyrinth seal created 5 rows of 4 cavities. There configuration is similar to the FP 6 bladed PDS, but differs in that the clearance of the seal blades are constant whereas the FP PDS is made up of diverging and converging cavities. Also, their seal contained 4 circumferential pockets and the FP design possesses 8 pockets. The test rig they used and the seal tested are shown in Figure 99. Static pressure probes were installed in 30 degree angle location around the periphery of the test seal chambers and were used to determine the resultant force on the seal housing. The rotor is overhung and is supported by two back to back mounted angular contact ball bearings and one cylindrical 
roller bearing. Also, Benchert and Wachter used pre-swirl rings to impose gas swirl into the inlet chamber of the seal. The rotor is driven by a variable speed drive and the test rig has the ability to offset (displace) the rotating assembly relative to the housing in one direction. This is illustrated in Figure 99, where the relative eccentricity $\varepsilon$ is plotted versus the lateral force Q. The right side graph shows results for a labyrinth seal without the swirl webs and their modified labyrinth with the swirl webs. Their results show that the addition of the swirl webs increased the lateral force Q, which would resolve in the direction of rotor whirl. They conclude that if a seal generates a lateral force or a force perpendicular to rotor displacement, that the seal possess a destabilizing stiffness cross-coupling force. Since the tests conducted for their work only were administered in one direction, only one of the stiffness cross-coupling coefficients can be determined, which cannot be used to determine the presence of destabilizing forces unless the other stiffness cross-coupled coefficient is known. The knowledge of the other coefficient requires a separate test in the orthogonal direction, which was not conducted in their work.

The static pressure measurements for the following experiments were used to determine the sign of the cross-coupled stiffness coefficient for two independent displacement patterns in the $\mathrm{X}$ and $\mathrm{Y}$ directions. The two tests conducted for cavities 3 of the 6 bladed PDS are shown in Figure 100. Tests were conducted at 15,200 rpm, which yields a surface speed of $597 \mathrm{ft} / \mathrm{sec}(182 \mathrm{~m} / \mathrm{s})$. Test 1 consisted of offsetting the rotor in the positive $\mathrm{Y}$ direction (position 1) and then traversing in the positive $\mathrm{X}$ direction, while measuring the static pressure in cavity 3 at the 315 degree and 45 degree location. Test 2 offset the rotor in the positive $\mathrm{X}$ direction and then moving in the positive $\mathrm{Y}$ direction. Static pressure measurements for test 2 were taken at 45 degrees and 135 degrees. The summary of the results are shown in Table 15. Recall that the radial clearance of the test seals from the rotor is 0.005 in or $0.127 \mathrm{~mm}$. First, consider test 1 where position 0 is the centered position. Of course the seal will always be offset and a truly centered configuration is not feasible; therefore position 0 represents the closet position to the theoretical center of the stator. At position 0 the cavity pressures are close to the same value. Position 1 shows a relative rotor position of 0.001 inches in the positive $\mathrm{Y}$ direction. At this point the pressure in the 45 degree angle cavity has decreased while the static pressure in the 315 degree cavity increased. 

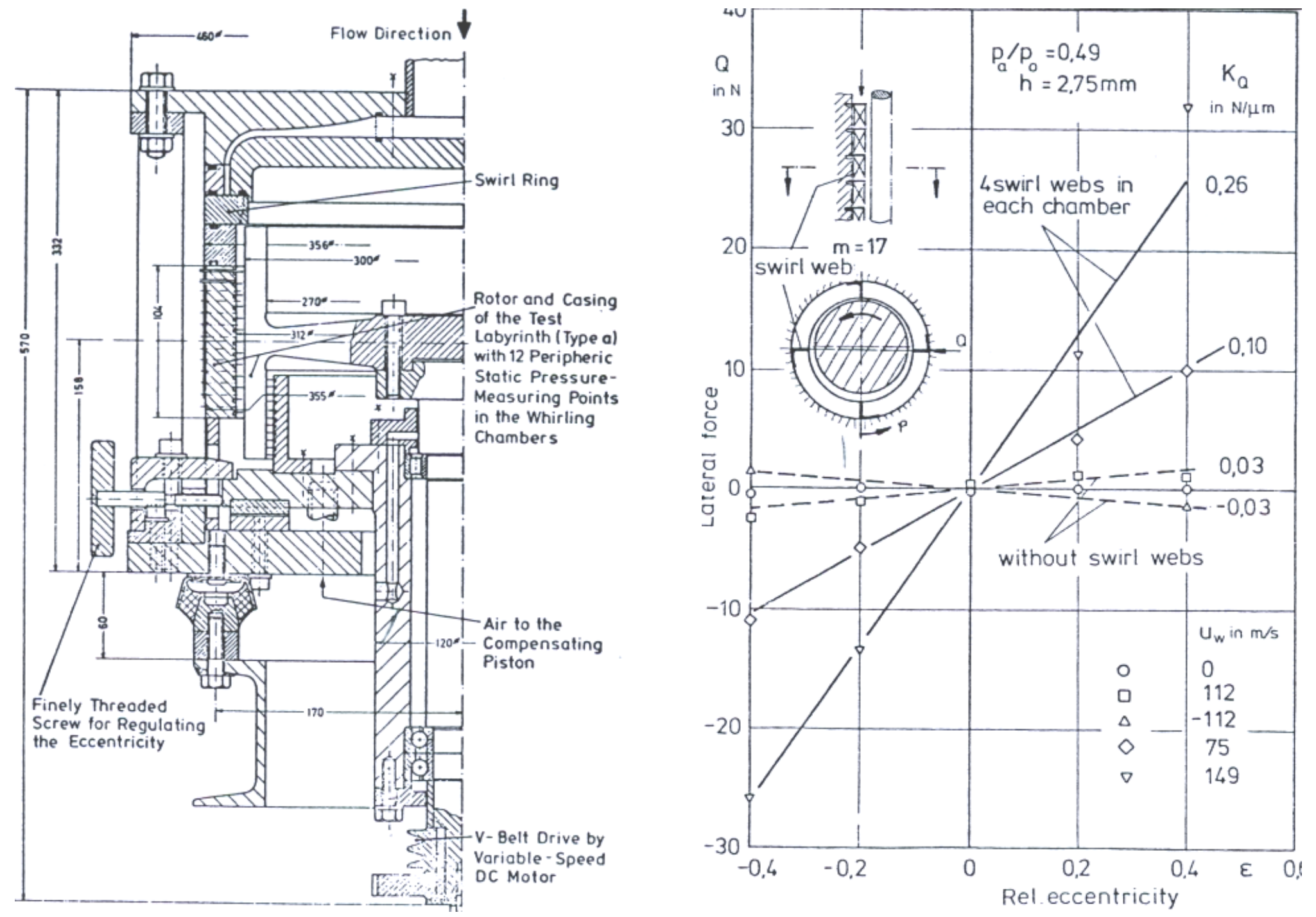

Figure 99. Benckert and Wachter's Test Rig and Experimental Results (1980) 

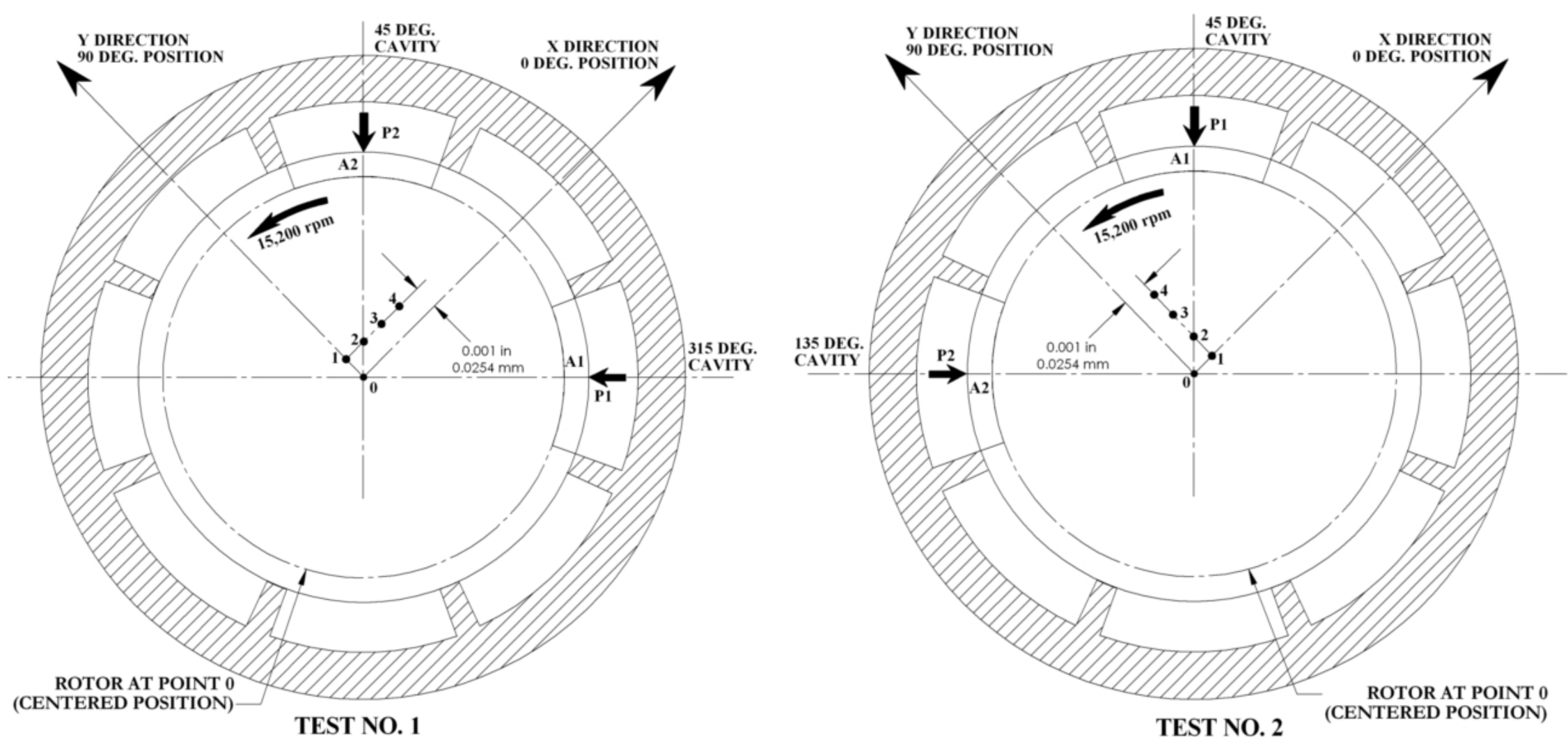

Figure 100. Static Pressure Measurements Displacement Pattern: 15,200 rpm 
Next, for points 2-4 the stator is moved in the negative $\mathrm{X}$ direction, which is a relative rotor movement in the positive $\mathrm{X}$ direction. Table 15 and Figure 101 show that the static pressure in the 45 degree cavity is lower than the 315 degree position cavity for these locations and in the pressure at 45 degrees is decreasing at a faster rate. The deviation in static pressures in these two pockets suggests that there is a force imposed on the system in the positive $\mathrm{Y}$ direction. A positive force in the $\mathrm{Y}$ direction $\left(\mathrm{F}_{\mathrm{Y}}\right)$ is related to a positive displacement in the $\mathrm{X}$ direction $(\mathrm{X})$ through a negative $\mathrm{Y}$ direction stiffness cross-coupled coefficient $\left(-\mathrm{K}_{\mathrm{YX}}\right)$.

Next, Test 2 was performed to determine the $\mathrm{X}$ direction stiffness cross-coupled coefficient. In this test the rotor was offset in the positive $\mathrm{X}$ direction and positioned in the positive $\mathrm{Y}$ direction, while the static pressures were measured in the 135 degree cavity and the 45 degree cavity. The results of these tests produced lower pressures at the 45 degree position for positions 2-4, compared to the pressure at 135 degrees. The rate of pressure decline in the 45 degree location is also faster for this test. Considering the static pressure evolution in these two cavities, the resultant pressure force is imposed in the positive $\mathrm{X}$ direction $\left(\mathrm{F}_{\mathrm{X}}\right)$, implying a negative sign $\mathrm{X}$ direction cross-coupled stiffness coefficient $\left(-\mathrm{K}_{\mathrm{XY}}\right)$.

Table 15. Cavity Pressure Measurements: Rotating Displacement Test Results

\begin{tabular}{|c|c|c|c|c|c|}
\hline \multirow{2}{*}{\begin{tabular}{|c|} 
TEST NO. 1 \\
POINT \\
\end{tabular}} & \multicolumn{4}{|c|}{ STATIC CAVITY PRESSURES AND DISPLACEMENT COORDINATES } & \multirow[b]{2}{*}{ P1-P2 } \\
\hline & X COORDINATE & Y COORDINATE & P1 (PSI) & P2 (PSI) & \\
\hline 0 & 0 & 0 & 807.34 & 806.01 & 1.33 \\
\hline 1 & 0 & $0.001 \mathrm{in} / 0.0254 \mathrm{~mm}$ & 808.8 & 803.88 & 4.92 \\
\hline 2 & $0.0005 \mathrm{in} / 0.0127 \mathrm{~mm}$ & $0.001 \mathrm{in} / 0.0254 \mathrm{~mm}$ & 808.1 & 802.64 & 5.46 \\
\hline 3 & $0.001 \mathrm{in} / 0.0254 \mathrm{~mm}$ & $0.001 \mathrm{in} \mathrm{/} 0.0254 \mathrm{~mm}$ & 807.01 & 800.35 & 6.66 \\
\hline 4 & $0.002 \mathrm{in} / 0.0508 \mathrm{~mm}$ & $0.001 \mathrm{in} / 0.0254 \mathrm{~mm}$ & 803.81 & 794.11 & 9.7 \\
\hline \multicolumn{5}{|c|}{\begin{tabular}{|l|l|l|} 
TEST NO. 2 & STATIC CAVITY PRESSURES AND DISPLACEMENT COORDINATES \\
\end{tabular}} & \\
\hline POINT & X COORDINATE & Y COORDINATE & P1 (PSI) & P2 (PSI) & P2-P1 \\
\hline 0 & 0 & 0 & 796.91 & 797.85 & 0.94 \\
\hline 1 & $0.001 \mathrm{in} / 0.0254 \mathrm{~mm}$ & 0 & 793.27 & 798.79 & 5.52 \\
\hline 2 & $0.001 \mathrm{in} / 0.0254 \mathrm{~mm}$ & $0.0005 \mathrm{in} / 0.0127 \mathrm{~mm}$ & 791.98 & 797.71 & 5.73 \\
\hline 3 & $0.001 \mathrm{in} \mathrm{/} 0.0254 \mathrm{~mm}$ & $0.001 \mathrm{in} \mathrm{/} 0.0254 \mathrm{~mm}$ & 788.36 & 796.72 & 8.36 \\
\hline 4 & $0.001 \mathrm{in} / 0.0254 \mathrm{~mm}$ & $0.002 \mathrm{in} / 0.0508 \mathrm{~mm}$ & 783.02 & 793.05 & 10.03 \\
\hline \multicolumn{5}{|c|}{\begin{tabular}{|l|l|} 
TEST NO. 1 & C \\
\end{tabular}} & \\
\hline POINT & AREA 1 & AREA 2 & $\%$ CHANGE , A1 & $\%$ CHANGE , A2 & \\
\hline 0 & $0.00885 \mathrm{in}^{\wedge} 2 / 0.2248 \mathrm{~mm}^{\wedge} 2$ & $0.00885 \mathrm{in}^{\wedge} 2 / 0.2248 \mathrm{~mm}^{\wedge} 2$ & 0 & 0 & \\
\hline 1 & $0.0101 \mathrm{in}^{\wedge} 2 / 0.2565 \mathrm{~mm}^{\wedge} 2$ & $0.00763 \mathrm{in}^{\wedge} 2 / 0.1938 \mathrm{~mm}^{\wedge} 2$ & 14.1 & -13.8 & \\
\hline 2 & $0.0095 \mathrm{in}^{\wedge} 2 / 0.2413 \mathrm{~mm}^{\wedge} 2$ & $0.00702 \mathrm{in}^{\wedge} 2 / 0.1783 \mathrm{~mm}^{\wedge} 2$ & -5.9 & -8.0 & \\
\hline 3 & $0.00885 \mathrm{in}^{\wedge} 2 / 0.2248 \mathrm{~mm}^{\wedge} 2$ & $0.00641 \mathrm{in}^{\wedge} 2 / 0.1628 \mathrm{~mm}^{\wedge} 2$ & -6.8 & -8.7 & \\
\hline 4 & $0.00763 \mathrm{in}^{\wedge} 2 / 0.1938 \mathrm{~mm}^{\wedge} 2$ & $0.00519 \mathrm{in}^{\wedge} 2 / 0.1318 \mathrm{~mm}^{\wedge} 2$ & -13.8 & -19.0 & \\
\hline \multicolumn{5}{|c|}{\begin{tabular}{|l|l|} 
TEST NO. 2 & CAVITY CLEARANCE AREAS \\
\end{tabular}} & \\
\hline POINT & AREA 1 & AREA 2 & $\%$ CHANGE , A1 & \% CHANGE , A2 & \\
\hline 0 & $0.00885 \mathrm{in}^{\wedge} 2 / 0.2248 \mathrm{~mm}^{\wedge} 2$ & $0.00885 \mathrm{in}^{\wedge} 2 / 0.2248 \mathrm{~mm}^{\wedge} 2$ & 0 & 0 & \\
\hline 1 & $0.00763 \mathrm{in}^{\wedge} 2 / 0.1938 \mathrm{~mm}^{\wedge} 2$ & $0.0101 \mathrm{in}^{\wedge} 2 / 0.2565 \mathrm{~mm}^{\wedge} 2$ & -13.8 & 14.1 & \\
\hline 2 & $0.00702 \mathrm{in}^{\wedge} 2 / 0.1783 \mathrm{~mm}^{\wedge} 2$ & $0.0095 \mathrm{in}^{\wedge} 2 / 0.2413 \mathrm{~mm}^{\wedge} 2$ & -8.0 & -5.9 & \\
\hline 3 & $0.00641 \mathrm{in}^{\wedge} 2 / 0.1628 \mathrm{~mm}^{\wedge} 2$ & $0.00885 \mathrm{in}^{\wedge} 2 / 0.2248 \mathrm{~mm}^{\wedge} 2$ & -8.7 & -6.8 & \\
\hline 4 & $0.00519 \mathrm{in}^{\wedge} 2 / 0.1318 \mathrm{~mm}^{\wedge} 2$ & $0.00763 \mathrm{in}^{\wedge} 2 / 0.1938 \mathrm{~mm}^{\wedge} 2$ & -19.0 & -13.8 & \\
\hline
\end{tabular}


The result in Tests 1-2 coincides with the discussion in Section III on same sign cross-coupled coefficients, and also agrees with the force diagram shown at position 2 in Figure 25 (a). In addition, the results of the cross-coupled coefficient signs support the experimental results in Figures 96-97. To better understand this effect, consider a simple example using a 2 bladed diverging PDS (Figure 102). Part a of Figure 102 shows the rotor centered in the seal where P1 is the pressure in the top pocket, P2 is the pressure in the bottom pocket, $\mathrm{Ph}$ is the high inlet pressure and $\mathrm{Pb}$ is the lower back pressure.

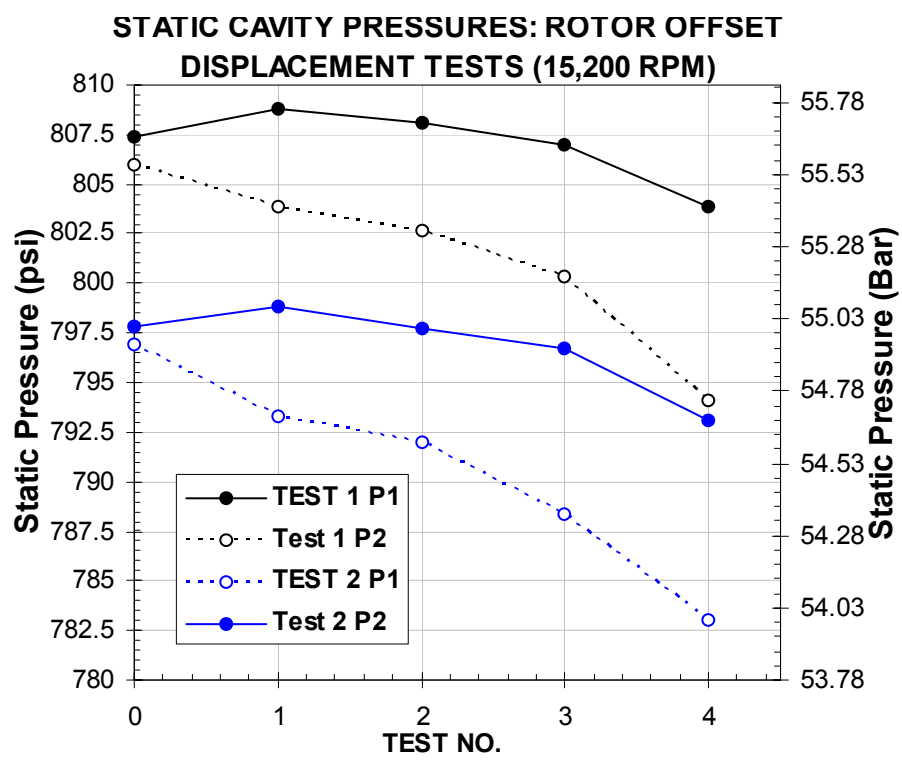

Figure 101. Static Pressure Measurements: Rotor Offset Displacement Tests

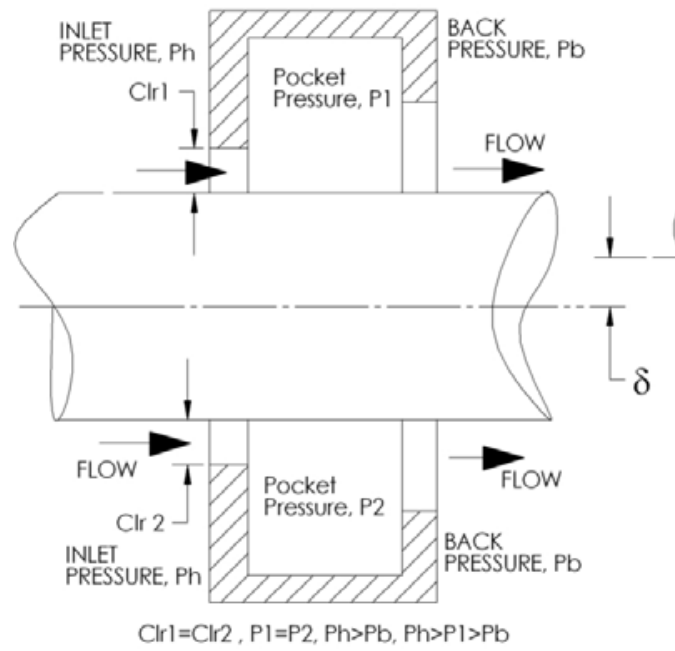

(a) CENTERED ROTOR

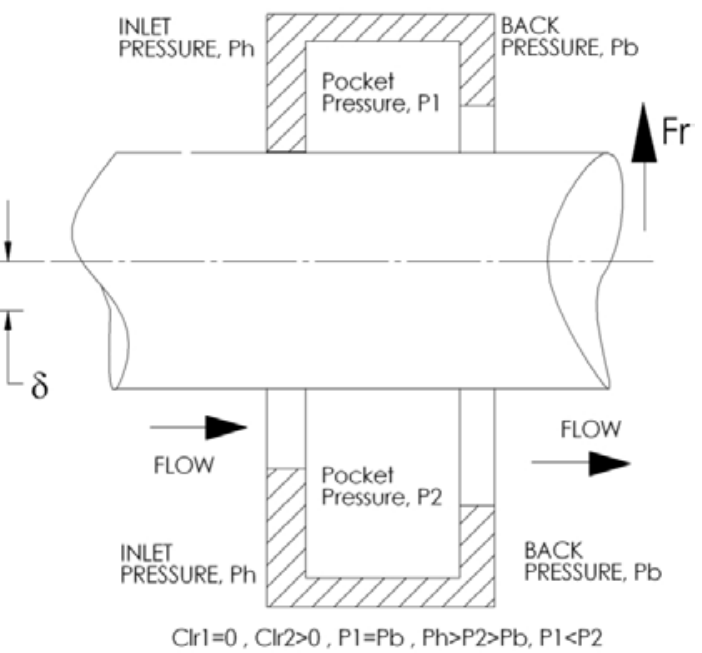

(b) OFFSET ROTOR-LIMITING CASE

Figure 102. Negative Stiffness Effect in Diverging Seals 
For case (a) the cavity clearance for the bottom and top pocket are the same and therefore there is no force imposed on the rotor. Now, consider the limiting case where the rotor is displaced by $\delta$ towards the top cavity and the top cavity clearance goes to zero. The limiting case results in the top cavity pressure $\mathrm{P} 1$ equaling the back pressure $\mathrm{Pb}$ and the bottom cavity pressure $\mathrm{P} 2$ is between $\mathrm{Ph}$ and $\mathrm{Pb}$, therefore resulting in a force $\mathrm{F}_{\mathrm{r}}$ in the direction of displacement (negative stiffness). Table 18 shows the cavity clearance areas for each of the positions (1-4). The area results show that for smaller clearance areas the pressure is lower, and that the percent change in area from point to point is different between the two instrumented pockets. 


\section{DAMPER SEAL THEORY}

The following section is focused on the derivation of stiffness and damping for a FP PDS. Figure 103 shows a cross sectional view of the two types of damper seals where the upper portion of the figure represents a conventional pocket damper seal composed of active plenums separated by inactive plenums. An active plenum for a PDS is defined as an annular plenum that has partition walls or barriers inserted in eight equally spaced angular locations creating 8 pressurized pockets. These pockets of pressurized gas can be modeled as individual control volumes that contain a dynamic pressure, which varies with time. An inactive plenum does not have partition walls, therefore possessing small dynamic pressure modulations for the applicable frequency ranges of rotating equipment. For the conventional PDS analysis each control volume is separated from adjacent control volumes by a constant pressure, therefore allowing one to solve the conservation of mass equations for each control volume separately. The configuration shown in the bottom portion of Figure 103 must utilize a coupled analysis between the conservation of mass equations.

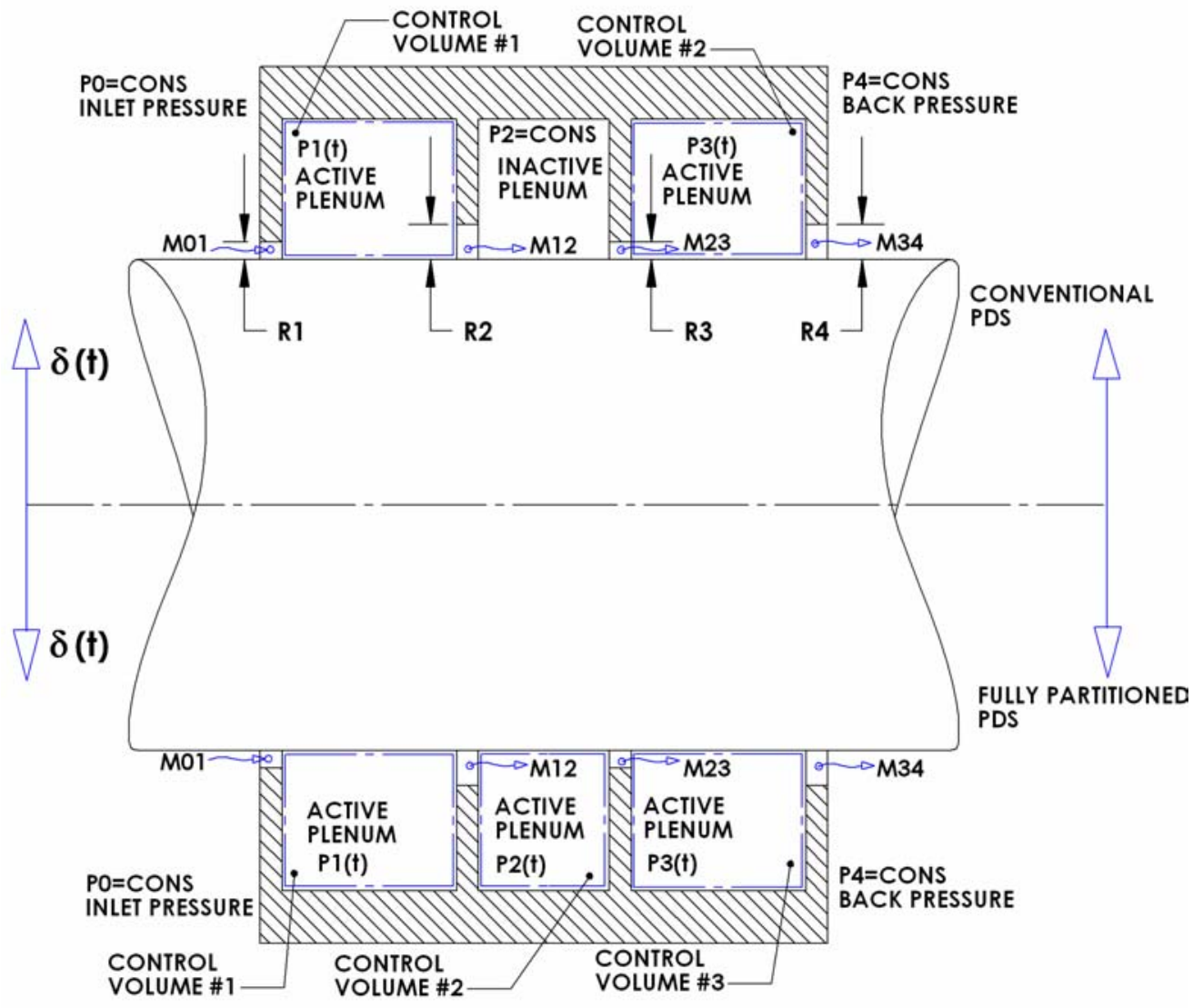

Figure 103. Stand-Alone Cavities vs. Sequential Cavities 
Implementing walls throughout the seal length requires all the internal pressures in the seal to be time dependent (dynamic) resulting in the mass flow equations for the internal blades to depend on two dynamic pressures, therefore coupling the analysis between each control volume. This ultimately requires the simultaneous solution of $2 \mathrm{n}$ ( $\mathrm{n}$ equals the number of control volumes) equations to determine stiffness and damping, rather than the single equation solution for the conventional PDS analysis.

\subsection{Mass Flow Derivation and Static Analysis}

To determine the force coefficients of the fully partitioned seal, first consider a single row of three pressurized cavities, shown in Figure 104. For this case the gas flows from left to right and the rotor is in static equilibrium. Each pocket or cavity can be defined as a control volume that contains a certain amount of a compressible ideal gas undergoing an adiabatic reversible process. The derivation of stiffness and damping will begin with the static conservation of mass equation for each control volume. For no displacement of the rotor the flow through the seal is in a steady state condition and can be described using Equation 6.1.

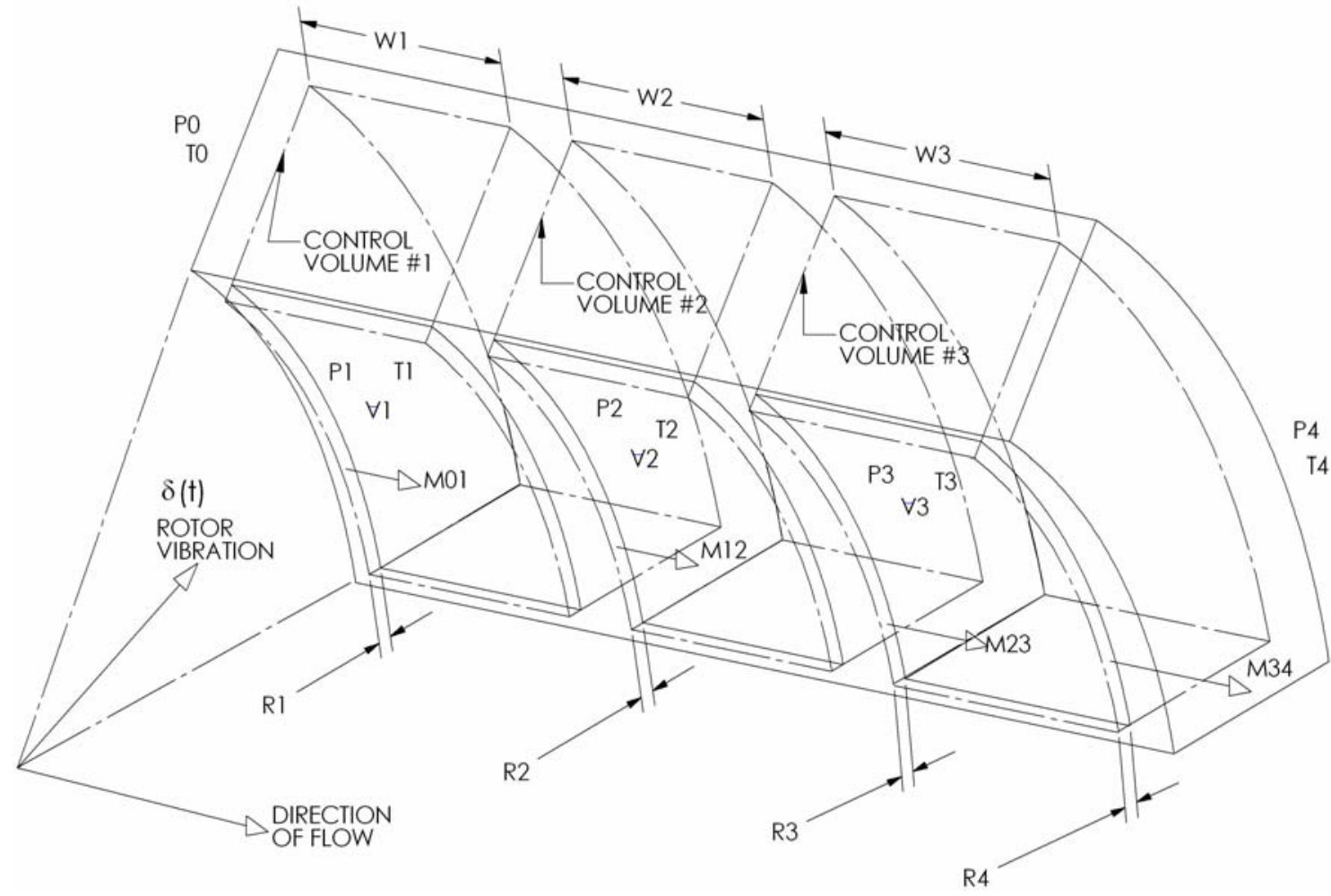

Figure 104. Single Row of Three Pressurized Pockets 
$\dot{m}=\dot{m}_{01}=\dot{m}_{12}=\dot{m}_{23}=\dot{m}_{34}$

Deriving the equations for mass flow through a PDS begins by considering the simple single constriction case as shown in Figure 105. This figure shows a large pressurized reservoir where the supply pressure is higher than the back pressure, which forces the gas to flow from the left to the right through a constriction. The supply pressure $\left(\mathrm{P}_{0}\right)$ and the backpressure $\left(\mathrm{P}_{1}\right)$ are constants (do not vary with time). Also the gas at station 0 can be considered to have zero velocity $\left(V_{0}=0\right)$ due to the assumption that $A_{0}>>A$. The mass flow rate (Equation 6.2) can be defined as the product of the density, flow area, and flow velocity. $\dot{m}=\rho \cdot A \cdot V$

The governing equation (Equation 6.3) for deriving the velocity at station 1 is the first law of thermodynamics; also know as the conservation law of energy. This law states that the amount of thermal energy (Q) transferred to the closed system must equal the sum of the energy change $\left(\mathrm{E}_{2}-\mathrm{E}_{1}\right)$ of the system and the amount of energy transferred from the system by means of mechanical work (W). For our case the $\mathrm{Q}$ and $\mathrm{W}$ term are zero, yielding Equation 6.4.

$Q=\left(E_{1}-E_{0}\right)+W$

$E_{1}=E_{0}$

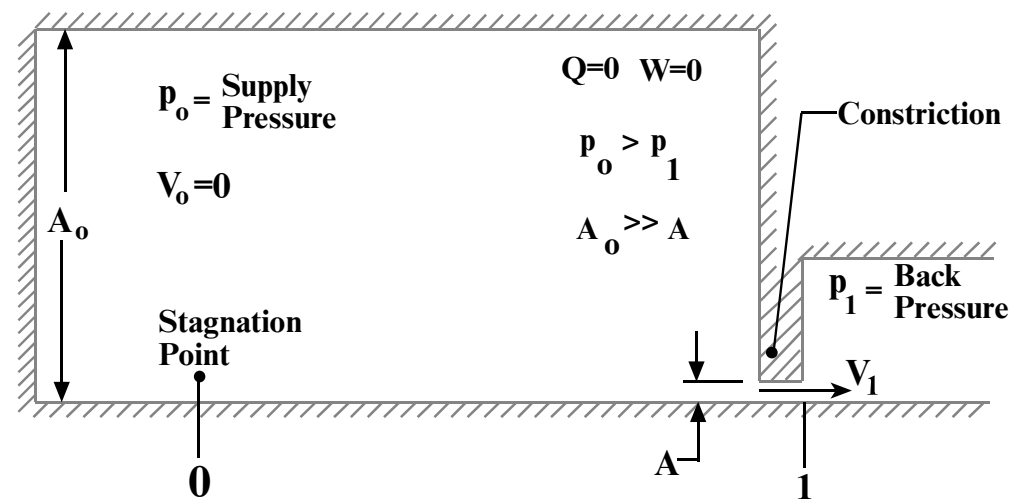

Figure 105. Mass Flow through a Single Constriction

The total energy (E) shown in Equation 6.5 is the sum of the potential, kinetic, and internal energies and then is reduced to the specific total energy (e) in Equation 6.6. Next one can equate the specific total energies (Equation 6.7) for the two stations in Figure 105. Neglecting potential energy effects, noting $\mathrm{V}_{0}=0$, and assuming an isentropic process yields 
Equation 6.8, where $\mathrm{h}$ is the enthalpy. Next, assuming the condition of a calorically perfect gas, the enthalpy (Equation 6.9) can be manipulated using the ideal gas law, definitions for specific heats, and the universal gas constant.

$$
\begin{aligned}
& E=U+\frac{1}{2} m V^{2}+m g z \\
& e=\frac{E}{m}=u+\frac{1}{2} V^{2}+g z \\
& u_{0}+\frac{1}{2} V_{0}^{2}+g z_{0}=u_{1}+\frac{1}{2} V_{1}^{2}+g z_{1} \\
& h_{0}=h_{1}+\frac{1}{2} V_{1}^{2} \\
& h=c_{p} T \\
& R=c_{p}-c_{v} \text { and } \gamma=\frac{c_{p}}{c_{v}} \text { and } P=\rho R T \\
& R=c_{p}-c_{v} \cdot\left(\frac{c_{p}}{c_{p}}\right)=c_{p}-\frac{c_{p}}{\gamma}=c_{p} \cdot\left(1-\frac{1}{\gamma}\right) \\
& c_{p}=\frac{R}{1-\frac{1}{\gamma}}=\left(\frac{R}{1-\frac{1}{\gamma}}\right) \cdot \frac{c_{p}}{c_{p}}=\frac{c_{p} \cdot R}{c_{p}-c_{v}}=\left(\frac{c_{p} \cdot R}{c_{p}-c_{v}}\right) \cdot \frac{c_{v}}{c_{v}}=\frac{c_{v}}{c_{p}-c_{v}} \\
& \frac{c_{v}}{\gamma}
\end{aligned}
$$

Substituting (6.12) into (6.9) and using the ideal gas law:

$$
h=c_{p} T=\frac{\gamma}{\gamma-1} \cdot R T=\frac{\gamma}{\gamma-1} \cdot \frac{P}{\rho}
$$

Equation 6.13 defines the enthalpy in terms of the pressure and then is inserted into (6.8) to yield (6.14) which is the energy equation for a calorically perfect gas:

$$
\frac{\gamma}{\gamma-1} \cdot \frac{P_{o}}{\rho_{o}}=\frac{\gamma}{\gamma-1} \cdot \frac{P_{1}}{\rho_{1}}+\frac{1}{2} V_{1}^{2}
$$

The next step is to define the isentropic condition $\frac{P}{\rho^{\gamma}}=$ Const. and use this condition to expand the right hand side term $\frac{P_{1}}{\rho_{1}}$ of Equation 14 to eliminate the density at station 1: 


$$
\frac{P_{1}}{\rho_{1}}=\left(\frac{P_{0}}{\rho_{0}}\right) \cdot\left(\frac{P_{1}}{P_{0}}\right) \cdot\left(\frac{\rho_{0}}{\rho_{1}}\right)=\left(\frac{P_{0}}{\rho_{0}}\right) \cdot\left(\frac{\rho_{1}}{\rho_{0}}\right)^{\gamma} \cdot\left(\frac{\rho_{0}}{\rho_{1}}\right)=\left(\frac{P_{0}}{\rho_{0}}\right) \cdot\left(\frac{\rho_{1}}{\rho_{0}}\right)^{\gamma-1}=\left(\frac{P_{0}}{\rho_{0}}\right)\left(\left(\frac{P_{1}}{P_{0}}\right)^{\frac{1}{\gamma}}\right)^{\gamma-1}=\frac{P_{0}}{\rho_{0}} \cdot\left(\frac{P_{1}}{P_{0}}\right)^{\frac{\gamma-1}{\gamma}}
$$

Substituting (6.15) into (6.14) yields Equation 6.16 and solving for the velocity at station 1 results in Equation 6.17. Now the velocity has been defined at the constriction in terms of the pressure ratio, constriction area, and gas properties of the system. Next, inserting the result from (6.17) into (6.2) yields the mass flow for the constriction (Equation 6.18). Using the ideal gas law (6.18) can be written as Equation 6.19.

$$
\begin{aligned}
& \frac{\gamma}{\gamma-1} \cdot \frac{P_{o}}{\rho_{o}}=\frac{\gamma}{\gamma-1} \cdot \frac{P_{0}}{\rho_{0}} \cdot\left(\frac{P_{1}}{P_{0}}\right)^{\frac{\gamma-1}{\gamma}}+\frac{1}{2} V_{1}^{2} \\
& V_{1}=\sqrt{\frac{2 \cdot \gamma}{\gamma-1} \cdot \frac{P_{0}}{\rho_{o}}\left[1-\left(\frac{P_{1}}{P_{0}}\right)^{\frac{\gamma-1}{\gamma}}\right]}
\end{aligned}
$$

A useful parameter to note from Equation 6.19 is the non-dimensional flow coefficient $(\psi)$, defined in Equation 6.20. The flow coefficient is helpful when determining the choked flow condition across a constriction. Choking through a nozzle or an area constriction occurs when the critical pressure ratio between the supply pressure and back pressure is reached. Once the critical pressure ratio is reached the constriction mass flow becomes a constant that does not vary with increasing pressure ratio. Figure 106 shows a plot of the flow coefficient as a function of pressure ratio for various gases with different specific heat ratios. Using the definitions from (6.20) and (6.21) the mass flow equation can be rewritten (Equation 6.22) in terms of the flow coefficient. Similarly, the mass flow for the choked case (6.23) can be defined in terms of the maximum valued flow coefficient.

$$
\begin{aligned}
& \dot{m}=A \cdot \rho_{0} \cdot \sqrt{\frac{2 \cdot \gamma}{\gamma-1} \cdot \frac{P_{0}}{\rho_{o}}\left[1-\left(\frac{P_{1}}{P_{0}}\right)^{\frac{\gamma-1}{\gamma}}\right]} \\
& \dot{m}=\frac{A \cdot P_{0}}{\sqrt{\gamma \cdot R \cdot T}} \sqrt{\frac{2 \cdot \gamma^{2}}{\gamma-1} \cdot\left[\left(\frac{P_{1}}{P_{0}}\right)^{\frac{2}{\gamma}}-\left(\frac{P_{1}}{P_{0}}\right)^{\frac{\gamma+1}{\gamma}}\right]}
\end{aligned}
$$


$\psi=\sqrt{\left[\left(\frac{P_{1}}{P_{0}}\right)^{\frac{2}{\gamma}}-\left(\frac{P_{1}}{P_{0}}\right)^{\frac{\gamma+1}{\gamma}}\right]}$

Flow Coefficient Versus Pressure Ratio

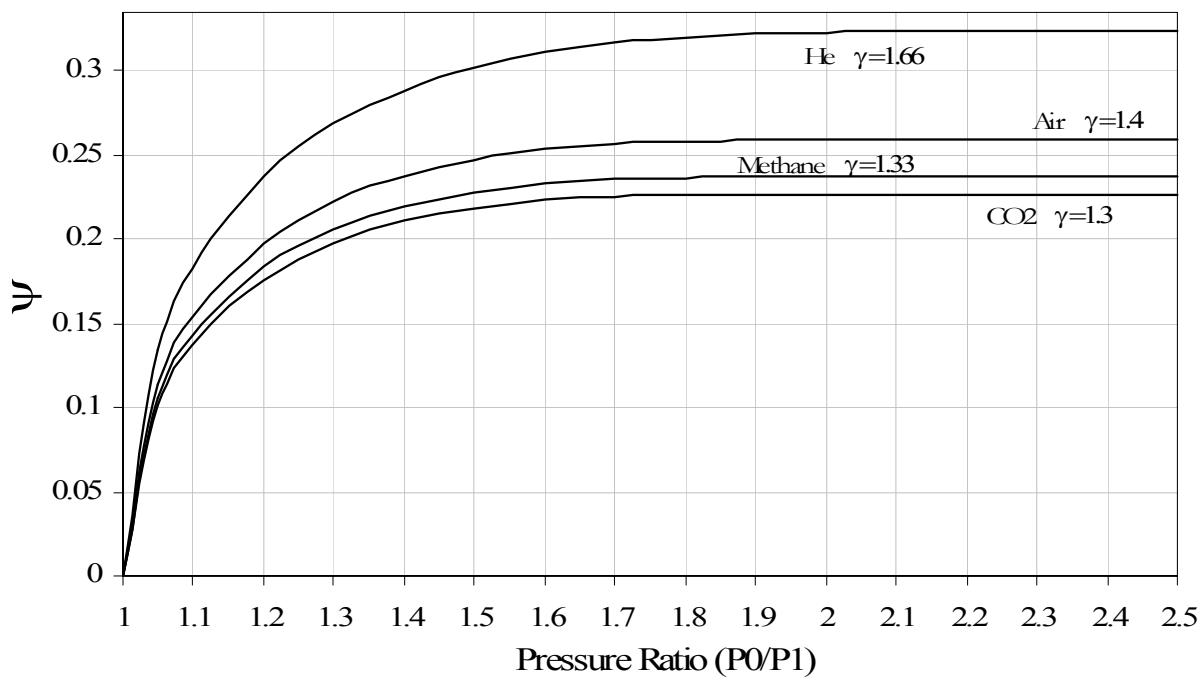

Figure 106. Flow Coefficient for Various Gases

$\psi_{\max }=\left(\frac{2}{\gamma+1}\right)^{\frac{1}{\gamma-1}} \cdot \sqrt{\frac{\gamma-1}{\gamma+1}}$

$\dot{m}=\psi \cdot \frac{A \cdot P_{0}}{\sqrt{\gamma \cdot R \cdot T}} \sqrt{\frac{2 \cdot \gamma^{2}}{\gamma-1}}$

$\dot{m}_{\text {choked }}=\psi_{\max } \cdot \frac{A \cdot P_{0}}{\sqrt{\gamma \cdot R \cdot T}} \sqrt{\frac{2 \cdot \gamma^{2}}{\gamma-1}}$

The mass flow equation in 6.23 can now be applied to the sequentially pressurized cavities shown in Figure 104, yielding the 4 mass flow equations shown in 6.24-6.27. These equations represent the mass flow for each of the four blades for a static steady state case (no rotor vibration). The cavity clearance area terms for a PDS with 8 circumferential pockets are defined in 6.24-6.27 and can be calculated using Equations 6.28-6.31, where D is the rotor diameter. Before proceeding with the dynamic analysis, the static pressures for each 
cavity are calculated using (6.24-6.27) in an iterative method, where $C_{d}$ is the discharge coefficient.

$$
\begin{aligned}
& \dot{m}_{01}=C_{d} \cdot \psi_{01} \cdot \frac{P_{0} A_{1}}{\sqrt{\gamma R T_{o}}} \cdot \sqrt{\frac{2 \gamma^{2}}{\gamma-1}} \\
& \dot{m}_{12}=C_{d} \cdot \psi_{12} \cdot \frac{P_{1} A_{2}}{\sqrt{\gamma R T_{1}}} \cdot \sqrt{\frac{2 \gamma^{2}}{\gamma-1}} \\
& \dot{m}_{23}=C_{d} \cdot \psi_{23} \cdot \frac{P_{2} A_{3}}{\sqrt{\gamma R T_{2}}} \cdot \sqrt{\frac{2 \gamma^{2}}{\gamma-1}} \\
& \dot{m}_{34}=C_{d} \cdot \psi_{34} \cdot \frac{P_{3} A_{4}}{\sqrt{\gamma R T_{3}}} \cdot \sqrt{\frac{2 \gamma^{2}}{\gamma-1}} \\
& A_{1}=\frac{\pi \cdot R_{1} \cdot\left[D+R_{1}\right]}{8} \\
& A_{2}=\frac{\pi \cdot R_{2} \cdot\left[D+R_{2}\right]}{8} \\
& A_{3}=\frac{\pi \cdot R_{3} \cdot\left[D+R_{3}\right]}{8} \\
& A_{4}=\frac{\pi \cdot R_{4} \cdot\left[D+R_{4}\right]}{8}
\end{aligned}
$$

\subsection{Control Volume Analysis for Compressible Flow}

After determining the static cavity pressures from the steady state analysis, Equation 6.1 is transformed into three dynamic conservation of mass equations (Equations 6.32-6.34) describing the mass balance for each of the three cavities in Figure 104. The mass in the pocket is expressed as the partial derivative of density and volume respect to time and implies that the mass in the pocket can change based on two factors: (1) density changes (which can be expressed in terms of pressure) or (2) volume changes. Expanding the third term using the product rule results in Equations 6.35-6.37.

$$
\begin{aligned}
& \dot{m}_{01}(t)=\dot{m}_{12}(t)+\frac{\partial}{\partial t}\left(\rho_{1} \cdot \forall_{1}\right) \\
& \dot{m}_{12}(t)=\dot{m}_{23}(t)+\frac{\partial}{\partial t}\left(\rho_{2} \cdot \forall_{2}\right)
\end{aligned}
$$


$\dot{m}_{23}(t)=\dot{m}_{34}(t)+\frac{\partial}{\partial t}\left(\rho_{3} \cdot \forall_{3}\right)$

$\frac{\partial}{\partial t}\left(\rho_{1} \forall_{1}\right)=\frac{\partial \rho_{1}}{\partial t} \cdot \forall_{1}+\frac{\partial \forall_{1}}{\partial t} \cdot \rho_{1}=\frac{\partial \rho_{1}}{\partial P_{1}} \cdot \frac{\partial P_{1}}{\partial t}+\frac{\partial \forall_{1}}{\partial t} \cdot \rho_{1}$

$\frac{\partial}{\partial t}\left(\rho_{2} \forall_{2}\right)=\frac{\partial \rho_{2}}{\partial t} \cdot \forall_{2}+\frac{\partial \forall_{2}}{\partial t} \cdot \rho_{2}=\frac{\partial \rho_{2}}{\partial P_{2}} \cdot \frac{\partial P_{2}}{\partial t}+\frac{\partial \forall_{2}}{\partial t} \cdot \rho_{2}$

$\frac{\partial}{\partial t}\left(\rho_{3} \forall_{3}\right)=\frac{\partial \rho_{3}}{\partial t} \cdot \forall_{3}+\frac{\partial \forall_{3}}{\partial t} \cdot \rho_{3}=\frac{\partial \rho_{3}}{\partial P_{3}} \cdot \frac{\partial P_{3}}{\partial t}+\frac{\partial \forall_{3}}{\partial t} \cdot \rho_{3}$

The next step is to define the $\frac{\partial \rho_{i}}{\partial P_{i}}$ term shown in Equations 6.35-6.37. For an adiabatic isentropic thermodynamic process the relation shown in Equation 6.38 holds. Rewriting Equation 6.38 in the form shown in Equation 6.39 and then taking the partial derivative of Equation 6.39 respect to density generates Equation 6.40. Rearranging and using the equation of state for an ideal gas $(\mathrm{P}=\rho \mathrm{RT})$ results in Equations 6.41-6.43. Then substitute Equations 6.41-6.43 into 6.35-6.37 and into 6.32-6.34 to yield Equations 6.44-6.46.

$\frac{P}{\rho^{\gamma}}=C$

$\ln P-\gamma \ln \rho=C$

$\frac{1}{P} \cdot \frac{\partial P}{\partial \rho}-\frac{\gamma}{\rho}=0$

$\frac{\partial \rho_{1}}{\partial P_{1}}=\frac{1}{R T_{1} \gamma}$

$\frac{\partial \rho_{2}}{\partial P_{2}}=\frac{1}{R T_{2} \gamma}$

$\frac{\partial \rho_{3}}{\partial P_{3}}=\frac{1}{R T_{3} \gamma}$

$\dot{m}_{01}(t)-\dot{m}_{12}(t)=\frac{\forall_{1}}{R T_{1} \gamma} \cdot \frac{\partial P_{1}}{\partial t}+\frac{\partial \forall_{1}}{\partial t} \cdot \frac{P_{1}}{R T_{1}}$ 


$$
\begin{aligned}
& \dot{m}_{12}(t)-\dot{m}_{23}(t)=\frac{\forall_{2}}{R T_{2} \gamma} \cdot \frac{\partial P_{2}}{\partial t}+\frac{\partial \forall_{2}}{\partial t} \cdot \frac{P_{2}}{R T_{2}} \\
& \dot{m}_{23}(t)-\dot{m}_{34}(t)=\frac{\forall_{3}}{R T_{3} \gamma} \cdot \frac{\partial P_{3}}{\partial t}+\frac{\partial \forall_{3}}{\partial t} \cdot \frac{P_{3}}{R T_{3}}
\end{aligned}
$$

Recall Equations 6.35-6.37 and consider the $\frac{\partial \forall_{i}}{\partial t}$ term. This term represents the change in the control volume respect to time and is directly related to the time dependent rotor motion and displacement dependent change in cavity clearance area. The rotor displacement (Equation 6.47) can be defined as a harmonic function with amplitude $(\mathrm{X})$ and frequency ( $\omega)$. The velocity is then defined in Equation 6.48. Using the result from (6.48) one can express the $\frac{\partial \forall_{i}}{\partial t}$ parameter as a function of vibration velocity and the partial derivative $\frac{\partial A}{\partial \delta}$ (Equations 6.49-6.51). It is now required that the cavity clearance area change be derived as a function of arbitrary rotor displacement.

$$
\begin{aligned}
& \delta(t)=\mathrm{X} \sin (\omega \cdot t) \\
& \dot{\delta}(t)=\frac{\partial \delta}{\partial t}=\omega \cdot \mathrm{X} \cos (\omega \cdot t) \\
& \frac{\partial \forall_{1}}{\partial t}=w_{1} \cdot\left(\frac{\partial A}{\partial \delta}\right)\left(\frac{\partial \delta}{\partial t}\right)=w_{1} \cdot\left(\frac{\partial A}{\partial \delta}\right) \cdot \omega \cdot \mathrm{X} \cos (\omega \cdot t) \\
& \frac{\partial \forall_{2}}{\partial t}=w_{2} \cdot\left(\frac{\partial A}{\partial \delta}\right)\left(\frac{\partial \delta}{\partial t}\right)=w_{2} \cdot\left(\frac{\partial A}{\partial \delta}\right) \cdot \omega \cdot \mathrm{X} \cos (\omega \cdot t) \\
& \frac{\partial \forall_{3}}{\partial t}=w_{3} \cdot\left(\frac{\partial A}{\partial \delta}\right)\left(\frac{\partial \delta}{\partial t}\right)=w_{3} \cdot\left(\frac{\partial A}{\partial \delta}\right) \cdot \omega \cdot \mathrm{X} \cos (\omega \cdot t)
\end{aligned}
$$

The calculation of the change in cavity clearance area for a pocket damper seal (PDS) experiencing off-center rotor displacement begins by illustrating (Figure 107) two cases of rotor position. The case on the left shows the rotor in the centered position with a constant clearance from 0-360 degrees. The calculation of area for case (a) is straight forward and can be determined using the basic equations for a circle. Given the seal radius $\left(R_{s}\right)$, the rotor radius $R$, and the blue hatched area between $\theta_{1}$ and $\theta_{2}$ in Figure 107-a, the cavity clearance area for the rotor in the centered position is defined by Equation 6.52, where $\theta$ is in radians. 
$A_{c e n}=\frac{\left(\theta_{2}-\theta_{1}\right)}{2} \cdot\left(R_{s}^{2}-R^{2}\right)$

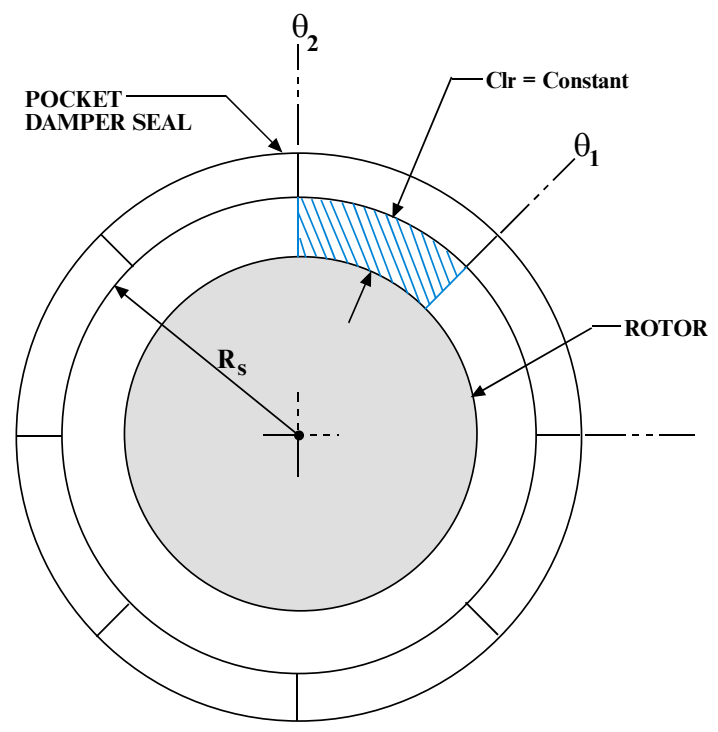

(a) ROTOR IN CENTERED POSITION

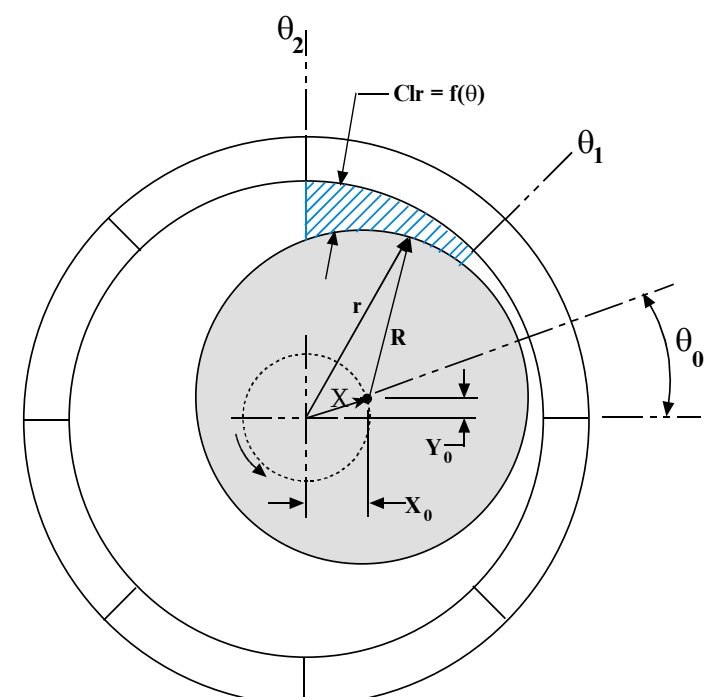

(b) ROTOR WITH DISPLACEMENT OFFSET

Figure 107. PDS Cavity Clearance Area for Arbitrary Rotor Displacement

Case (b) illustrates that when the rotor is displaced from the centered position, the clearance becomes a function of angular location $(\theta)$. To better understand the derivations of the clearance area for an off centered rotor refer to Figure 108. Figure 108 shows an arbitrary function with boundaries $\theta_{1}$ and $\theta_{2}$. Considering a differential angle $\mathrm{d} \theta$ and a radius $(\mathrm{r})$, which is a function of $\theta$, one can calculate the area within the sector using Equation 6.53.

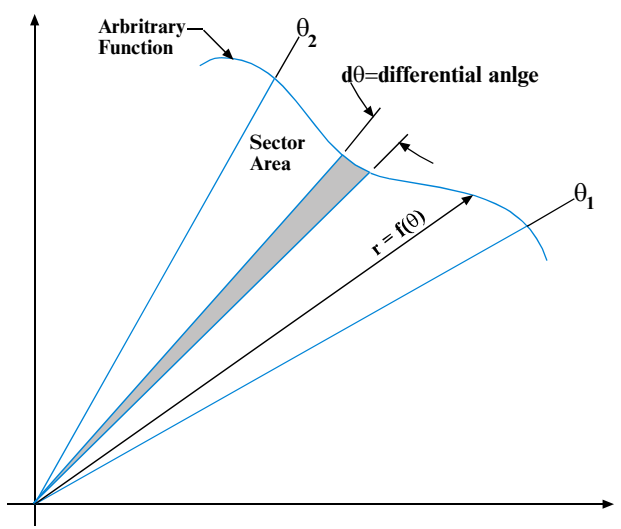

Figure 108. Area of a Sector Using Polar Coordinates 
Area $=\frac{1}{2} \int_{\theta_{1}}^{\theta_{2}} r^{2} d \theta$

The main task in implementing (6.53) involves solving the boundary equation for an off centered rotor displacement in terms of the variable r. Consider Equation 6.54 for the off centered circle case, where the terms $\mathrm{x}_{0}$ and $\mathrm{y}_{0}$ can be expressed using $\mathrm{X}$ and $\theta_{0}(6.55)$. Factoring Equation 6.55 we arrive at a second order polynomial equation (Equation 6.56) in terms of $\mathrm{r}$ and $\theta$, where $C=R^{2}-\mathrm{X}^{2} \cos ^{2} \theta_{0}-\mathrm{X}^{2} \sin ^{2} \theta_{0}$.

$\left(x-x_{o}\right)^{2}+\left(y-y_{o}\right)^{2}=R^{2}$

$\left(r \cos \theta-\mathrm{X} \cos \theta_{0}\right)^{2}+\left(r \sin \theta-\mathrm{X} \sin \theta_{0}\right)^{2}=R^{2}$

$r^{2}-2 r\left(\mathrm{X} \cos \theta_{0} \cos \theta+\mathrm{X} \sin \theta_{0} \sin \theta\right)-C=0$

Utilizing the quadratic formula and only considering the positive solution, the final equation for $r$ in terms of $\theta$ is shown in Equation 6.58. Since we have generated an equation for $r$ in terms of $\theta$, the result can be found by substituting into (6.53), which yields Equation 6.59. Note that Equation 6.59 represents the entire sector area from the origin. To calculate the blue hatched area shown in Figure 107-b, we need to subtract (6.59) from the seal bore area between $\theta_{1}$ and $\theta_{2}$. Finally the clearance area for an arbitrary rotor displacement is defined in Equation 6.60.

Quadratic Formula: $\quad a r^{2}+b r+c=0 ; \quad r=\frac{-b \pm \sqrt{\left(b^{2}-4 a c\right)}}{2 a}$

$r=\left(X \cos \theta_{0} \cos \theta+X \sin \theta_{0} \sin \theta\right)+$ $\sqrt{\left(X^{2} \cos ^{2} \theta_{0} \cdot \cos ^{2} \theta+X^{2} \sin ^{2} \theta_{0} \cdot \sin ^{2} \theta+2 X^{2} \cos \theta_{0} \cdot \sin \theta_{0} \cdot \cos \theta \cdot \sin \theta\right)+C}$

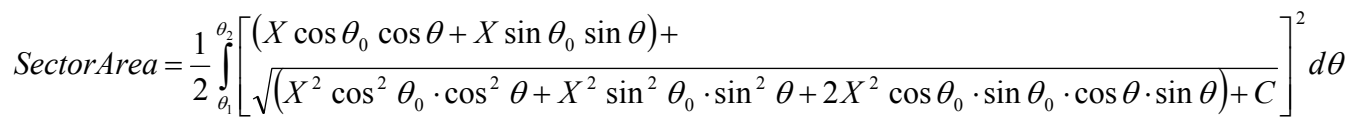

Clearance area for a single cavity for an arbitrary rotor displacement:

$A=\frac{1}{2} \int_{\theta_{1}}^{\theta_{2}} R_{s}^{2} d \theta-\frac{1}{2} \int_{\theta_{1}}^{\theta_{2}}\left[\begin{array}{l}\left(X \cos \theta_{0} \cos \theta+X \sin \theta_{0} \sin \theta\right)+ \\ \sqrt{\left(X^{2} \cos ^{2} \theta_{0} \cdot \cos ^{2} \theta+X^{2} \sin ^{2} \theta_{0} \cdot \sin ^{2} \theta+2 X^{2} \cos \theta_{0} \cdot \sin \theta_{0} \cdot \cos \theta \cdot \sin \theta\right)+C}\end{array}\right]^{2} d \theta$ 
Now consider a rotor displacement where the displacement $\delta$ is centered (Equation 6.61) with a cavity as shown in Figure 109. The change in area respect to centered rotor displacement (Equation 6.63) is now calculated noting the change in area (Equation 6.62) and the cavity centered displacement amplitude X.

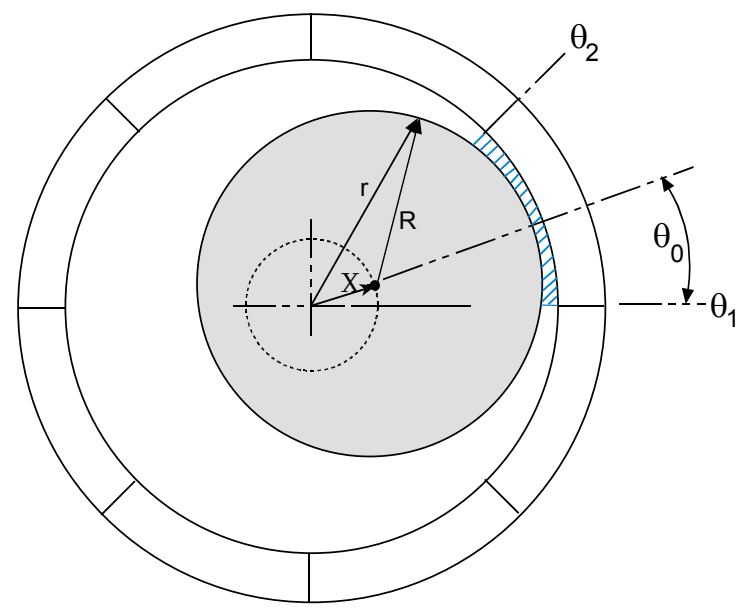

Figure 109. PDS Clearance Area for Cavity-Centered Rotor Displacement

$\left(\frac{\theta_{2}+\theta_{1}}{2}\right)=\theta_{0}$

Change in PDS cavity clearance area:

$\Delta A=\frac{\left(\theta_{2}-\theta_{1}\right)}{2} \cdot\left(R_{s}^{2}-R^{2}\right)-\frac{1}{2} \int_{\theta_{1}}^{\theta_{2}} R_{s}^{2} d \theta-\frac{1}{2} \int_{\theta_{1}}^{\theta_{2}}\left[\begin{array}{l}\left(X \cos \theta_{0} \cos \theta+X \sin \theta_{0} \sin \theta\right)+ \\ \sqrt{\left(X^{2} \cos ^{2} \theta_{0} \cdot \cos ^{2} \theta+X^{2} \sin ^{2} \theta_{0} \cdot \sin ^{2} \theta+2 X^{2} \cos \theta_{0} \cdot \sin \theta_{0} \cdot \cos \theta \cdot \sin \theta\right)+C}\end{array}\right]^{2} d \theta(6.62$

$\frac{\partial A}{\partial \delta}=-\frac{\Delta A}{\mathrm{X}}$

The remaining terms that require definition in Equations 6.30-6.32 are the time dependent mass flow rate terms. The time dependent mass flow rates can vary based on two factors: 1) changes in cavity pressure and 2) changes in rotor displacement (cavity clearance change). Equations 6.64-6.75 show the dependency for the mass flows that can be expressed as a steady state component (no rotor modulation) plus a dynamic component (rotor modulating seal). The pressure can also be thought of as a steady state (static) component $P_{i}$ and a dynamic component $\bar{P}_{i}(t)$ (Equations 6.68-6.70).

$\dot{m}_{01}(t)=\dot{m}_{01}\left(\bar{P}_{1}(t), \delta(t)\right)$ 


$$
\begin{aligned}
& \dot{m}_{12}(t)=\dot{m}_{12}\left(\bar{P}_{1}(t), \bar{P}_{2}(t), \delta(t)\right) \\
& \dot{m}_{23}(t)=\dot{m}_{23}\left(\bar{P}_{2}(t), \bar{P}_{3}(t), \delta(t)\right) \\
& \dot{m}_{34}(t)=\dot{m}_{34}\left(\bar{P}_{3}(t), \delta(t)\right) \\
& P_{1}(t)=P_{1}+\bar{P}_{1}(t) \\
& P_{2}(t)=P_{2}+\bar{P}_{2}(t) \\
& P_{3}(t)=P_{3}+\bar{P}_{3}(t)
\end{aligned}
$$

The total flow rate (steady state and dynamic) can be found by expanding Equations 6.646.67 using a Taylor Series expansion (Equations 6.71-6.74). Substituting Equations 6.71-6.74 into Equations 6.44-6.46 yields the refined conservation of mass equations (Equations 6.756.77) for the three control volumes. Next, the partial derivatives of the mass flow rates respect to cavity pressure in Equations 6.75-6.77 must be derived. A generalized form for the partial derivatives respect to cavity is shown in Equations 6.78-6.79.

$$
\begin{aligned}
& \dot{m}_{01}=\dot{m}+\left.\frac{\partial \dot{m}_{01}}{\partial P_{1}}\right|_{P_{1}} \cdot \bar{P}_{1}(t)+\left.\frac{\partial \dot{m}_{01}}{\partial \delta}\right|_{\delta_{o}} \cdot \delta(t) \\
& \dot{m}_{12}=\dot{m}+\left.\frac{\partial \dot{m}_{12}}{\partial P_{1}}\right|_{P_{1}} \cdot \bar{P}_{1}(t)+\left.\frac{\partial \dot{m}_{12}}{\partial P_{2}}\right|_{P_{2}} \cdot \bar{P}_{2}(t)+\left.\frac{\partial \dot{m}_{12}}{\partial \delta}\right|_{\delta_{o}} \cdot \delta(t) \\
& \dot{m}_{23}=\dot{m}+\left.\frac{\partial \dot{m}_{23}}{\partial P_{2}}\right|_{P_{2}} \cdot \bar{P}_{2}(t)+\left.\frac{\partial \dot{m}_{23}}{\partial P_{3}}\right|_{P_{3}} \cdot \bar{P}_{3}(t)+\left.\frac{\partial \dot{m}_{23}}{\partial \delta}\right|_{\delta_{o}} \cdot \delta(t) \\
& \dot{m}_{34}=\dot{m}+\left.\frac{\partial \dot{m}_{34}}{\partial P_{3}}\right|_{P_{3}} \cdot \bar{P}_{3}(t)+\left.\frac{\partial \dot{m}_{34}}{\partial \delta}\right|_{\delta_{o}} \cdot \delta(t) \\
& \left(\frac{\partial \dot{m}_{01}}{\partial P_{1}}-\frac{\partial \dot{m}_{12}}{\partial P_{1}}\right) \cdot \bar{P}_{1}(t)+\left(\frac{\partial \dot{m}_{01}}{\partial \delta}-\frac{\partial \dot{m}_{12}}{\partial \delta}\right) \cdot \delta(t)-\left(\frac{\partial \dot{m}_{12}}{\partial P_{2}}\right) \cdot \bar{P}_{2}(t)-\left(\frac{P_{1} \cdot w_{1} \cdot L_{1}}{R \cdot T_{1}}\right) \cdot \frac{\partial \delta}{\partial t}-\left(\frac{\forall_{1}}{\gamma \cdot R \cdot T_{1}}\right) \cdot \frac{\partial P_{1}}{\partial t}=0 \\
& \left(\frac{\partial \dot{m}_{12}}{\partial P_{2}}-\frac{\partial \dot{m}_{23}}{\partial P_{2}}\right) \cdot \bar{P}_{2}(t)+\left(\frac{\partial \dot{m}_{12}}{\partial \delta}-\frac{\partial \dot{m}_{23}}{\partial \delta}\right) \cdot \delta(t)-\left(\frac{\partial \dot{m}_{23}}{\partial P_{3}}\right) \cdot \bar{P}_{3}(t)+\left(\frac{\partial \dot{m}_{12}}{\partial P_{1}}\right) \cdot \bar{P}_{1}(t)-\left(\frac{P_{2} \cdot w_{2} \cdot L_{2}}{R \cdot T_{2}}\right) \cdot \frac{\partial \delta}{\partial t}-\left(\frac{\forall \cdot}{\gamma \cdot R \cdot T_{2}}\right) \cdot \frac{\partial P_{2}}{\partial t}=0 \\
& \left(\frac{\partial \dot{m}_{23}}{\partial P_{3}}-\frac{\partial \dot{m}_{34}}{\partial P_{3}}\right) \cdot \bar{P}_{3}(t)+\left(\frac{\partial \dot{m}_{23}}{\partial \delta}-\frac{\partial \dot{m}_{34}}{\partial \delta}\right) \cdot \delta(t)+\left(\frac{\partial \dot{m}_{23}}{\partial P_{2}}\right) \cdot \bar{P}_{2}(t)-\left(\frac{P_{2} \cdot w_{3} \cdot L_{3}}{R \cdot T_{3}}\right) \cdot \frac{\partial \delta}{\partial t}-\left(\frac{\forall_{3}}{\gamma \cdot R \cdot T_{3}}\right) \cdot \frac{\partial P_{3}}{\partial t}=0 \\
& \left.\frac{\partial \dot{m}_{i j}}{\partial P_{j}}=\frac{P_{i} \cdot A_{j}}{2 \cdot \sqrt{\gamma \cdot R \cdot T} \cdot\left[\left(\frac{P_{j}}{P_{i}}\right)^{\frac{2}{\gamma}}-\left(\frac{P_{j}}{P_{i}}\right)^{\frac{\gamma+1}{\gamma}}\right.}\right]^{\frac{1}{2}} \cdot\left[\frac{2}{\gamma \cdot P_{i}} \cdot\left(\frac{P_{j}}{P_{i}}\right)^{1-\frac{\gamma}{2}}-\frac{\gamma+1}{\gamma \cdot P_{i}} \cdot\left(\frac{P_{j}}{P_{i}}\right)^{1-\frac{\gamma+1}{\gamma}}\right]
\end{aligned}
$$


$\left.\frac{\partial \dot{m}_{i j}}{\partial P_{i}}=\frac{A_{j}}{\sqrt{\gamma \cdot R \cdot T}} \cdot\left[\left(\frac{P_{j}}{P_{i}}\right)^{\frac{2}{\gamma}}-\left(\frac{P_{j}}{P_{i}}\right)^{\frac{\gamma+1}{\gamma}}\right]^{\frac{1}{2}}+\frac{P_{i}}{\left[\left(\frac{P_{j}}{P_{i}}\right)^{\frac{2}{\gamma}}-\left(\frac{P_{j}}{P_{i}}\right)^{\frac{\gamma+1}{\gamma}}\right]^{\frac{1}{2}}} \cdot\left[\frac{(\gamma+1) \cdot P_{j}}{\gamma \cdot P_{i}^{2}} \cdot\left(\frac{P_{j}}{P_{i}}\right)^{1-\frac{\gamma+1}{\gamma}}-\frac{\gamma}{2} \cdot \frac{P_{j}}{P_{i}^{2}} \cdot\left(\frac{P_{j}}{P_{i}}\right)^{1-\frac{\gamma}{2}}\right]\right]$

The remaining terms from 6.75-6.77 are defined in 6.80-6.83.

$\frac{\partial \dot{m}_{01}}{\partial \delta}=\frac{\partial \dot{m}_{01}}{\partial A_{1}} \cdot \frac{\partial A_{1}}{\partial \delta}=\frac{\dot{m}_{01}}{A_{1}} \cdot \frac{\partial A_{1}}{\partial \delta}$

$\frac{\partial \dot{m}_{12}}{\partial \delta}=\frac{\partial \dot{m}_{12}}{\partial A_{2}} \cdot \frac{\partial A_{2}}{\partial \delta}=\frac{\dot{m}_{12}}{A_{2}} \cdot \frac{\partial A_{2}}{\partial \delta}$

$\frac{\partial \dot{m}_{23}}{\partial \delta}=\frac{\partial \dot{m}_{23}}{\partial A_{3}} \cdot \frac{\partial A_{3}}{\partial \delta}=\frac{\dot{m}_{23}}{A_{3}} \cdot \frac{\partial A_{3}}{\partial \delta}$

$\frac{\partial \dot{m}_{34}}{\partial \delta}=\frac{\partial \dot{m}_{34}}{\partial A_{4}} \cdot \frac{\partial A_{4}}{\partial \delta}=\frac{\dot{m}_{34}}{A_{4}} \cdot \frac{\partial A_{4}}{\partial \delta}$

The resultant dynamic cavity pressures are composed of a sine and a cosine component, as shown in Equations 6.84-6.86. Taking the time derivative yields Equations 6.87-6.89. Next, rewrite Equations 6.75-6.77 in the form shown in (6.90-6.92). Substituting the results from Equations 6.84-6.89 into Equations 6.90-6.92 results in Equations 6.93-6.95. Note that Equations 6.93-6.95 contain sine and cosine components that can be separated to form a set of $2 \mathrm{n}$ equations (Equations 6.96-6.101), where $\mathrm{n}$ is the number of cavities. The set of $2 \mathrm{n}$ equations can also be expressed in matrix form, where $P$ is the pressure component matrix, $\Lambda$ is the pressure coefficient matrix, and $\Theta$ represents the right side element matrix.

$\bar{P}_{1}(t)=\bar{P}_{1 C} \cdot \cos \omega t+\bar{P}_{1 S} \cdot \sin \omega t$

$\bar{P}_{2}(t)=\bar{P}_{2 C} \cdot \cos \omega t+\bar{P}_{2 S} \cdot \sin \omega t$

$\bar{P}_{3}(t)=\bar{P}_{3 C} \cdot \cos \omega t+\bar{P}_{3 S} \cdot \sin \omega t$

$\frac{\partial \bar{P}_{1}}{\partial t}=-\omega \cdot \bar{P}_{1 C} \cdot \sin \omega t+\omega \cdot \bar{P}_{1 S} \cdot \cos \omega t$

$\frac{\partial \bar{P}_{2}}{\partial t}=-\omega \cdot \bar{P}_{2 C} \cdot \sin \omega t+\omega \cdot \bar{P}_{2 S} \cdot \cos \omega t$

$\frac{\partial \bar{P}_{3}}{\partial t}=-\omega \cdot \bar{P}_{3 C} \cdot \sin \omega t+\omega \cdot \bar{P}_{3 S} \cdot \cos \omega t$ 


$$
\begin{aligned}
& e_{1} \cdot \frac{\partial \delta}{\partial t}+c_{1} \cdot \frac{\partial \bar{P}_{1}}{\partial t}+\left(f_{1}-f_{2}\right) \cdot \bar{P}_{1}(t)+f_{3} \bar{P}_{2}(t)-d_{1} \cdot \delta(t)=0 \\
& e_{2} \cdot \frac{\partial \delta}{\partial t}+c_{2} \cdot \frac{\partial \bar{P}_{2}}{\partial t}+f_{2} \cdot \bar{P}_{1}(t)+\left(f_{3}-f_{4}\right) \bar{P}_{2}(t)-f_{5} \cdot \bar{P}_{3}(t)-d_{2} \cdot \delta(t)=0 \\
& e_{3} \cdot \frac{\partial \delta}{\partial t}+c_{3} \cdot \frac{\partial \bar{P}_{3}}{\partial t}+f_{4} \cdot \bar{P}_{2}(t)+\left(f_{5}-f_{6}\right) \bar{P}_{3}(t)-d_{3} \cdot \delta(t)=0
\end{aligned}
$$

$e_{1} \cdot \mathrm{X} \cdot \omega \cdot \cos \omega t+c_{1} \cdot \omega \cdot \bar{P}_{1 S} \cdot \cos \omega t-c_{1} \cdot \omega \cdot \bar{P}_{1 C} \cdot \sin \omega t+\left(f_{1}-f_{2}\right) \cdot \bar{P}_{1 C} \cdot \cos \omega t+$ $\left(f_{1}-f_{2}\right) \cdot \bar{P}_{1 S} \cdot \sin \omega t+f_{3} \cdot \bar{P}_{2 C} \cdot \cos \omega t+f_{3} \cdot \bar{P}_{2 S} \cdot \sin \omega t+d_{1} \cdot \mathrm{X} \sin \omega t=0$

$e_{2} \cdot \mathrm{X} \cdot \omega \cdot \cos \omega t+c_{2} \cdot \omega \cdot \bar{P}_{2 S} \cdot \cos \omega t-c_{2} \cdot \omega \cdot \bar{P}_{2 C} \cdot \sin \omega t+f_{2} \cdot \bar{P}_{1 C} \cdot \cos \omega t+f_{2} \cdot \bar{P}_{1 S} \cdot \sin \omega t+$ $\left(f_{3}-f_{4}\right) \cdot \bar{P}_{2 C} \cdot \cos \omega t+\left(f_{3}-f_{4}\right)_{2} \cdot \bar{P}_{2 S} \cdot \sin \omega t-f_{5} \cdot \bar{P}_{3 C} \cdot \cos \omega t-f_{5} \cdot \bar{P}_{3 S} \cdot \sin \omega t-d_{2} \cdot \mathrm{X} \sin \omega t=0$ $e_{3} \cdot \mathrm{X} \cdot \omega \cdot \cos \omega t+c_{3} \cdot \omega \cdot \bar{P}_{3 S} \cdot \cos \omega t-c_{3} \cdot \omega \cdot \bar{P}_{3 C} \cdot \sin \omega t+f_{4} \cdot \bar{P}_{2 C} \cdot \cos \omega t+$ $f_{4} \cdot \bar{P}_{2 S} \cdot \sin \omega t+\left(f_{5}-f_{6}\right) \cdot \bar{P}_{3 C} \cdot \cos \omega t+\left(f_{5}-f_{6}\right) \cdot \bar{P}_{3 S} \cdot \sin \omega t-d_{3} \cdot \mathrm{X} \sin \omega t=0$

$\left(e_{1} \cdot \omega \cdot \mathrm{X}+c_{1} \cdot \omega \cdot \bar{P}_{1 S}+\left(f_{1}-f_{2}\right)_{1} \cdot \bar{P}_{1 C}+f_{3} \cdot \bar{P}_{2 C}\right) \cdot \cos \omega t=0$

$\left(-c_{1} \cdot \omega \cdot \bar{P}_{1 C}+\left(f_{1}-f_{2}\right) \cdot \bar{P}_{1 S}+f_{3} \cdot \bar{P}_{2 S}-d_{1} \cdot \mathrm{X}\right) \cdot \sin \omega t=0$

$\left(e_{2} \cdot \omega \cdot \mathrm{X}+c_{2} \cdot \omega \cdot \bar{P}_{2 S}+f_{2} \cdot \bar{P}_{1 C}+\left(f_{3}-f_{4}\right) \cdot \bar{P}_{2 C}-f_{5} \cdot \bar{P}_{3 C}\right) \cdot \cos \omega t=0$

$\left(-c_{2} \cdot \omega \cdot \bar{P}_{2 C}+f_{2} \cdot \bar{P}_{1 S}+\left(f_{3}-f_{4}\right) \cdot \bar{P}_{2 S}-f_{5} \cdot \bar{P}_{3 S}-d_{2} \cdot \mathrm{X}\right) \cdot \sin \omega t=0$

$\left(e_{3} \cdot \omega \cdot \mathrm{X}+c_{3} \cdot \omega \cdot \bar{P}_{3 S}+f_{4} \cdot \bar{P}_{2 C}+\left(f_{5}-f_{6}\right) \cdot \bar{P}_{3 C}\right) \cdot \cos \omega t=0$

$\left(-c_{3} \cdot \omega \cdot \bar{P}_{3 C}+f_{4} \cdot \bar{P}_{2 S}+\left(f_{5}-f_{6}\right)_{3} \cdot \bar{P}_{3 S}-d_{3} \cdot \mathrm{X}\right) \cdot \sin \omega t=0$

$$
\left[\begin{array}{cccccc}
\left(f_{1}-f_{2}\right) & -c_{1} \cdot \omega & -f_{3} & 0 & 0 & 0 \\
c_{1} \cdot \omega & \left(f_{1}-f_{2}\right) & 0 & -f_{3} & 0 & 0 \\
f_{2} & 0 & \left(f_{3}-f_{4}\right) & -c_{2} \cdot \omega & -f_{5} & 0 \\
0 & f_{2} & c_{2} \cdot \omega & \left(f_{3}-f_{4}\right) & 0 & -f_{5} \\
0 & 0 & f_{4} & 0 & \left(f_{5}-f_{6}\right) & -c_{3} \cdot \omega \\
0 & 0 & 0 & f_{4} & c_{3} \cdot \omega & \left(f_{5}-f_{6}\right)
\end{array}\right]\left[\begin{array}{c}
\bar{P}_{1 C} \\
\bar{P}_{1 S} \\
\bar{P}_{2 C} \\
\bar{P}_{2 S} \\
\bar{P}_{3 C} \\
\bar{P}_{3 S}
\end{array}\right]=\left[\begin{array}{c}
-e_{1} \cdot \omega \cdot \mathrm{X} \\
d_{1} \cdot \mathrm{X} \\
-e_{2} \cdot \omega \cdot \mathrm{X} \\
d_{2} \cdot \mathrm{X} \\
-e_{3} \cdot \omega \cdot \mathrm{X} \\
d_{3} \cdot \mathrm{X}
\end{array}\right]
$$


$\Lambda \cdot P=\Theta$

$\mathrm{P}=\Lambda^{-1} \cdot \Theta$

The solution matrix $P$ (Equation 6.104) is composed of 2 pressure components per cavity, $\bar{P}_{i C}$ and $\bar{P}_{i S}$, which then can be used to calculate the resultant dynamic cavity pressure magnitude (Equation 6.105) and phase (Equation 6.106). The pressure magnitude and phase are directly related to the dynamic force coefficients. Finally, the damper seal stiffness and damping coefficients are defined in equations 6.107-6.108, where $A_{i p}$ is the projected area of the cavity on the rotor. A plot showing the dynamic pressures for the FP six bladed PDS using this theory is shown in Figure 110 . This $20 \mathrm{~Hz}$ case shows the 3 diverging cavities to have a pressure phase between 90 and 180 degrees and the two converging cavities are showing a pressure phase between 0 and 90 degrees. This results in the diverging cavities to have positive damping and negative stiffness, whereas the converging cavities have positive damping and stiffness, which agrees with the experimental results.

$$
\begin{aligned}
& \left|\bar{P}_{i}\right|=\sqrt{\left(\bar{P}_{i c}\right)^{2}+\left(\bar{P}_{i S}\right)^{2}} \\
& \phi_{i}=\tan ^{-1}\left(\frac{\bar{P}_{i S}}{\bar{P}_{i C}}\right)
\end{aligned}
$$

Direct Damping $=C=\sum_{i=1}^{n} \frac{\bar{P}_{i C} \cdot A_{i p}}{\omega \cdot \mathrm{X}}$

Direct Stiffness $=K=\sum_{i=1}^{n} \frac{\bar{P}_{i S} \cdot A_{i p}}{\mathrm{X}}$

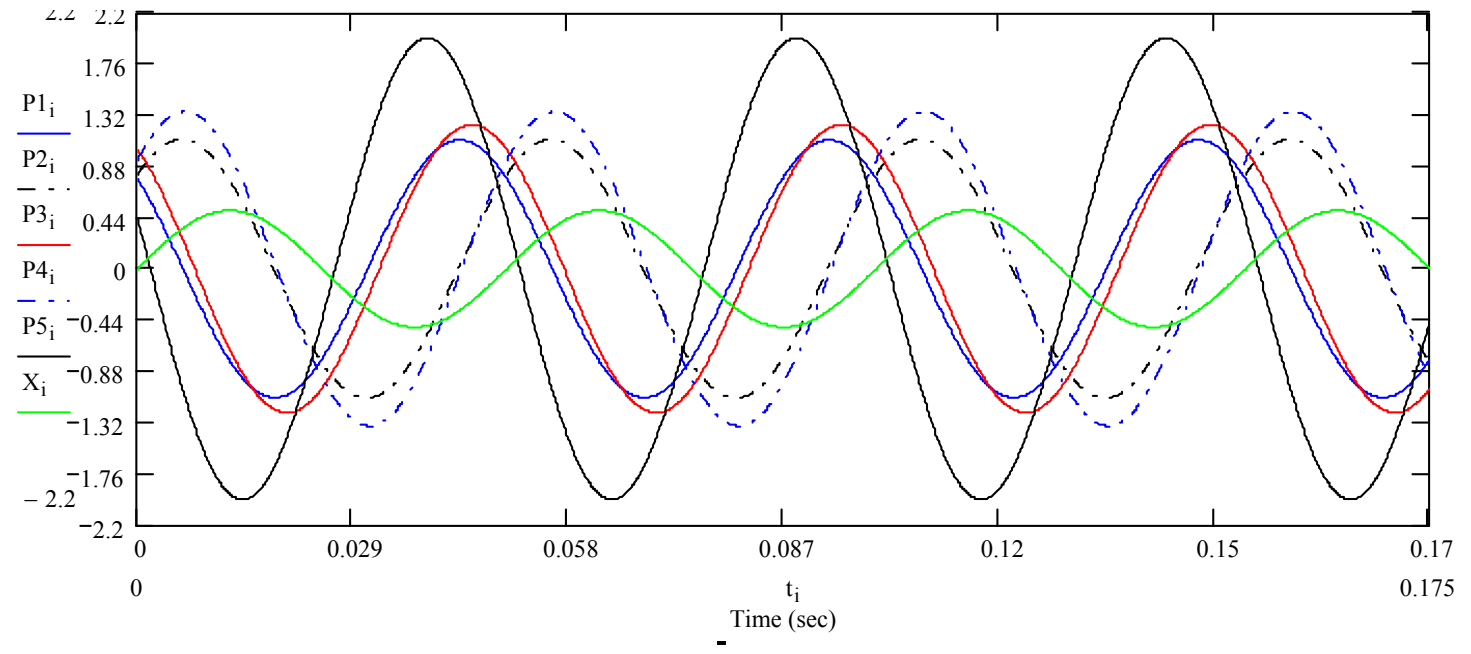

Figure 110. Dynamic Cavity Pressures-Theoretical Predications Fully Partitioned PDS 
Using the MathCad code in the Appendix, several simulations were performed. Table 16 lists the different configurations, and Figures 111-112 show the direct stiffness and direct damping. Cases 1-5 were performed for the FP design with different pitch ratios. The pitch ratio is defined as the diverging cavity pitch divided by the converging cavity pitch. Case 6 was performed for the conventional 6 bladed PDS presented in Section II. The results in Figure 111 indicate that the FP design possesses significantly more damping compared to the conventional PDS at low frequencies, but crosses over and drops below the damping levels (at $\sim 75 \mathrm{~Hz}$ ) produced by the conventional PDS design. This trend was observed in the impedance testing and also the dynamic pressure response testing, but the cross-over frequency was higher at $150 \mathrm{~Hz}$.

Table 16. Cases for Fully Partitioned PDS-Variable Pitch Ratio

\begin{tabular}{|c|c|c|c|c|}
\hline CASE & & AXIAL PITCH (IN) & PITCH RATIO & SEAL TYPE \\
\hline \multirow[t]{2}{*}{1} & DIV.CAVITY & 0.833 & \multirow[t]{2}{*}{13.33} & FULLY \\
\hline & \begin{tabular}{|l} 
CON.CAVITY \\
\end{tabular} & 0.0625 & & PARTITIONED \\
\hline \multirow[t]{2}{*}{2} & DIV.CAVITY & 0.7916 & \multirow[t]{2}{*}{6.33} & FULLY \\
\hline & CON.CAVITY & 0.125 & & PARTITIONED \\
\hline \multirow[t]{2}{*}{3} & DIV.CAVITY & 0.742 & \multirow[t]{2}{*}{3.71} & FULLY \\
\hline & CON.CAVITY & 0.2 & & PARTITIONED \\
\hline \multirow[t]{2}{*}{4} & DIV.CAVITY & 0.7 & \multirow[t]{2}{*}{2.67} & FULLY \\
\hline & CON.CAVITY & 0.2625 & & PARTITIONED \\
\hline \multirow[t]{2}{*}{5} & DIV.CAVITY & 0.625 & \multirow[t]{2}{*}{1.67} & FULLY \\
\hline & CON.CAVITY & 0.375 & & PARTITIONED \\
\hline \multirow[t]{2}{*}{6} & DIV.CAVITY & 0.742 & \multirow[t]{2}{*}{3.71} & \multirow{2}{*}{$\begin{array}{c}\text { CONVENTIONAI } \\
\text { PDS }\end{array}$} \\
\hline & INACTIVE PITCH & 0.2 & & \\
\hline
\end{tabular}

* Axial Pitch Ratio=(Diverging Cavity Axial Pitch)/(Converging Cavity Axial Pitch)

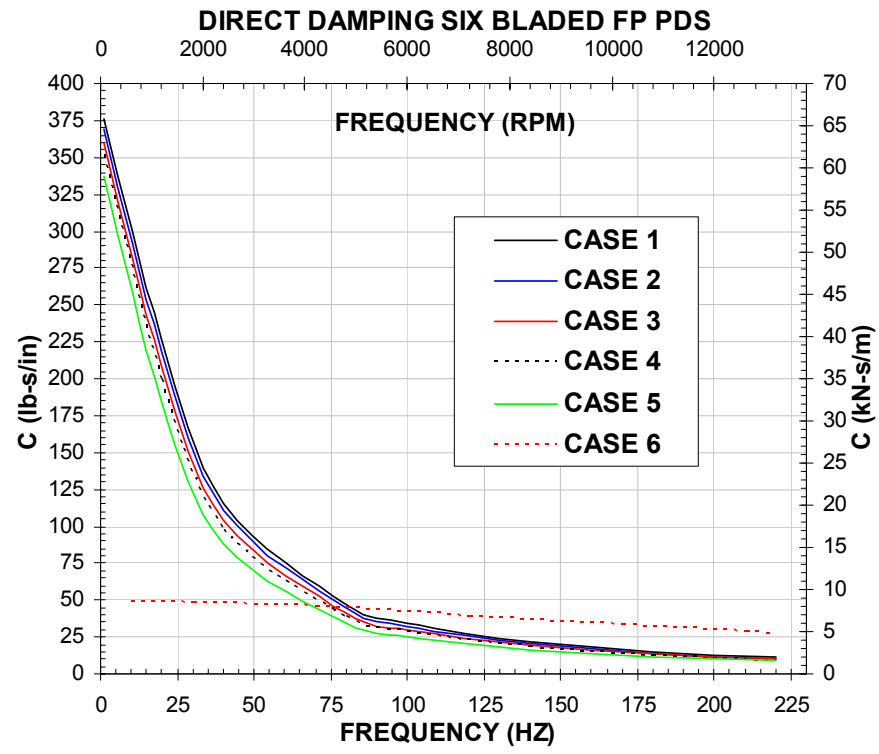

Figure 111. Direct Damping Coefficients for Varying Pitch Ratios 
The direct stiffness (Figure 112) of the FP design is comparable to the direct stiffness of the conventional design at low frequencies, but increases rapidly to more positive values and crosses over the 0 stiffness mark around $50 \mathrm{~Hz}$, resulting in a seal with significantly more positive direct stiffness at higher frequencies.

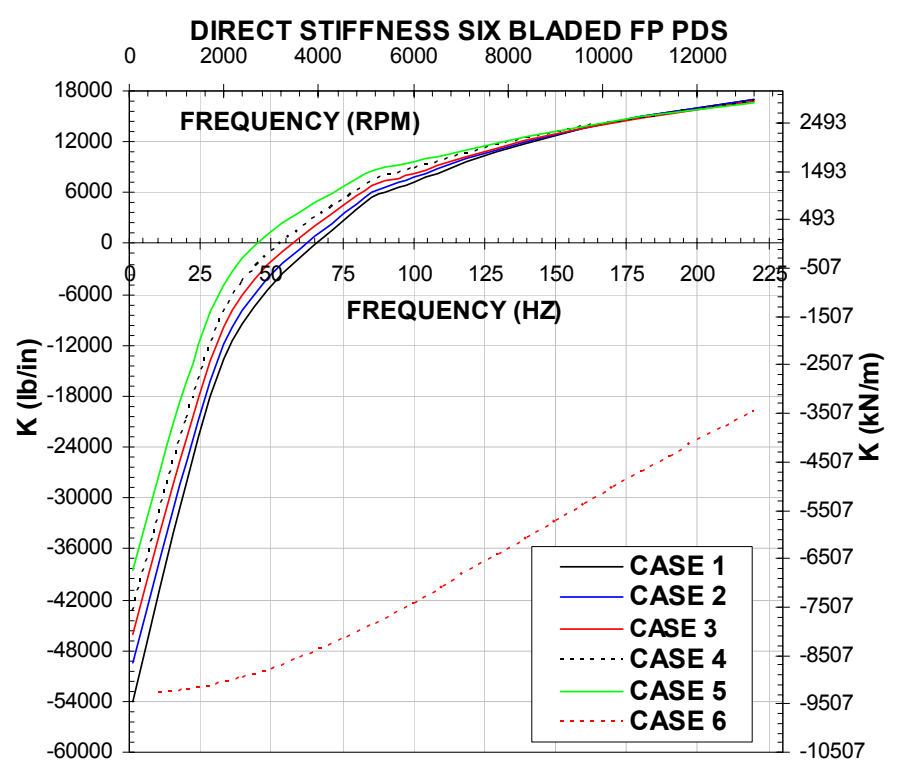

Figure 112. Direct Stiffness Coefficients for Varying Pitch Ratios 


\subsection{Theory vs. Experiment: 8 and 12 Bladed PDS}

The last section in this section focuses on how the theory compares with the experiments. The theory presented in this section only uses the continuity equations for determining force coefficients. The conventional PDS designs are compared to Shultz's theory and the fully partitioned results are compared to coefficients generated from the analysis shown in previous sections. Also, the impedance method is compared to the dynamic pressure method for the six bladed PDS. Figure 113 compares the 12 bladed 1:2 clearance ratio direct damping measured using the impedance method to the theory, which shows good agreement with the experiments. The direct stiffness (Figure 114) results however do not agree well with the theory.
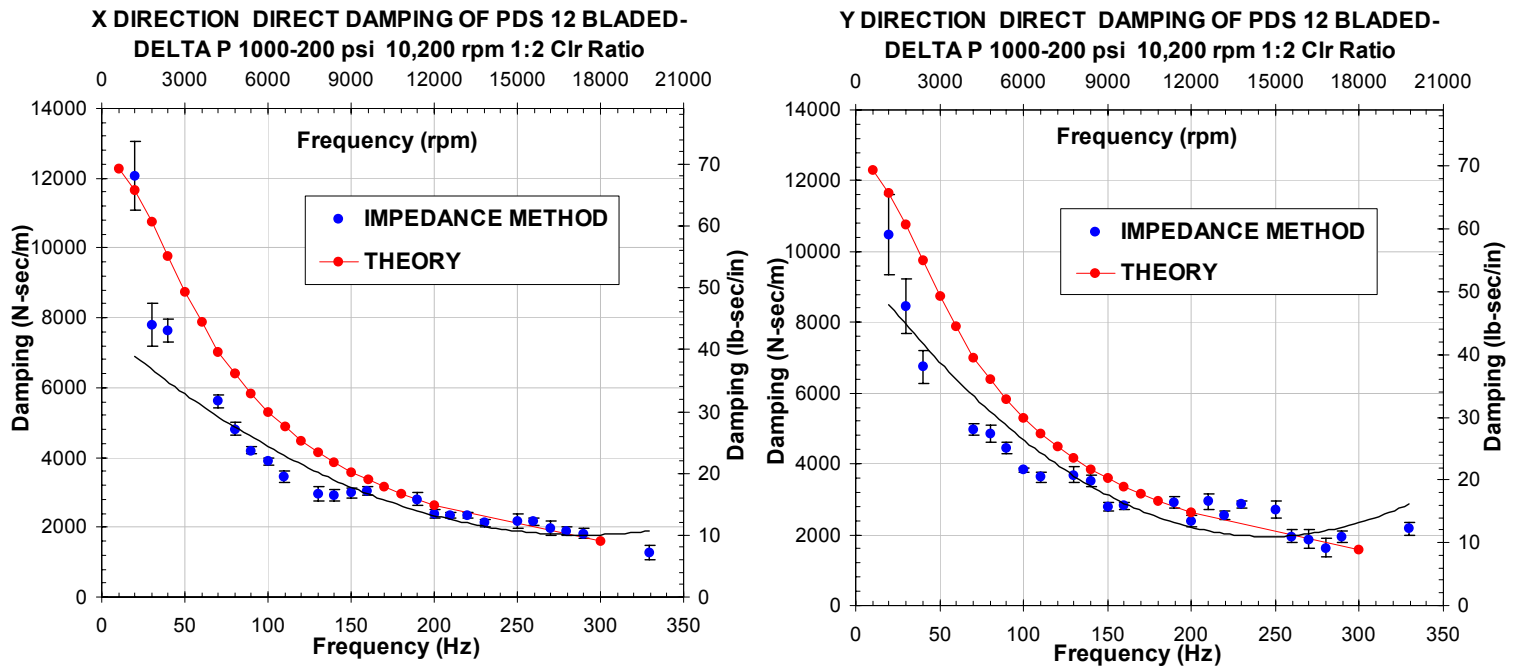

Figure 113. Theory vs. Impedance Method: 12 Bladed PDS Direct Damping
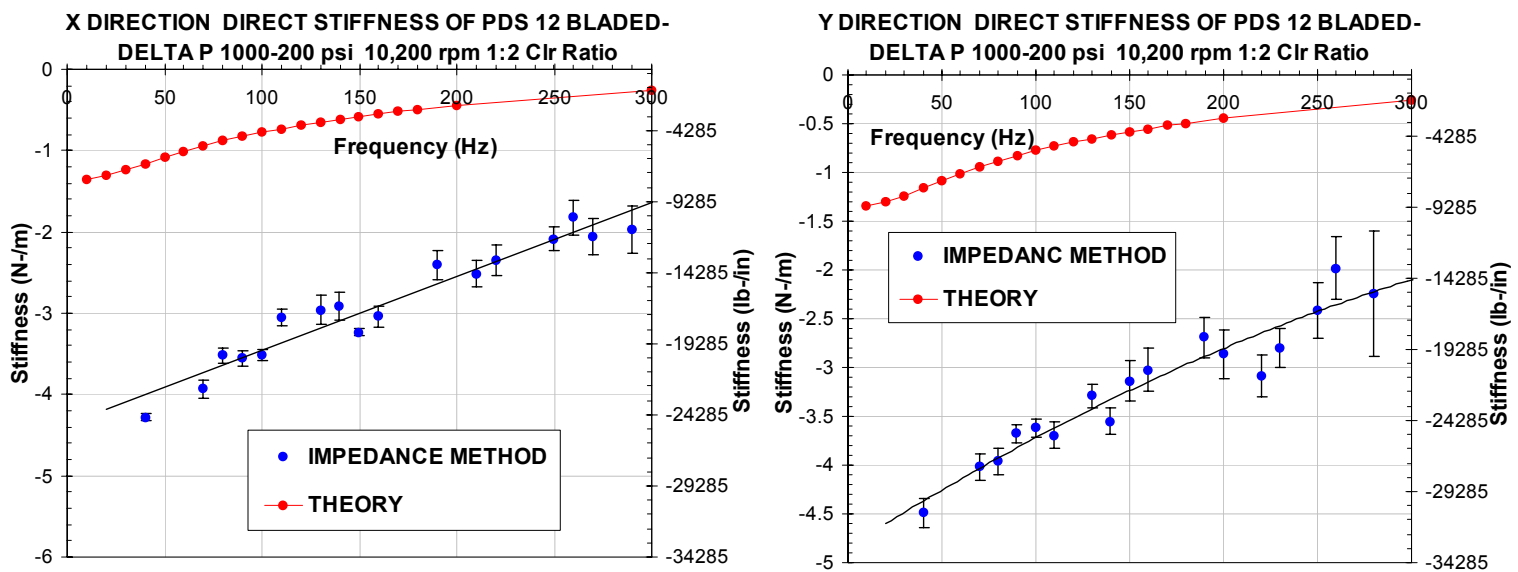

Figure 114. Theory vs. Impedance Method: 12 Bladed PDS Direct Stiffness 
The experimentally measured direct stiffness for the 12 bladed 1:2 clearance ratio PDS was measured to be 3.5 times more negative than the theory predictions. The large variation between experiments and theory could possibly be attributed to errors in the circular notches machined on the downstream blades. Notches fabricated using a ball end mill with a significant diameter can result in significant manufacturing errors associated with the notch area. The next graph compares the impedance results for the 8 bladed 1:1.5 clearance ratio PDS with the theory (Figure 115). The damping in this figure is under predicting the experimental measurements but the stiffness agrees much better compared to the 12 bladed seal case. The stiffness values are moderately close to the experimental results until $200 \mathrm{~Hz}$ where the experiment starts to deviate away from the theory.
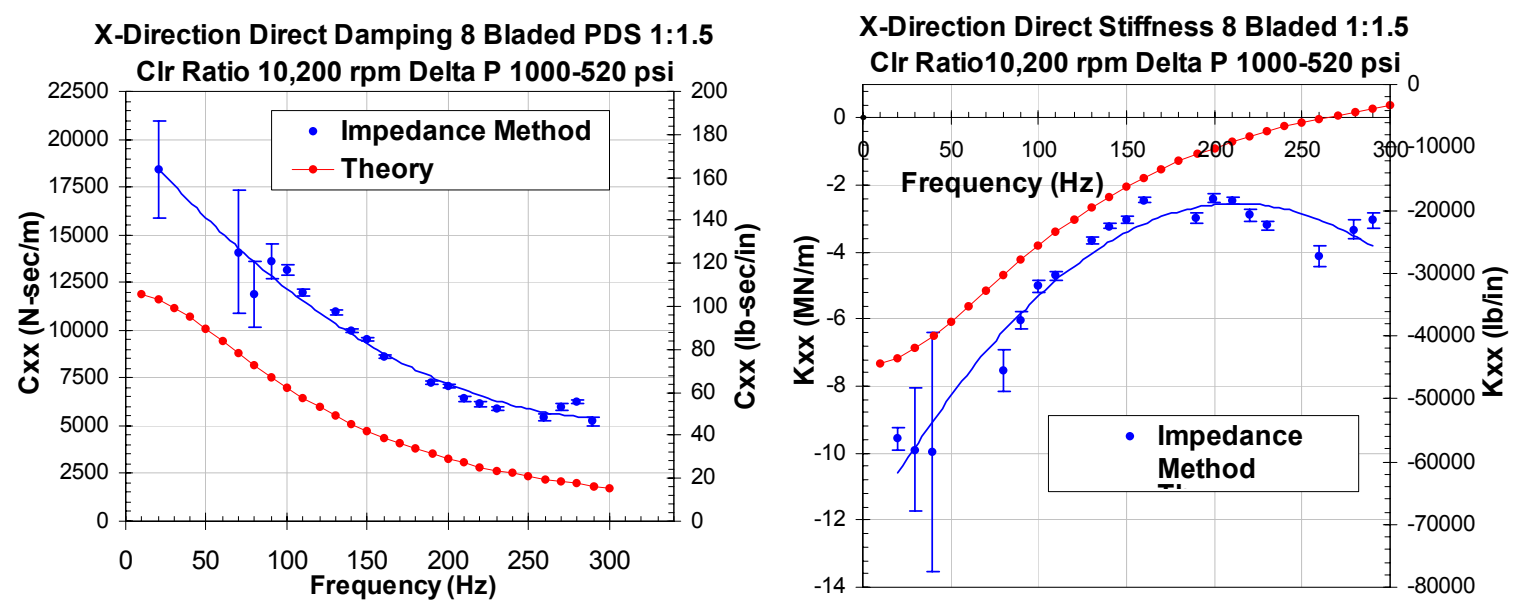

Figure 115. Theory vs. Impedance Method: 8 Bladed PDS Stiffness and Damping 


\section{FULLY PARTITIONED DAMPER SEAL VS CONVENTIONAL PDS}

Figures 116-117 display the stiffness and damping coefficients for the 6 bladed PDS configurations. In these graphs the impedance method and the dynamic pressure method are compared to the theory. Figure 116 shows excellent agreement with the theory and the dynamic pressure method. The impedance method generated coefficients that were larger than the pressure method. The direct stiffness measurements from the dynamic pressure method were more positive compared to the theory while the impedance method yielded direct stiffness coefficients that were more negative than the theory. The FP PDS results (Figure 117) show good agreement between the impedance method and the dynamic pressure method, especially the direct stiffness. The theory is under predicting the damping from $60-200 \mathrm{~Hz}$ and the theoretical stiffness crosses over to positive stiffness at $60 \mathrm{~Hz}$; whereas, the experimental results show a cross-over at $100 \mathrm{~Hz}$. The theory is indicating a stronger dependence to frequency for the stiffness and damping. Figure 118 compares the experimental results for stiffness and damping of the conventional PDS and the FP PDS. The FP PDS results in Figure 118 show good agreement between the impedance method and the dynamic pressure method, where as the results for the conventional PDS show that the impedance method has higher damping coefficients and lower valued stiffness coefficients.
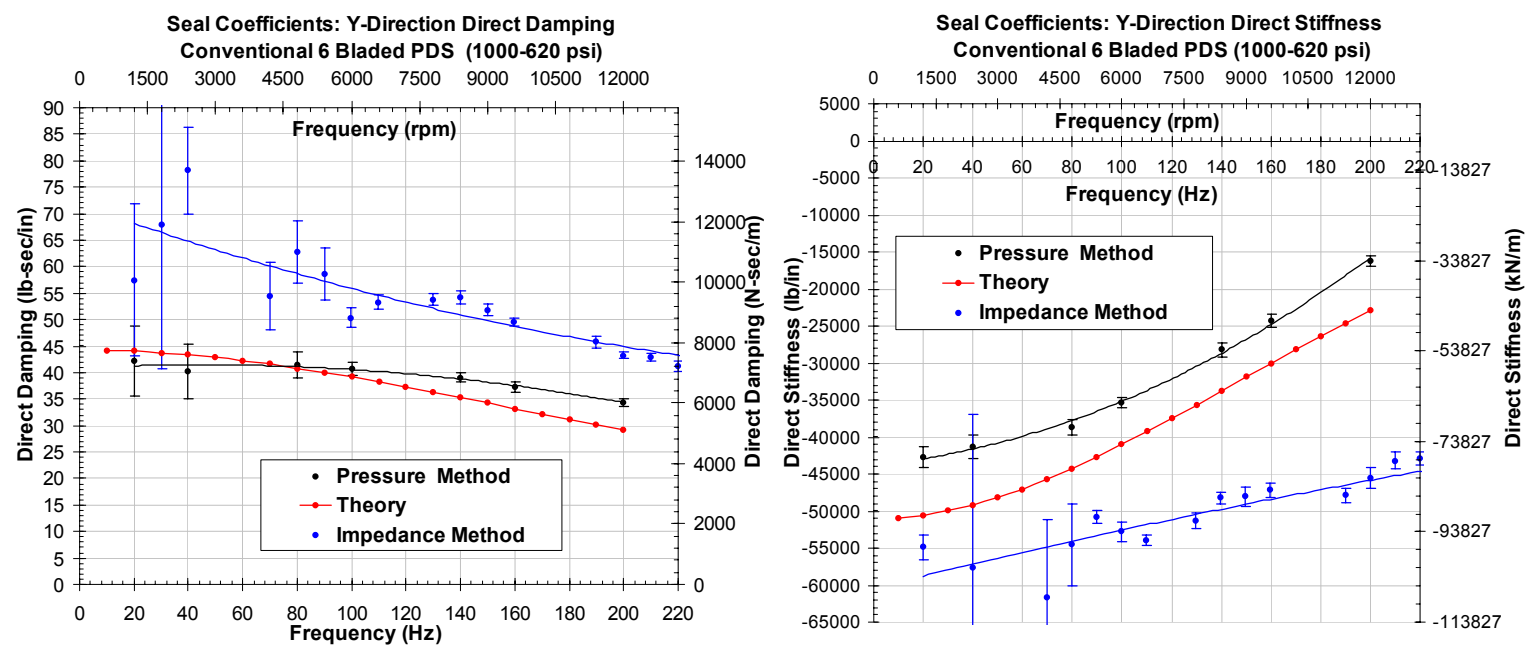

Figure 116. Theory vs. Experiments: Conventional 6 Bladed PDS Stiffness and Damping 

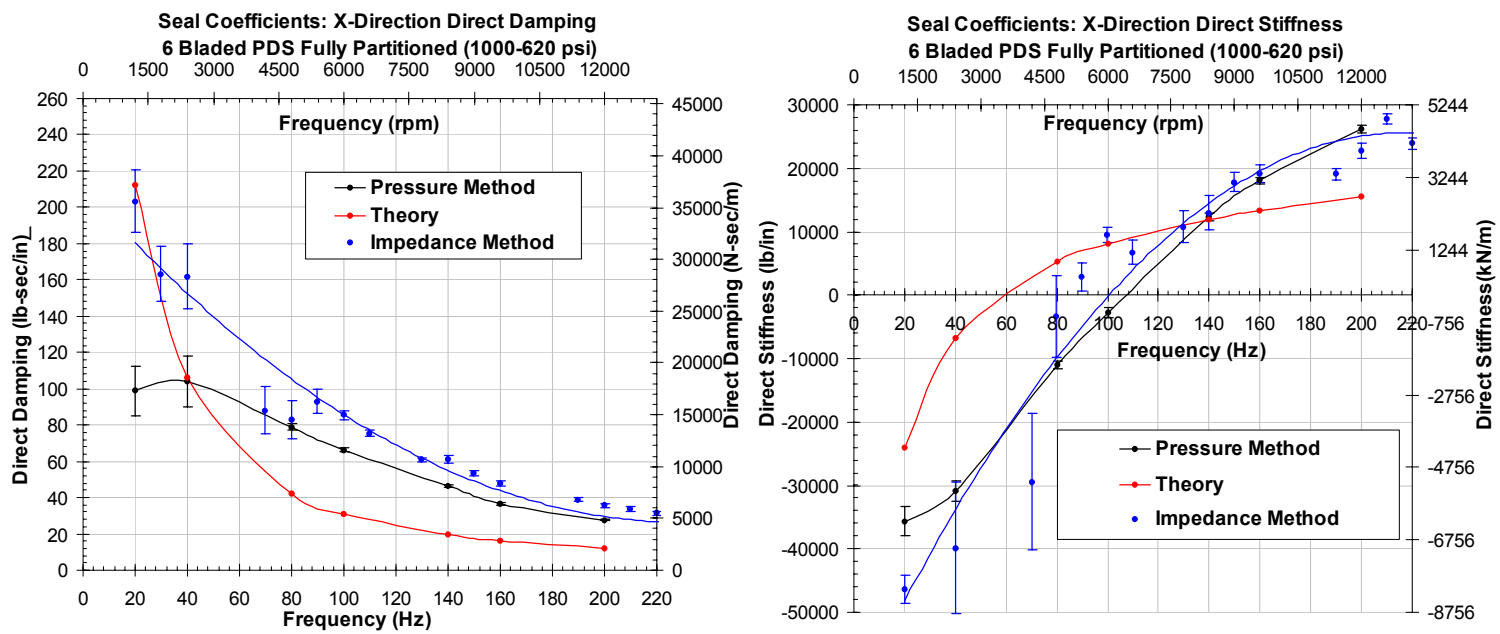

Figure 117. Theory vs. Experiments: FP 6 Bladed PDS Stiffness and Damping
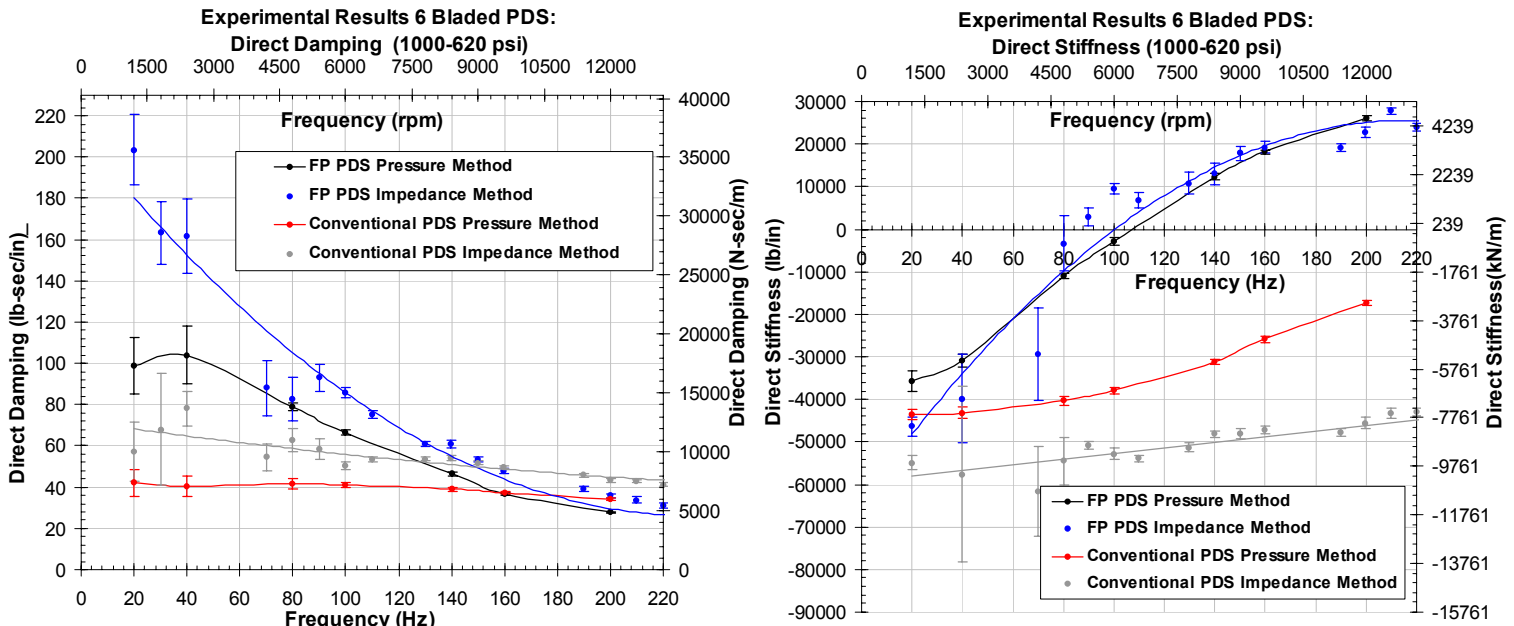

Figure 118. Six Bladed PDS Experimental Results 


\section{SEAL LEAKAGE}

The seal leakage for the 12 bladed PDS and the 8 bladed clearance ratio PDS were measured and analyzed in detail by Gamal (2003). The tests by Gamal showed that the 12 bladed PDS leaked more than the 8 bladed PDS for the same pressure ratio. This result was contradictory to the theoretical predications of seal leakage. One major difference between the 12 bladed PDS and the 8 bladed PDS was the blade profile, but concrete understanding of the results was restricted by several factors that made for an uncontrolled experiment. These factors were the difference in the clearance, the axial pitch of the cavities, and the pocket depth. The reduction in leakage for the 8 bladed PDS compared to the 12 bladed PDS could not be confidently explained by the bladed profile because of the previously mentioned factors.

The following leakage tests were conducted for three different PDS configurations: (1) conventional 6 bladed PDS with rectangular profile blades, (2) FP 6 bladed PDS with rectangular profile blades and, (3) conventional 6 bladed PDS with beveled blades as shown in Figure 12. These seals possessed the exact same clearance, cavity depth, and axial cavity pitch. The only parameters modified between the configurations were the partition walls in the inactive plenums and the blade profile of the seals. Table 17 lists the leakage results for the three configurations. The results show that the FP PDS and the conventional PDS with rectangular bladed profiles have similar leakage rates for the same pressure differential. But when comparing the beveled profile bladed seals the results show leakage values that are 1520\% higher than the conventional PDS design with the rectangular blades. Engineers often design chamfers or bevels on seal blades in anticipation of possible stator-rotor interaction during operation, which may prevent and limit damage to the rotor during rubs, but for PDS the leakage is higher for the beveled profile blades. Using the leakage results in combination with static cavity pressures, the inlet blade and exit blade discharge coefficients were determined. Figure 119 shows one example static cavity pressure measurement and the predicted pressures from the PDS theory. Table 18 contains the resultant discharge coefficients three six bladed PDS configurations. 
O Degree Position Static Pressures

6 Bladed Fully Partitioned PDS (1000-615 psi)

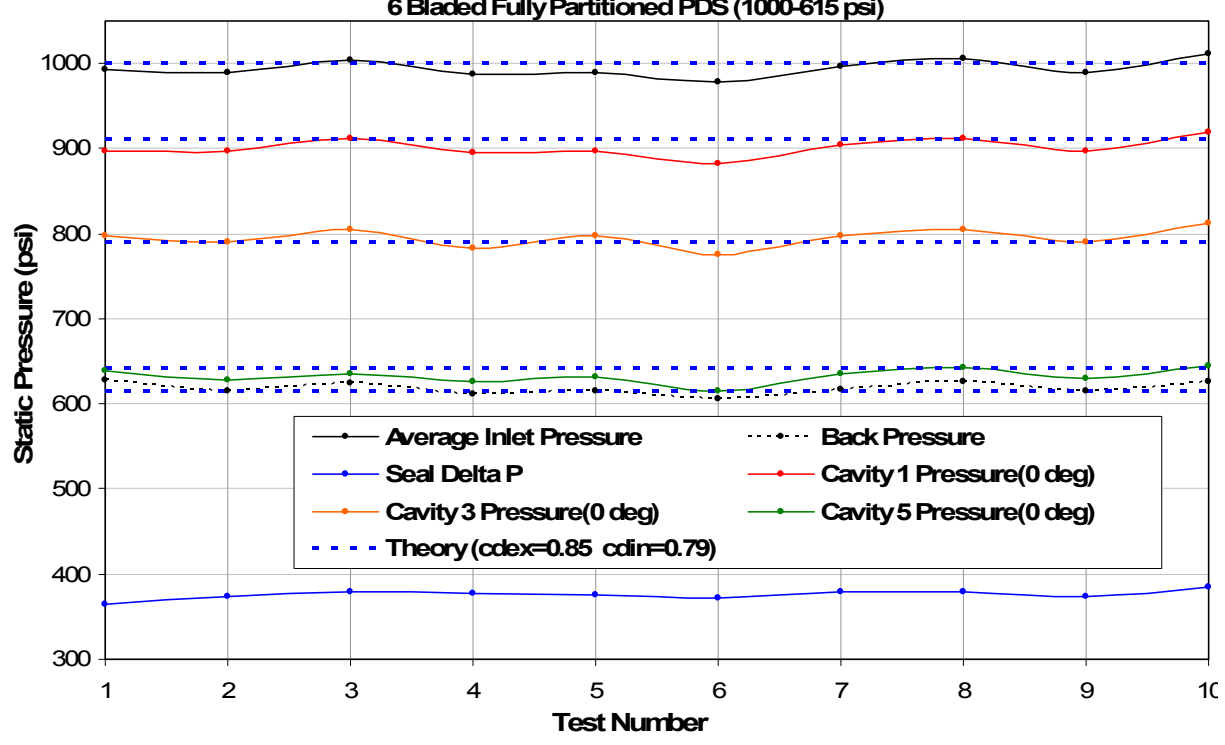

Figure 119. Static Cavity Pressure Measurements vs Theory: FP PDS 
Table 17. Leakage Results for 6 Bladed PDS Configurations

\begin{tabular}{|c|c|c|c|c|c|c|c|c|}
\hline TEST & BLADE PROFILE & SEAL DESIGN & NO. BLADES & PRESSURE $(\mathrm{psi})$ & DELTA P & TEMP (F) & FLOW RATE (kg/s) & RPM \\
\hline A & Rectangular & Conventional & 6 & $1007-603$ & 403 & 50 & 0.374 & 10,200 \\
\hline B & Rectangular & Conventional & 6 & $993-581$ & 411 & 51 & 0.359 & 15,200 \\
\hline C & Rectangular & FP & 6 & $1001-602$ & 399 & 46 & 0.362 & 10,200 \\
\hline D & Rectangular & FP & 6 & $1013-600$ & 414 & 49 & 0.348 & 15,200 \\
\hline E & Beveled & Conventional & 6 & $1005-603$ & 402 & 58 & 0.439 & 10,200 \\
\hline F & Beveled & Conventional & 6 & $998-597$ & 401 & 62 & 0.426 & 15,200 \\
\hline
\end{tabular}

Table 18. Inlet Blade and Exit Blade Discharge Coefficients

\begin{tabular}{|l|l|c|c|c|}
\hline BLADE PROFILE & PDS DESIGN & NO. BLADES & INLET Cd & EXIT Cd \\
\hline RECTANGULAR & CONVENTIONAL & 6 & 0.71 & 0.78 \\
\hline RECTANGULAR & FULLY PARTITIONED & 6 & 0.65 & 0.78 \\
\hline BEVELED & CONVENTIONAL & 6 & 0.965 & 1.2 \\
\hline
\end{tabular}




\section{CONCLUSION}

The present work has focused on determining rotordynamic coefficients and leakage characteristics for several PDS configurations. Two parameter identification methods were used to determine the frequency dependent coefficients. A new PDS design configuration was tested and analyzed using a compressible flow model. The tests conducted in the present work have successfully answered the main research objective questions listed in Section I.

The main conclusions of this research are of the following:

(1) All pocket damper seal configurations possessed positive damping over the frequency range of 20-280 Hz. The twelve bladed PDS showed the least amount of direct damping and the six bladed PDS configurations were shown to have the highest amount of direct damping.

(2) The conventional PDS with diverging clearances exhibited large negative values of direct stiffness that increased (became more positive) with increasing frequency. The straight through configurations produced negative stiffness at low frequencies and then crossed over to positive stiffness for higher frequencies.

(3) Pressure ratio was shown to have the strongest effect at low frequencies. Pocket damper seal clearance ratio was shown to have the strongest influence on coefficients, as shown with the twelve and eight bladed seal results. Rotor speed seemed to have little or no effect on cross-coupled stiffness coefficients.

(4) The cavity coefficients for the 8 bladed 1:1 clearance ratio seal did not agree well with the theory. Direct stiffness was measured to be negative for low frequencies and positive for higher frequencies. The damping was under-predicted for both cavities. The 8 bladed 1:1.5 clearance ratio seal compared much better with the conventional theory for both damping and stiffness.

(5) The inactive plenums in the conventional six bladed PDS were tested for pressure modulation and phase resolution. It was concluded that the contribution from the plenums was greatest at the highest test frequency of $200 \mathrm{~Hz}$. Although there is pressure modulation in these plenums and the pressure has repeatable phase resolution above $100 \mathrm{~Hz}$, the coefficients are small compared to the overall seal 
coefficients. The effects of the plenums are most likely amplified and become significant at frequencies above $200 \mathrm{~Hz}$.

(6) The FP PDS yielded significantly larger direct damping (1.5-2 times more) coefficients for frequencies between 20 and $100 \mathrm{~Hz}$. The direct damping of the FP PDS becomes less than the conventional PDS after $150 \mathrm{~Hz}$. The direct stiffness for the FP design was comparable to the direct stiffness of the conventional PDS at low frequencies, but increased rapidly with frequency crossing over to positive stiffness around $100 \mathrm{~Hz}$. These conclusions support the test results Li et al. (2002) reported.

(7) Both the impedance tests and the dynamic pressure response tests indicated same sign cross-coupled coefficients. The static pressure tests for the offset rotor displacements also revealed forces that imply same sign stiffness cross-coupling. It was also shown that same sign cross-coupling coefficients are not destabilizing, but impose a distortion force on the orbit making it more asymmetric.

(8) The impedance method compared well with the dynamic pressure response method as shown in Figure 117. It also matched the theory closely for the direct damping of the 12 bladed PDS. The bulk flow model utilizing only the continuity equation seems to be an adequate theory when considering the diverging 8 bladed cavity coefficients and the comparison with the dynamic pressure method as shown in Figure 116. The theory for the FP configuration is showing to be more frequency dependent and nonlinear than the experimental results.

(9) The beveled blade profile yielded higher leakage compared to the PDS with the rectangular profile blade for the same pressure differential. 


\section{REFERENCES}

Aguilar, R., 2002, "Rotordynamic Analysis of a Pocket Damper Seal Using Circular Orbits of Large Amplitudes,” Ph.D. Dissertation, Texas A\&M University, College Station.

Alford, J.S., 1965, "Protecting Turbomachinery from Self Excited Rotor Whirl," ASME Journal of Engineering for Power, Vol. 87, No. 4, pp. 333-344.

Benckert, H. and Wachter, J., 1980, "Flow Induced Spring Coefficients of Labyrinth Seals for Application in Rotordynamics," NASA CP-21333, Proceedings of a Workshop on Rotordynamic Instability Problems in High Performance Turbomachinery, Texas A\&M University, College Station, pp. 189-212.

Childs, D.W., 1993, Turbomachinery Rotordynamics, John Wiley and Sons, New York.

Childs, D.W. and Hale, K., 1994, "A Test Apparatus and Facitlity to Identify the Rotordynamic Coefficients of High Speed Hydrostatic Bearings," ASME Journal of Tribology, Vol. 116, pp.337-334.

Dawson, M., 2000, “A Comparison of the Static and Dynamic Characteristics of StraightBore and Convergent Tapered-Bore Honeycomb Annular Gas Seals,” M.S. Thesis, Texas A\&M University, College Station.

Droste, M.R., 1995, "Evaluation of Instability Forces of Labyrinth Seals in Turbines or Compressors,"NASA CP No. 2133, Proceedings of a Workshop on Rotordynamic Instability Problems in High Performance Turbomachinery, Texas A\&M University, College Station, pp. 139-167.

Ertas, B.H.. and Vance, J.M.., 2004, "Effect of Static and Dynamic Misalignment on Ball Bearing Radial Stiffness," AIA A Journal of Propulsion and Power, Vol. 20, No. 4, July-August, pp. 634-647. 
Gamal, A., 2003, “Analytical and Experimental Evaluation of the Leakage and Stiffness Characteristics of High Pressure Pocket Damper Seals," M.S. Thesis, Texas A\&M University, College Station.

Hibner, D. and Bansal, P., 1979, "Effects of Fluid Compressibility on Viscous Damper Characteristics," Proceedings of the Conference on the Stability and Dynamic Response of Rotors with Squeeze Film Bearings, University of Virginia, pp. 348-355

Kaneko, S., Takashi, I., Takuro, S., and Shin, I., 2003, “Dynamic Characteristics of Liquid Annular Convergent-Tapered Damper Seals with Honeycomb Roughness Pattern," ASME Transactions of the ASME, Vol. 125, July, pp. 592-599.

Kwanka , K., Ortinger, W., and Steckel, J., 1993, "Calculation and Measurement of the Influence of Flow Parameters on Rotordynamic Coefficients in Labyrinth Seals," Proceedings of a Workshop on Rotordynamic Instability Problems in High Performance Turbomachinery, Texas A\&M University, College Station, pp. 121-129.

Laos, H. E., 1999, "Rotordynamic Effect of Pocket Damper Seals," Ph.D. Dissertation, Texas A\&M University, College Station.

Li, J., and Vance, J.M., 1995, "Effects of Clearance and Clearance Ratio on Two and Three Bladed TAMSEALS, TRC-Seal-4-95", Turbomachinery Laboratory Research Progress Report, Texas A\&M University, College Station.

Li, J., Aguilar, R., San Andrés, L., and Vance, J. M., 2000, "Dynamic Force Coefficients of a Multiple Blade, Multiple-Pocket Gas Damper Seal: Test Results and Predictions," ASME Journal of Tribology, Vol. 122, pp. 317-322.

Li, J., Kushner, F., and DeChoudhury, P., 2002,” Experimental Evaluation of Slotted Pocket Damper Seals on a Rotating Test Rig," The $47^{\text {th }}$ ASME Turbo Expo Land Sea \& Air, pp 230240. 
Li, J., San Andrés, L., and Vance, J. M., 1998, “A Bulk Flow model Analysis of MultiplePocket Gas Damper seals," ASME Paper 98-GT-13.

Lucas, M.J., Noreen, R.A., Sutherland, L.C., Cole, J.E., and Junger, M.C., 1997, Handbook of the Characteristics of Turbomachinery Cavities, The American Society of Mechanical Engineers, New York.

Millsaps , K. and Sanchez, M., 1993, "Rotordynamic Forces in Labyrinth Seals: Theory and Experiment," Proceedings of a Workshop on Rotordynamic Instability Problems in High Performance Turbomachinery, Texas A\&M University, College Station.

Murphy, B. T., 1984, “Eigenvalues of Rotating Machinery,” Ph.D. Dissertation, Texas A\&M University, College Station.

Picardo, A., 2003, "High Pressure Testing of See-Through Labyrinth Seals," M.S. Thesis, Texas A\&M University, College Station.

Ransom, D., Li, J., San Andrés, L., and Vance, J. M., 1998, “Experimental Force Coefficients for a Two-Bladed Labyrinth Seal and a Four Pocket Damper Seal," ASME Journal of Tribology, Vol. 28, pp. 98-109.

San Andres, L. A., 1985, "Effect of Fluid Inertia on Squeeze Film Damper Force Response," Ph.D. Dissertation, Texas A\&M University, College Station.

Sundararajan, P., and Vance, J. M., 1993a, "A Theoretical and Experimental Investigation of a Gas Operated Bearing Damper for Turbomachinery - Part I: Theoretical Model and Predictions," Proceedings of the $14^{\text {th }}$ Vibration and Noise Conference, Albuquerque, NM, ASME DE-Vol. 60, pp. 67-83.

Sundararajan, P., and Vance, J. M., 1993b, “A Theoretical and Experimental Investigation of a Gas Operated Bearing Damper for Turbomachinery - Part II: Experimental Results and 
Comparison with Theory," Proceedings of the $14^{\text {th }}$ Vibration and Noise Conference, Albuquerque, NM, ASME DE-Vol. 60, pp. 85-119.

Vance, J. M., Cardon, B. P., San Andrés, L., and Storace, A. F., 1993, "A Gas Operated Bearing Damper for Turbomachinery," ASME Journal of Engineering for Gas Turbines and Power, Vol. 115, pp. 383-389.

Vance, J. M. and Kirton, A. J., 1975, "Experimental Measurement of the Dynamic Force Response of A Squeeze-Film Damper," Journal of Engineering for Industry, November, pp. $1282-1290$.

Vance, J.M., Sharma , A, and Jayakar, N., 2002, "Effect of Frequency and Design Parameters on Pocket Damper Seal Performance," Proceedings of Fluid Structure Interactions, New Orleans, LA, No. 23, pp. 208-217.

Vance, J. M., and Shultz, R. R., 1993, “A New Damper Seal for Turbomachinery,” Proceedings of the $14^{\text {th }}$ Vibration and Noise Conference, Albuquerque, NM, ASME DE-Vol. 60, pp. 139-148.

Zeidan, F., 1989, "Cavitation Effects on the Rotordynamic Performance of SFD Bearings," Ph.D. Dissertation, Texas A\&M University, College Station. 
APPENDIX A

\section{LABVIEW PROGRAMS}




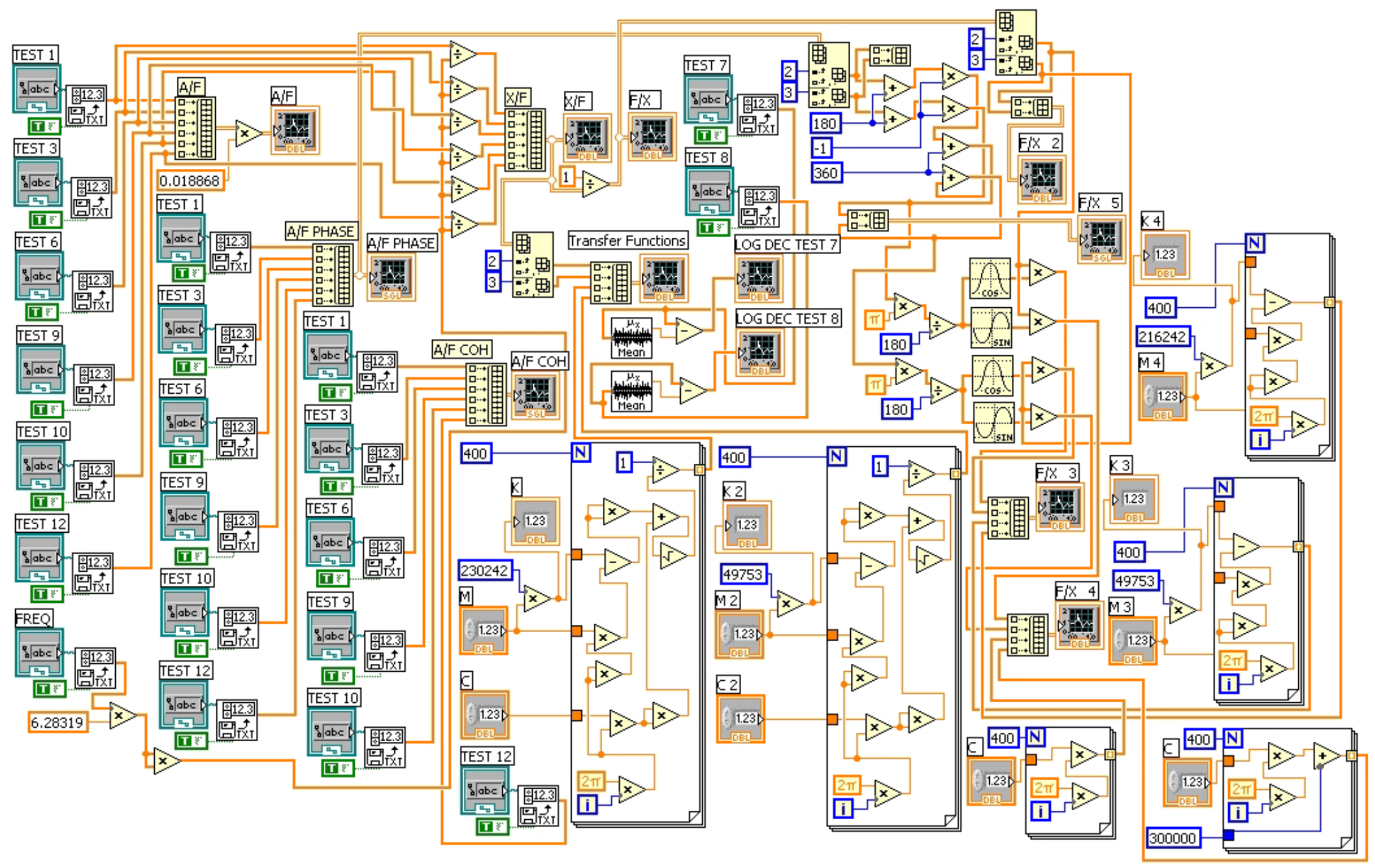

Curve Fitting Program for Modal Vibration Tests 


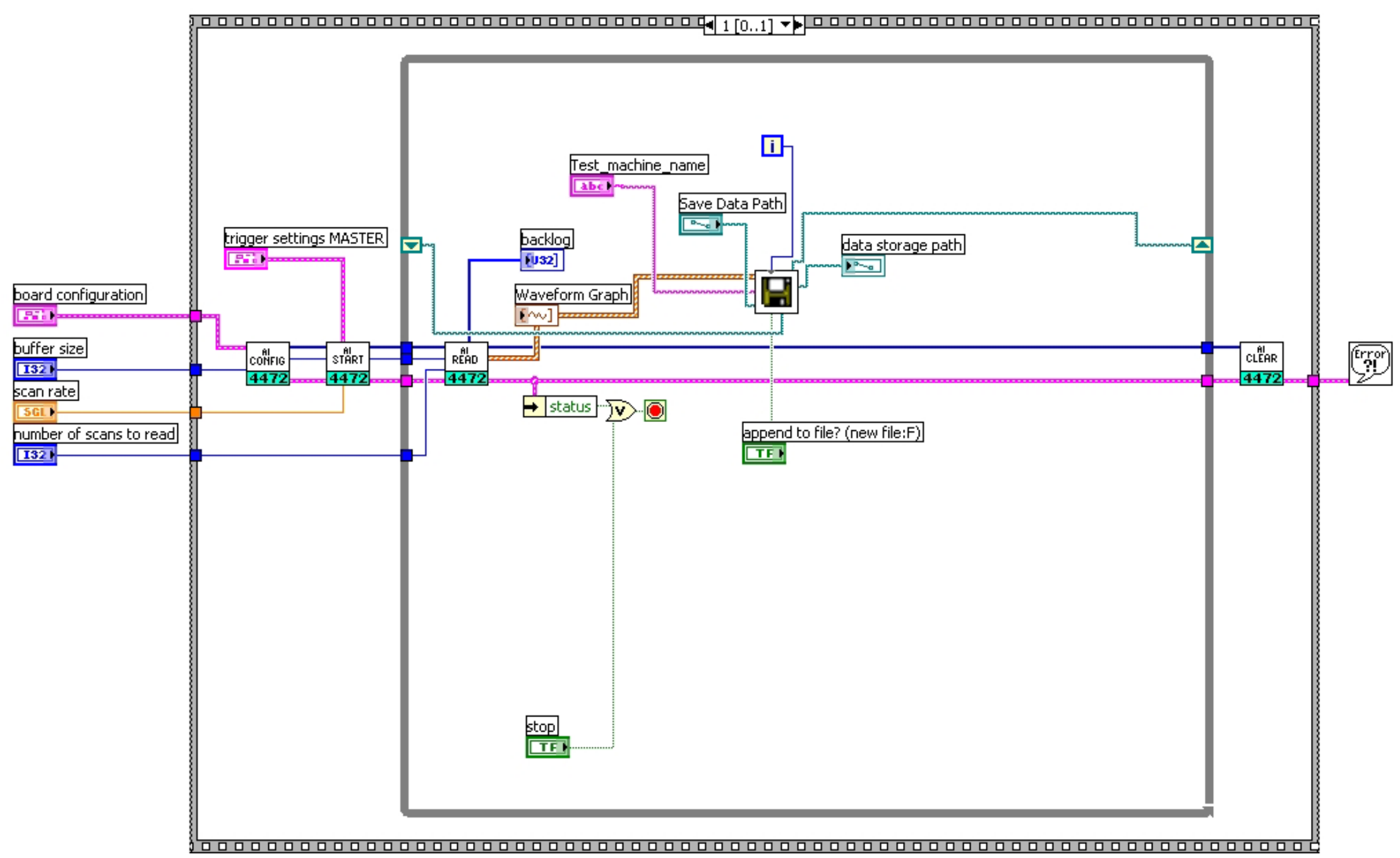

Data Acquisitions Block Diagram NI 4472 With Trigger 


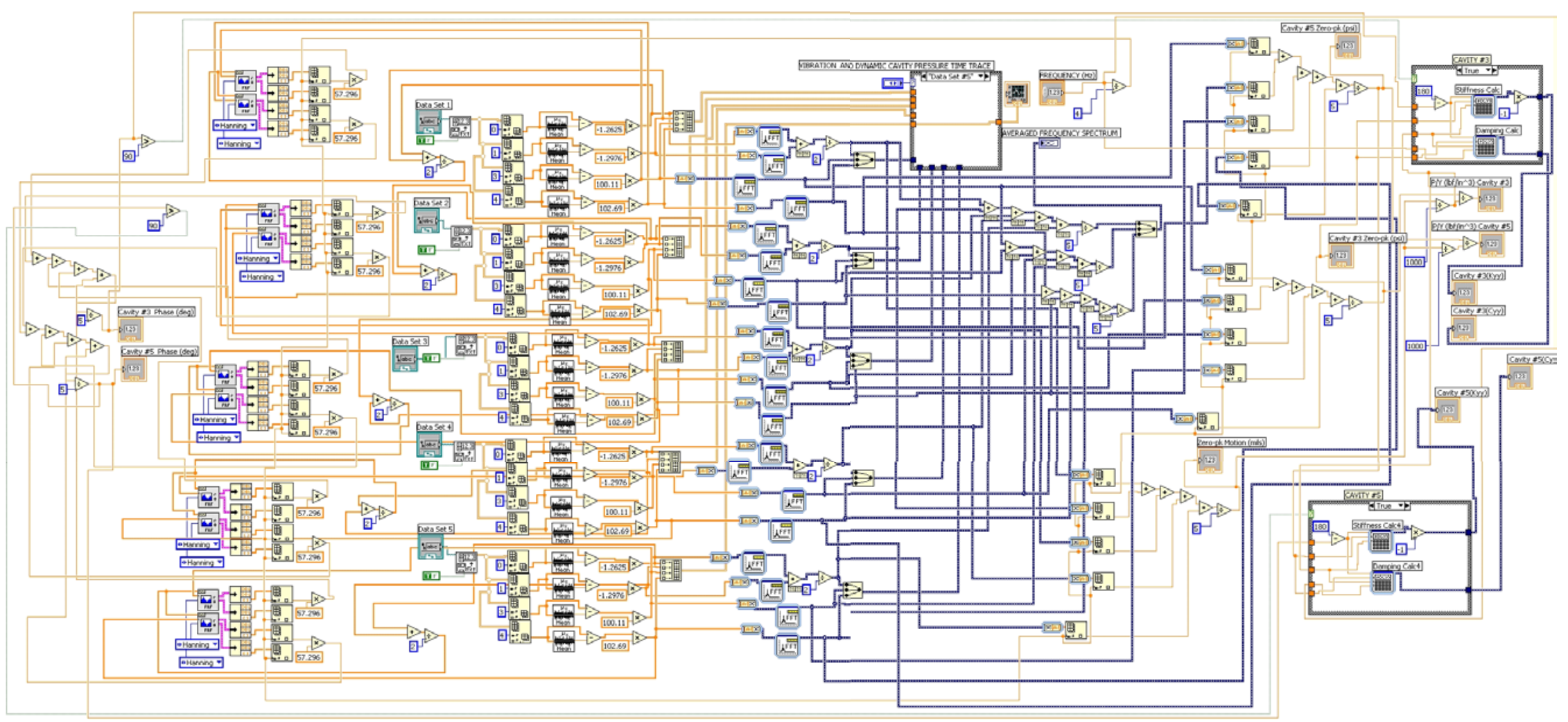

8 Bladed PDS Cavity Coefficients Block Diagram- Data Reduction 


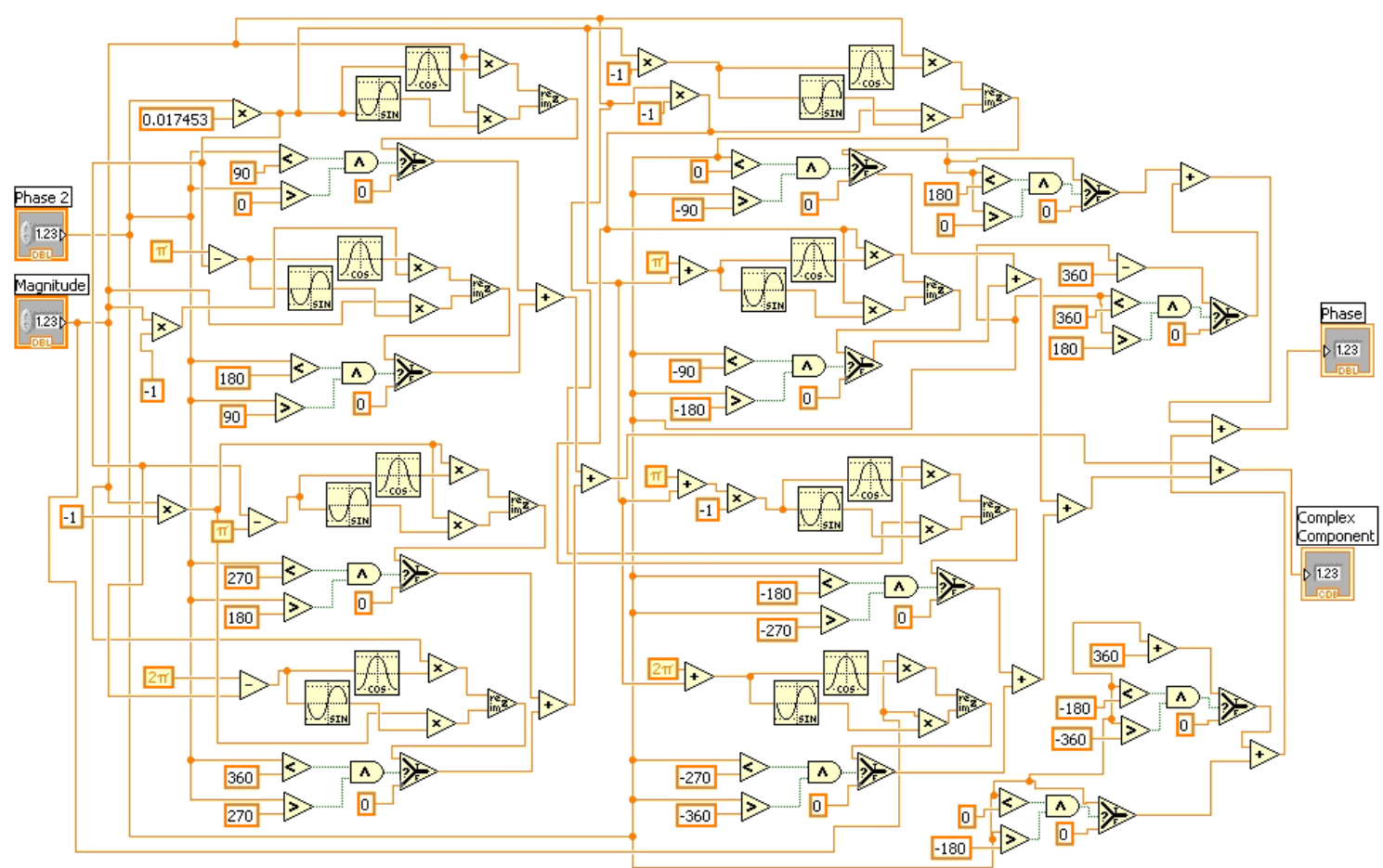

Phase Conversion and Complex Number Generation

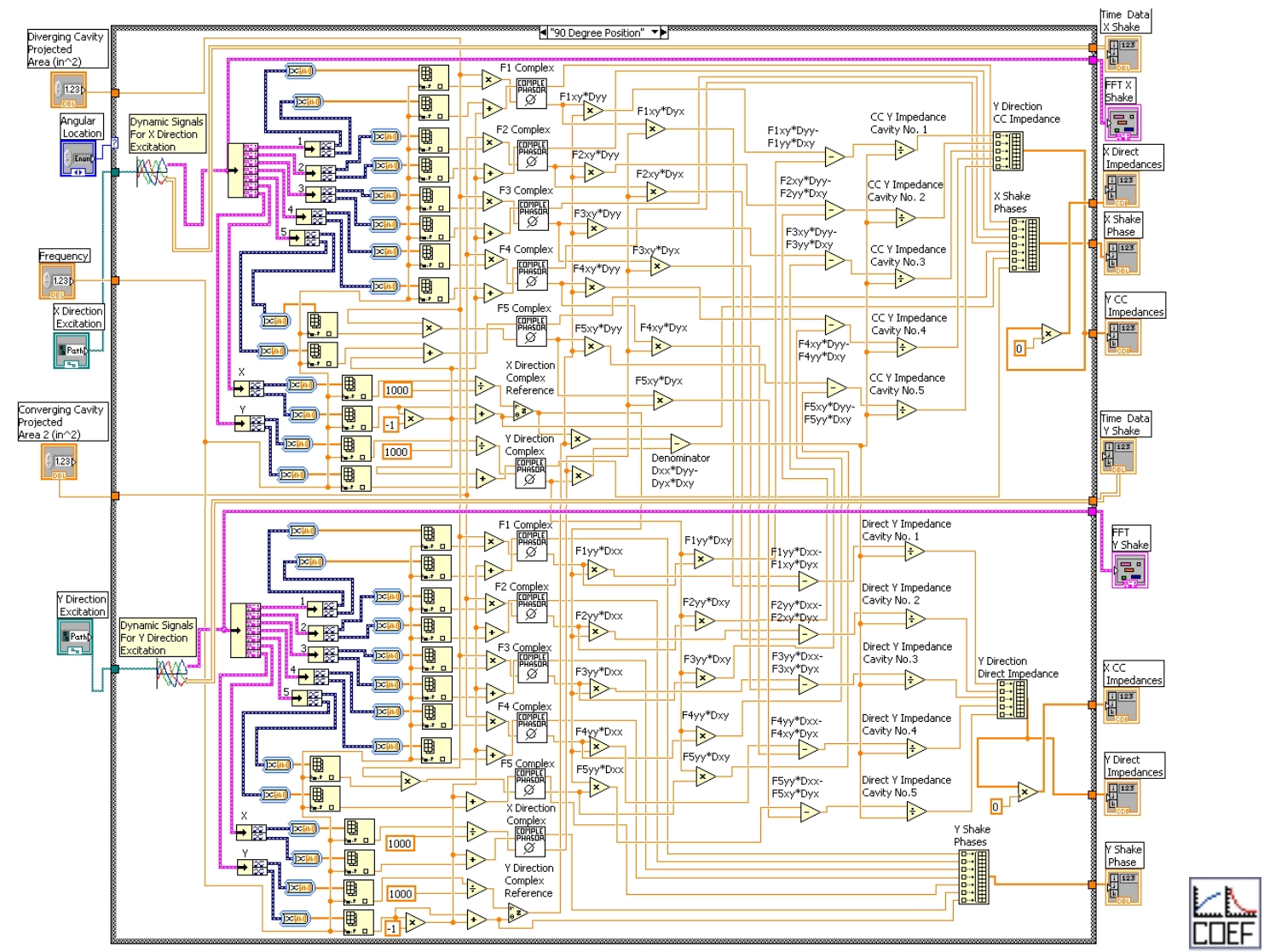

Direct and Cross-Coupled Impedance Calculations (90, 270 Degree position) 

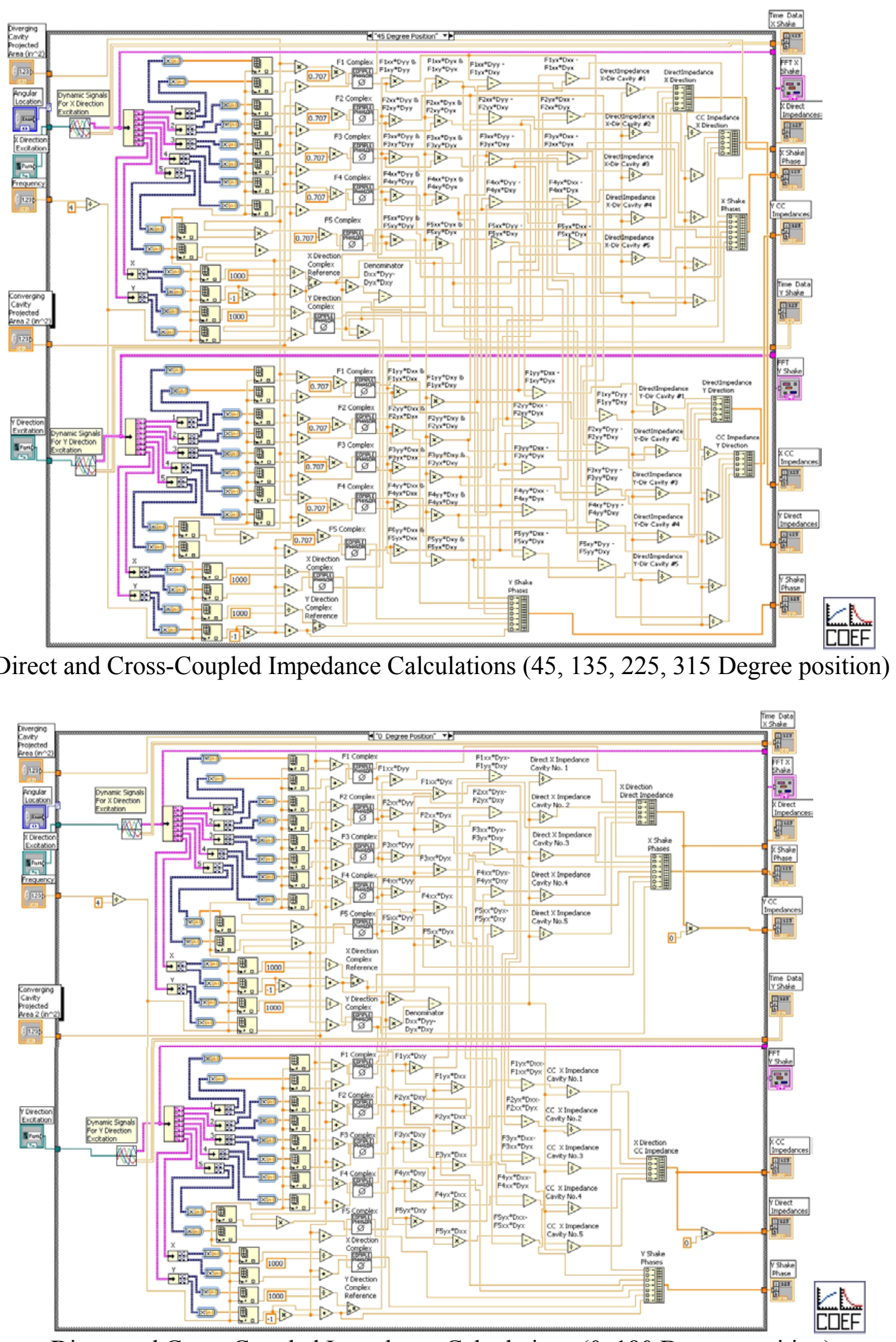

Direct and Cross-Coupled Impedance Calculations (0, 180 Degree position) 


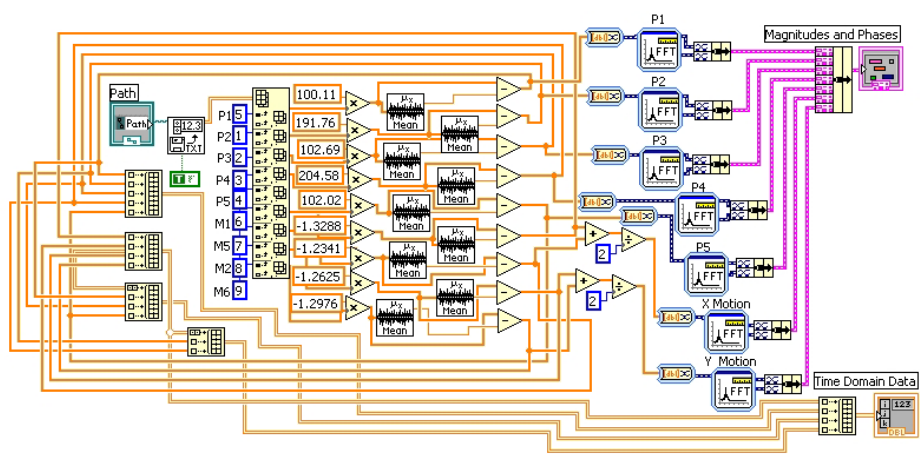

Conversion of Time Data to FFT Magnitudes and Phases (0, 45, 90 degree)

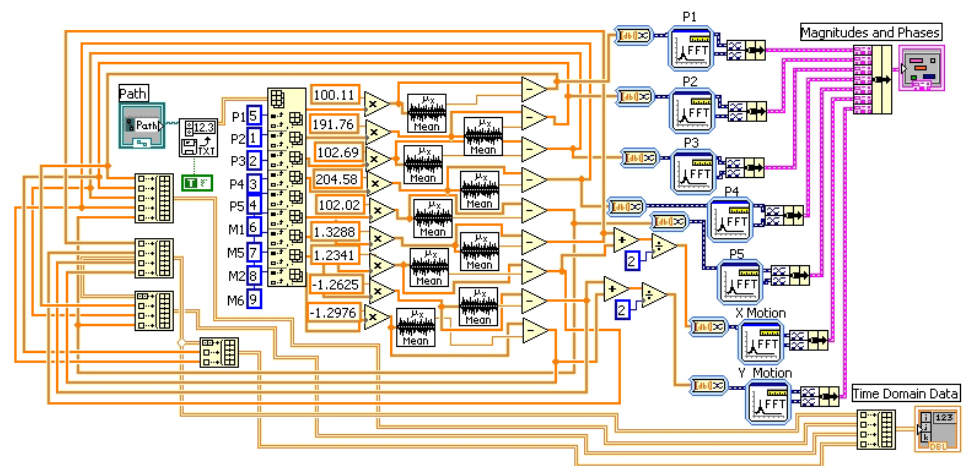

Conversion of Time Data to FFT Magnitudes and Phases (135 degree)

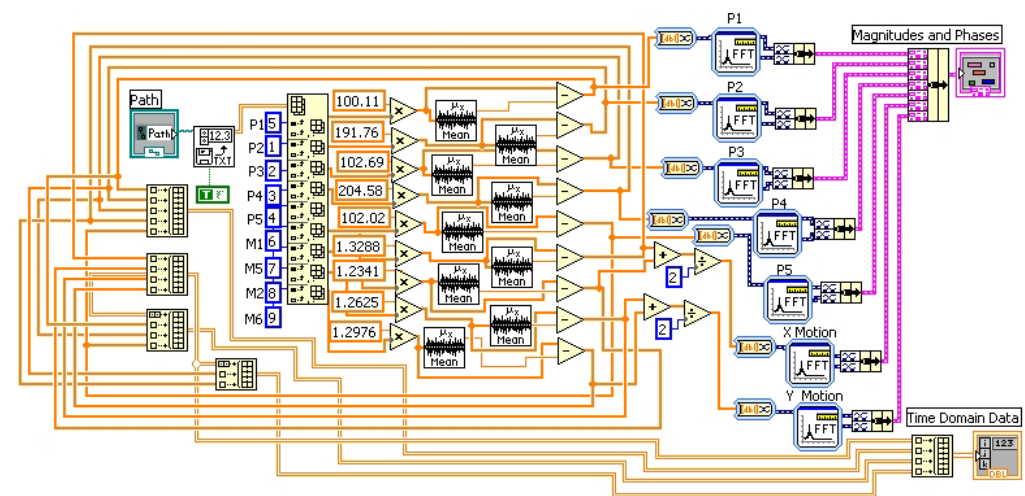

Conversion of Time Data to FFT Magnitudes and Phases (180, 225, 270 degree)

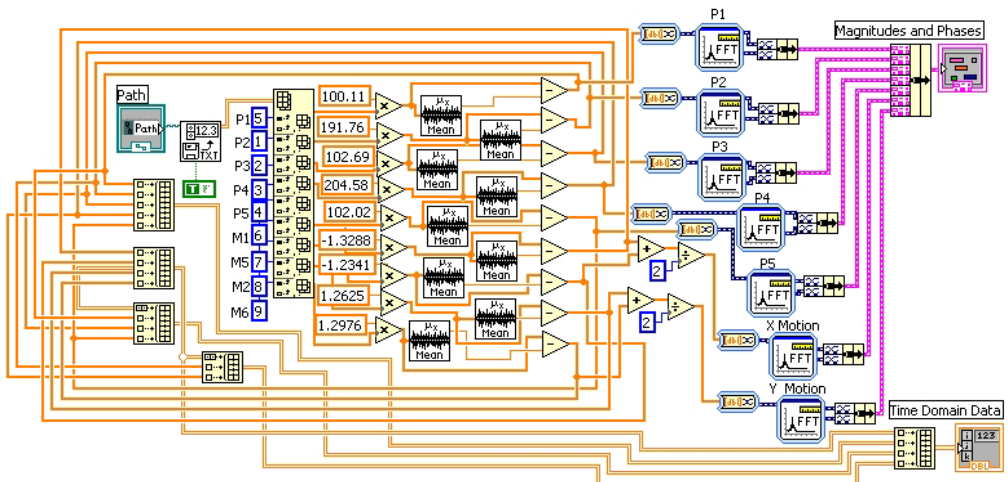

Conversion of Time Data to FFT Magnitudes and Phases (315 degree) 
APPENDIX B

MATHCAD PROGRAMS 


\section{PDS THEORY FOR A SINGLE POCKET CAVITY (1:1.5 cIr ratio) CAVITY \#3}

\section{INPUT :}

Inlet Pressure (psi) $\quad \mathrm{P0}:=884.1$

Cavity Pressure (psi) $\quad$ P1 $:=808$

Exit Pressure (psi) $\quad$ P2 $:=780.85$

Rotor Diameter (in) $\quad \mathrm{D}:=4.5$

Ratio of Specific Heats $\quad y:=1.4$

Gas Constant $\mathrm{R}:=247029.517$

Temperature ( $R$ ) $\mathrm{T}:=535$

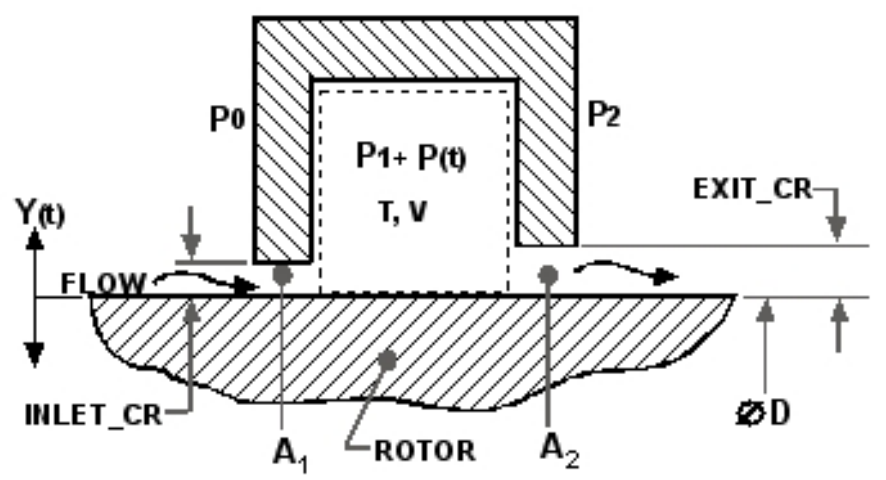

$\gamma \cdot \mathrm{T} \cdot \mathrm{R}=1.85 \times 10^{8} \quad$ Vibration Amplitude (in) $\mathrm{y}:=0.00094$

Inlet Blade Radial Clearance (in) inlet_cr: $: 0.005$

Exit Blade Radial Clearance (in) exit_cr $:=0.0075$

Rotor Frequency ( $\mathrm{rpm}) \quad$ Rotor_Freq $:=12000$

$A 1:=\frac{(\pi \cdot D \cdot \text { inlet_cr })}{8} \quad A 2:=\frac{\left(\pi \cdot D \cdot e^{2 x i t}+c r\right)}{8} \quad$ Pocket Volume $\left(i^{n} 3\right) \quad$ PV $:=0.9281$

A1 $=8.836 \times 10^{-3}$ in $^{2} \quad$ A2 $=0.013 \quad$ in $^{2} \quad$ Pressure Area $\left(i n^{n} 2\right) \quad$ Area_P $:=0.7176$

MASS FLOW THROUGH INLET BLADE:

$\mathrm{m} 01:=0.75 \mathrm{P} 0 \cdot \frac{\mathrm{A} 1 \cdot\left[2 \cdot \frac{\gamma^{2}}{(\gamma-1)}\right]^{5} \cdot\left[\left(\frac{\mathrm{P} 1}{\mathrm{P} 0}\right)^{\frac{2}{\gamma}}-\left(\frac{\mathrm{P} 1}{\mathrm{P} 0}\right)^{\frac{(\gamma+1)}{\gamma}}\right]^{.5}}{(\gamma \cdot \mathrm{R} \cdot \mathrm{T})^{5}} \quad \mathrm{~m} 01=2.015 \times 10^{-4}$

MASS FLOW THROUGH EXIT BLADE:

$\mathrm{m} 12:=85 \mathrm{P} 1 \cdot \frac{\mathrm{A} 2 \cdot\left[2 \cdot \frac{\gamma^{2}}{(\gamma-1)}\right]^{5} \cdot\left[\left(\frac{\mathrm{P} 2}{\mathrm{P} 1}\right)^{\frac{2}{\gamma}}-\left(\frac{\mathrm{P} 2}{\mathrm{P} 1}\right)^{\frac{(\gamma+1)}{\gamma}}\right]^{.5}}{(\gamma \cdot \mathrm{R} \cdot \mathrm{T})^{5}} \quad \begin{aligned} & \text { TOTAL MASS FLOW : } \\ & \mathrm{mtot}:=\mathrm{m} 01.8 \cdot 386.4 \quad \text { Mtot }=0.623 \frac{\mathrm{lb}}{\mathrm{sec}}\end{aligned}$ 
PARTIAL DERIVATIVE OF m01 RESPECT TO P1

$\mathrm{T} 1:=\frac{\mathrm{m} 01 \cdot\left[2 \cdot\left(\frac{\mathrm{P} 1}{\mathrm{P} 0}\right)^{\frac{(2-\gamma)}{\gamma}}-(\gamma+1) \cdot\left(\frac{\mathrm{P} 1}{\mathrm{P} 0}\right)^{\frac{1}{\gamma}}\right]}{\mathrm{P} 0 \cdot 2 \cdot \gamma \cdot\left[\left(\frac{\mathrm{P} 1}{\mathrm{P} 0}\right)^{\frac{2}{\gamma}}-\left(\frac{\mathrm{P} 1}{\mathrm{P} 0}\right)^{\frac{(\gamma+1)}{\gamma}}\right]}$
PARTIAL DERIVATIVE OF m12 RESPECT TO P1

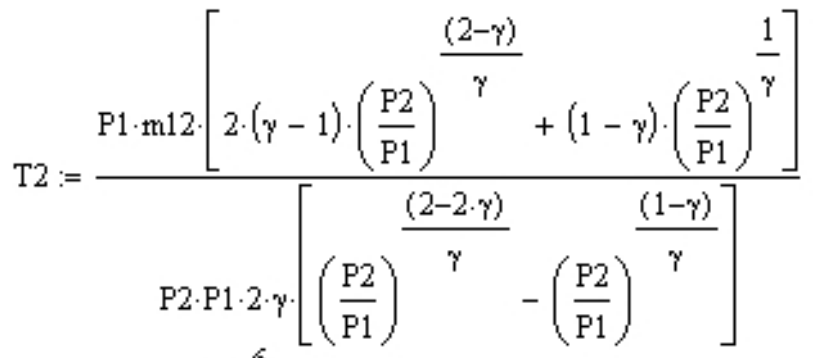

$\mathrm{T} 2=3.702 \times 10^{-6}$

\section{Defining Constants:}

$\mathrm{C} 1:=$ Area_P $\frac{\mathrm{P} 2}{\mathrm{R} \cdot \mathrm{T}} \quad \mathrm{C} 2:=\frac{\mathrm{PV}}{\gamma \cdot \mathrm{R} \cdot \mathrm{T}} \quad \mathrm{C} 3:=(\mathrm{T} 1-\mathrm{T} 2) \mathrm{C} 4:=\left(\frac{1}{\text { inlet_cr }}-\frac{1}{\text { exit_cr }}\right) \cdot \mathrm{m01} \omega:=$ Rotor_Freq $2 \cdot \frac{\pi}{60}$

Damping (lb-sec/in) $\mathrm{Cyy}:=(\mathrm{C} 2 \cdot \mathrm{C} 4-\mathrm{C} 3 \cdot \mathrm{C} 1) \cdot \frac{\text { Area_P }}{\left(\mathrm{C}^{2}+\mathrm{C}^{2} \cdot \omega^{2}\right)} \quad \mathrm{Cyy}=0.993$

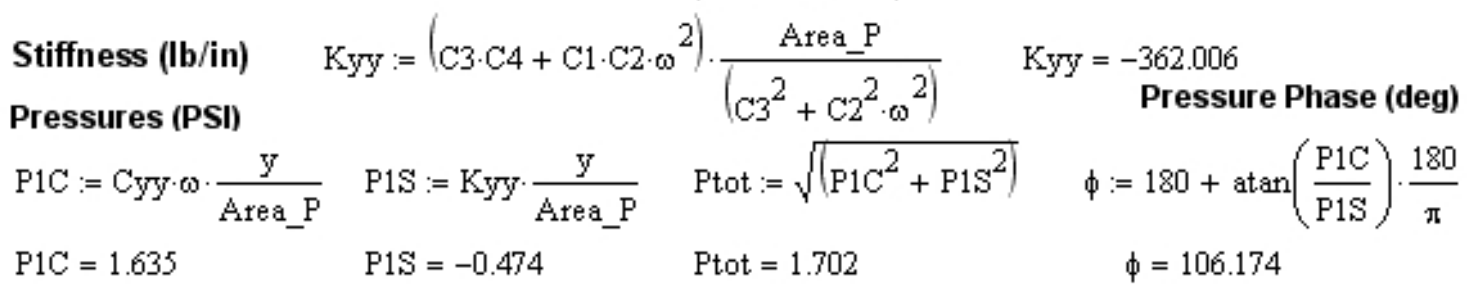

Cavity Pressure (psi)/ Vibration (mils) Pressure Impedance Function (Ibf/in^3)

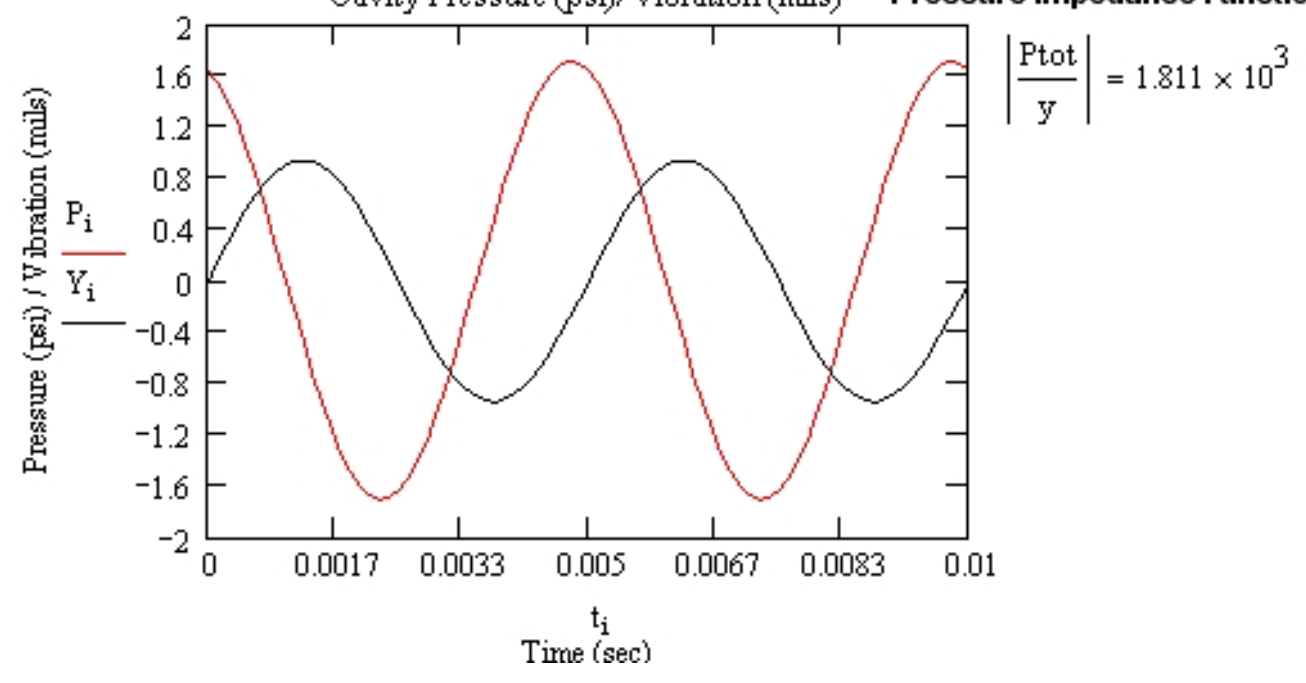




\section{PDS THEORY FOR A SINGLE POCKET CAVITY (1:1.5 clr ratio) CAVITY \#5}

INPUT :

Inlet Pressure (psi) $\quad \mathrm{P} 0:=773.3$

Cavity Pressure (psi) P1 $:=683$

Exit Pressure (psi) P2 $:=650.38$

Rotor Diameter (in) $\quad \mathrm{D}:=4.5$

Ratio of Specific Heats $\quad y:=1.4$

Gas Constant

$\mathrm{R}:=247029.517$

Temperature ( $R$ )

$\mathrm{T}:=535$

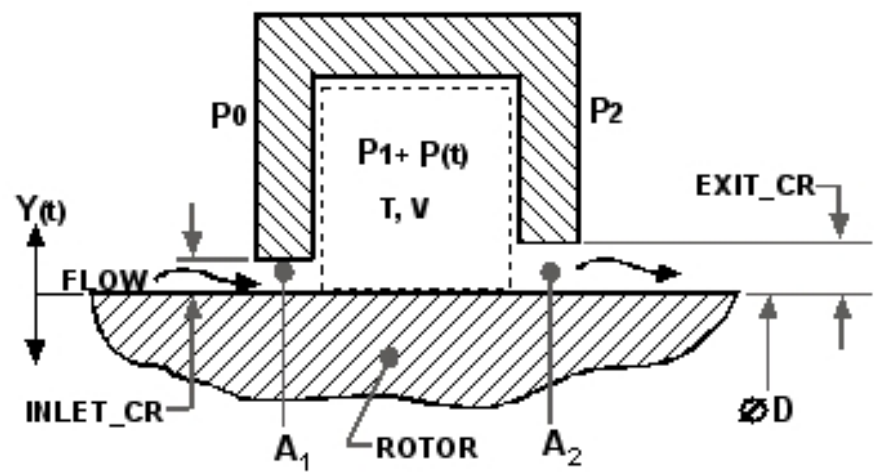

$\gamma \cdot \mathrm{T} \cdot \mathrm{R}=1.85 \times 10^{8} \quad$ Vibration Amplitude (in) $\mathrm{y}:=0.00094$

Inlet Blade Radial Clearance (in) inlet_cr $:=0.005$

Exit Blade Radial Clearance (in) exit_cr: $: 0.0075$

Rotor Frequency (rpm) Rotor_Freq : $=12000$
$A 1:=\frac{(\pi \cdot D \cdot \text { inlet_cr })}{8}$
$\mathrm{A} 2:=\frac{(\pi \cdot \mathrm{D} \cdot \text { exit_ct })}{8}$
A1 $=8.836 \times 10^{-3}$ in $^{2}$
$\mathrm{A} 2=0.013$
in $^{2}$
Pocket Volume (in 3 ) PV $:=0.9281$

MASS FLOW THROUGH INLET BLADE:

$\mathrm{m} 01:=0.75 \mathrm{PO} \cdot \frac{\mathrm{A} 1 \cdot\left[2 \cdot \frac{\gamma^{2}}{(\gamma-1)}\right]^{5} \cdot\left[\left(\frac{\mathrm{P} 1}{\mathrm{P} 0}\right)^{\frac{2}{\gamma}}-\left(\frac{\mathrm{P} 1}{\mathrm{P} 0}\right)^{\left.\frac{(\gamma+1)}{\gamma}\right]^{5}}\right.}{(\gamma \cdot \mathrm{R} \cdot \mathrm{T})^{5}} \quad \mathrm{~m} 01=2.015 \times 10^{-4}$

MASS FLOW THROUGH EXIT BLADE:

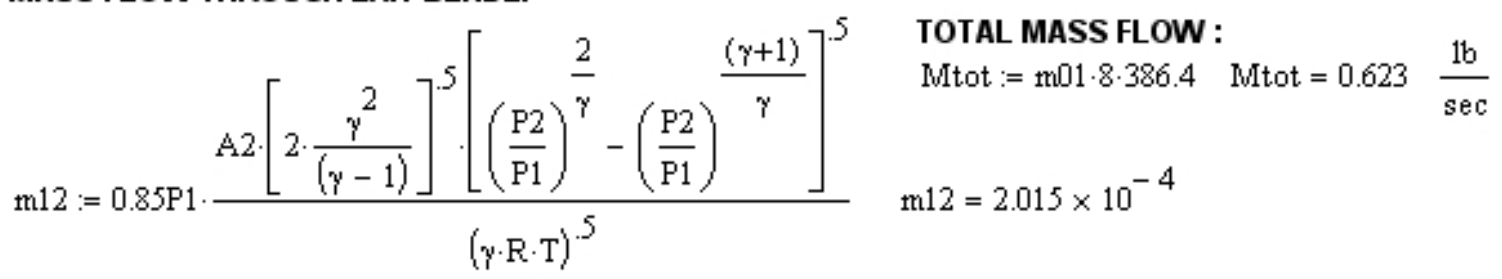


PARTIAL DERIVATIVE OF m01 RESPECT TO P1

$\mathrm{T} 1:=\frac{\mathrm{m} 01 \cdot\left[2 \cdot\left(\frac{\mathrm{P} 1}{\mathrm{P} 0}\right)^{\frac{(2-\gamma)}{\gamma}}-(\gamma+1) \cdot\left(\frac{\mathrm{P} 1}{\mathrm{P} 0}\right)^{\frac{1}{\gamma}}\right]}{\mathrm{P} 0 \cdot 2 \cdot \gamma \cdot\left[\left(\frac{\mathrm{P} 1}{\mathrm{P} 0}\right)^{\frac{2}{\gamma}}-\left(\frac{\mathrm{P} 1}{\mathrm{P} 0}\right)^{\frac{(\gamma+1)}{\gamma}}\right]}$

$\mathrm{T} 1=-9.563 \times 10^{-7}$
PARTIAL DERIVATIVE OF m12 RESPECT TO P1

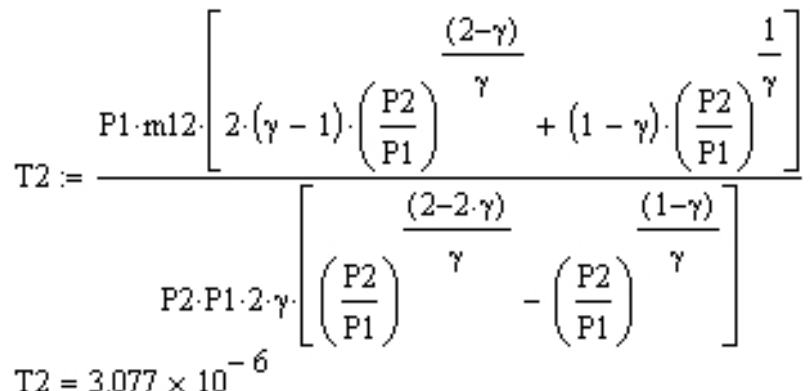

\section{Defining Constants:}

$\mathrm{C} 1:=$ Area_P $\cdot \frac{\mathrm{P} 2}{\mathrm{R} \cdot \mathrm{T}} \quad \mathrm{C} 2:=\frac{\mathrm{PV}}{\gamma \cdot \mathrm{R} \cdot \mathrm{T}} \quad \mathrm{C} 3:=(\mathrm{T} 1-\mathrm{T} 2) \mathrm{C} 4:=\left(\frac{1}{\text { inlet_cr }}-\frac{1}{\text { exit_cr }}\right) \cdot \mathrm{m} 01 \omega:=$ Rotor_Freq $2 \cdot \frac{\pi}{60}$

Damping (lb-sec/in) $\mathrm{Cyy}:=(\mathrm{C} 2 \cdot \mathrm{C} 4-\mathrm{C} 3 \cdot \mathrm{C} 1) \cdot \frac{\text { Area_P }}{\left(\mathrm{C}^{2}+\mathrm{C}^{2} \cdot{ }^{2}\right)} \quad \mathrm{Cyy}=1.046$

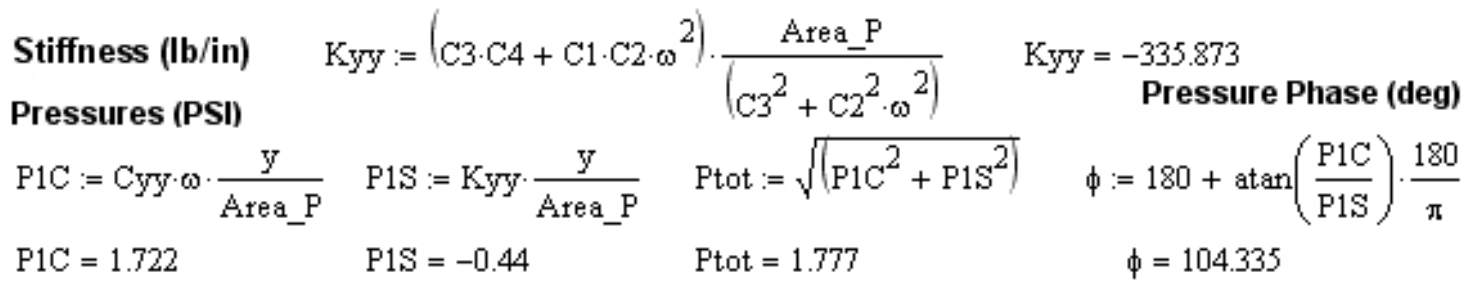

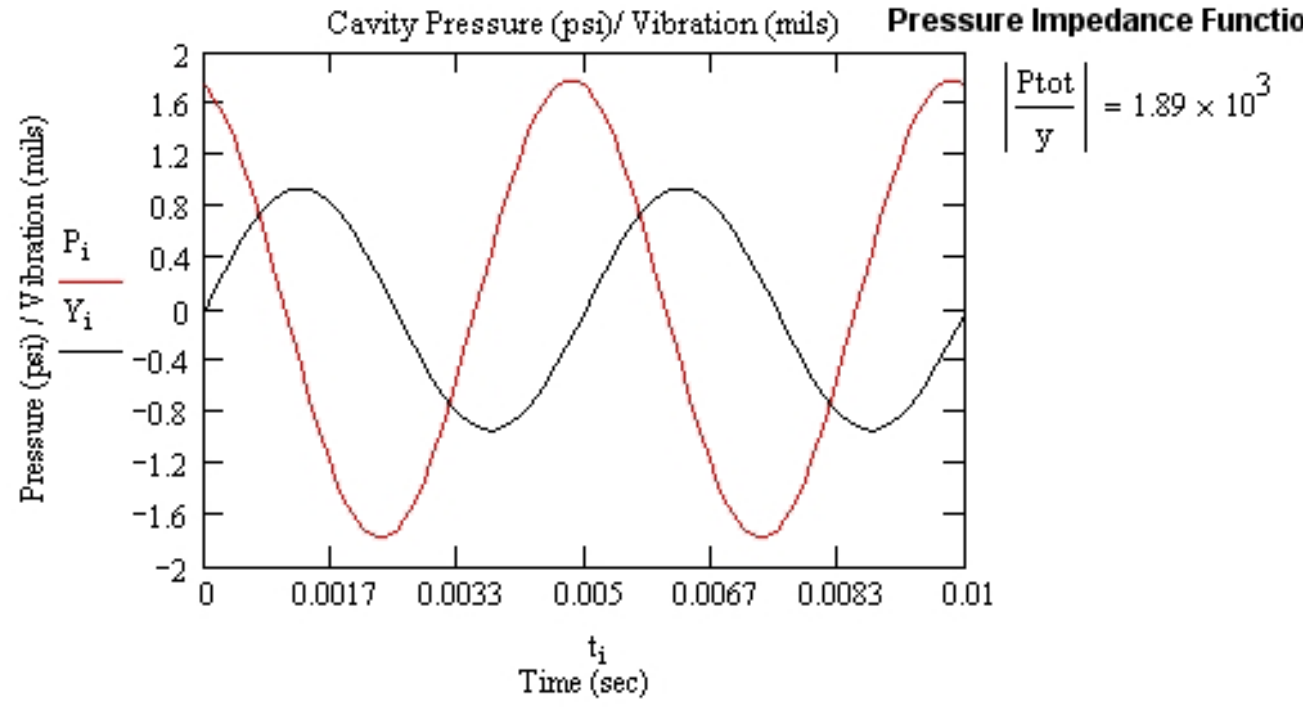


following program calculates dynamic coefficients for sequentially placed pressurized cavities in a

fully partitioned pocket damper seal. In addition to the coefficients the program also calculates the dynamic

pressure phase and magnitude respect to rotor vibration. The inputs to this code are highlighted in gray and

the results have borders.

GAS PROPERTIES :

Ratio of Specific Heats

Back Pressure (psi)

$\mathrm{Pb}:=615$

Inlet Pressure (psi)

$\mathrm{P} 0:=1000$

Gas Constant

\section{$\mathrm{R}:=247029.517$}

Exit Discharge Coeff. cdex $:=.85$

The critical pressure ratio is the upstream pressure divided by the downstream pressure. The following

Inlet Discharge Coeff. $\quad$ cdin $:=.7994$

chart shows the Pcrit for various gases. Also shown is the ratio of specific heats $(\gamma)$.

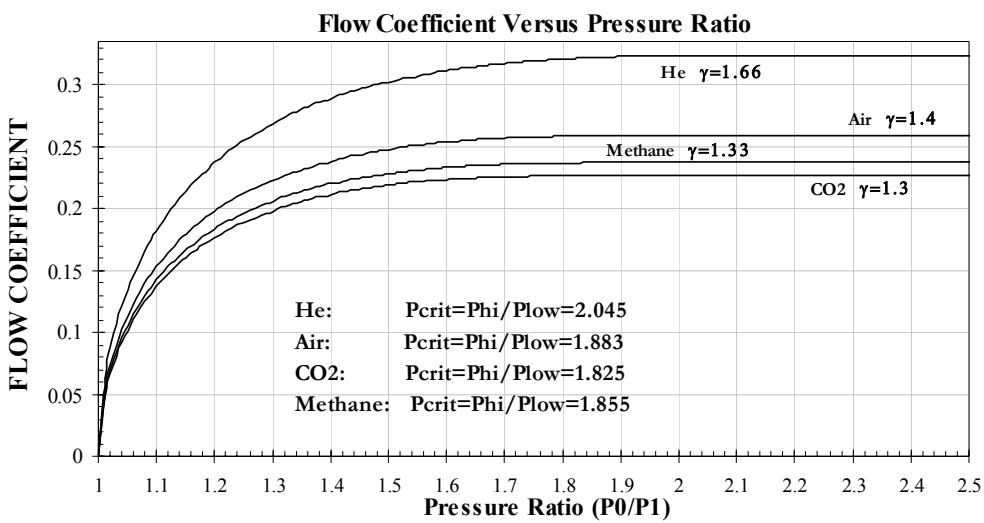

ROTOR AND SEAL GEOMETRY:

Rotor Diameter (in)

$\mathrm{D}:=4.50$

Rotor Frequency (rpm) Rotor_Freq := 1200

$\omega:=$ Rotor_Freq $\cdot \frac{2 \pi}{60}$

Vibration Amplitude (in) $\quad \mathrm{X}:=0.0005$

Average Seal Temperature (R) $\mathrm{T}:=501.67$

Axial Cavtiy Length (in):

w1 $:=0.742 \quad$ w2 $:=0.20 \quad$ w3 $:=0.742 \quad$ w4 $:=0.20 \quad$ w5 $:=0.742$

Cavtiy Depth (in):

$\mathrm{d} 1:=.56 \quad \mathrm{~d} 2:=.56 \quad \mathrm{~d} 3:=.56 \quad \mathrm{~d} 4:=.56 \quad \mathrm{~d} 5:=.56$

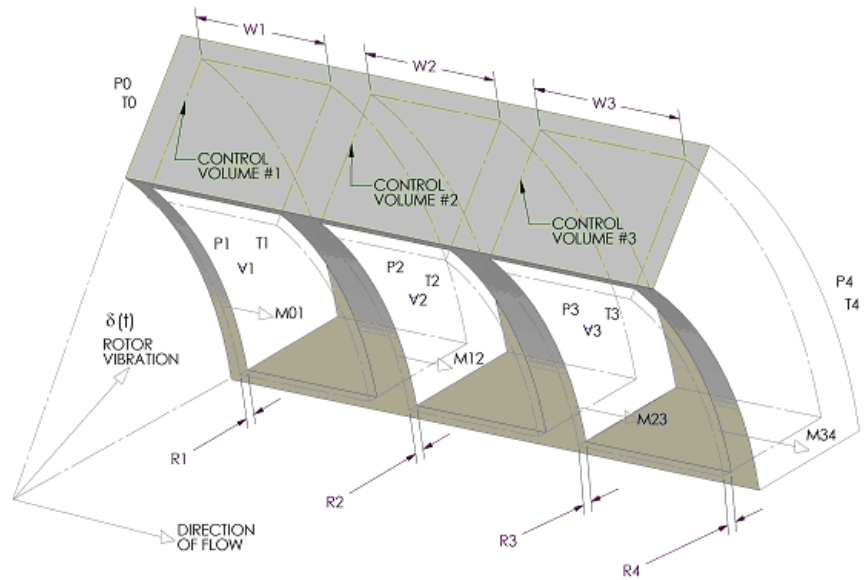


Radial Blade Clearances :

Blade1 Radial Clearance (in) $\quad \mathrm{R} 1:=0.005 \quad$ Blade4 Radial Clearance (in) $\mathrm{R} 4:=0.01$

Blade2 Radial Clearance (in) $\quad$ R2 $:=0.01 \quad$ Blade5 Radial Clearance (in) $\quad$ R5 $:=0.00$

Blade3 Radial Clearance (in) $\mathrm{R} 3:=0.005$

Blade6 Radial Clearance (in) $\quad \mathrm{R} 6:=0.01$

\section{Rotor-Stator Cavtiy Clearance Area Calculations:}

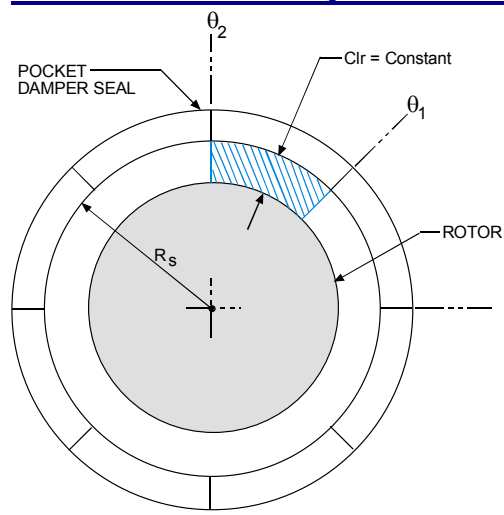

(a) ROTOR IN CENTERED POSITION

Blade 1 (in)

Seal Radius

Rs $1:=\mathrm{R} 1+\frac{\mathrm{D}}{2}$

Blade 4 (in)
Seal Radius $\quad$ Rs $4:=\mathrm{R} 4+\frac{\mathrm{D}}{2}$

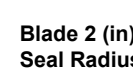

Seal Radius

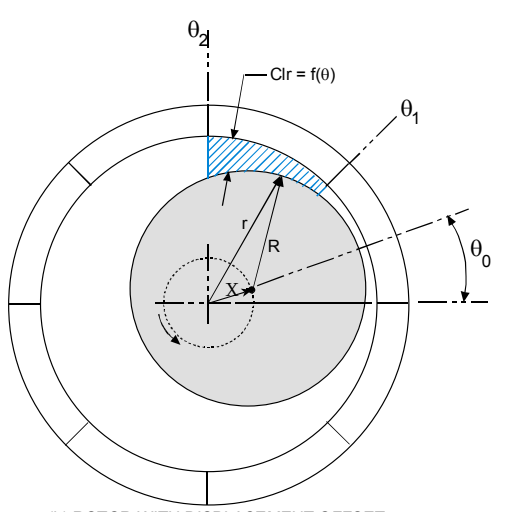

Cavity Stop Angle (Deg)

$\theta 2:=67.5$

Displacement Angle (Deg)

$\theta 0:=45$

$\theta 1 \mathrm{r}:=\theta 1 \cdot \frac{\pi}{180} \quad \theta 2 \mathrm{r}:=\theta 2 \cdot \frac{\pi}{180} \quad \theta 0 \mathrm{r}:=\theta 0 \cdot \frac{\pi}{180}$

Blade 1 (in^2) Clearance Area

Blade 2 (in^2) Clearance Area

Blade 3 (in^2) Clearance Area

Blade 4 (in^2) Clearance Area

Blade 5 (in^2) Clearance Area

Blade 6 (in^2) Clearance Area
$\mathrm{A} 1:=\frac{(\theta 2 \mathrm{r}-\theta 1 \mathrm{r}) \cdot\left[\mathrm{Rs}^{2}-\left(\frac{\mathrm{D}}{2}\right)^{2}\right]}{2} \quad \mathrm{~A} 1=8.846 \times 10^{-3}$

$\mathrm{A} 2:=\frac{(\theta 2 \mathrm{r}-\theta 1 \mathrm{r}) \cdot\left[\mathrm{Rs}^{2}-\left(\frac{\mathrm{D}}{2}\right)^{2}\right]}{2} \quad \mathrm{~A} 2=0.018$ 


\section{Seal Sector Area}

Asec $:=\frac{1}{2} \cdot\left(\int_{\theta 1 \mathrm{r}}^{\theta 2 \mathrm{r}} \mathrm{Rs}^{2} \mathrm{~d} \theta\right)$ Asec $=1.997 \quad \mathrm{C}:=\left(\frac{\mathrm{D}}{2}\right)^{2}-(\mathrm{X} \cdot \sin (\theta 0 \mathrm{r}))^{2}-(\mathrm{X} \cdot \cos (\theta 0 \mathrm{r}))^{2}$

\section{Area Of Rotor Between $\theta 1$ and $\theta^{2}$}

$$
\begin{aligned}
\text { Arot }:=\frac{1}{2} \cdot\left[\int_{\theta 1 \mathrm{r}}^{\theta 2 \mathrm{r}}\left[(\mathrm{X} \cdot \cos (\theta 0 \mathrm{r}) \cdot \cos (\theta)+\mathrm{X} \cdot \sin (\theta 0 \mathrm{r}) \cdot \sin (\theta))+\sqrt{(\mathrm{X} \cdot \cos (\theta 0 \mathrm{r}))^{2}(\cos (\theta))^{2}+(\mathrm{X} \cdot \sin (\theta 0 \mathrm{r}))^{2}(\sin (\theta))^{2}+2 \cdot(\mathrm{X} \cdot \cos (\theta 0 \mathrm{r})) \cdot(\mathrm{X} \cdot \sin (\theta 0 \mathrm{r})) \cdot \cos (\theta) \cdot \sin (\theta)+\mathrm{C}}\right]^{2} \mathrm{~d} \theta\right] \\
\mathrm{A}:=\mathrm{Asec}-\operatorname{Arot} \quad \mathrm{A}=7.984 \times 10^{-3} \quad \Delta \mathrm{A}:=\mathrm{A} 1-\mathrm{A} \quad \Delta \mathrm{A}=8.611 \times 10^{-4}
\end{aligned}
$$

Cavity Volumes (in^3)

$$
\begin{aligned}
& \text { PV1 := w1 } \cdot\left[\frac{(\theta 2-\theta 1)}{360} \cdot\left[\left(\frac{\mathrm{Rs} 1+\mathrm{Rs} 2}{2}+\mathrm{d} 1\right)^{2} \cdot \pi-\left(\frac{\mathrm{Rs} 1+\mathrm{Rs} 2}{2}\right)^{2} \cdot \pi\right]\right] \quad \text { PV1 }=0.828 \\
& \mathrm{PV} 2:=\mathrm{w} 2 \cdot\left[\frac{(\theta 2-\theta 1)}{360} \cdot\left[\left(\frac{\mathrm{Rs} 2+\mathrm{Rs} 3}{2}+\mathrm{d} 2\right)^{2} \cdot \pi-\left(\frac{\mathrm{Rs} 2+\mathrm{Rs} 3}{2}\right)^{2} \cdot \pi\right]\right] \quad \mathrm{PV} 2=0.223 \\
& \text { PV3 := w3. }\left[\frac{(\theta 2-\theta 1)}{360} \cdot\left[\left(\frac{\mathrm{Rs} 3+\mathrm{Rs} 4}{2}+\mathrm{d} 3\right)^{2} \cdot \pi-\left(\frac{\mathrm{Rs} 3+\mathrm{Rs} 4}{2}\right)^{2} \cdot \pi\right]\right] \quad \text { PV } 3=0.828 \\
& \text { PV4:= w4 }\left[\frac{(\theta 2-\theta 1)}{360} \cdot\left[\left(\frac{\mathrm{Rs} 4+\mathrm{Rs} 5}{2}+\mathrm{d} 4\right)^{2} \cdot \pi-\left(\frac{\mathrm{Rs} 4+\mathrm{Rs} 5}{2}\right)^{2} \cdot \pi\right]\right] \quad \text { PV4 }=0.223 \\
& \text { PV5:= w5. }\left[\frac{(\theta 2-\theta 1)}{360} \cdot\left[\left(\frac{\text { Rs5 }+ \text { Rs6 }}{2}+\mathrm{d} 5\right)^{2} \cdot \pi-\left(\frac{\text { Rs5 }+ \text { Rs } 6}{2}\right)^{2} \cdot \pi\right]\right] \quad \text { PV5 }=0.828
\end{aligned}
$$

\section{Cavity Pressure Areas (in^2)}

Area_P1 $:=\mathrm{w} 1 \cdot 2 \cdot\left[\frac{(\mathrm{Rs} 1+\mathrm{Rs} 2)}{2} \cdot \sin \left(\frac{\theta 2 \mathrm{r}-\theta 1 \mathrm{r}}{2}\right)\right] \quad$ Area_P1 $=1.282$

Area_P2 $:=\mathrm{w} 2 \cdot 2 \cdot\left[\frac{(\mathrm{Rs} 2+\mathrm{Rs} 3)}{2} \cdot \sin \left(\frac{\theta 2 \mathrm{r}-\theta 1 \mathrm{r}}{2}\right)\right] \quad$ Area_P2 $=0.346$

Area_P3 $:=\mathrm{w} 3 \cdot 2 \cdot\left[\frac{(\mathrm{Rs} 3+\mathrm{Rs} 4)}{2} \cdot \sin \left(\frac{\theta 2 \mathrm{r}-\theta 1 \mathrm{r}}{2}\right)\right] \quad$ Area_P3 $=1.282$

Area_P4 $:=\mathrm{w} 4 \cdot 2 \cdot\left[\frac{(\mathrm{Rs} 4+\mathrm{Rs} 5)}{2} \cdot \sin \left(\frac{\theta 2 \mathrm{r}-\theta 1 \mathrm{r}}{2}\right)\right] \quad$ Area_P4 $=0.346$

Area_P5 $:=w 5 \cdot 2 \cdot\left[\frac{(\mathrm{Rs} 5+\mathrm{Rs} 6)}{2} \cdot \sin \left(\frac{\theta 2 \mathrm{r}-\theta 1 \mathrm{r}}{2}\right)\right] \quad$ Area_P5 $=1.282$

INTERNAL STATIC CAVITY PRESSURE CALCULATIONS :

pi $:=\frac{\mathrm{P} 0-\mathrm{Pb}}{6}$

P1i $:=$ P0 - pi $\quad$ P2i $:=$ P0 - 2pi $\quad$ P3i $:=$ P0 - 3pi $\quad$ P4i := P0 - 4pi P5i $:=$ P0 - 5pi

Given 


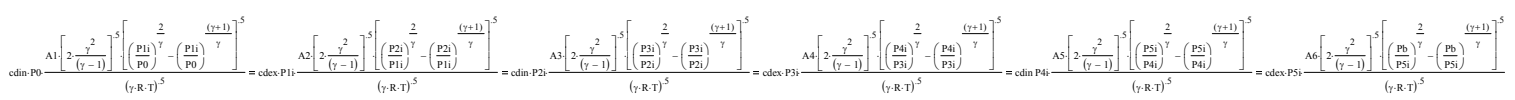

Ps := Find(P1i, P2i, P3i, P4i, P5i)

Ps $=\left(\begin{array}{l}912.383 \\ 892.731 \\ 791.502 \\ 768.665 \\ 643.577\end{array}\right)$

$\mathrm{Ps}_{0}=912.383 \mathrm{Ps}_{1}=892.731 \quad \mathrm{Ps}_{2}=791.502 \quad \mathrm{Ps}_{3}=768.665 \quad \mathrm{Ps}_{4}=643.577$

$\mathrm{pj}:=\frac{\mathrm{P} 0-\mathrm{Pb}}{6}$

$\mathrm{P} 1 \mathrm{j}:=\mathrm{P} 0-$ pj $\quad \mathrm{P} 2 \mathrm{j}:=\mathrm{P} 0-2 \mathrm{pj} \quad \mathrm{P} 3 \mathrm{j}:=\mathrm{P} 0-3 \mathrm{pj} \quad \mathrm{P} 4 \mathrm{j}:=\mathrm{P} 0-4 \mathrm{pj} \quad \mathrm{P} 5 \mathrm{j}:=\mathrm{P} 0-5 \mathrm{pj}$

Given

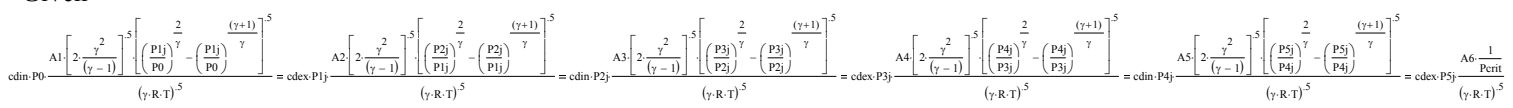

$P c:=\operatorname{Find}(P 1 j, P 2 j, P 3 j, P 4 j, P 5 j)$

$P c=\left(\begin{array}{c}886.39 \\ 860.758 \\ 720.279 \\ 688.181 \\ 467.512\end{array}\right)$

$\begin{array}{lllll}\mathrm{Pc}_{0}=886.39 & \mathrm{Pc}_{1}=860.758 & \mathrm{Pc}_{2}=720.279 & \mathrm{Pc}_{3}=688.181 & \mathrm{Pc}_{4}=467.512\end{array}$

$\mathrm{P} 1:=\mid \begin{array}{ll}\mathrm{Ps}_{0} & \text { if } \frac{\mathrm{Ps}_{4}}{\mathrm{~Pb}}<\mathrm{Pcrit} \quad \mathrm{P} 2:=\mid \begin{array}{l}\mathrm{Ps}_{1} \\ \mathrm{Pc}_{0} \text { otherwise }\end{array} \text { 年 } \frac{\mathrm{Ps}_{4}}{\mathrm{~Pb}}<\mathrm{Pcrit} \quad \mathrm{P} 3:=\mid \begin{array}{l}\mathrm{Ps}_{2} \\ \mathrm{Pc}_{1} \text { otherwise }\end{array} \text { if } \frac{\mathrm{Ps}_{4}}{\mathrm{~Pb}}<\mathrm{Pcrit} \quad \mathrm{P} 4:= \\ \mathrm{Pc}_{2} \text { otherwise }\end{array}$

$\begin{array}{llll}\mathrm{P} 1=912.383 & \mathrm{P} 2=892.731 & \mathrm{P} 3=791.502 & \mathrm{P} 4=768.665\end{array}$

\section{MASS FLOW EQUATIONS:}

Blade \#1

$$
\mathrm{m} 01:=\operatorname{cdin} \mathrm{P} 0 \cdot \frac{\mathrm{A} 1 \cdot\left[2 \cdot \frac{\gamma^{2}}{(\gamma-1)}\right]^{.5} \cdot\left[\left(\frac{\mathrm{P} 1}{\mathrm{P} 0}\right)^{\frac{2}{\gamma}}-\left(\frac{\mathrm{P} 1}{\mathrm{P} 0}\right)^{\frac{(\gamma+1)}{\gamma}}\right]^{.5}}{(\gamma \cdot \mathrm{R} \cdot \mathrm{T})^{.5}} \mathrm{~m} 01=2.531 \times 10^{-4}
$$

Blade \#2

$$
\mathrm{m} 12:=\operatorname{cdexP} 1 \cdot \frac{\mathrm{A} 2 \cdot\left[2 \cdot \frac{\gamma^{2}}{(\gamma-1)}\right]^{5} \cdot\left[\left(\frac{\mathrm{P} 2}{\mathrm{P} 1}\right)^{\frac{2}{\gamma}}-\left(\frac{\mathrm{P} 2}{\mathrm{P} 1}\right)^{\frac{(\gamma+1)}{\gamma}}\right]^{.5}}{(\gamma \cdot \mathrm{R} \cdot \mathrm{T})^{.5}} \mathrm{~m} 12=2.531 \times 10^{-4}
$$

Blade \#3

$$
\mathrm{m} 23:=\operatorname{cdin} \mathrm{P} 2 \cdot \frac{\mathrm{A} 3 \cdot\left[2 \cdot \frac{\gamma^{2}}{(\gamma-1)}\right]^{.5} \cdot\left[\left(\frac{\mathrm{P} 3}{\mathrm{P} 2}\right)^{\frac{2}{\gamma}}-\left(\frac{\mathrm{P} 3}{\mathrm{P} 2}\right)^{\left.\frac{(\gamma+1)}{\gamma}\right]^{.5}}\right.}{(\gamma \cdot \mathrm{R} \cdot \mathrm{T})^{.5}} \mathrm{~m} 23=2.531 \times 10^{-4}
$$


Blade \#4

$$
\mathrm{m} 34:=\operatorname{cdexP} 3 \cdot \frac{\mathrm{A} 4 \cdot\left[2 \cdot \frac{\gamma^{2}}{(\gamma-1)}\right]^{.5} \cdot\left[\left(\frac{\mathrm{P} 4}{\mathrm{P} 3}\right)^{\frac{2}{\gamma}}-\left(\frac{\mathrm{P} 4}{\mathrm{P} 3}\right)^{\left.\frac{(\gamma+1)}{\gamma}\right]^{.5}}\right.}{(\gamma \cdot \mathrm{R} \cdot \mathrm{T})^{.5}} \mathrm{~m} 34=2.531 \times 10^{-4}
$$

Blade \#5

$$
\mathrm{m} 45:=\operatorname{cdin} \mathrm{P} 4 \cdot \frac{\mathrm{A} 5 \cdot\left[2 \cdot \frac{\gamma^{2}}{(\gamma-1)}\right]^{.5} \cdot\left[\left(\frac{\mathrm{P} 5}{\mathrm{P} 4}\right)^{\frac{2}{\gamma}}-\left(\frac{\mathrm{P} 5}{\mathrm{P} 4}\right)^{\left.\frac{(\gamma+1)}{\gamma}\right]^{.5}}\right.}{(\gamma \cdot \mathrm{R} \cdot \mathrm{T})^{.5}} \mathrm{~m} 45=2.531 \times 10^{-4}
$$

Blade \#6

$$
\mathrm{m} 56:=\operatorname{cdexP} 5 \cdot \frac{\mathrm{A} 6 \cdot\left[2 \cdot \frac{\gamma^{2}}{(\gamma-1)}\right]^{.5} \cdot\left[\left(\frac{\mathrm{Pb}}{\mathrm{P} 5}\right)^{\frac{2}{\gamma}}-\left(\frac{\mathrm{Pb}}{\mathrm{P} 5}\right)^{\left.\frac{(\gamma+1)}{\gamma}\right]^{.5}}\right.}{(\gamma \cdot \mathrm{R} \cdot \mathrm{T})^{.5}} \mathrm{~m} 56=2.531 \times 10^{-4}
$$

\section{MASS FLOW PARTIAL DERIVATIVE}

PARTIAL DERIVATIVE OF m01 RESPECT TO P1 PARTIAL DERIVATIVE OF m12 RESPECT TO P1

$\mathrm{F} 1:=\frac{\mathrm{m} 01 \cdot\left[2 \cdot\left(\frac{\mathrm{P} 1}{\mathrm{P} 0}\right)^{\frac{(2-\gamma)}{\gamma}}-(\gamma+1) \cdot\left(\frac{\mathrm{P} 1}{\mathrm{P} 0}\right)^{\frac{1}{\gamma}}\right]}{\mathrm{P} 0 \cdot 2 \cdot \gamma \cdot\left[\left(\frac{\mathrm{P} 1}{\mathrm{P} 0}\right)^{\frac{2}{\gamma}}-\left(\frac{\mathrm{P} 1}{\mathrm{P} 0}\right)^{\frac{(\gamma+1)}{\gamma}}\right]}$

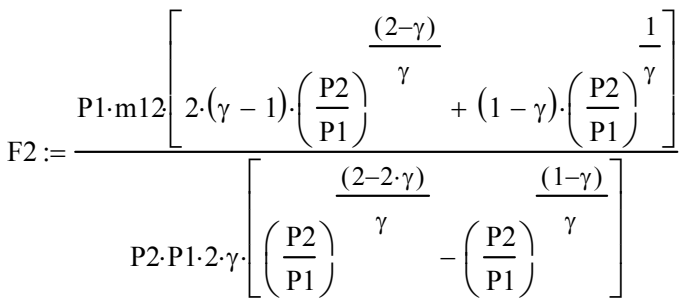

$\mathrm{F} 1=-1.295 \times 10^{-6}$

$\mathrm{F} 2=6.43 \times 10^{-6}$

PARTIAL DERIVATIVE OF m12 RESPECT TO P2

PARTIAL DERIVATIVE OF m23 RESPECT TO P2

$\mathrm{F} 3:=\frac{\mathrm{m} 12 \cdot\left[2 \cdot\left(\frac{\mathrm{P} 2}{\mathrm{P} 1}\right)^{\frac{(2-\gamma)}{\gamma}}-(\gamma+1) \cdot\left(\frac{\mathrm{P} 2}{\mathrm{P} 1}\right)^{\frac{1}{\gamma}}\right]}{\mathrm{P} 1 \cdot 2 \cdot \gamma \cdot\left[\left(\frac{\mathrm{P} 2}{\mathrm{P} 1}\right)^{\frac{2}{\gamma}}-\left(\frac{\mathrm{P} 2}{\mathrm{P} 1}\right)^{\frac{(\gamma+1)}{\gamma}}\right]}$

$\mathrm{F} 4:=\frac{\mathrm{P} 2 \cdot \mathrm{m} 23\left[2 \cdot(\gamma-1) \cdot\left(\frac{\mathrm{P} 3}{\mathrm{P} 2}\right)^{\frac{(2-\gamma)}{\gamma}}+(1-\gamma) \cdot\left(\frac{\mathrm{P} 3}{\mathrm{P} 2}\right)^{\frac{1}{\gamma}}\right]}{\mathrm{P} 3 \cdot \mathrm{P} 2 \cdot 2 \cdot \gamma \cdot\left[\left(\frac{\mathrm{P} 3}{\mathrm{P} 2}\right)^{\frac{(2-2 \cdot \gamma)}{\gamma}}-\left(\frac{\mathrm{P} 3}{\mathrm{P} 2}\right)^{\frac{(1-\gamma)}{\gamma}}\right]}$

$\mathrm{F} 3=-6.288 \times 10^{-6}$

$\mathrm{F} 4=1.239 \times 10^{-6}$

PARTIAL DERIVATIVE OF m23 RESPECT TO P3 PARTIAL DERIVATIVE OF m34 RESPECT TO P3

$$
\mathrm{F} 5:=\frac{\mathrm{m} 23 \cdot\left[2 \cdot\left(\frac{\mathrm{P} 3}{\mathrm{P} 2}\right)^{\frac{(2-\gamma)}{\gamma}}-(\gamma+1) \cdot\left(\frac{\mathrm{P} 3}{\mathrm{P} 2}\right)^{\frac{1}{\gamma}}\right]}{\mathrm{P} 2 \cdot 2 \cdot \gamma \cdot\left[\left(\frac{\mathrm{P} 3}{\mathrm{P} 2}\right)^{\frac{2}{\gamma}}-\left(\frac{\mathrm{P} 3}{\mathrm{P} 2}\right)^{\frac{(\gamma+1)}{\gamma}}\right]}
$$$$
\mathrm{F} 6:=\frac{\mathrm{P} 3 \cdot \mathrm{m} 34\left[2 \cdot(\gamma-1) \cdot\left(\frac{\mathrm{P} 4}{\mathrm{P} 3}\right)^{\frac{(2-\gamma)}{\gamma}}+(1-\gamma) \cdot\left(\frac{\mathrm{P} 4}{\mathrm{P} 3}\right)^{\frac{1}{\gamma}}\right]}{\mathrm{P} 4 \cdot \mathrm{P} 3 \cdot 2 \cdot \gamma \cdot\left[\left(\frac{\mathrm{P} 4}{\mathrm{P} 3}\right)^{\frac{(2-2 \cdot \gamma)}{\gamma}}-\left(\frac{\mathrm{P} 4}{\mathrm{P} 3}\right)^{\frac{(1-\gamma)}{\gamma}}\right]}
$$

$\mathrm{F} 5=-1.077 \times 10^{-6}$

$\mathrm{F} 6=5.53 \times 10^{-6}$ 
PARTIAL DERIVATIVE OF m34 RESPECT TO P4

PARTIAL DERIVATIVE OF m45 RESPECT TO P4

$\mathrm{F} 7:=\frac{\mathrm{m} 34\left[2 \cdot\left(\frac{\mathrm{P} 4}{\mathrm{P} 3}\right)^{\frac{(2-\gamma)}{\gamma}}-(\gamma+1) \cdot\left(\frac{\mathrm{P} 4}{\mathrm{P} 3}\right)^{\frac{1}{\gamma}}\right]}{\mathrm{P} 3 \cdot 2 \cdot \gamma \cdot\left[\left(\frac{\mathrm{P} 4}{\mathrm{P} 3}\right)^{\frac{2}{\gamma}}-\left(\frac{\mathrm{P} 4}{\mathrm{P} 3}\right)^{\frac{(\gamma+1)}{\gamma}}\right]}$

$\mathrm{F} 8:=\frac{\mathrm{P} 4 \cdot \mathrm{m} 45\left[2 \cdot(\gamma-1) \cdot\left(\frac{\mathrm{P} 5}{\mathrm{P} 4}\right)^{\frac{(2-\gamma)}{\gamma}}+(1-\gamma) \cdot\left(\frac{\mathrm{P} 5}{\mathrm{P} 4}\right)^{\frac{1}{\gamma}}\right]}{\mathrm{P} 5 \cdot \mathrm{P} 4 \cdot 2 \cdot \gamma \cdot\left[\left(\frac{\mathrm{P} 5}{\mathrm{P} 4}\right)^{\frac{(2-2 \cdot \gamma)}{\gamma}}-\left(\frac{\mathrm{P} 5}{\mathrm{P} 4}\right)^{\frac{(1-\gamma)}{\gamma}}\right]}$

$\mathrm{F} 7=-5.365 \times 10^{-6}$

$\mathrm{F} 8=9.977 \times 10^{-7}$

PARTIAL DERIVATIVE OF m45 RESPECT TO P5

PARTIAL DERIVATIVE OF m56 RESPECT TO P5

$\mathrm{F} 9:=\frac{\mathrm{m} 45 \cdot\left[2 \cdot\left(\frac{\mathrm{P} 5}{\mathrm{P} 4}\right)^{\frac{(2-\gamma)}{\gamma}}-(\gamma+1) \cdot\left(\frac{\mathrm{P} 5}{\mathrm{P} 4}\right)^{\frac{1}{\gamma}}\right]}{\mathrm{P} 4 \cdot 2 \cdot \gamma \cdot\left[\left(\frac{\mathrm{P} 5}{\mathrm{P} 4}\right)^{\frac{2}{\gamma}}-\left(\frac{\mathrm{P} 5}{\mathrm{P} 4}\right)^{\frac{(\gamma+1)}{\gamma}}\right]}$

$\mathrm{F} 10:=\frac{\mathrm{P} 5 \cdot \mathrm{m} 56\left[2 \cdot(\gamma-1) \cdot\left(\frac{\mathrm{Pb}}{\mathrm{P} 5}\right)^{\frac{(2-\gamma)}{\gamma}}+(1-\gamma) \cdot\left(\frac{\mathrm{Pb}}{\mathrm{P} 5}\right)^{\frac{1}{\gamma}}\right]}{\mathrm{Pb} \cdot \mathrm{P} 5 \cdot 2 \cdot \gamma \cdot\left[\left(\frac{\mathrm{Pb}}{\mathrm{P} 5}\right)^{\frac{(2-2 \cdot \gamma)}{\gamma}}-\left(\frac{\mathrm{Pb}}{\mathrm{P} 5}\right)^{\frac{(1-\gamma)}{\gamma}}\right]}$

$\mathrm{F} 9=-7.984 \times 10^{-7}$

$\mathrm{F} 10=4.414 \times 10^{-6}$

\section{Defining Constants:}

$\mathrm{C} 1:=\frac{\mathrm{PV} 1}{\gamma \cdot \mathrm{R} \cdot \mathrm{T}} \quad \mathrm{C} 1=4.773 \times 10^{-9} \quad \mathrm{C} 2:=\frac{\mathrm{PV} 2}{\gamma \cdot \mathrm{R} \cdot \mathrm{T}} \quad \mathrm{C} 2=1.287 \times 10^{-9}$

$\mathrm{C} 3:=\frac{\mathrm{PV} 3}{\gamma \cdot \mathrm{R} \cdot \mathrm{T}} \quad \mathrm{C} 3=4.773 \times 10^{-9} \quad \mathrm{C} 4:=\frac{\mathrm{PV} 4}{\gamma \cdot \mathrm{R} \cdot \mathrm{T}} \quad \mathrm{C} 4=1.287 \times 10^{-9}$

$\mathrm{C} 5:=\frac{\mathrm{PV} 5}{\gamma \cdot \mathrm{R} \cdot \mathrm{T}} \quad \mathrm{C} 5=4.773 \times 10^{-9} \quad \mathrm{E} 1:=\mathrm{w} 1 \cdot \frac{\Delta \mathrm{A}}{\mathrm{X}} \cdot \frac{\mathrm{P} 1}{\mathrm{R} \cdot \mathrm{T}} \quad \mathrm{E} 1=9.408 \times 10^{-6}$

$\mathrm{E} 2:=\mathrm{w} 2 \cdot \frac{\Delta \mathrm{A}}{\mathrm{X}} \cdot \frac{\mathrm{P} 2}{\mathrm{R} \cdot \mathrm{T}} \quad \mathrm{E} 2=2.481 \times 10^{-6} \quad \mathrm{E} 3:=\mathrm{w} 3 \cdot \frac{\Delta \mathrm{A}}{\mathrm{X}} \cdot \frac{\mathrm{P} 3}{\mathrm{R} \cdot \mathrm{T}} \quad \mathrm{E} 3=8.162 \times 10^{-6}$

$\mathrm{E} 4:=\mathrm{w} 4 \cdot \frac{\Delta \mathrm{A}}{\mathrm{X}} \cdot \frac{\mathrm{P} 4}{\mathrm{R} \cdot \mathrm{T}} \quad \mathrm{E} 4=2.136 \times 10^{-6} \quad \mathrm{E} 5:=\mathrm{w} 5 \cdot \frac{\Delta \mathrm{A}}{\mathrm{X}} \cdot \frac{\mathrm{P} 5}{\mathrm{R} \cdot \mathrm{T}} \quad \mathrm{E} 5=6.636 \times 10^{-6}$

Subtraction of Partial Derivatives of Massflow Respect To Rotor Vibration:

$$
\begin{aligned}
& \mathrm{D} 1:=\left(\frac{\Delta \mathrm{A}}{\mathrm{X} \cdot \mathrm{A} 1}-\frac{\Delta \mathrm{A}}{\mathrm{X} \cdot \mathrm{A} 2}\right) \cdot \mathrm{m} 01 \quad \mathrm{D} 1=0.025 \quad \mathrm{D} 2:=\left(\frac{\Delta \mathrm{A}}{\mathrm{X} \cdot \mathrm{A} 2}-\frac{\Delta \mathrm{A}}{\mathrm{X} \cdot \mathrm{A} 3}\right) \cdot \mathrm{m} 12 \quad \mathrm{D} 2=-0.025 \\
& \mathrm{D} 3:=\left(\frac{\Delta \mathrm{A}}{\mathrm{X} \cdot \mathrm{A} 3}-\frac{\Delta \mathrm{A}}{\mathrm{X} \cdot \mathrm{A} 4}\right) \cdot \mathrm{m} 23 \quad \mathrm{D} 3=0.025 \quad \mathrm{D} 4:=\left(\frac{\Delta \mathrm{A}}{\mathrm{X} \cdot \mathrm{A} 4}-\frac{\Delta \mathrm{A}}{\mathrm{X} \cdot \mathrm{A} 5}\right) \cdot \mathrm{m} 34 \quad \mathrm{D} 4=-0.025 \\
& \mathrm{D} 5:=\left(\frac{\Delta \mathrm{A}}{\mathrm{X} \cdot \mathrm{A} 5}-\frac{\Delta \mathrm{A}}{\mathrm{X} \cdot \mathrm{A} 6}\right) \cdot \mathrm{m} 45 \quad \mathrm{D} 5=0.025
\end{aligned}
$$


Conservation of Mass

Matrix Equation: $\quad \Lambda * \mathrm{P}=\Theta$

Pressure Coefficient Matrix:

$$
\Lambda:=\left(\begin{array}{cccccccccc}
\mathrm{F} 1-\mathrm{F} 2 & -\omega \cdot \mathrm{C} 1 & -\mathrm{F} 3 & 0 & 0 & 0 & 0 & 0 & 0 & 0 \\
\omega \cdot \mathrm{C} 1 & \mathrm{~F} 1-\mathrm{F} 2 & 0 & -\mathrm{F} 3 & 0 & 0 & 0 & 0 & 0 & 0 \\
\mathrm{~F} 2 & 0 & \mathrm{~F} 3-\mathrm{F} 4 & -\omega \cdot \mathrm{C} 2 & -\mathrm{F} 5 & 0 & 0 & 0 & 0 & 0 \\
0 & \mathrm{~F} 2 & \omega \cdot \mathrm{C} 2 & \mathrm{~F} 3-\mathrm{F} 4 & 0 & -\mathrm{F} 5 & 0 & 0 & 0 & 0 \\
0 & 0 & \mathrm{~F} 4 & 0 & \mathrm{~F} 5-\mathrm{F} 6 & -\omega \cdot \mathrm{C} 3 & -\mathrm{F} 7 & 0 & 0 & 0 \\
0 & 0 & 0 & \mathrm{~F} 4 & \omega \cdot \mathrm{C} 3 & \mathrm{~F} 5-\mathrm{F} 6 & 0 & -\mathrm{F} 7 & 0 & 0 \\
0 & 0 & 0 & 0 & \mathrm{~F} 6 & 0 & \mathrm{~F} 7-\mathrm{F} 8 & -\omega \cdot \mathrm{C} 4 & -\mathrm{F} 9 & 0 \\
0 & 0 & 0 & 0 & 0 & \mathrm{~F} 6 & \omega \cdot \mathrm{C} 4 & \mathrm{~F} 7-\mathrm{F} 8 & 0 & -\mathrm{F} 9 \\
0 & 0 & 0 & 0 & 0 & 0 & \mathrm{~F} 8 & 0 & \mathrm{~F} 9-\mathrm{F} 10 & -\omega \cdot \mathrm{C} 5 \\
0 & 0 & 0 & 0 & 0 & 0 & 0 & \mathrm{~F} 8 & \omega \cdot \mathrm{C} 5 & \mathrm{~F} 9-\mathrm{F} 10
\end{array}\right)
$$

Right Side Element Matrix:

$$
\Theta:=\left(\begin{array}{c}
-\omega \cdot \mathrm{X} \cdot \mathrm{E} 1 \\
\mathrm{X} \cdot \mathrm{D} 1 \\
-\omega \cdot \mathrm{X} \cdot \mathrm{E} 2 \\
\mathrm{X} \cdot \mathrm{D} 2 \\
-\omega \cdot \mathrm{X} \cdot \mathrm{E} 3 \\
\mathrm{X} \cdot \mathrm{D} 3 \\
-\omega \cdot \mathrm{X} \cdot \mathrm{E} 4 \\
\mathrm{X} \cdot \mathrm{D} 4 \\
-\omega \cdot \mathrm{X} \cdot \mathrm{E} 5 \\
\mathrm{X} \cdot \mathrm{D} 5
\end{array}\right)
$$

Solution Matrix-Dynamic Pressure Components $\quad P:=\Lambda^{-1} \cdot \Theta$

$P=$\begin{tabular}{|r|r|}
\hline & \multicolumn{1}{|c|}{0} \\
\hline 0 & 0.961 \\
\hline 1 & -0.825 \\
\hline 2 & 1.008 \\
\hline 3 & 0.857 \\
\hline 4 & 1.29 \\
\hline 5 & -0.692 \\
\hline 6 & 1.182 \\
\hline 7 & 1.104 \\
\hline 8 & 0.547 \\
\hline 9 & -2.092 \\
\hline
\end{tabular}




\section{Cavity Coefficients:}

Cavity 1

$\mathrm{C} 1 \mathrm{xx}:=\mathrm{P}_{0} \cdot \frac{\text { Area_P1 }}{\omega \cdot \mathrm{X}} \mathrm{C} 1 \mathrm{xx}=19.607 \mathrm{~b} \cdot \frac{\mathrm{sec}}{\mathrm{in}}$

$\mathrm{K} 1 \mathrm{xx}:=\mathrm{P}_{1} \cdot \frac{\text { Area_P1 }}{\mathrm{X}} \mathrm{K} 1 \mathrm{xx}=-2.115 \times 10^{3} \quad \frac{\mathrm{lb}}{\mathrm{in}}$

Cavity 3

$\mathrm{C} 3 \mathrm{xx}:=\mathrm{P}_{4} \cdot \frac{\text { Area_P3 }}{\omega \cdot \mathrm{X}}$

$\mathrm{K} 3 \mathrm{xx}:=\mathrm{P}_{5} \cdot \frac{\text { Area_P3 }}{\mathrm{X}}$
Cavity 2

$\mathrm{C} 2 \mathrm{xx}:=\mathrm{P}_{2} \cdot \frac{\text { Area_P2 }}{\omega \cdot \mathrm{X}} \mathrm{C} 2 \mathrm{xx}=5.543 \quad \mathrm{lb} \cdot \frac{\mathrm{sec}}{\mathrm{in}}$

$\mathrm{K} 2 \mathrm{xx}:=\mathrm{P}_{3} \cdot \frac{\text { Area_P2 }}{\mathrm{X}} \quad \mathrm{K} 2 \mathrm{xx}=592.055 \quad \frac{\mathrm{lb}}{\mathrm{in}}$

Cavity 4

$\mathrm{C} 3 \mathrm{xx}=26.312 \quad \mathrm{lb} \cdot \frac{\mathrm{sec}}{\mathrm{in}} \quad \mathrm{C} 4 \mathrm{xx}:=\mathrm{P}_{6} \cdot \frac{\text { Area_P4 }}{\omega \cdot \mathrm{X}} \quad \mathrm{C} 4 \mathrm{xx}=6.503 \quad \mathrm{lb} \cdot \frac{\mathrm{sec}}{\mathrm{in}}$

$\mathrm{K} 3 \mathrm{xx}=-1.776 \times 10^{3} \quad \frac{\mathrm{lb}}{\text { in }} \mathrm{K} 4 \mathrm{xx}:=\mathrm{P}_{7} \cdot \frac{\text { Area_P4 }}{\mathrm{X}} \quad \mathrm{K} 4 \mathrm{xx}=763.116 \quad \frac{\mathrm{lb}}{\text { in }}$

Cavity 5

$\mathrm{C} 5 \mathrm{xx}:=\mathrm{P}_{8} \cdot\left(\frac{\text { Area_P5 }}{\mathrm{X} \cdot \omega}\right) \quad \mathrm{C} 5 \mathrm{xx}=11.163 \quad \mathrm{lb} \cdot \frac{\mathrm{sec}}{\mathrm{in}}$

$\mathrm{K} 5 \mathrm{xx}:=\mathrm{P}_{9} \cdot \frac{\text { Area_P5 }}{\mathrm{X}} \quad \mathrm{K} 5 \mathrm{xx}=-5.364 \times 10^{3} \quad \frac{\mathrm{lb}}{\mathrm{in}}$

\section{Total Row Coefficients:}

Total Direct Stiffness For 5

Sequential Cavities

Ktot $=-7.9 \times 10^{3} \quad \frac{1 \mathrm{~b}}{\text { in }}$

Ktot := K1 $x x+K 2 x x+K 3 x x+K 4 x x+K 5 x x$

Total Direct Damping For 5

Sequential Cavities

Ctot $=69.127 \quad \frac{\mathrm{lb} \cdot \mathrm{sec}}{\text { in }} \quad \mathrm{Ctot}:=\mathrm{C} 1 \mathrm{xx}+\mathrm{C} 2 \mathrm{xx}+\mathrm{C} 3 \mathrm{xx}+\mathrm{C} 4 \mathrm{xx}+\mathrm{C} 5 \mathrm{xx}$

TOTAL MASS FLOW:

Mtot $:=\operatorname{m01} \cdot\left(\frac{360}{\theta 2-\theta 1}\right) \cdot 386.4$

Mtot $=0.782 \frac{\mathrm{lb}}{\mathrm{sec}}$

\section{Dynamic Pressures and Phases:}

$$
\begin{aligned}
& \text { DynPressure } 1:=\sqrt{\left[\left(\mathrm{P}_{0}\right)^{2}+\left(\mathrm{P}_{1}\right)^{2}\right.} \quad \text { DynPressure1 }=1.266 \text { psi 0-pk } \\
& \text { DynPressure }:=\sqrt{\left[\left(\mathrm{P}_{2}\right)^{2}+\left(\mathrm{P}_{3}\right)^{2}\right.} \quad \text { DynPressure2 }=1.323 \text { psi 0-pk } \\
& \text { DynPressure3 }:=\sqrt{\left[\left(\mathrm{P}_{4}\right)^{2}+\left(\mathrm{P}_{5}\right)^{2}\right]} \text { DynPressure3 }=1.464 \text { psi 0-pk } \\
& \text { DynPressure4 }:=\sqrt{\left[\left(\mathrm{P}_{6}\right)^{2}+\left(\mathrm{P}_{7}\right)^{2}\right.} \quad \text { DynPressure4 }=1.618 \text { psi 0-pk } \\
& \text { DynPressure5 }:=\sqrt{\left[\left(\mathrm{P}_{8}\right)^{2}+\left(\mathrm{P}_{9}\right)^{2}\right.} \quad \text { DynPressure5 }=2.162 \text { psi 0-pk }
\end{aligned}
$$

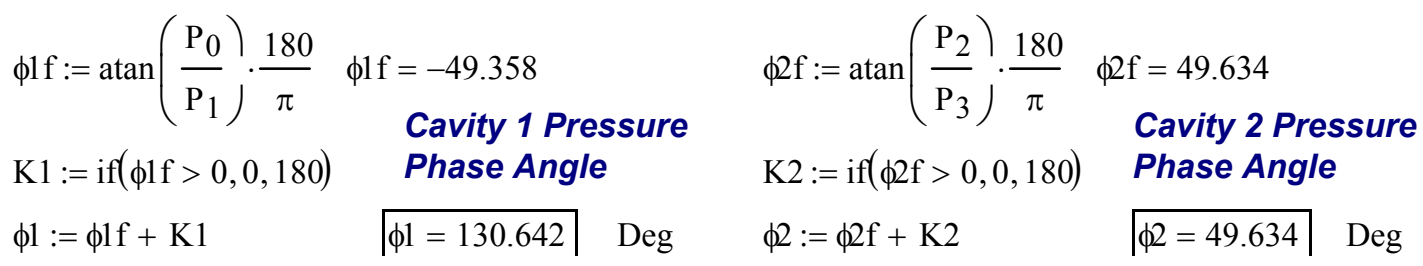




$$
\begin{aligned}
& \phi 3 \mathrm{f}:=\operatorname{atan}\left(\frac{\mathrm{P}_{4}}{\mathrm{P}_{5}}\right) \cdot \frac{180}{\pi} \quad \begin{array}{c}
\phi \mathrm{f}=-61.763 \\
\text { Cavity } 3 \text { Pressure }
\end{array} \quad \phi 4 \mathrm{f}:=\operatorname{atan}\left(\frac{\mathrm{P}_{6}}{\mathrm{P}_{7}}\right) \cdot \frac{180}{\pi} \quad \phi 4 \mathrm{f}=46.961 \\
& \mathrm{~K} 3:=\mathrm{if}(\phi 3 \mathrm{f}>0,0,180) \quad \text { Phase Angle } \quad \mathrm{K} 4:=\mathrm{if}(\phi 4 \mathrm{f}>0,0,180) \quad \text { Phase Angle } \\
& \phi 3:=\phi 3 \mathrm{f}+\mathrm{K} 3 \quad \phi 3=118.237 \text { Deg } \phi 4:=\phi 4 \mathrm{f}+\mathrm{K} 4 \quad \phi 4=46.961 \text { Deg } \\
& \phi 5 \mathrm{f}:=\operatorname{atan}\left(\frac{\mathrm{P}_{8}}{\mathrm{P}_{9}}\right) \cdot \frac{180}{\pi} \phi 5 \mathrm{f}=-14.655 \\
& \begin{array}{ll}
\mathrm{K} 5:=\mathrm{if}(\phi 5 \mathrm{f}>0,0,180) & \begin{array}{l}
\text { Cavity } 5 \text { Pressure } \\
\text { Phase Angle }
\end{array} \\
\phi 5:=\phi 5 \mathrm{f}+\mathrm{K} 5 & \phi 5=165.345
\end{array}
\end{aligned}
$$

Cavity 1 Pressure Impedance (Ibf/in^3)

$$
\begin{array}{||l|l|}
\hline \frac{\text { DynPressure 1 }}{\mathrm{X}} & =2.533 \times 10^{3} \\
\hline
\end{array}
$$

Cavity 3 Pressure Impedance (Ibf/in^3)

$$
\begin{array}{||l|l|}
\hline \frac{\text { DynPressure3 }}{\mathrm{X}} & =2.927 \times 10^{3} \\
\hline
\end{array}
$$

\section{Cavity 5 Pressure Impedance (Ibf/in^3)}

$$
\begin{array}{||l|l|}
\hline \frac{\text { DynPressure }}{\mathrm{X}} & =4.325 \times 10^{3} \\
\hline
\end{array}
$$

\section{Plotting Commands for Cavity Pressures and Vibration}

$$
\begin{array}{ll}
\mathrm{N}:=2000 & \mathrm{~N}:=2000 \\
\mathrm{i}:=0 . . \mathrm{N} & \mathrm{i}:=0 . . \mathrm{N} \\
\mathrm{t}_{\mathrm{i}}:=\mathrm{i} \cdot \frac{0.25}{2000} & \mathrm{t}_{\mathrm{i}}:=\mathrm{i} \cdot \frac{0.25}{2000} \\
\mathrm{X}_{\mathrm{i}}:=\mathrm{X} \cdot 1000 \sin \left[[\omega \cdot(1 \cdot \mathrm{t})]_{\mathrm{i}}\right] & \mathrm{P}_{\mathrm{i}}:=\operatorname{DynPressure3} \cdot \sin \left[[\omega \cdot(1 \cdot \mathrm{t})]_{\mathrm{i}}+\phi 3 \cdot \frac{\pi}{180}\right] \\
\mathrm{N}:=2000 & \\
\mathrm{P}_{\mathrm{i}}:=\operatorname{DynPressure1} \cdot \sin \left[[\omega \cdot(1 \cdot \mathrm{t})]_{\mathrm{i}}+\phi 1 \cdot \frac{\pi}{180}\right] & \mathrm{P} 5_{\mathrm{i}}:=\operatorname{DynPressure} 5 \cdot \sin \left[[\omega \cdot(1 \cdot \mathrm{t})]_{\mathrm{i}}+\phi 5 \cdot \frac{\pi}{180}\right] \\
\mathrm{i}:=0 . . \mathrm{N} & \mathrm{N}:=2000 \\
\mathrm{t}_{\mathrm{i}}:=\mathrm{i} \cdot \frac{0.25}{2000} & \mathrm{i}:=0 . . \mathrm{N} \\
\mathrm{N}:=2000 & \mathrm{t}_{\mathrm{i}}:=\mathrm{i} \cdot \frac{0.25}{2000} \\
\mathrm{i}:=0 . . \mathrm{N} & \\
\mathrm{t}_{\mathrm{i}}:=\mathrm{i} \cdot \frac{0.25}{2000} & \\
\mathrm{P} 2_{\mathrm{i}}:=\operatorname{DynPressure} 2 \cdot \sin \left[[\omega \cdot(1 \cdot \mathrm{t})]_{\mathrm{i}}+\phi 2 \cdot \frac{\pi}{180}\right] & \mathrm{P} 4_{\mathrm{i}}:=\operatorname{DynPressure} 4 \cdot \sin \left[[\omega \cdot(1 \cdot \mathrm{t})]_{\mathrm{i}}+\phi 4 \cdot \frac{\pi}{180}\right]
\end{array}
$$

Cavity 4 Pressure Impedance (Ibf/in^3)

\begin{tabular}{||l|l|}
\hline$\frac{\text { DynPressure4 }}{\mathrm{X}}$ & $=3.236 \times 10^{3}$ \\
\hline
\end{tabular}


Diverging Cavities: Dynamic Cavity Pressure and Rotor Vibration

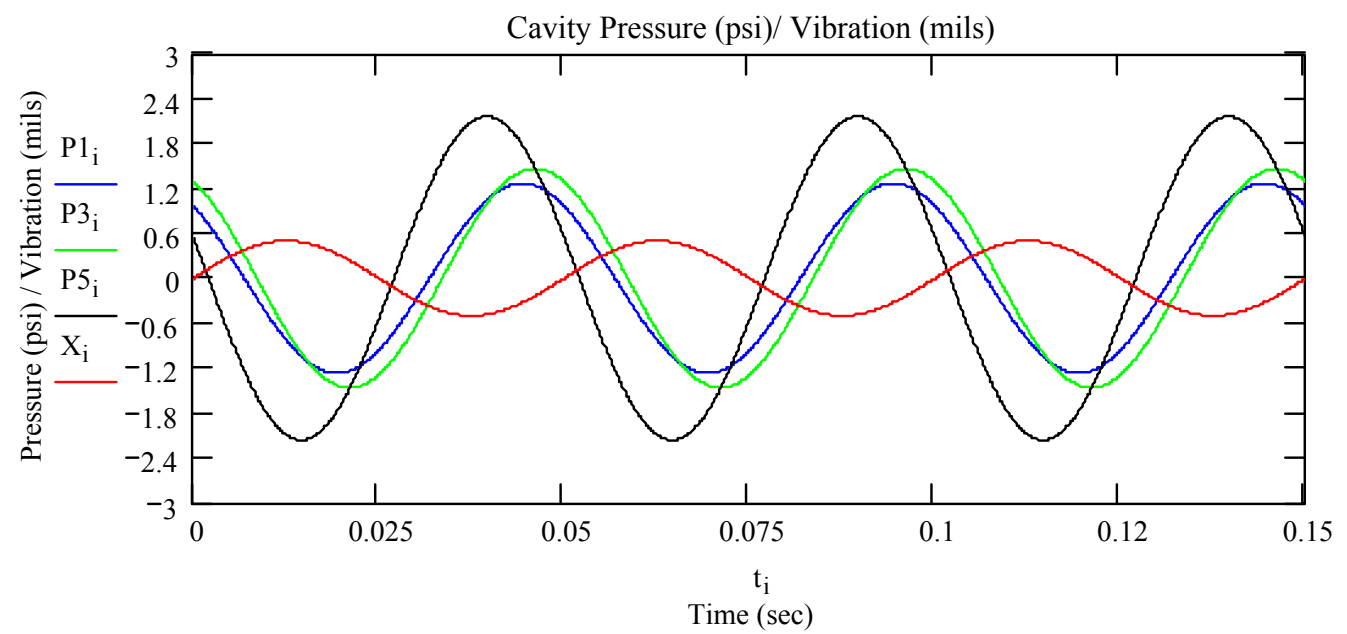

Converging Cavities: Dynamic Cavity Pressure and Rotor Vibration

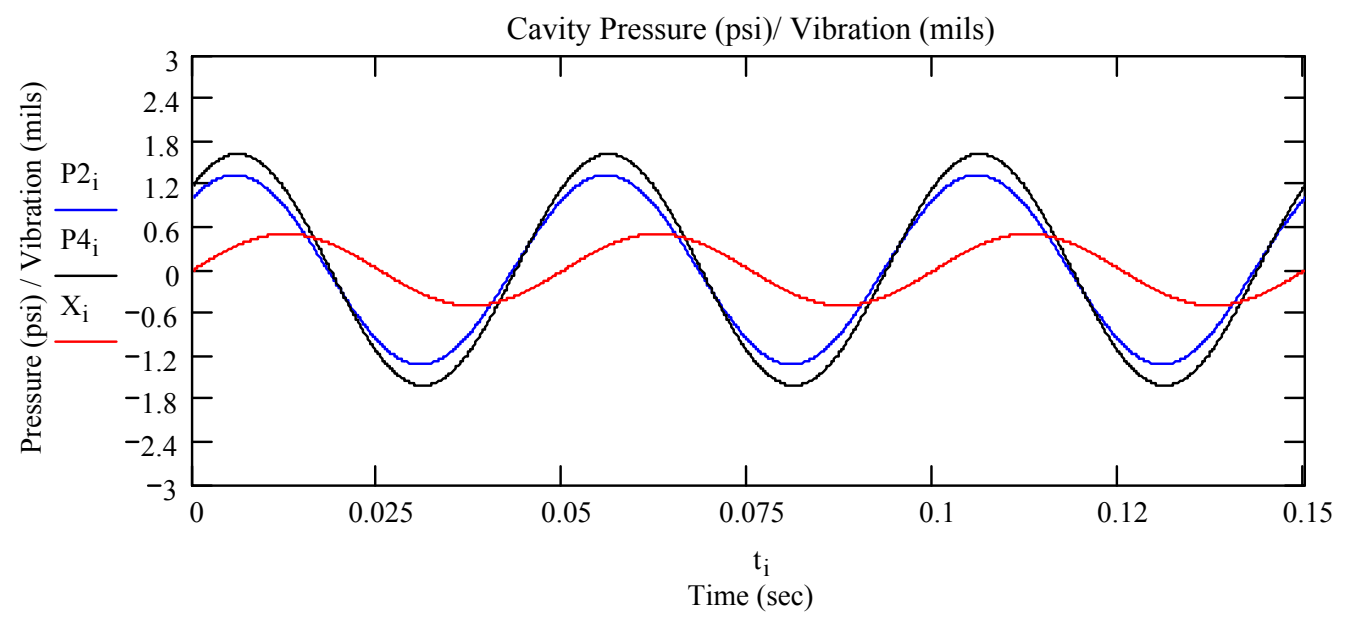

Dynamic Cavity Pressures and Rotor Vibration

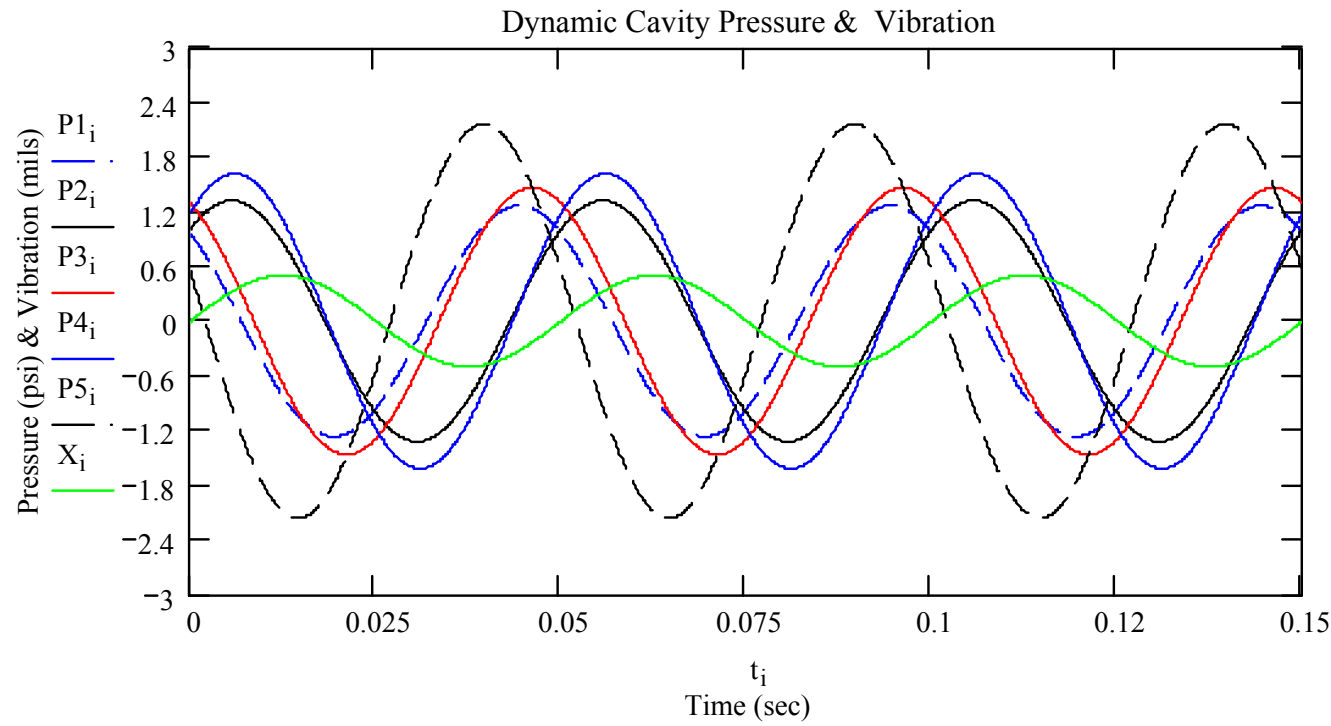


APPENDIX C

CAVITY COEFFICIENTS 
Conventional 6 Bladed PDS Cavity Coefficients: 0 Degree Position
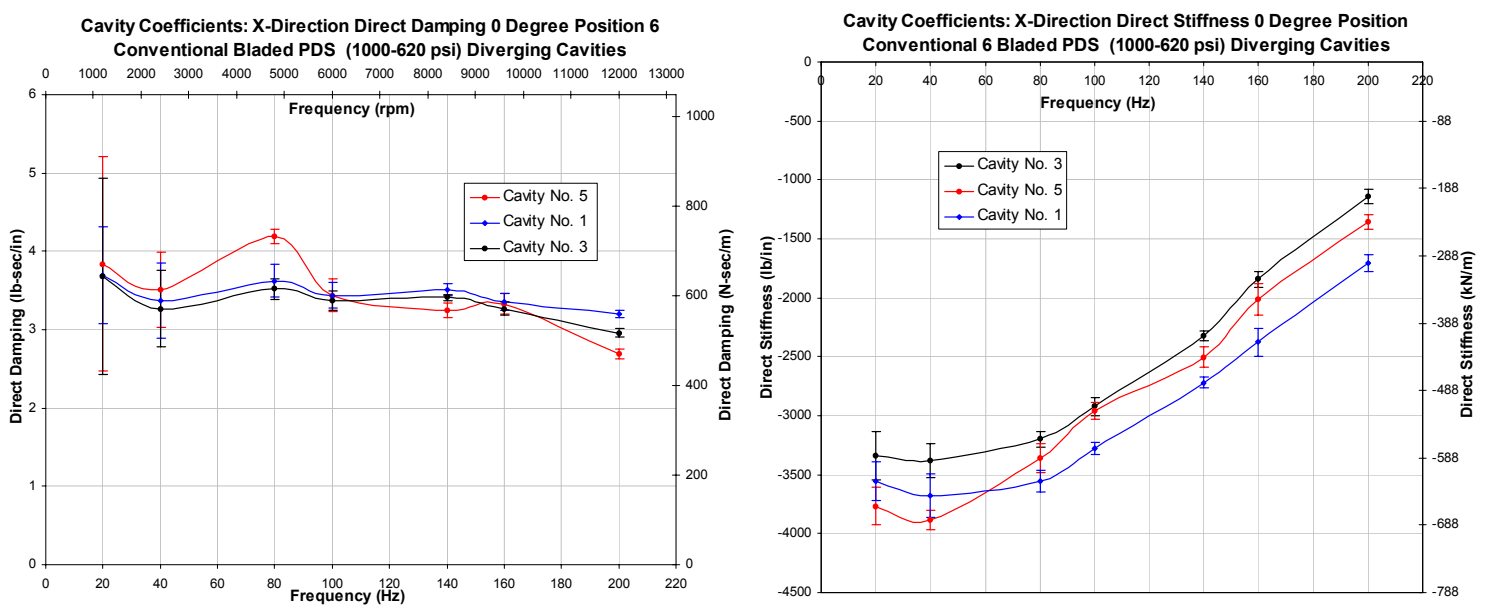

Cavity Direct Coefficients
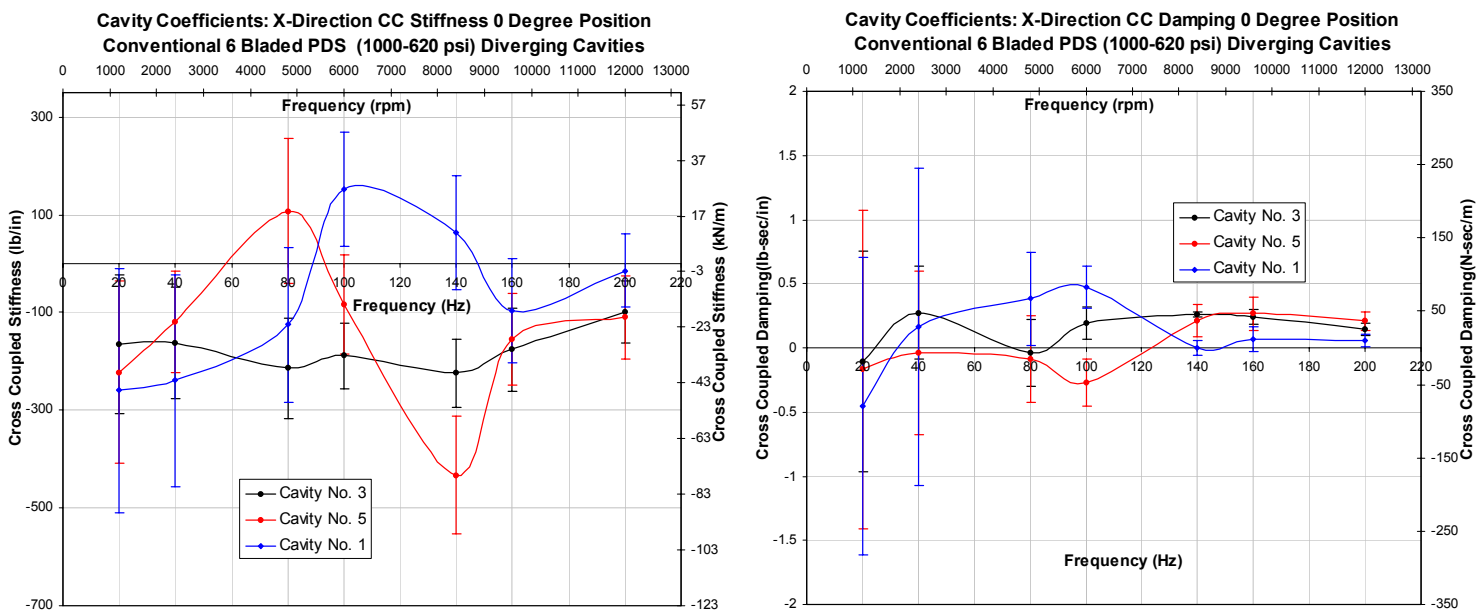

Cavity Cross-coupled Coefficients

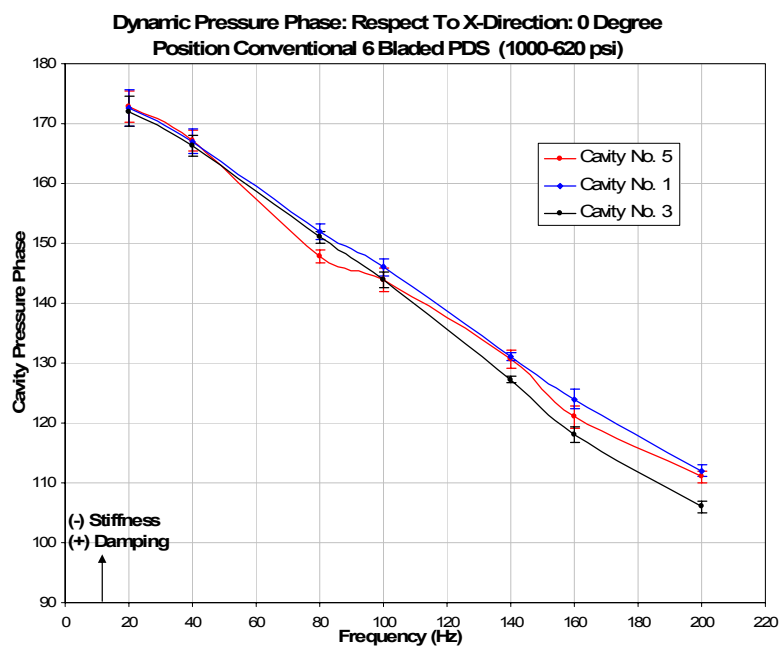

Cavity Pressure Phase Respect to X Direction 

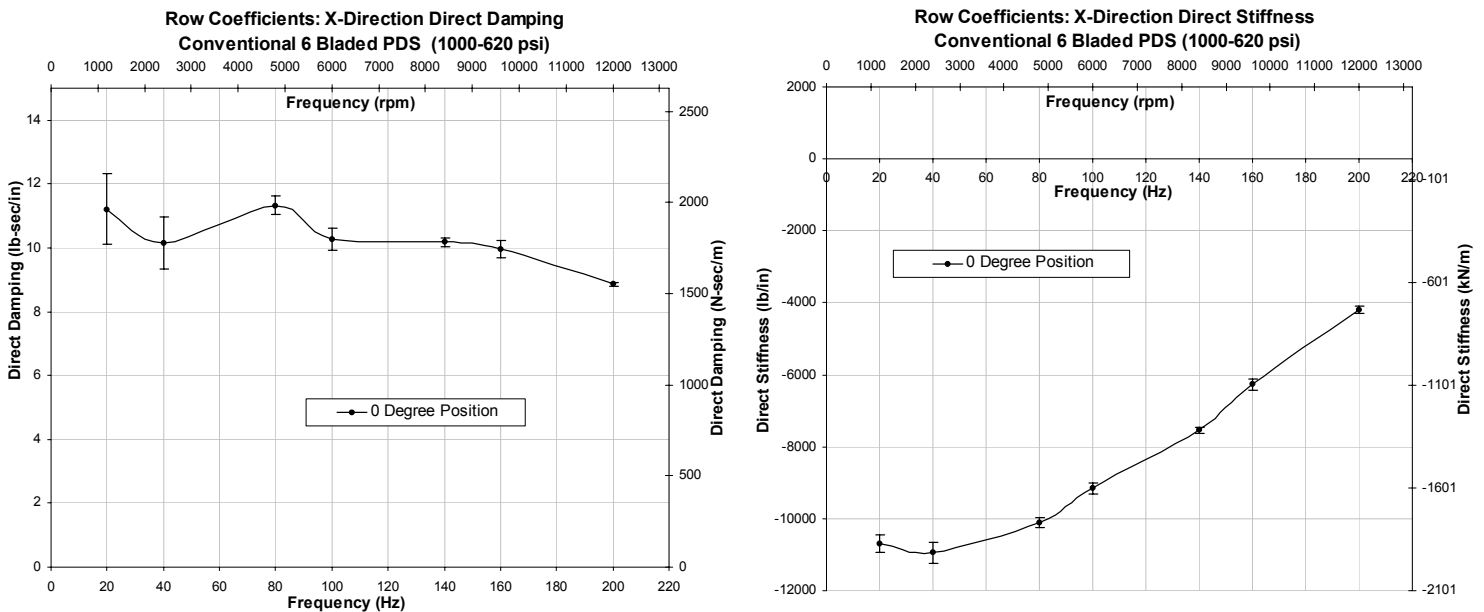

Total Row Direct Coefficients
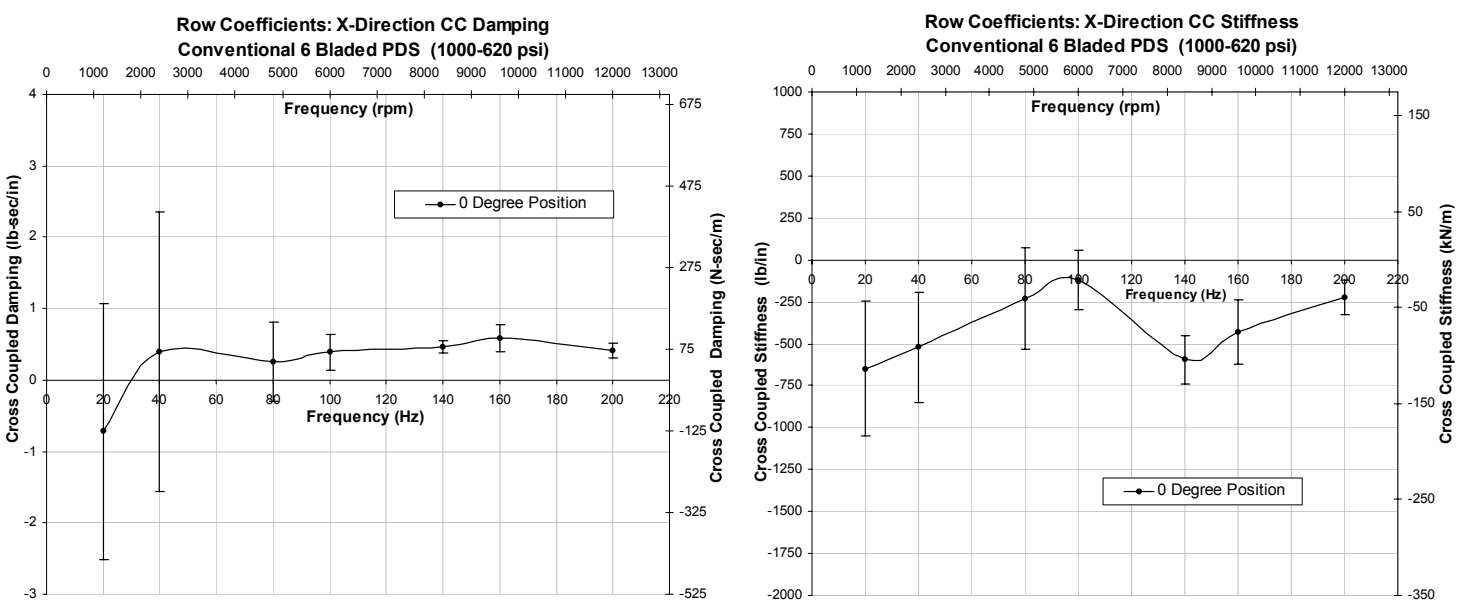

Total Row Cross-coupled Coefficients 
Conventional 6 Bladed PDS Cavity Coefficients: 90 Degree Position
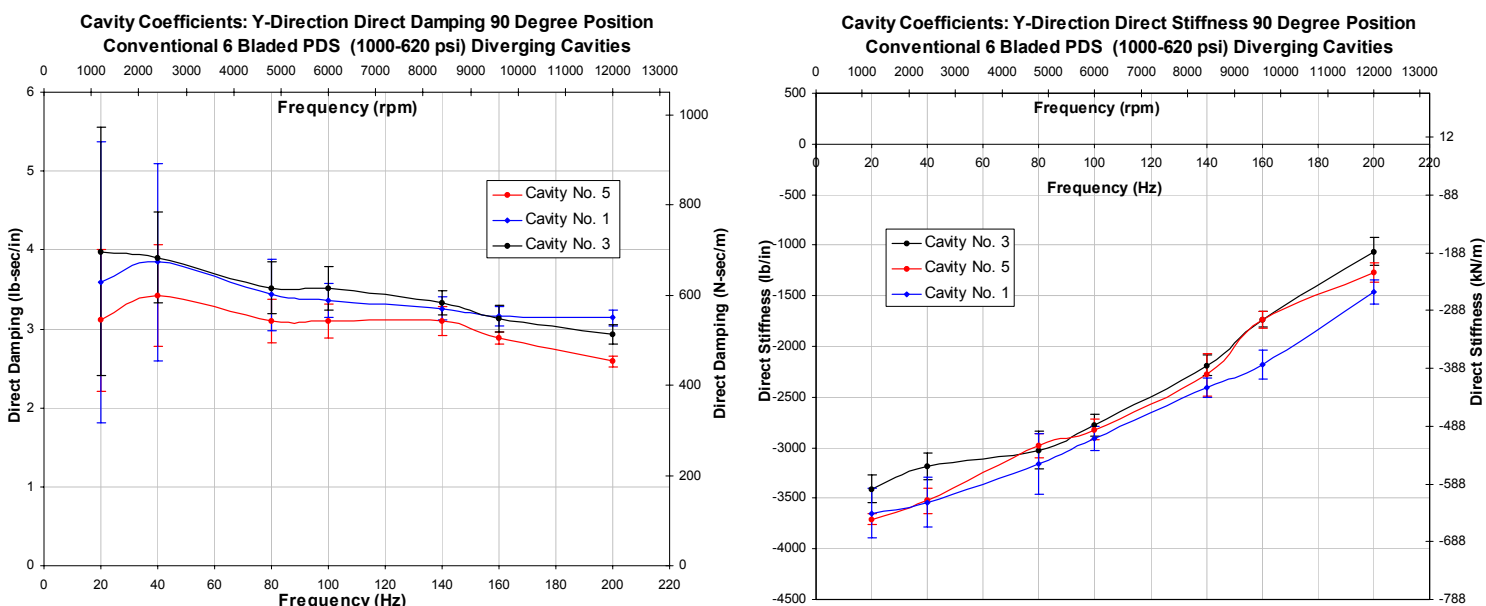

Cavity Direct Coefficients
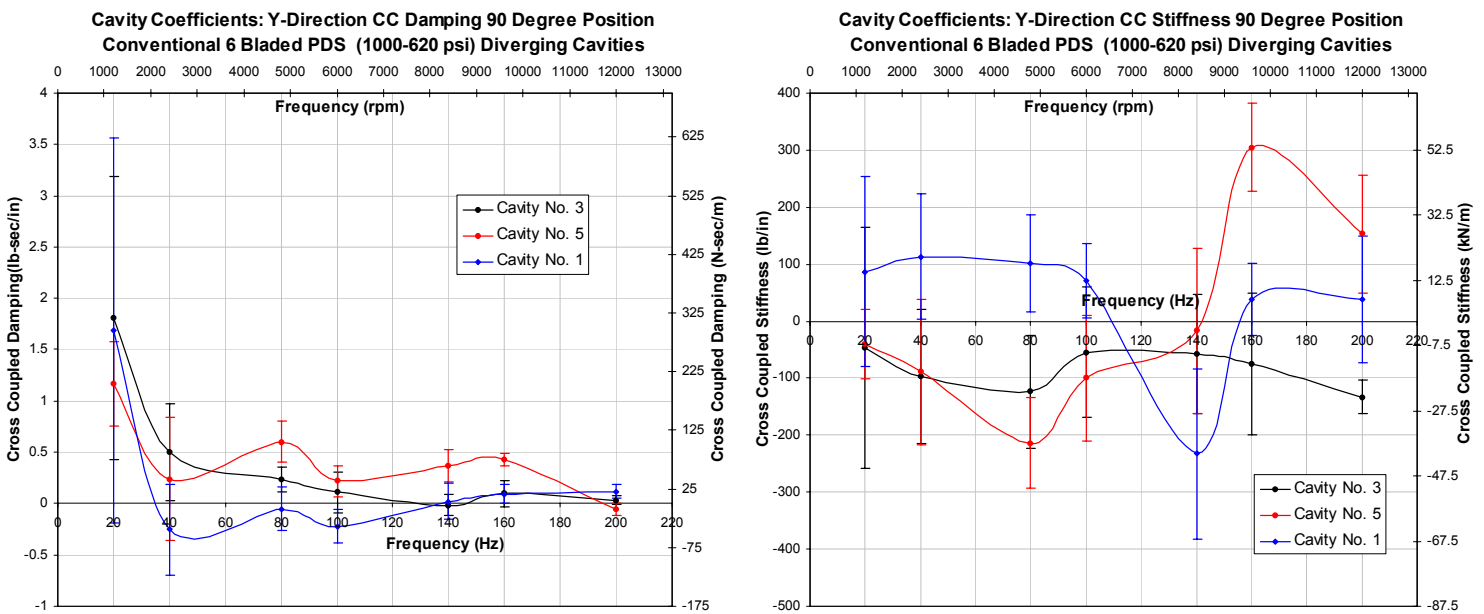

Cavity Cross-coupled Coefficients

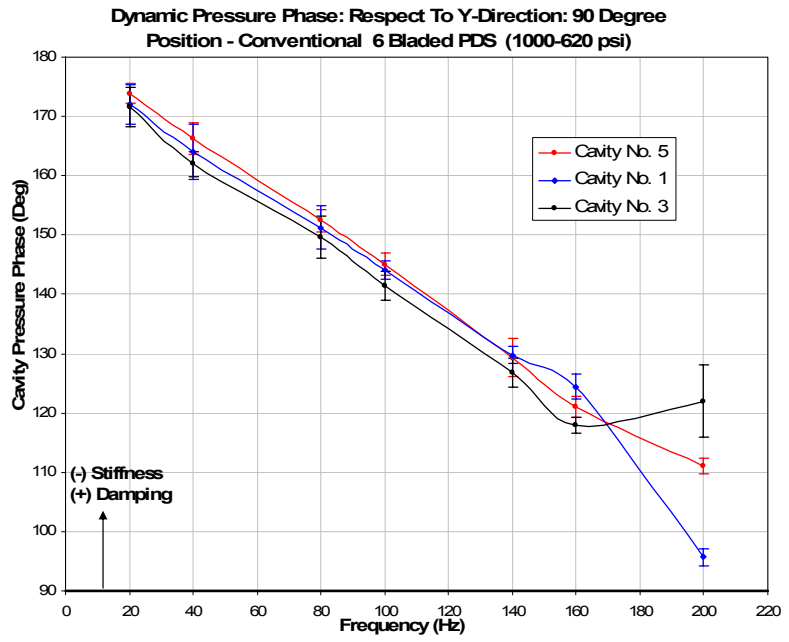

Cavity Pressure Phase Respect to Y Direction 

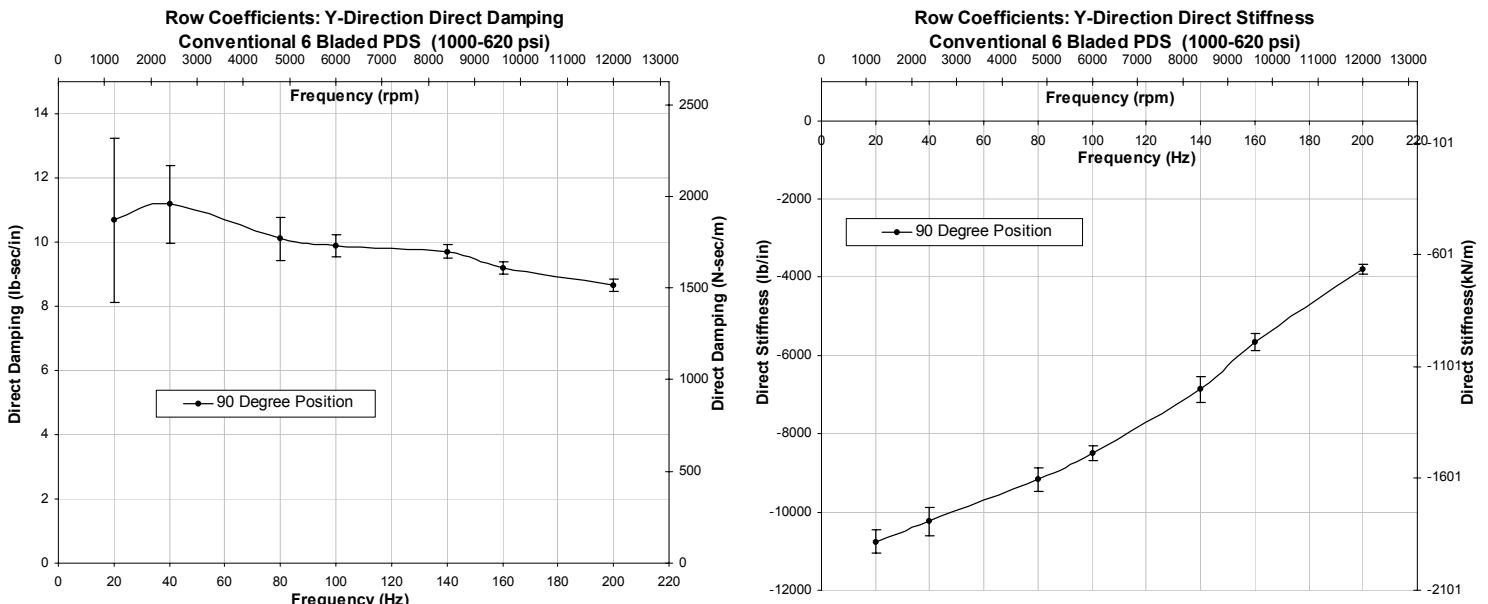

Total Row Direct Coefficients
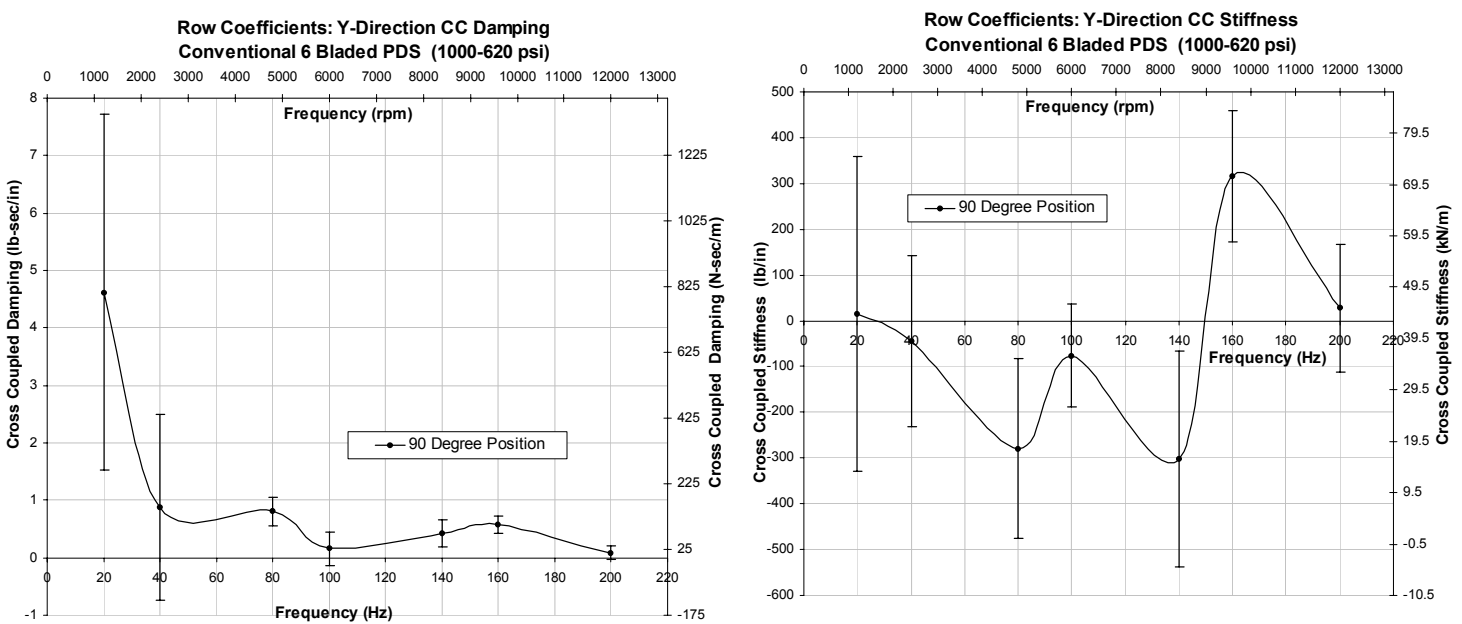

Total Row Cross-coupled Coefficients 


\section{Conventional 6 Bladed PDS Cavity Coefficients: 180 Degree Position}
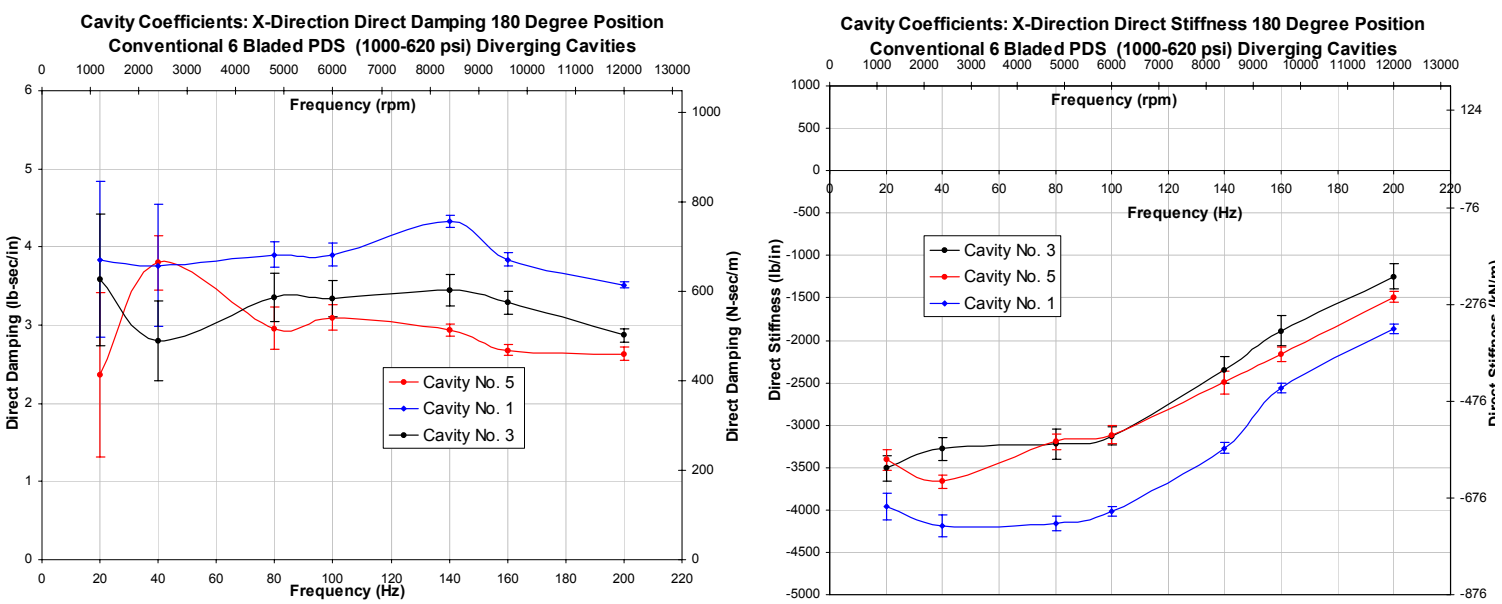

Cavity Direct Coefficients
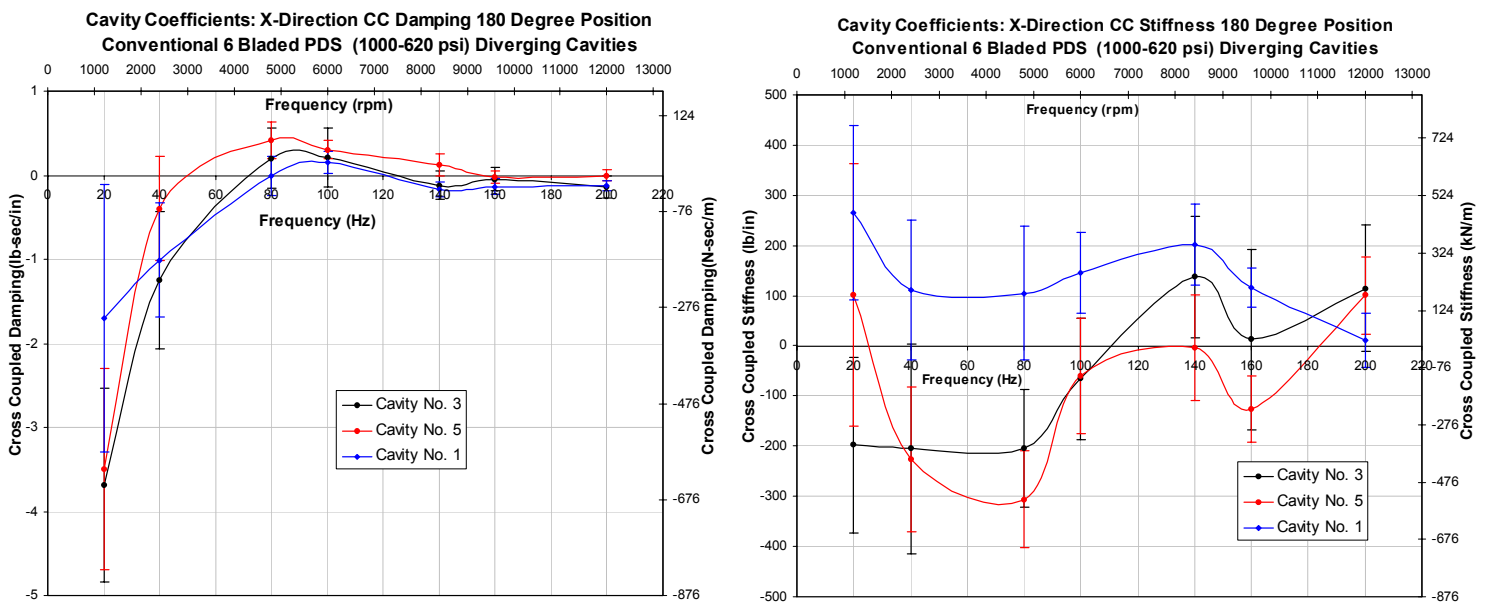

Cavity Cross-coupled Coefficients

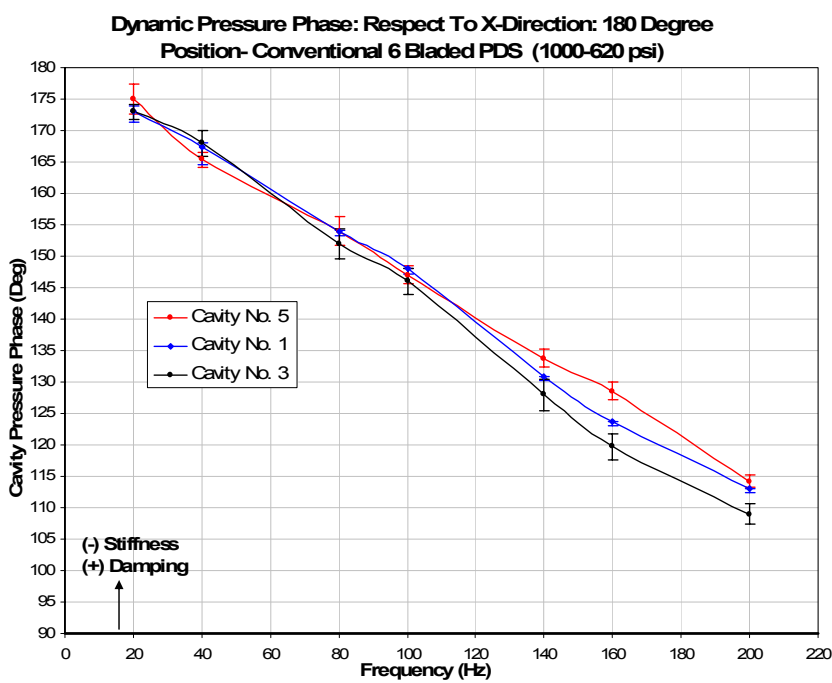

Cavity Pressure Phase Respect to X Direction 

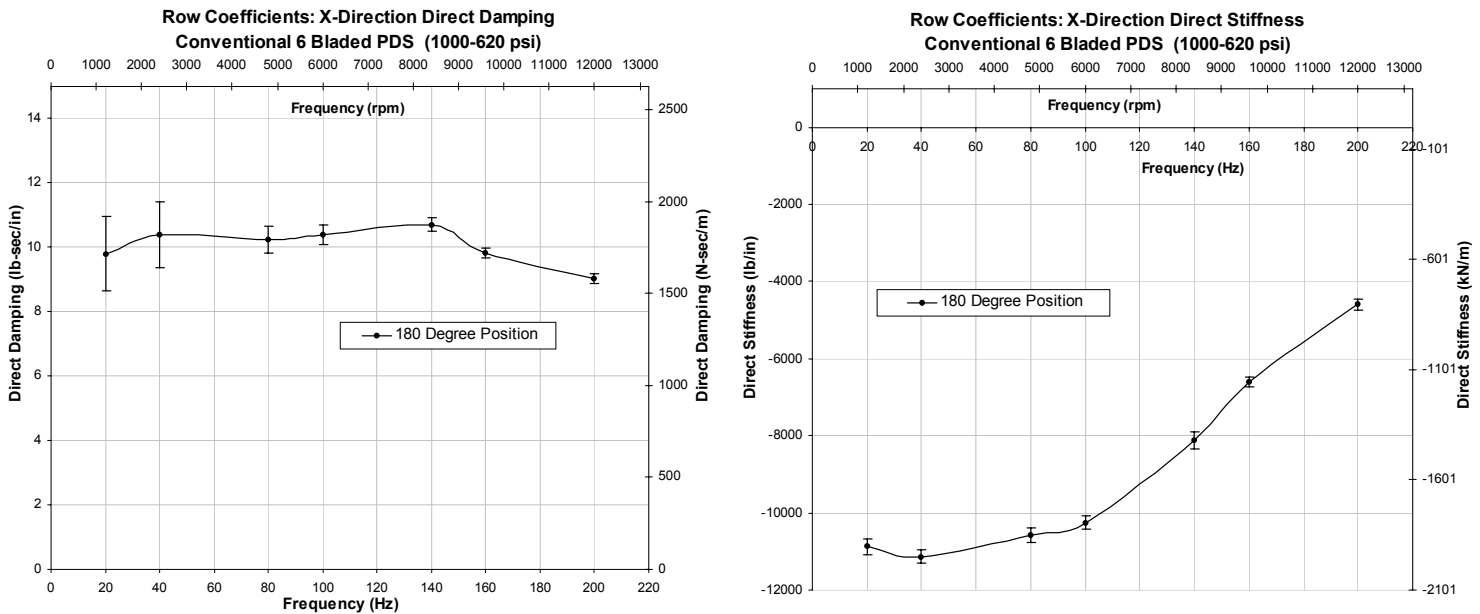

Total Row Direct Coefficients
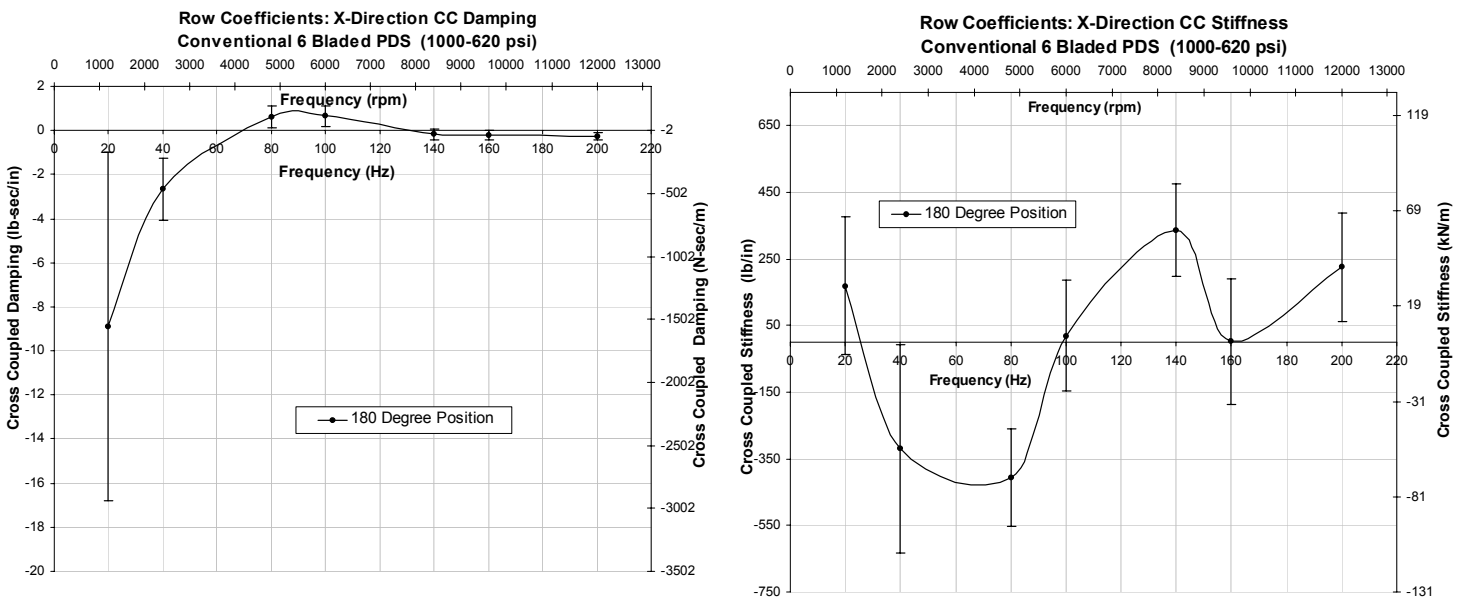

Total Row Cross-coupled Coefficients 
Conventional 6 Bladed PDS Cavity Coefficients: 270 Degree Position
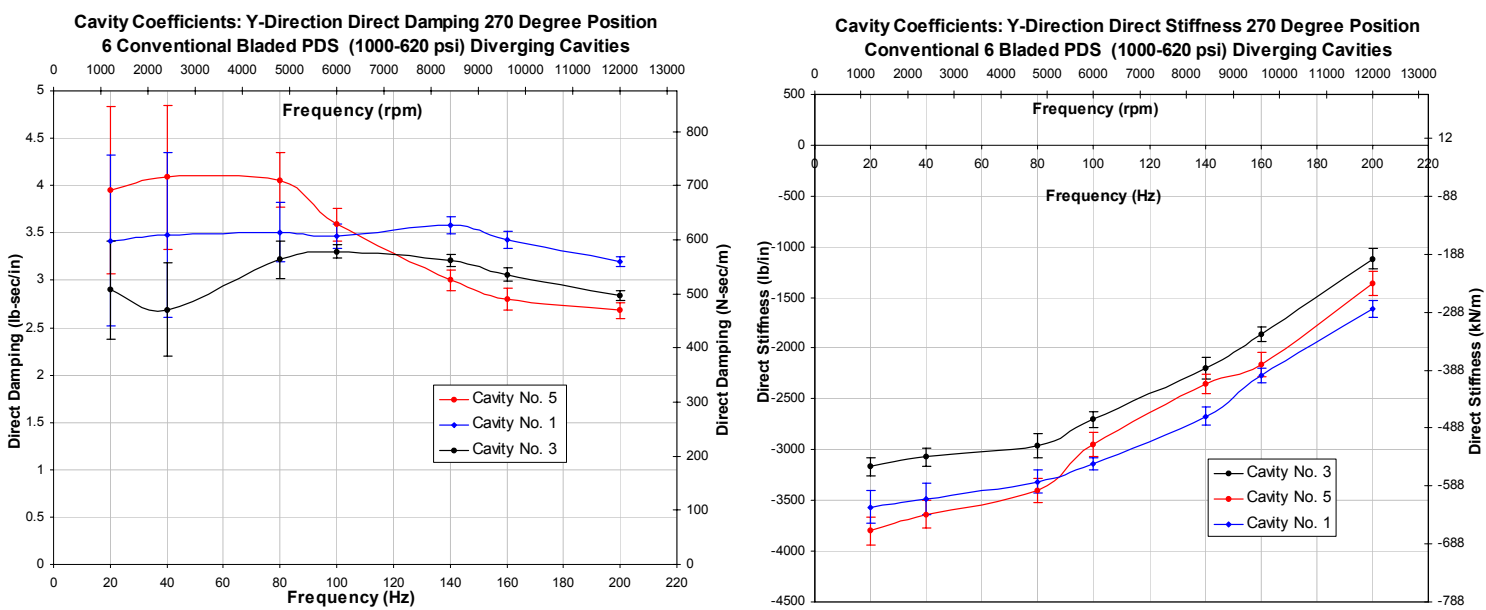

Cavity Direct Coefficients
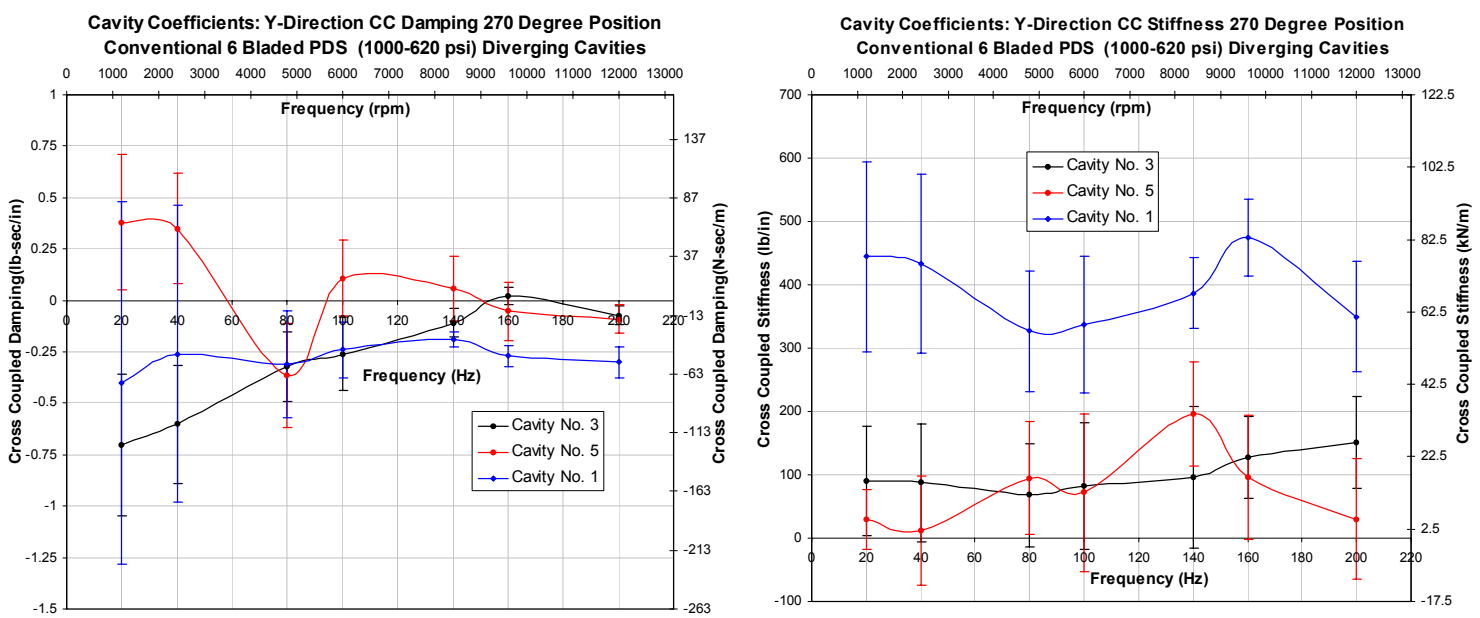

Cavity Cross-coupled Coefficients

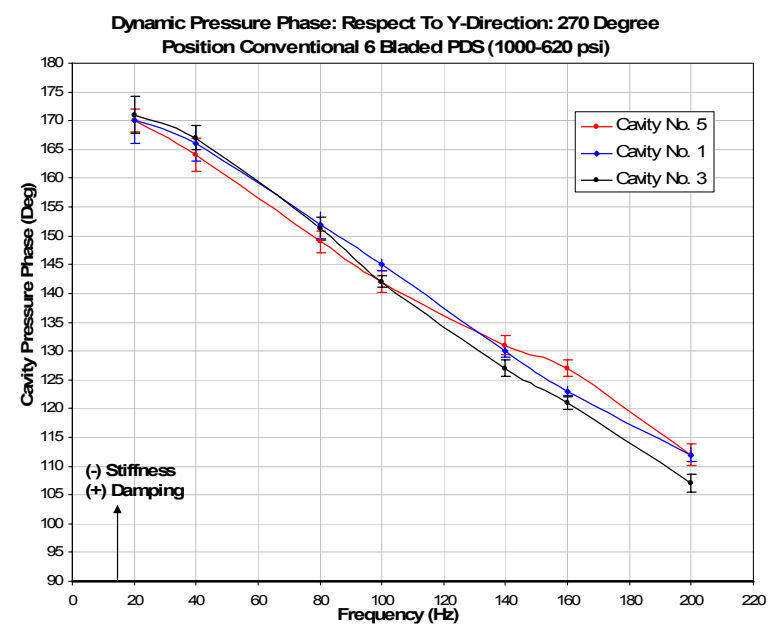

Cavity Pressure Phase Respect to Y Direction 

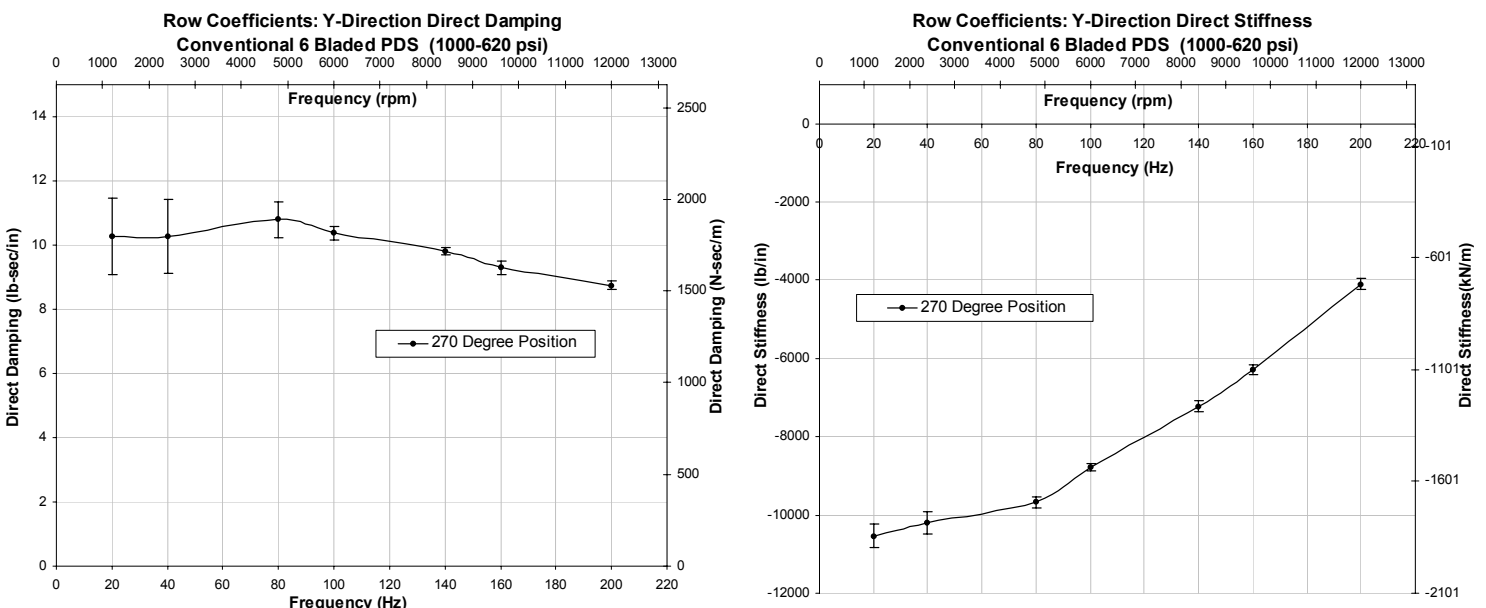

Total Row Direct Coefficients
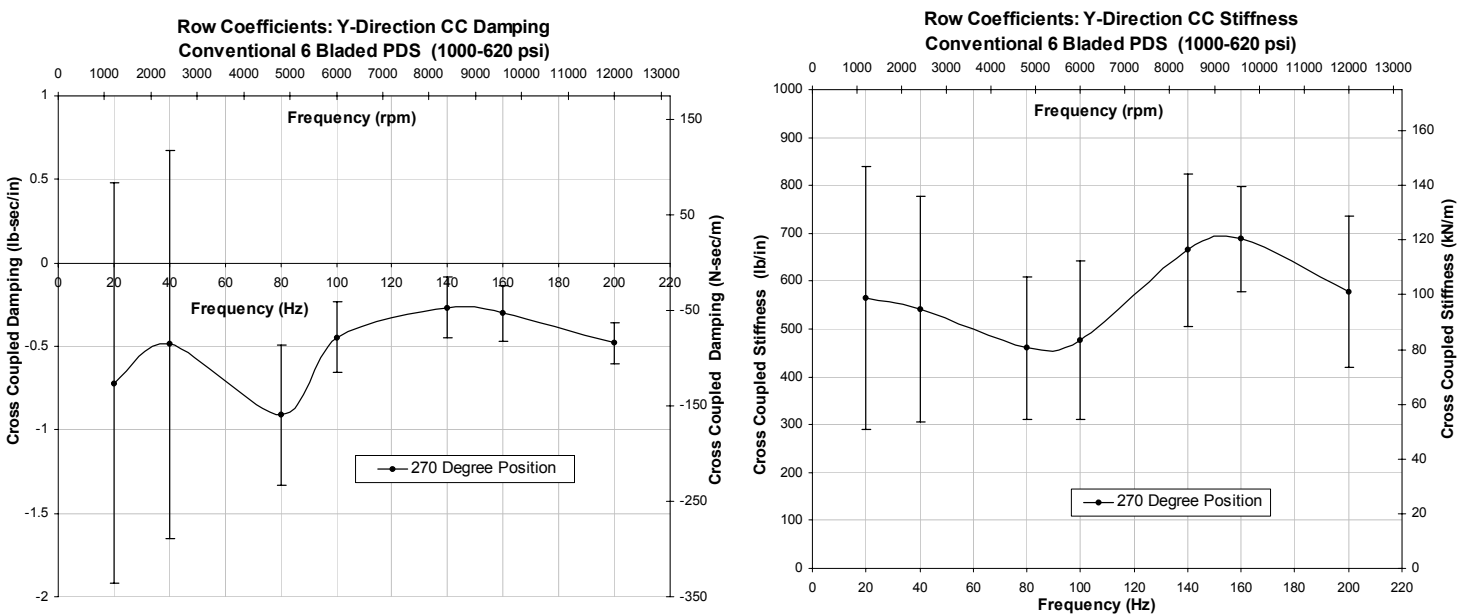

Total Row Cross-coupled Coefficients 


\section{Conventional 6 Bladed PDS Cavity Coefficients: 45 Degree Position}
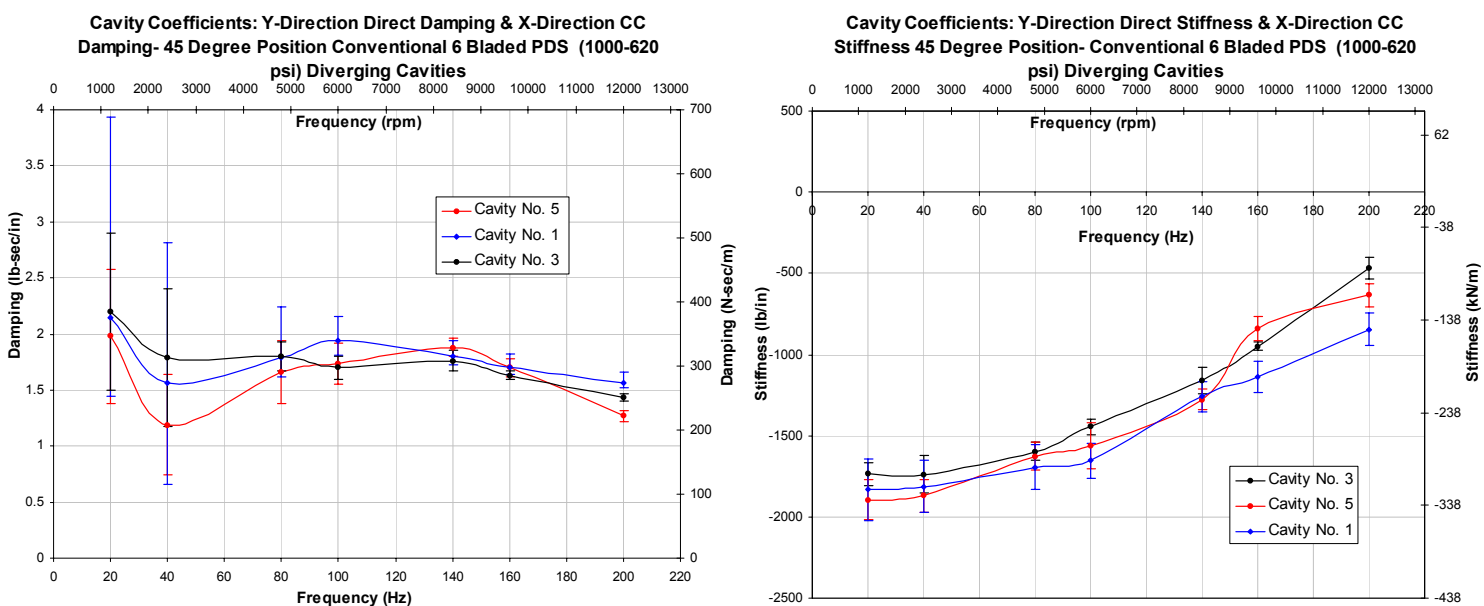

Y Direction Cavity Direct Coefficients \& X Direction Cavity Cross-coupled Coefficients
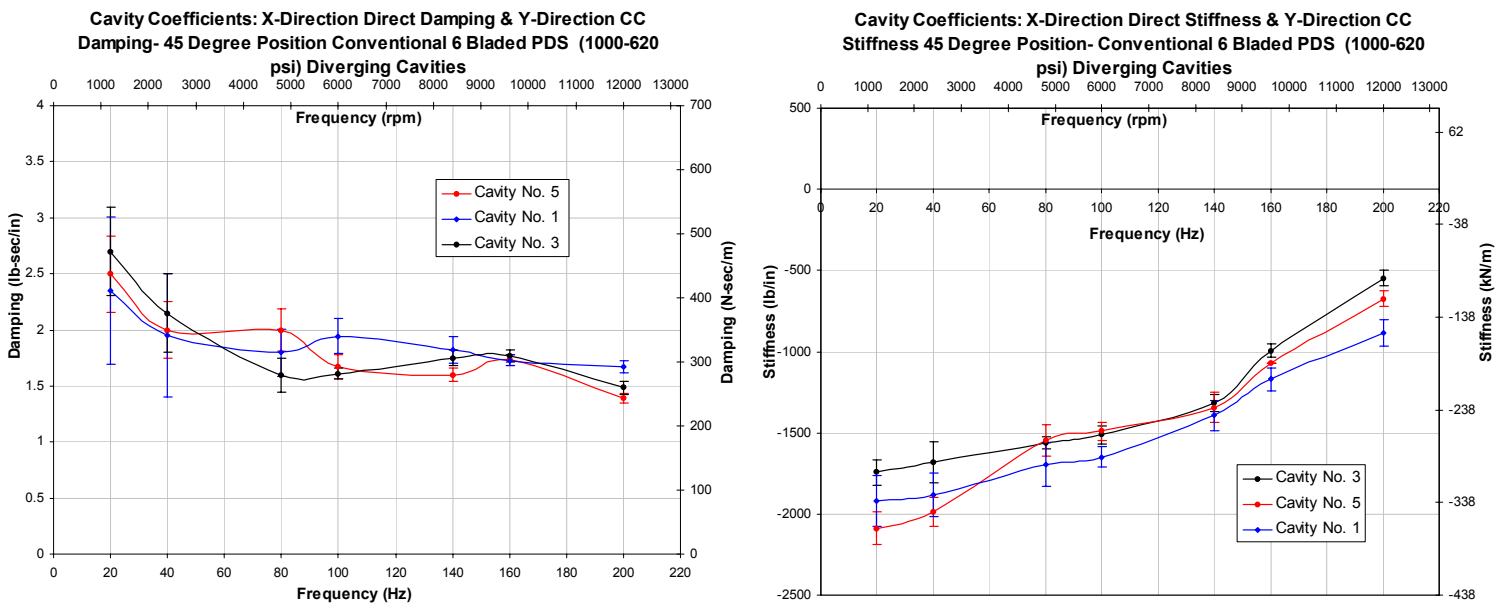

X Direction Cavity Direct Coefficients \& Y Direction Cavity Cross-coupled Coefficients
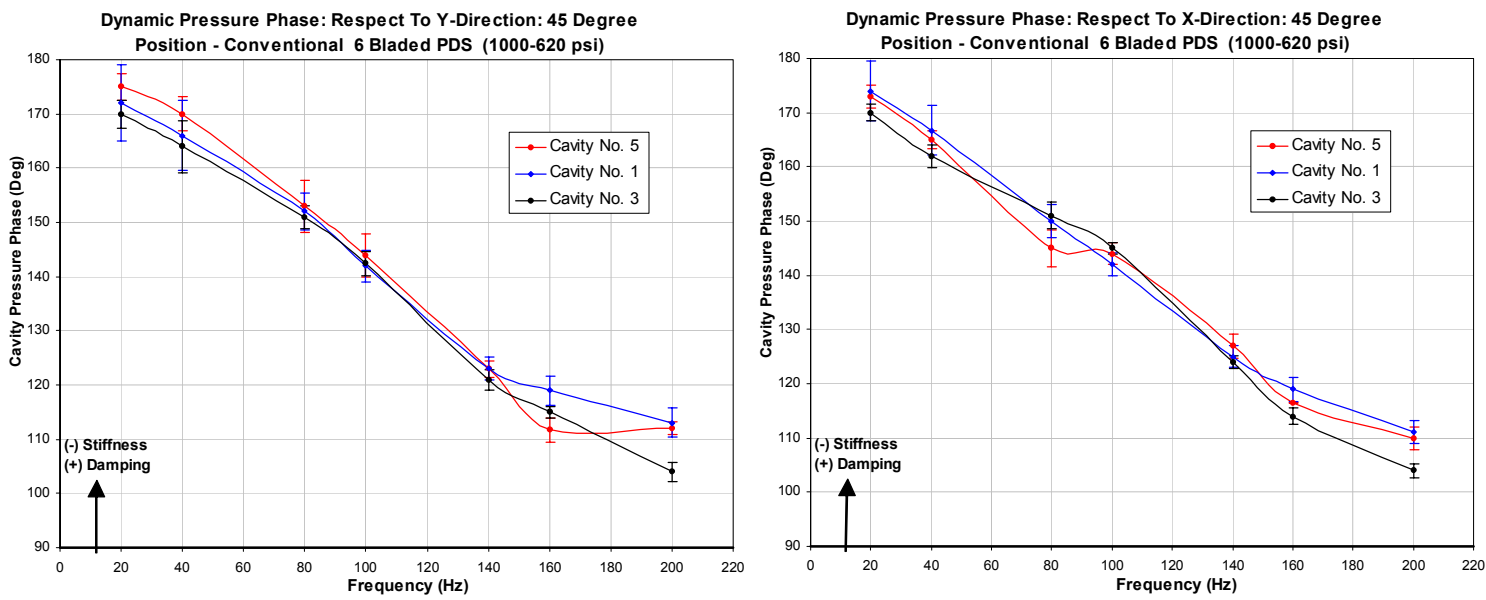

Dynamic Cavity Pressure Phase 

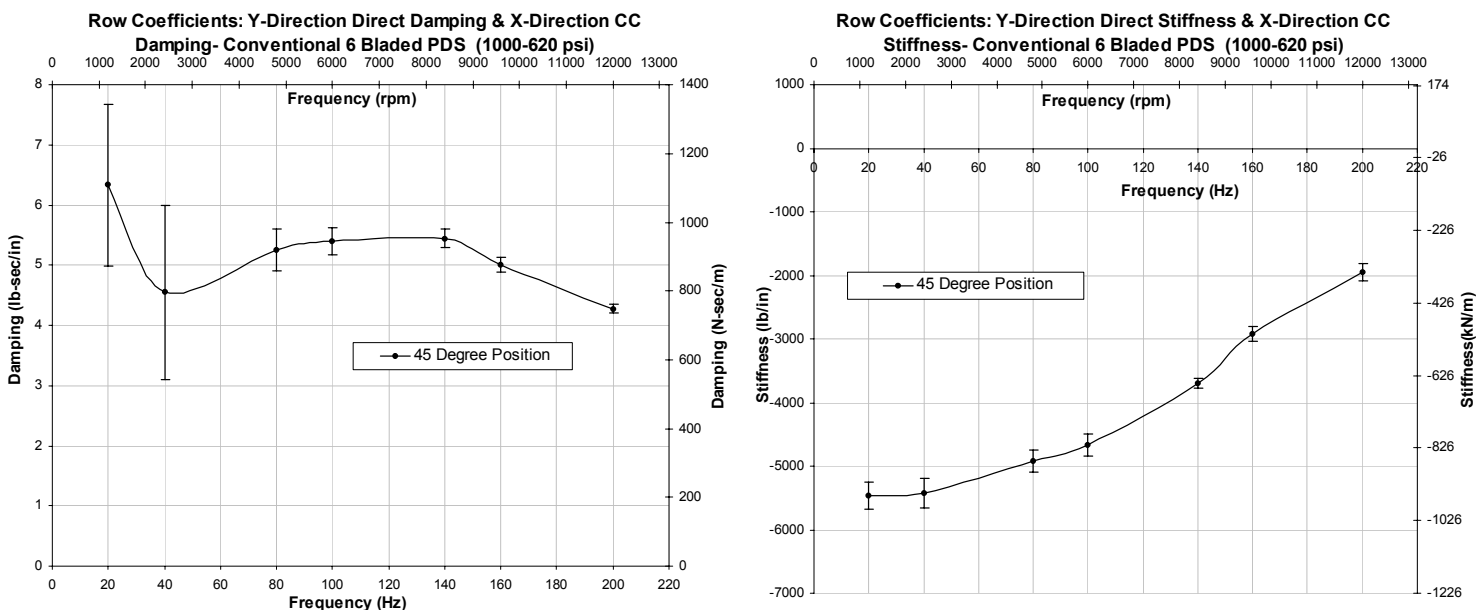

Total Row Coefficients- Y Direction Direct and X Direction Cross-coupled
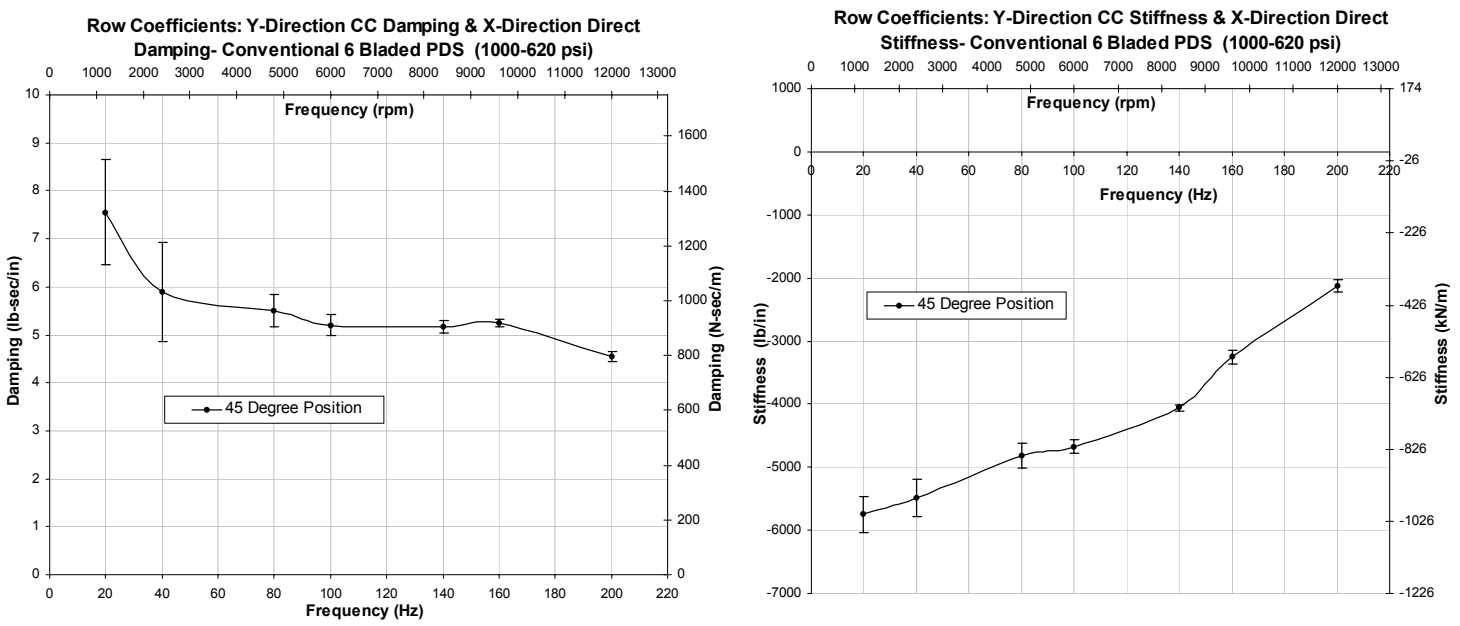

Total Row Coefficients- X Direction Direct and Y Direction Cross-coupled 


\section{Conventional 6 Bladed PDS Cavity Coefficients: 135 Degree Position}
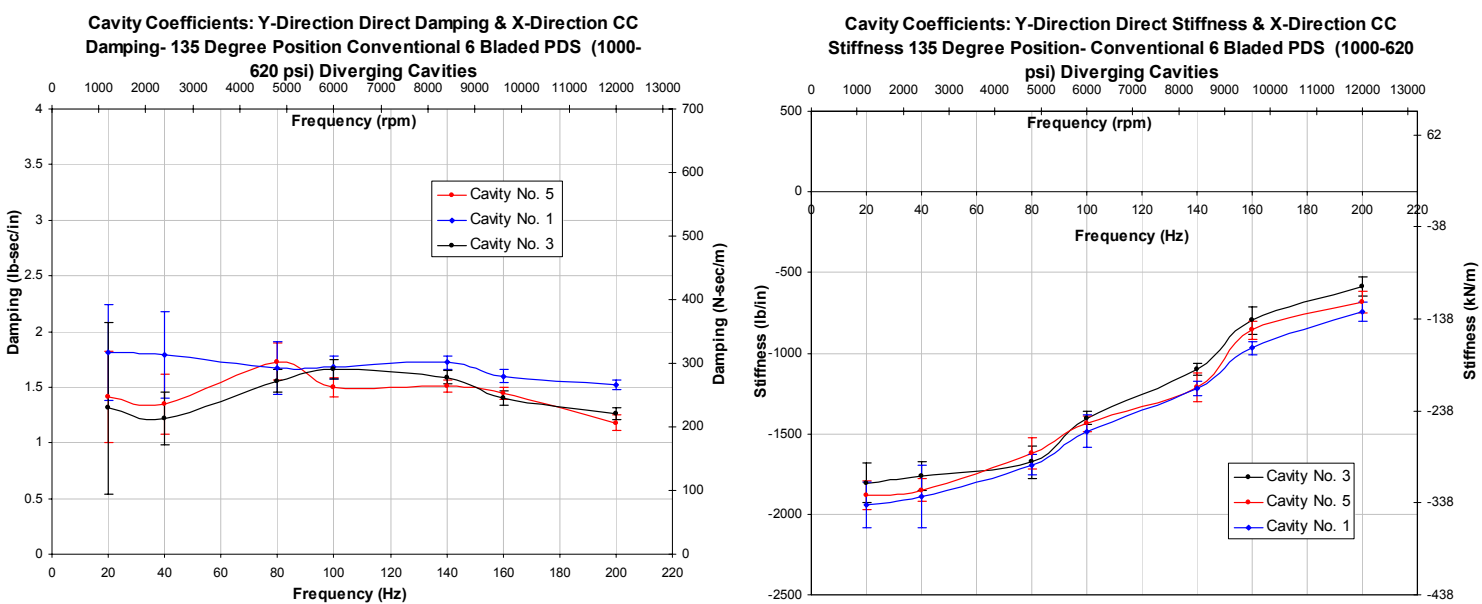

Y Direction Cavity Direct Coefficients \& X Direction Cavity Cross-coupled Coefficients
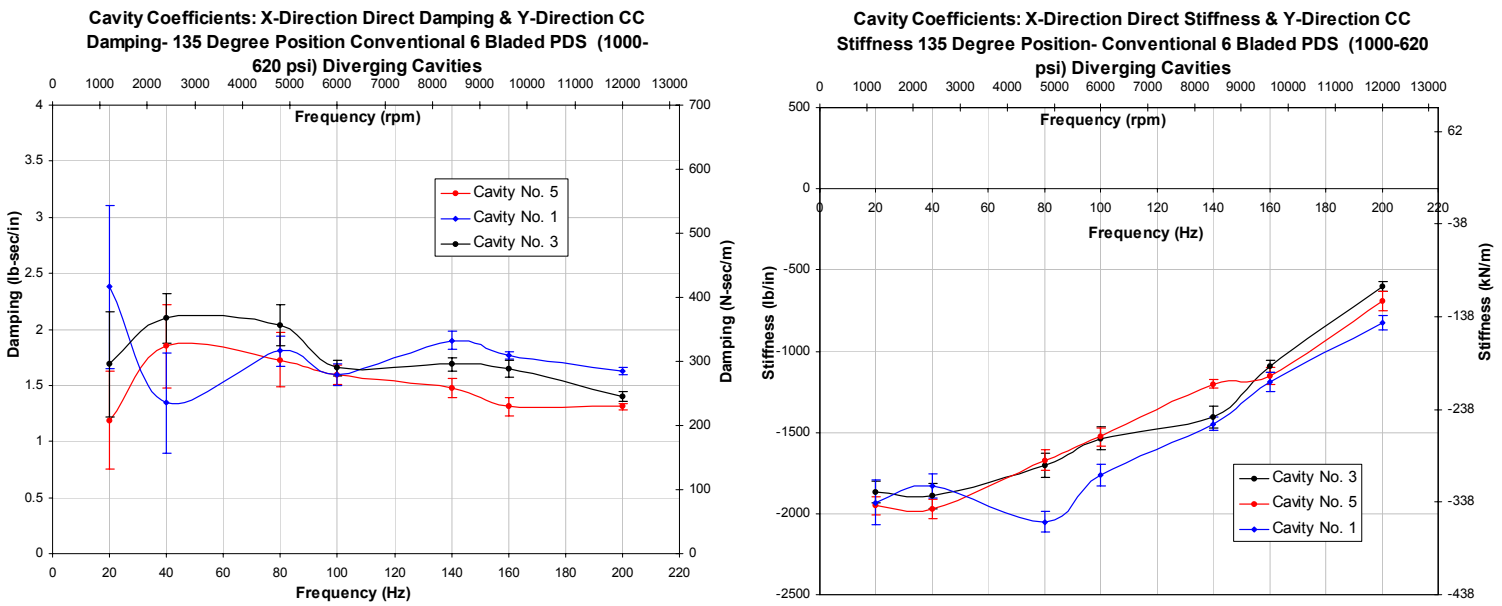

X Direction Cavity Direct Coefficients \& Y Direction Cavity Cross-coupled Coefficients
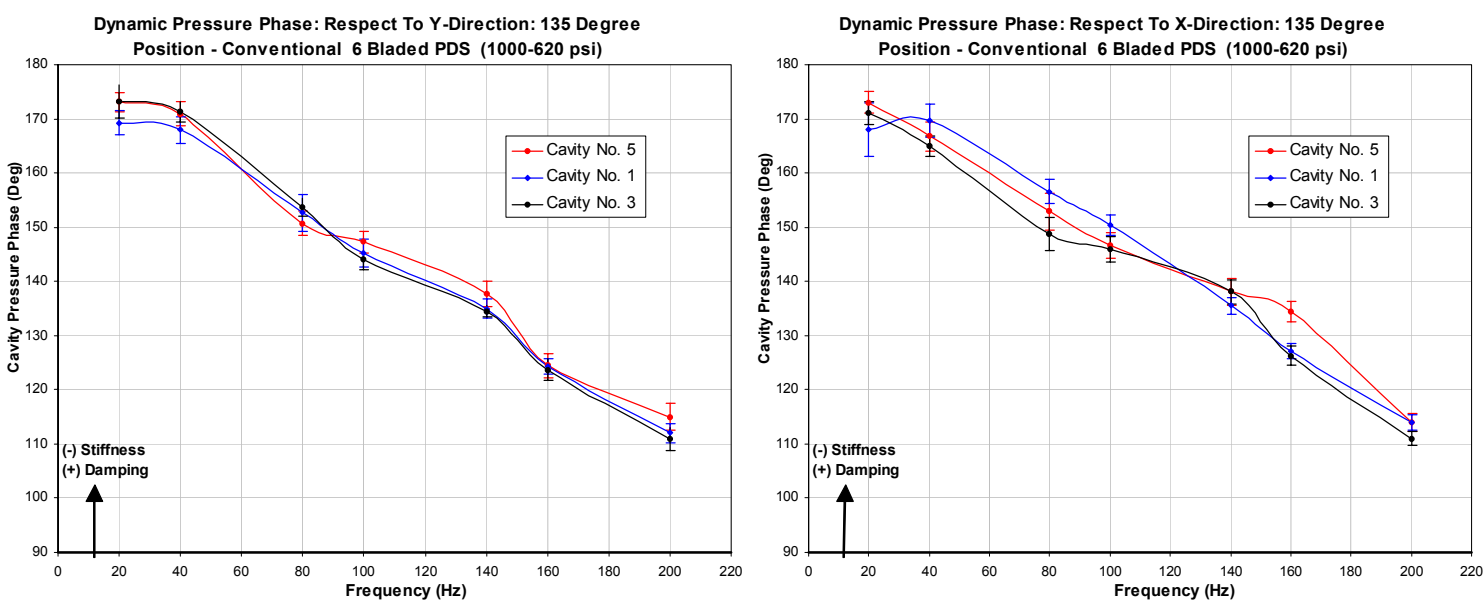

Dynamic Cavity Pressure Phase 

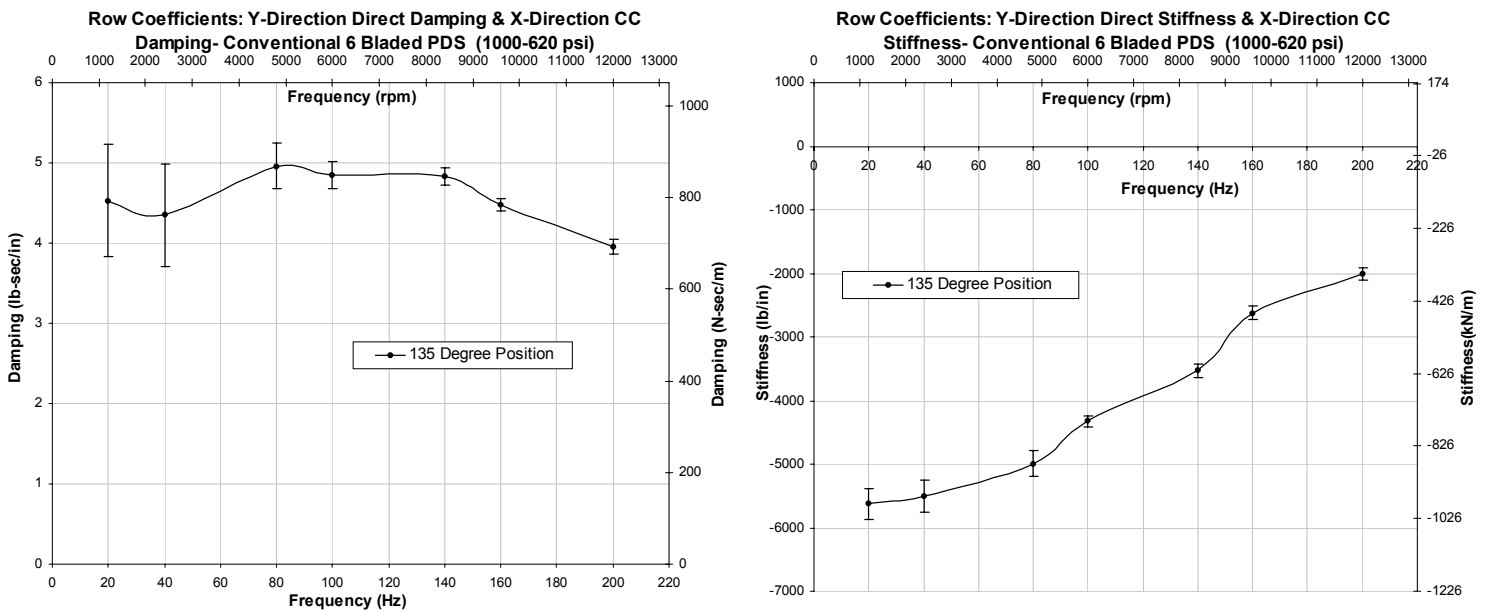

Total Row Coefficients- Y Direction Direct and X Direction Cross-coupled
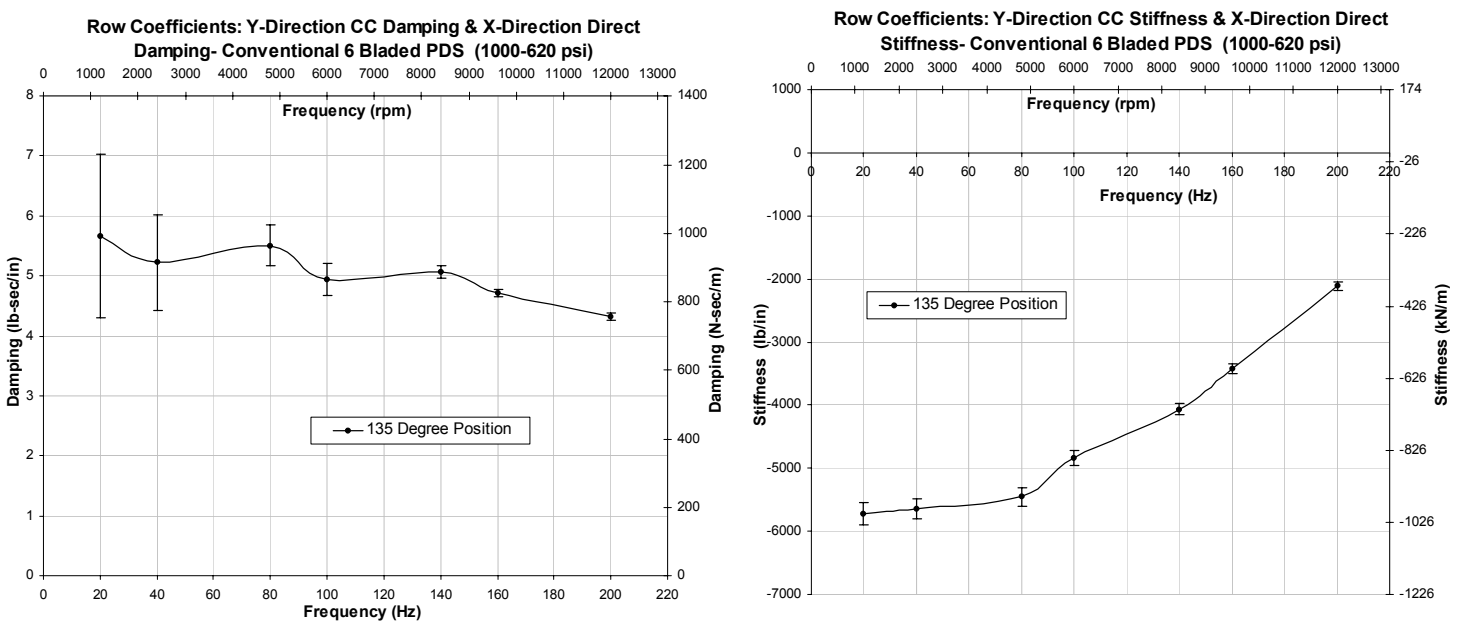

Total Row Coefficients- X Direction Direct and Y Direction Cross-coupled 
Conventional 6 Bladed PDS Cavity Coefficients: 225 Degree Position
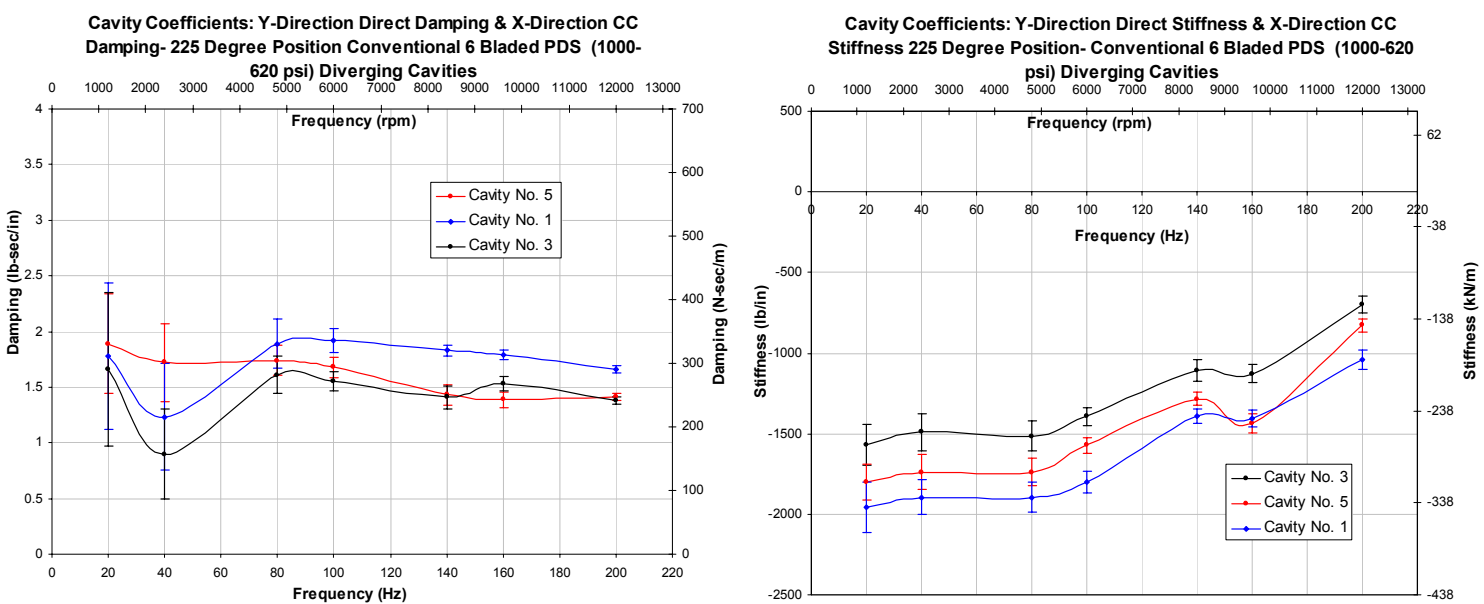

Y Direction Cavity Direct Coefficients \& X Direction Cavity Cross-coupled Coefficients
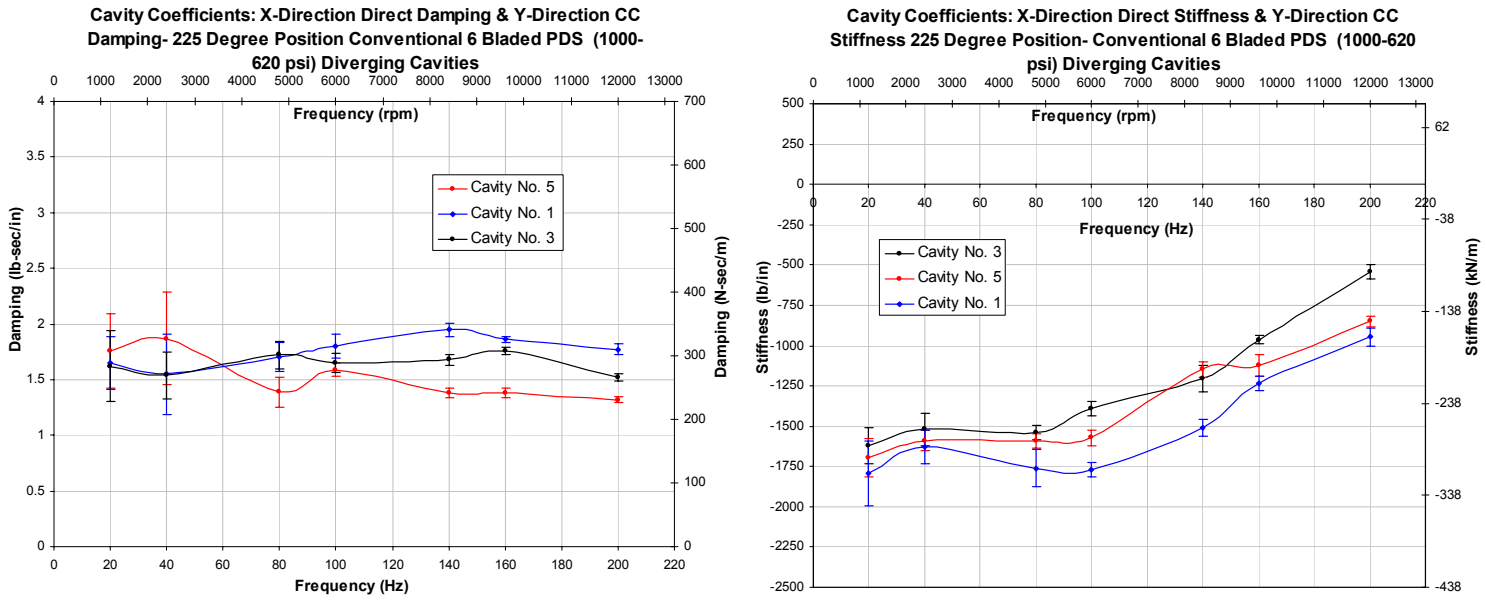

X Direction Cavity Direct Coefficients \& Y Direction Cavity Cross-coupled Coefficients
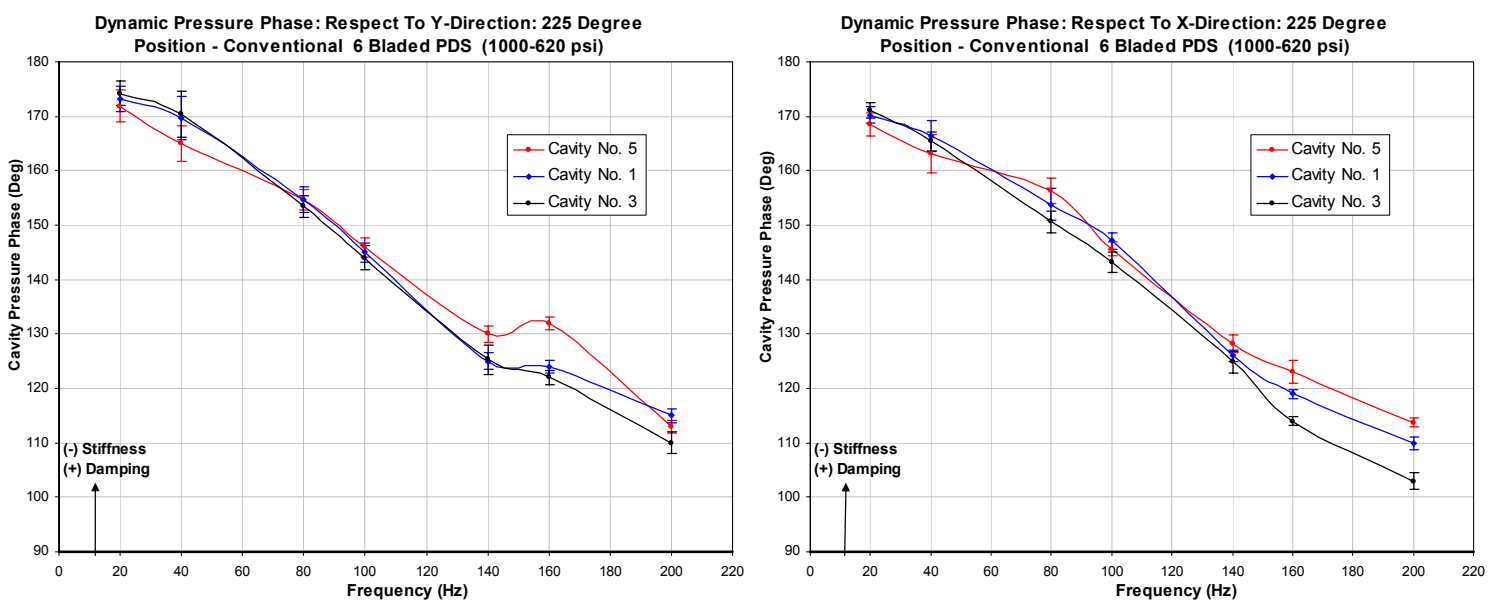

Dynamic Cavity Pressure Phase 

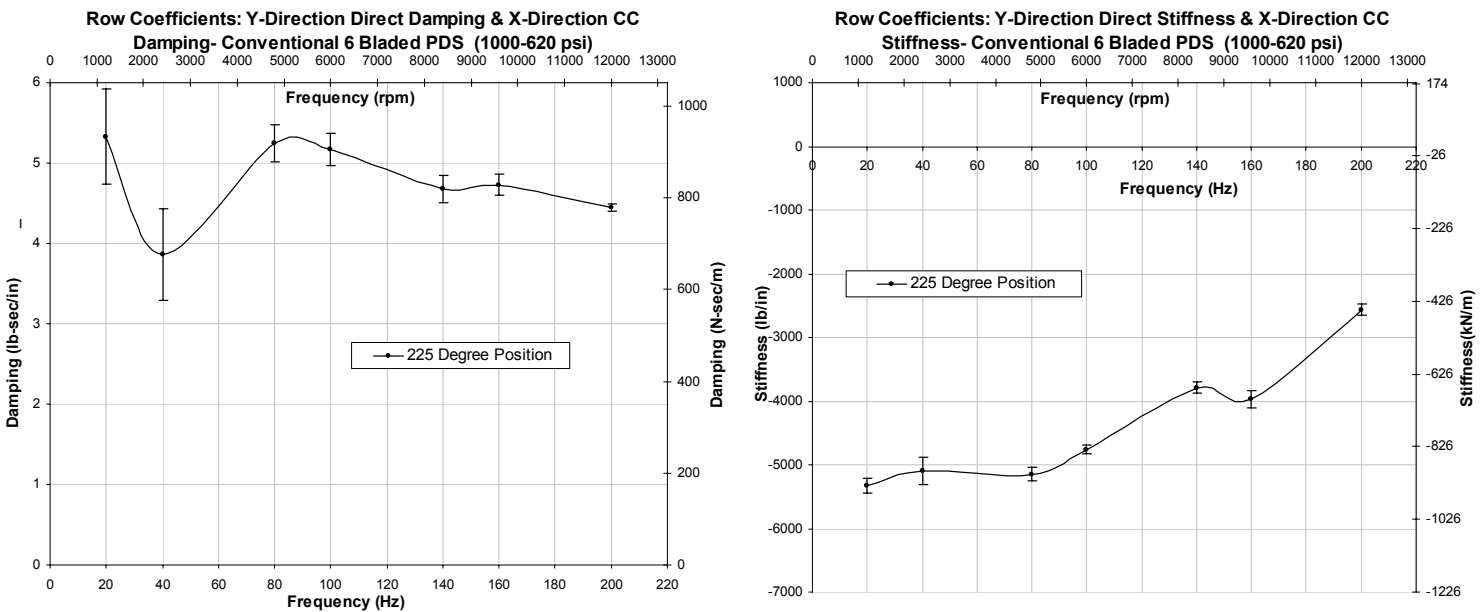

Total Row Coefficients- Y Direction Direct and X Direction Cross-coupled
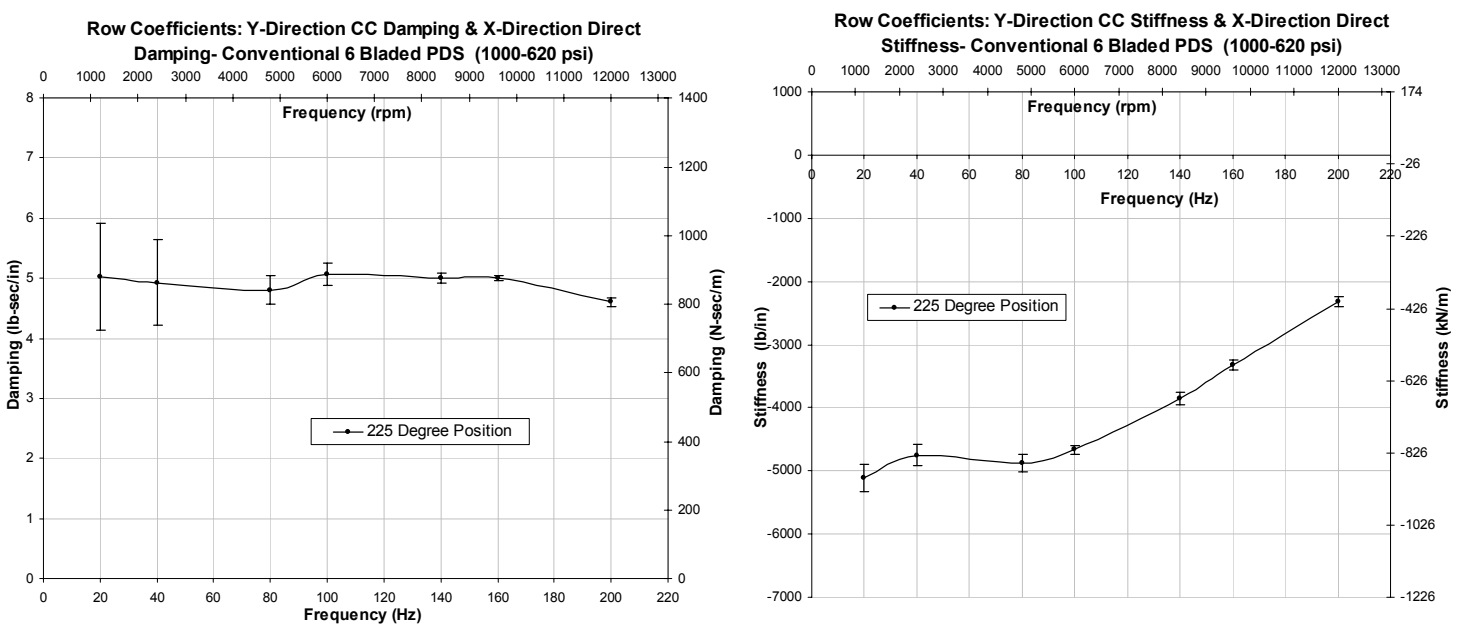

Total Row Coefficients- X Direction Direct and Y Direction Cross-coupled 


\section{Conventional 6 Bladed PDS Cavity Coefficients: 315 Degree Position}
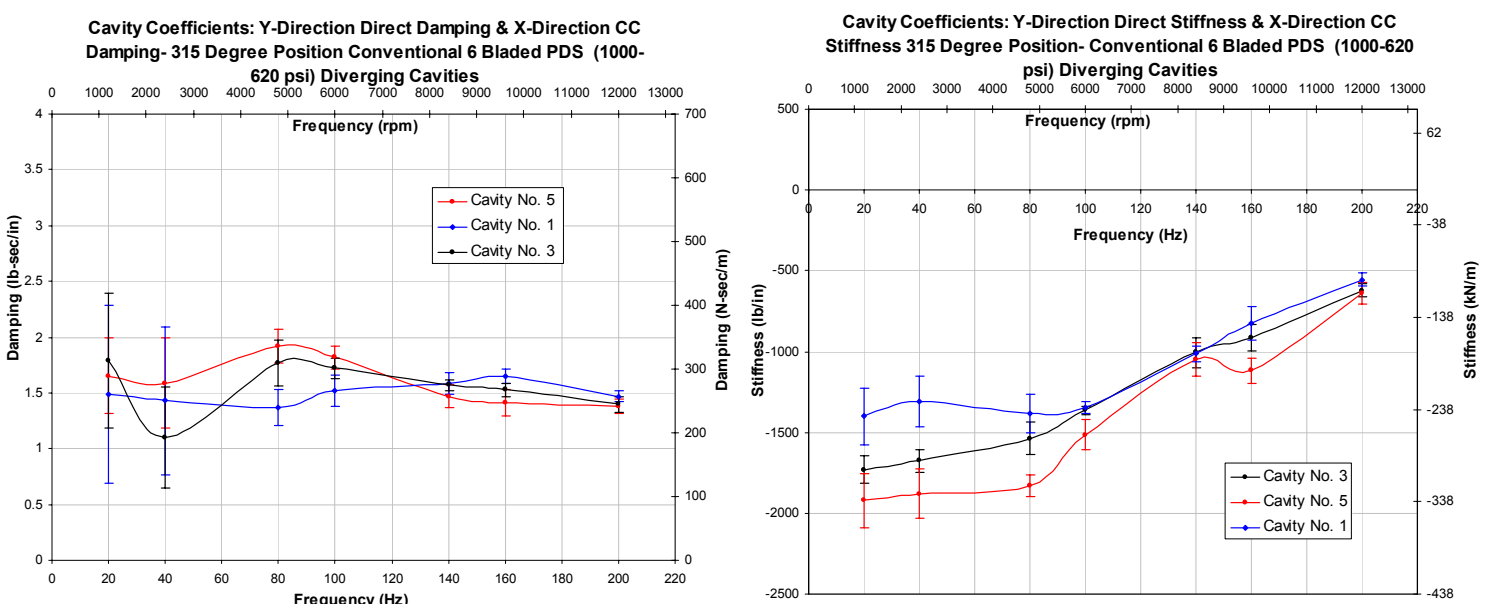

Y Direction Cavity Direct Coefficients \& X Direction Cavity Cross-coupled Coefficients
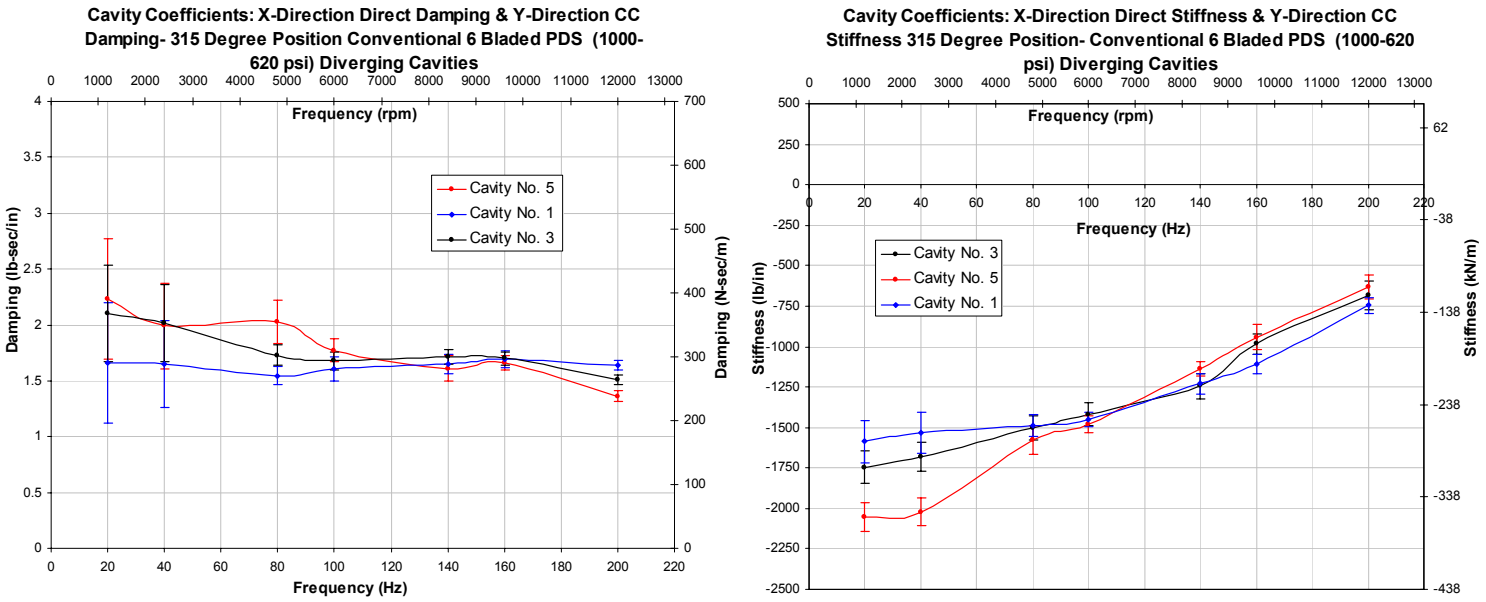

X Direction Cavity Direct Coefficients \& Y Direction Cavity Cross-coupled Coefficients
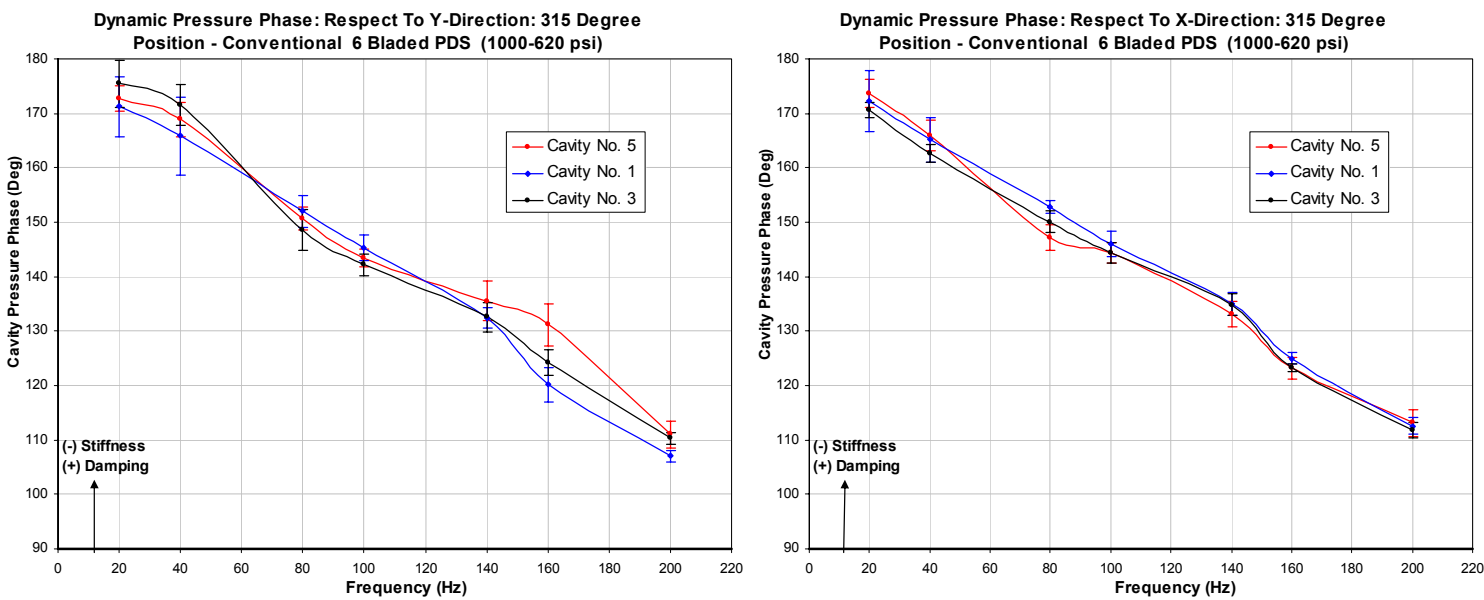

Dynamic Cavity Pressure Phase 

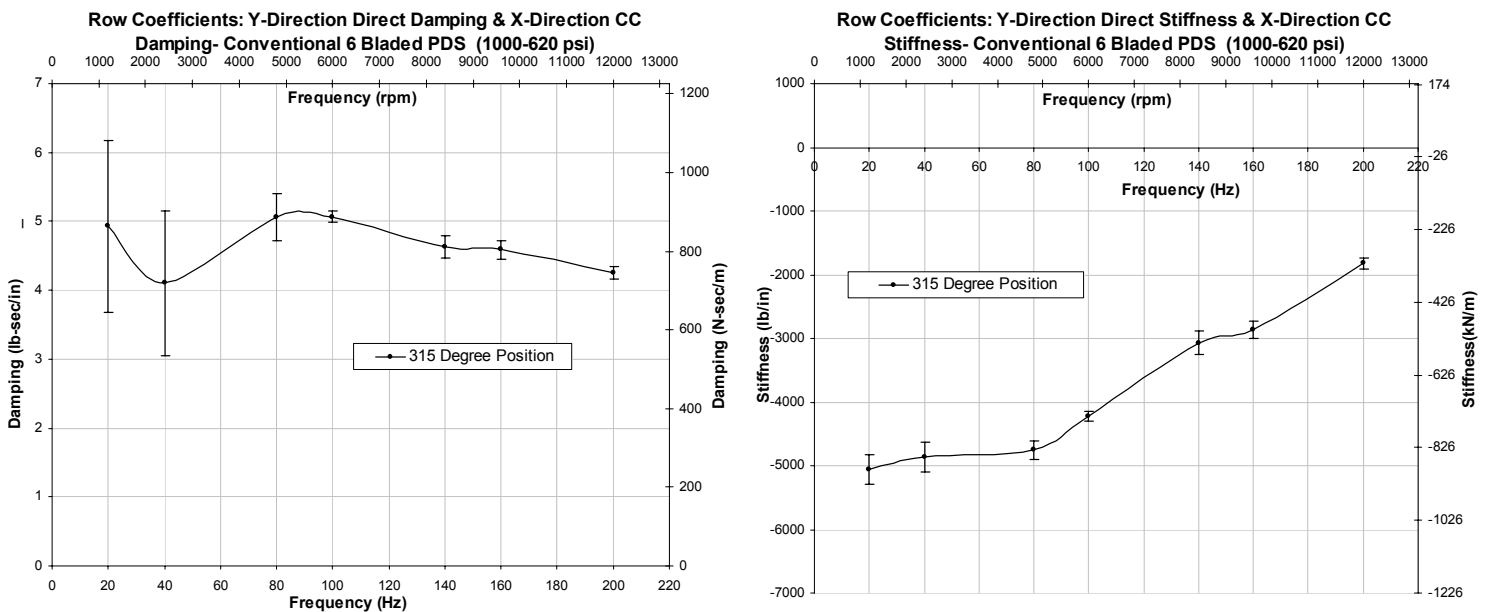

Total Row Coefficients- Y Direction Direct and X Direction Cross-coupled
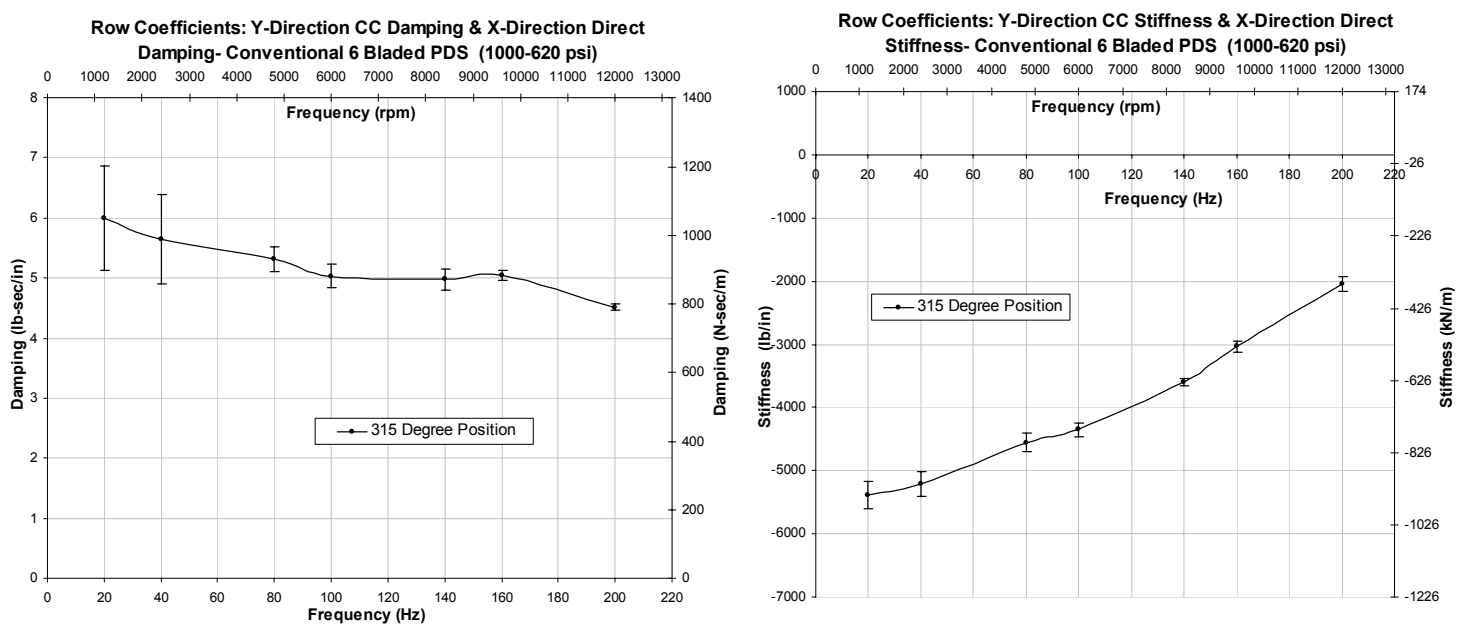

Total Row Coefficients- X Direction Direct and Y Direction Cross-coupled 


\section{Bladed Fully Partitioned Seal Cavity Coefficients: 0 Degree Position}
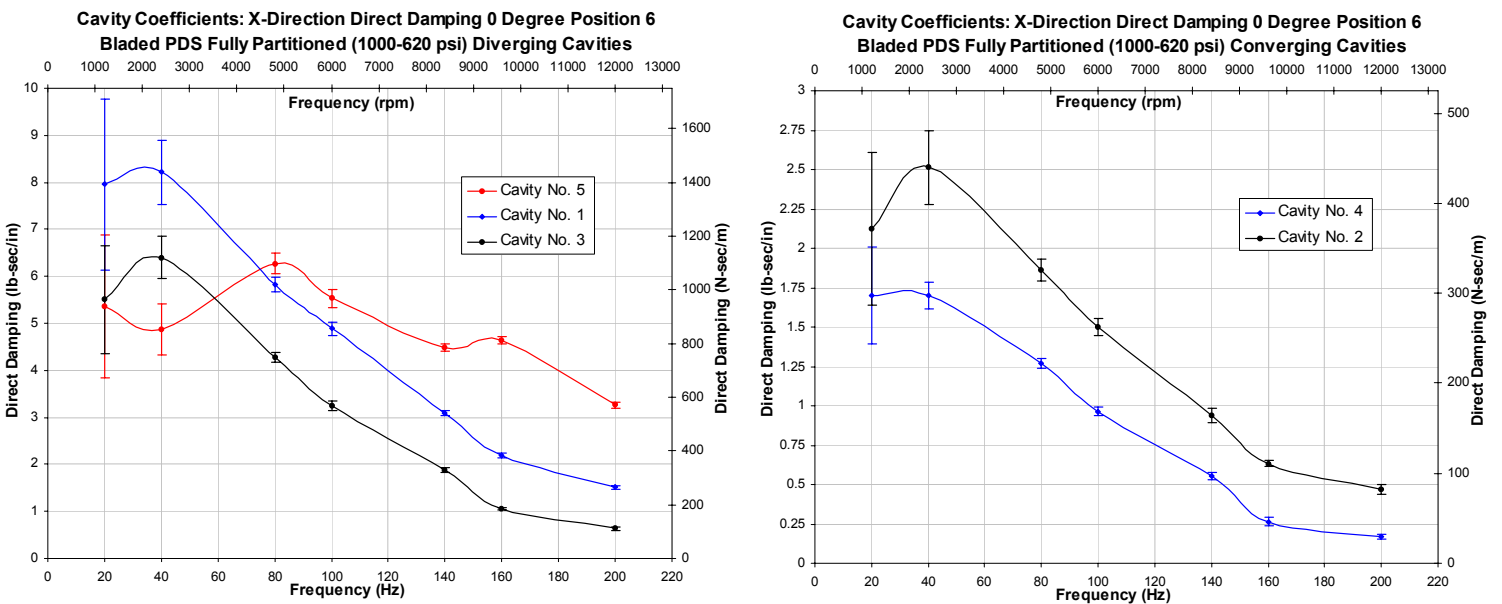

Cavity Direct Damping
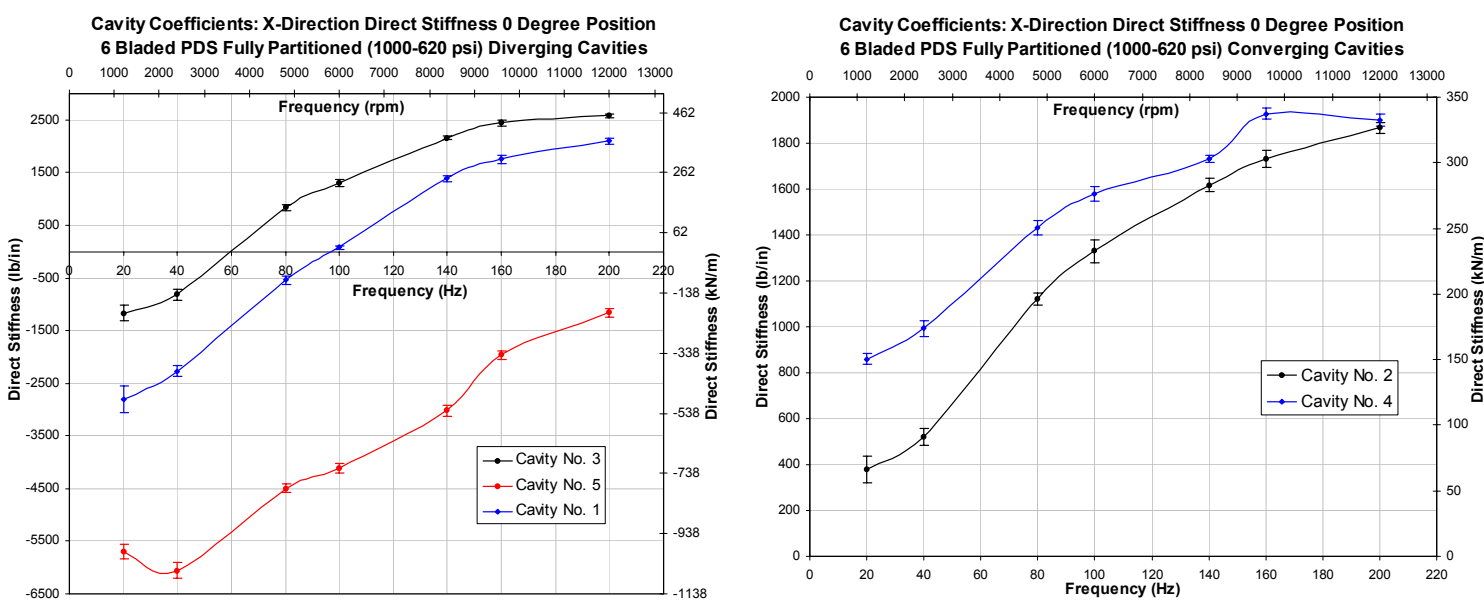

Cavity Direct Stiffness
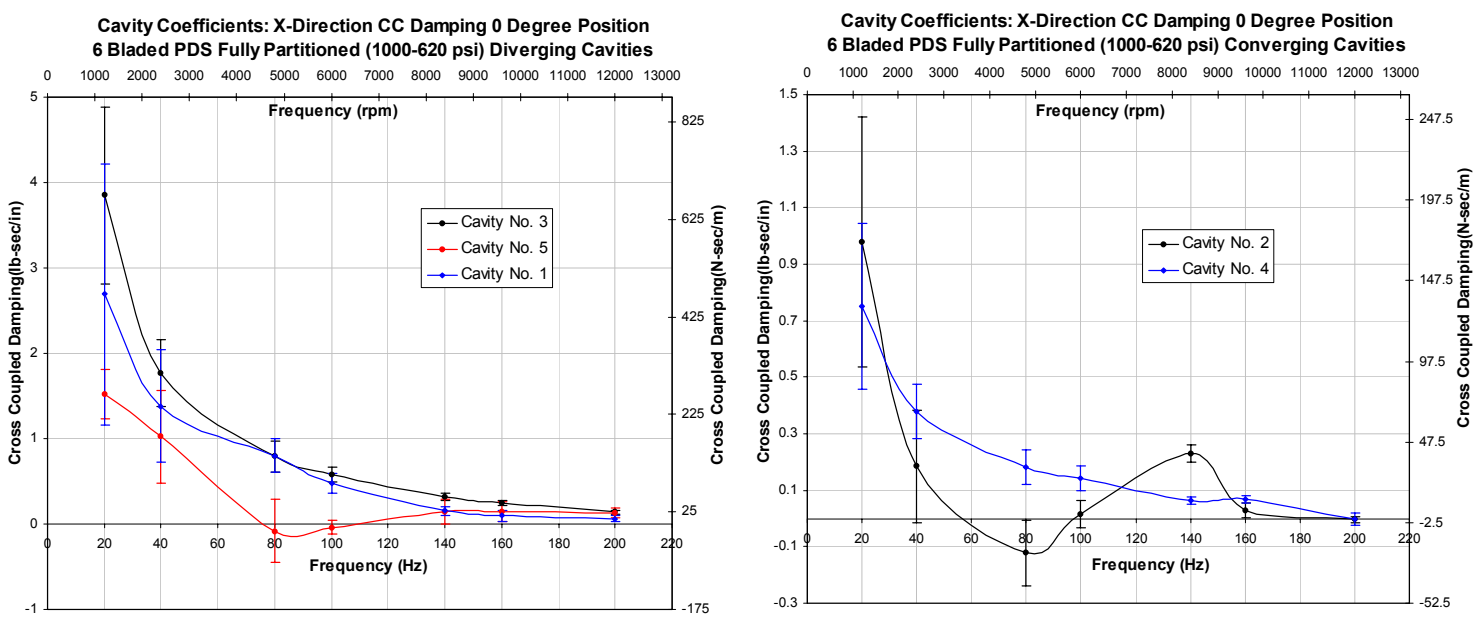

Cavity Cross-coupled Damping 

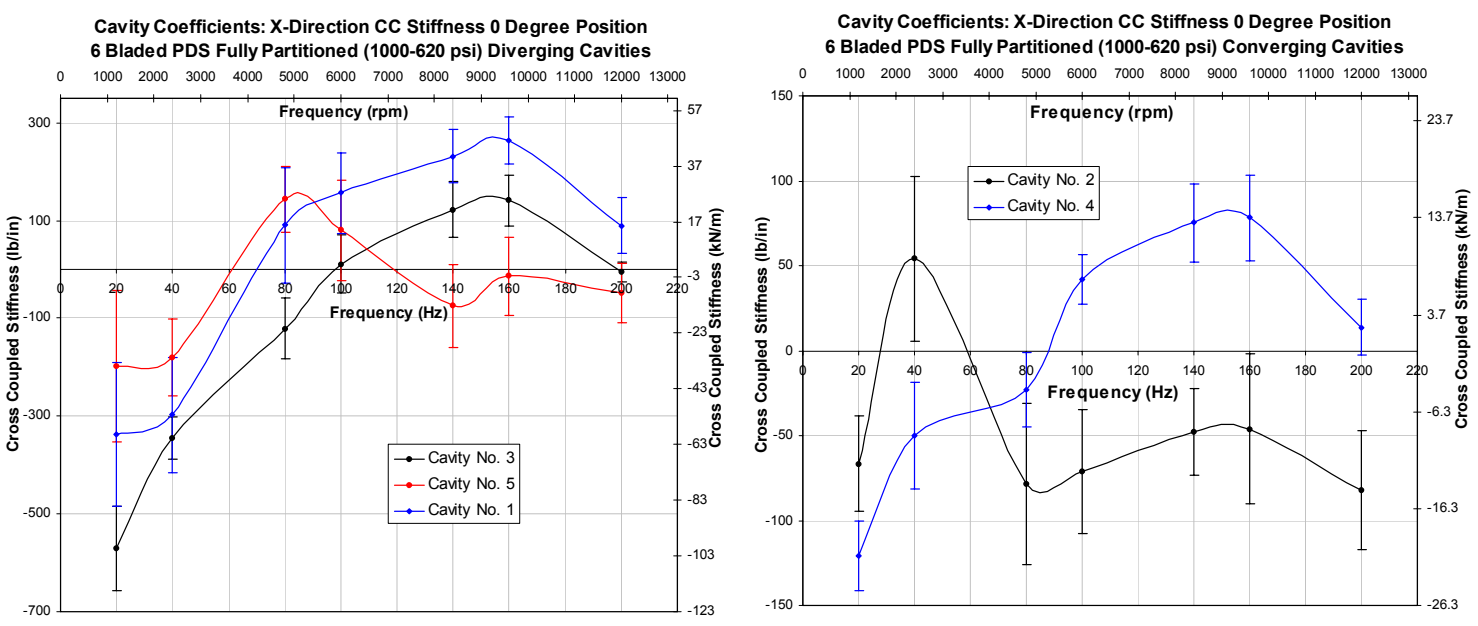

Cavity Cross-coupled Stiffness

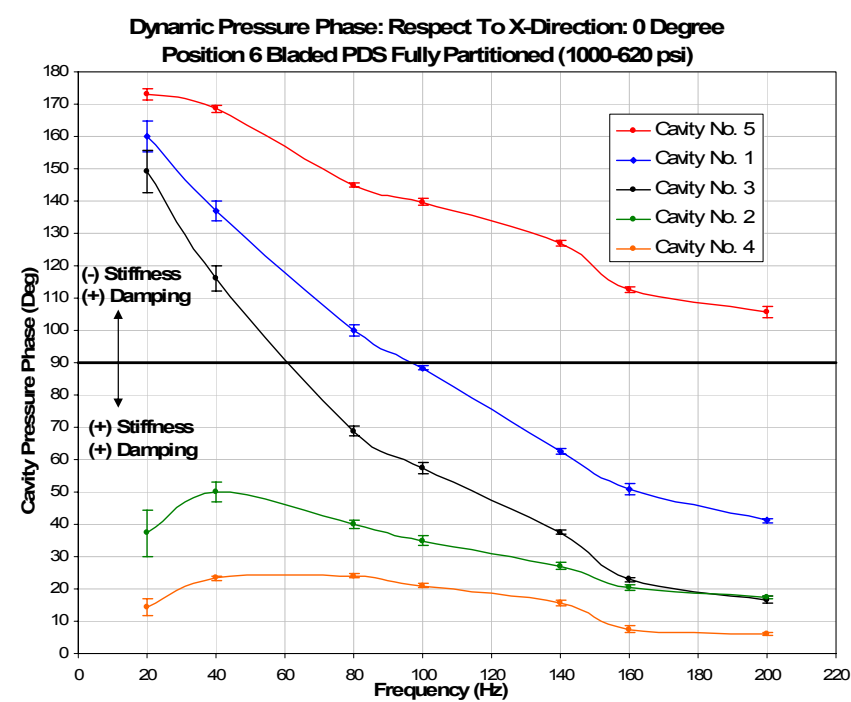

Cavity Phase Respect to X direction Excitation
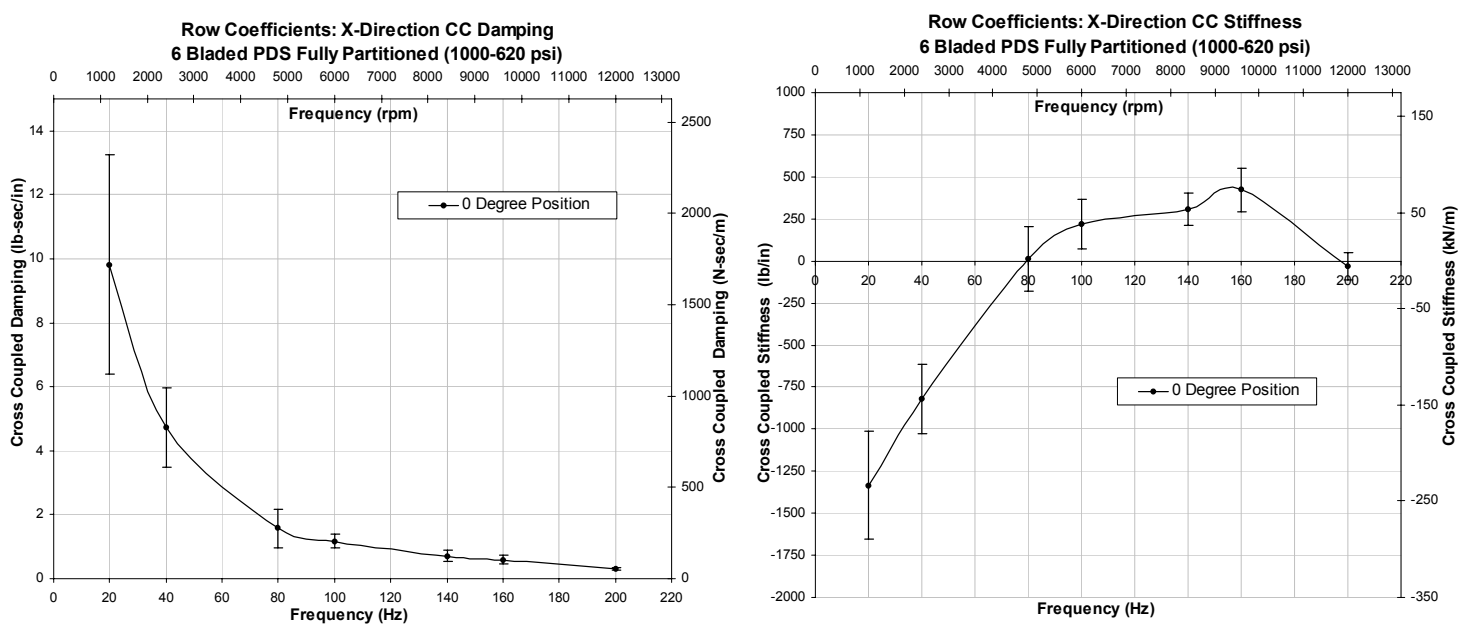

Total Row Cross-coupled Coefficients 

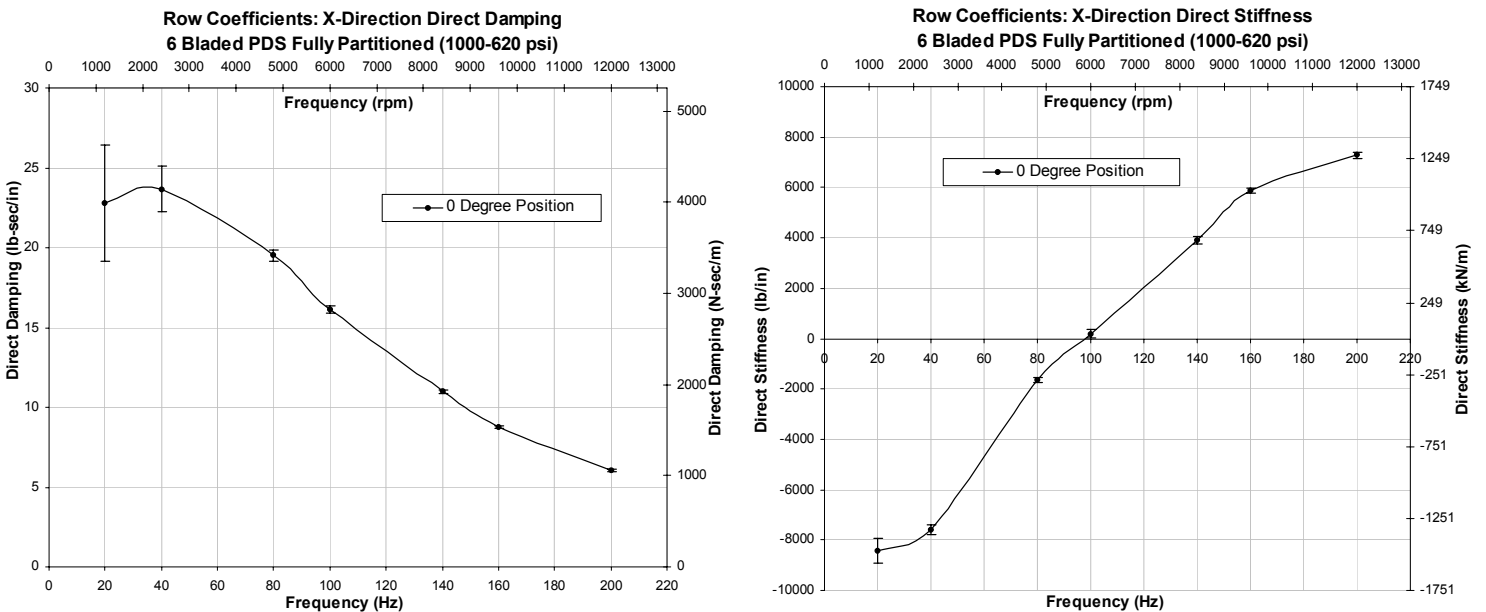

Total Row Direct Coefficients 


\section{Bladed Fully Partitioned Seal Cavity Coefficients: 90 Degree Position}
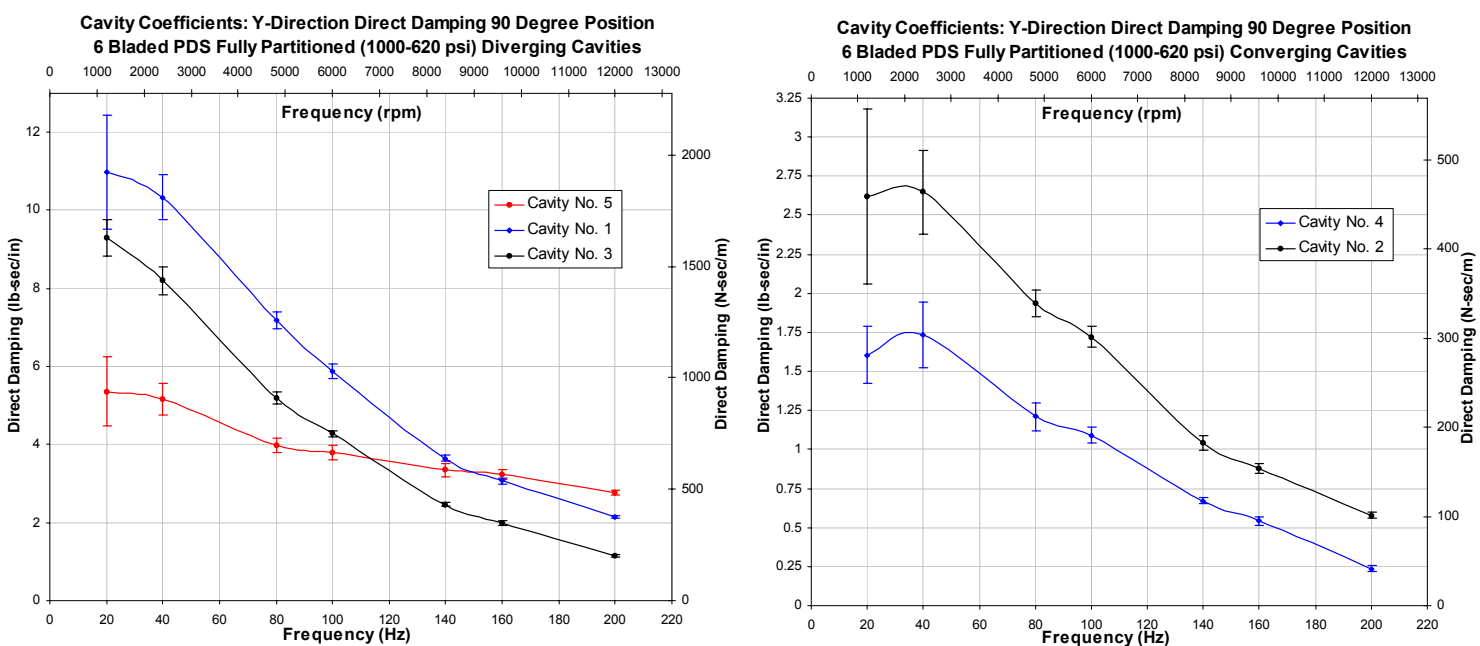

Cavity Direct Damping
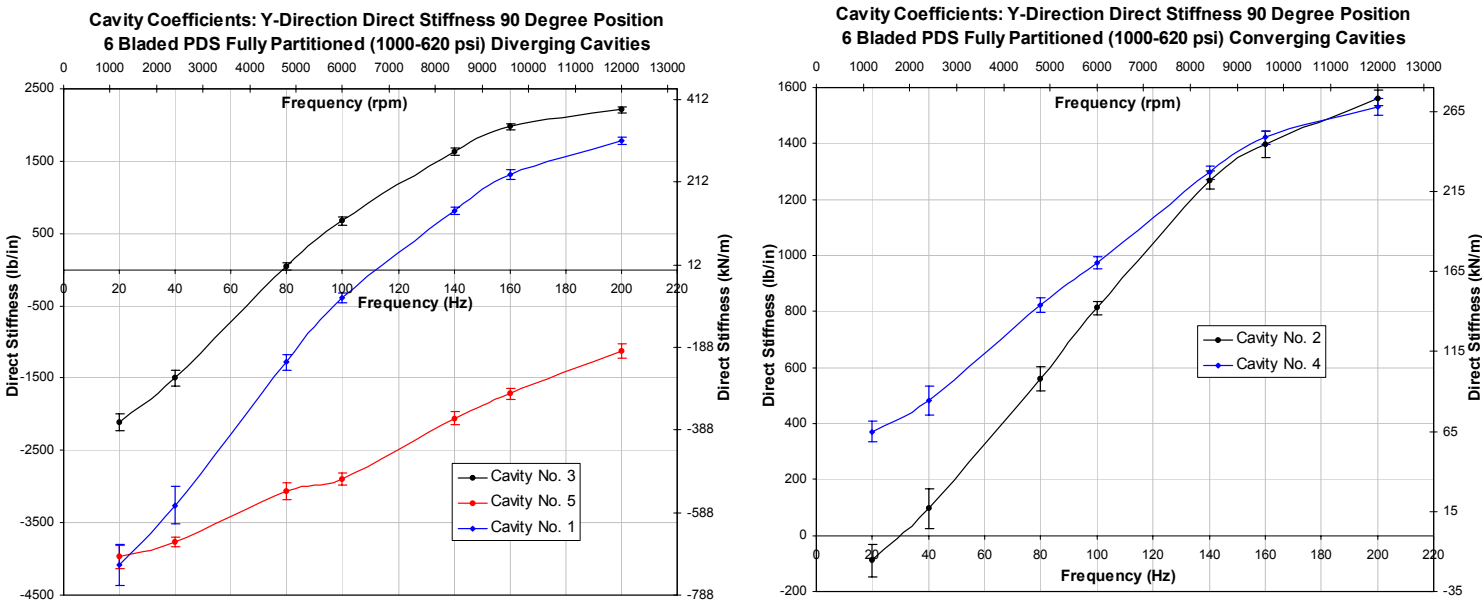

Cavity Direct Stiffness
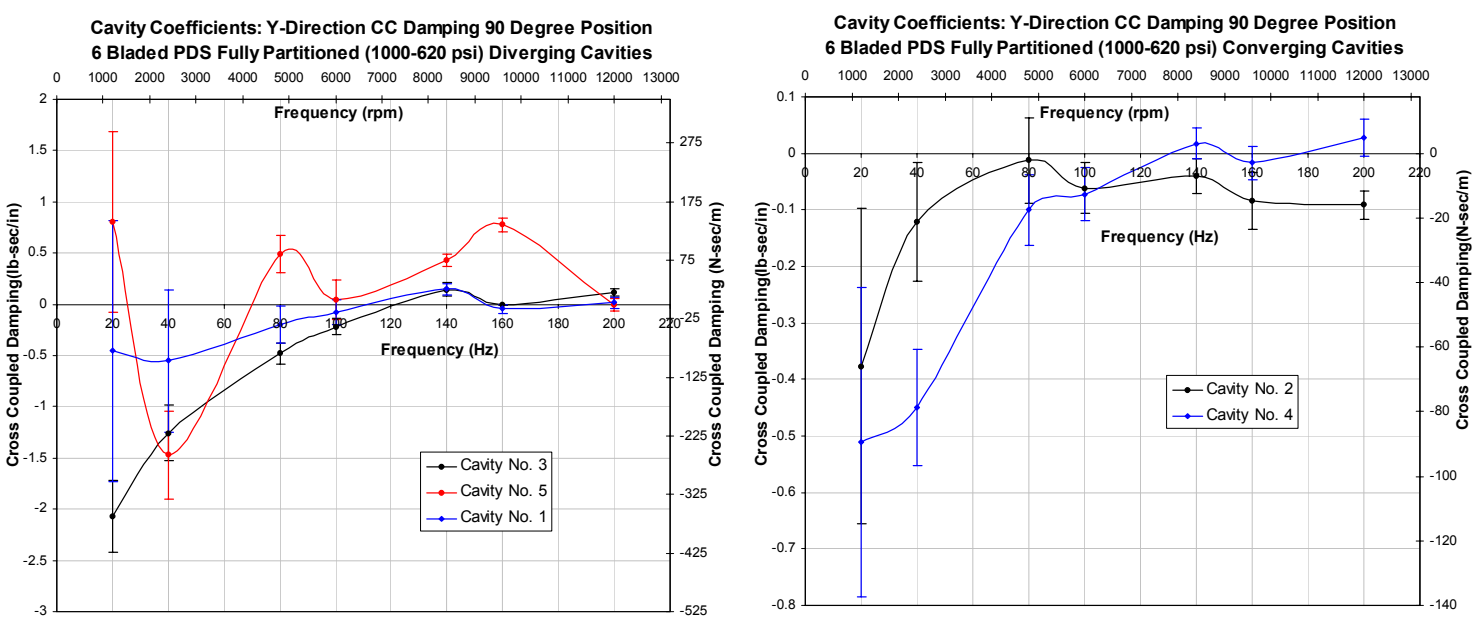

Cavity Cross-coupled Damping 

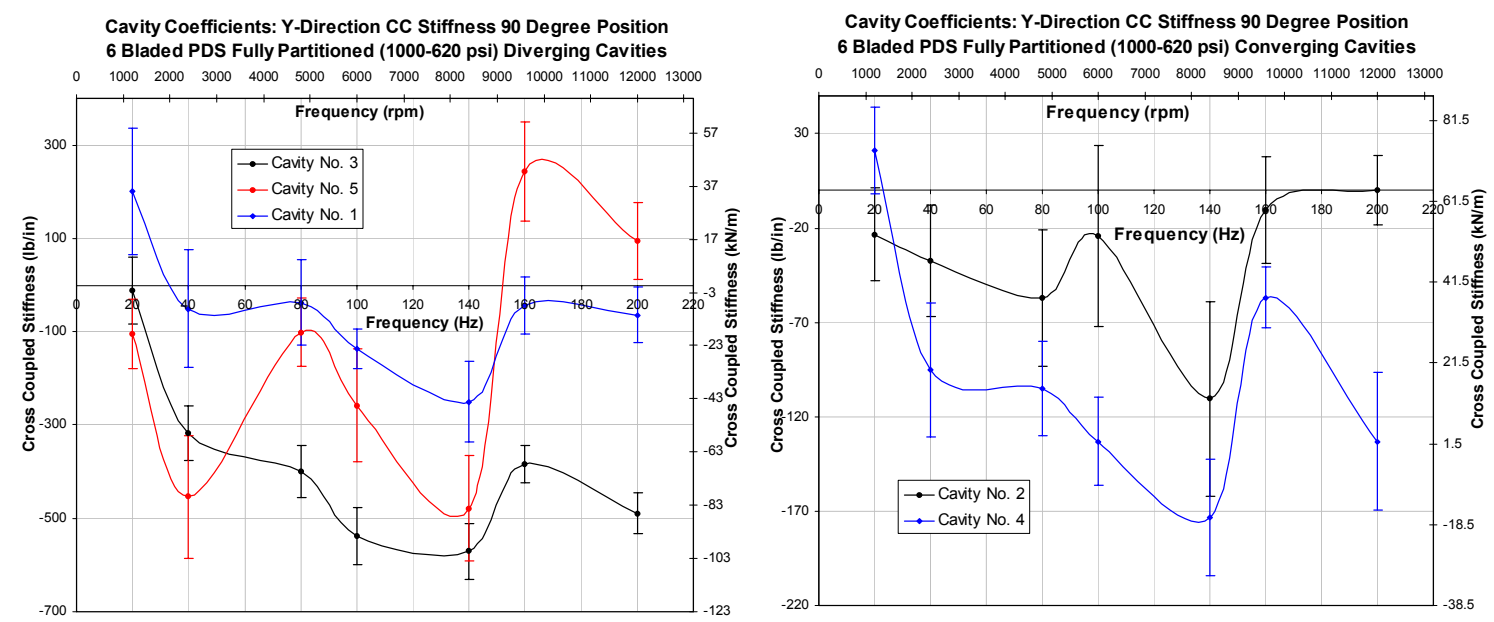

Cavity Cross-coupled Stiffness

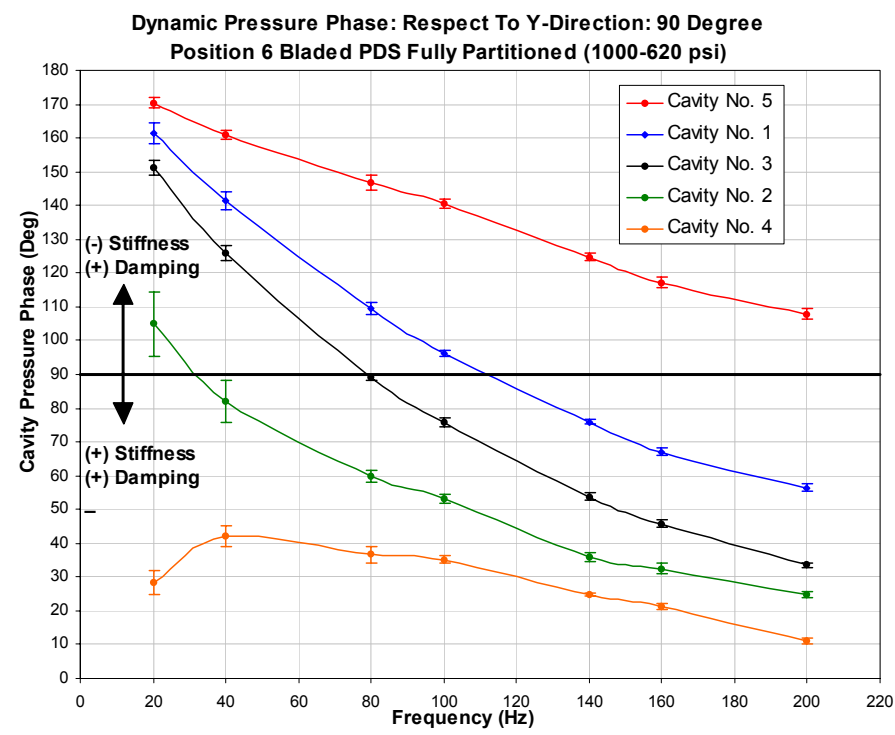

Cavity Phase Respect to Y direction Excitation
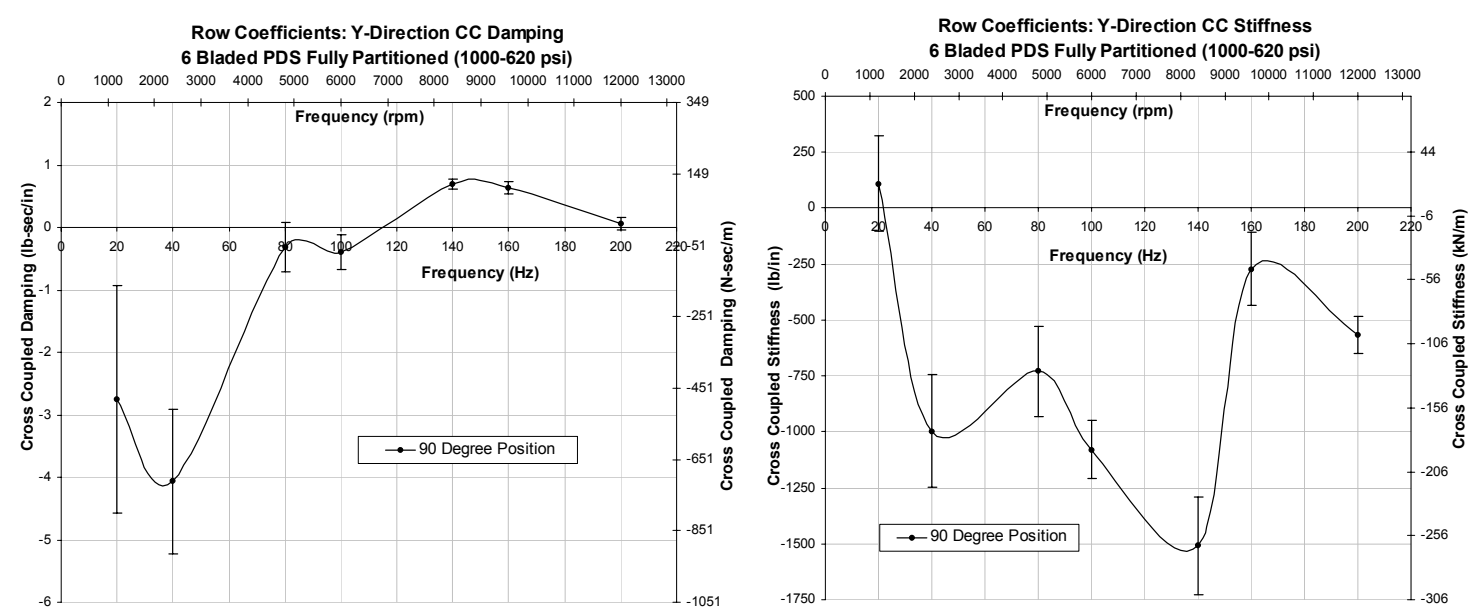

Total Row Cross-coupled Coefficients 

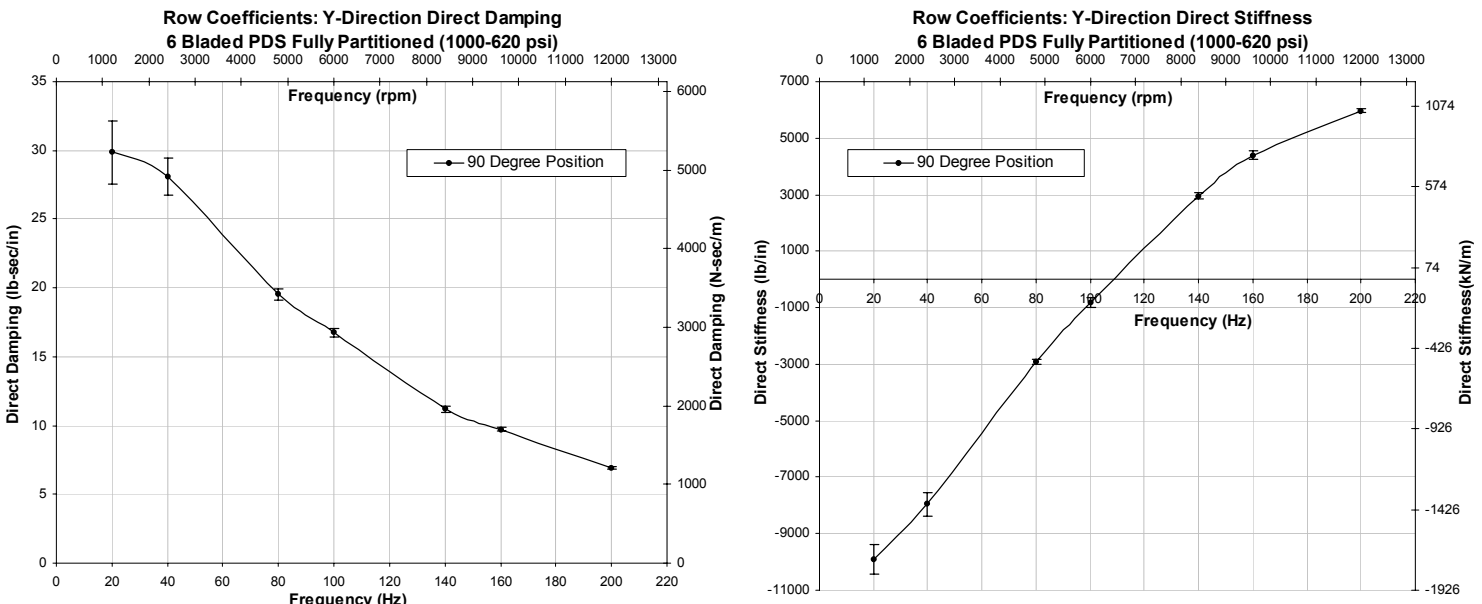

Total Row Direct Coefficients 


\section{Bladed Fully Partitioned Seal Cavity Coefficients: 180 Degree Position}
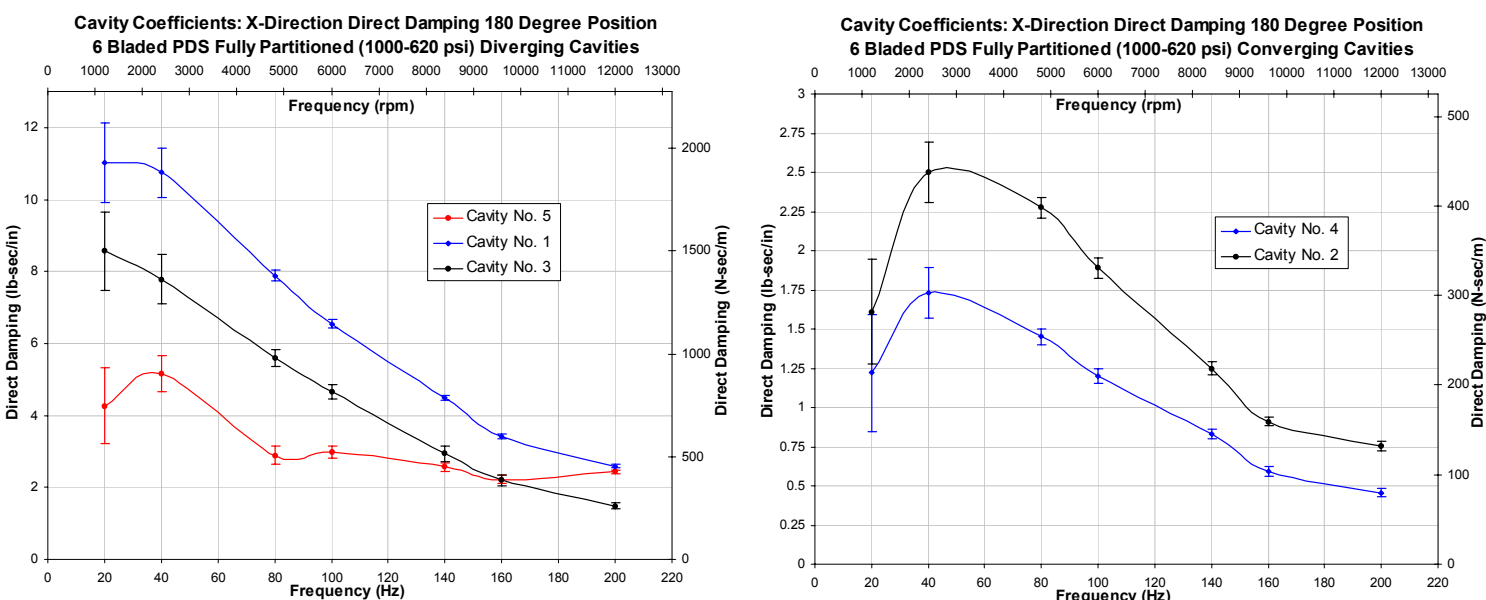

Cavity Direct Damping
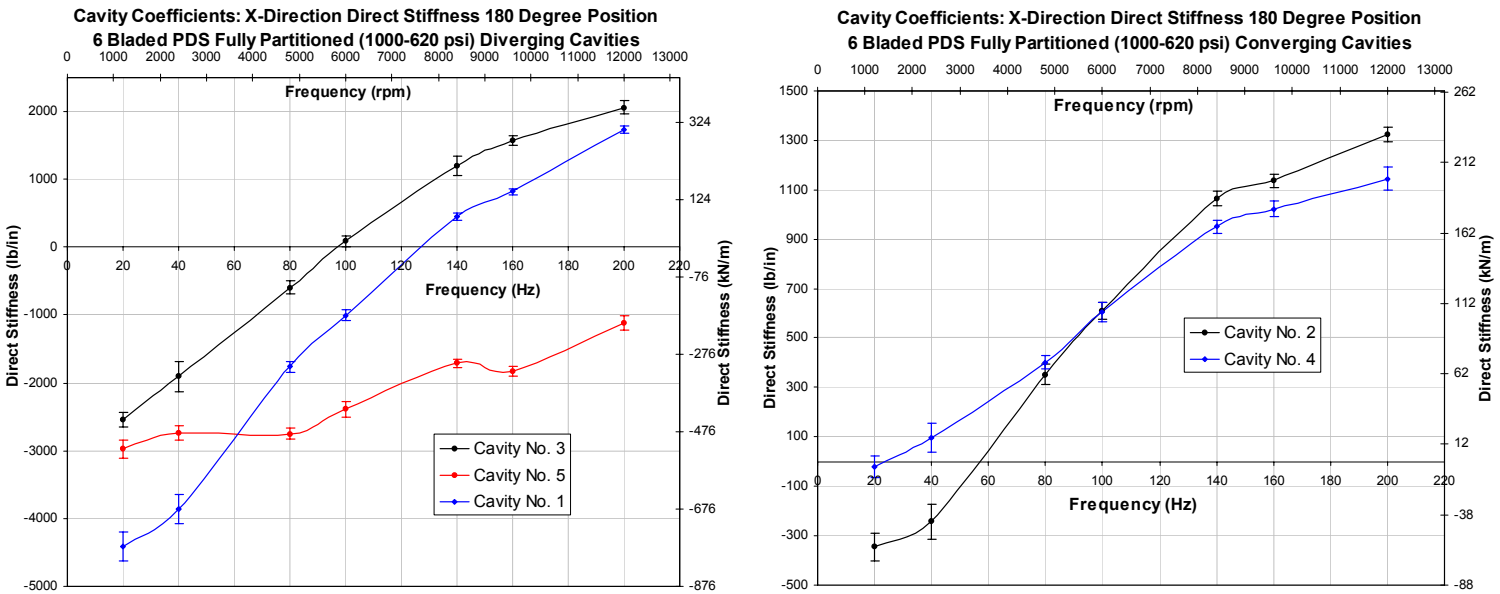

Cavity Direct Stiffness
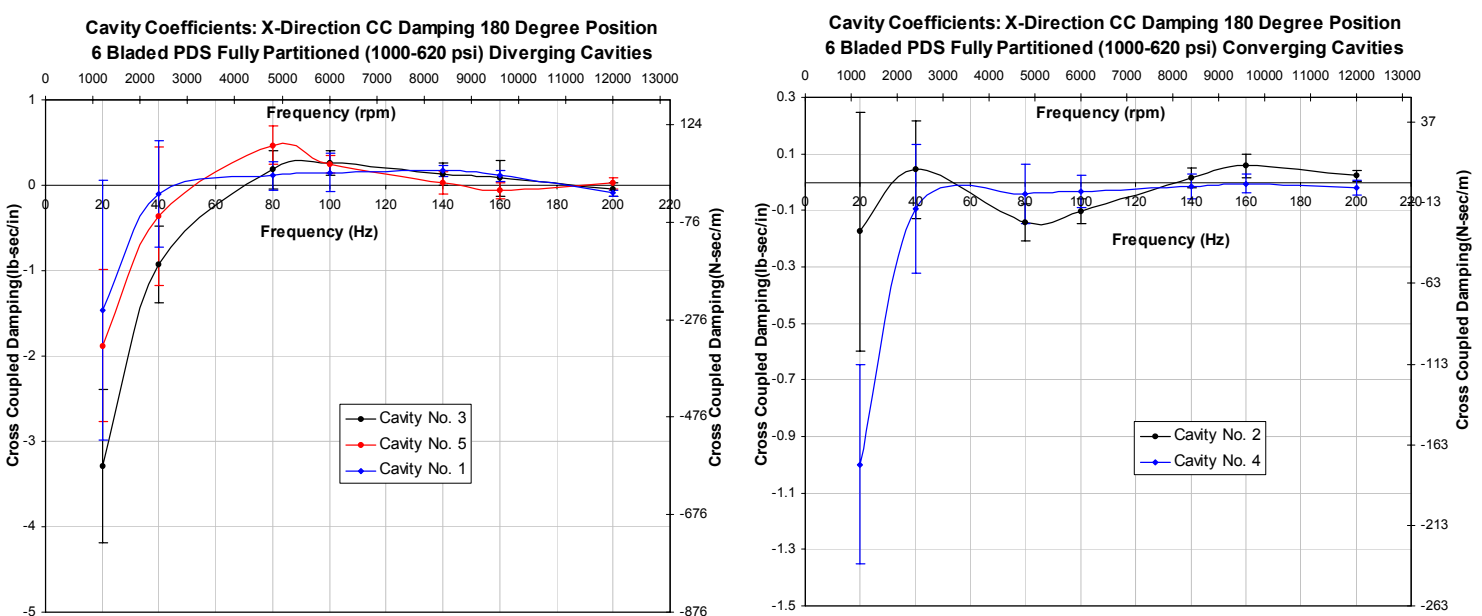

Cavity Cross-coupled Damping 

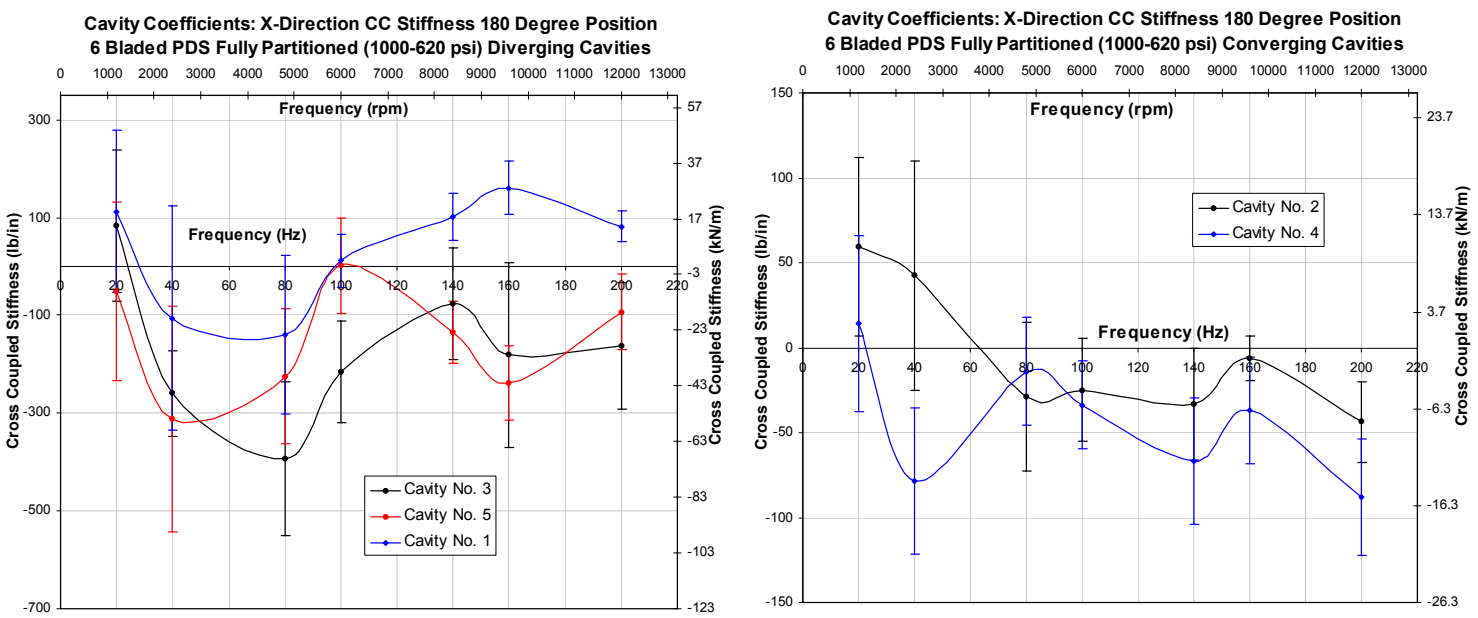

Cavity Cross-coupled Stiffness

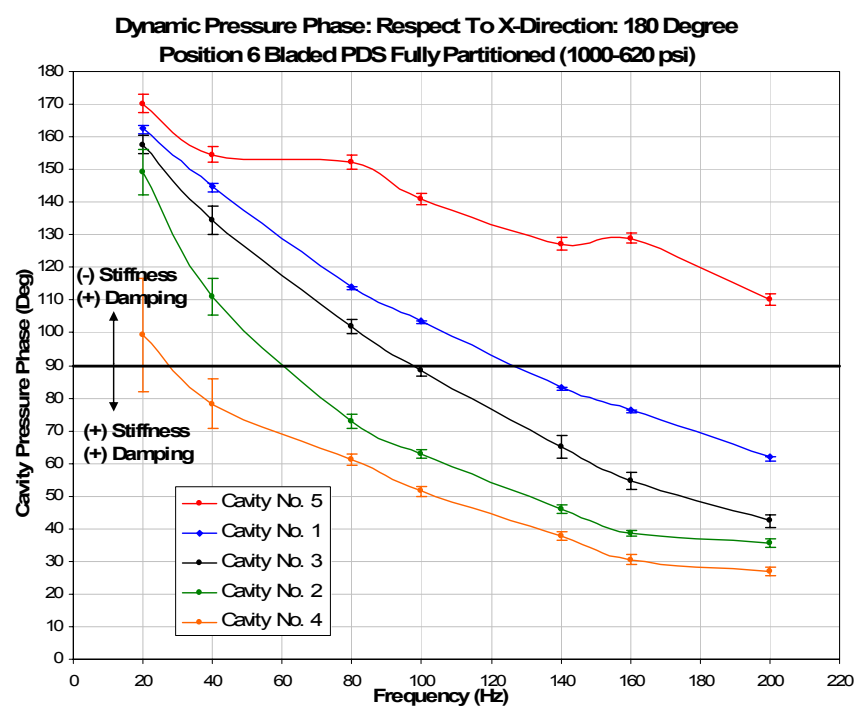

Cavity Phase Respect to X direction Excitation
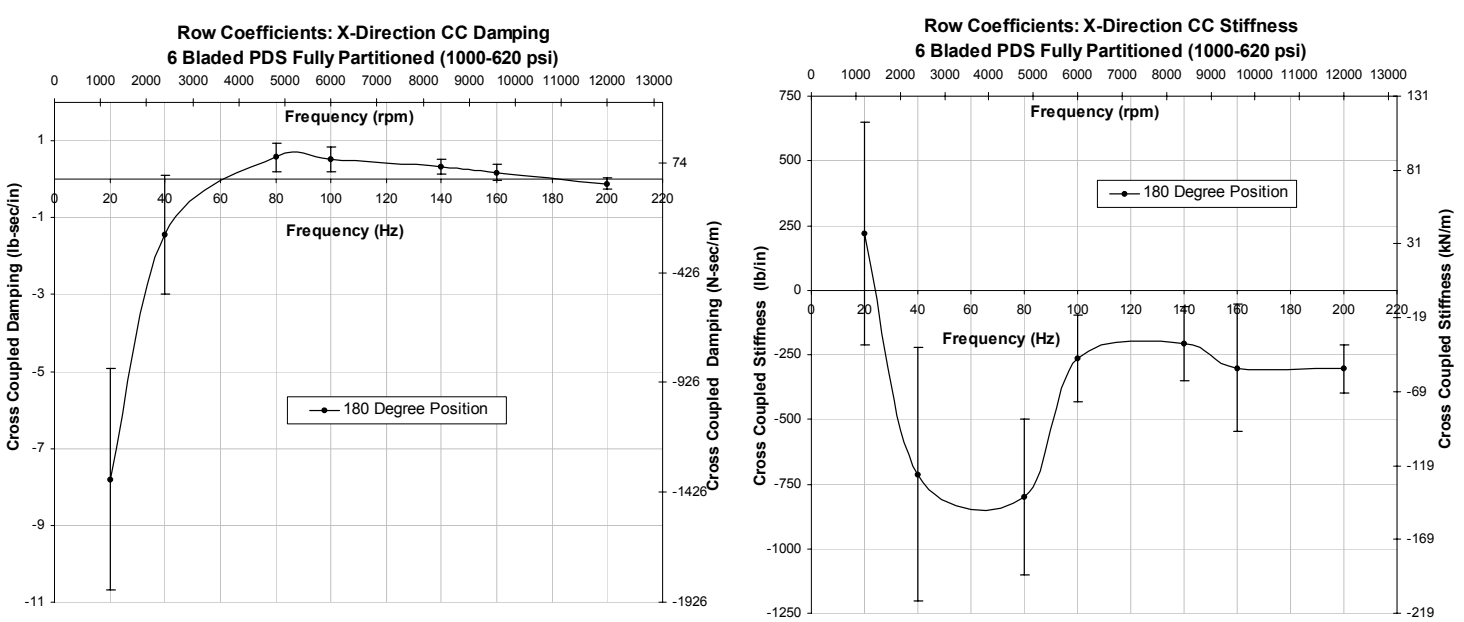

Total Row Cross-coupled Coefficients 

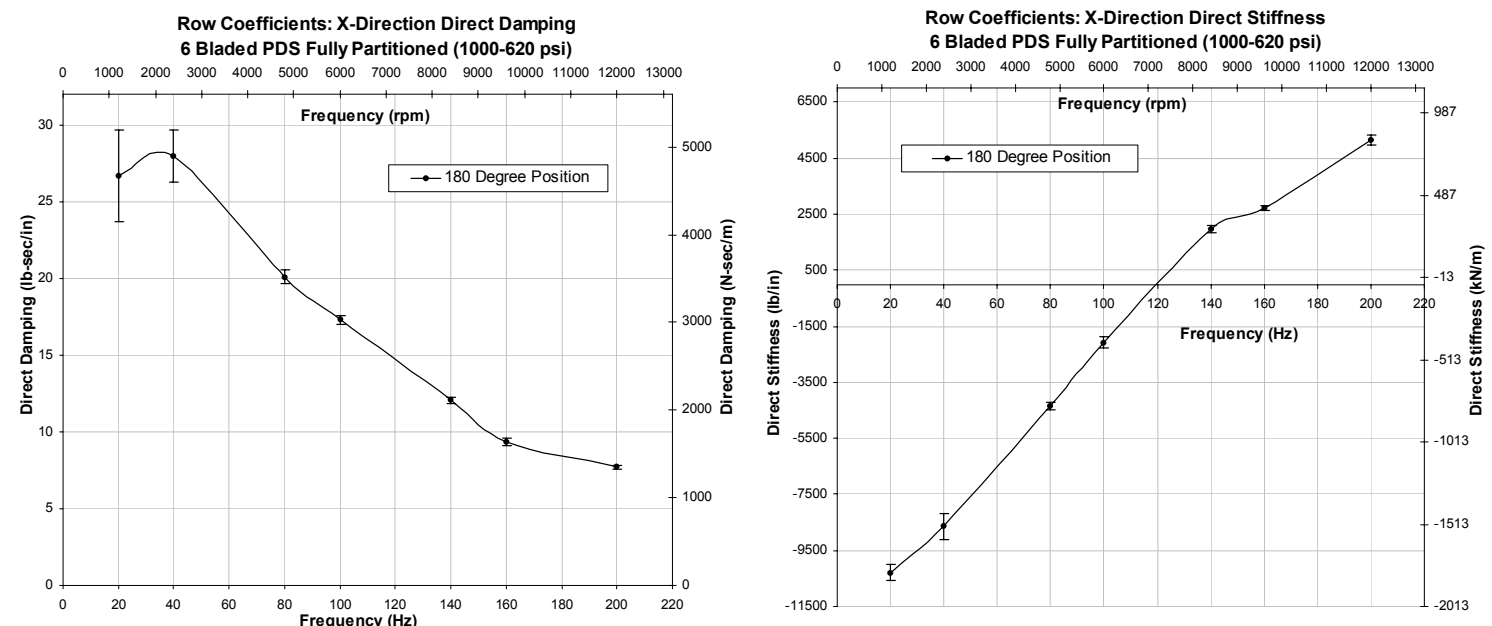

Total Row Direct Coefficients 


\section{Bladed Fully Partitioned Seal Cavity Coefficients: 270 Degree Position}
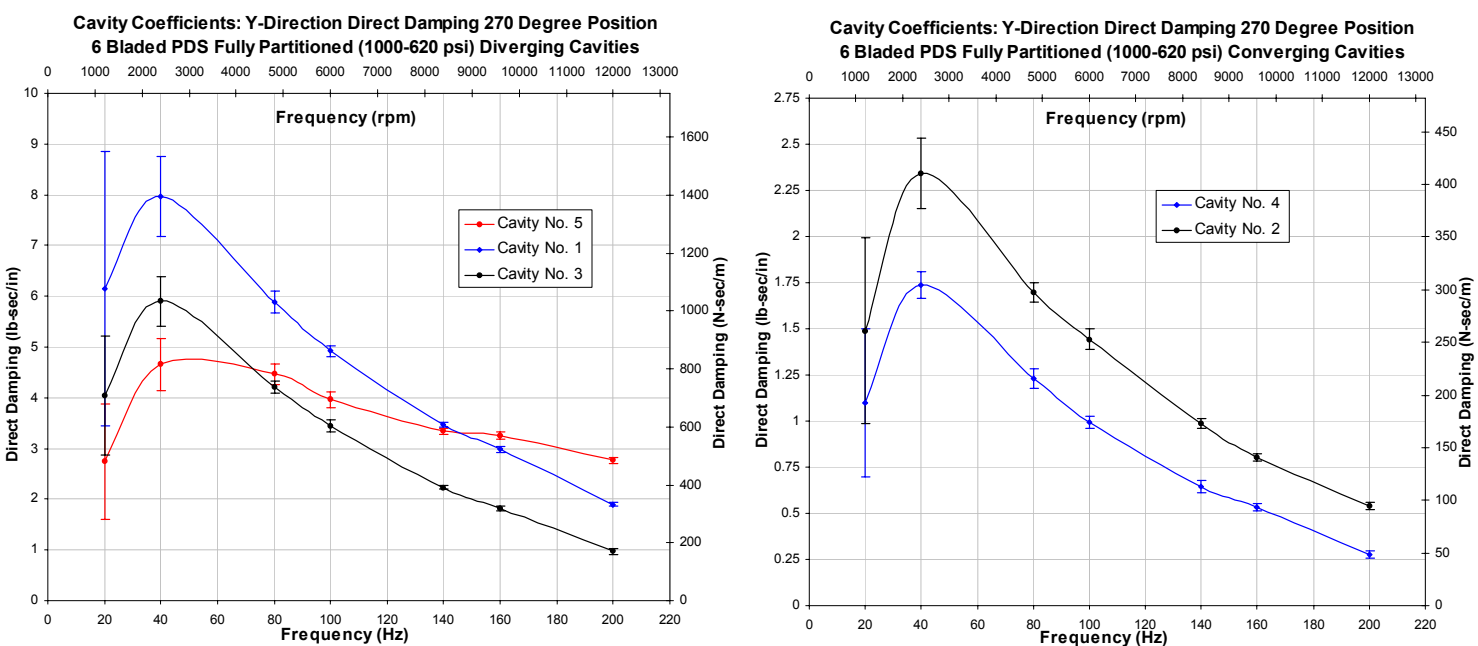

Cavity Direct Damping
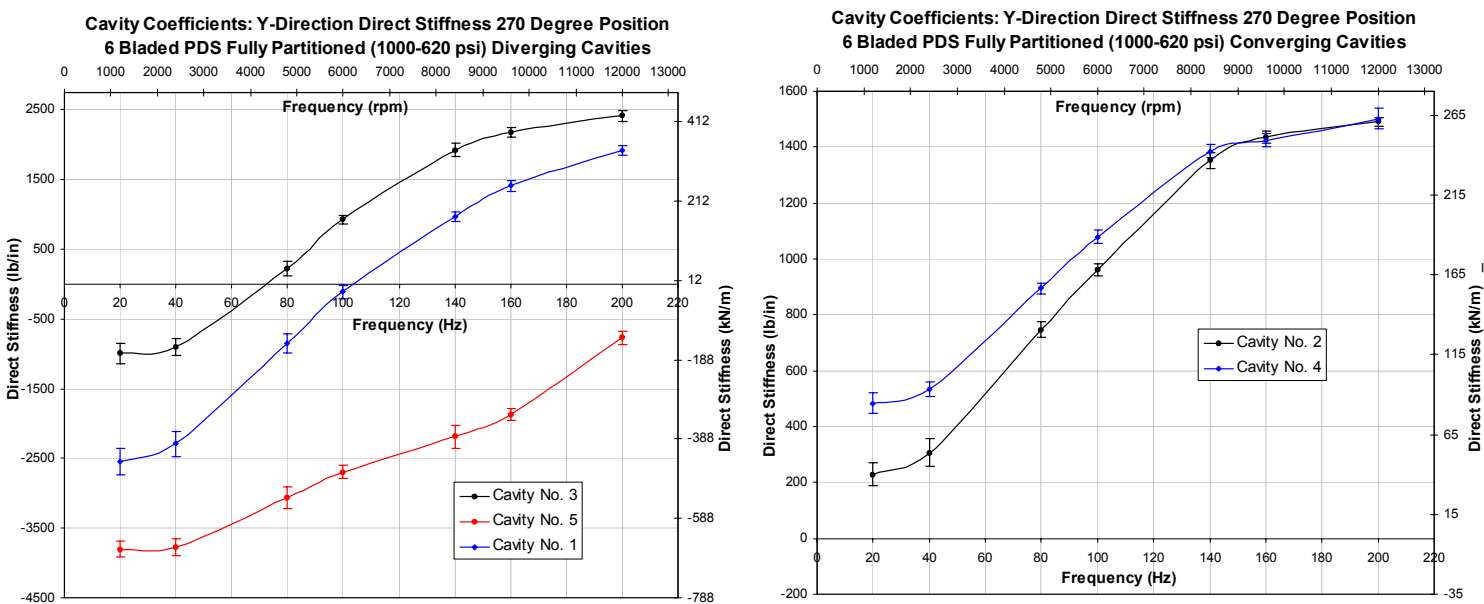

Cavity Direct Stiffness
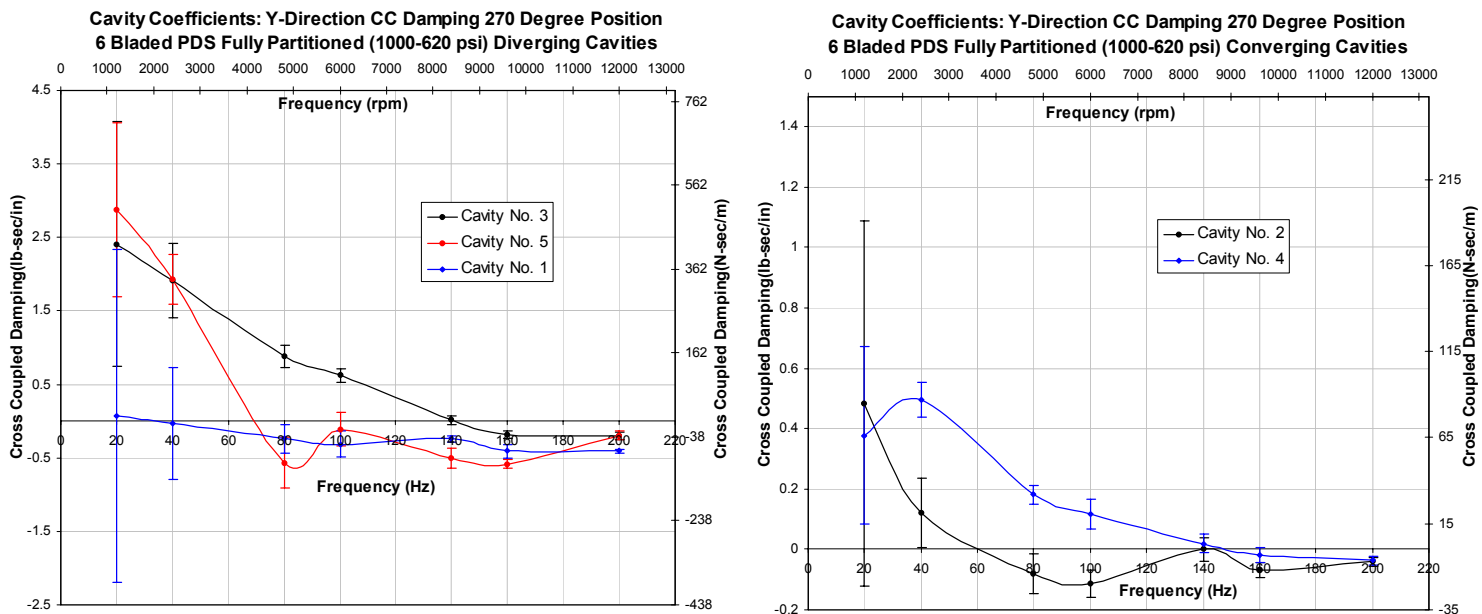

Cavity Cross-coupled Damping 

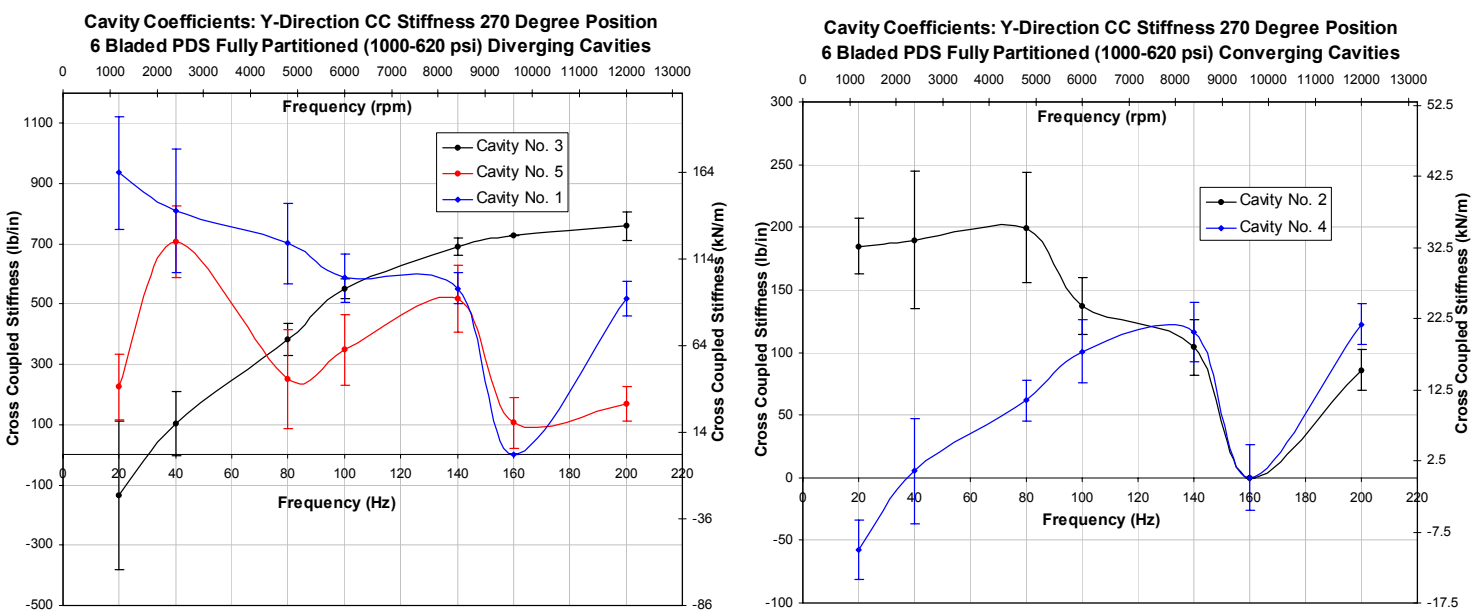

Cavity Cross-coupled Stiffness

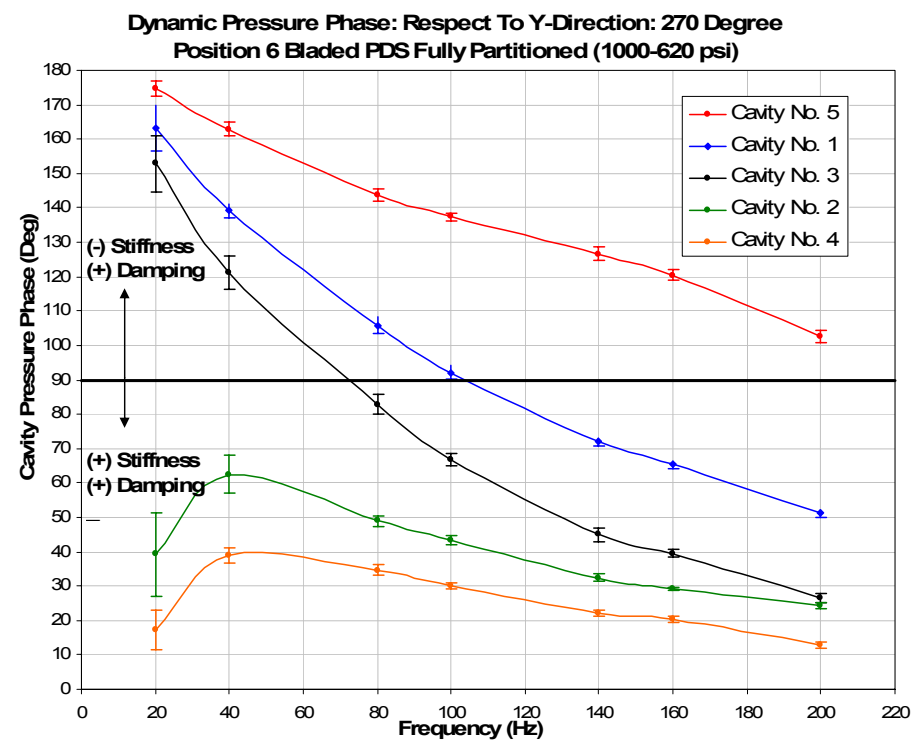

Cavity Phase Respect to Y direction Excitation
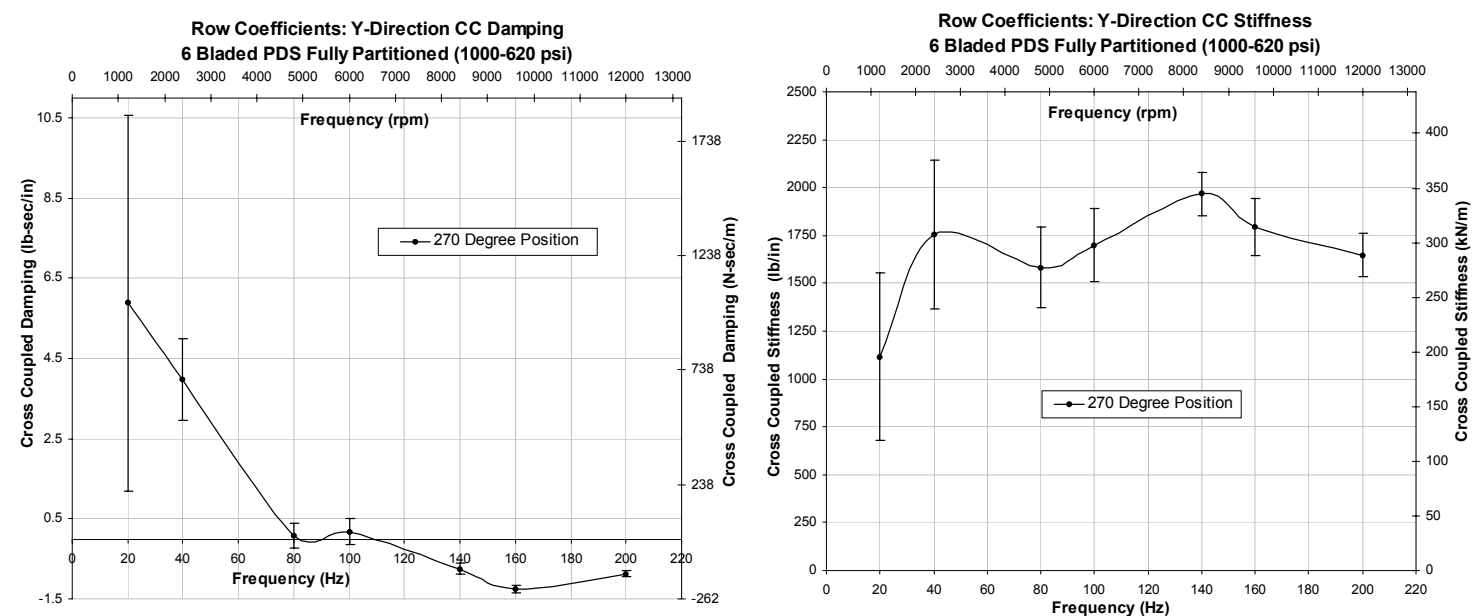

Total Row Cross-coupled Coefficients 

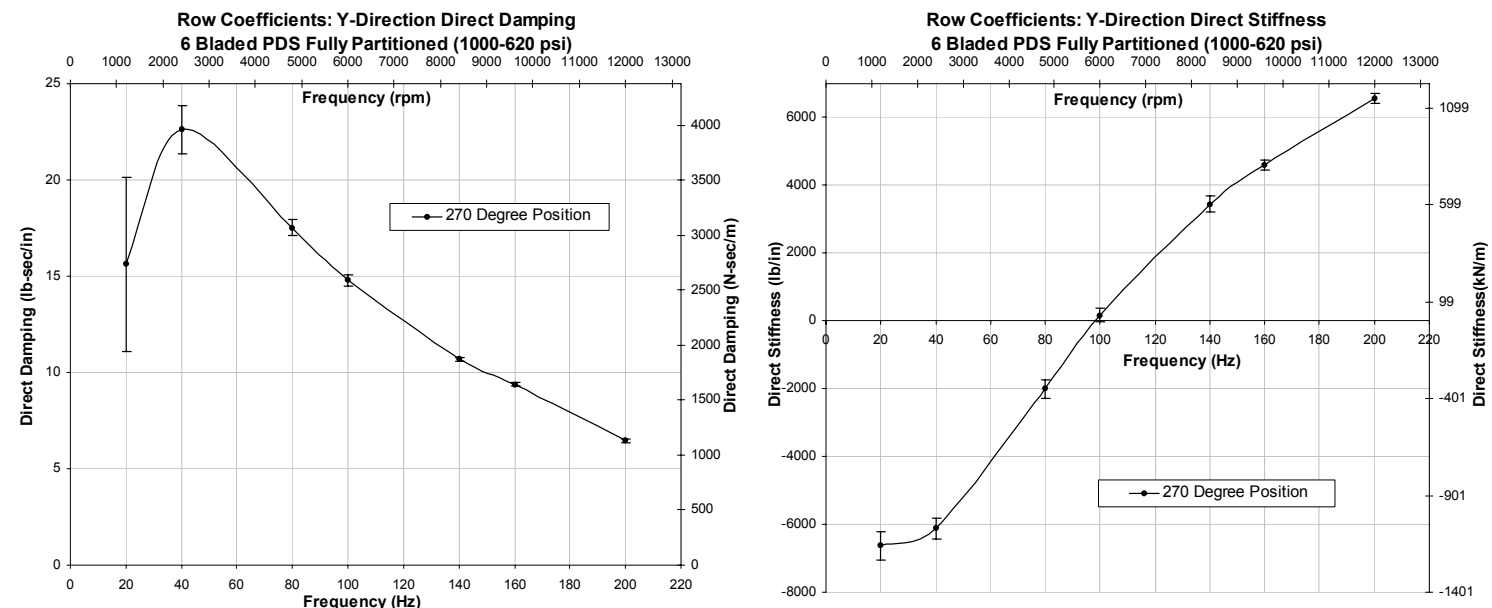

Total Row Direct Coefficients 


\section{Bladed Fully Partitioned Seal Cavity Coefficients: 45 Degree Position}
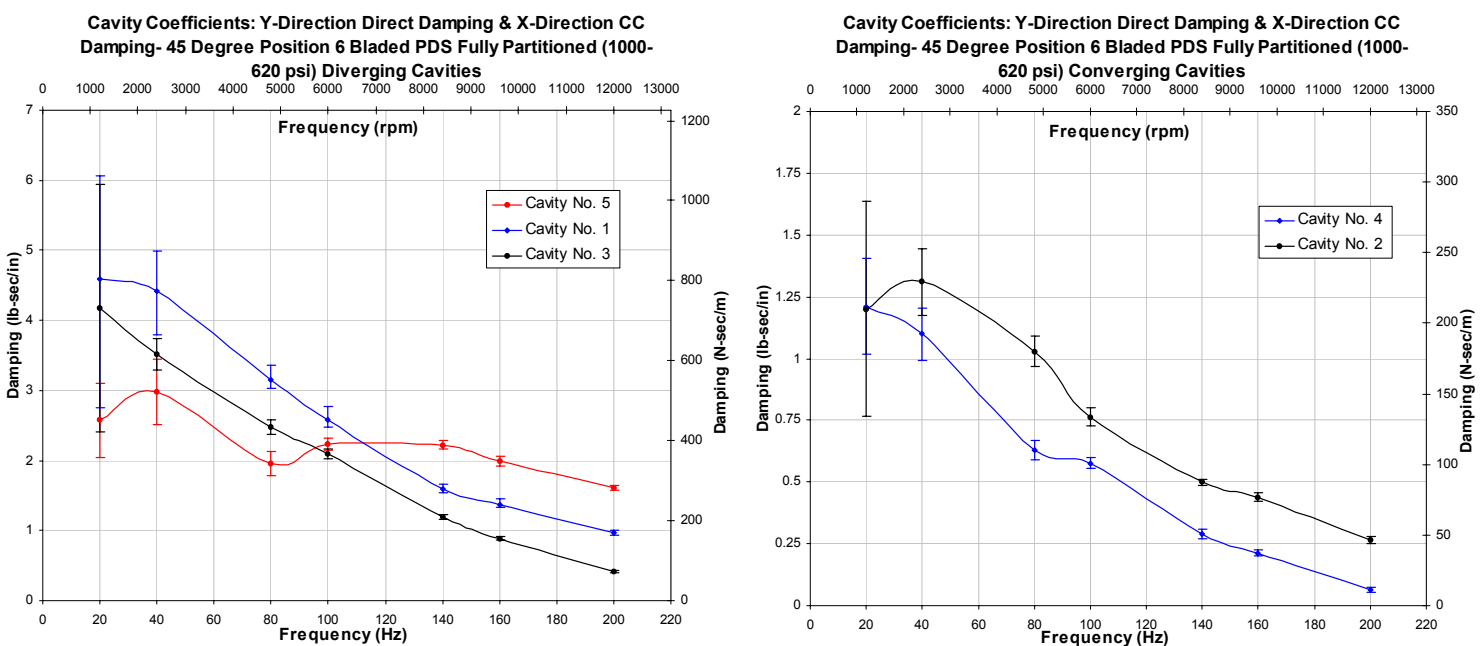

Y Direction Direct Damping \& X-Direction Cross-coupled Damping

Cavity Coefficients: Y-Direction Direct Stiffness \& X-Direction CC Stiffness 45 Degree Position- 6 Bladed PDS Fully Partitioned (1000
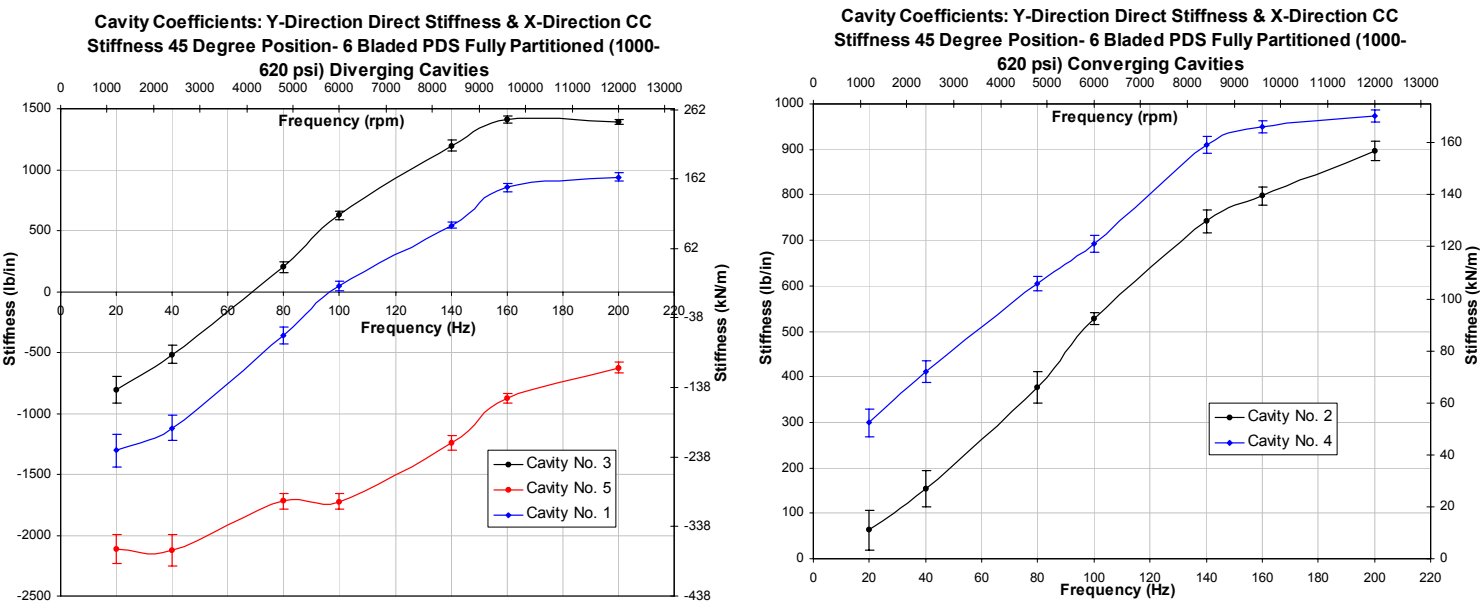

Y Direction Direct Stiffness \& X-Direction Cross-coupled Stiffness
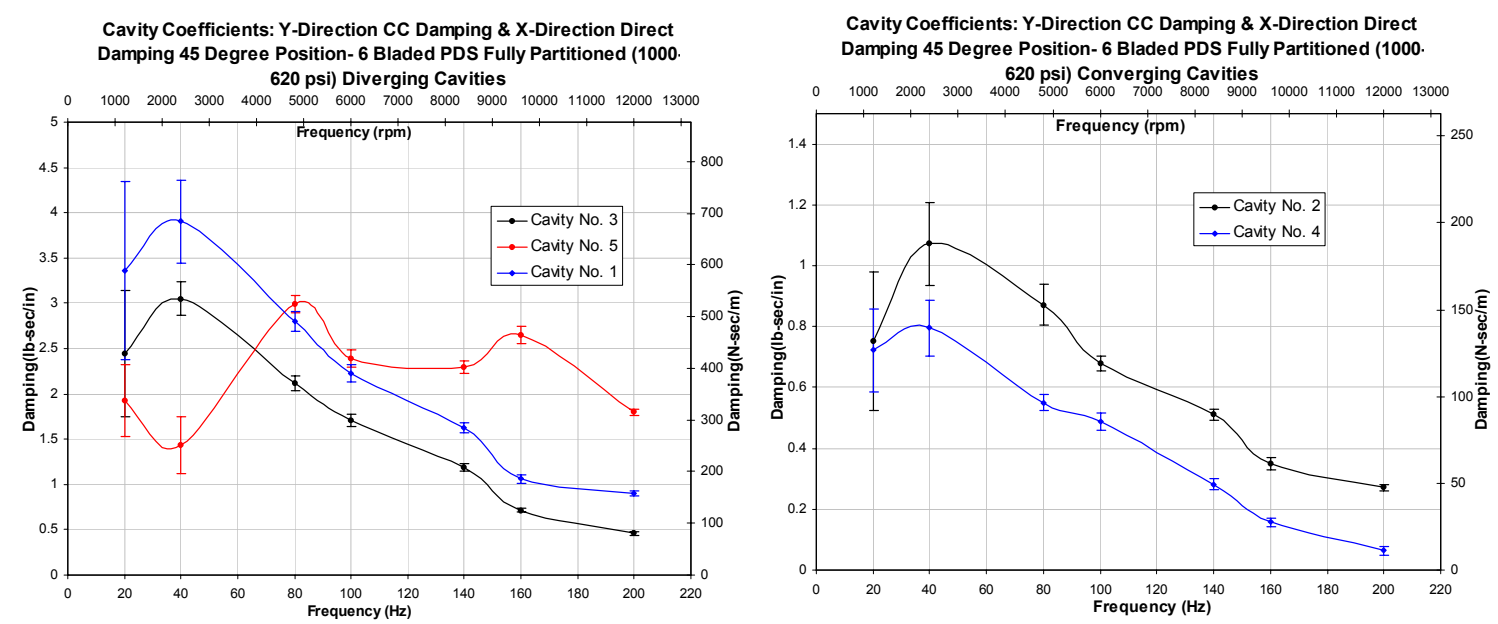

X Direction Direct Damping \& Y-Direction Cross-coupled Damping 

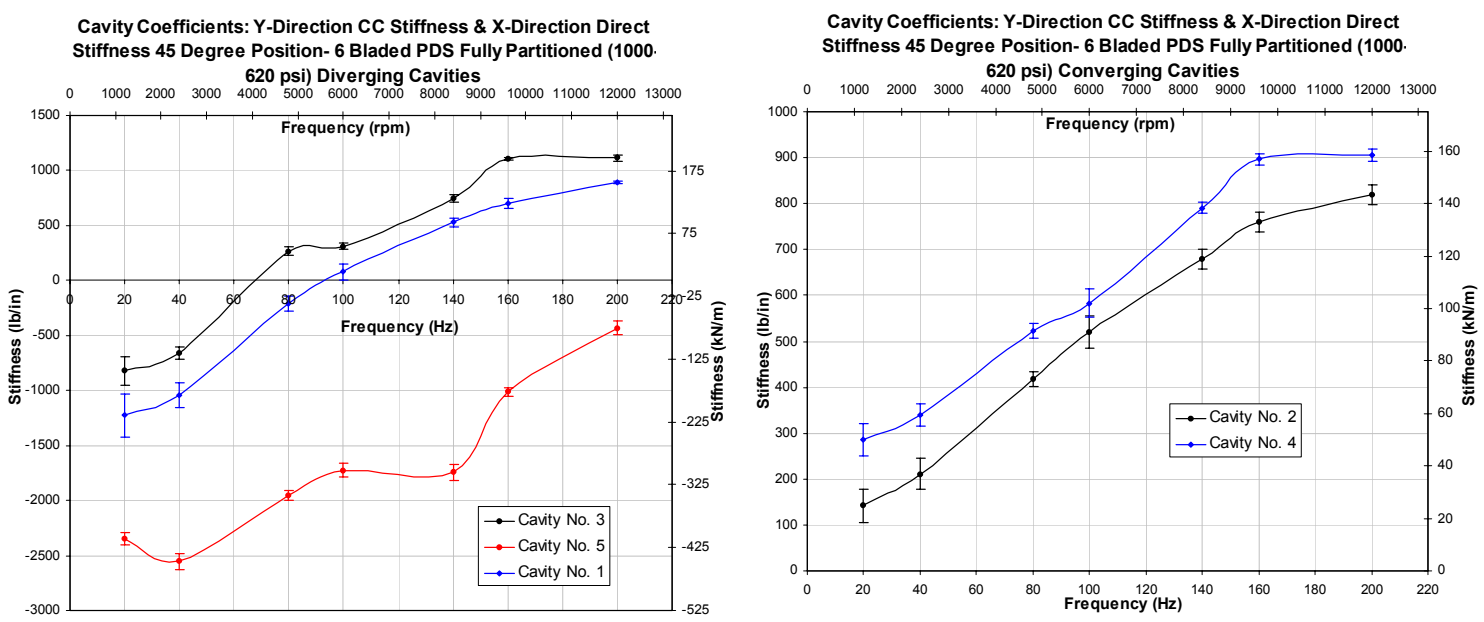

X Direction Direct Stiffness \& Y-Direction Cross-coupled Stiffness
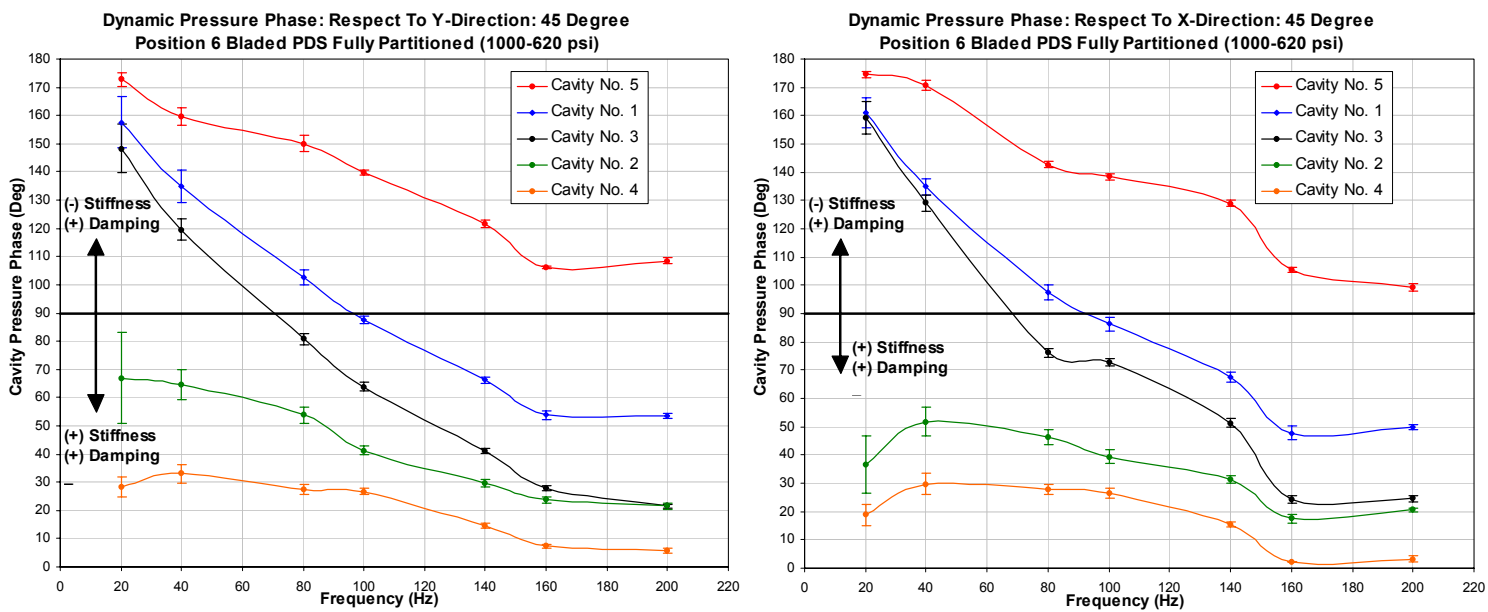

Dynamic Pressure Phase
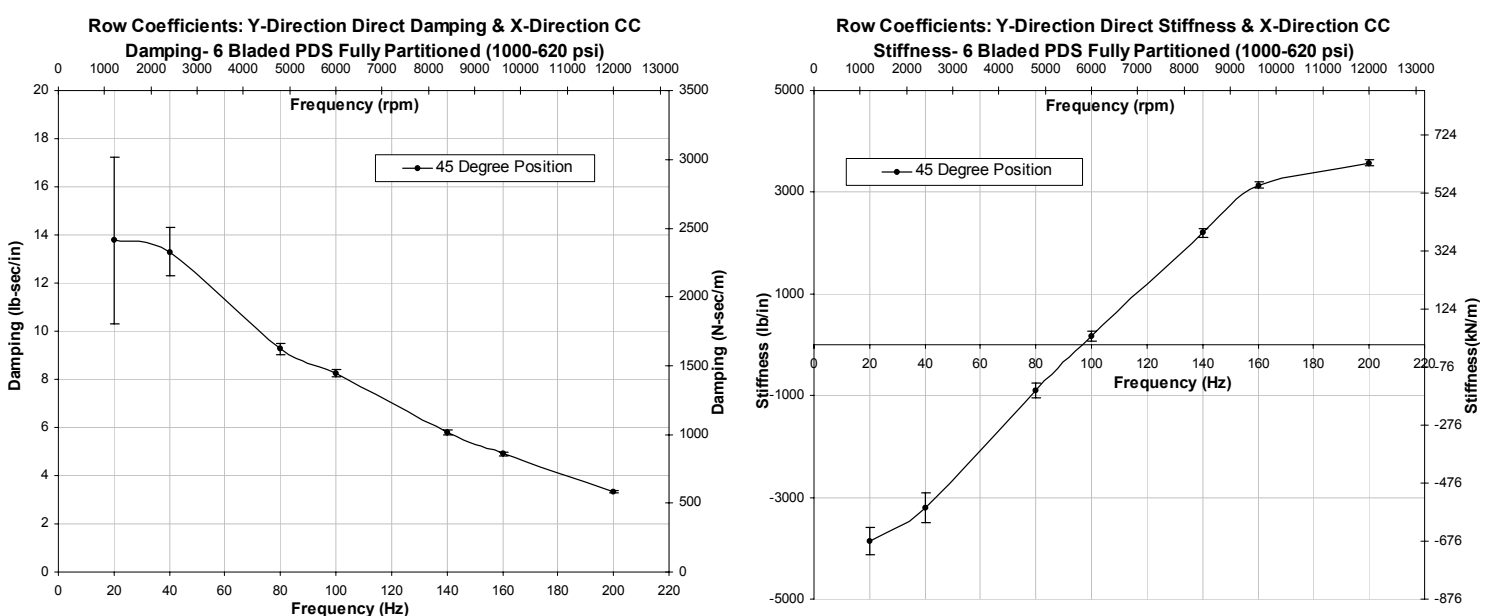

Y Direction Direct Coefficients \& X-Direction Cross-coupled Coefficients 

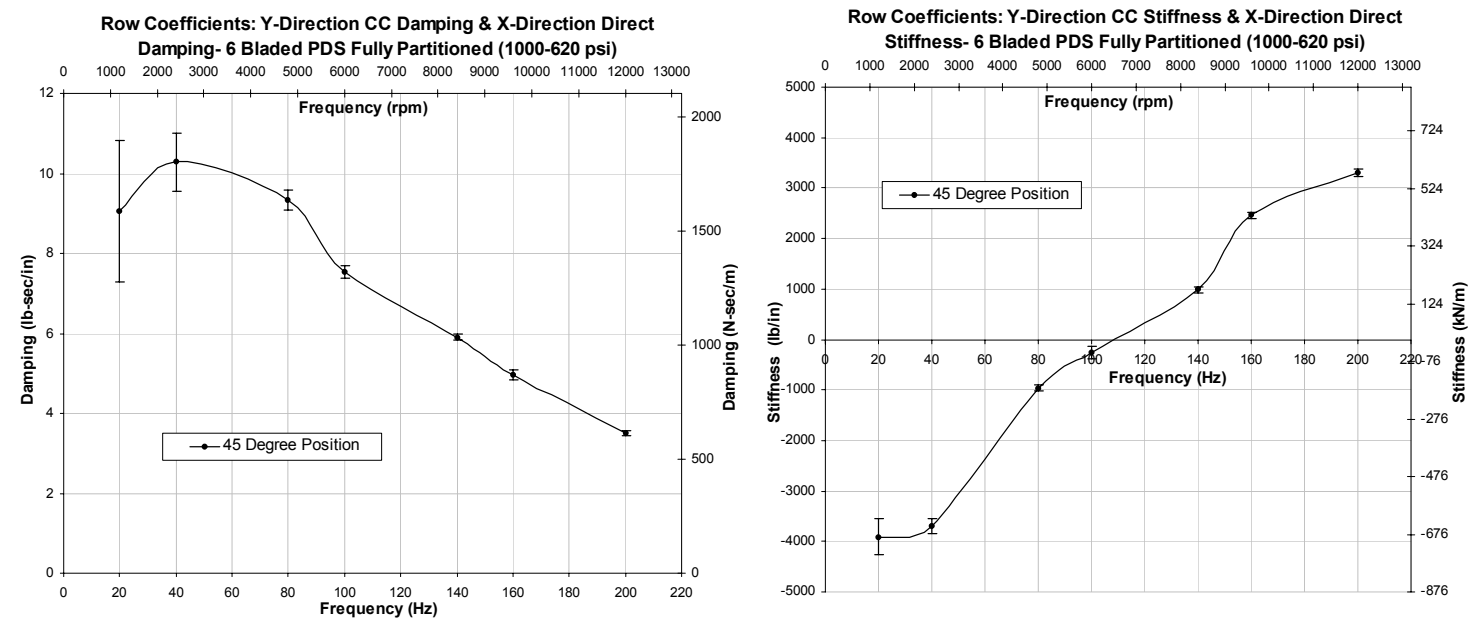

X Direction Direct Coefficients \& Y-Direction Cross-coupled Coefficients 


\section{Bladed Fully Partitioned Seal Cavity Coefficients: 135 Degree Position}
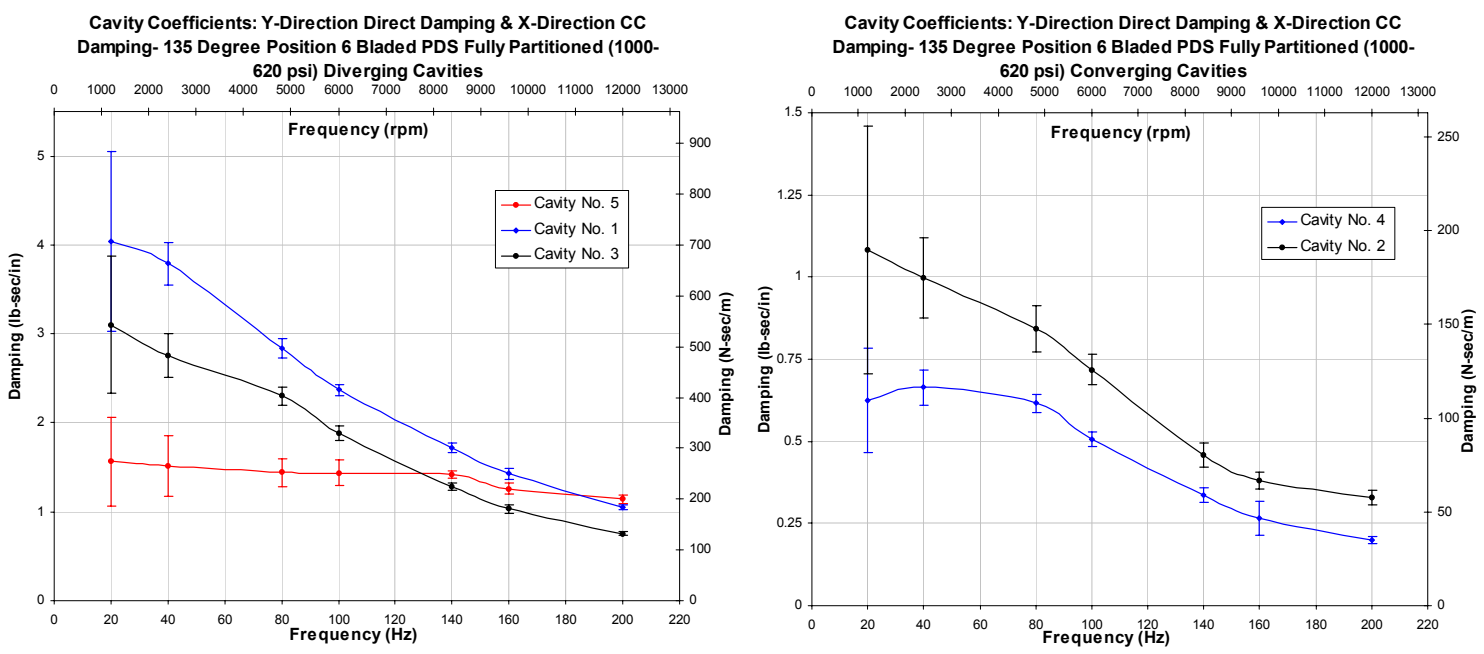

Y Direction Direct Damping \& X-Direction Cross-coupled Damping
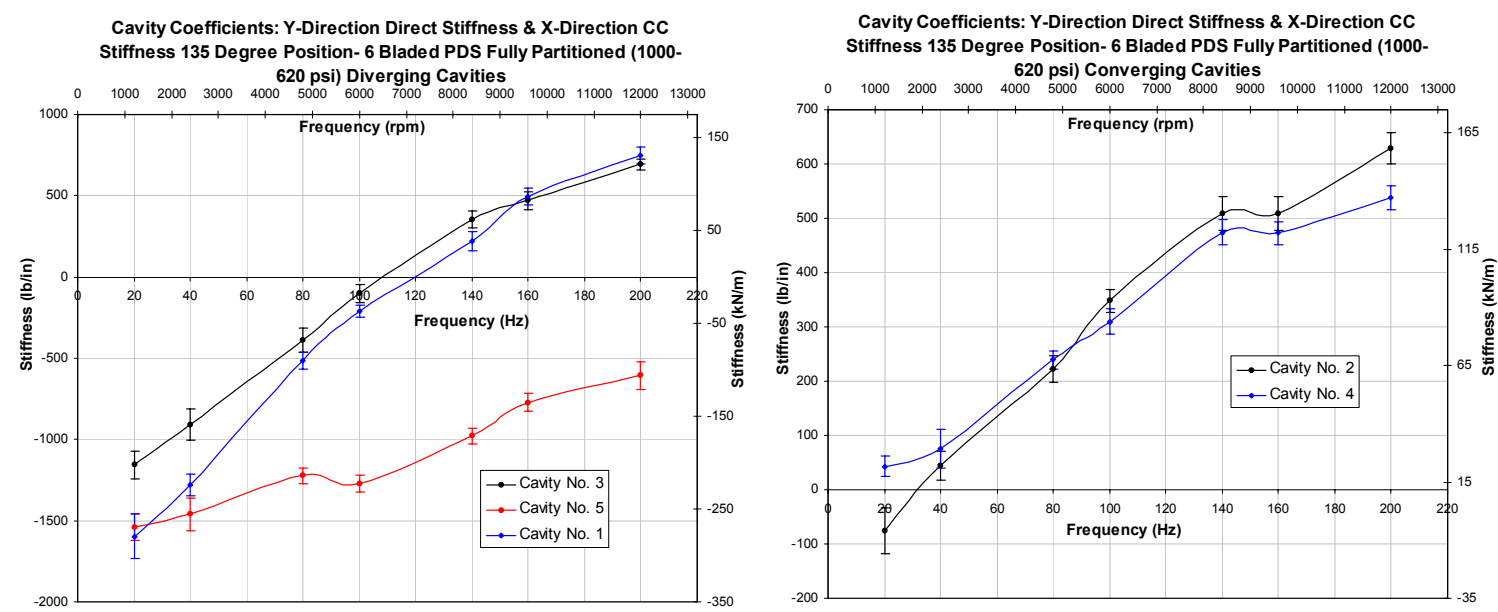

Y Direction Direct Stiffness \& X-Direction Cross-coupled Stiffness
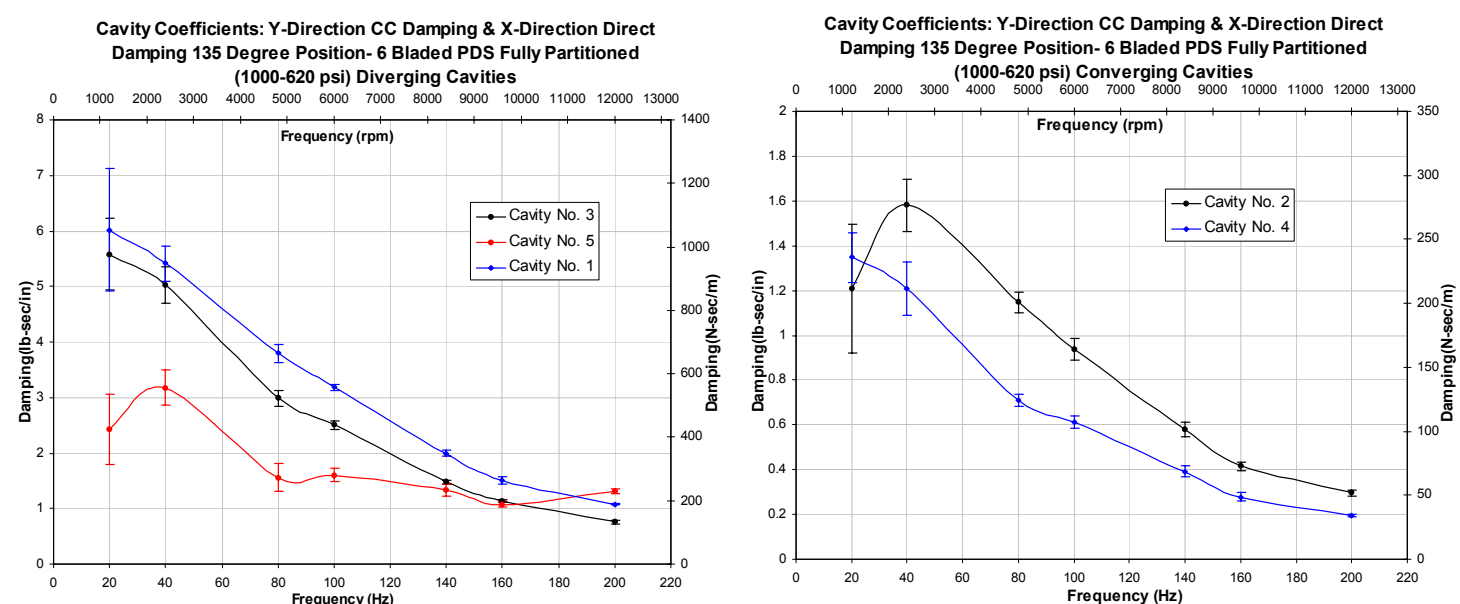

X Direction Direct Damping \& Y-Direction Cross-coupled Damping 

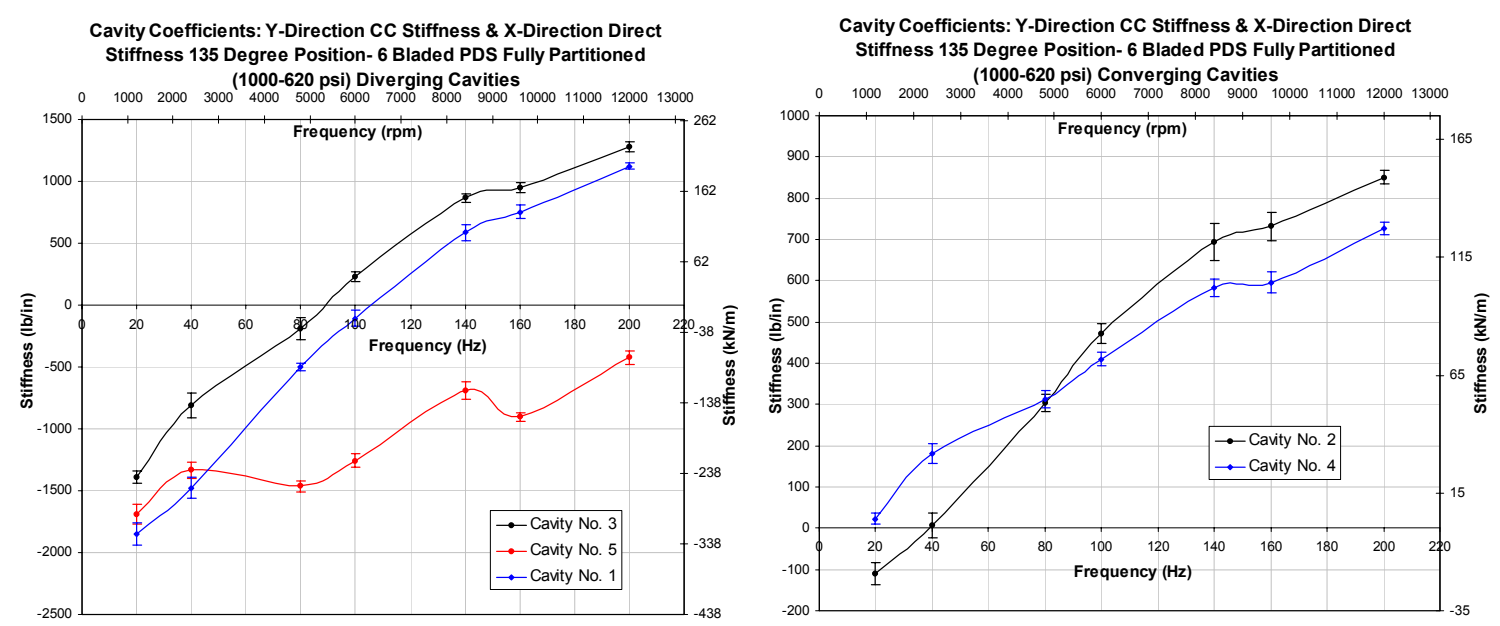

X Direction Direct Stiffness \& Y-Direction Cross-coupled Stiffness
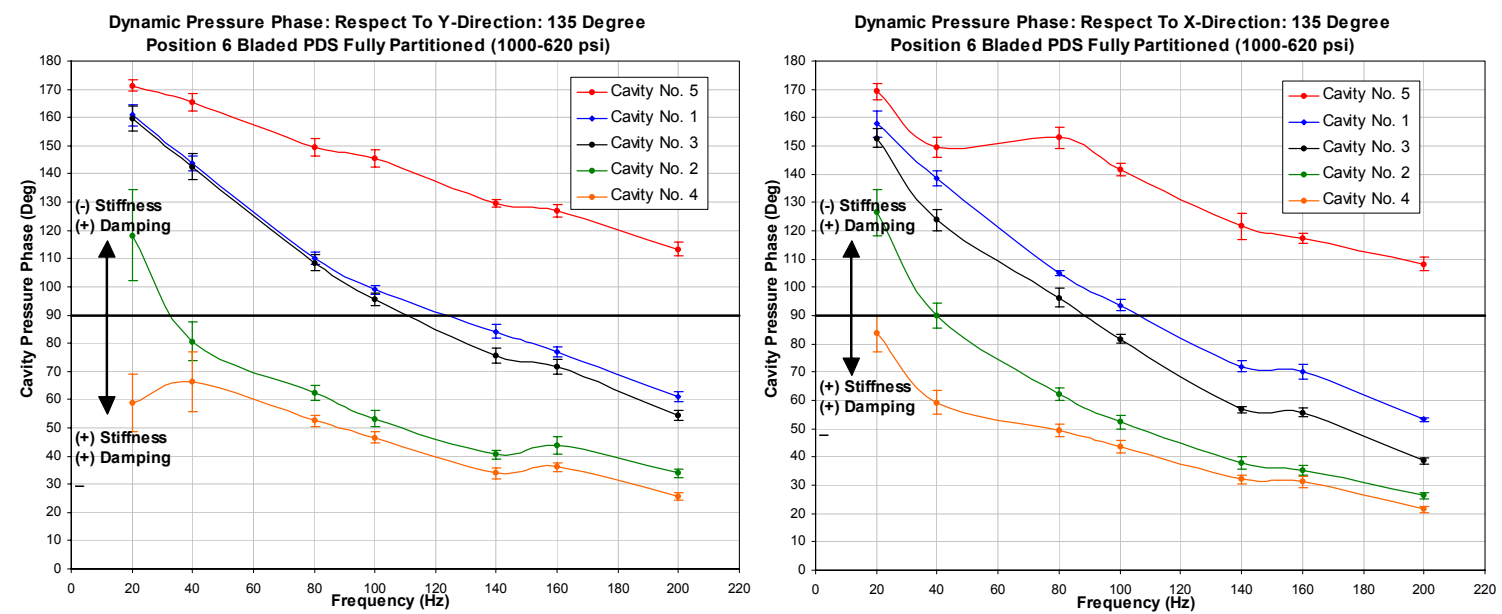

Dynamic Pressure Phase
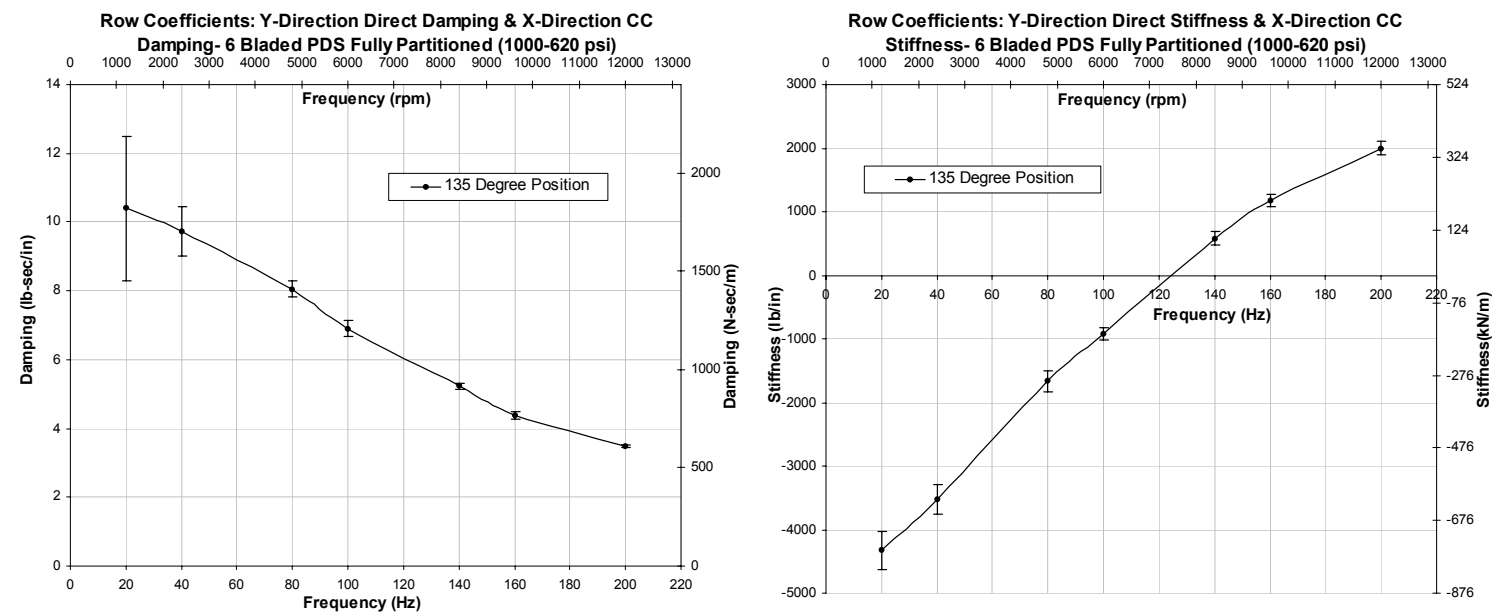

Y Direction Direct Coefficients \& X-Direction Cross-coupled Coefficients 

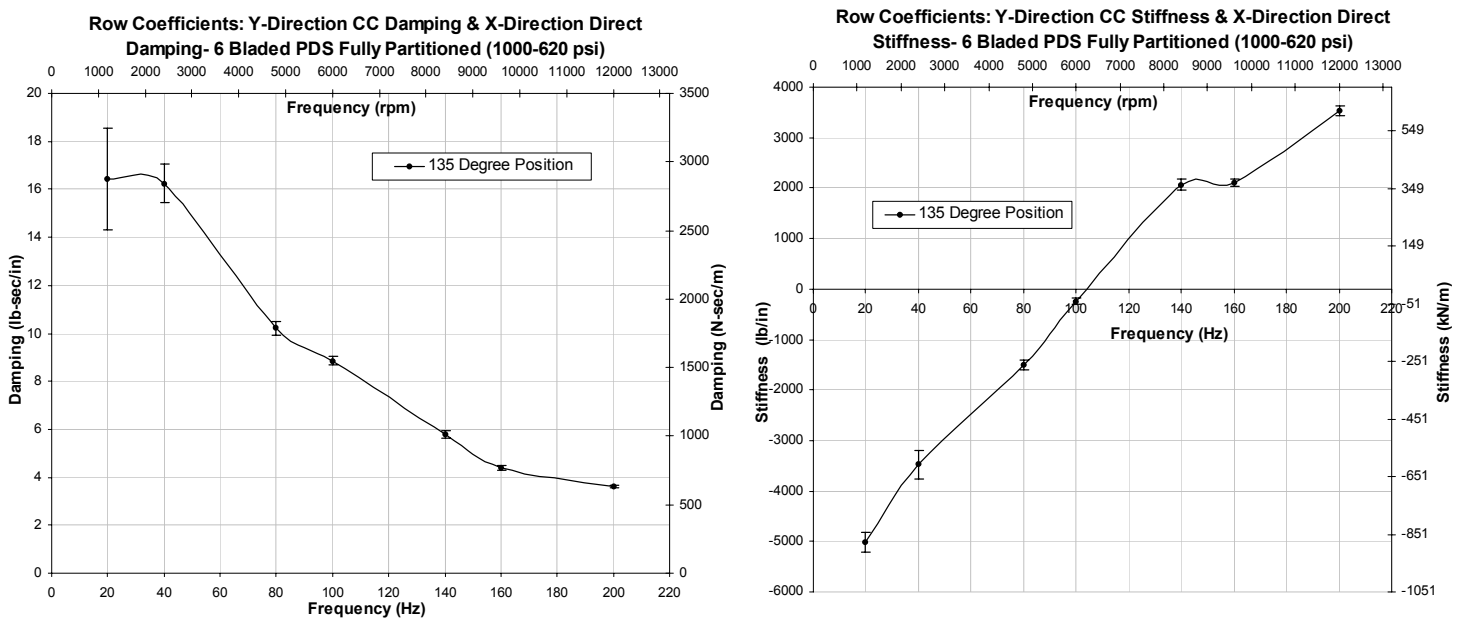

X Direction Direct Coefficients \& Y-Direction Cross-coupled Coefficients 


\section{Bladed Fully Partitioned Seal Cavity Coefficients: 225 Degree Position}
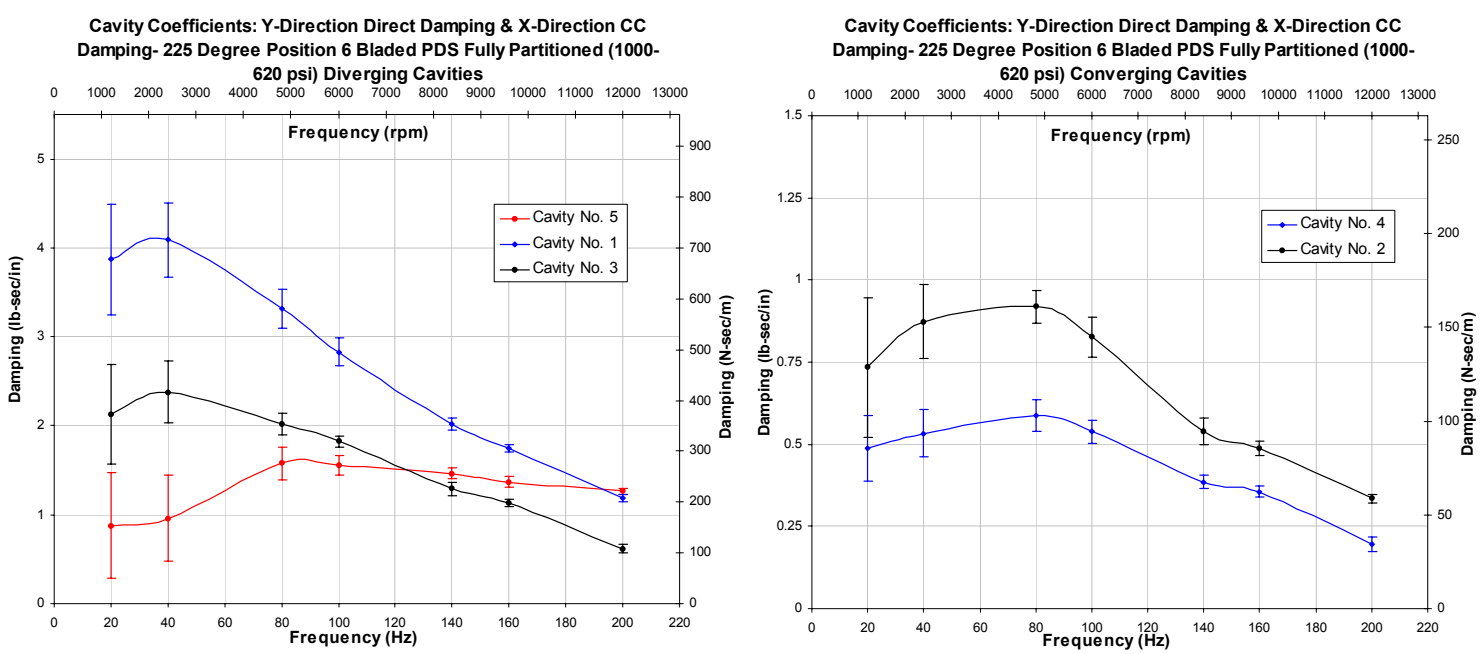

Y Direction Direct Damping \& X-Direction Cross-coupled Damping

Cavity Coefficients: Y-Direction Direct Stiffness \& X-Direction CC Stiffness 225 Degree Position- 6 Bladed PDS Fully Partitioned (1000-

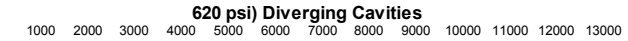
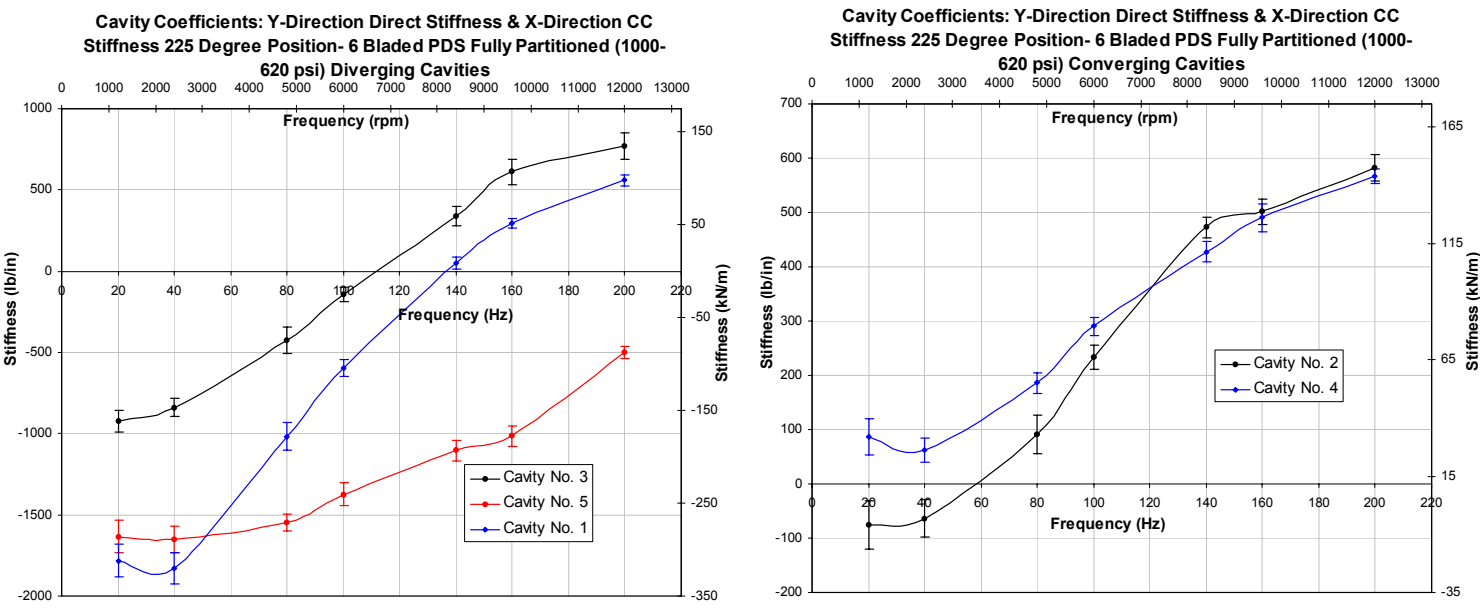

Y Direction Direct Stiffness \& X-Direction Cross-coupled Stiffness
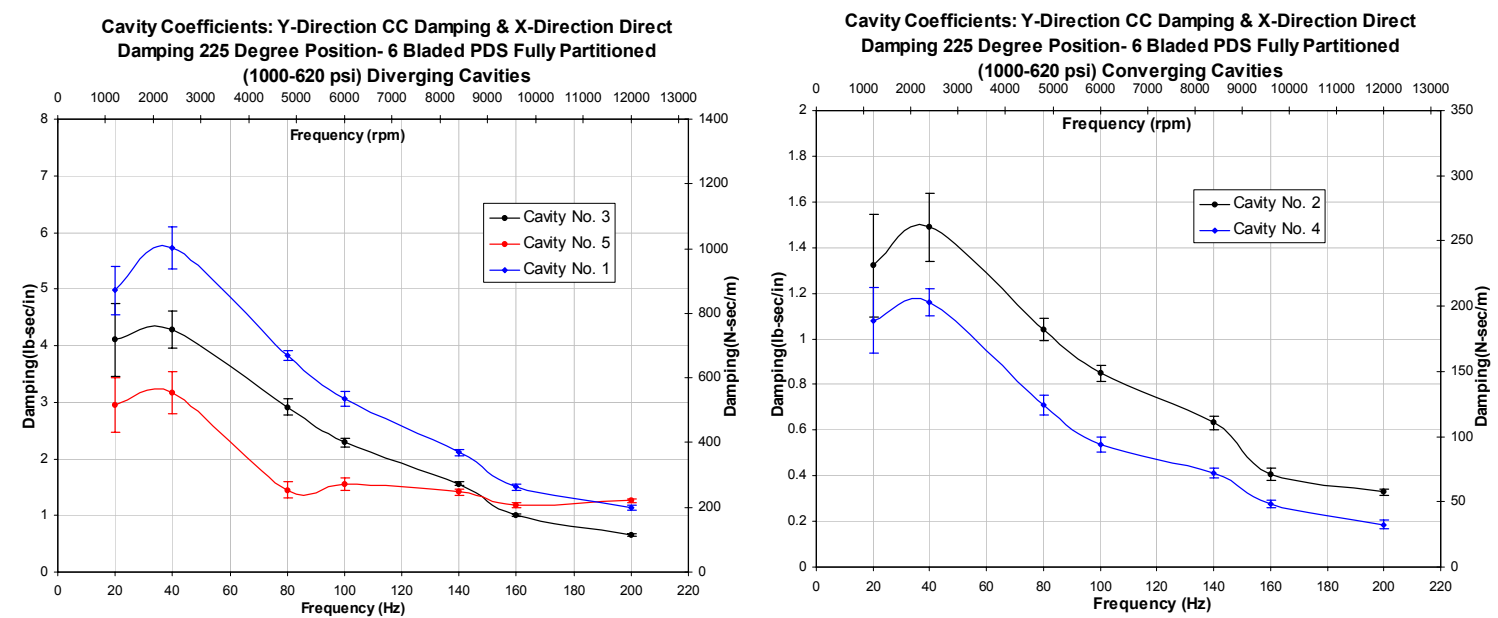

X Direction Direct Damping \& Y-Direction Cross-coupled Damping 

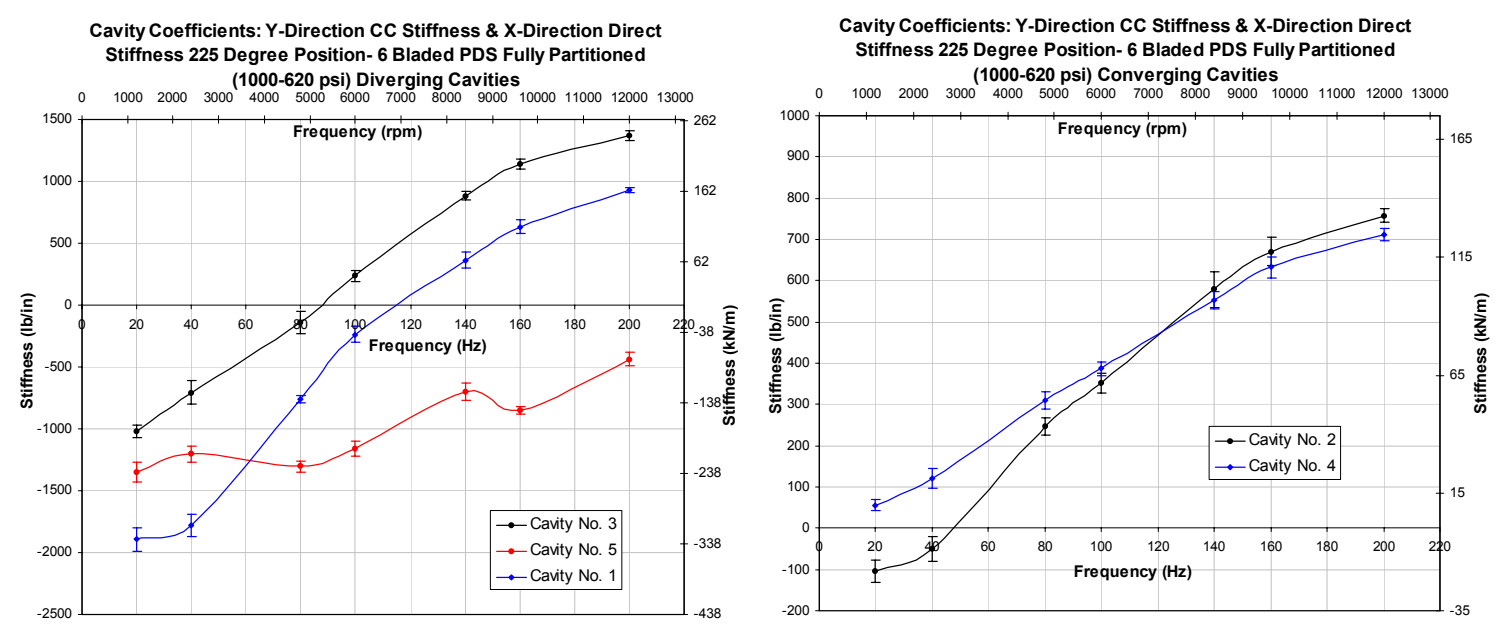

X Direction Direct Stiffness \& Y-Direction Cross-coupled Stiffness
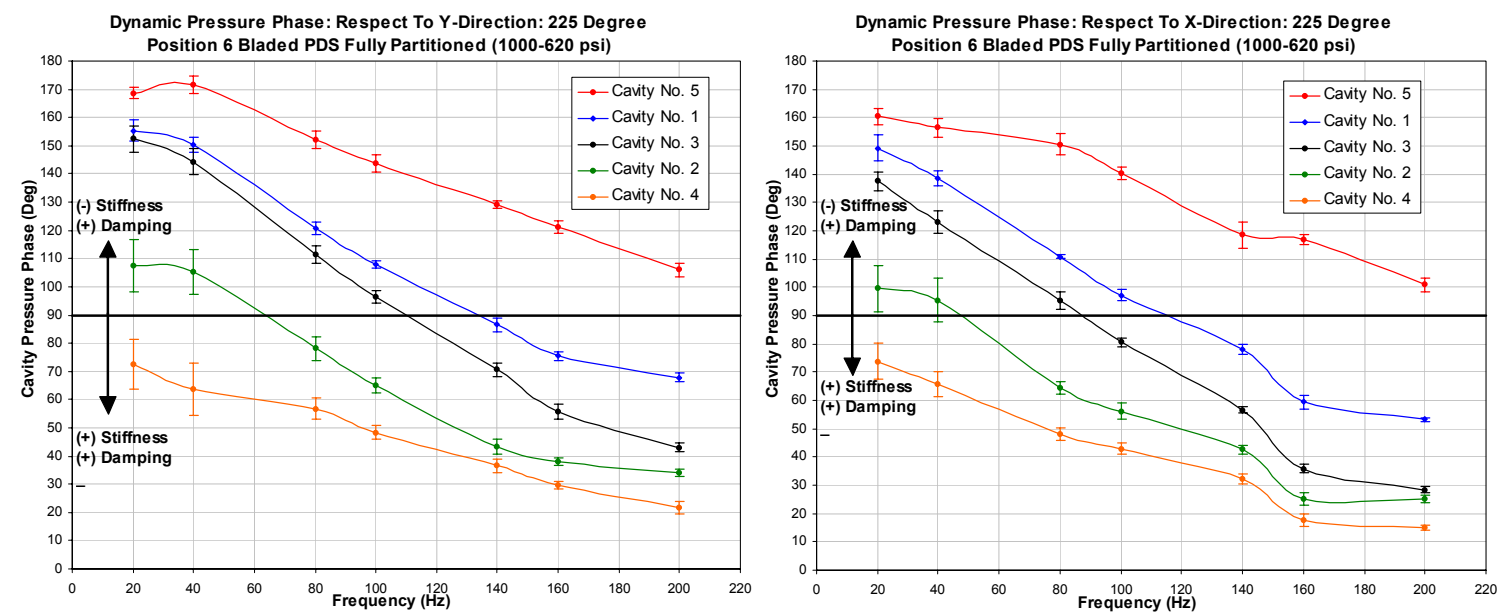

Dynamic Pressure Phase
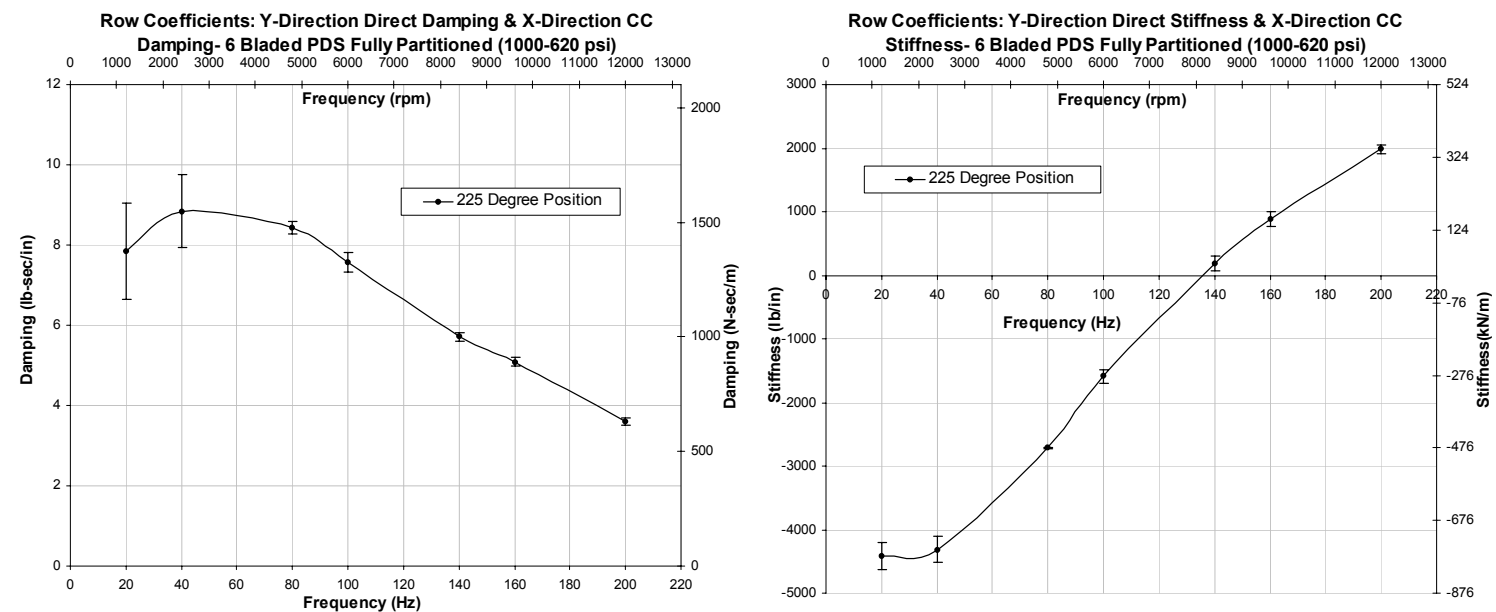

Y Direction Direct Coefficients \& X-Direction Cross-coupled Coefficients 

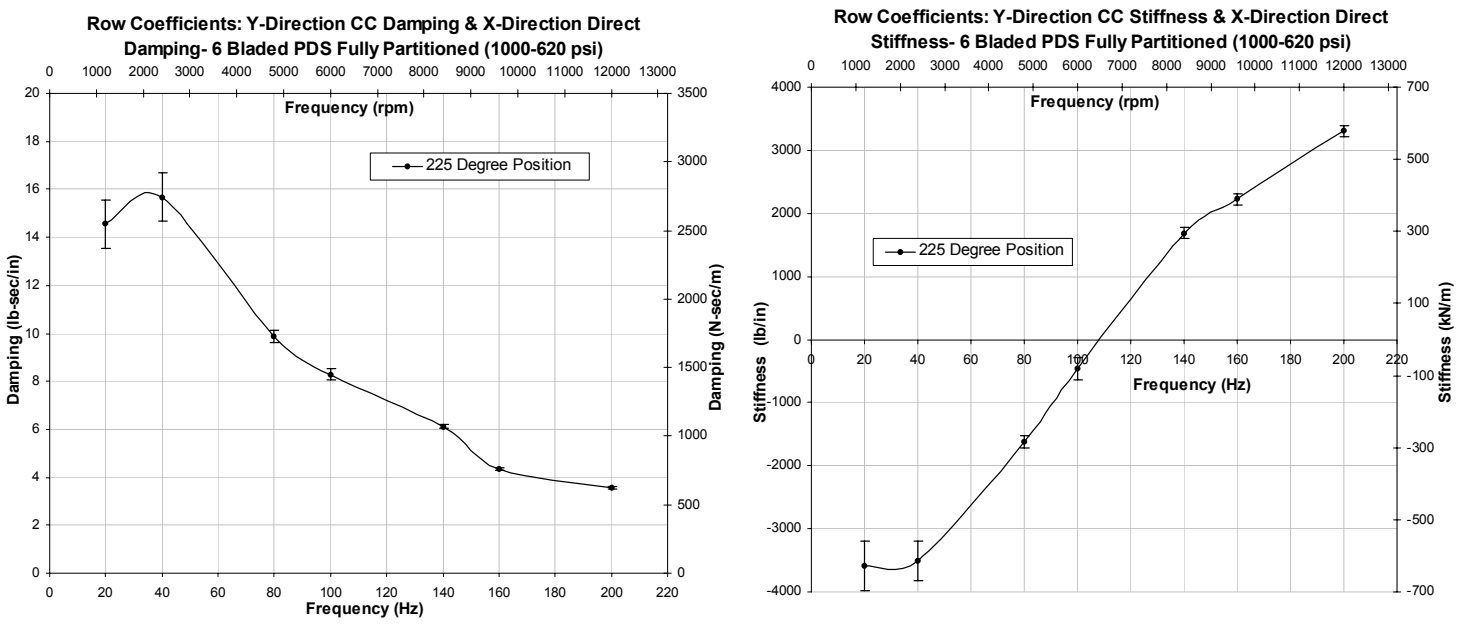

X Direction Direct Coefficients \& Y-Direction Cross-coupled Coefficients 


\section{Bladed Fully Partitioned Seal Cavity Coefficients: 315 Degree Position}
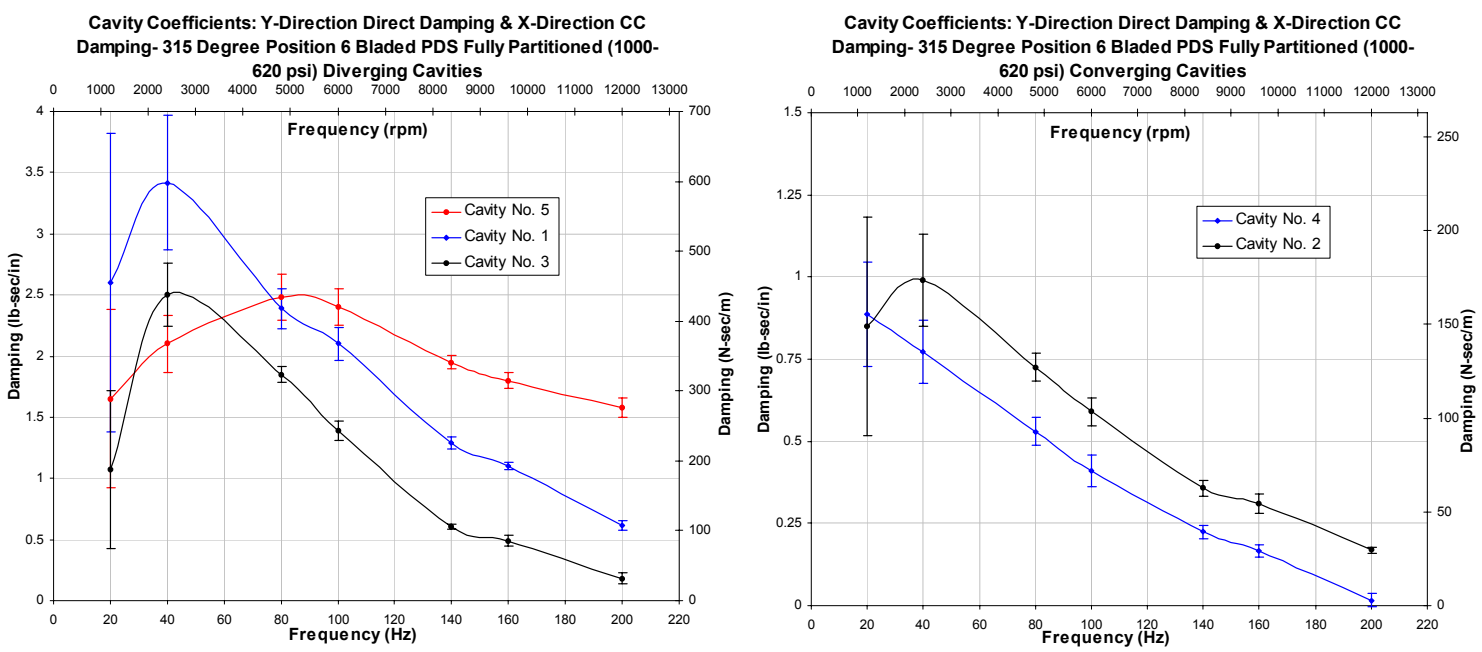

Y Direction Direct Damping \& X-Direction Cross-coupled Damping

Cavity Coefficients: Y-Direction Direct Stiffness \& X-Direction CC Stiffness 315 Degree Position- 6 Bladed PDS Fully Partitioned (1000-

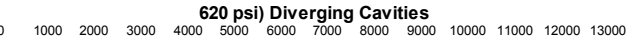

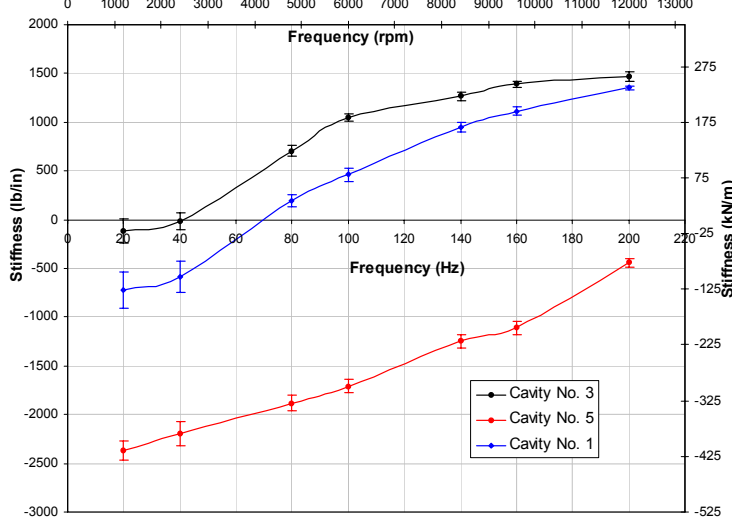

Y Direction Direct Stiffness \& X-Direction Cross-coupled Stiffness Stiffness 315 Degree Position- 6 Bladed PDS Fully Partitioned (1000-

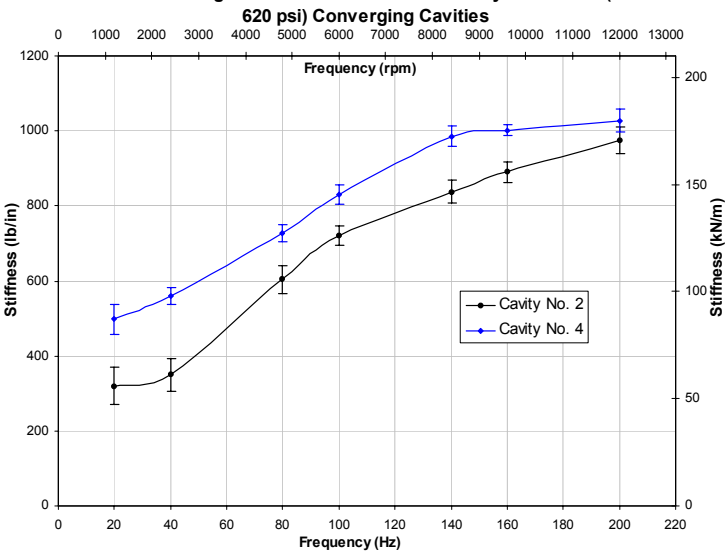

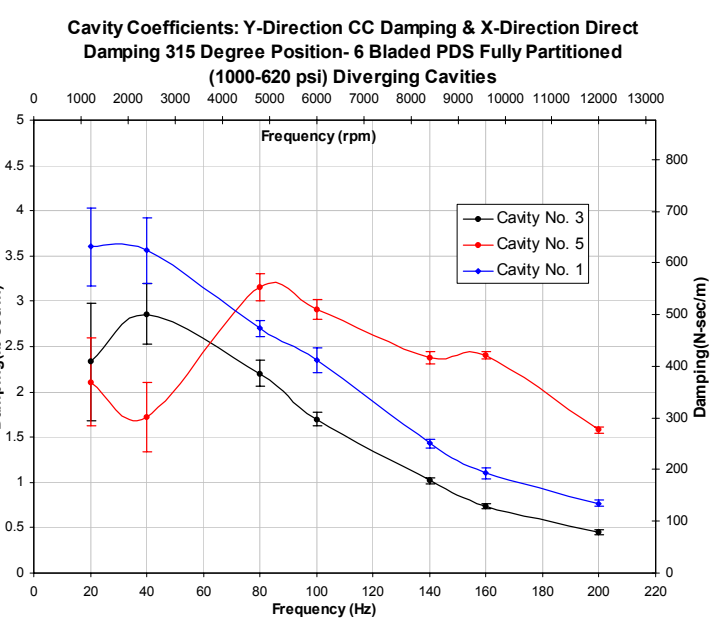

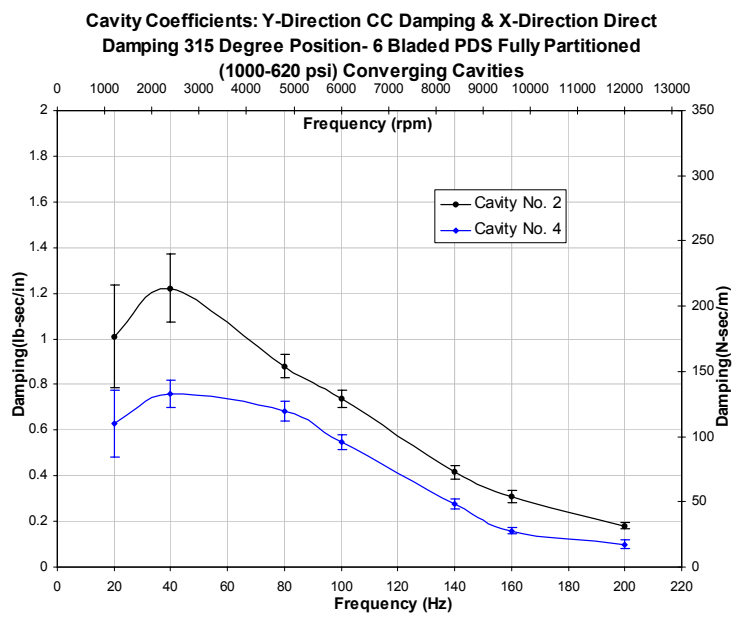

X Direction Direct Damping \& Y-Direction Cross-coupled Damping 

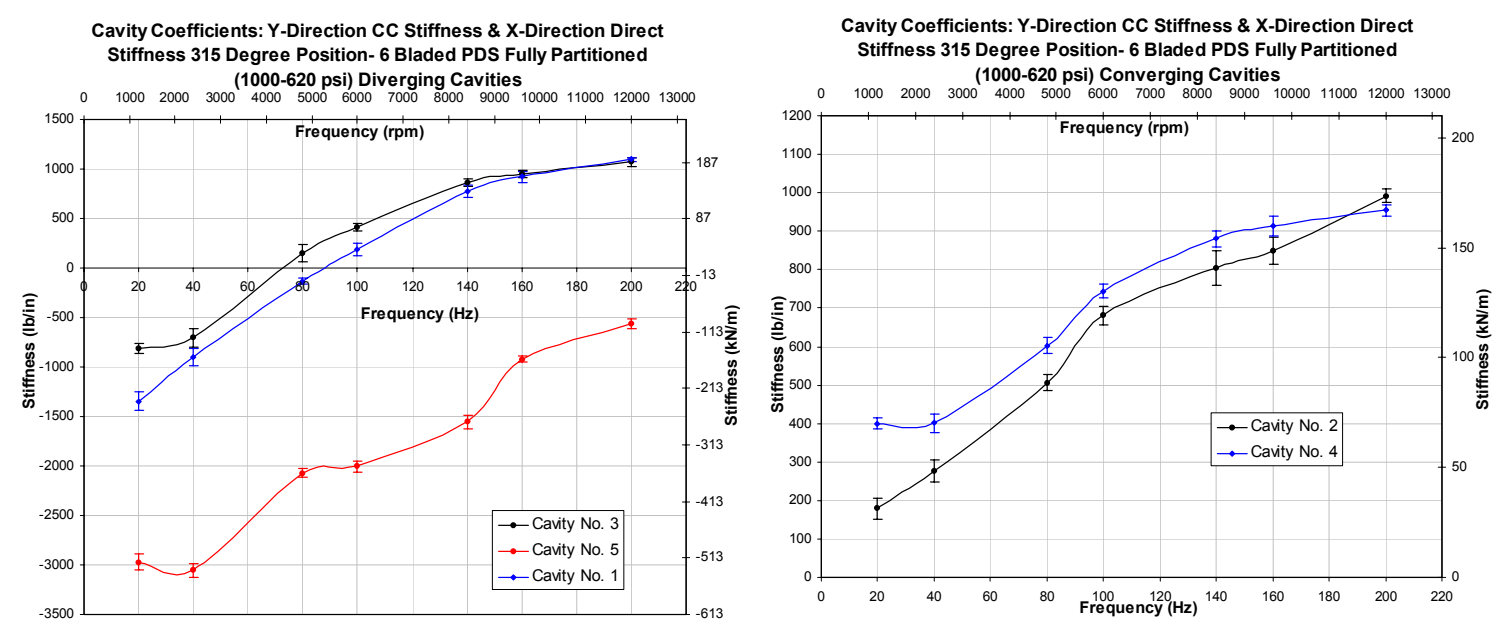

X Direction Direct Stiffness \& Y-Direction Cross-coupled Stiffness
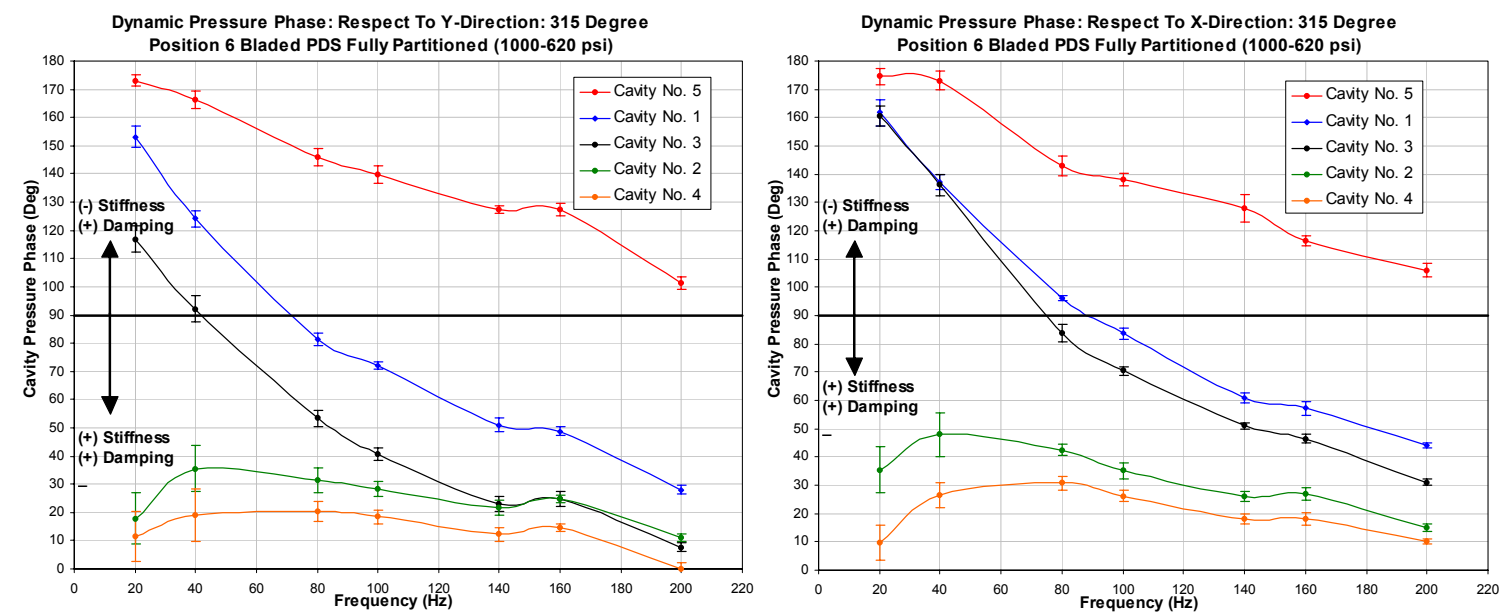

Dynamic Pressure Phase
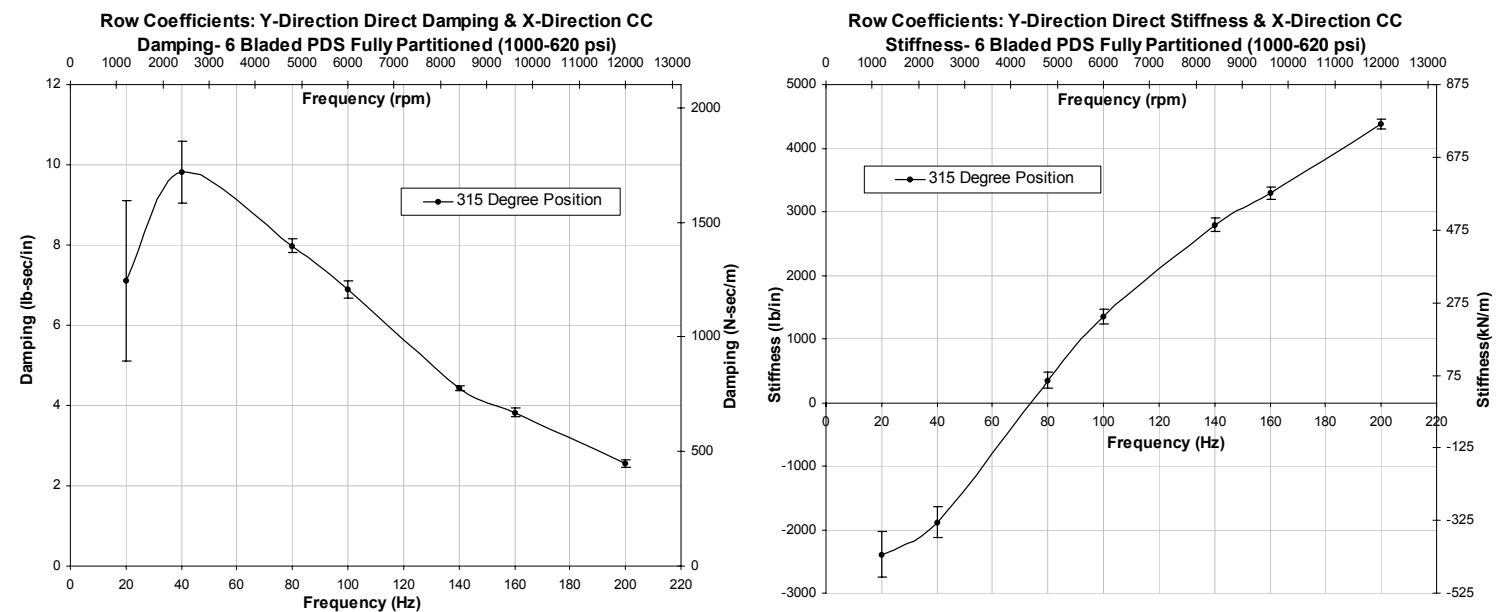

Y Direction Direct Coefficients \& X-Direction Cross-coupled Coefficients 

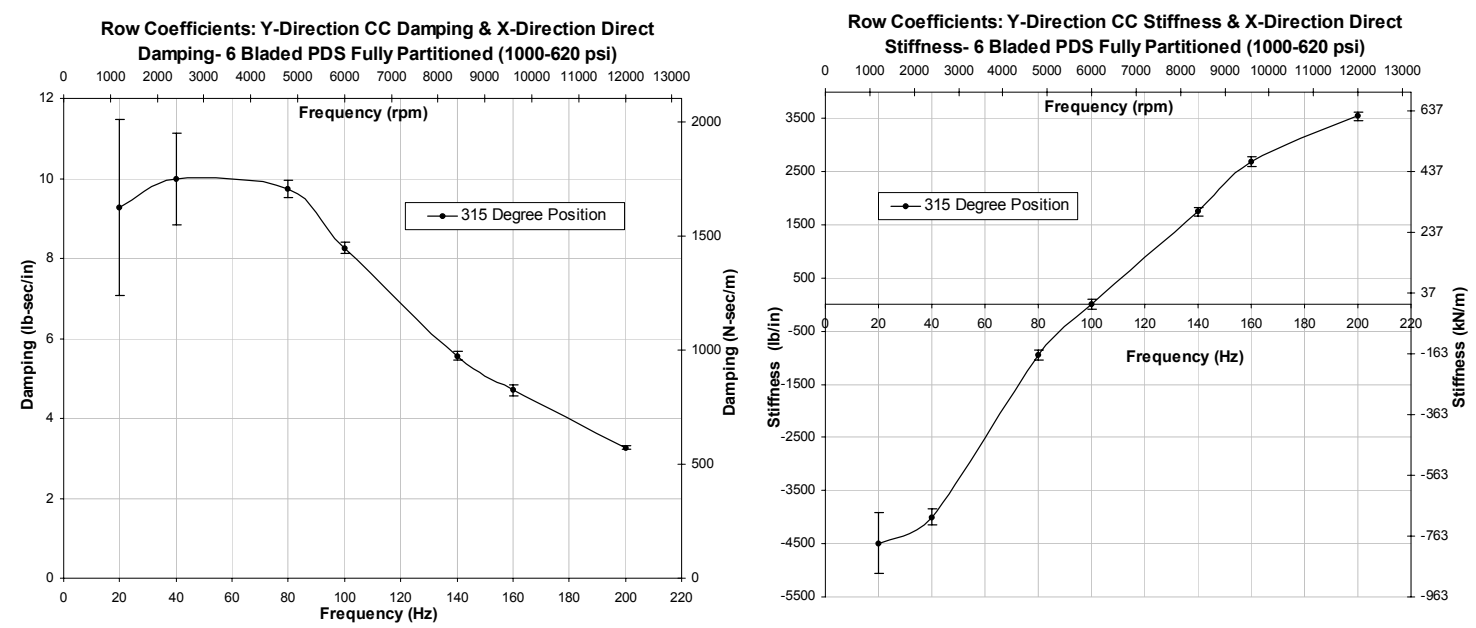

X Direction Direct Coefficients \& Y-Direction Cross-coupled Coefficients 
APPENDIX D

PDS DYNAMIC PRESSURE RESULTS 

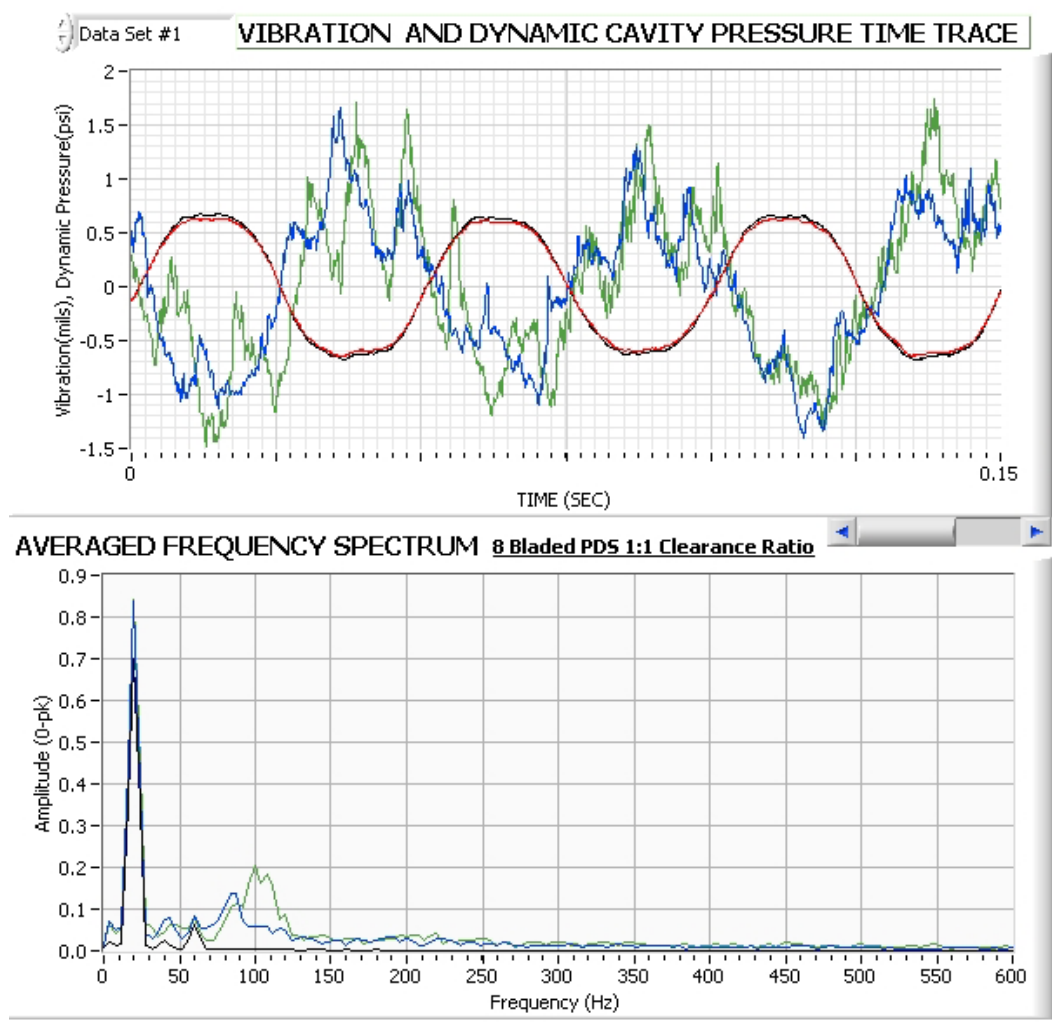

Cavity\#3

FREOUENCY (HZ)

1) 20

Zero-pk Motion (mils)

0.698066

Cavity \#3 Phase (dea)

165.361

Cavity \#3 Zero-pk (psi)

0.835489

Cavity \#5 Phase (dea)

159.836

Cavity \#5 Zero-pk (psi)

0.841834

P/Y (lbf/in`3) Cavity \#3

1196.86

$\mathrm{P} / \mathrm{Y}(\mathrm{lbf} / \mathrm{in} \wedge 3)$ Cavity $\# \mathbf{5}$

1205.95

Stiffness, Kyy (lb/in)

Cavity \#3(Kyy) Cavity \#5(Kyy)

$\begin{array}{ll}-830.986 & -812.35\end{array}$

Damping, Cyy (lb-sec/in)

Cavity \#3(Cyy) Cavity \#5(Cyy)

$1.72736 \quad 2.37389$

AVG -M28-MG 公

Cavity\#3

Cavity\#5

$20 \mathrm{~Hz} 8$ Bladed No Divergence

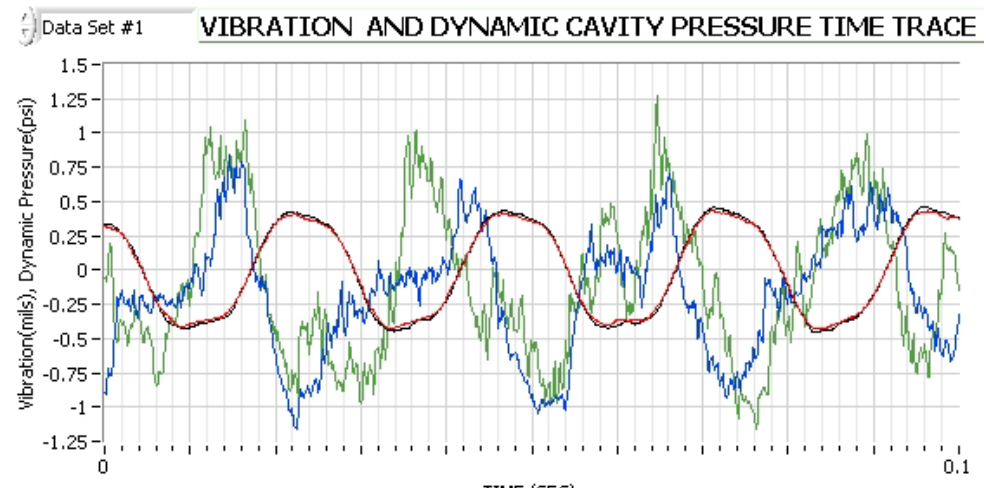

Cavity\#3

Cavity\#5

7) 40

Zero-pk Motion (mils)

0.472287

Cavity \#3 Phase (deq)

145.308

Cavity \#3 Zero-pk (psi)

0.612084

Cavity \#5 Phase (dea)

136.886

Cavity \#5 Zero-pk (psi)

AVERAGED FREQUENCY SPECTRUM 8 Bladed PDS 1:1 Clearance Ratio

-

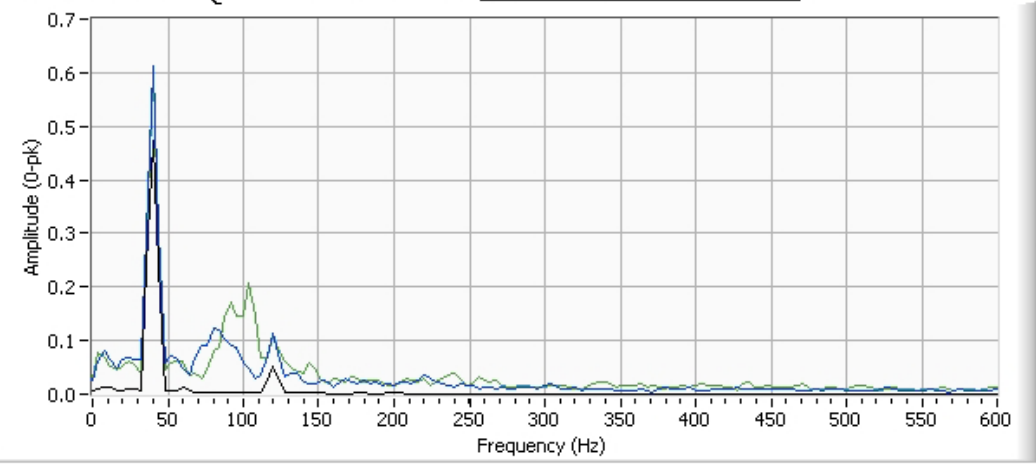

$\mathrm{P} / \mathrm{Y}(\mathrm{lbf} / \mathrm{in} \wedge 3)$ Cavity $\# \mathbf{3}$

1296

$\underline{P} / Y\left(\mathrm{lbf} / \mathrm{in}^{\wedge} 3\right)$ Cavity $\# \mathbf{5}$

1260.18

Stiffness, Kyy (lb/in)

Cavity \#3(Kyy) Cavity \#5(Kyy)

$-764.678-660.142$

Damping, Cyy (lb-sec/in)

Cavity \#3(Cyy) Cavity \#5(Cyy)

$\begin{array}{ll}2.10612 & 2.45914\end{array}$

AVG -M2\&-MG

Cavity\#3

Cavity\#5

$40 \mathrm{~Hz} 8$ Bladed No Divergence 

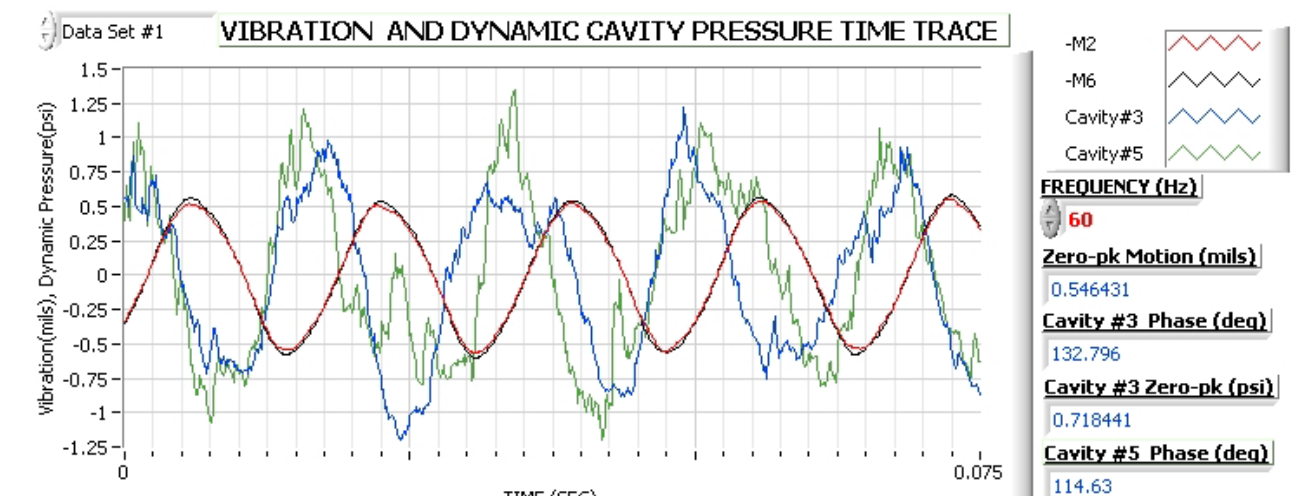

Zero-pk Motion (mils)

0.546431

Cavity \#3 Phase (deq)

132.796

Cavity \#3 Zero-pk (psi)

0.718441

Cavity \# 5 Phase (deq)

TIME (SEC)

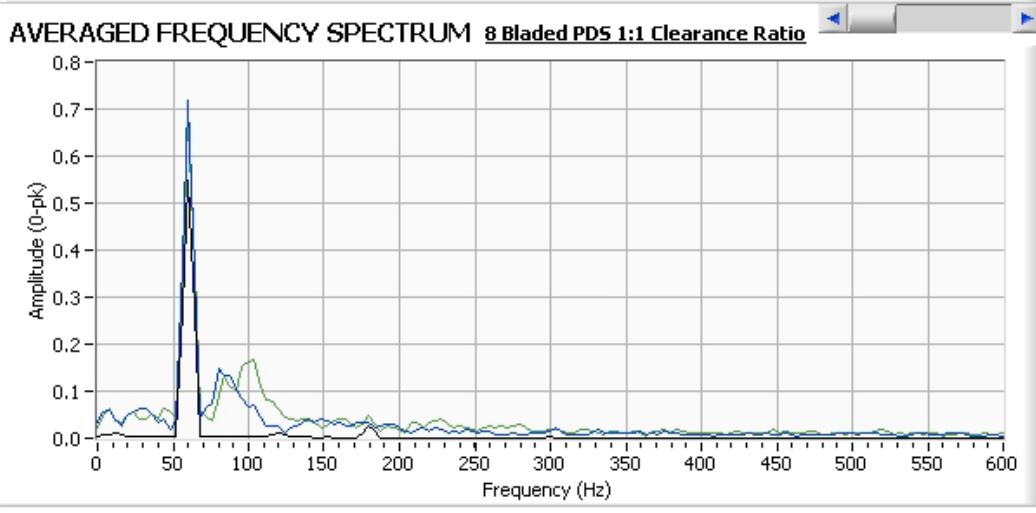

Cavity \#5 Zero-pk (psi)

0.688357

P/Y (lbf/in^3) Cavity \#3

1314.79

P/Y (lbf/in`3) Cavity \#5

1259.73

Stiffness, Kyy (lb/in)

Cavity \#3(Kyy) Cavity \#5(Kyy)

$-641.004-376.738$

Damping, Cyy (lb-sec/in)

Cavity \#3(Cyy) Cavity \#5(Cyy)

$\begin{array}{lll}1.83641 & 2.17973\end{array}$

AVG -M28-MG

Cavity\#3

$60 \mathrm{~Hz} 8$ Bladed No Divergence
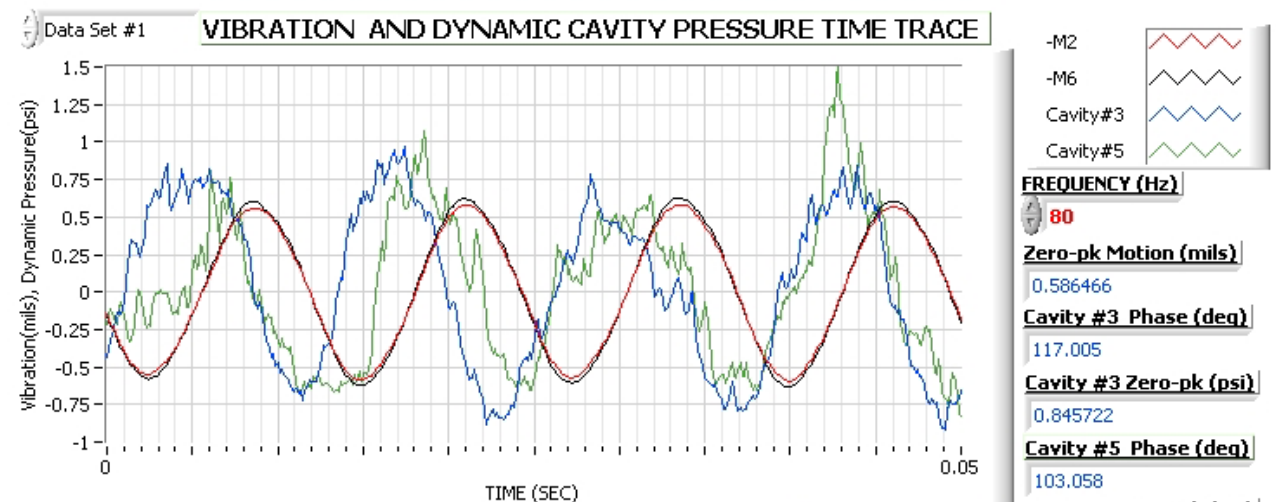

FREQUENCY (Hz)

광 80

Zero-pk Motion (mils)

0.586466

Cavity \#3 Phase (dea)

117.005

Cavity \#3 Zero-pk (psi)

0.845722

Cavity \#5 Phase (deq)

103.058

Cavity $\# 5$ Zero-pk (psi)

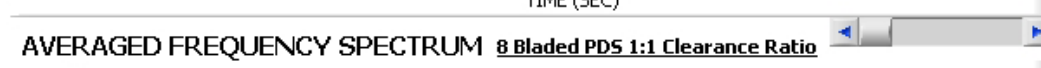

0.721229

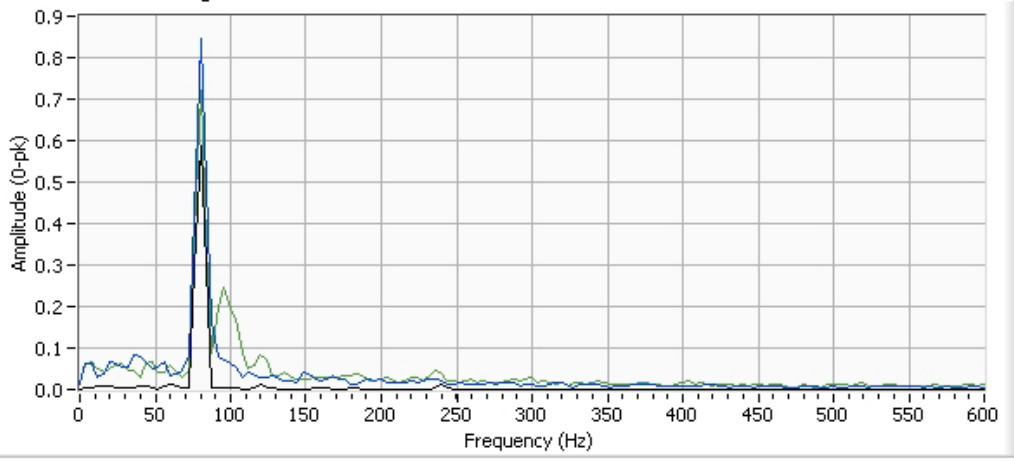

P/Y (lbf/in`3) Cavity\#3

1442.06

P/Y (lbf/in`3) Cavity \#5

1229.79

Stiffness, Kyy (lb/in)

Cavity \#3(Kyy) Cavity \#5(Kyy)

$-469.88 \quad-199.389$

Damping, Cyy (lb-sec/in)

Cavity \#3(Cyy) Cavity \#5(Cyy)

$1.83425 \quad 1.71027$

AVG -M2\&-MG

Cavity\#3

Cavity\#5

$80 \mathrm{~Hz} 8$ Bladed No Divergence 


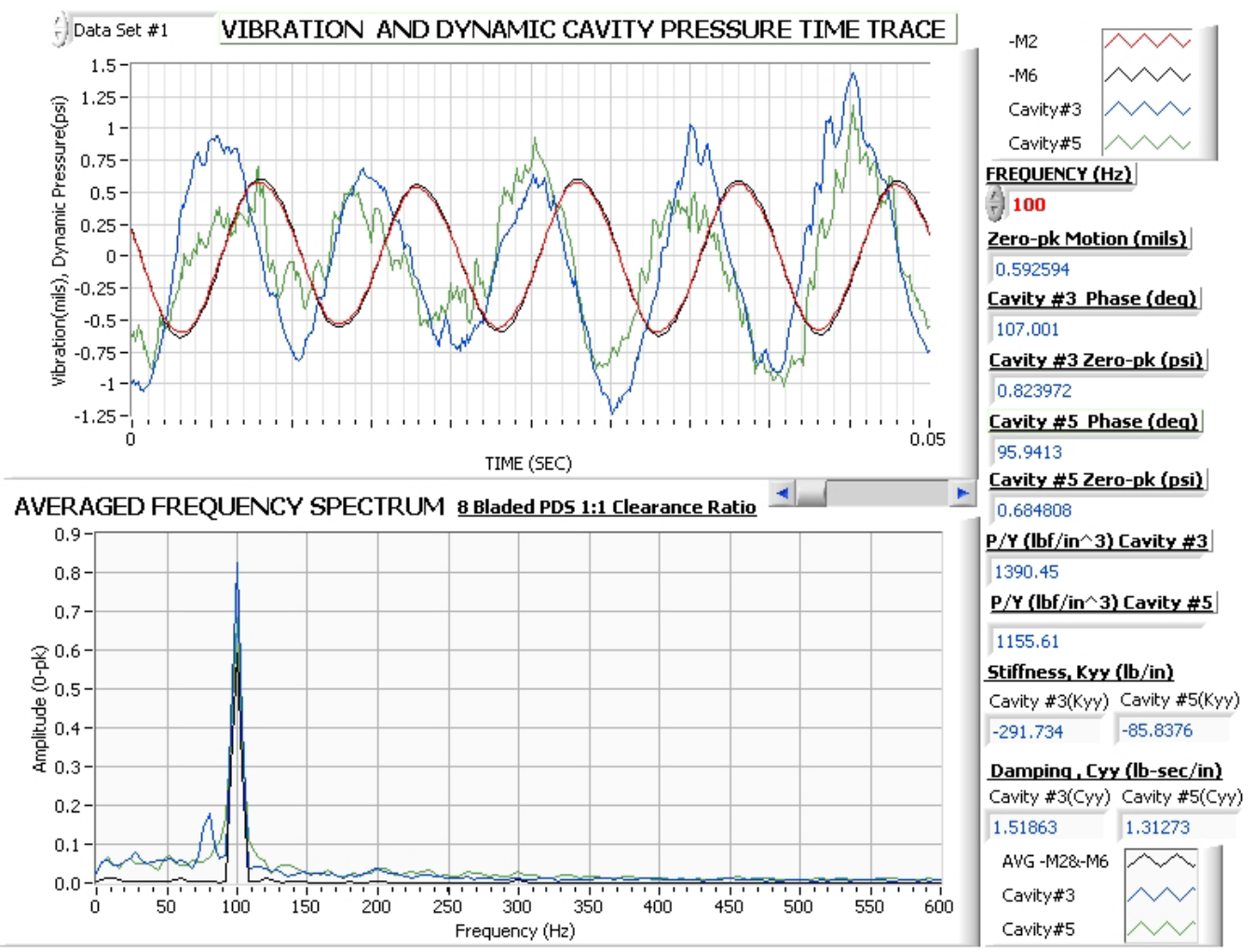

$100 \mathrm{~Hz} 8$ Bladed No Divergence
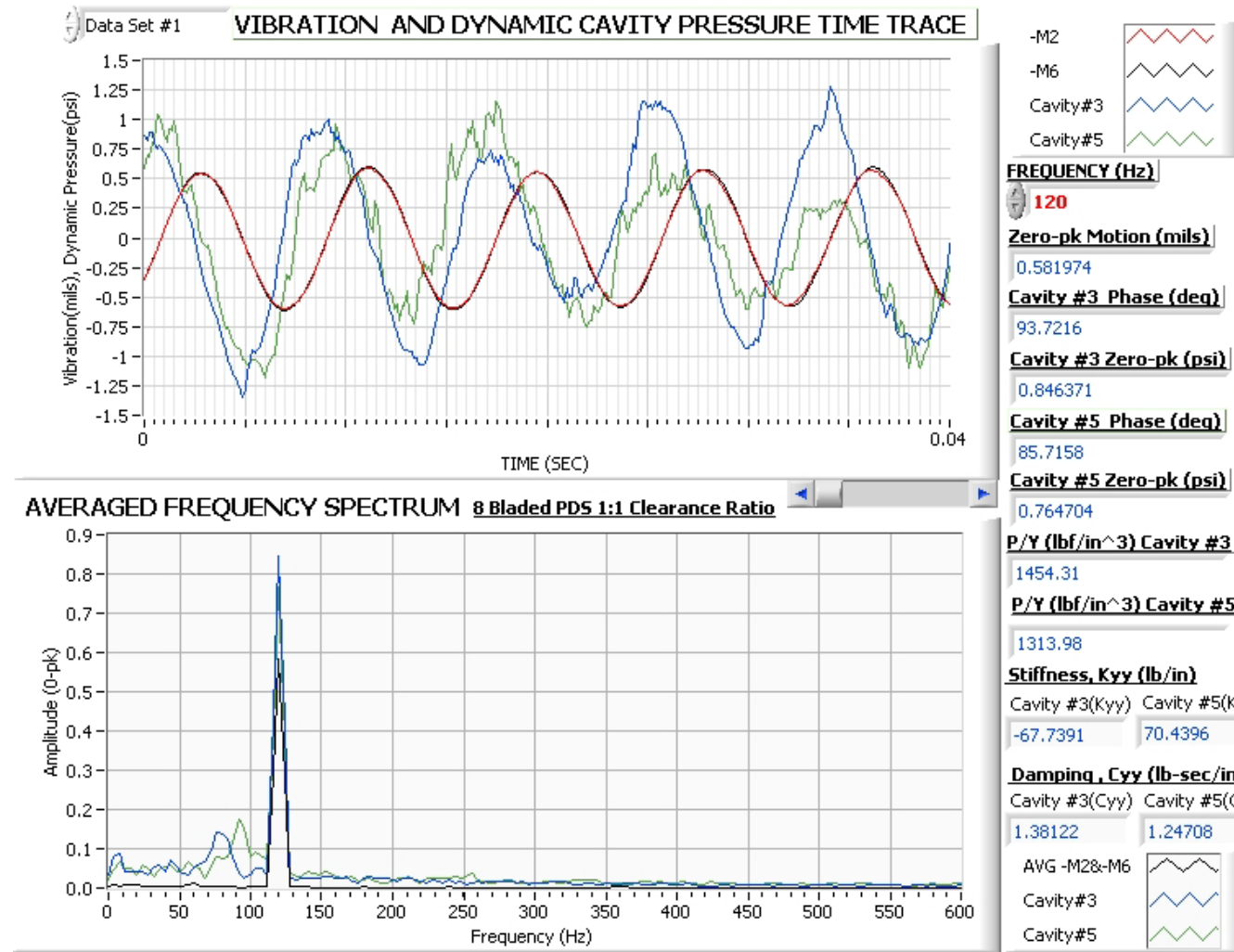

Cavity \#5 Zero-pk (psi)

0.764704

$\mathrm{P} / \mathrm{Y}\left(\mathrm{lbf} / \mathrm{in}^{\wedge} \mathbf{3}\right)$ Cavity \#3

1454.31

$\mathrm{P} / \mathrm{Y}(\mathrm{lbf} / \mathrm{in} \wedge 3)$ Cavity \#5

1313.98

Stiffness, Kyy (lb/in)

Cavity \#3(Kyy) Cavity \#5(Kyy)

$-67.7391 \quad 70.4396$

Damping, Cyy (lb-sec/in)

Cavity \#3(Cyy) Cavity \#5(Cyy)

$1.38122 \quad 1.24708$

AVG -M2\&-MG $\leadsto$

Cavity\#3

Cavity\#5

$120 \mathrm{~Hz} 8$ Bladed No Divergence 


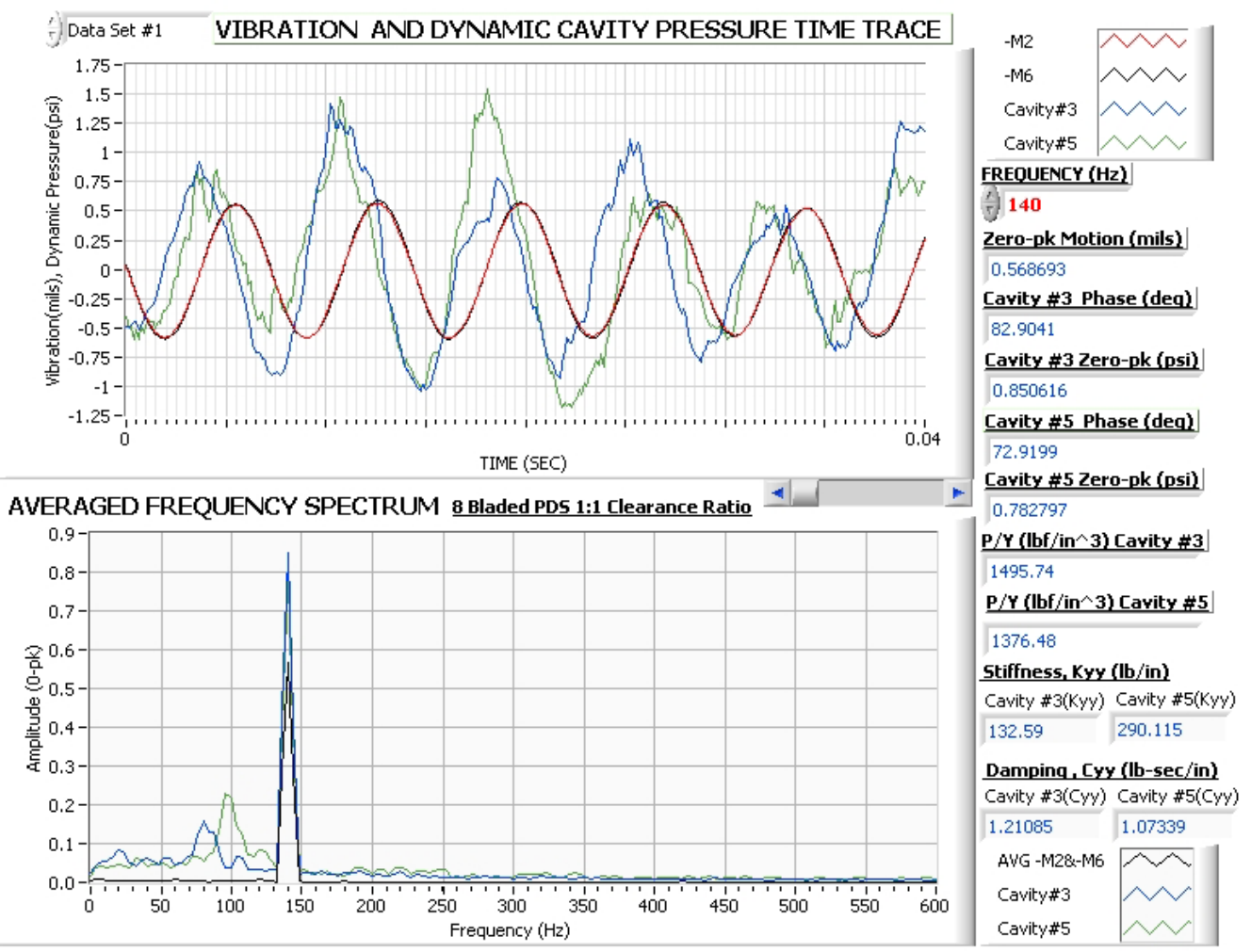

$140 \mathrm{~Hz} 8$ Bladed No Divergence
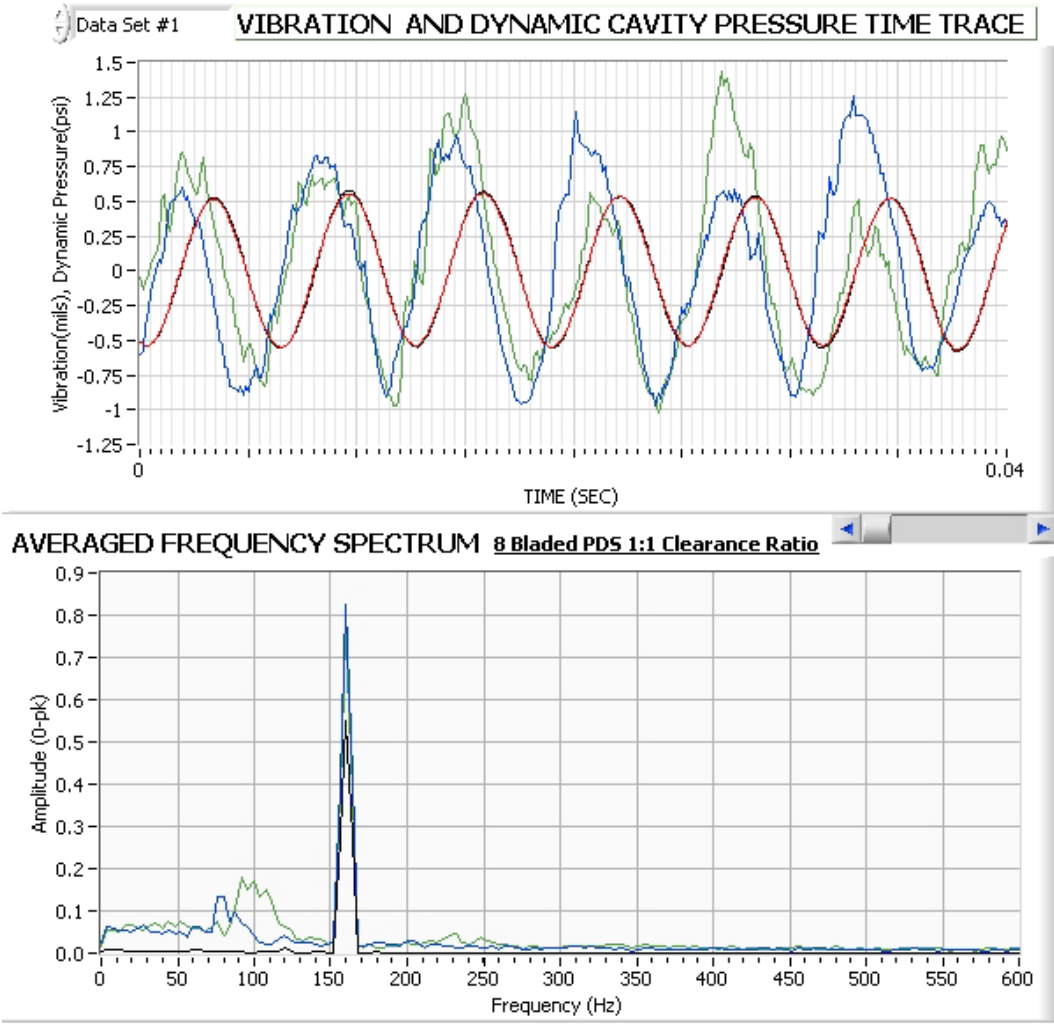

$-M 2$

-M6

Cavity\#3

$\curvearrowright$

Cavity\#5

$\leadsto$

FREOUENCY (Hz)

160

Zero-pk Motion (mils)

0.54903

Cavity \#3 Phase (deq)

75.8654

Cavity \#3 Zero-pk (psi)

0.825049

Cavity \#5 Phase (dea)

64.4636

Cavity $\# 5$ Zero-pk (psi)

0.758656

$\mathrm{P} / \mathrm{Y}$ (lbf/in`3) Cavity \#3

1502.74

P/Y (lbf/in^3) Cavity \#5

1381.81

Stiffness, Kyy (lb/in)

Cavity \#3(Kyy) Cavity \#5(Kyy)

$263.337 \quad 427.459$

Damping, Cyy (lb-sec/in)

Cavity \#3(Cyy) Cavity \#5(Cy)

$1.0402 \quad 0.889996$

AVG-M2\&-MG ఋ

Cavity\#3

Cavity\#5

$160 \mathrm{~Hz} 8$ Bladed No Divergence 

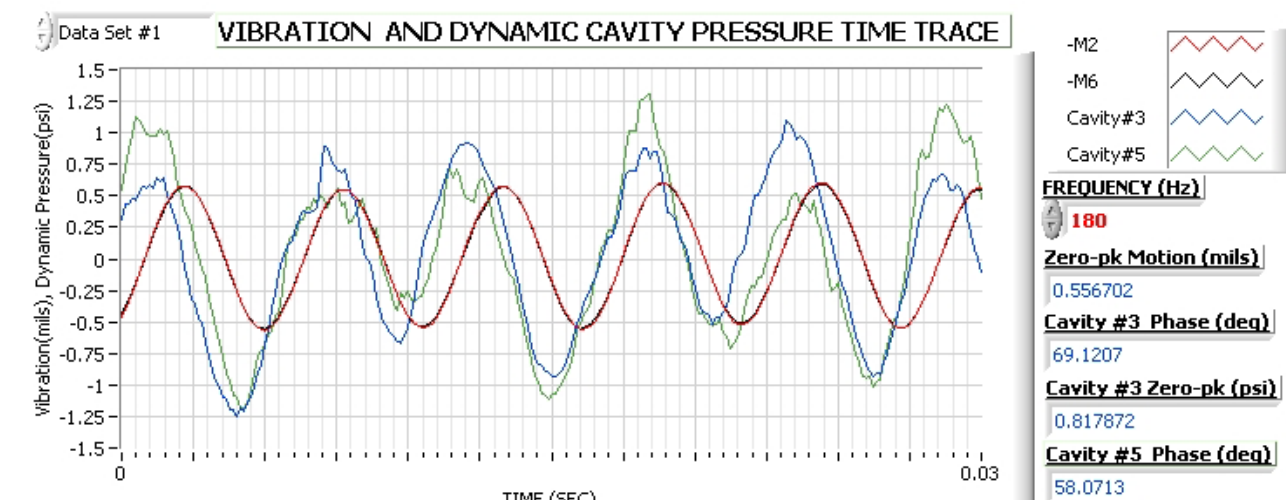

Zero-pk Motion (mils)

0.556702

Cavity \#3 Phase (deq)

69.1207

Cavity \#3 Zero-pk (psi)

0.817872

Cavity \#5 Phase (deq)

58.0713

Cavity \#5 Zero-pk (psi)

AVERAGED FREQUENCY SPECTRUM 8 Bladed PDS 1:1 Clearance Ratio

r

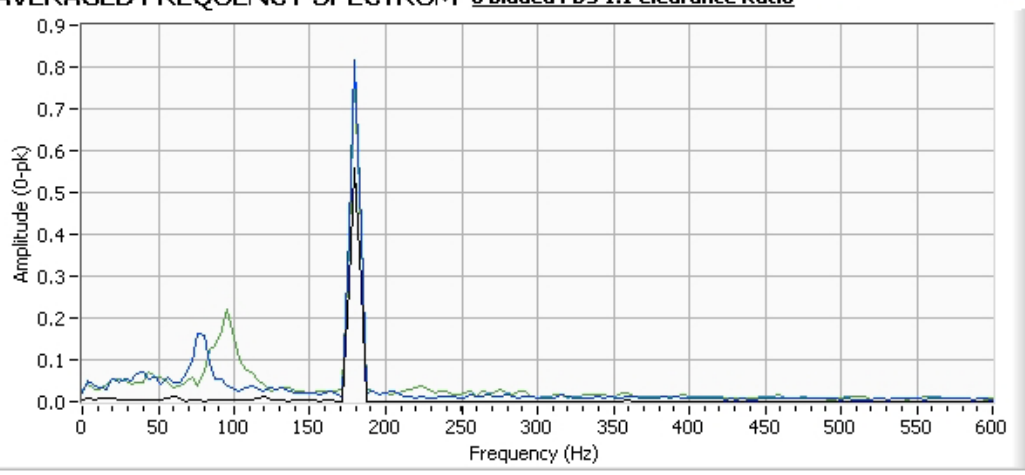

0.781449

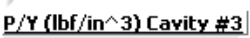

1469.14

$P / Y\left(\mathrm{lbf} / \mathrm{in}^{\wedge} 3\right)$ Cavity \#5

1403.71

Stiffness, Kyy (lb/in)

Cavity \#3(Kyy) Cavity \#5(Kyy)

$375.737 \quad 532.726$

Damping, Cyy (lb-sec/in)

Cavity \#3(Cyy) Cavity \#5(Cyy)

$\begin{array}{ll}0.870953 & 0.755904\end{array}$

AVG -M28-MG

Cavity\#3

Cavity\#5

$180 \mathrm{~Hz} 8$ Bladed No Divergence
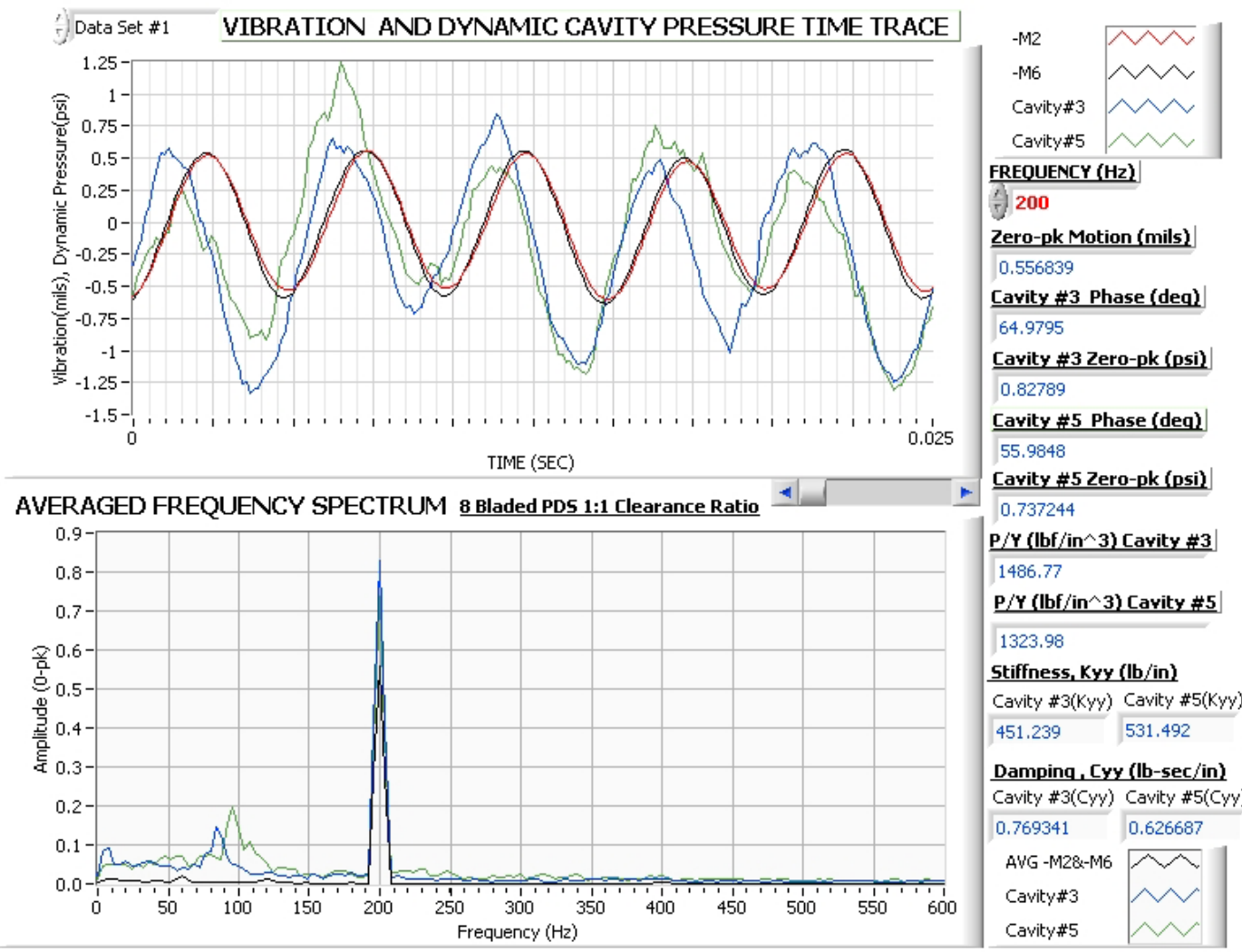

$200 \mathrm{~Hz} 8$ Bladed No Divergence 


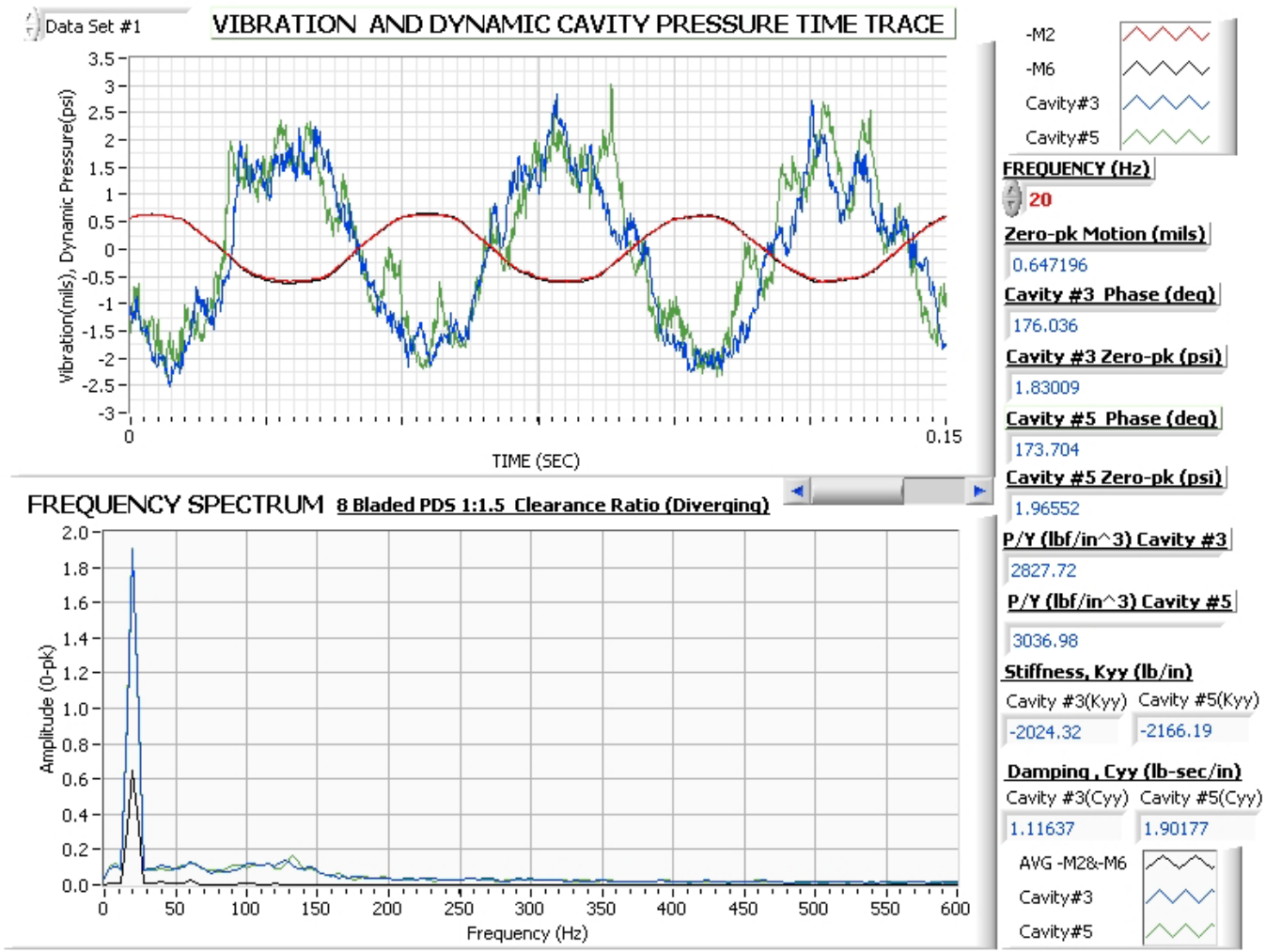

$20 \mathrm{~Hz} 8$ Bladed Diverging Seal 1:1.5 Clr Ratio (Vibration amp. 1.2 mils pk -pk)
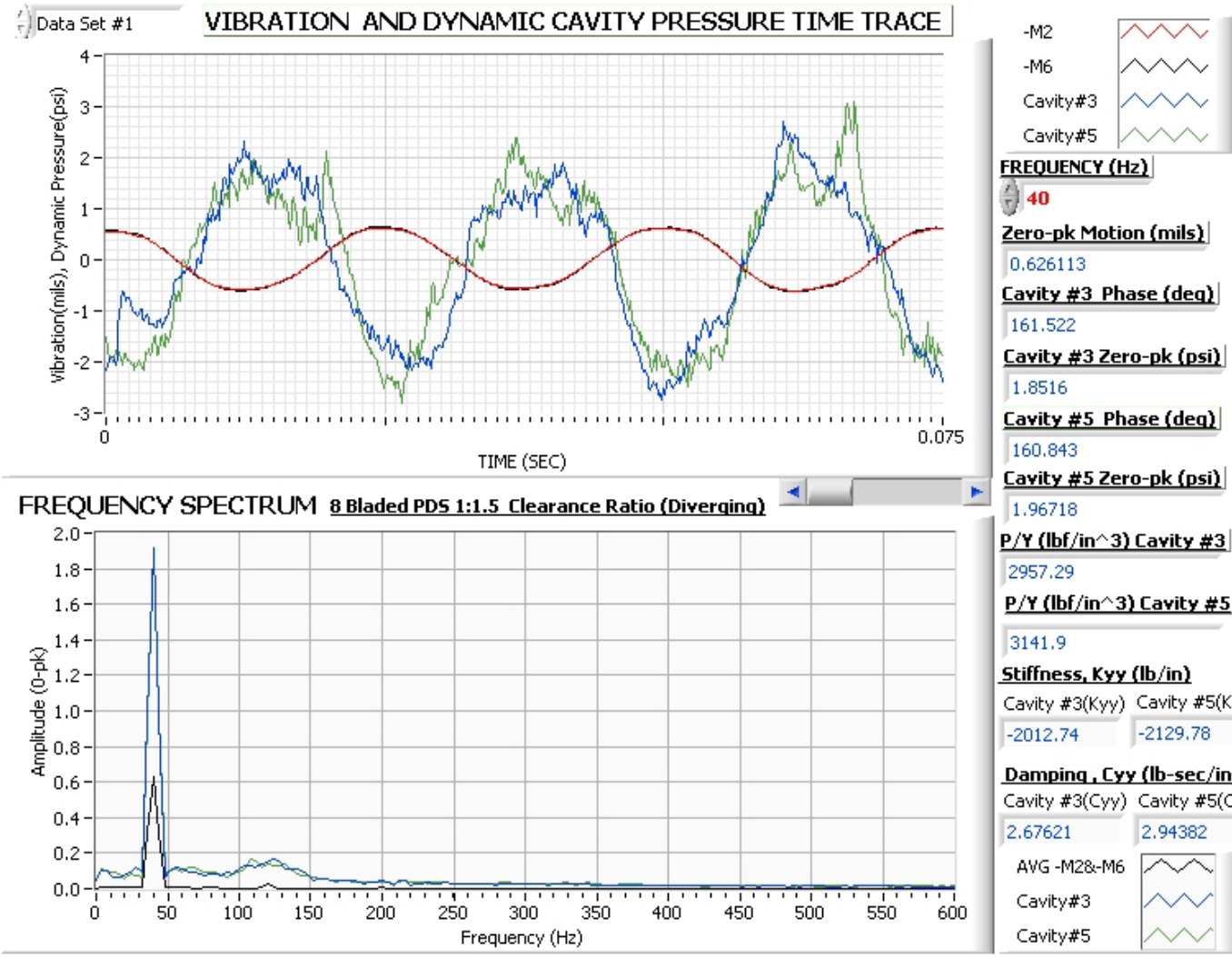

160.843

Cavity $\# 5$ Zero-pk (psi)

1.96718

P/Y (lbf/in`3) Cavity \#3

2957.29

$\mathrm{P} / \mathrm{Y}(\mathrm{lbf} / \mathrm{in} \wedge 3)$ Cavity \#5

3141.9

Stiffness, Kyy (lb/in)

Cavity \#3(Kyy) Cavity \#5(Kyy)

$-2012.74-2129.78$

Damping, Cyy (lb-sec/in)

Cavity \#3(Cyy) Cavity \#5(Cyy)

$2.67621 \quad 2.94382$

AVG -M28-MG

Cavity\#3

Cavity\#5

$40 \mathrm{~Hz} 8$ Bladed Diverging Seal 1:1.5 Clr Ratio (Vibration amp. 1.2 mils pk -pk) 


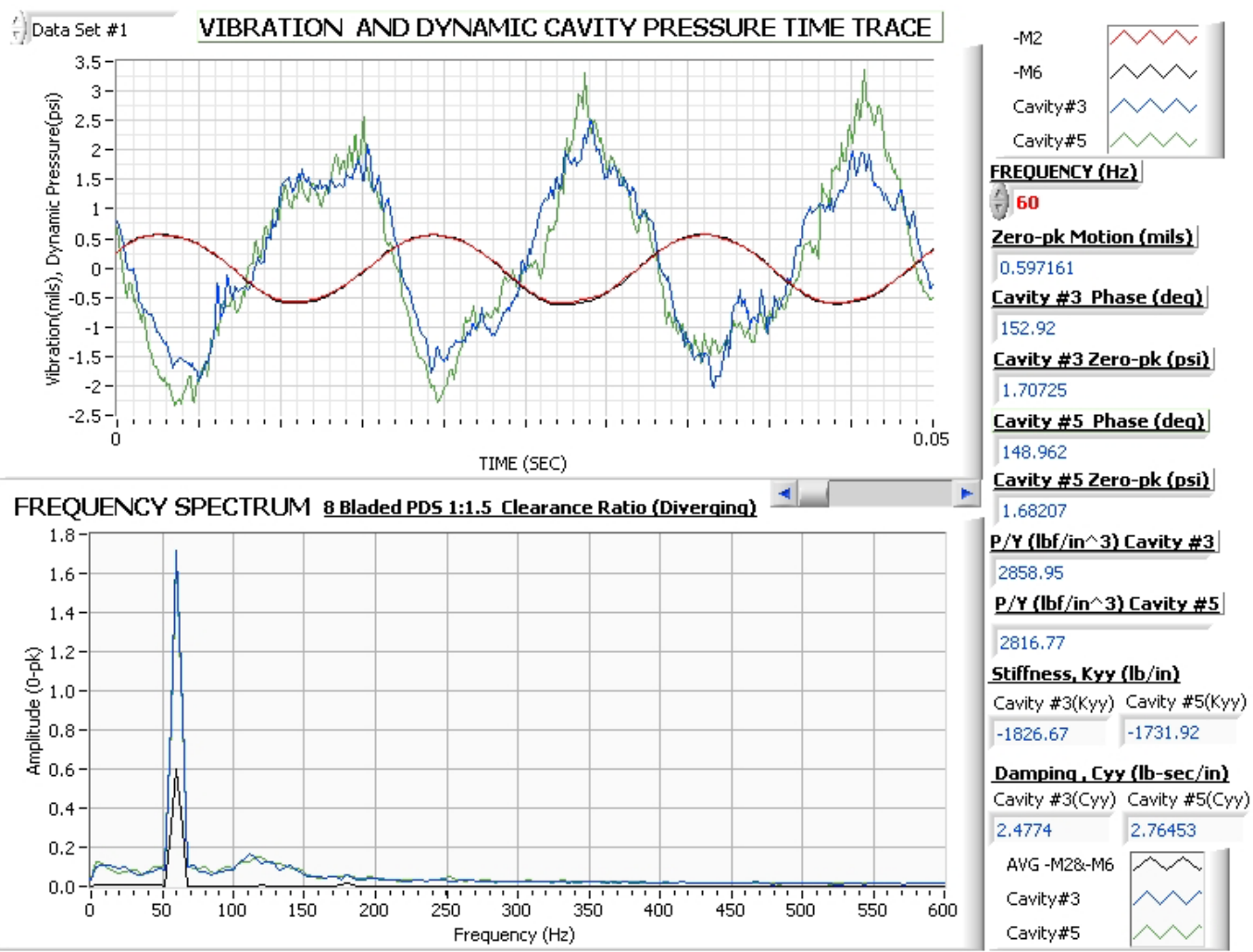

$60 \mathrm{~Hz} 8$ Bladed Diverging Seal 1:1.5 Clr Ratio (Vibration amp. 1.2 mils pk -pk)
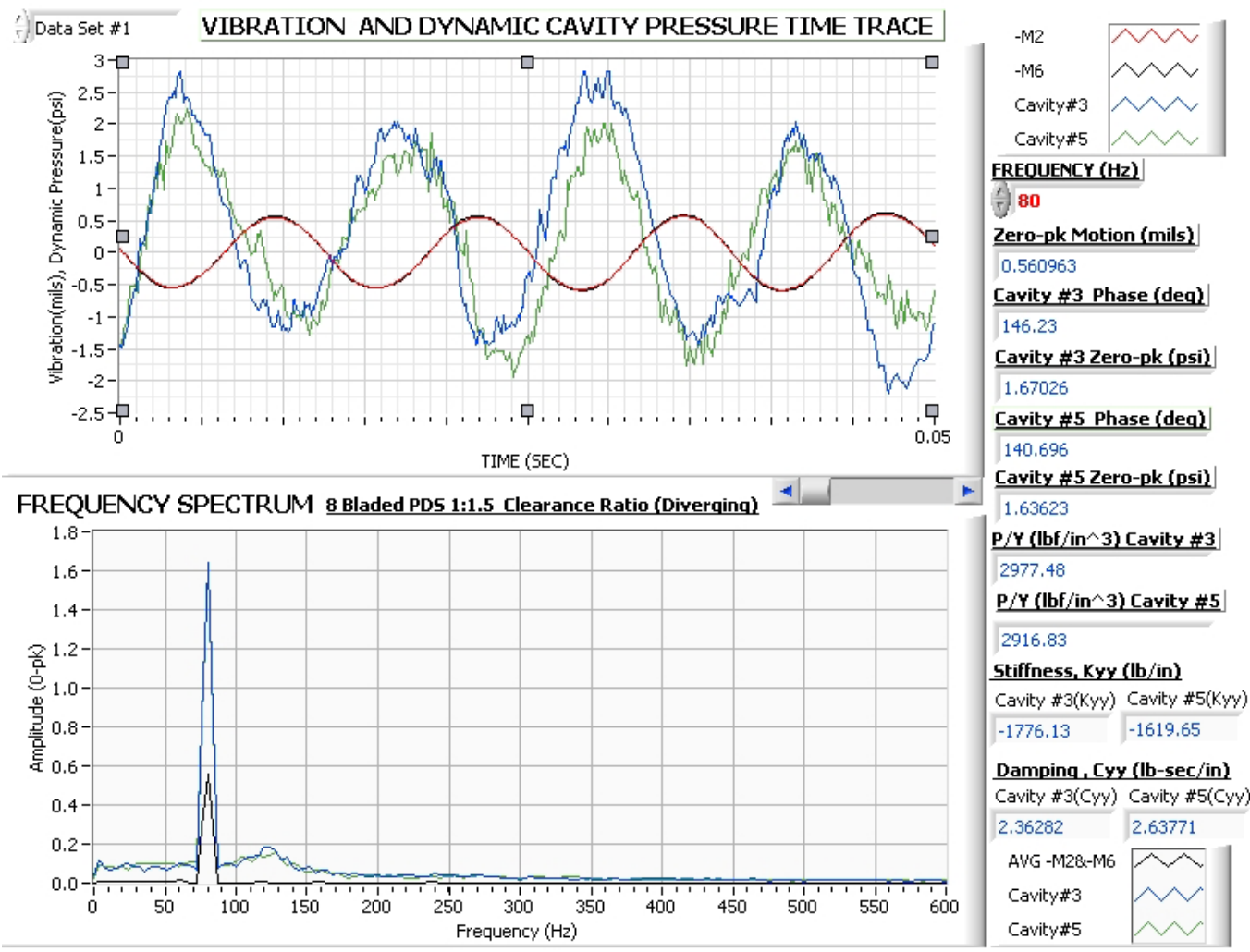

$80 \mathrm{~Hz} 8$ Bladed Diverging Seal 1:1.5 Clr Ratio (Vibration amp. 1.2 mils pk -pk) 


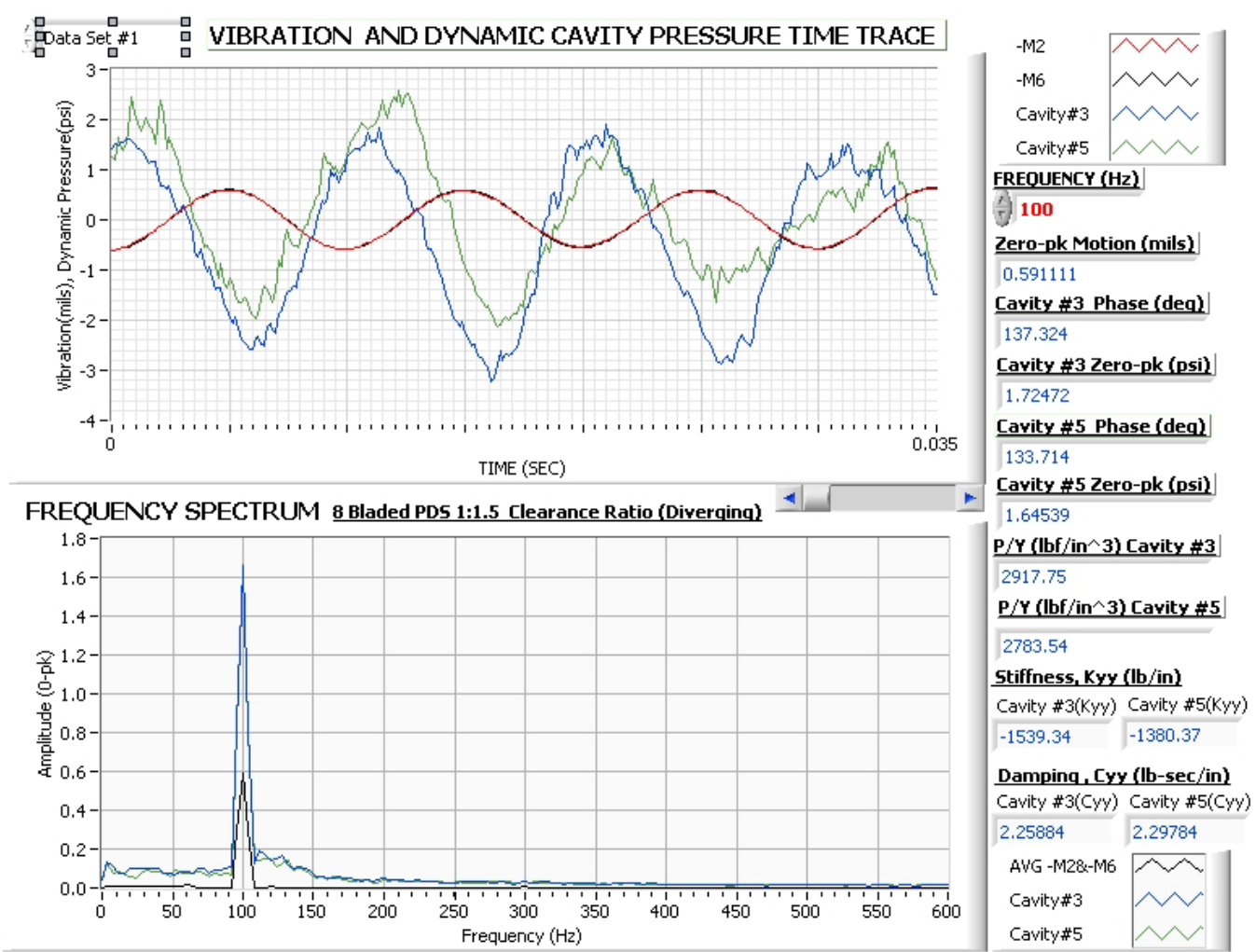

100 Hz 8 Bladed Diverging Seal 1:1.5 Clr Ratio (Vibration amp. 1.2 mils pk -pk)
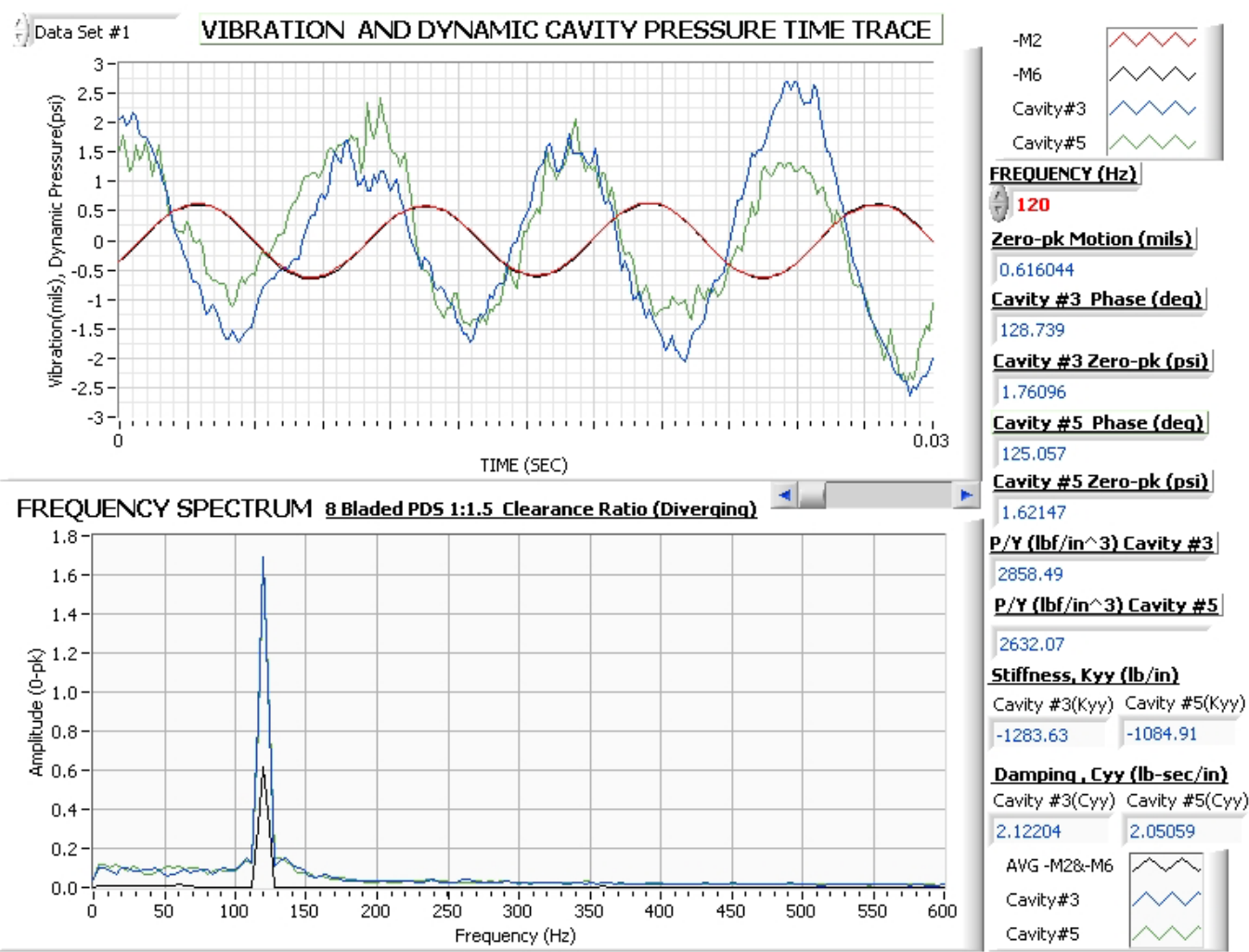

$120 \mathrm{~Hz} 8$ Bladed Diverging Seal 1:1.5 Clr Ratio (Vibration amp. 1.2 mils pk -pk) 


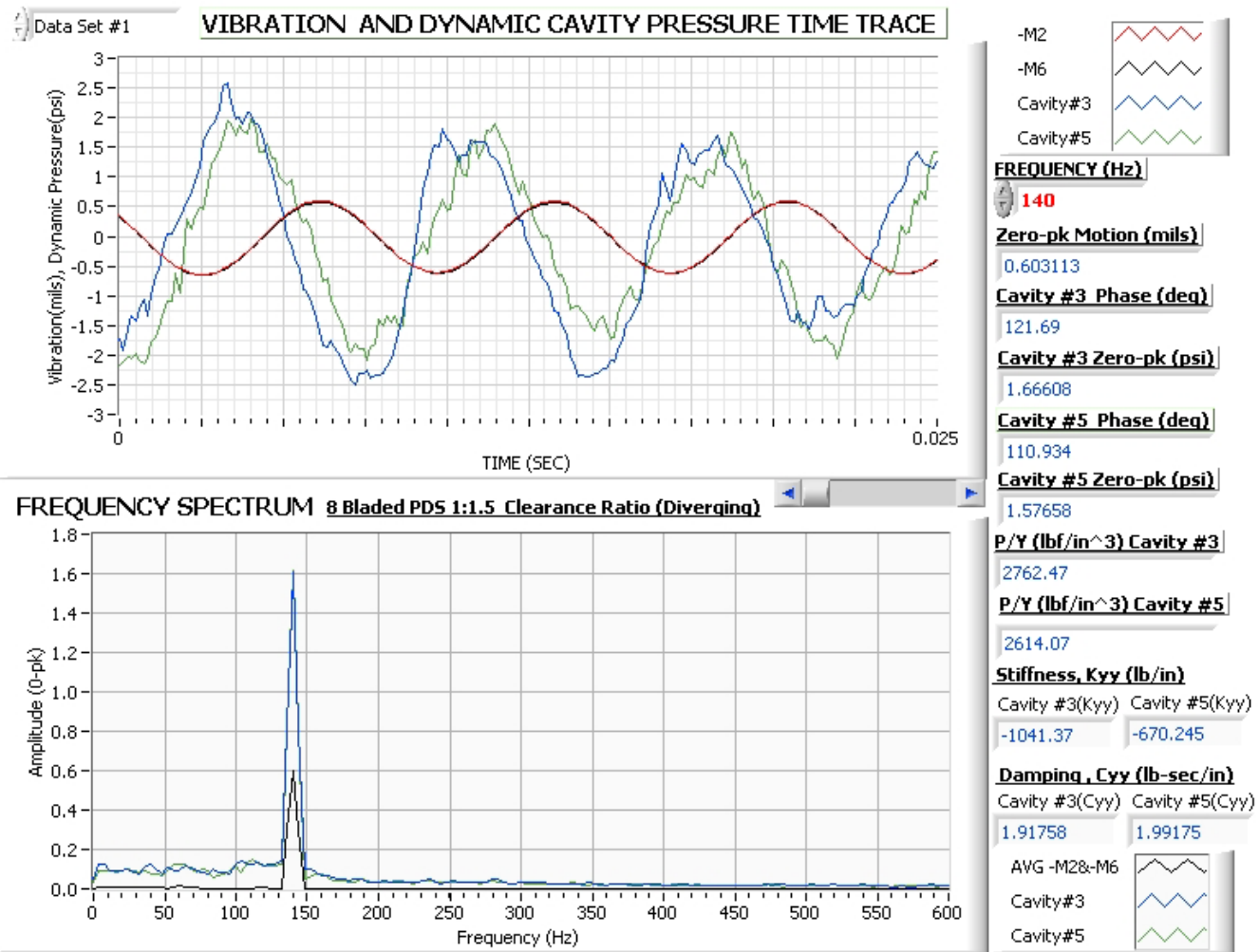

$140 \mathrm{~Hz} 8$ Bladed Diverging Seal 1:1.5 Clr Ratio (Vibration amp. 1.2 mils pk -pk)
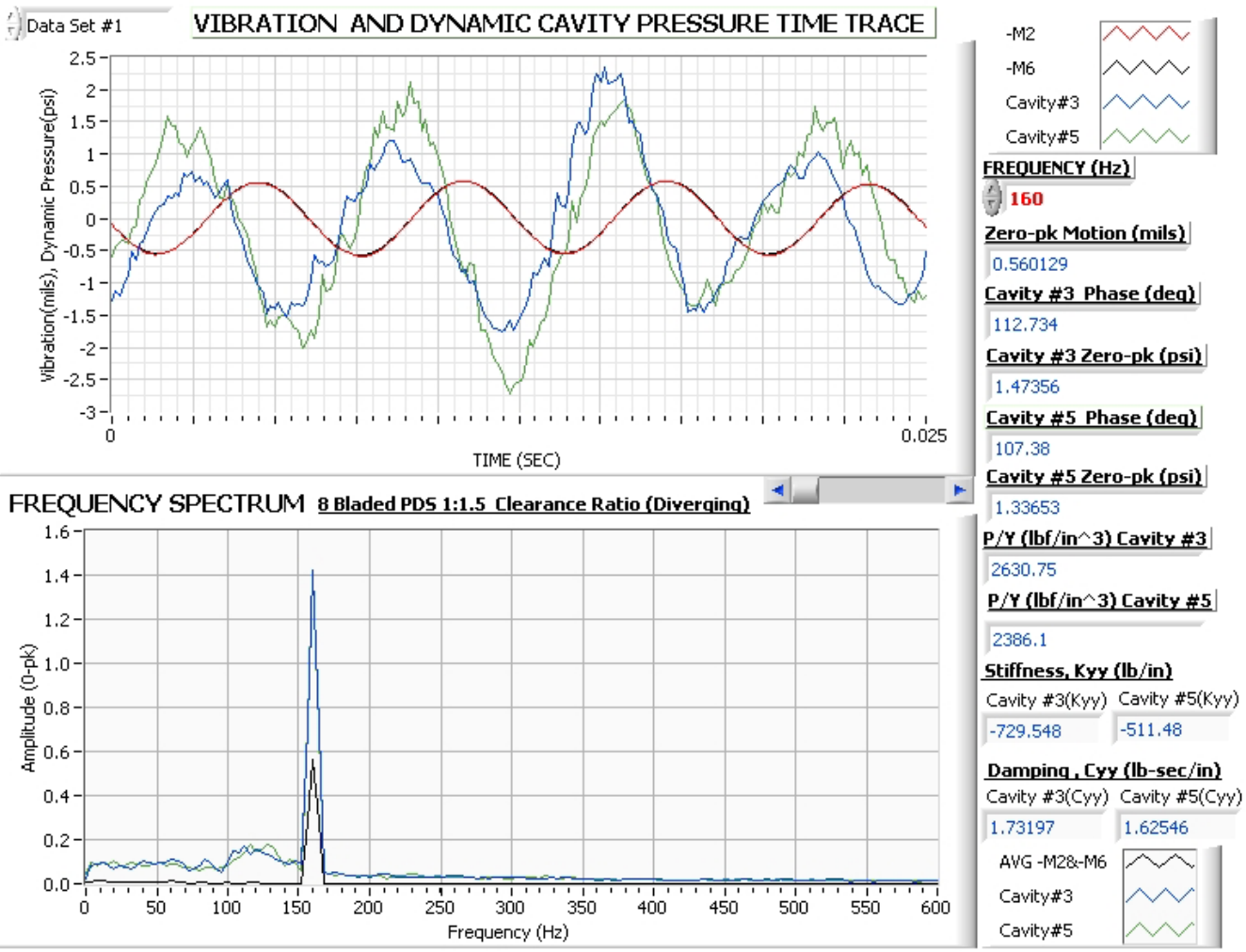

$160 \mathrm{~Hz} 8$ Bladed Diverging Seal 1:1.5 Clr Ratio (Vibration amp. 1.2 mils pk -pk) 


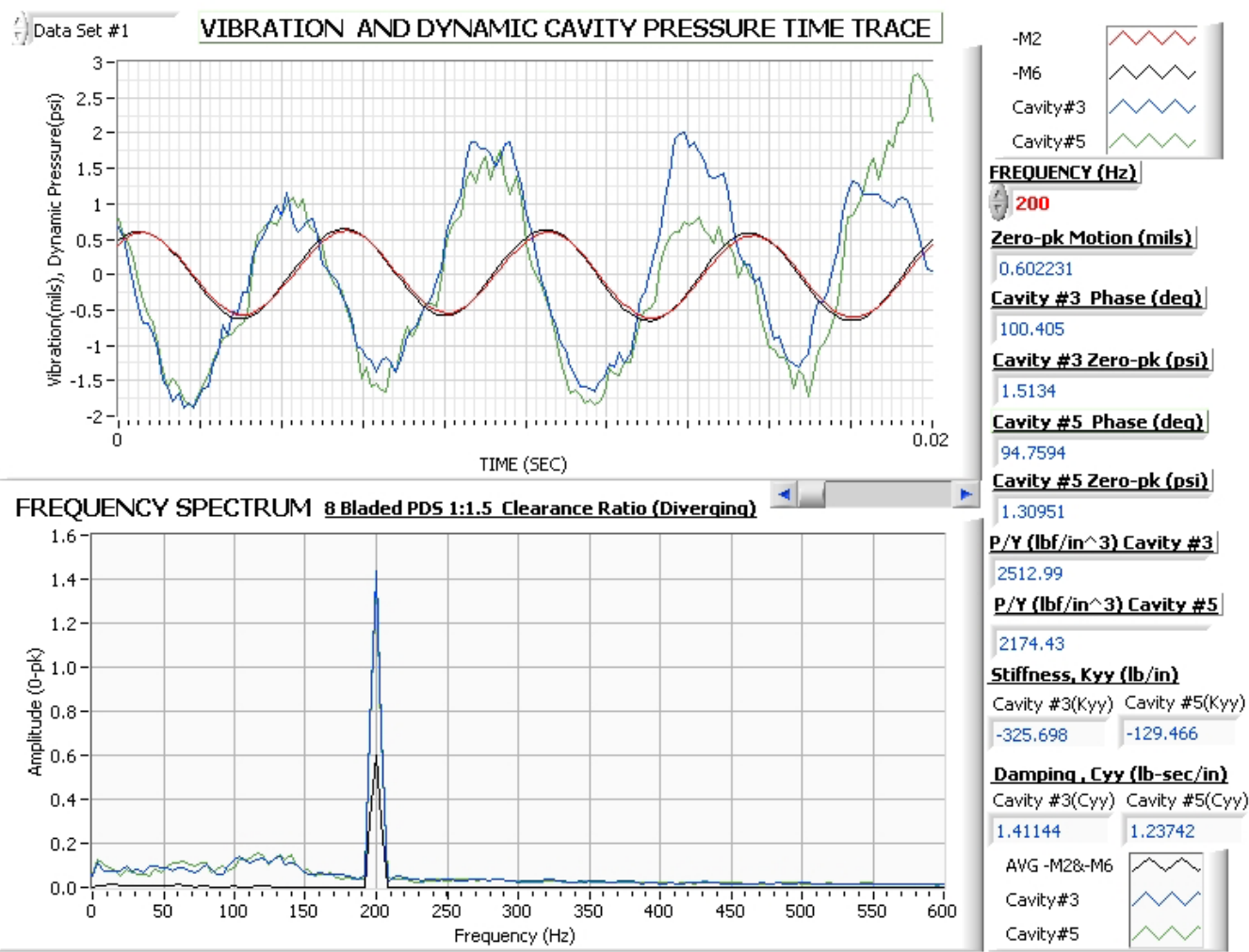

200 Hz 8 Bladed Diverging Seal 1:1.5 Clr Ratio (Vibration amp. 1.2 mils pk-pk)
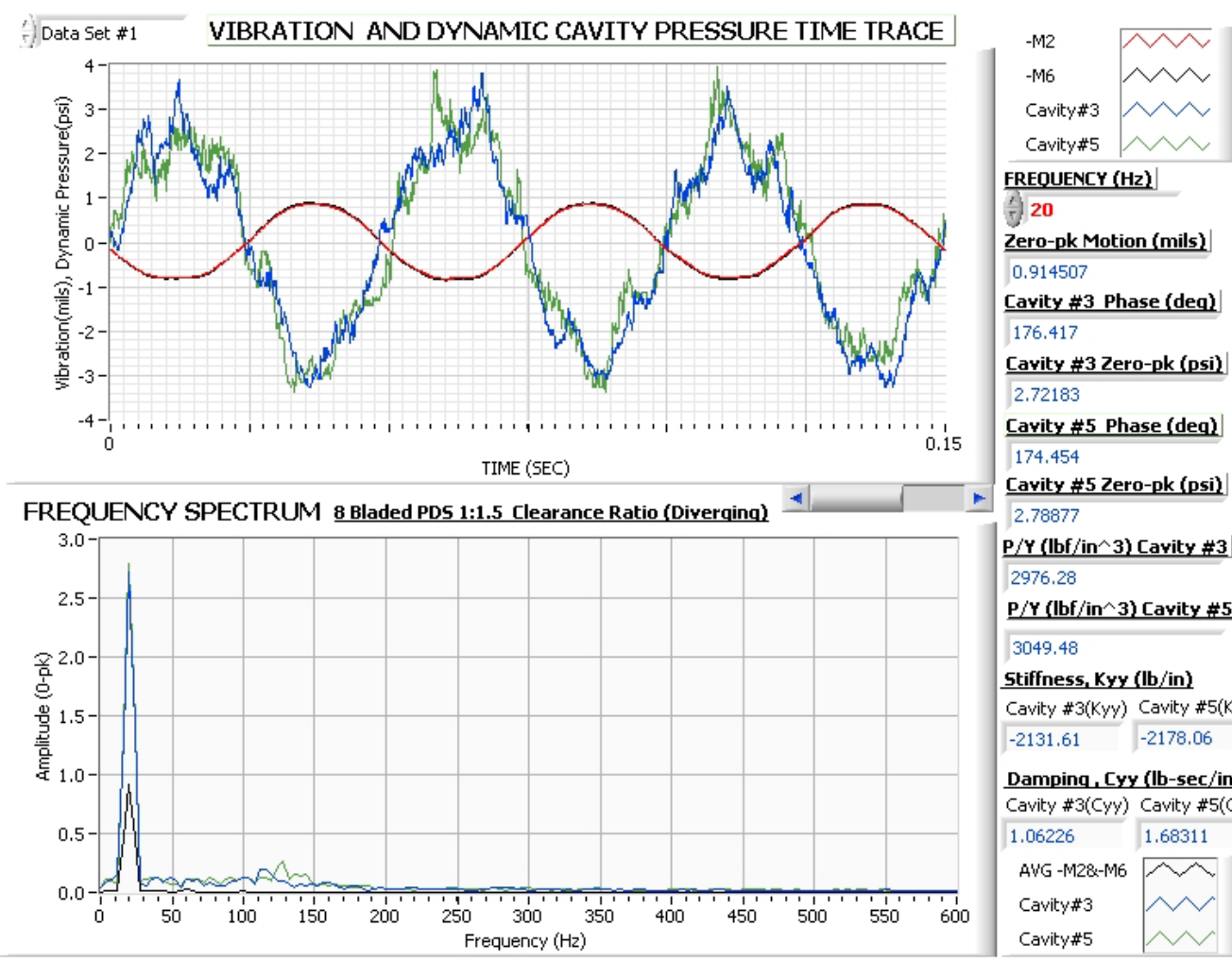

Cavity \#5 Zero-pk (psi)

2.78877

$\mathrm{P} / \mathrm{Y}\left(\mathrm{lbf} / \mathrm{in}^{\wedge} \mathbf{3}\right)$ Cavity \#3

2976.28

$\mathrm{P} / \mathrm{Y}(\mathrm{lbf} / \mathrm{in} \wedge 3)$ Cavity $\# \mathbf{5}$

3049.48

Stiffness, Kyy (lb/in)

Cavity \#3(Kyy) Cavity \#5(Ky)

$-2131.61-2178.06$

Damping, Cyy (lb-sec/in)

Cavity \#3(Cyy) Cavity \#5(Cyy)

$1.06226 \quad 1.68311$

AVG -M2\&-MG

Cavity\#3

Cavity\#5

$20 \mathrm{~Hz} 8$ Bladed Diverging Seal 1:1.5 Clr Ratio (Vibration amp. 1.8 mils pk-pk) 

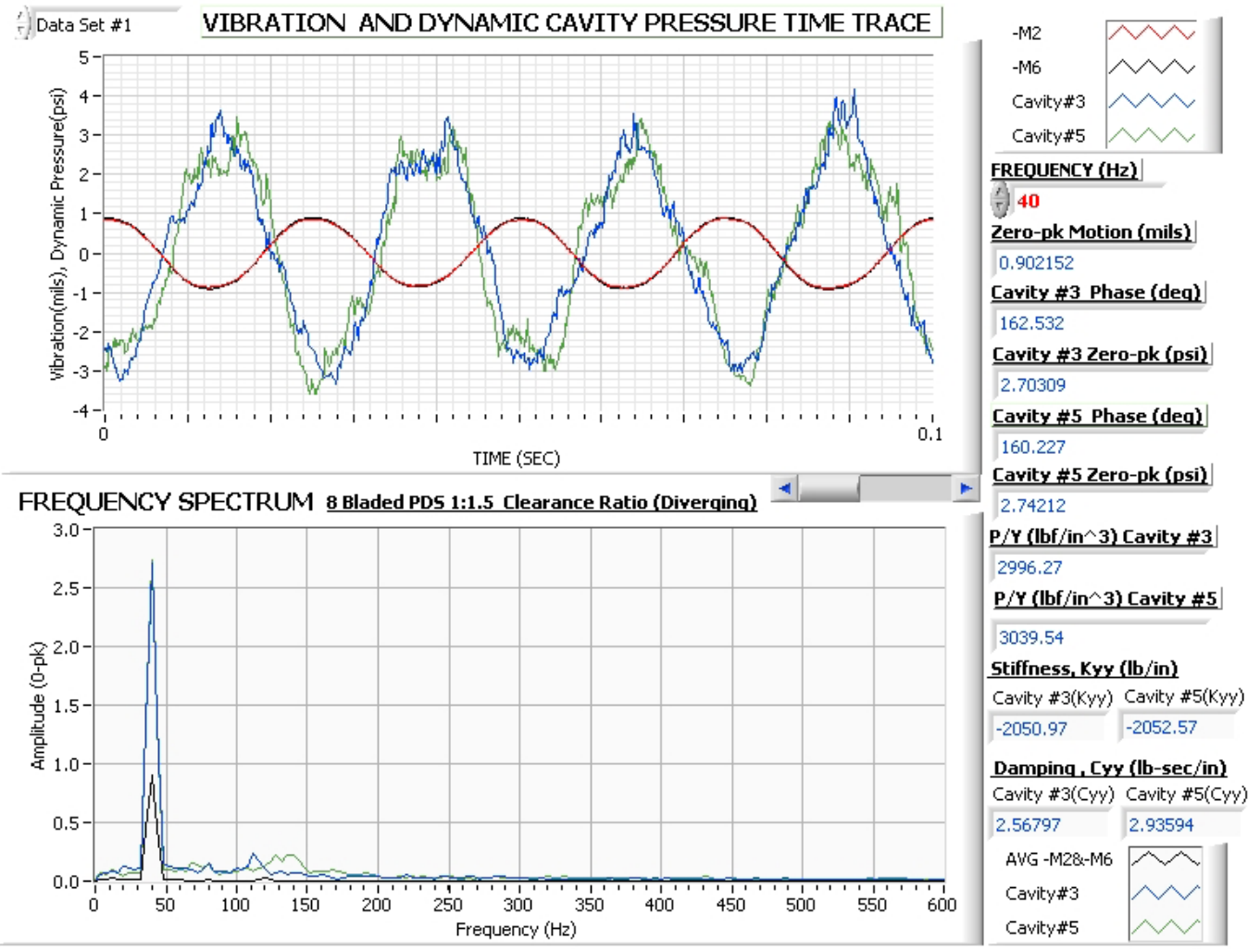

$40 \mathrm{~Hz} 8$ Bladed Diverging Seal 1:1.5 Clr Ratio (Vibration amp. 1.8 mils pk-pk)
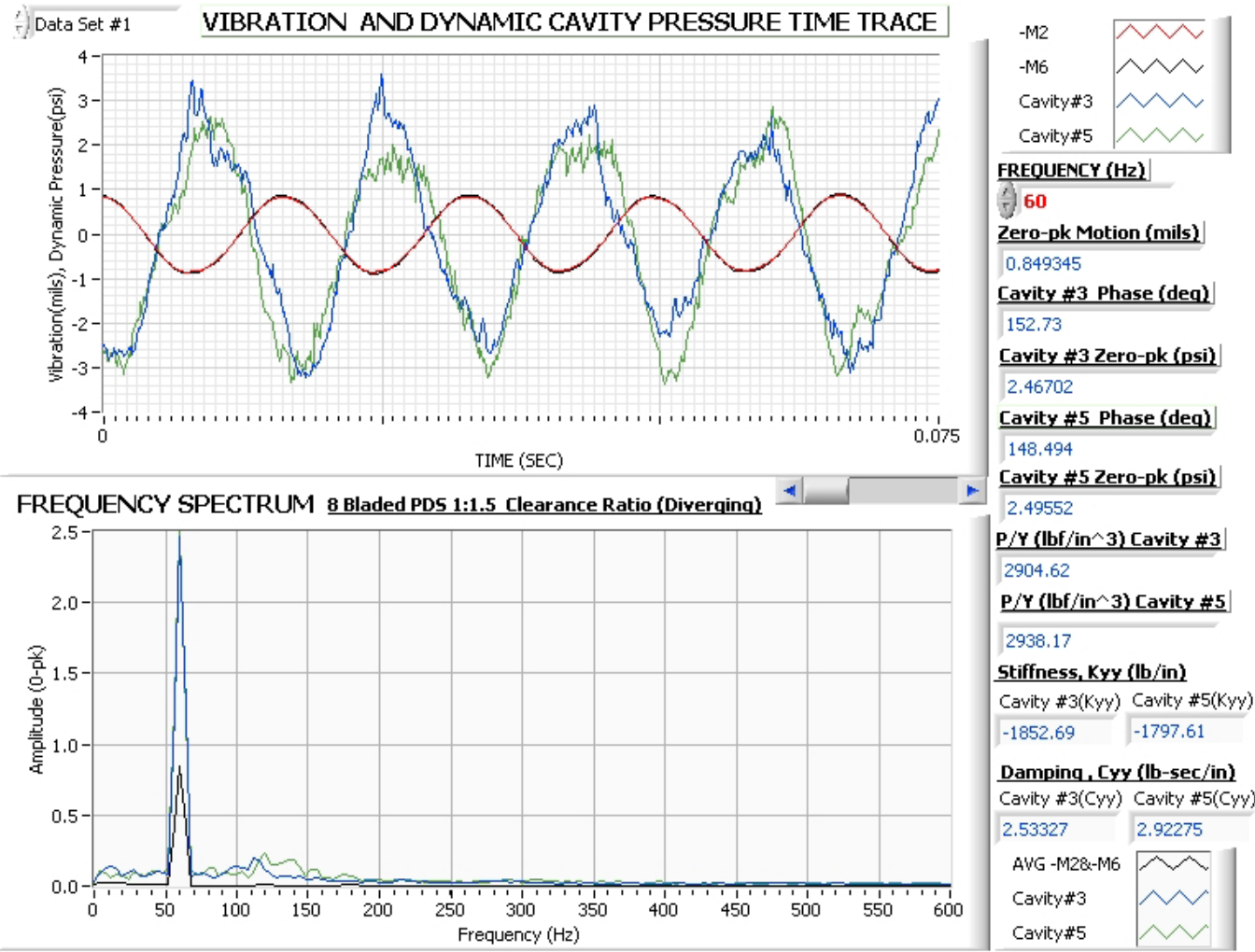

$60 \mathrm{~Hz} 8$ Bladed Diverging Seal 1:1.5 Clr Ratio (Vibration amp. 1.8 mils pk-pk) 

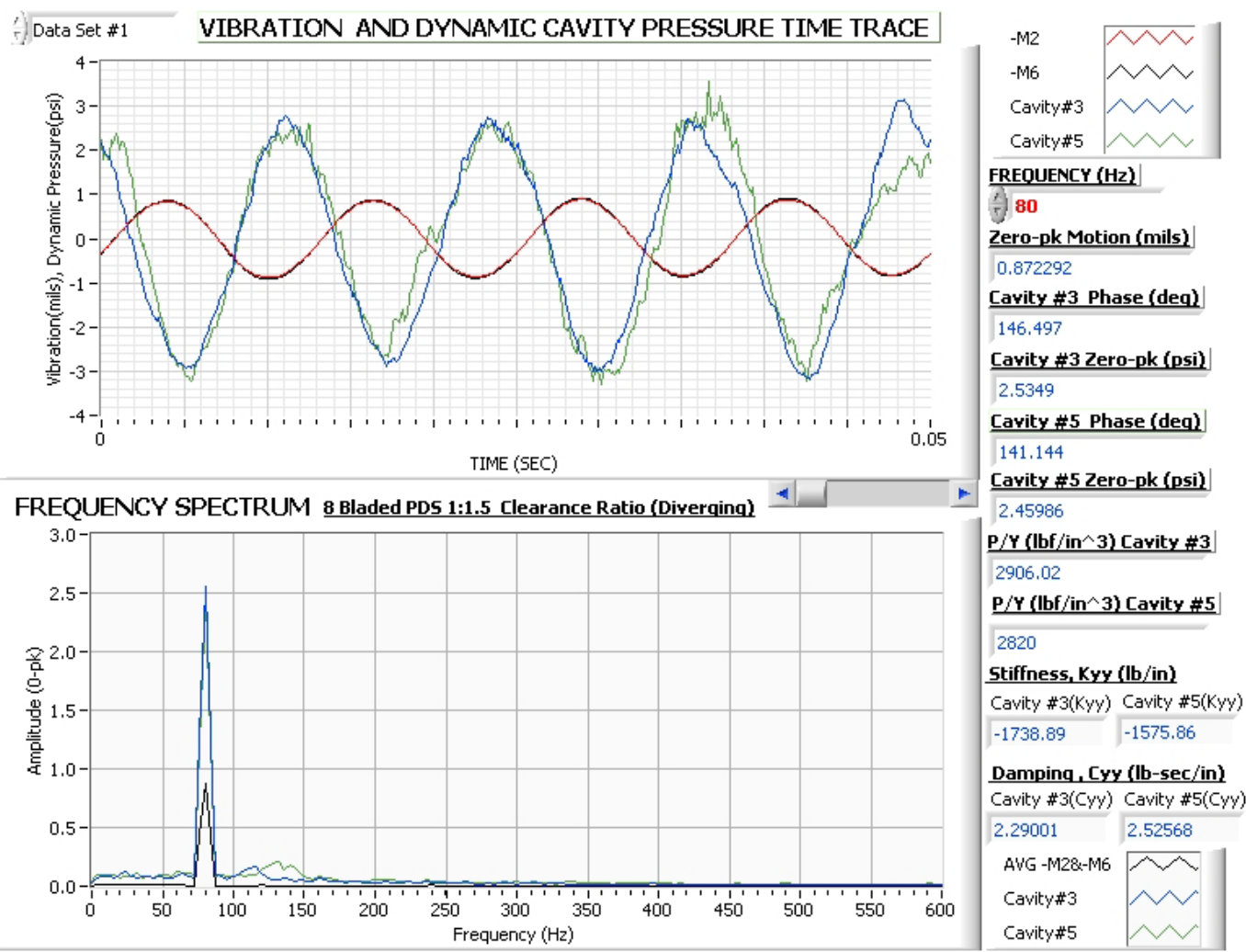

$80 \mathrm{~Hz} 8$ Bladed Diverging Seal 1:1.5 Clr Ratio (Vibration amp. 1.8 mils pk-pk)
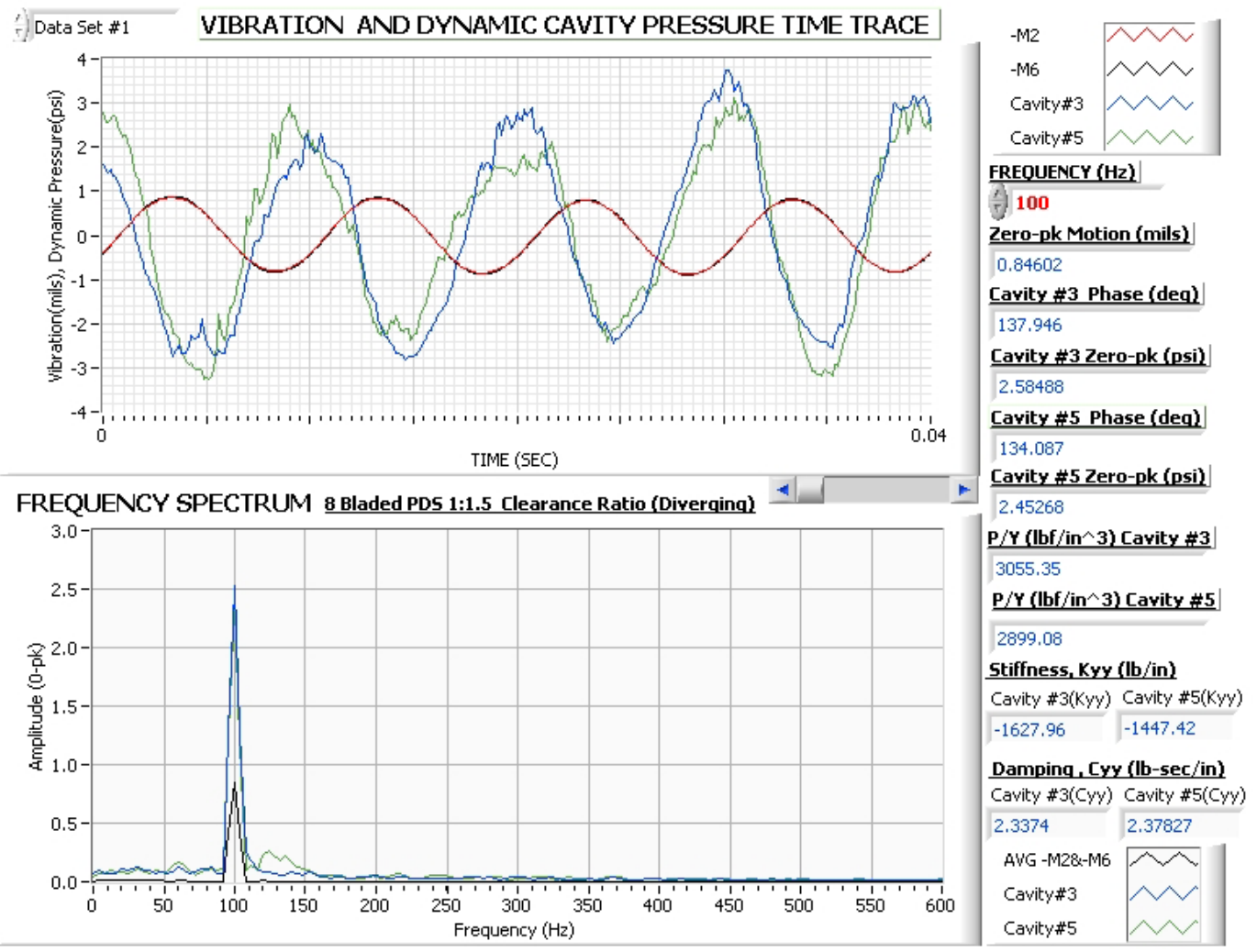

100 Hz 8 Bladed Diverging Seal 1:1.5 Clr Ratio (Vibration amp. 1.8 mils pk-pk) 


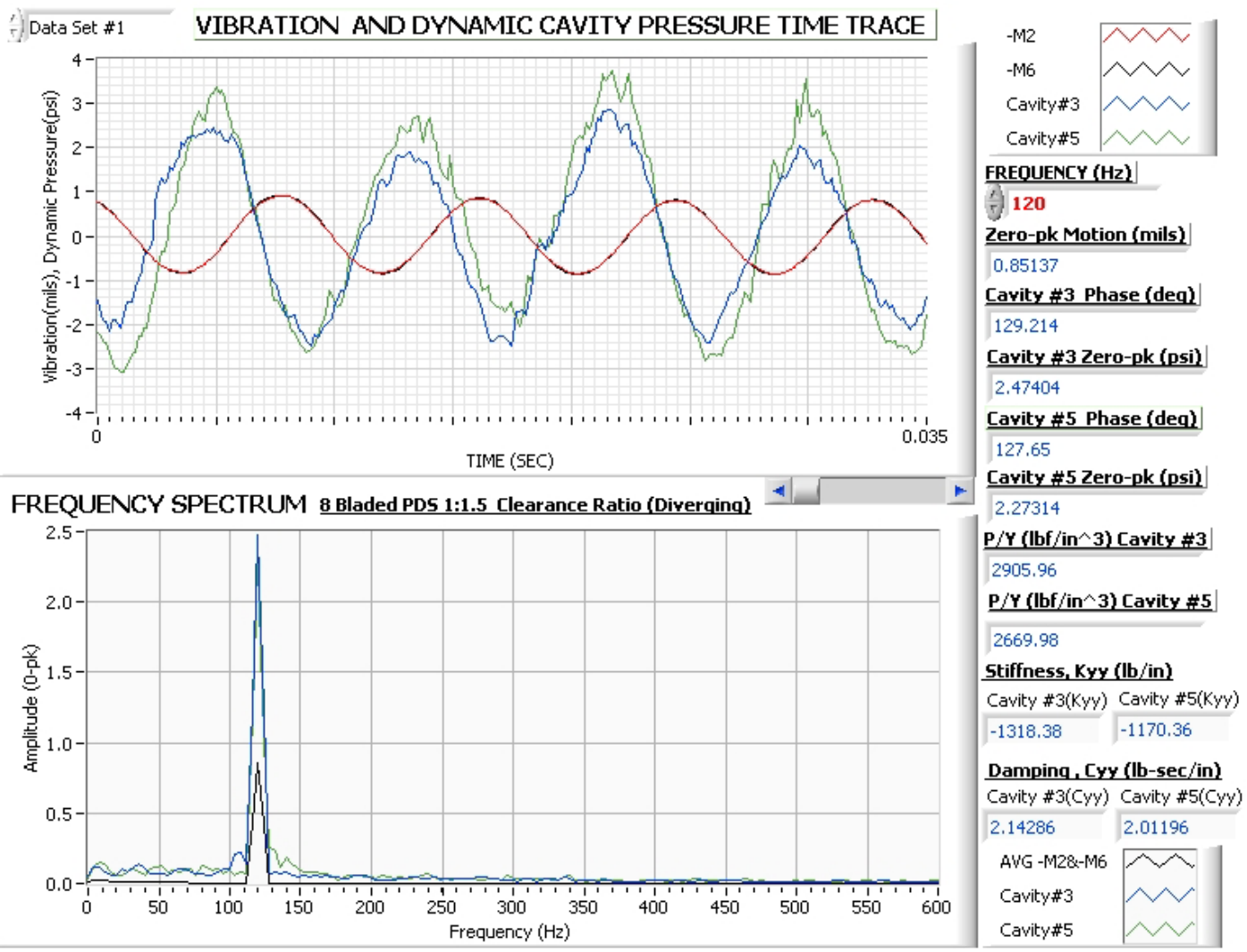

120 Hz 8 Bladed Diverging Seal 1:1.5 Clr Ratio (Vibration amp. 1.8 mils pk-pk)

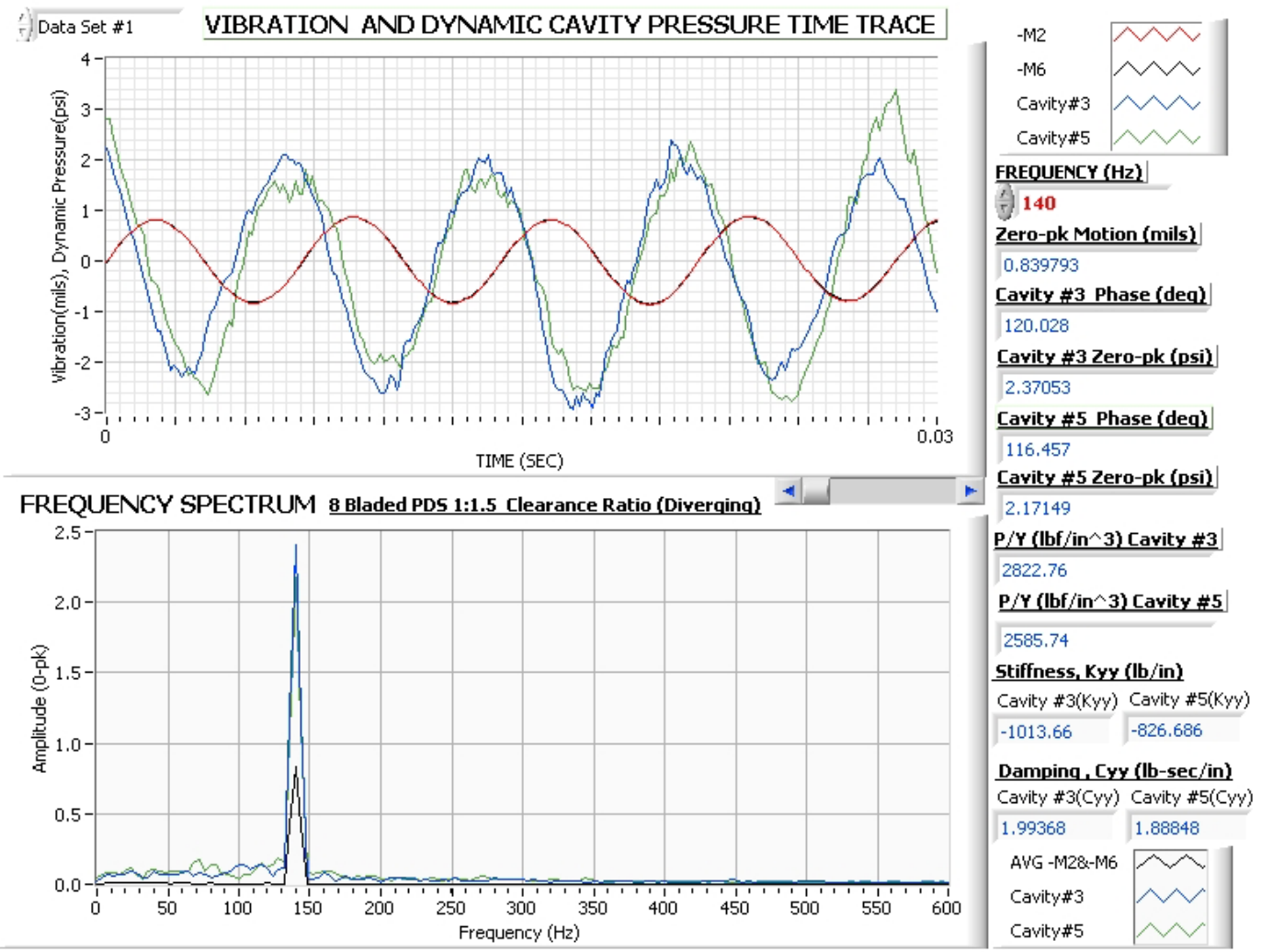

140 Hz 8 Bladed Diverging Seal 1:1.5 Clr Ratio (Vibration amp. 1.8 mils pk-pk) 


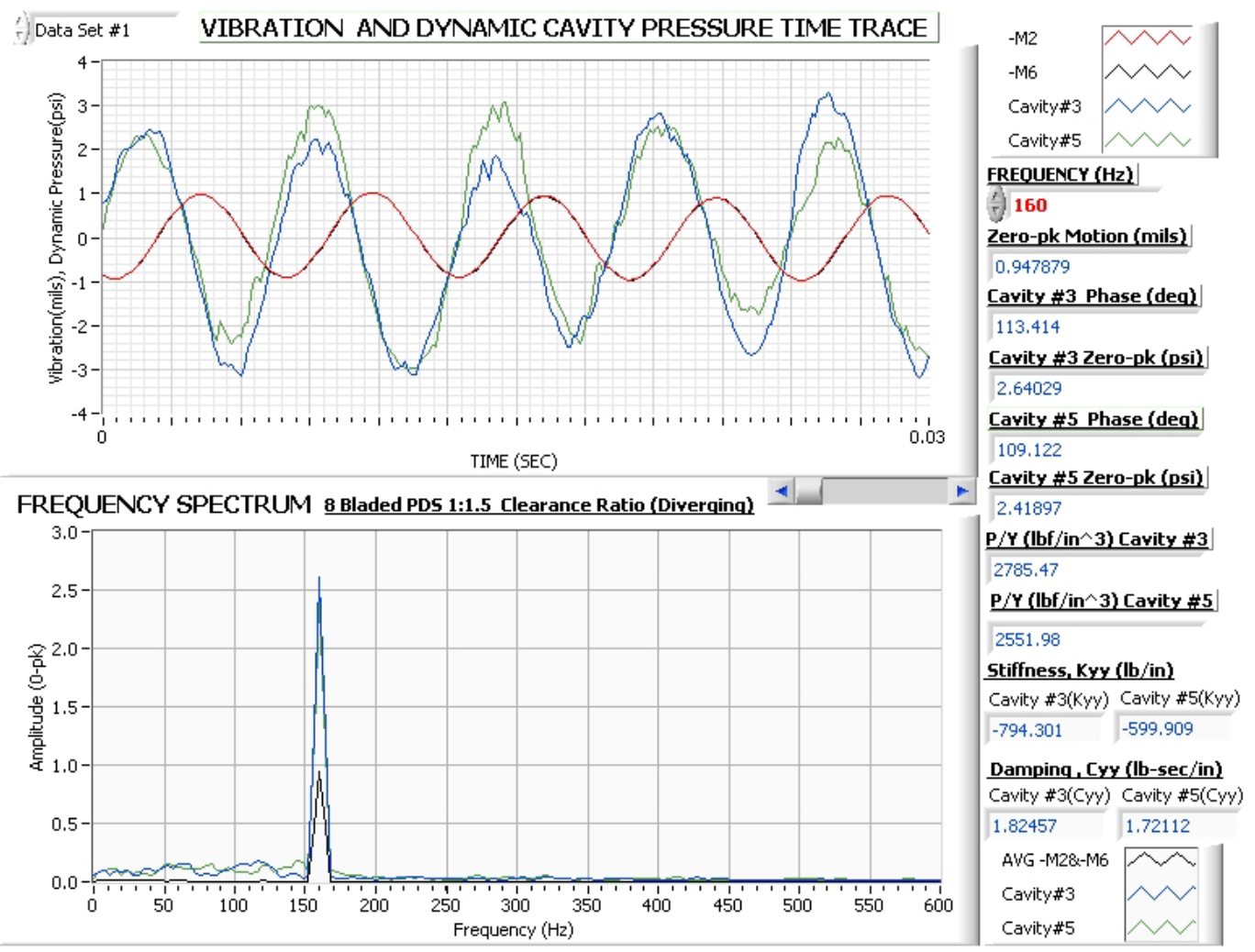

160 Hz 8 Bladed Diverging Seal 1:1.5 Clr Ratio (Vibration amp. 1.8 mils pk-pk)
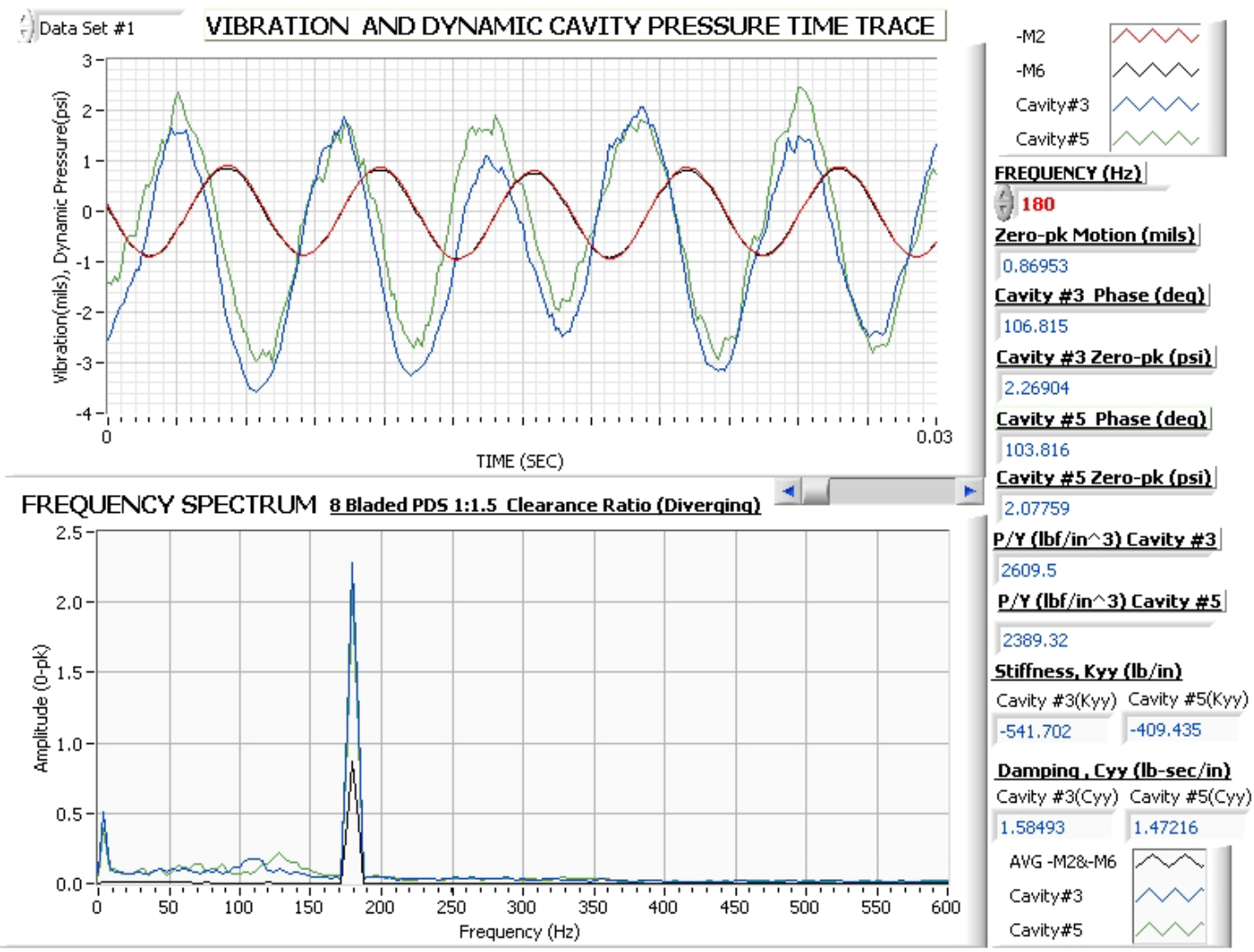

$180 \mathrm{~Hz} 8$ Bladed Diverging Seal 1:1.5 Clr Ratio (Vibration amp. 1.8 mils pk-pk) 

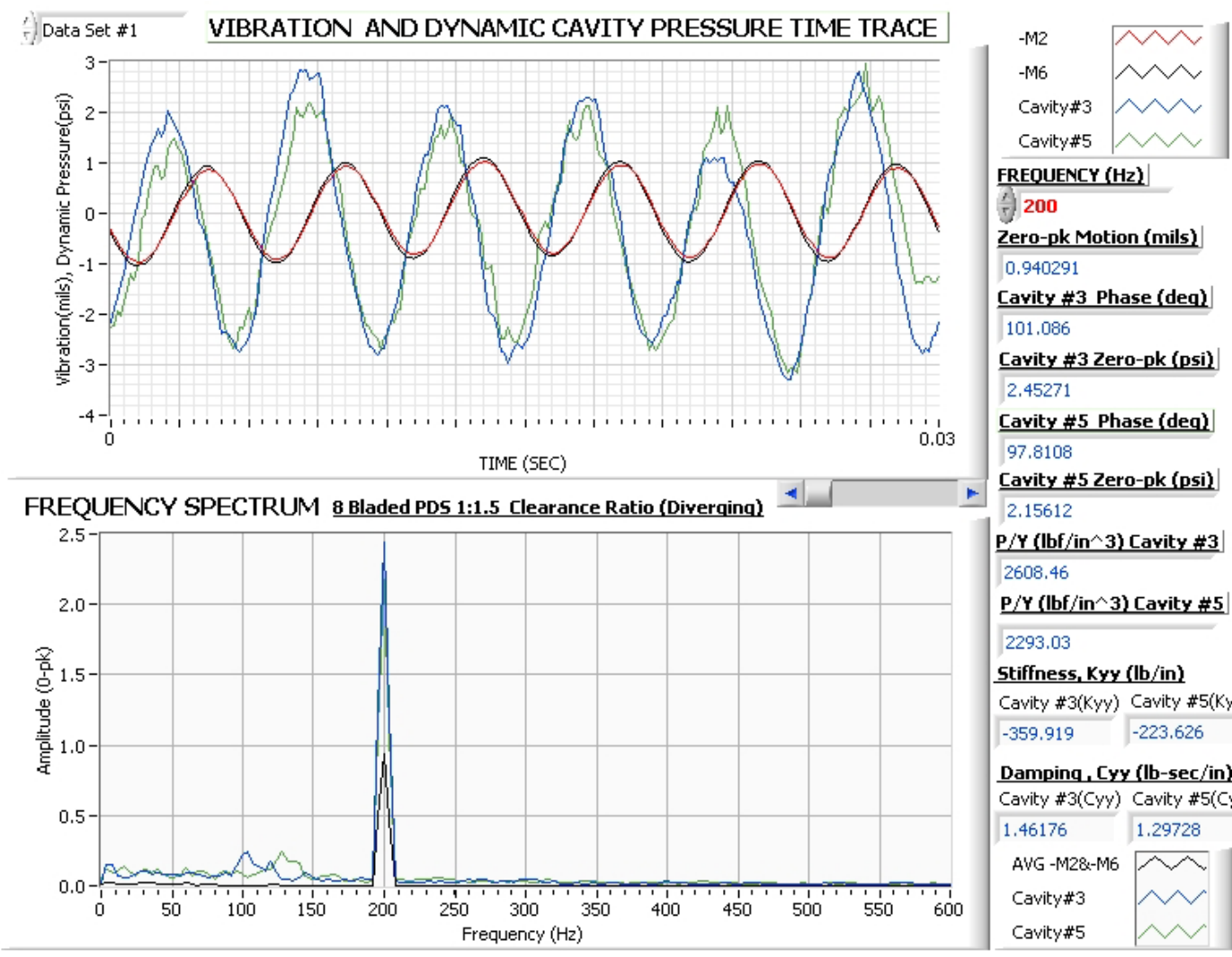

FREQUENCY (Hz)

곡 200

Zero-pk Motion (mils)

0.940291

Cavity \#3 Phase (deg)

101.086

Cavity \#3 Zero-pk (psi)

2.45271

Cavity \#5 Phase (dea)

97.8108

Cavity \#5 Zero-pk (psi)

2.15612

$\mathrm{P} / \mathrm{Y}\left(\mathrm{lbf} / \mathrm{in}^{\wedge} 3\right)$ Cavity \#3

2608.46

P/Y (lbf/in`3) Cavity \#5

2293.03

Stiffness, Kyy (lb/in)

Cavity \#3(Kyy) Cavity \#5(Kyy)

$-359.919-223.626$

Damping, Cyy (lb-sec/in)

Cavity \#3(Cy) Cavity \#5(Cyy)

$1.46176 \quad 1.29728$

AVG -M2\&-M6

Cavity\#3

Cavity\#5

200 Hz 8 Bladed Diverging Seal 1:1.5 Clr Ratio (Vibration amp. 1.8 mils pk-pk) 
Dynamic Cavity Pressure Tests

6 Bladed Conventional PDS

0 DEGREE POSITION 


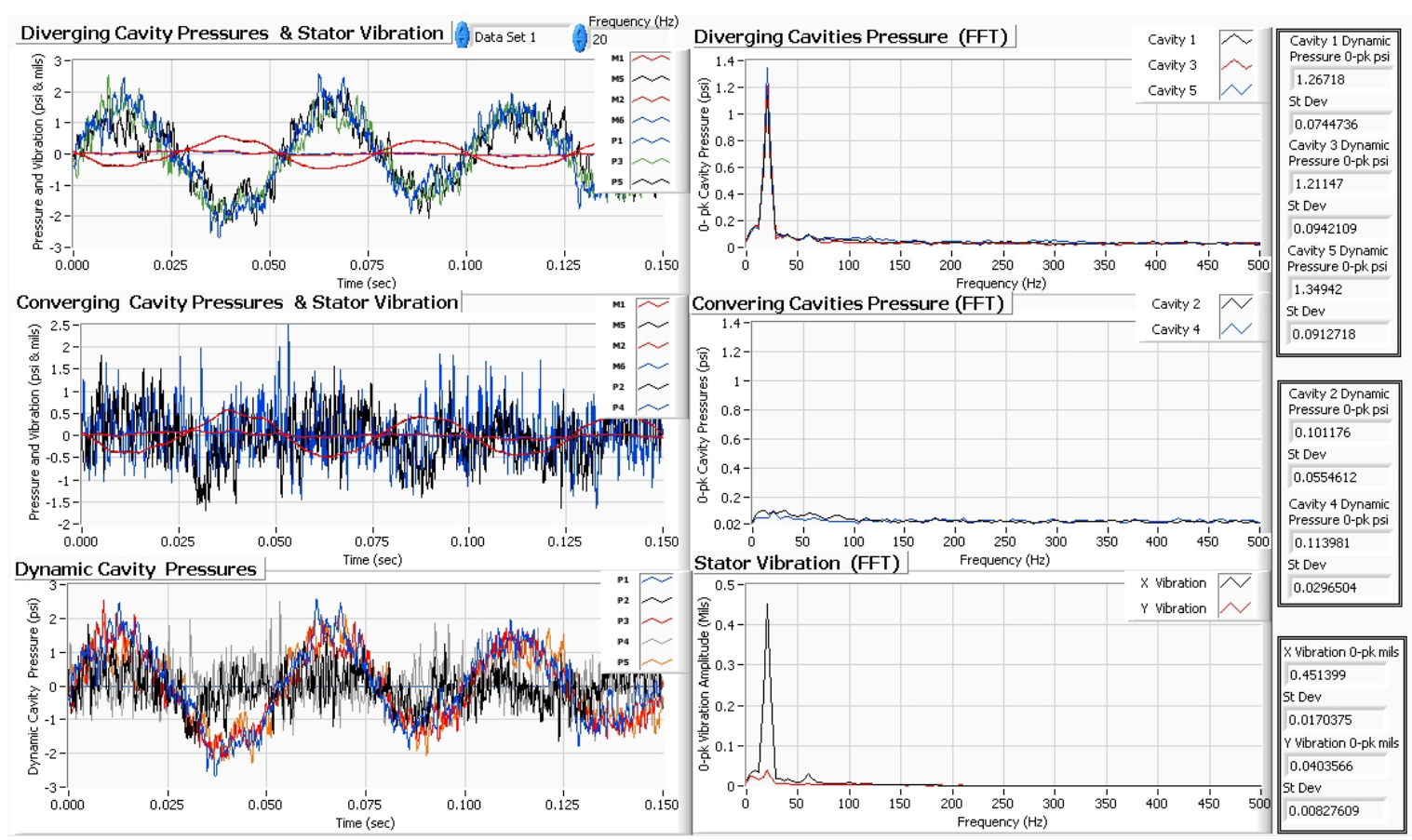

$20 \mathrm{~Hz} 0$ Degree Position X Direction Excitation
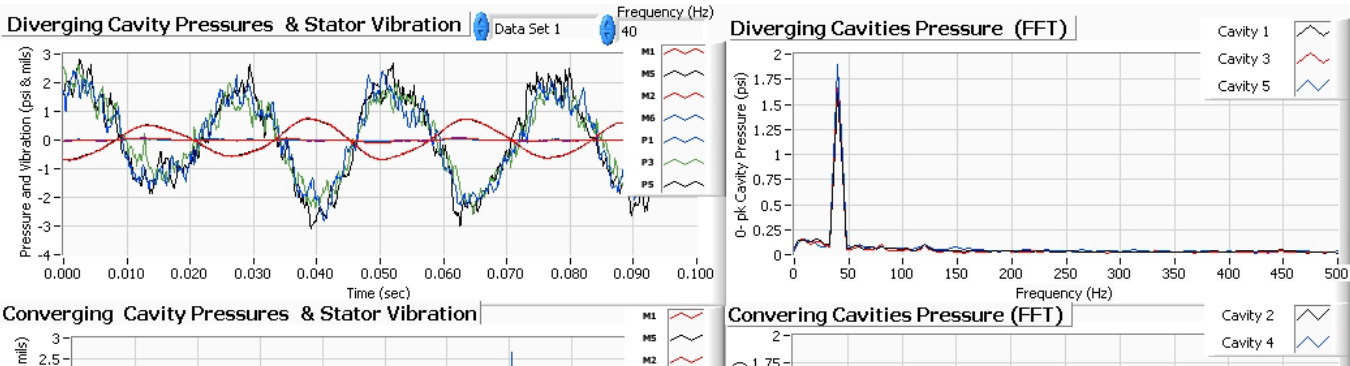

Cavity 1 Dynamic
Pressure 0-pk ps
1.7912 .

1.79812

St Dev

0.0966578

Cavity 3 Dynamic

1.65804

St Dev

0.103657

Cavity 5 Dynamic
Pressure 0 -pk psi

1.89613

Converging Cavity Pressures \& Stator Vibration
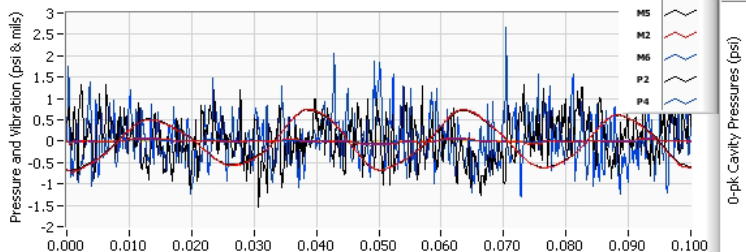

Dev

0.0590778

$\begin{array}{ccccccccccc}0.000 & 0.010 & 0.020 & 0.030 & 0.040 & 0.050 & 0.060 & 0.0070 & 0.080 & 0.090 & 0.100\end{array}$

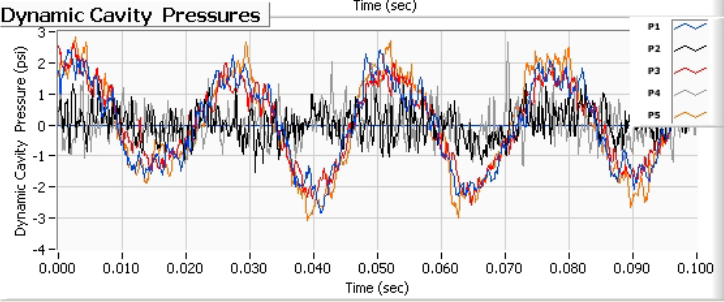

$40 \mathrm{~Hz} 0$ Degree Position X Direction Excitation

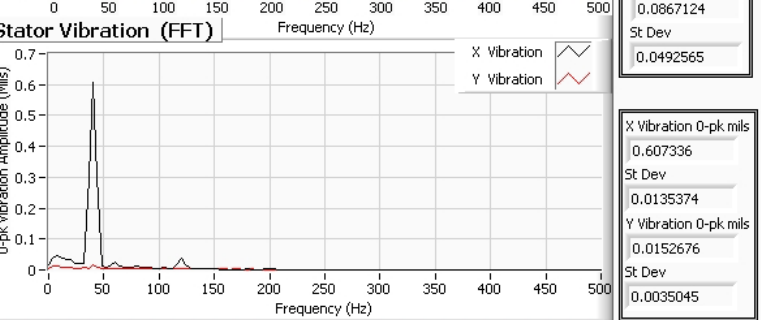

40 


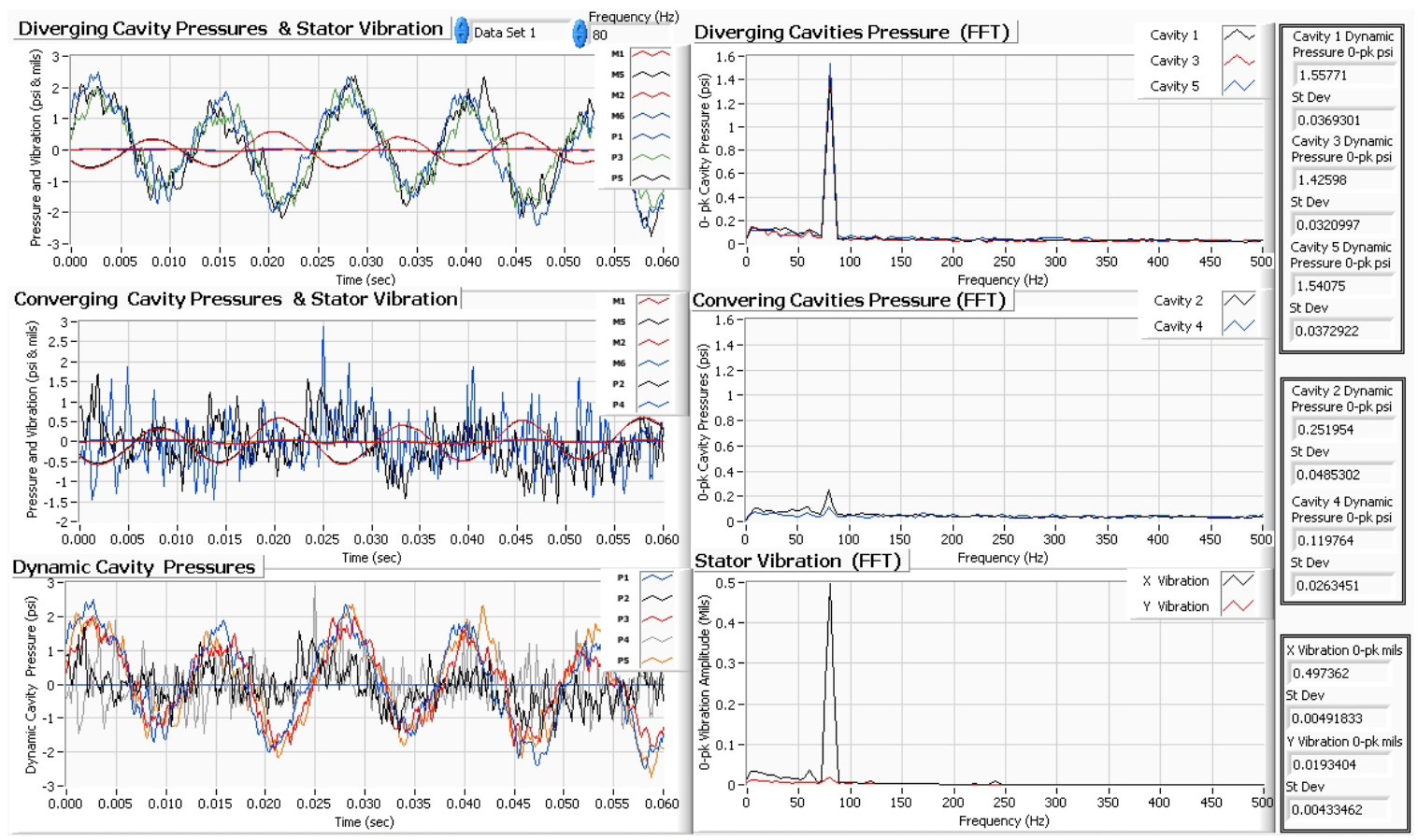

80 Hz 0 Degree Position X Direction Excitation

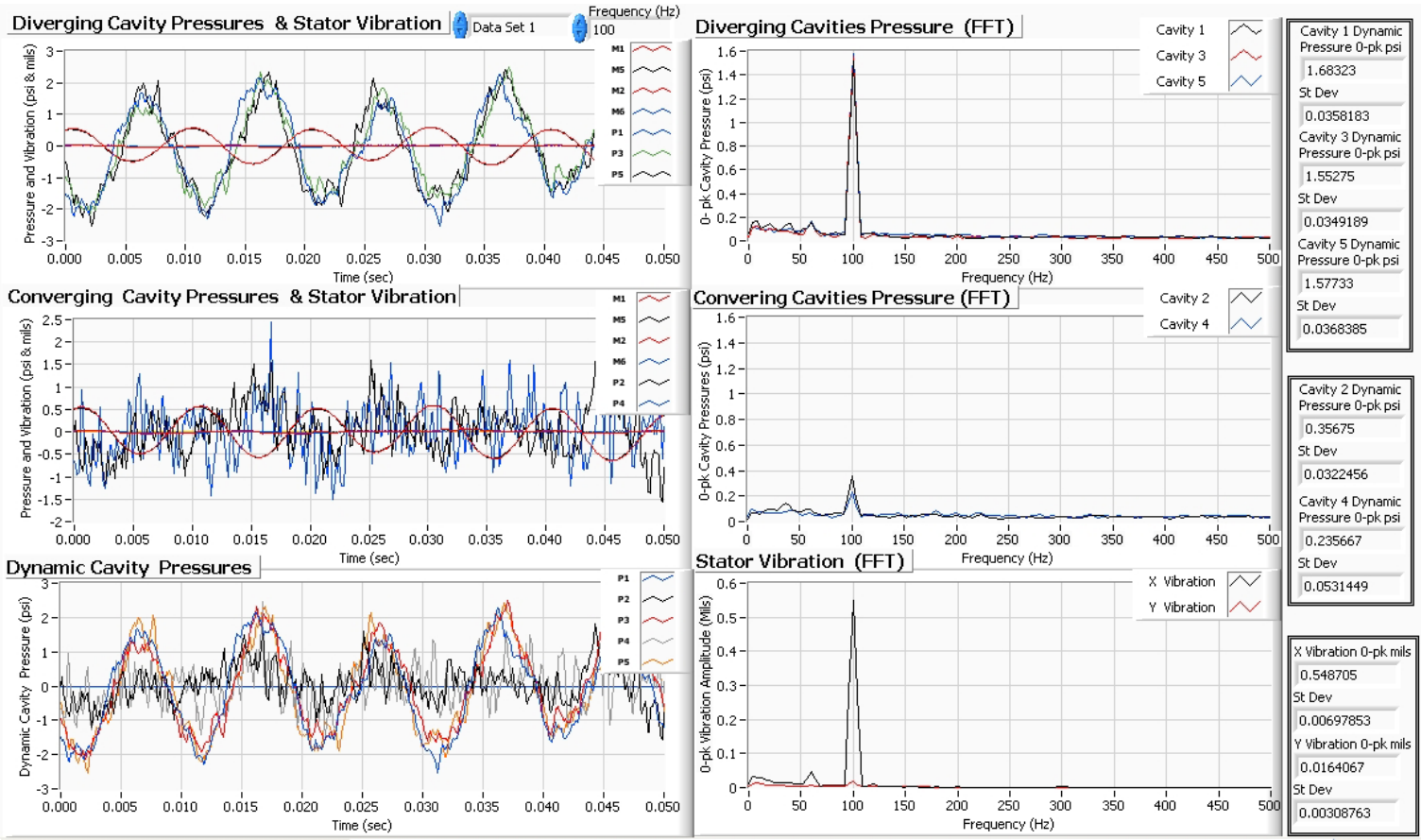

$100 \mathrm{~Hz} 0$ Degree Position X Direction Excitation 


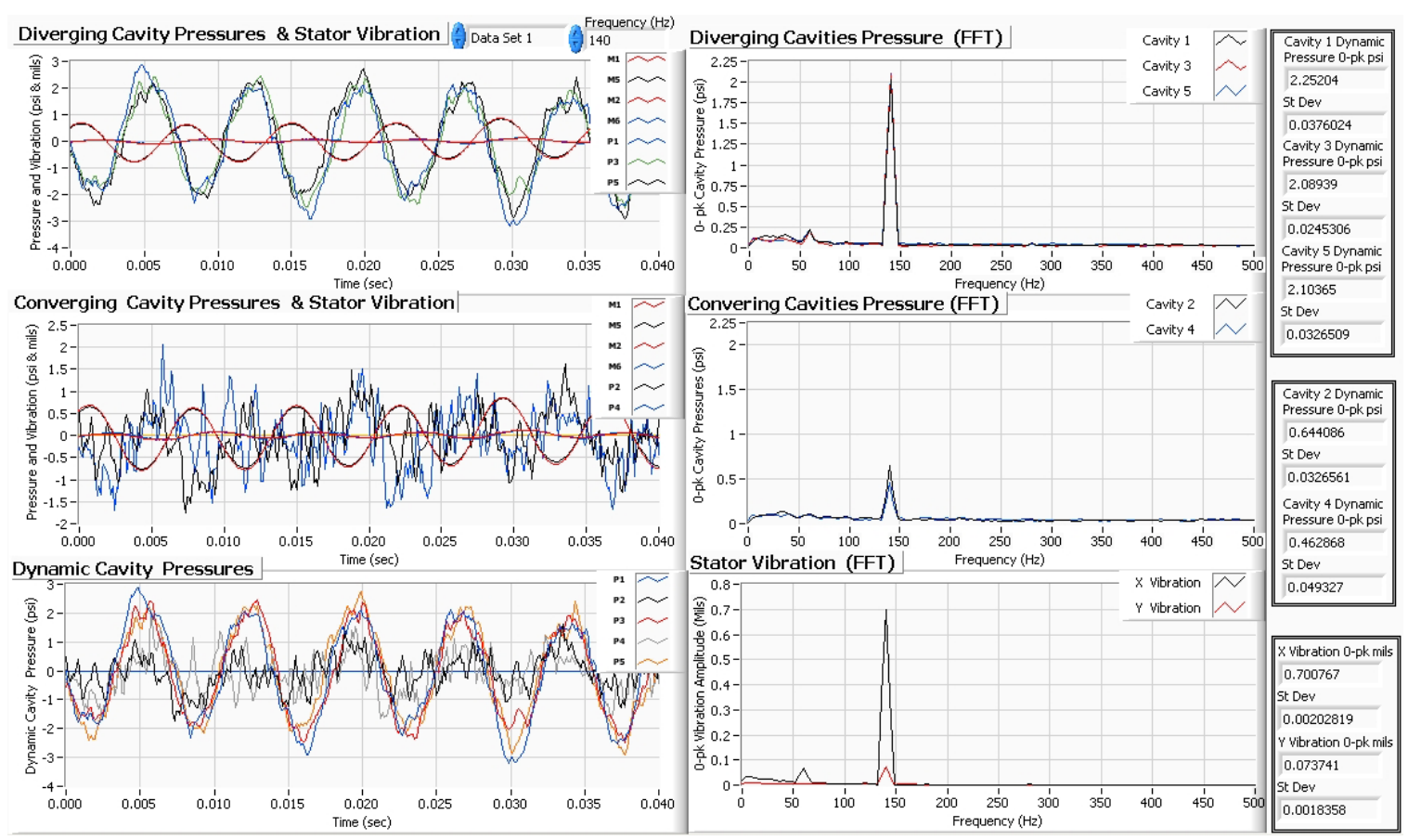

$140 \mathrm{~Hz} 0$ Degree Position X Direction Excitation

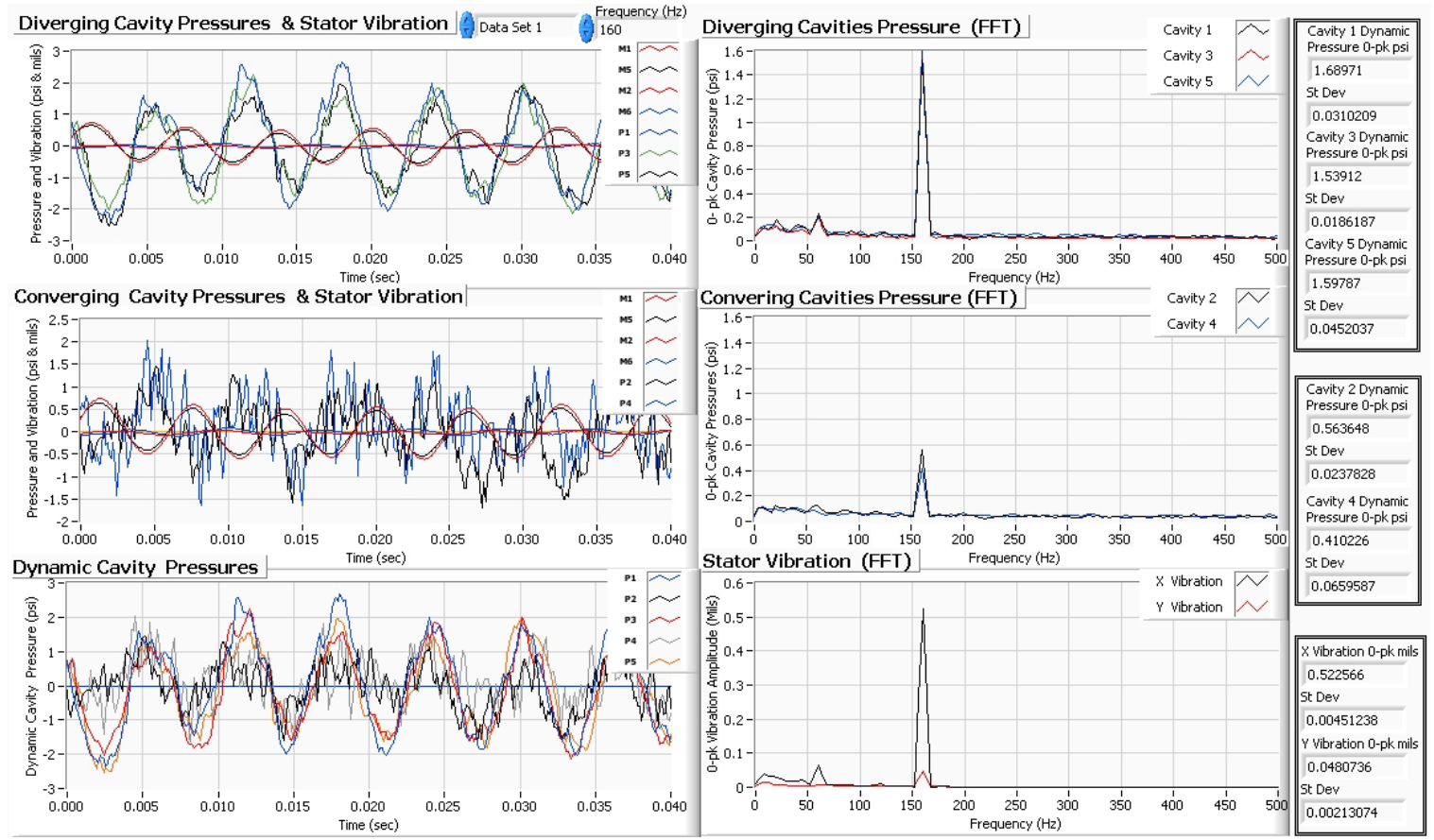

$160 \mathrm{~Hz} 0$ Degree Position X Direction Excitation 


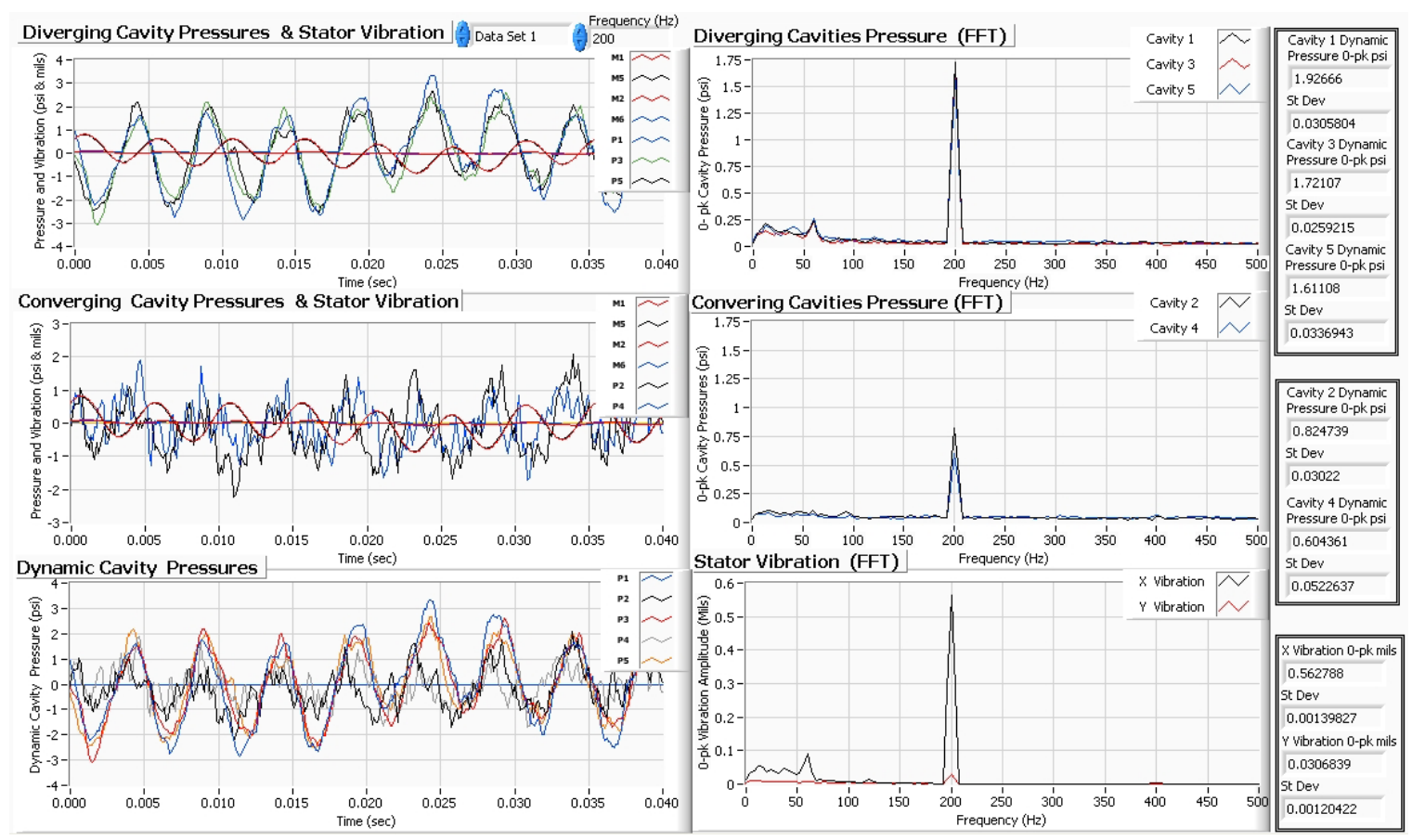

$200 \mathrm{~Hz} 0$ Degree Position X Direction Excitation

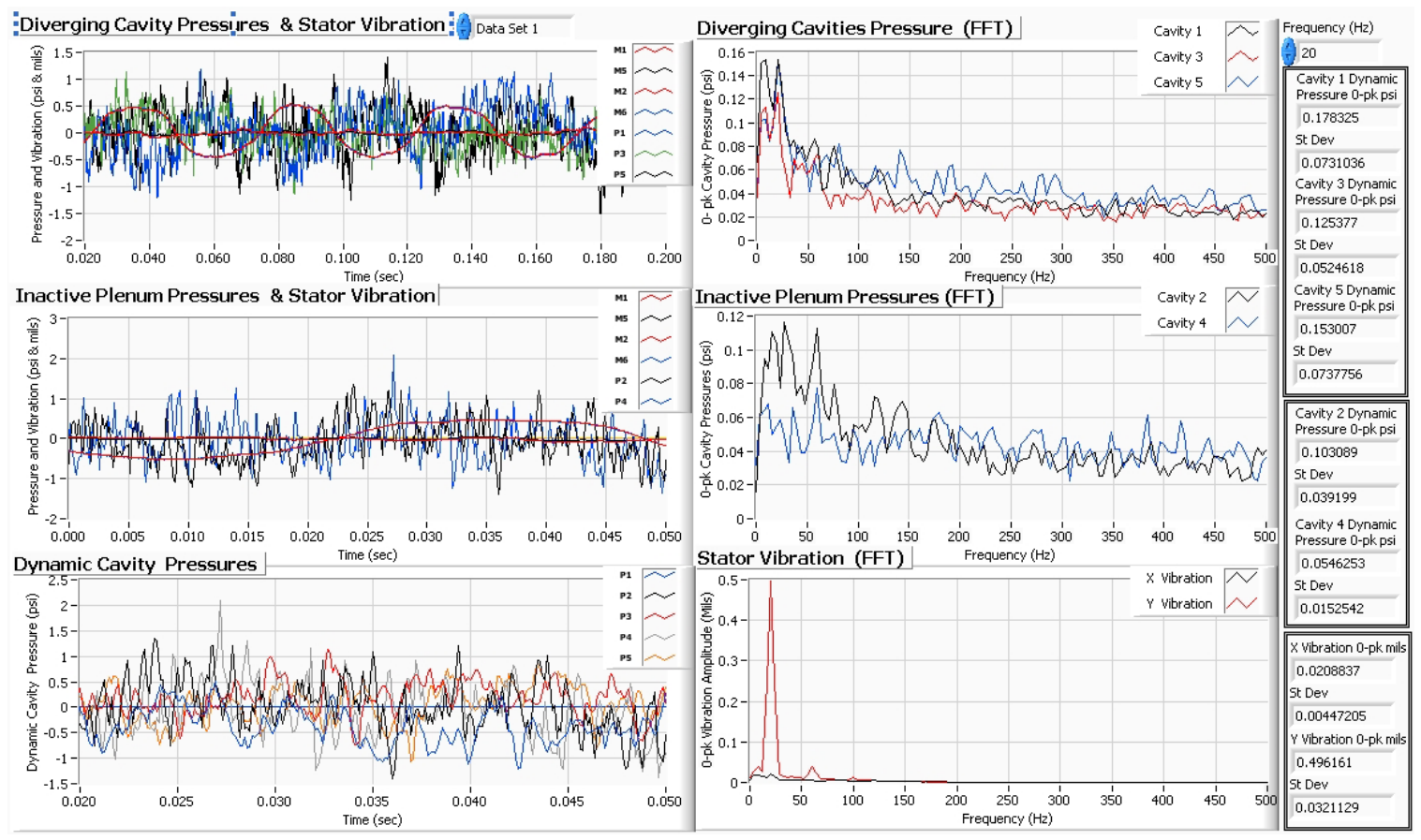

$20 \mathrm{~Hz} 0$ Degree Position Y Direction Excitation 

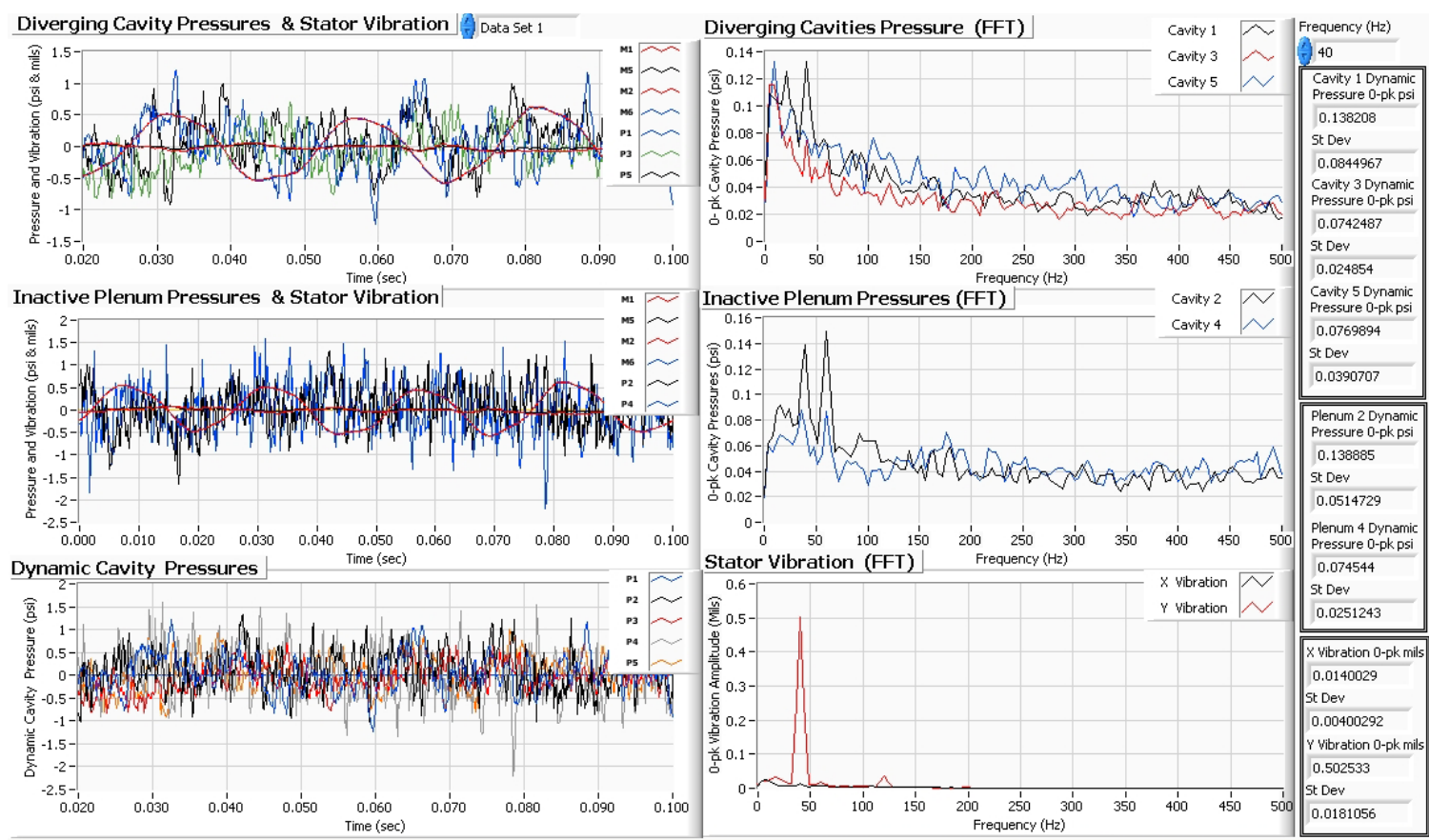

$40 \mathrm{~Hz} 0$ Degree Position Y Direction Excitation
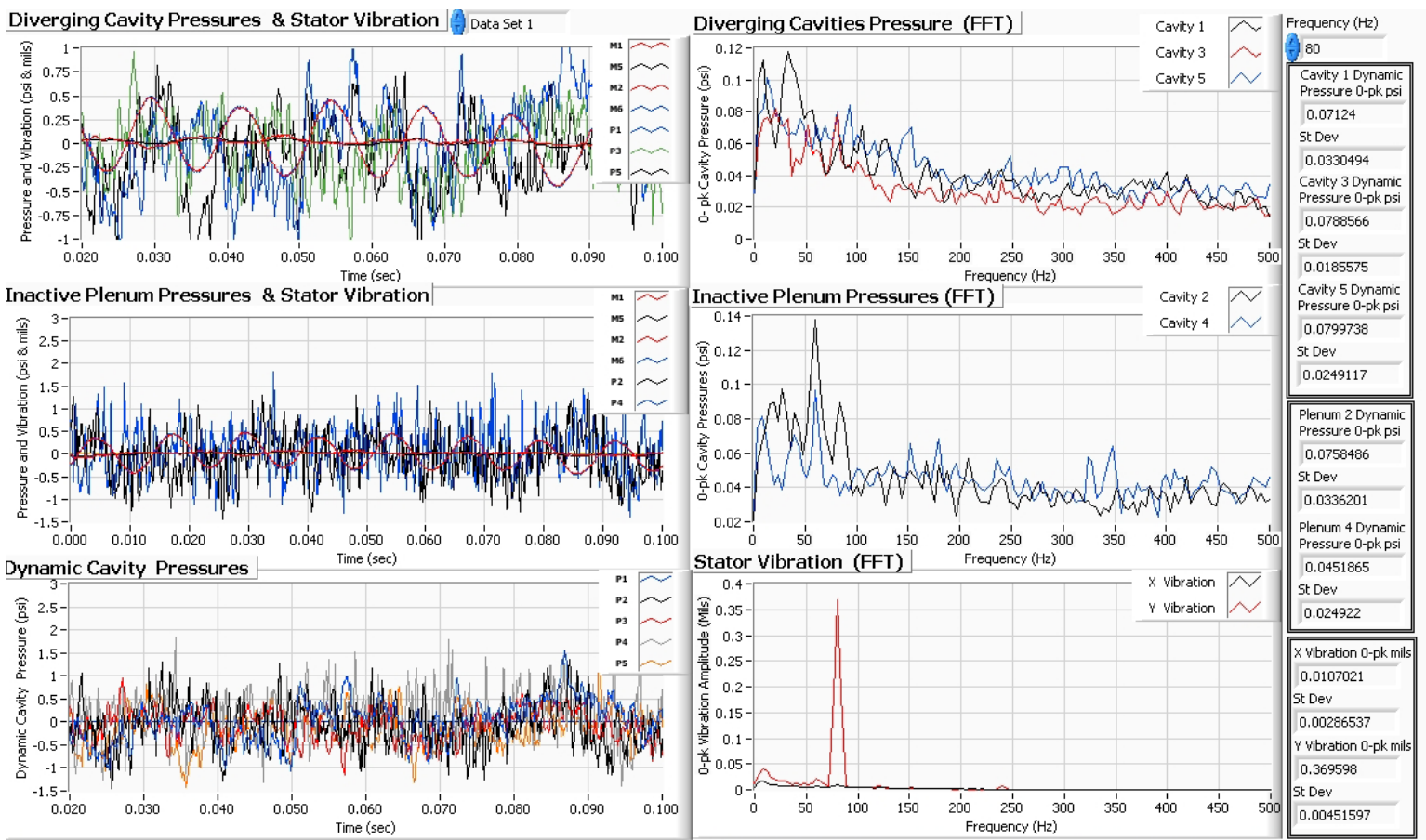

$80 \mathrm{~Hz} 0$ Degree Position Y Direction Excitation 


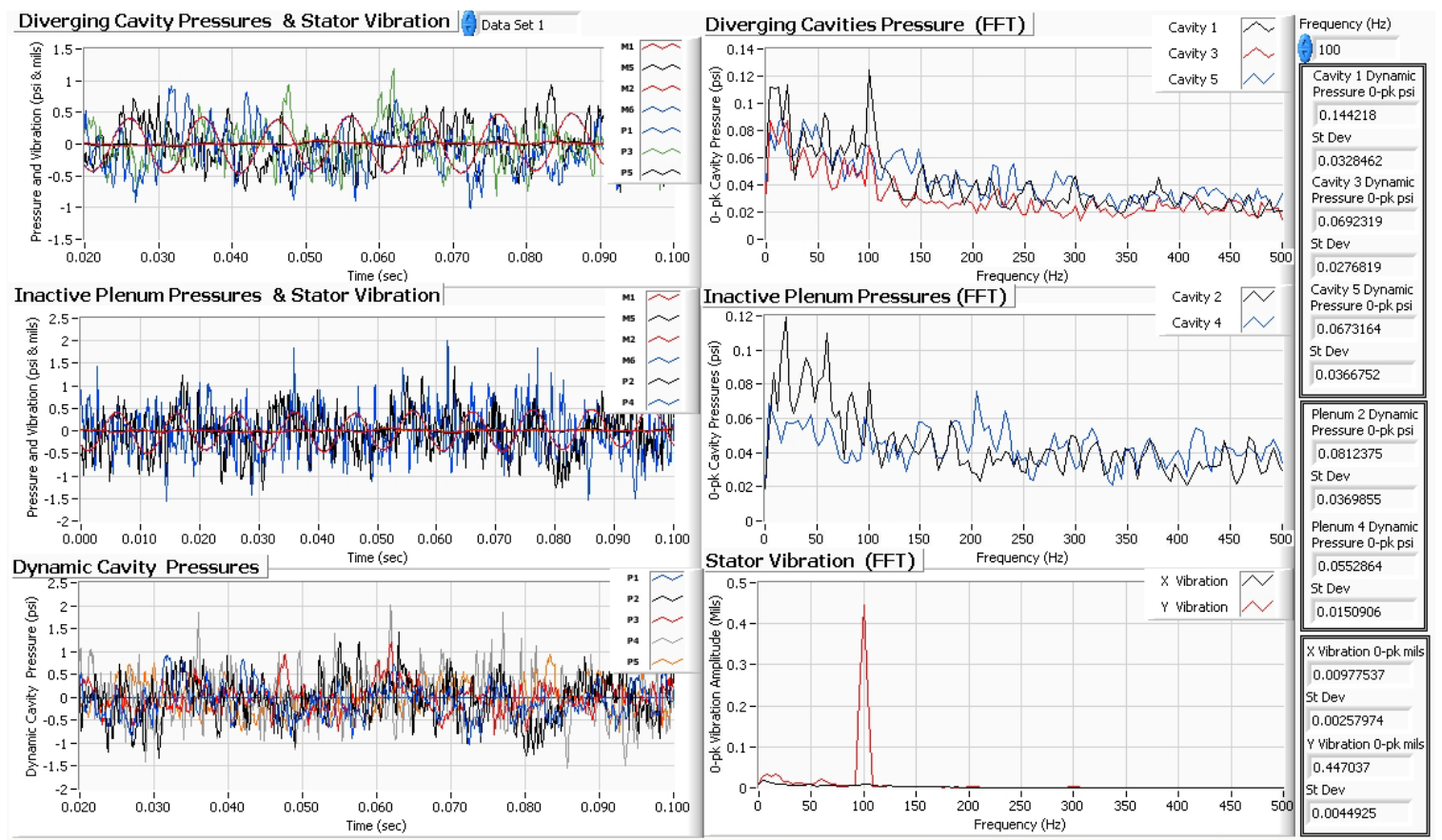

$100 \mathrm{~Hz} 0$ Degree Position Y Direction Excitation

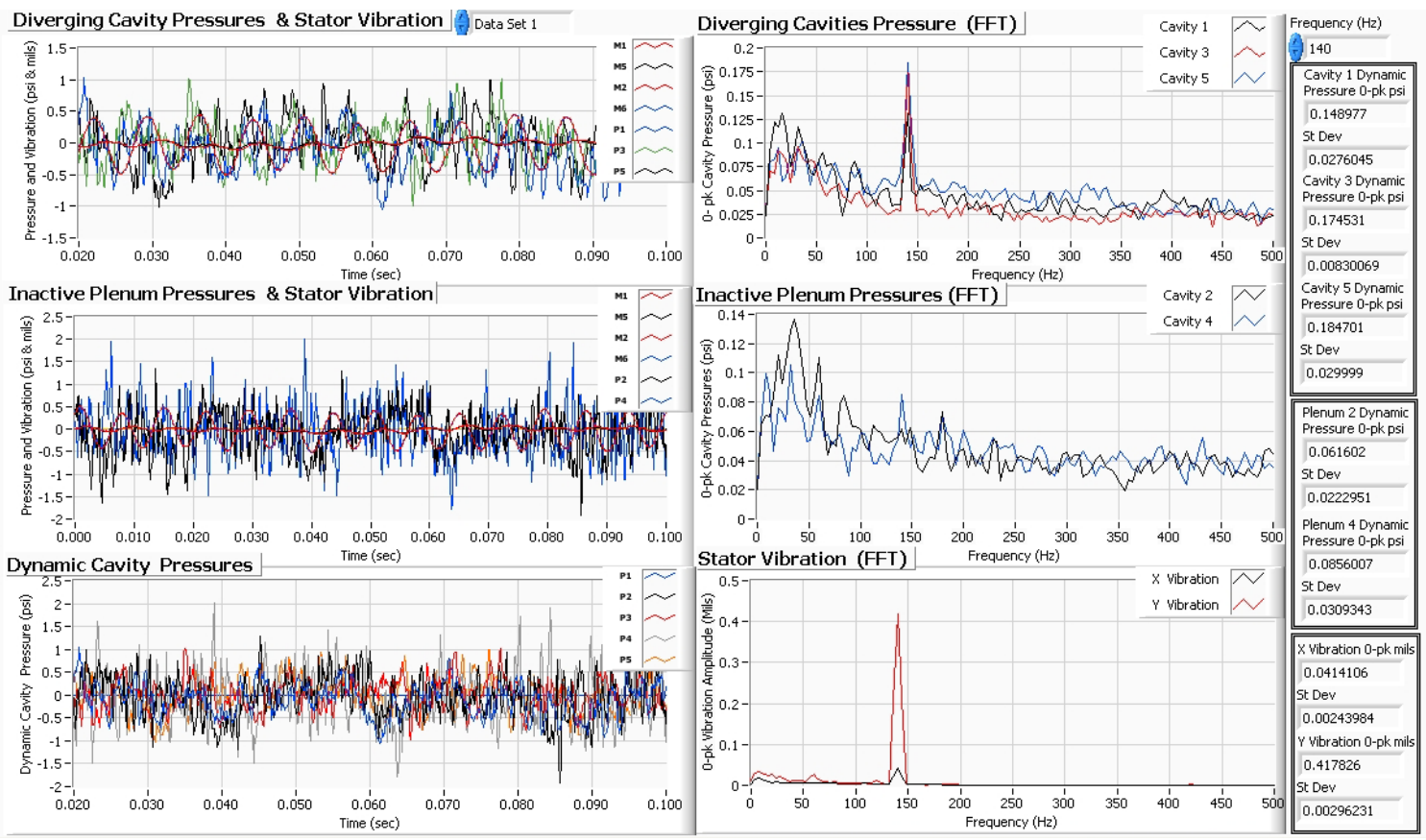

$140 \mathrm{~Hz} 0$ Degree Position Y Direction Excitation 

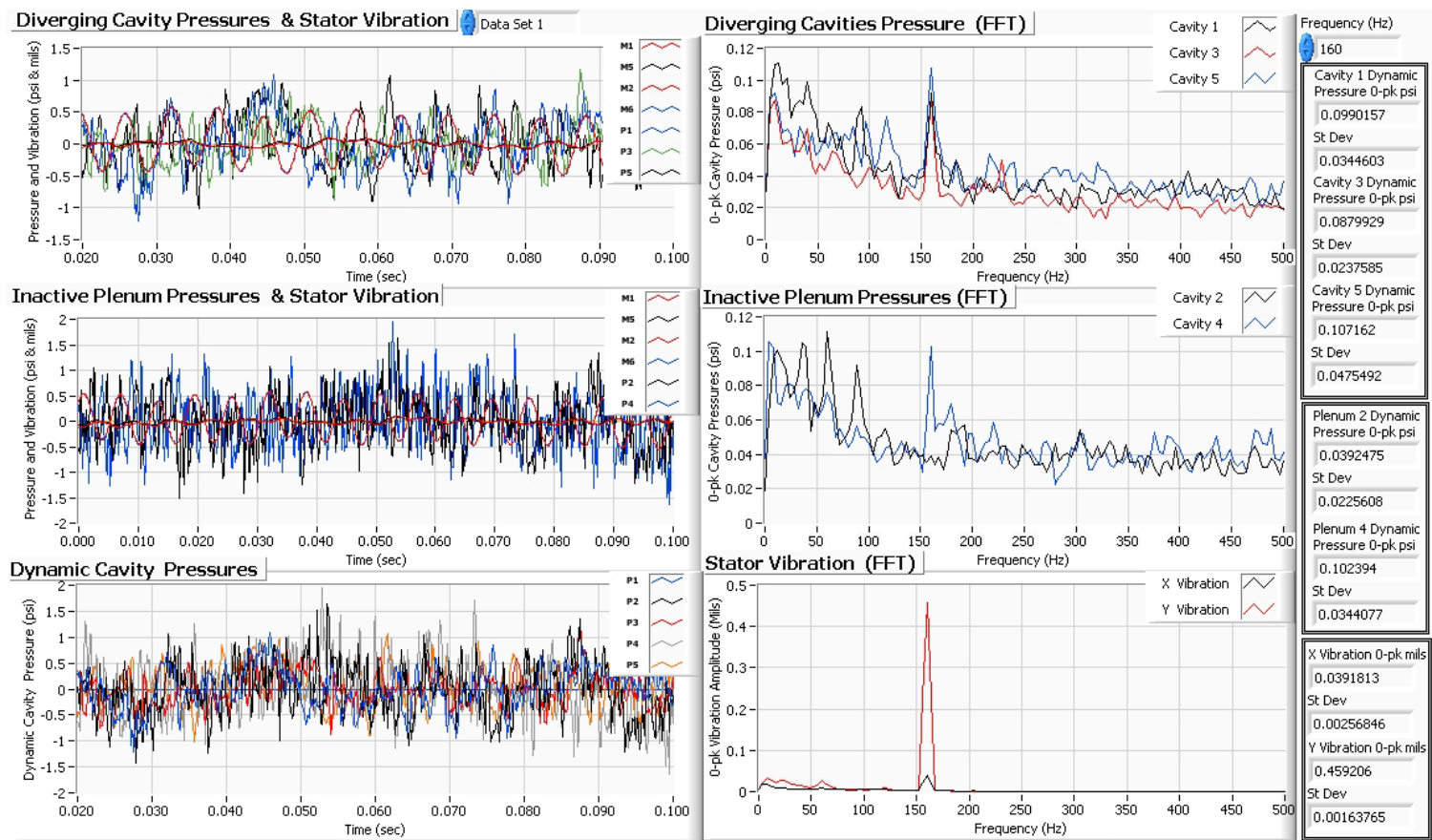

$160 \mathrm{~Hz} 0$ Degree Position Y Direction Excitation
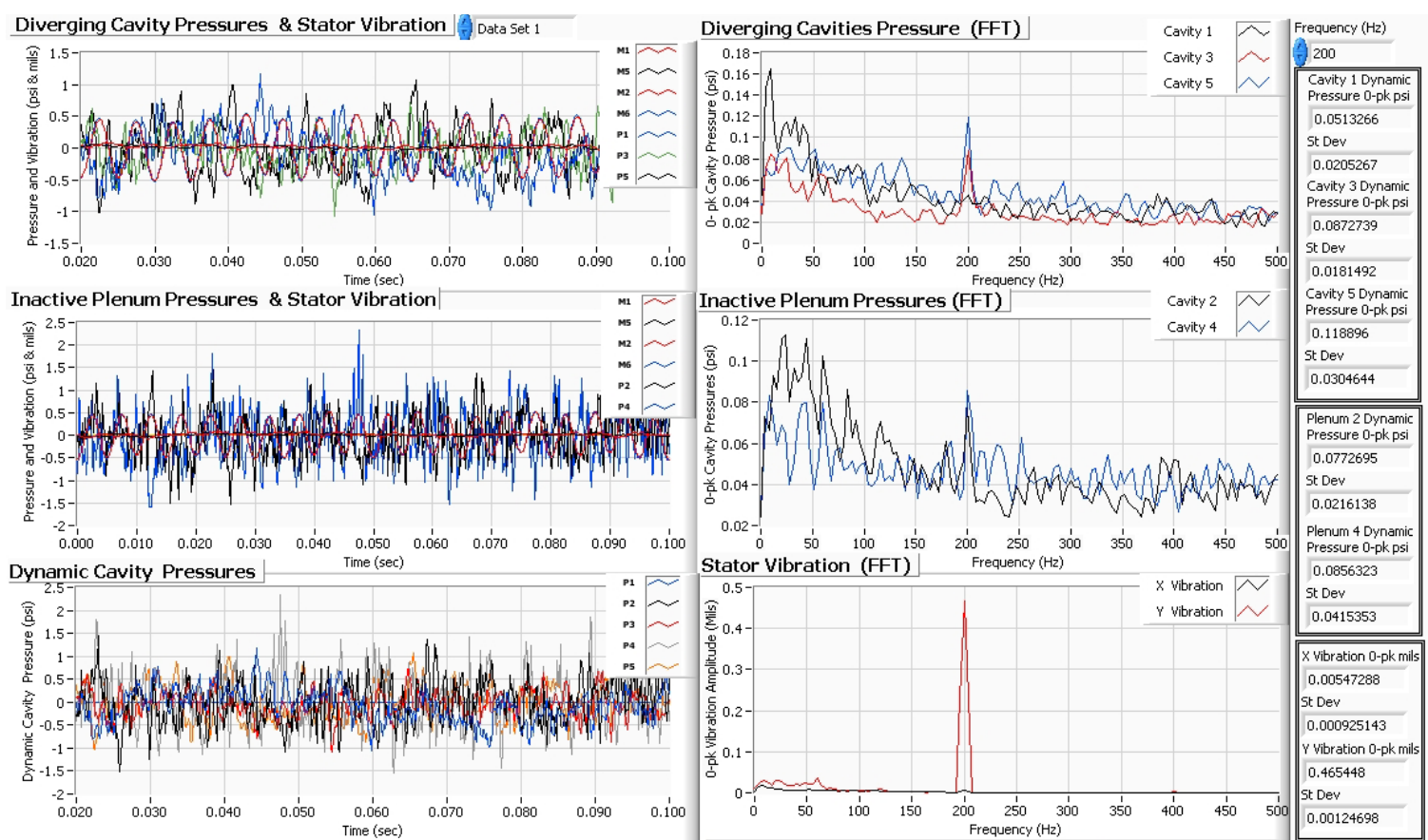

$200 \mathrm{~Hz} 0$ Degree Position Y Direction Excitation 
Dynamic Cavity Pressure Tests

6 Bladed Conventional PDS

\section{DEGREE POSITION}



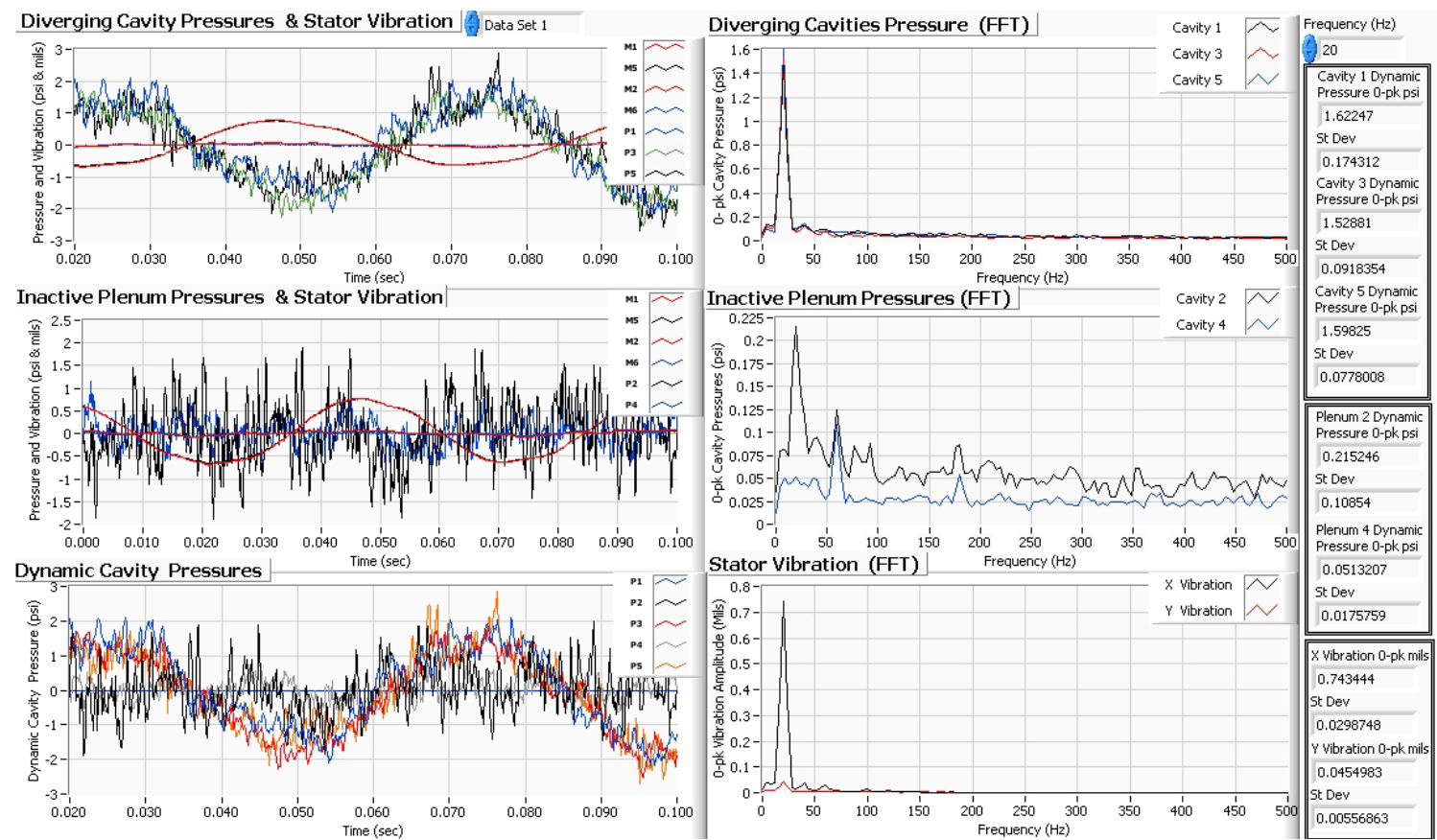

$20 \mathrm{~Hz} 45$ Degree Position X Direction Excitation

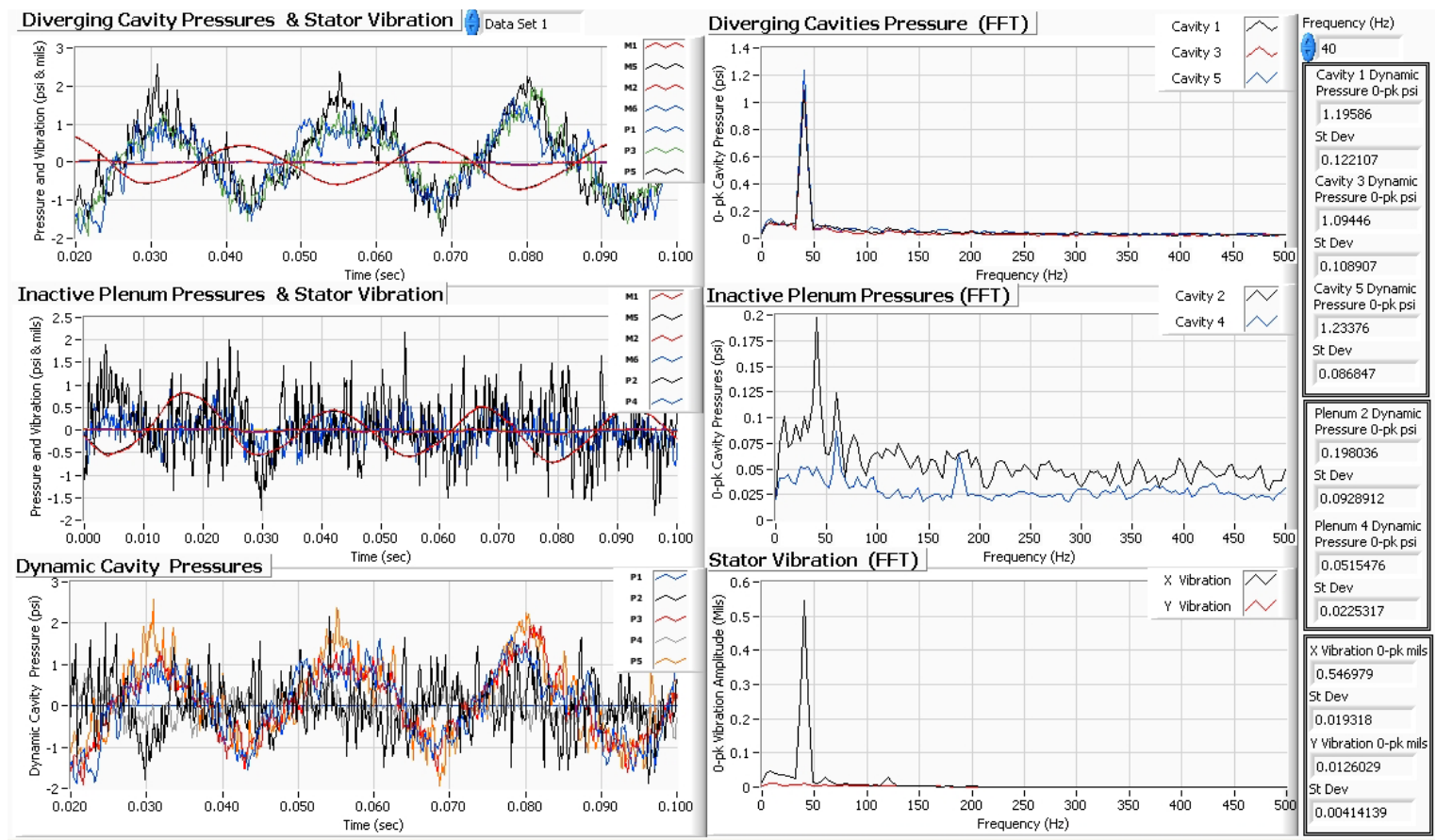

$40 \mathrm{~Hz} 45$ Degree Position X Direction Excitation 


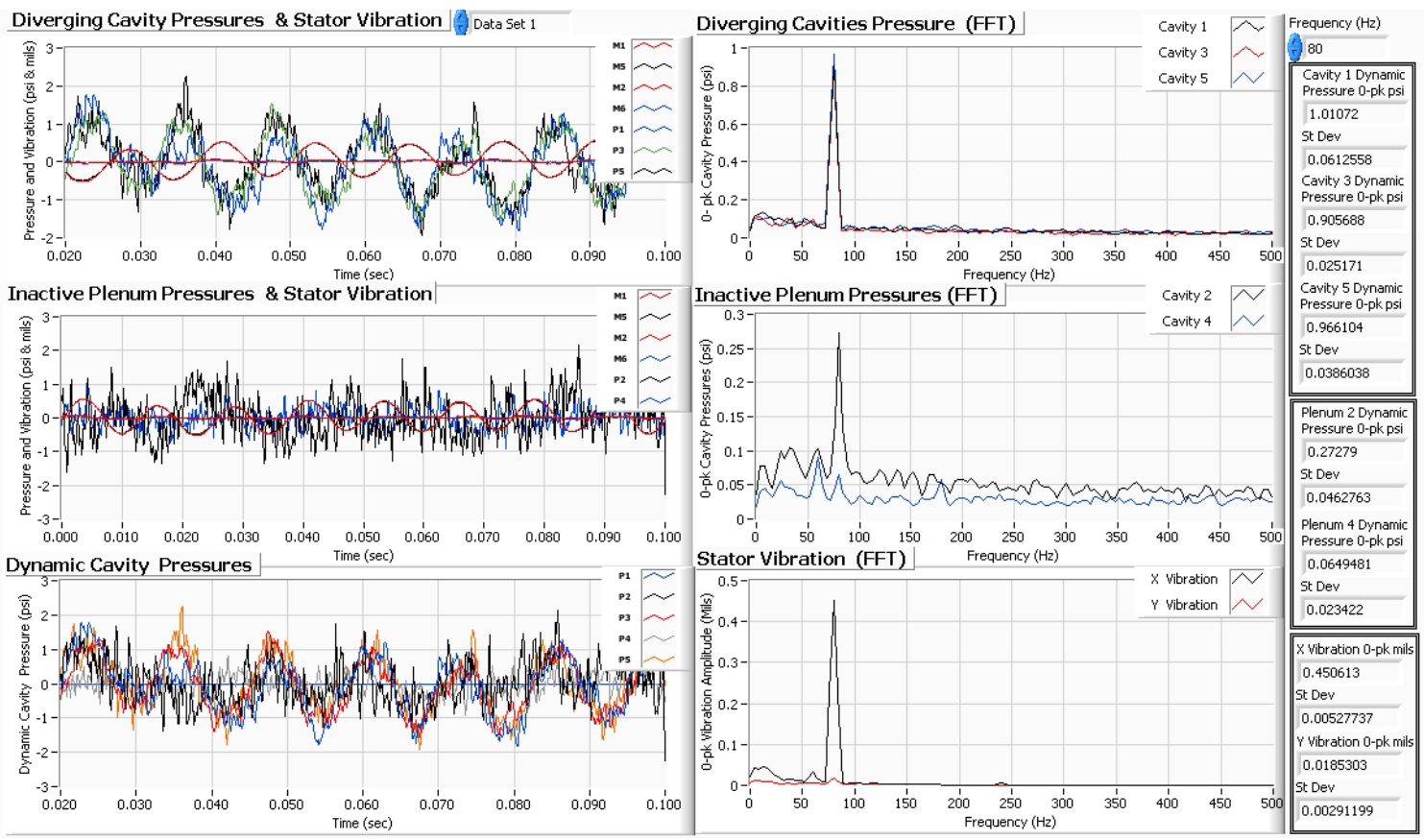

$80 \mathrm{~Hz} 45$ Degree Position X Direction Excitation

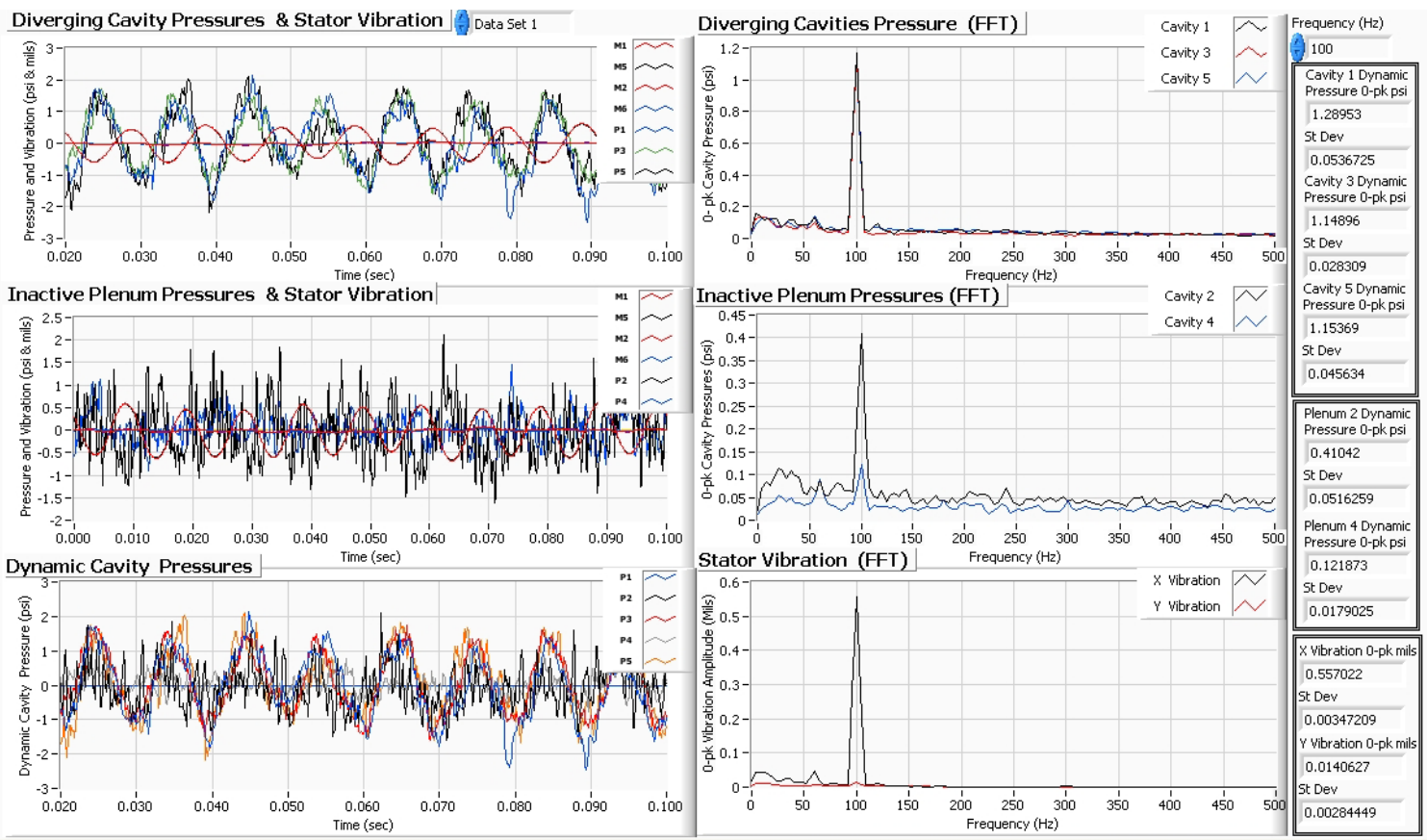

$100 \mathrm{~Hz} 45$ Degree Position X Direction Excitation 

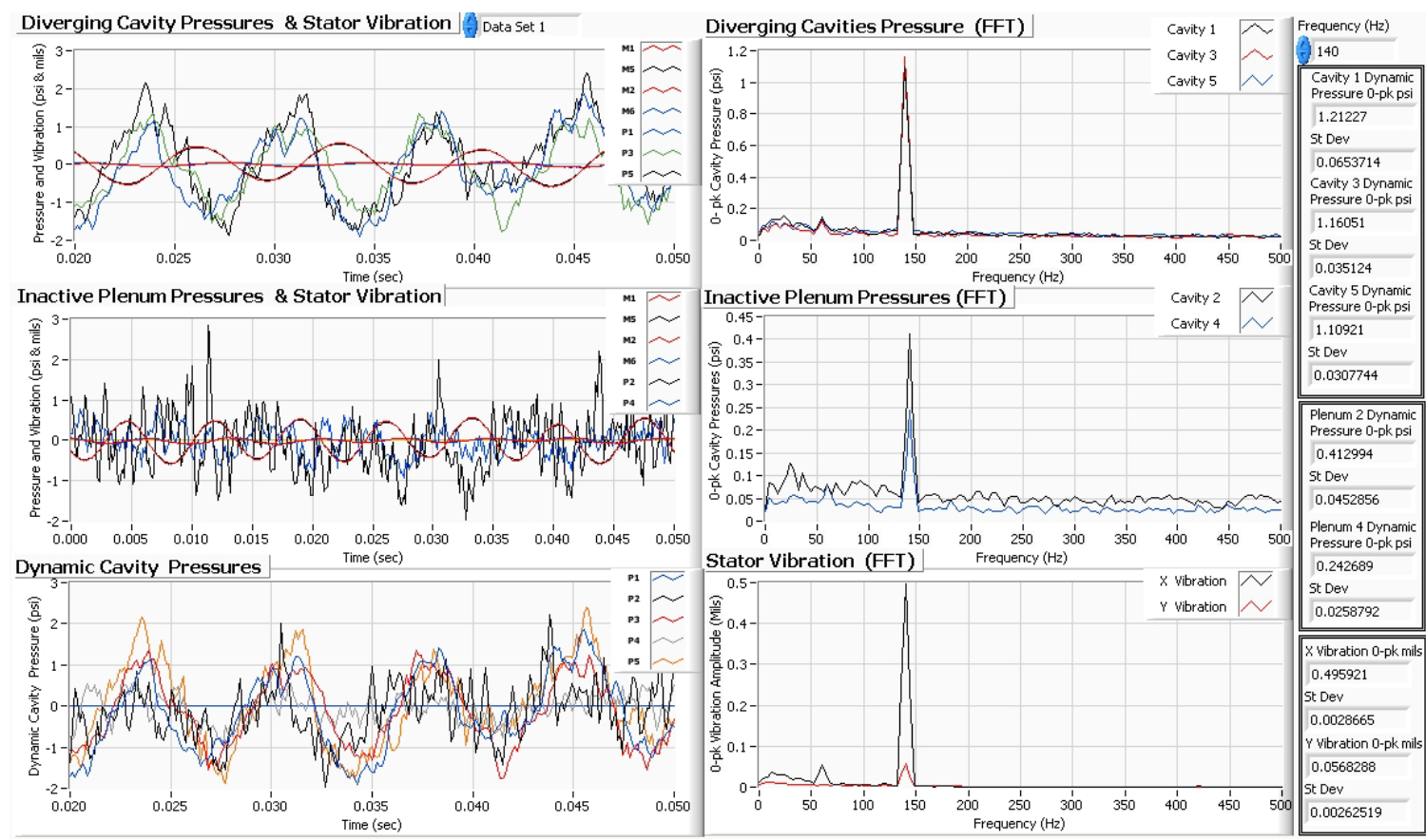

$140 \mathrm{~Hz} 45$ Degree Position X Direction Excitation
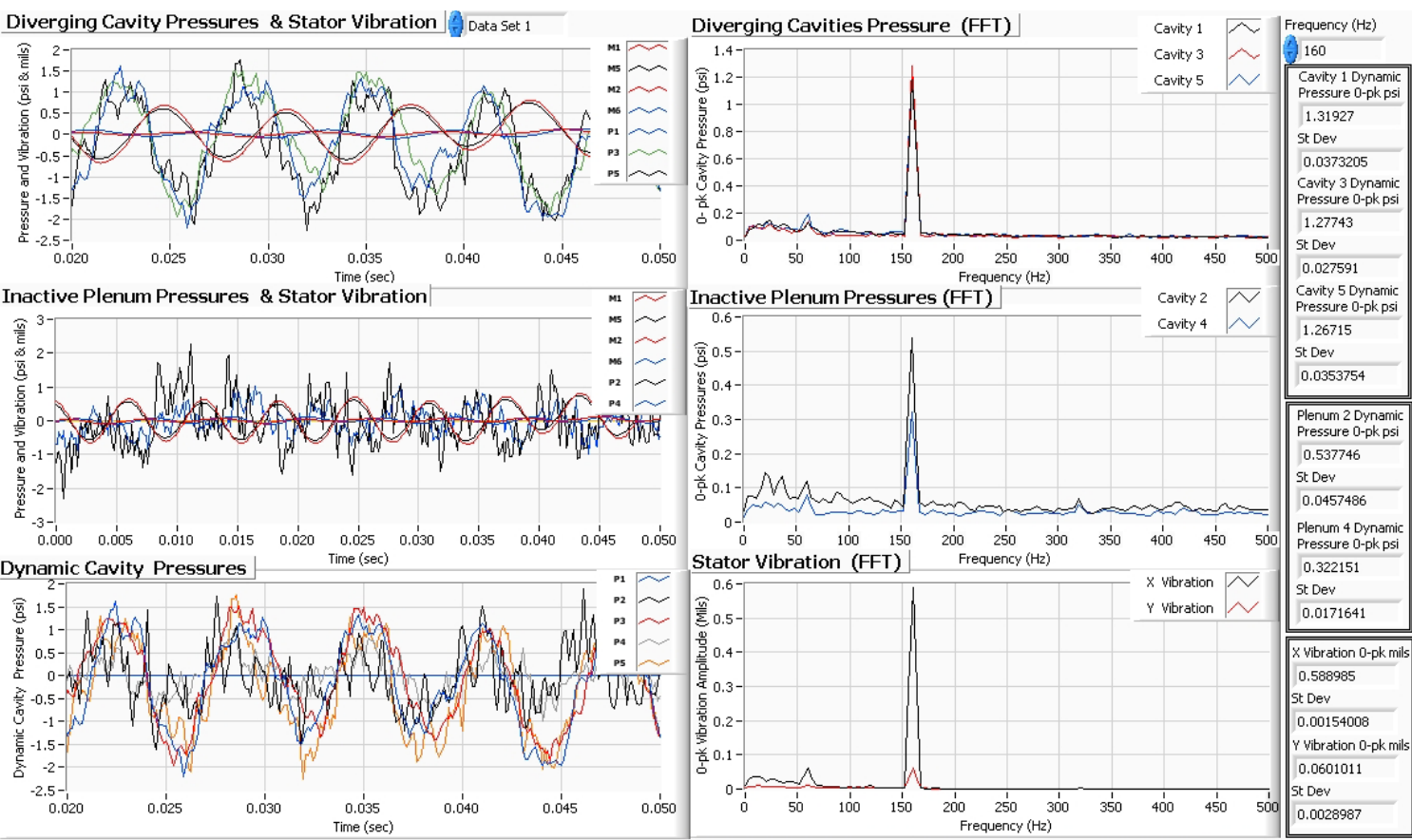

$160 \mathrm{~Hz} 45$ Degree Position X Direction Excitation 

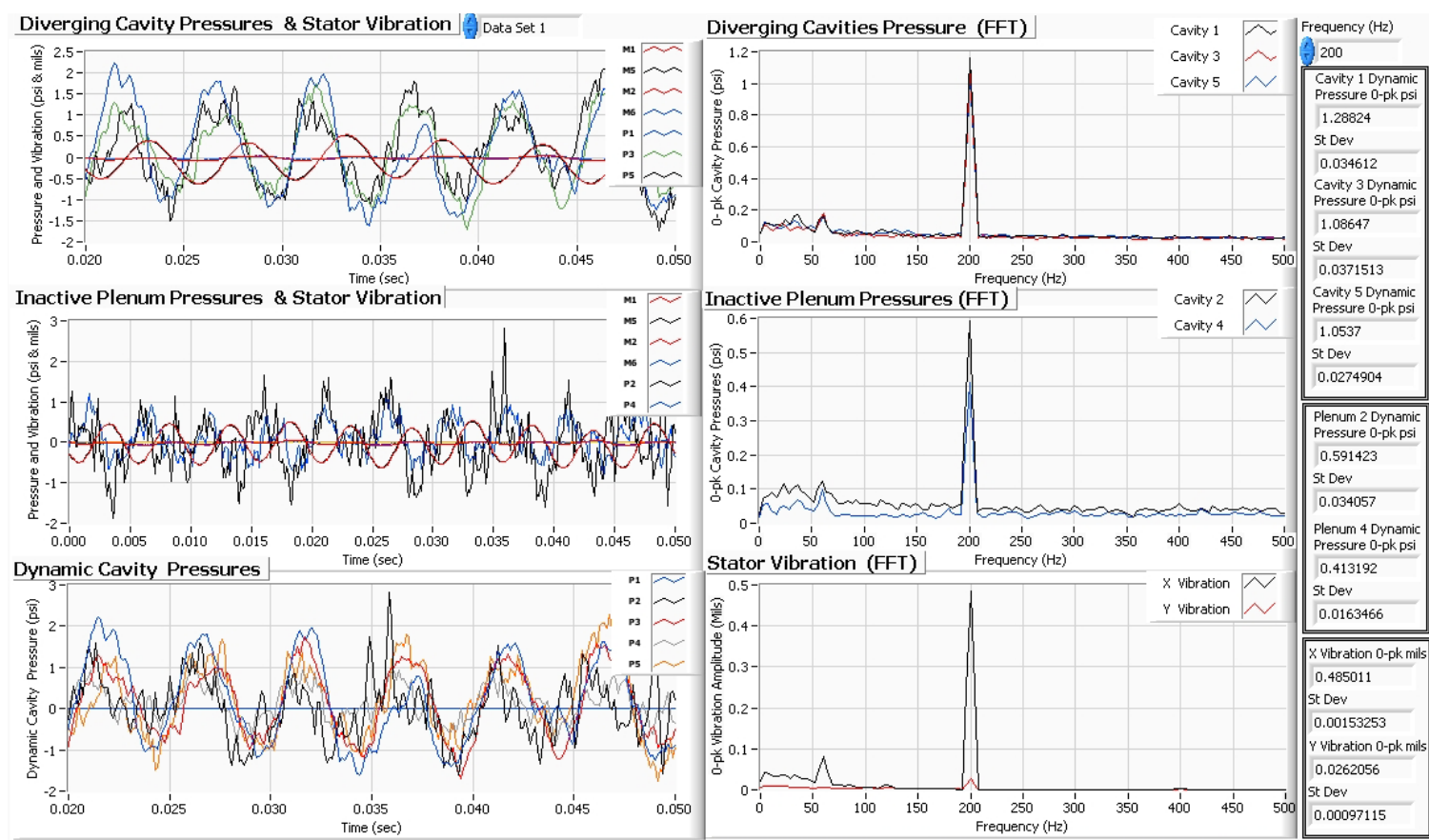

$200 \mathrm{~Hz} 45$ Degree Position X Direction Excitation

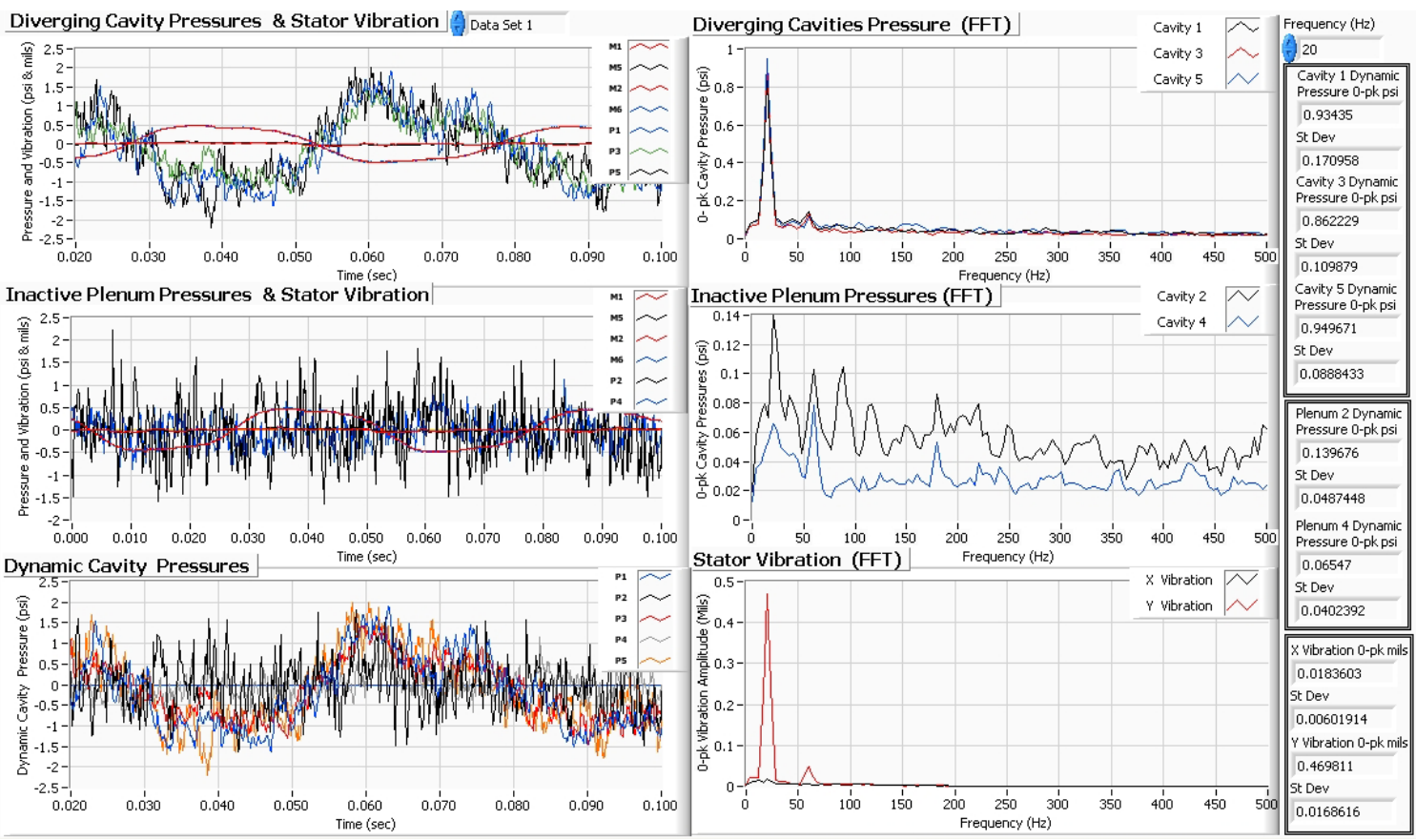

$20 \mathrm{~Hz} 45$ Degree Position Y Direction Excitation 

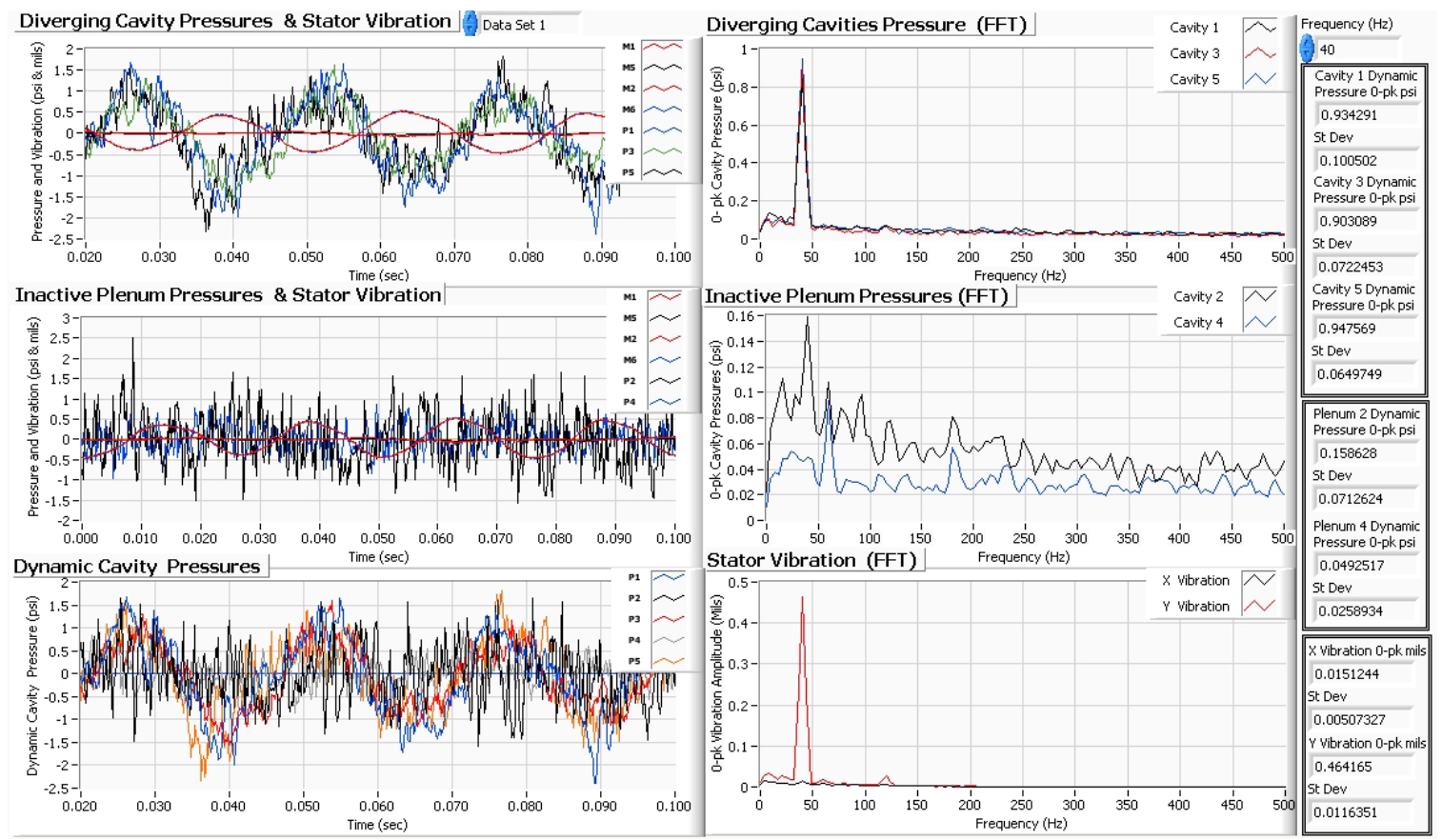

$40 \mathrm{~Hz} 45$ Degree Position Y Direction Excitation

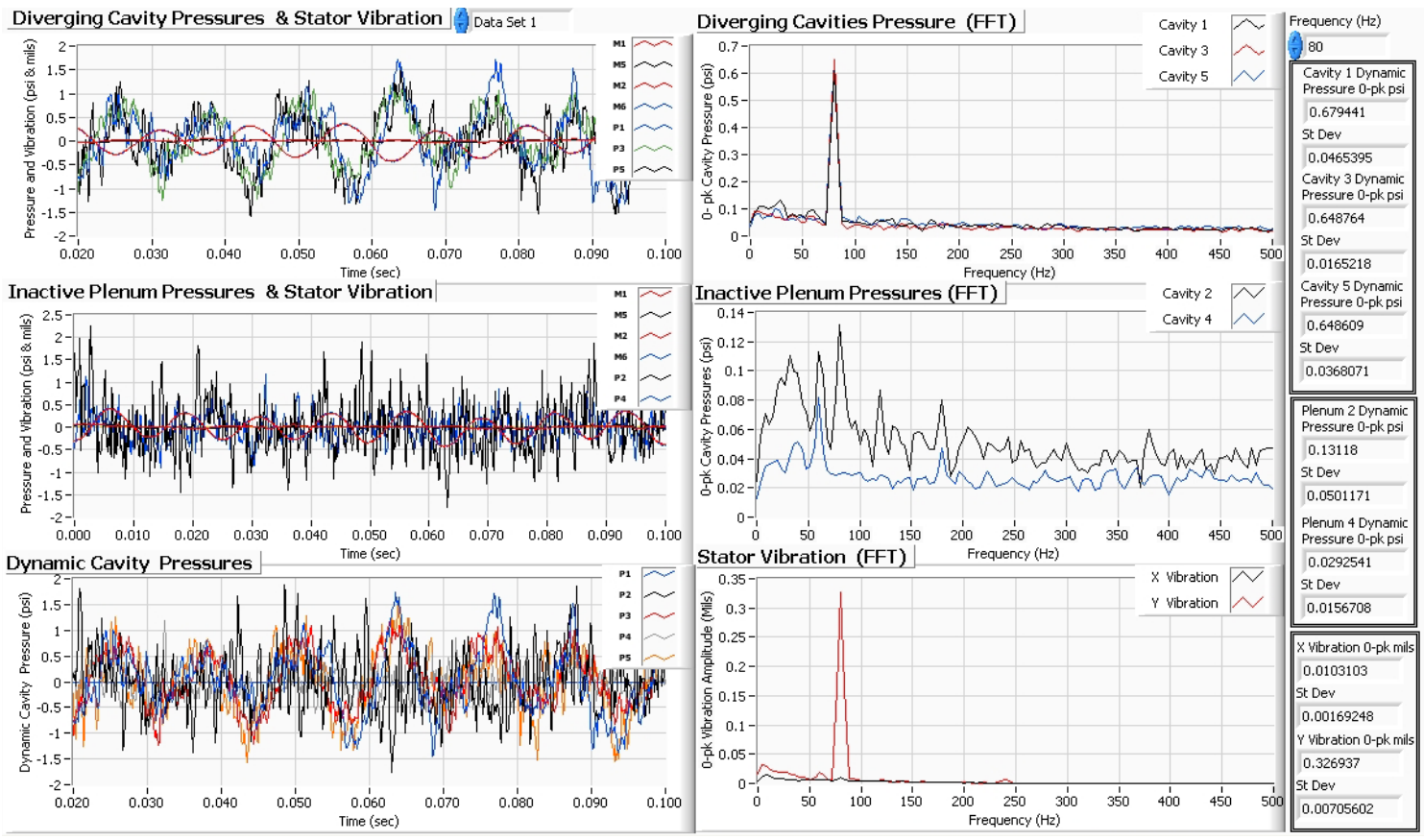

$80 \mathrm{~Hz} 45$ Degree Position Y Direction Excitation 


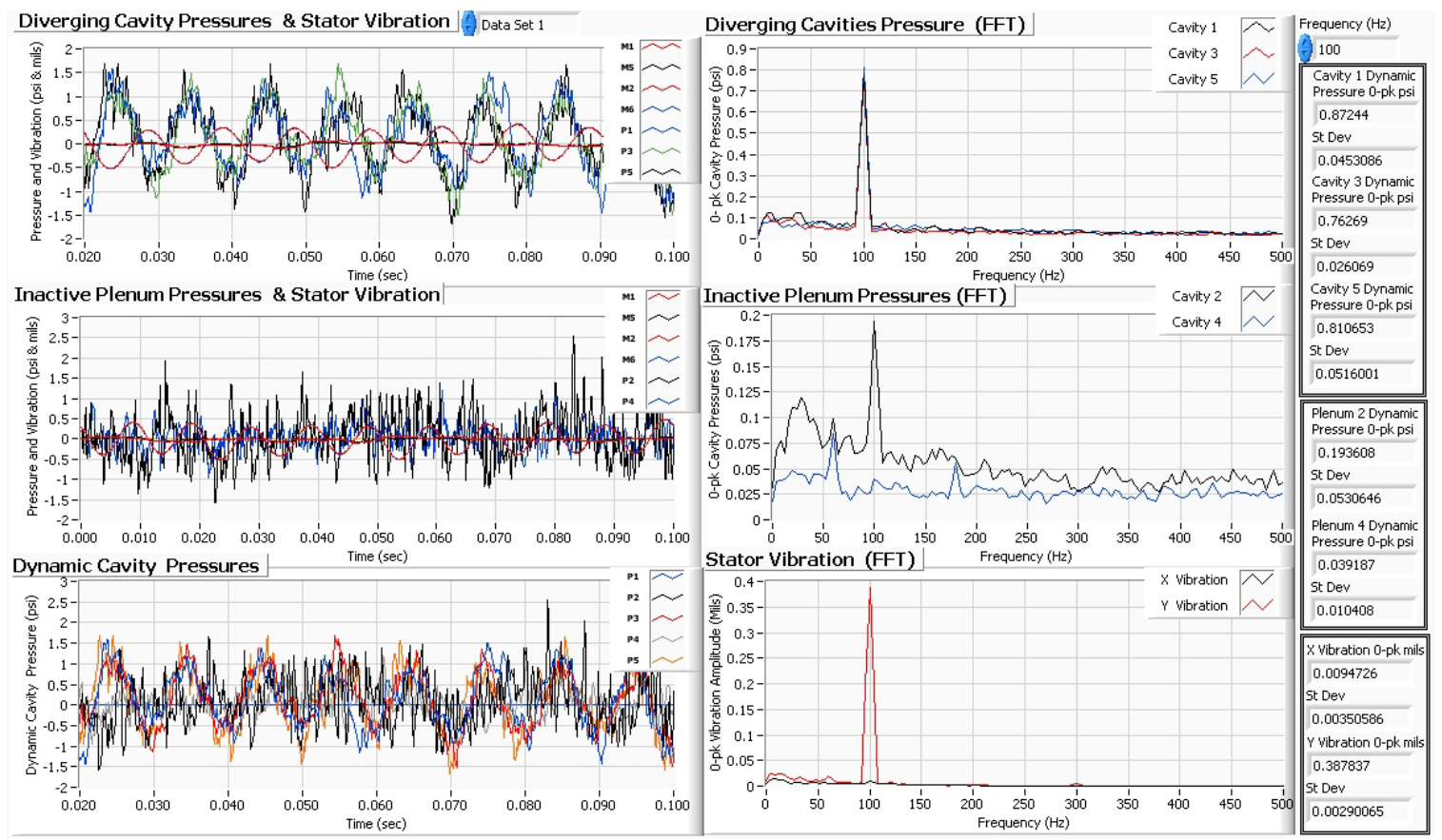

$100 \mathrm{~Hz} 45$ Degree Position Y Direction Excitation

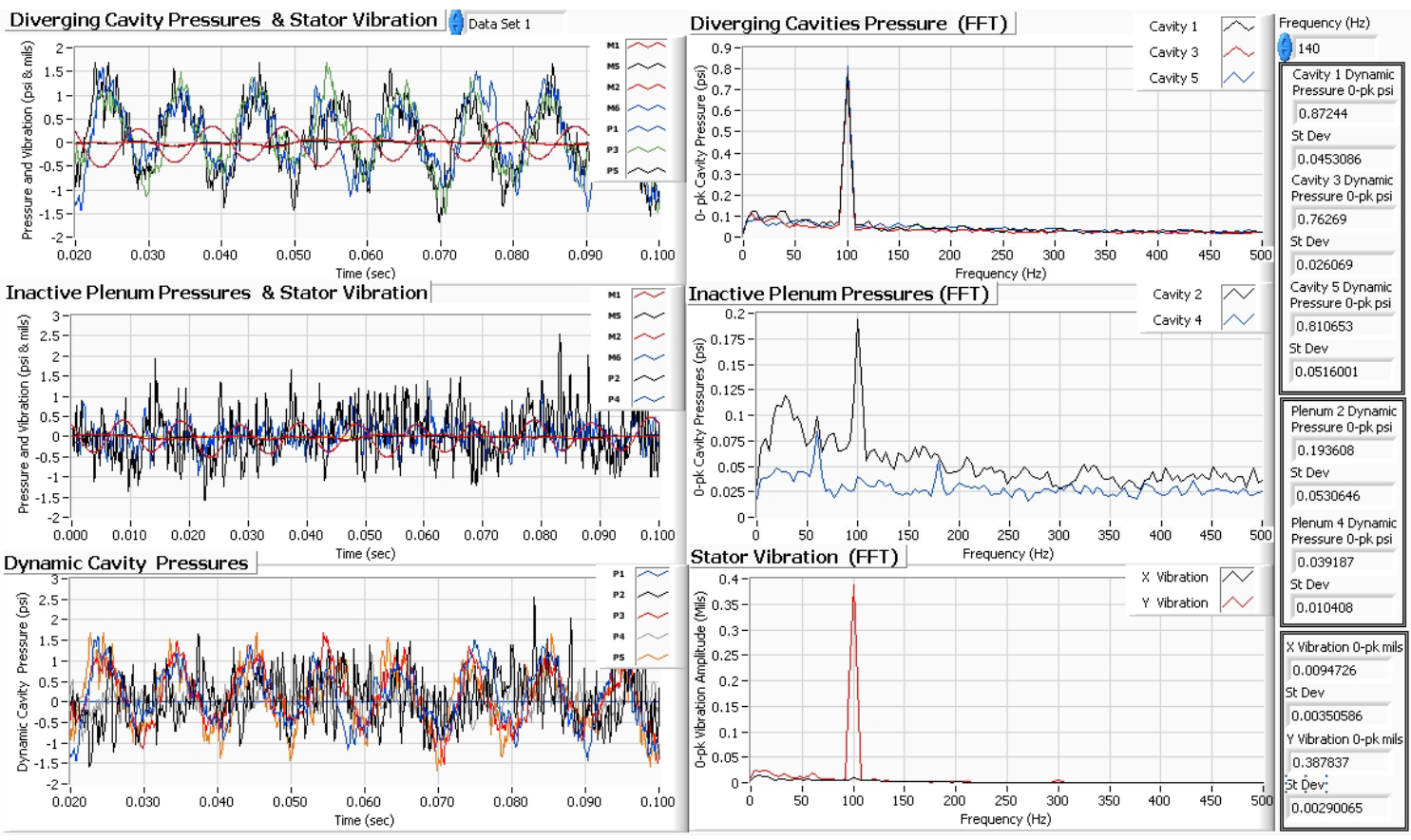

$140 \mathrm{~Hz} 45$ Degree Position Y Direction Excitation 


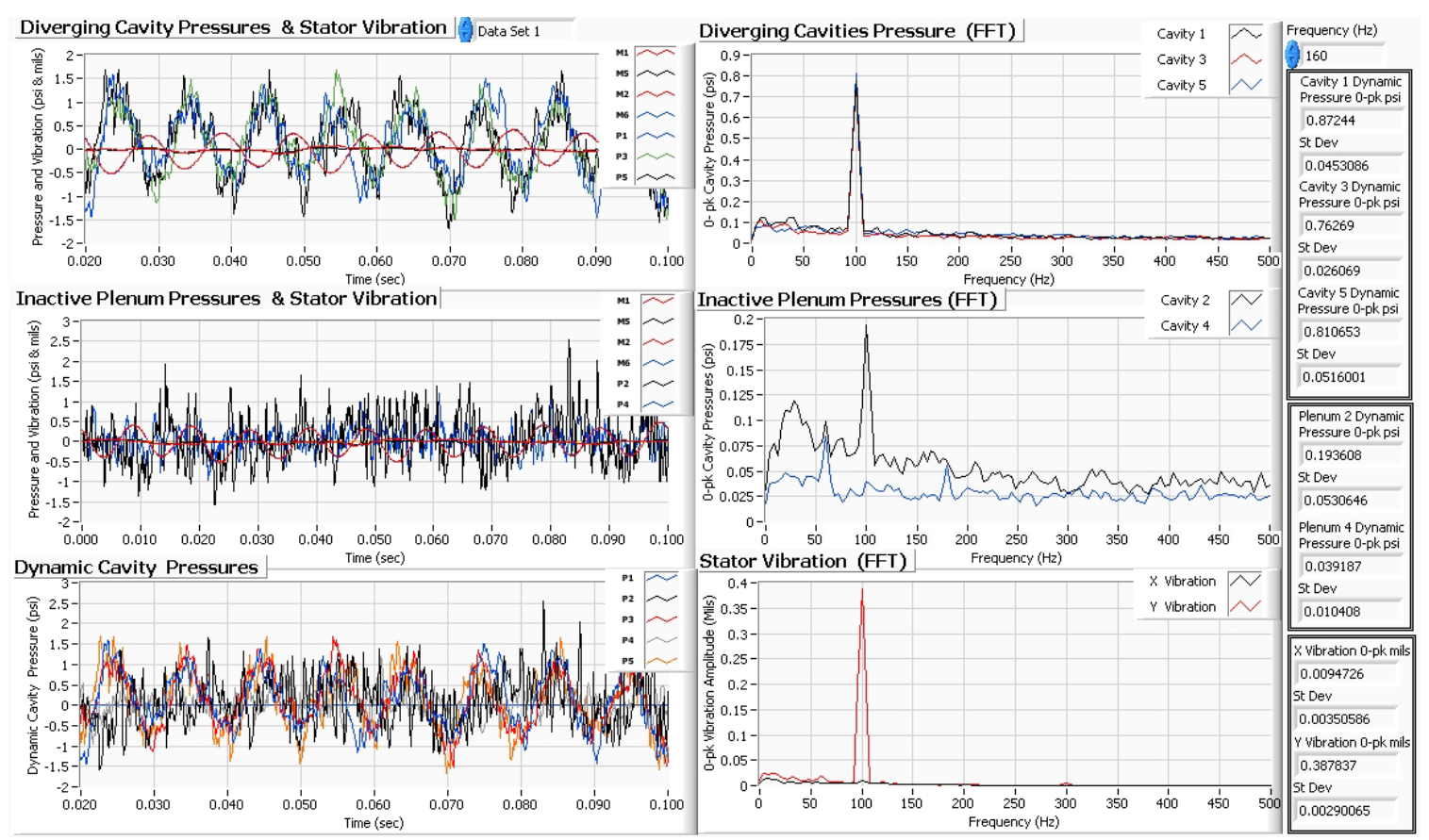

$160 \mathrm{~Hz} 45$ Degree Position Y Direction Excitation

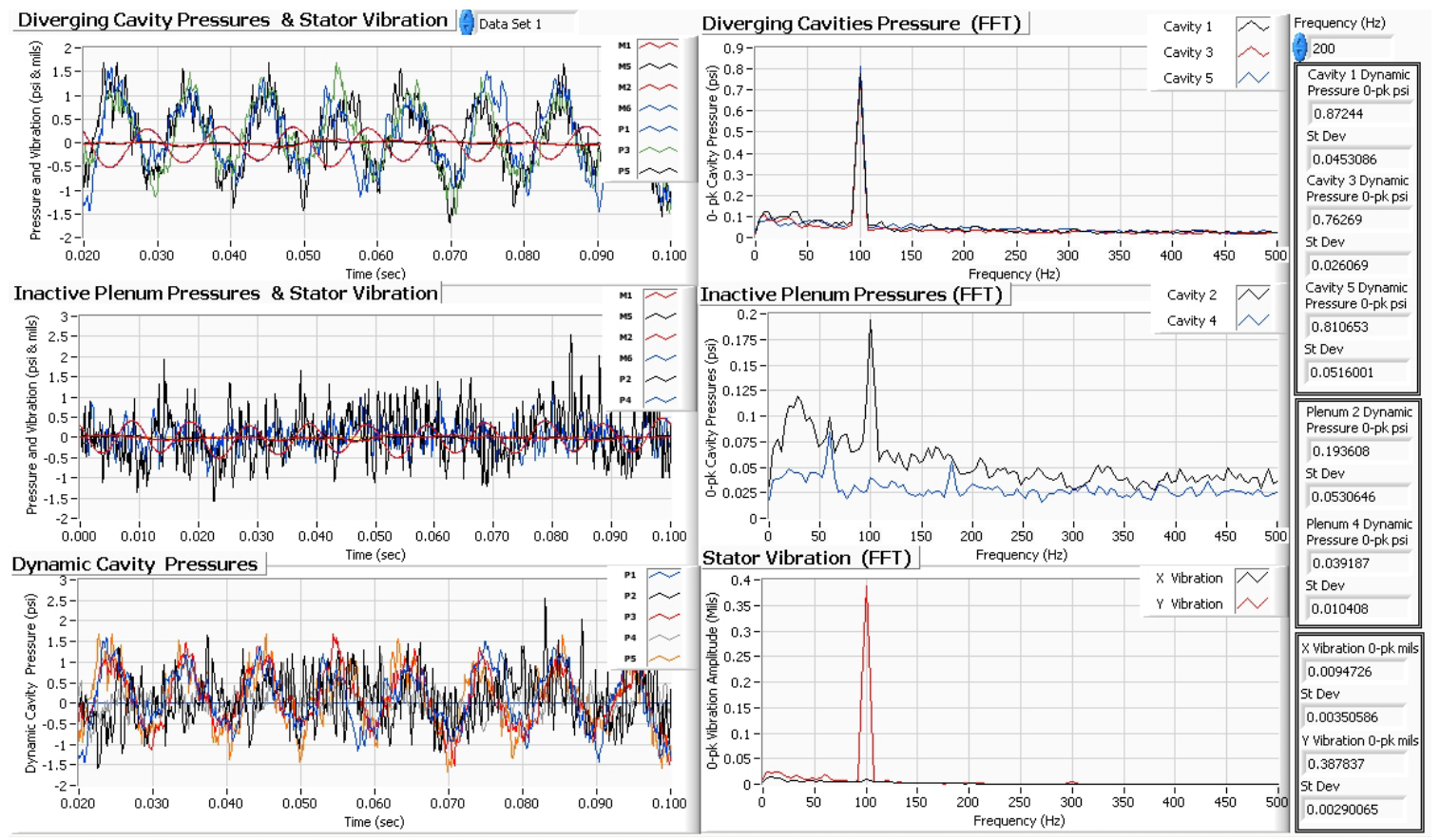

$200 \mathrm{~Hz} 45$ Degree Position Y Direction Excitation 
Dynamic Cavity Pressure Tests

6 Bladed Conventional PDS

90 DEGREE POSITION 


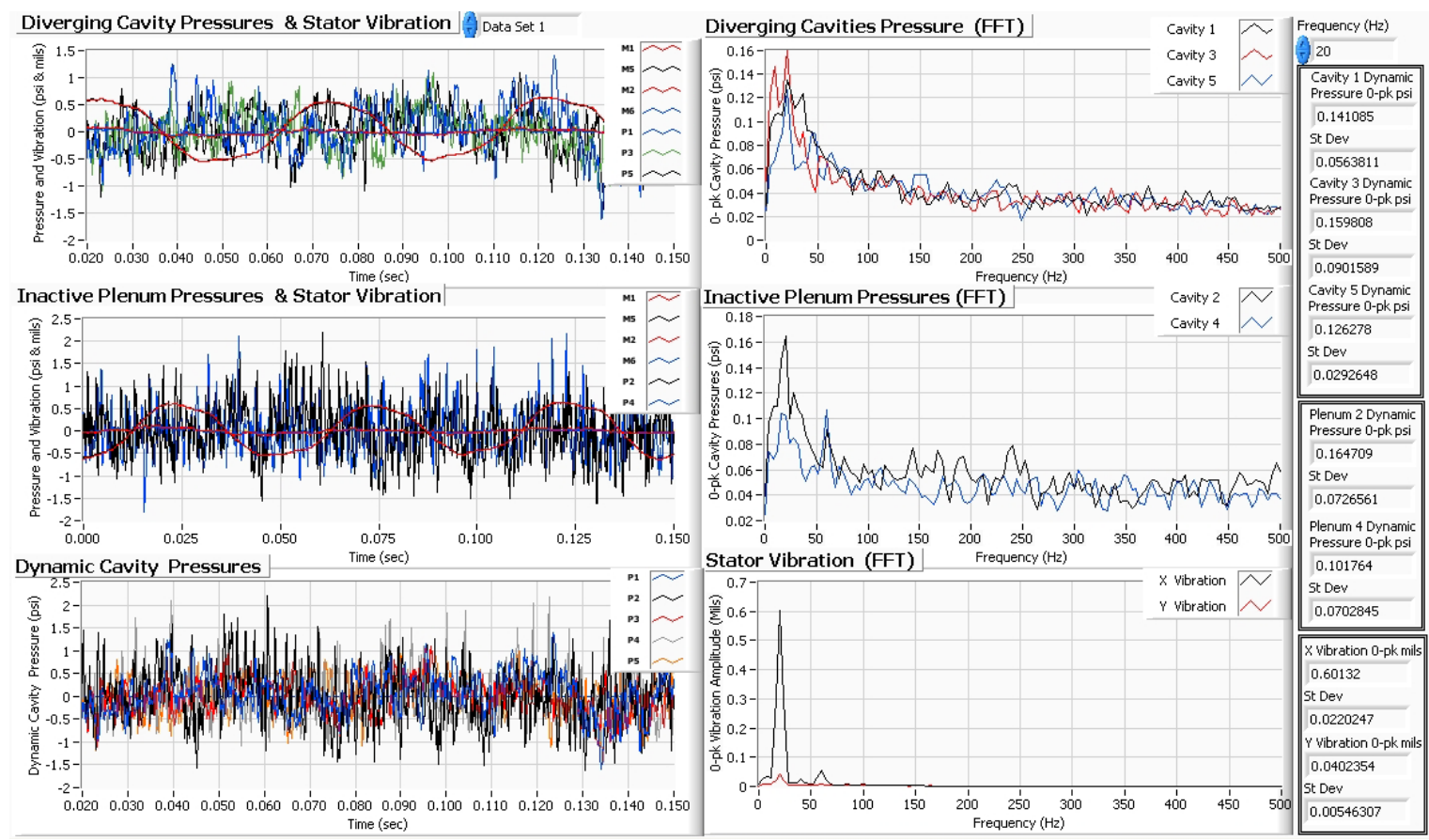

$20 \mathrm{~Hz} 90$ Degree Position X Direction Excitation
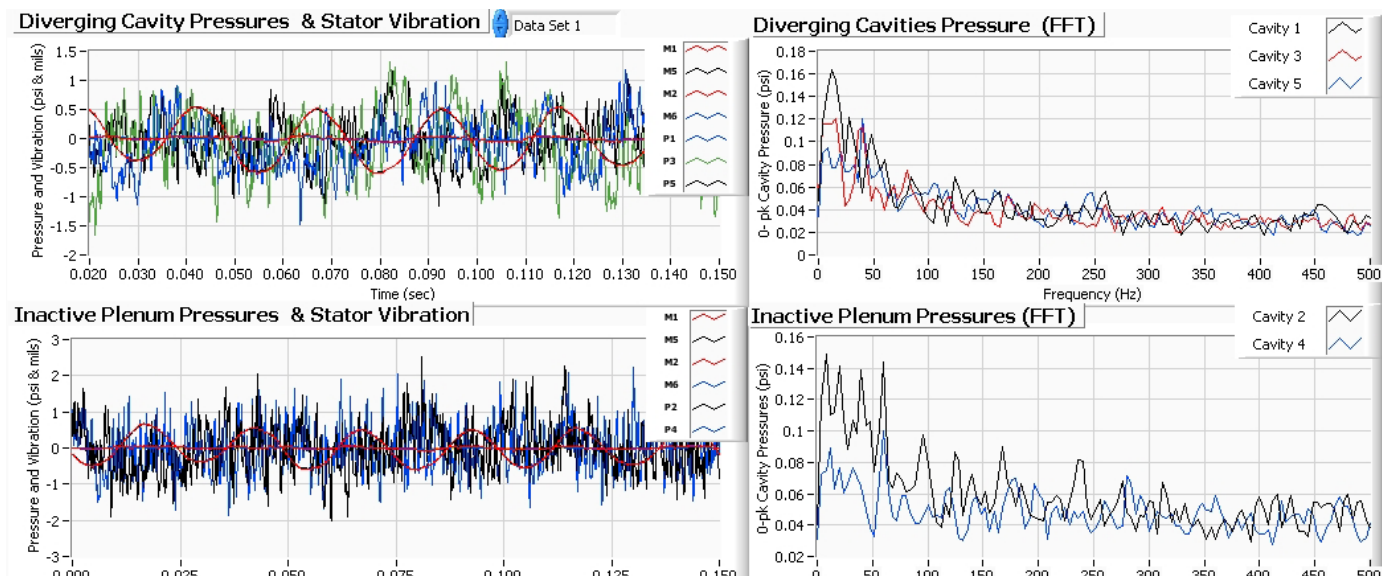

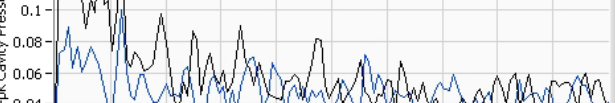

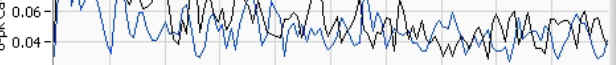
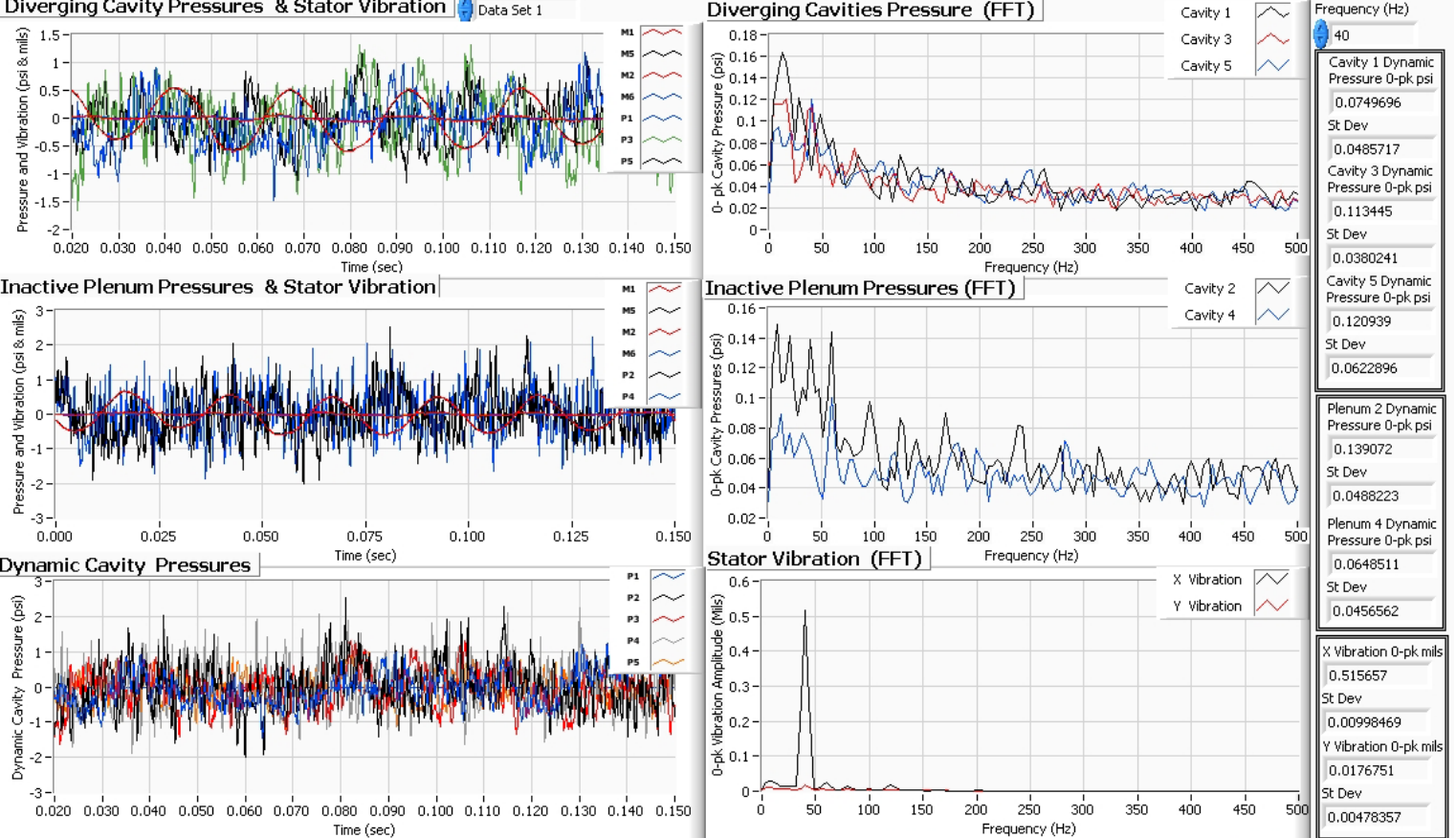

$40 \mathrm{~Hz} 90$ Degree Position X Direction Excitation 


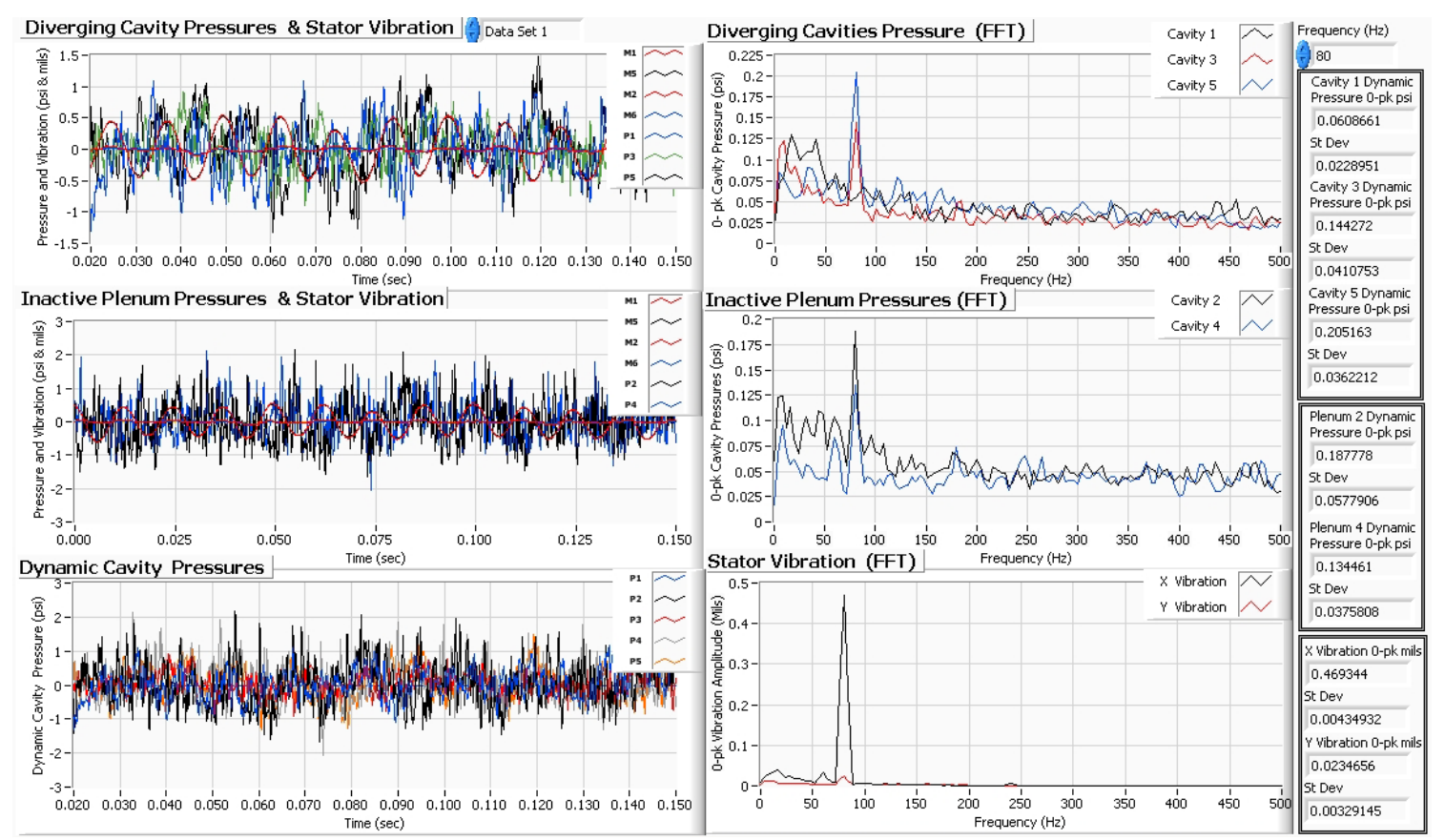

$80 \mathrm{~Hz} 90$ Degree Position X Direction Excitation
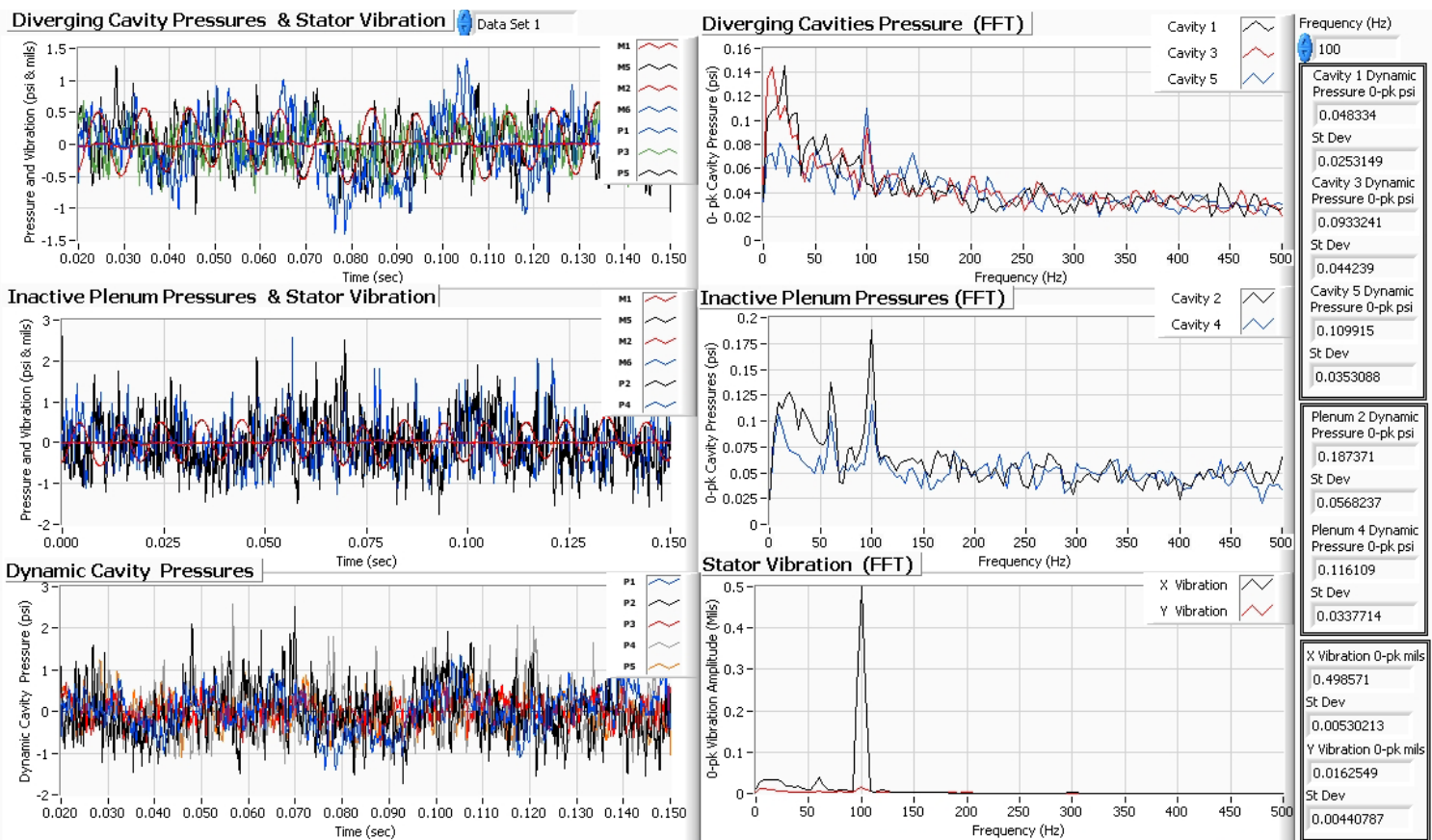

$100 \mathrm{~Hz} 90$ Degree Position X Direction Excitation 

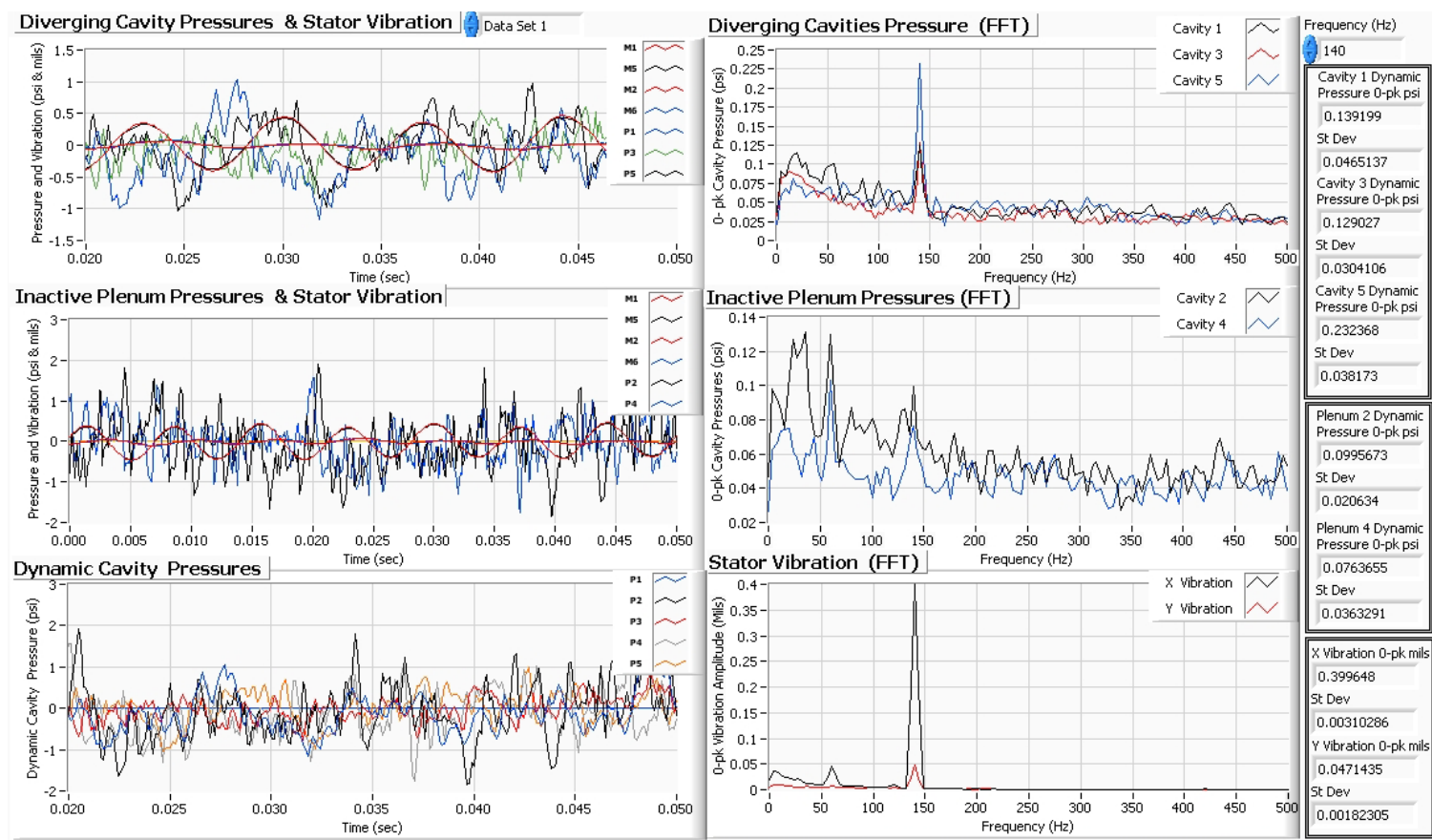

\section{$140 \mathrm{~Hz} 90$ Degree Position X Direction Excitation}
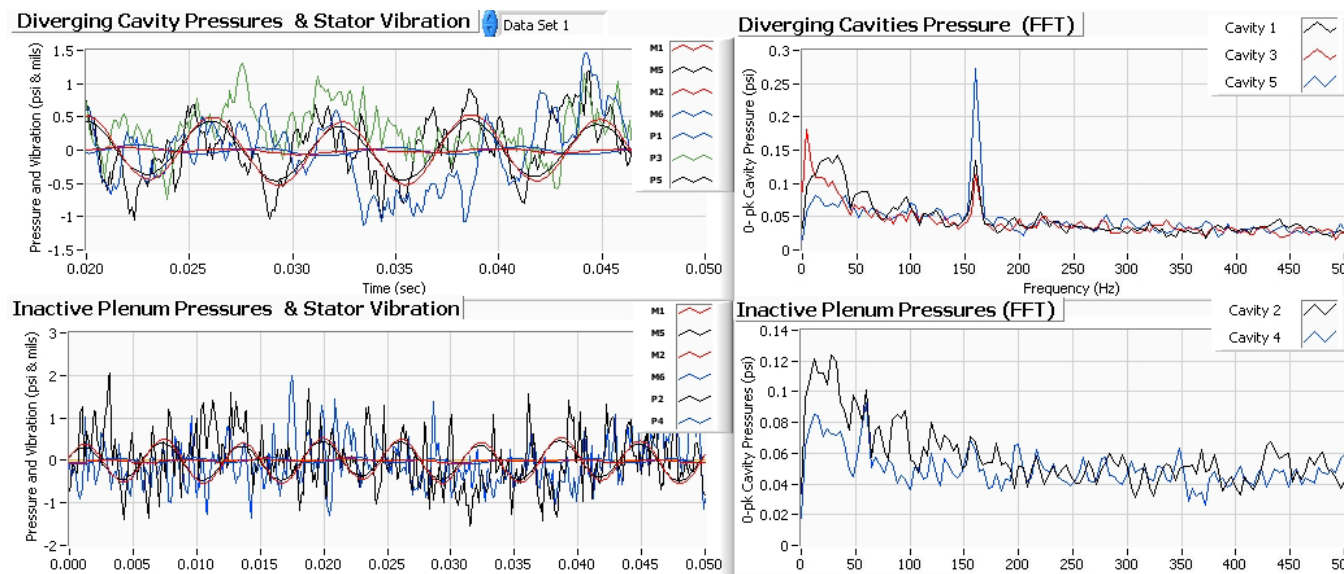

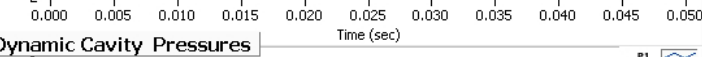
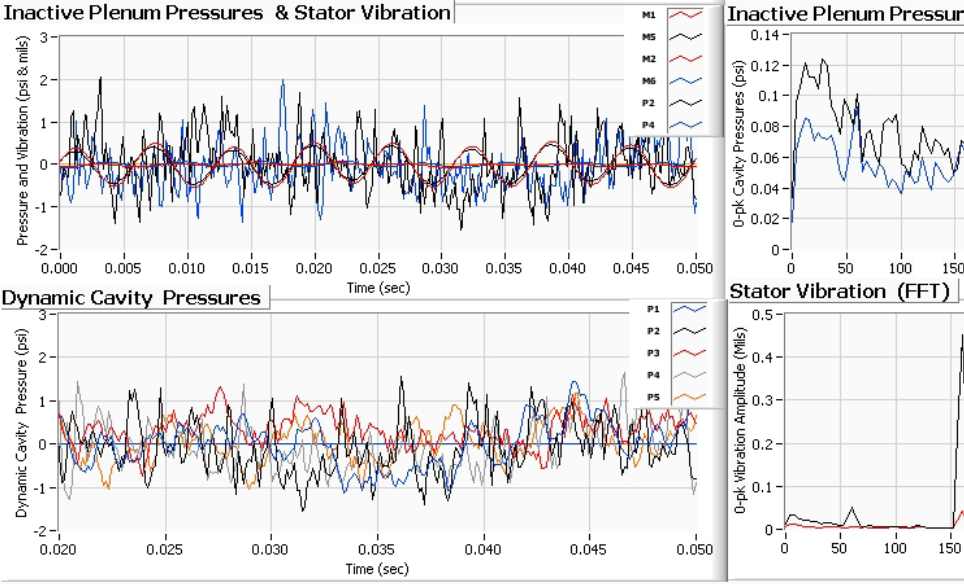

\section{FFT)}

cavity $2 \longdiv { }$

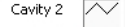

4 Pressure 0-ph ps

0.273114
St Dev

0.0322924

Plenum 2 Dynamic

0.067418

St Dev

0.0278041

Plenum 4 Dynamic
Pressure 0-pk psi

0.0623614

St Dev

0.0306693

Vibration 0-pk mils

0.450609

0.00155719

0.00155719

$160 \mathrm{~Hz} 90$ Degree Position X Direction Excitation 

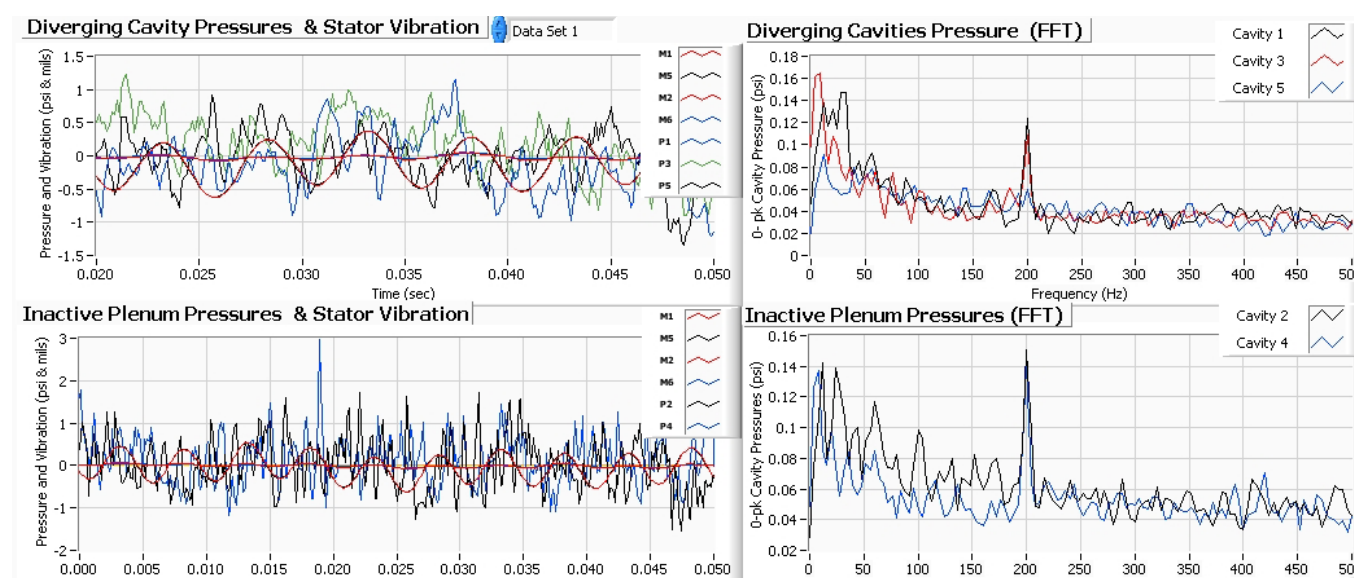

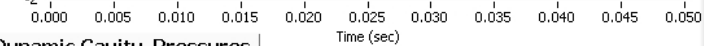
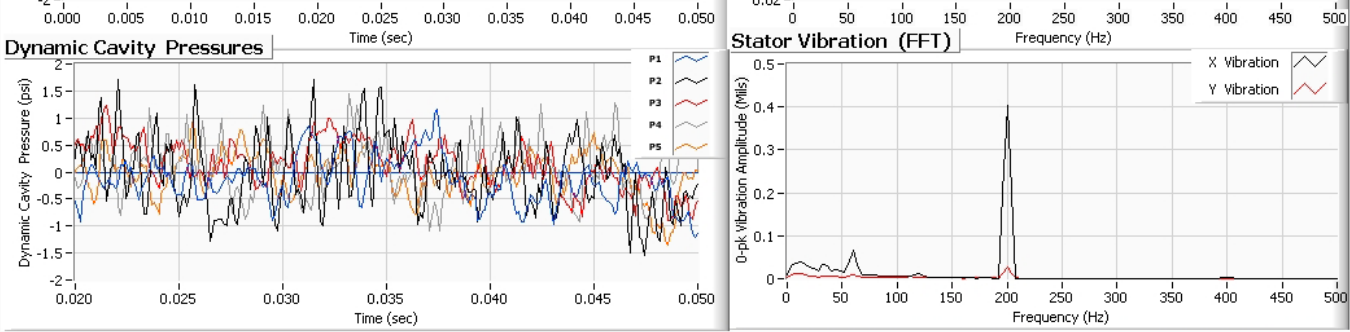

$200 \mathrm{~Hz} 90$ Degree Position X Direction Excitation
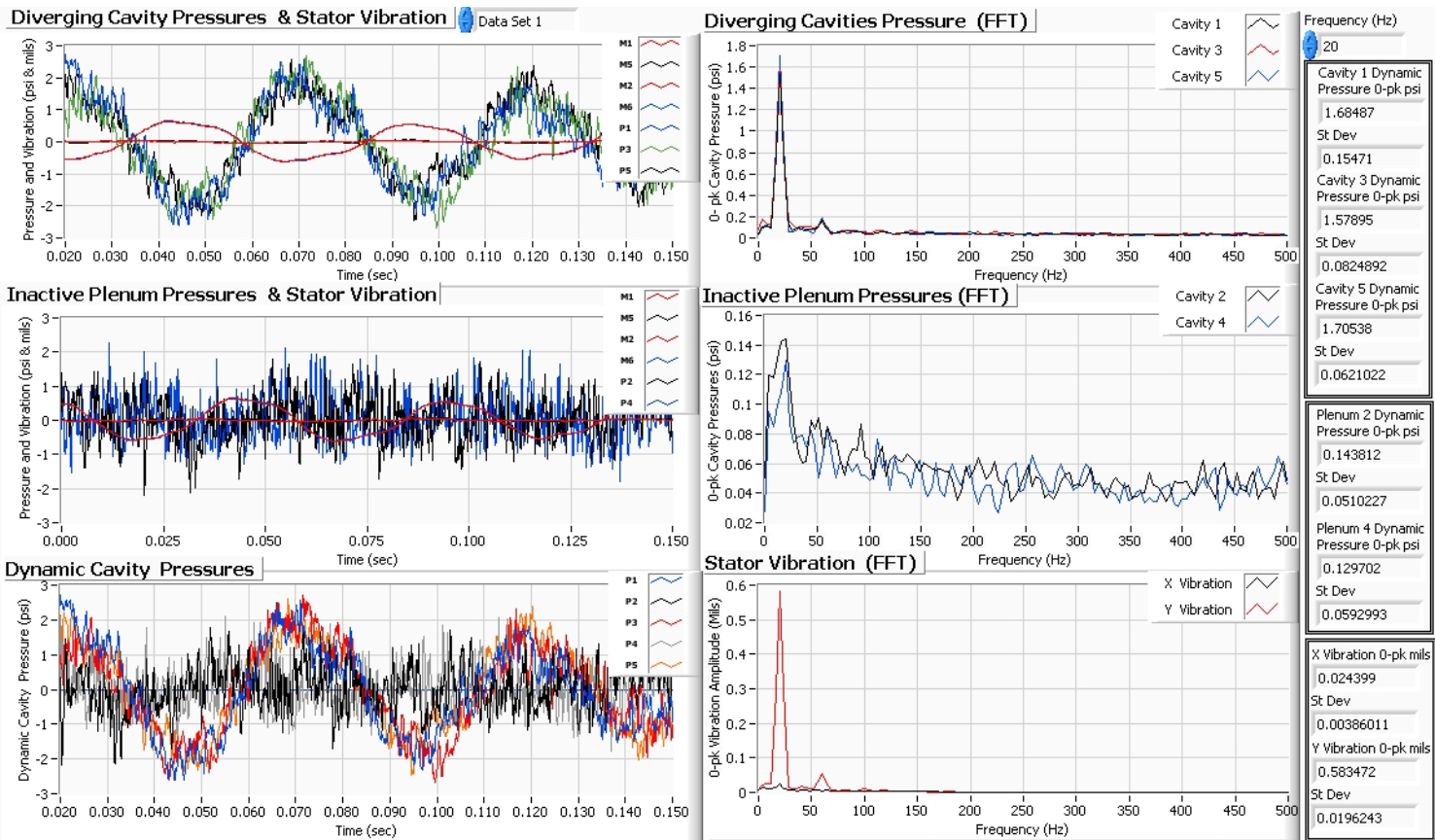

$20 \mathrm{~Hz} 90$ Degree Position Y Direction Excitation 

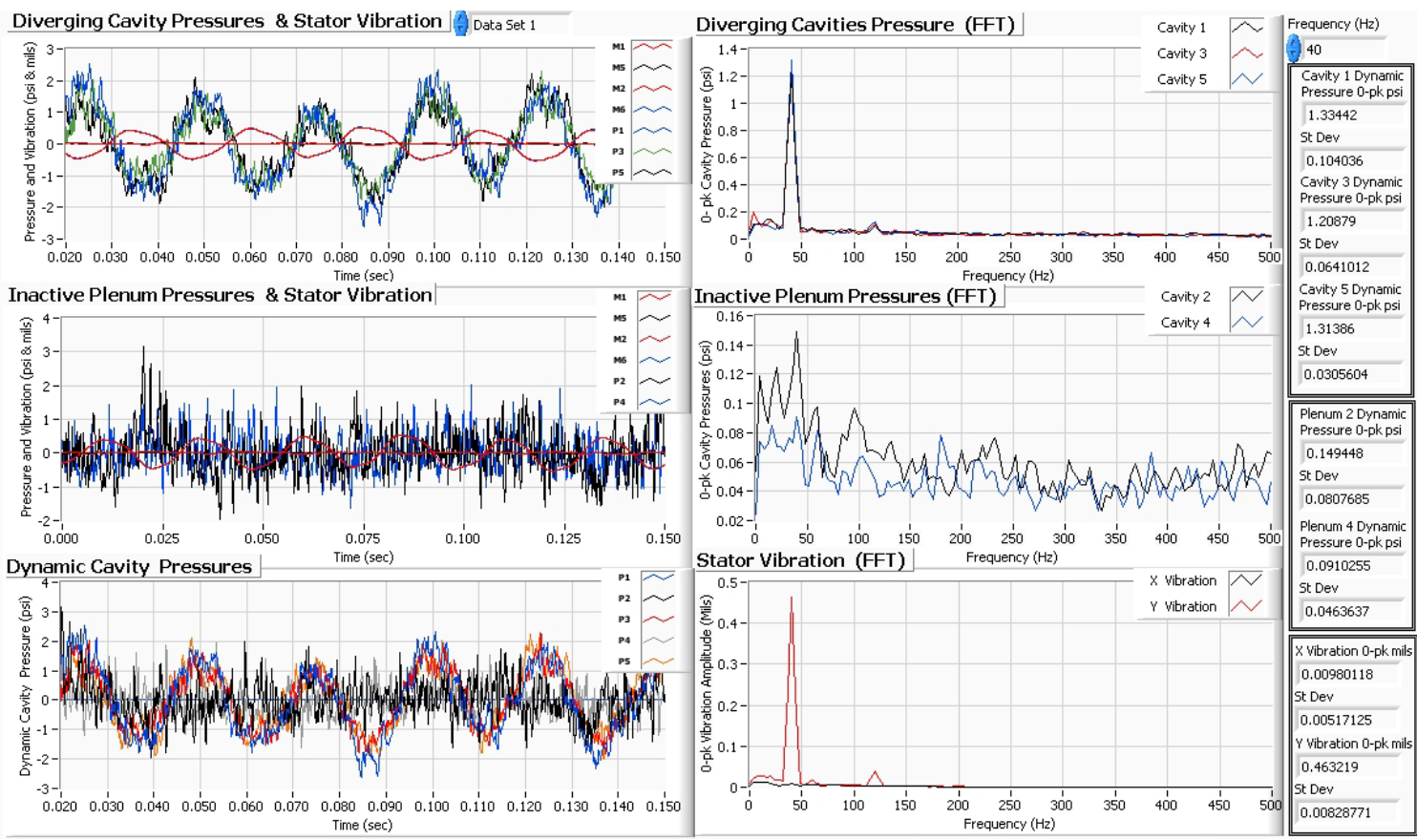

40 Hz 90 Degree Position Y Direction Excitation
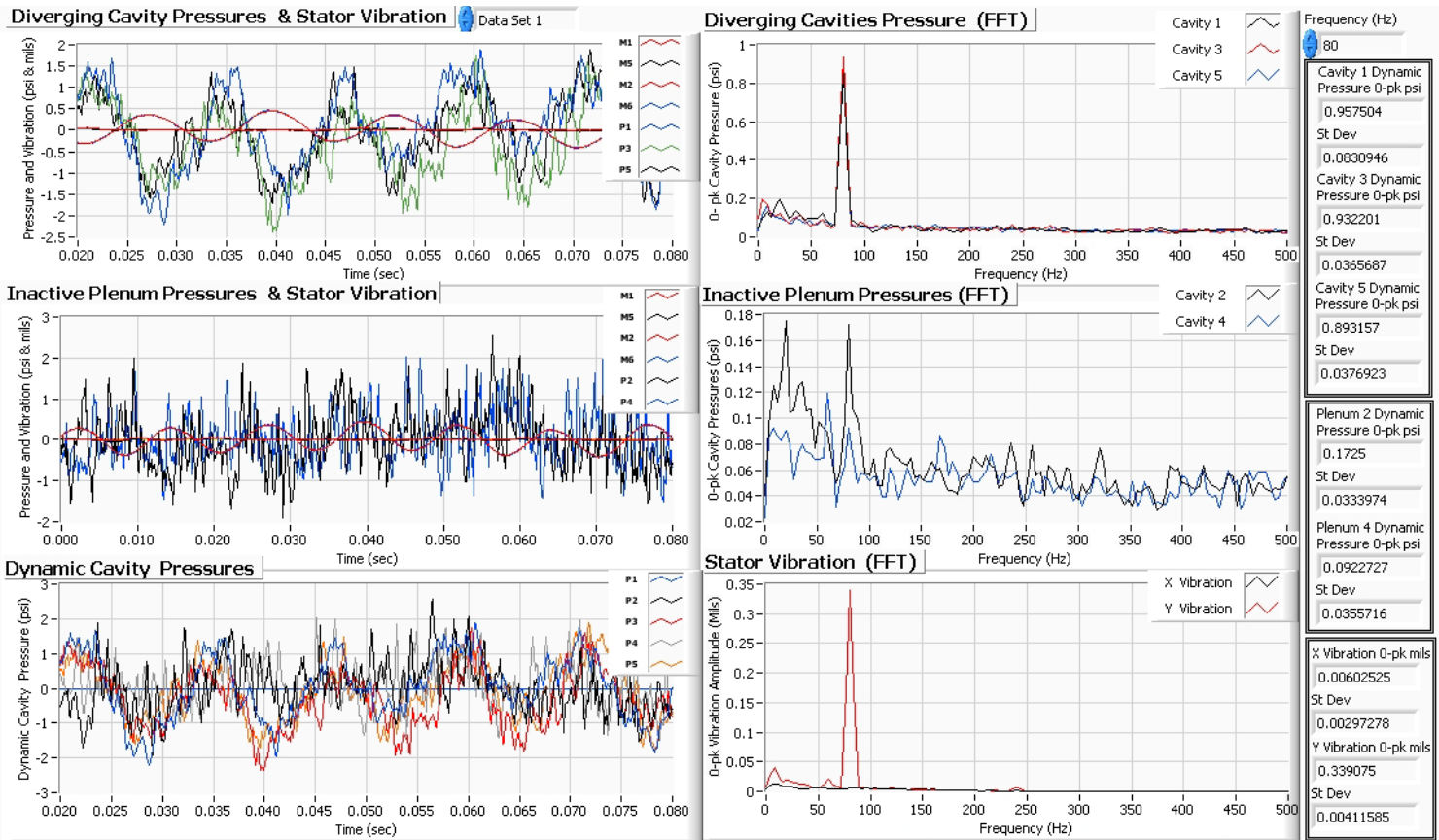

$80 \mathrm{~Hz} 90$ Degree Position Y Direction Excitation 

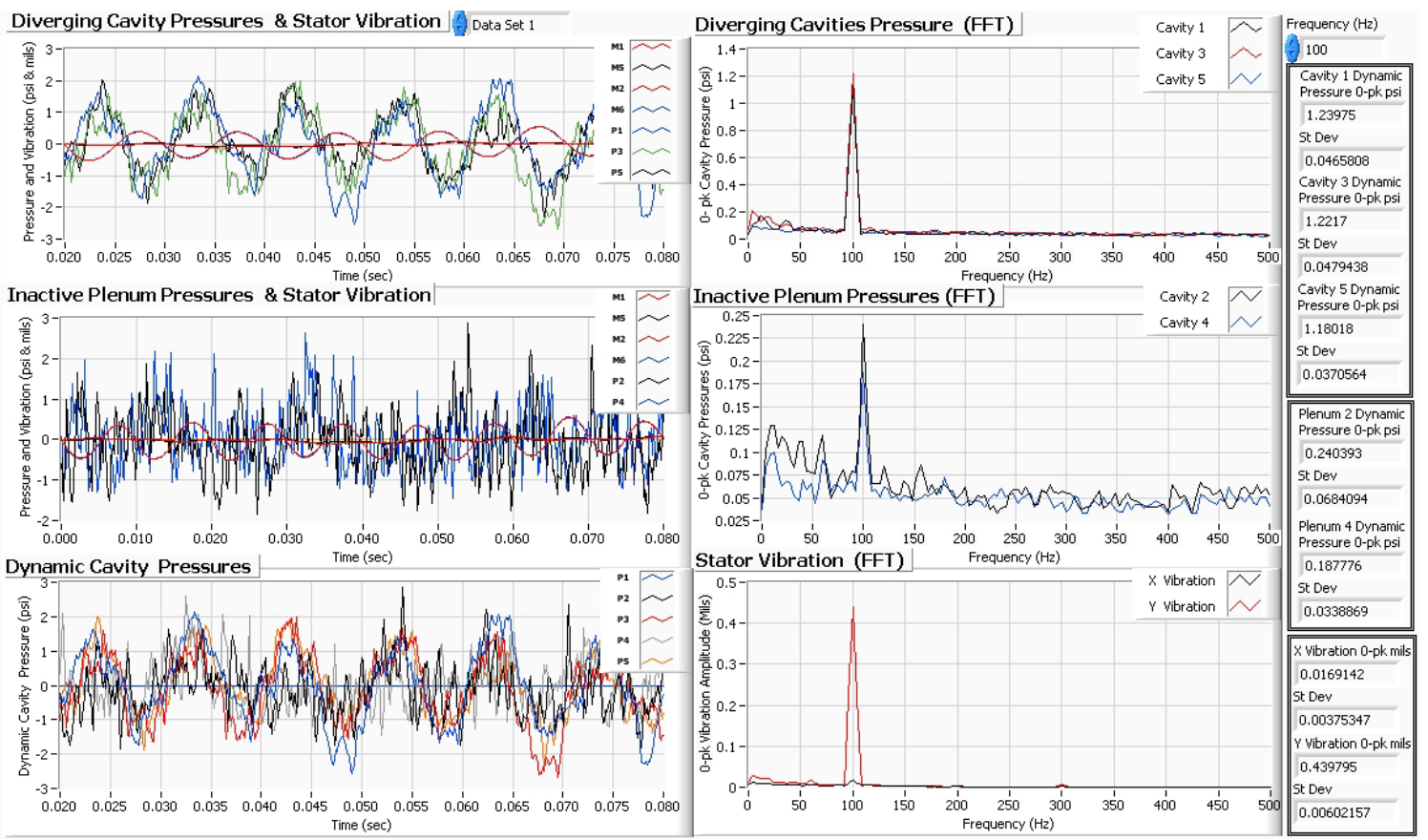

100 Hz 90 Degree Position Y Direction Excitation
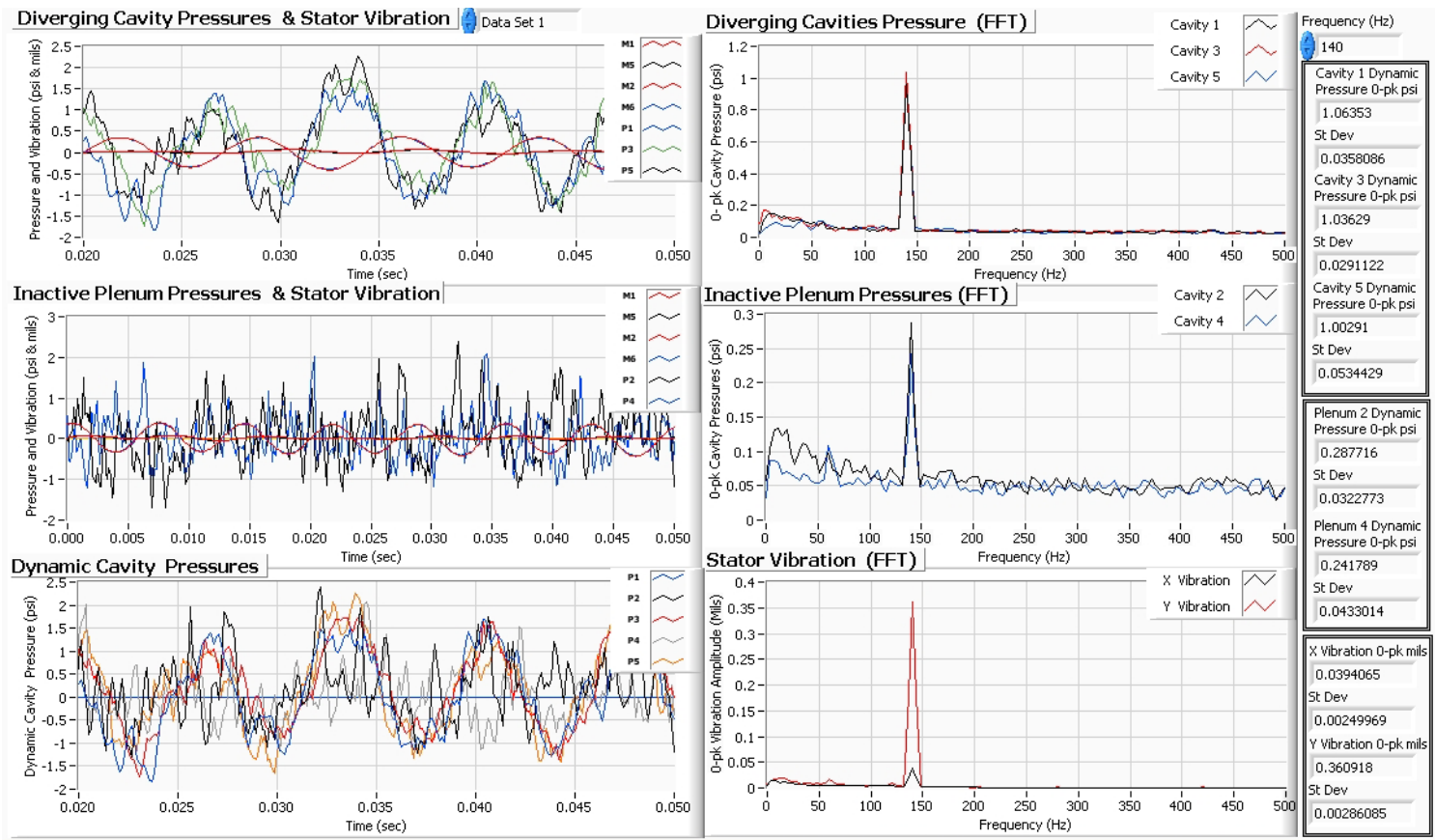

$140 \mathrm{~Hz} 90$ Degree Position Y Direction Excitation 

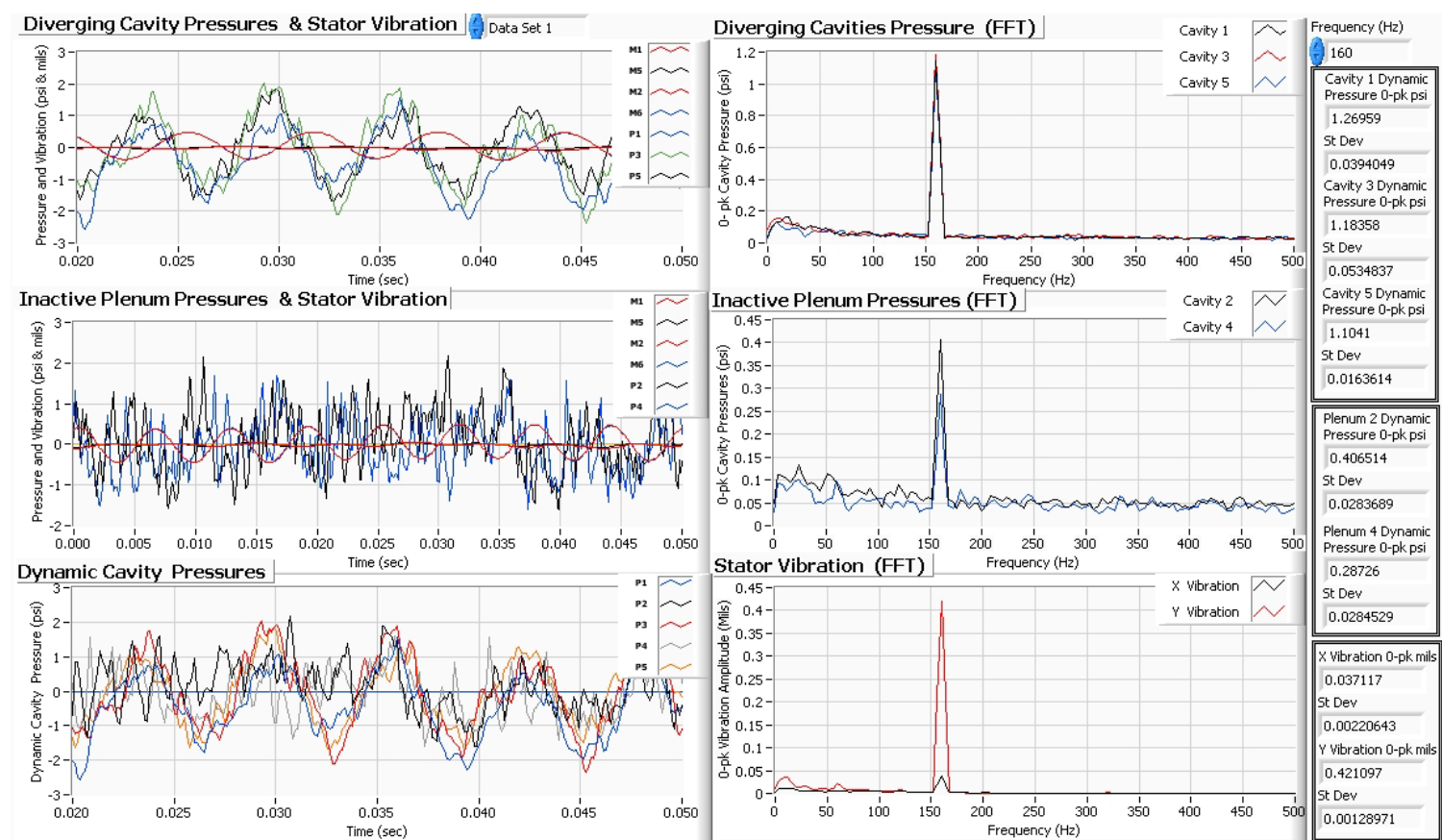

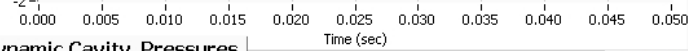

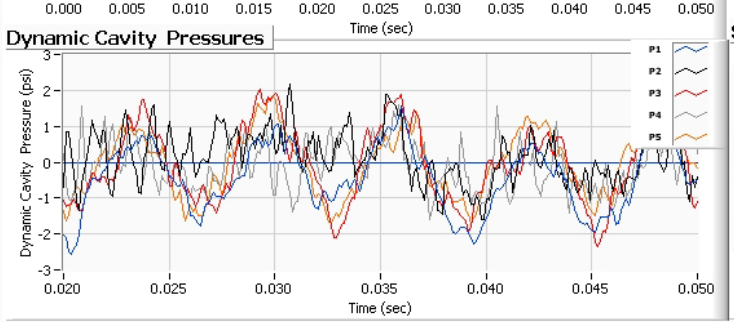

$160 \mathrm{~Hz} 90$ Degree Position Y Direction Excitation
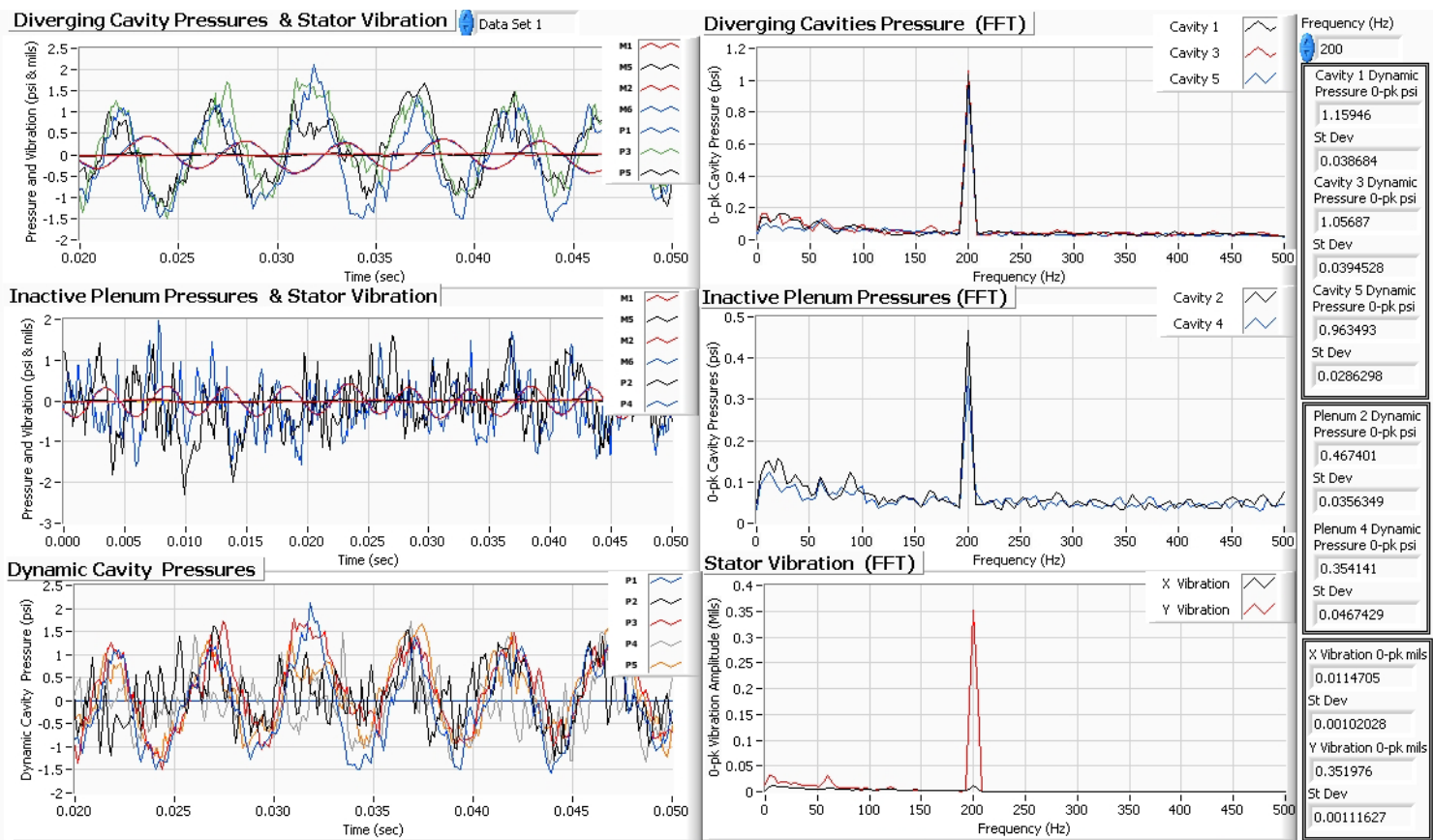

$200 \mathrm{~Hz} 90$ Degree Position Y Direction Excitation 
Dynamic Cavity Pressure Tests

6 Bladed Fully Partitioned PDS

\section{DEGREE POSITION}



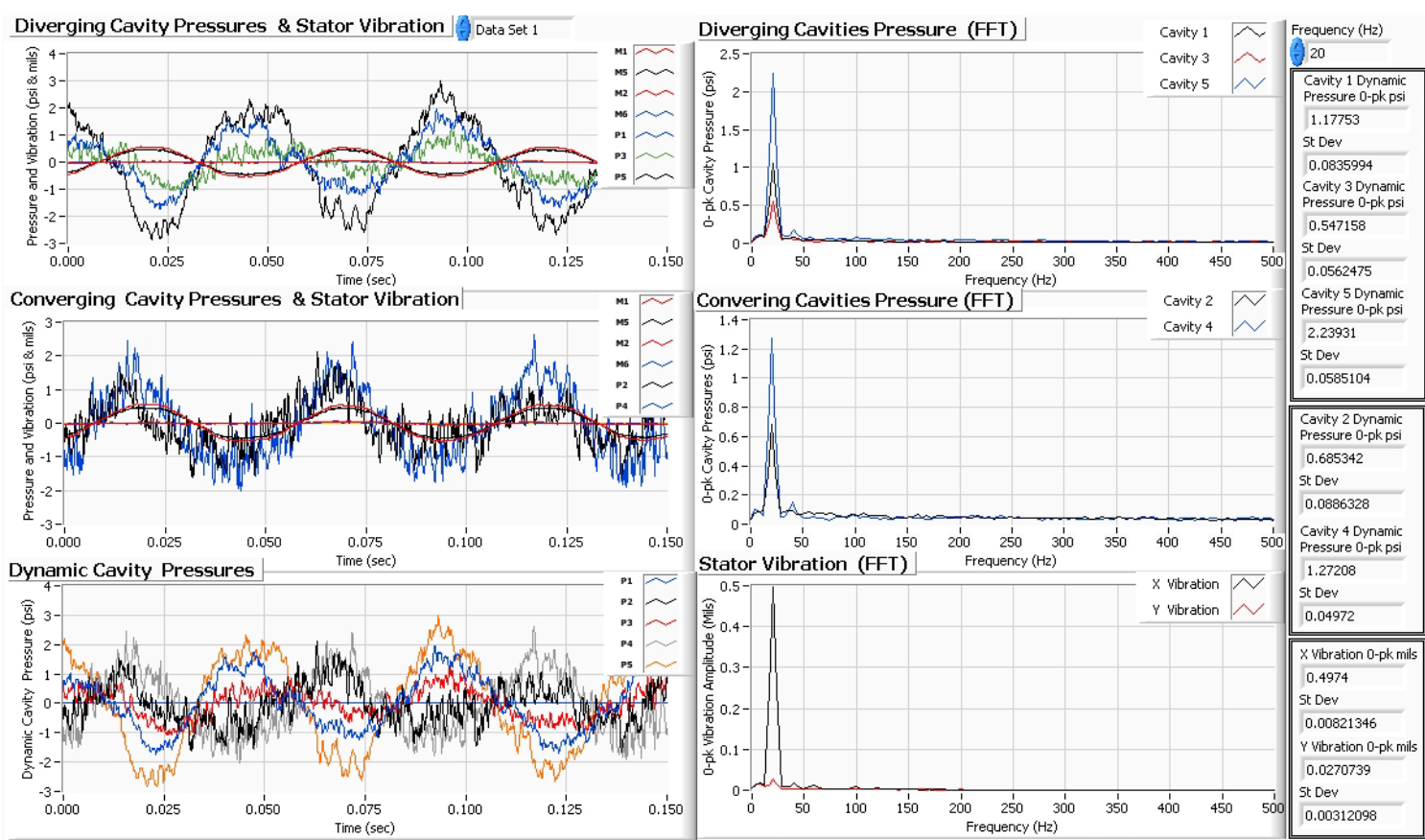

$20 \mathrm{~Hz} 0$ Degree Position X Direction Excitation
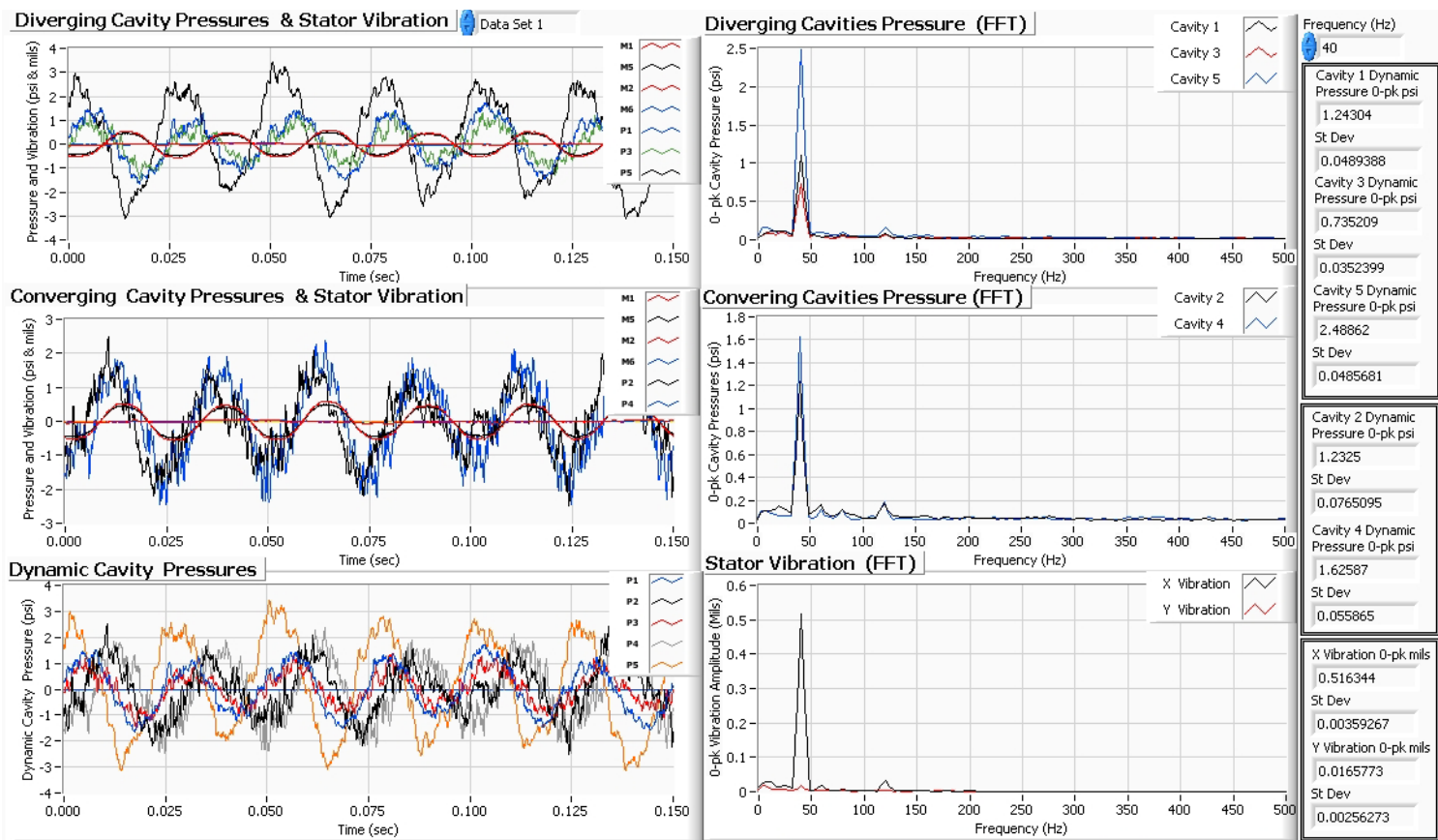

$40 \mathrm{~Hz} 0$ Degree Position X Direction Excitation 

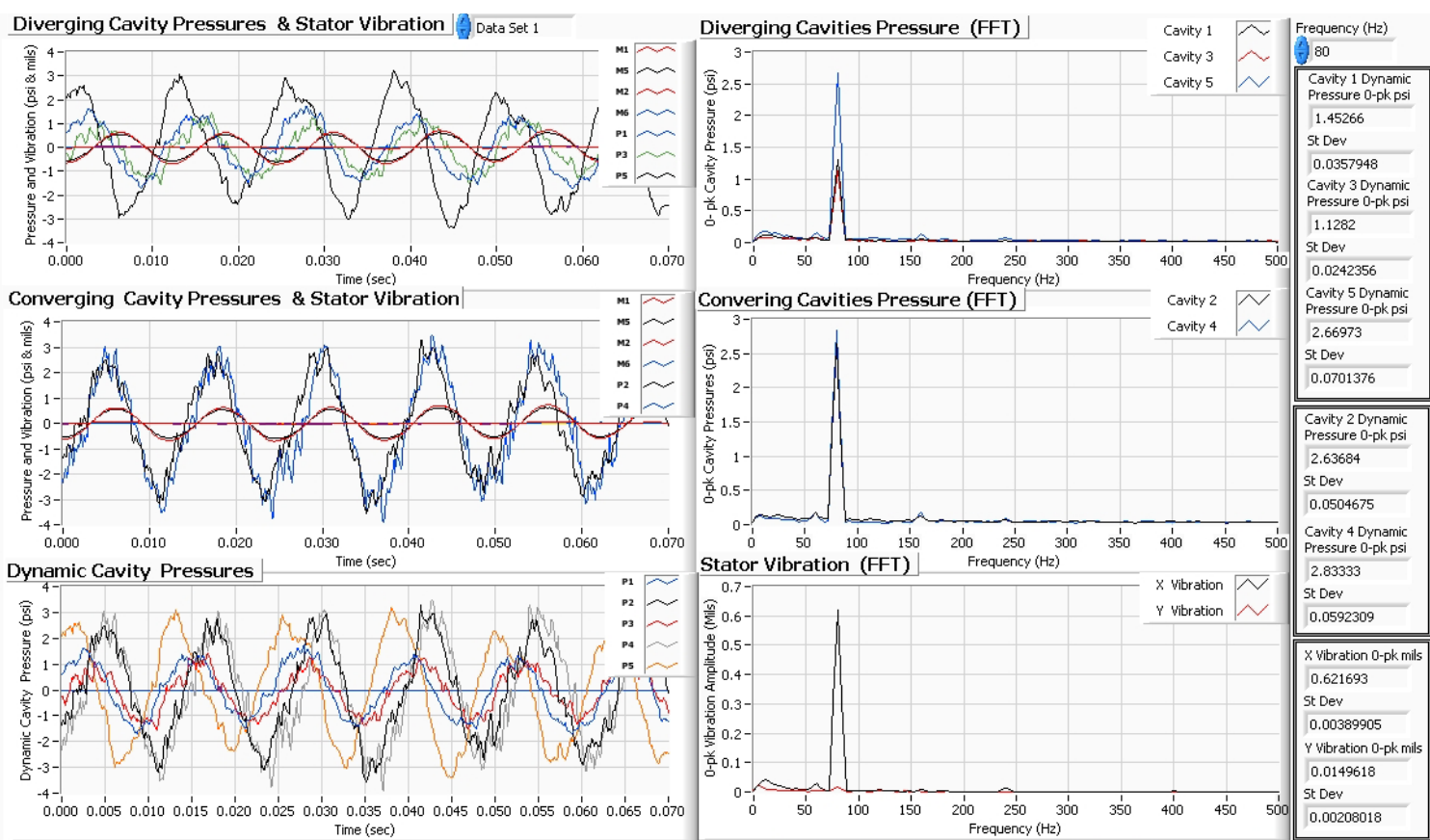

$80 \mathrm{~Hz} 0$ Degree Position X Direction Excitation
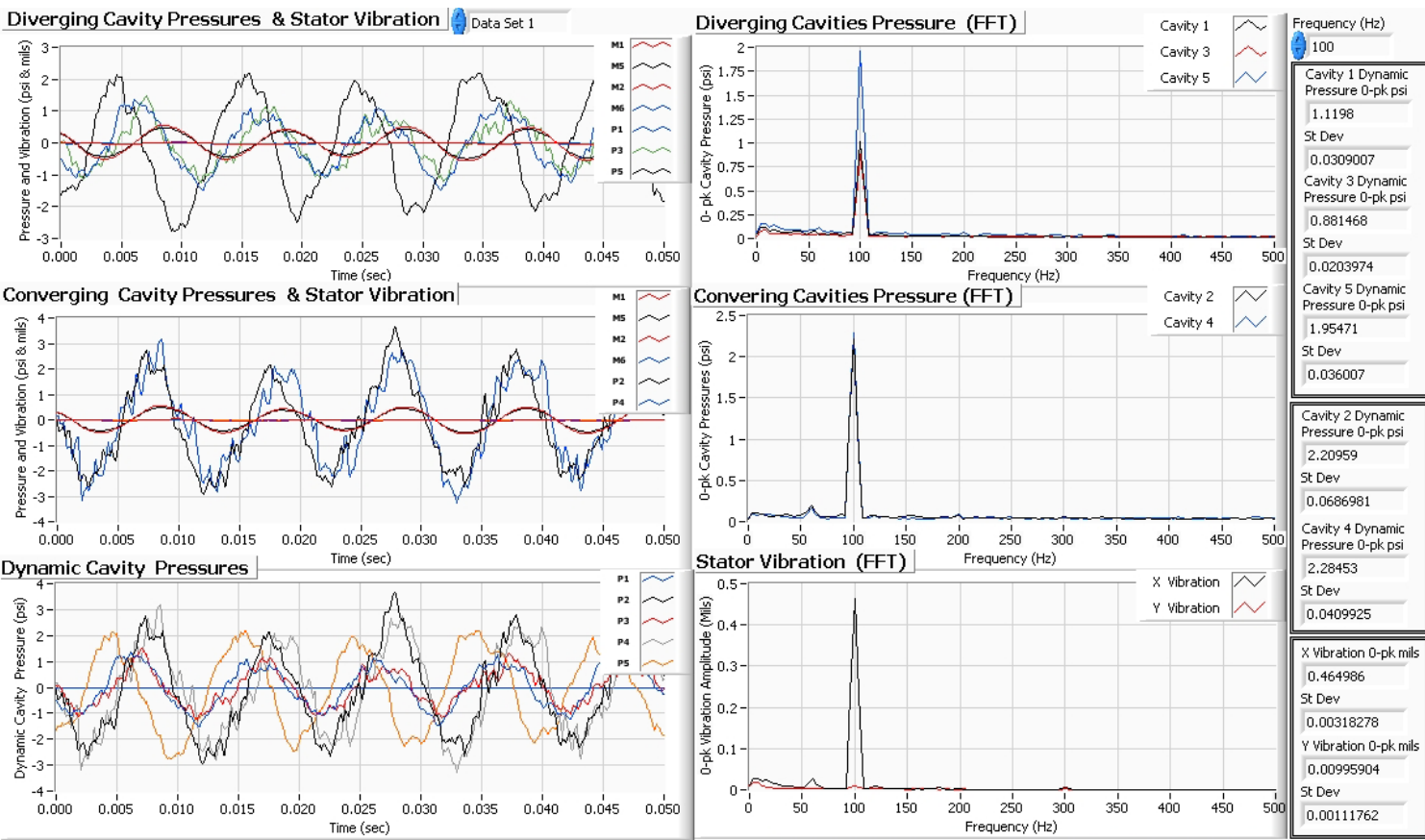

$100 \mathrm{~Hz} 0$ Degree Position X Direction Excitation 


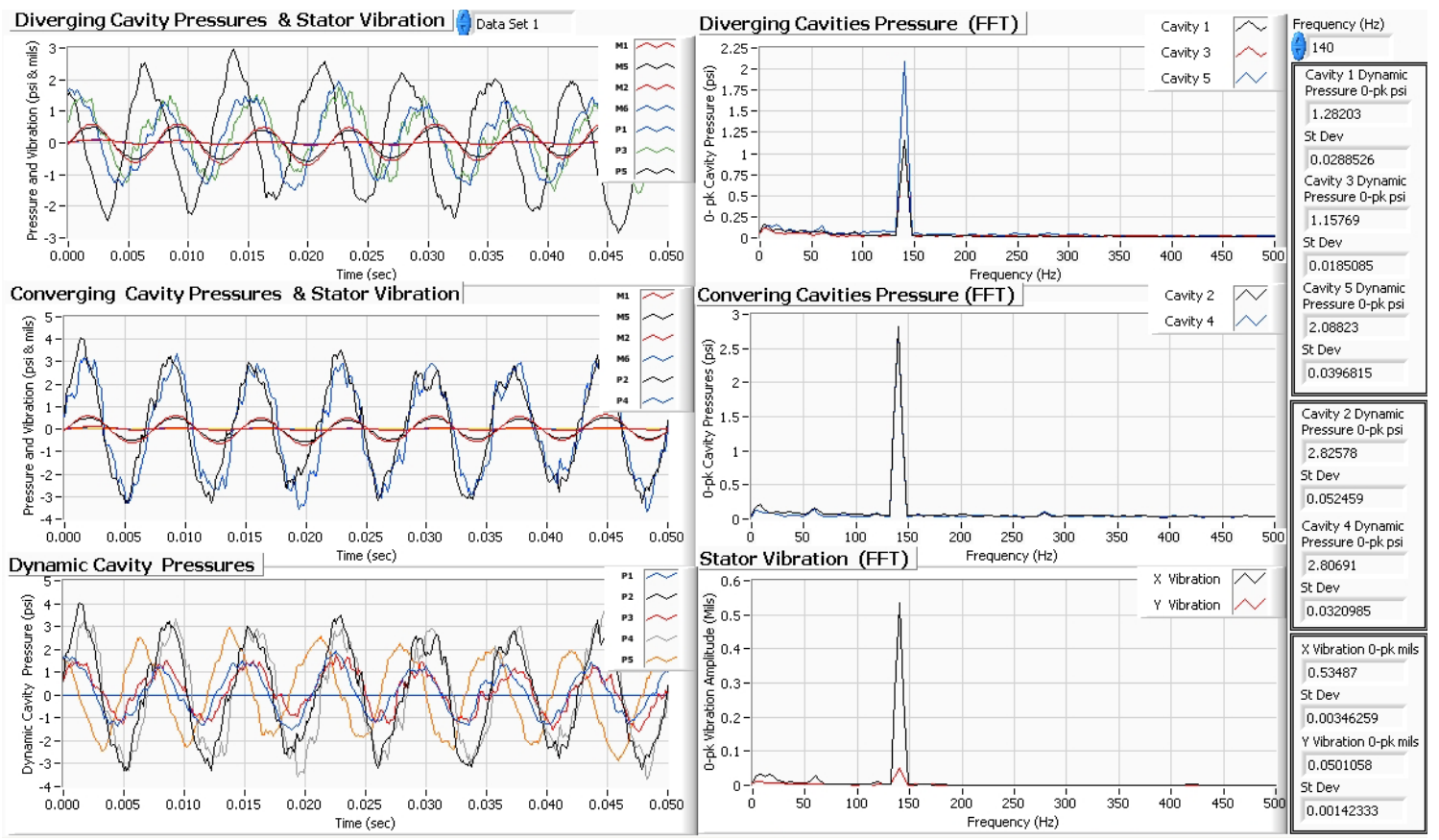

$140 \mathrm{~Hz} 0$ Degree Position X Direction Excitation
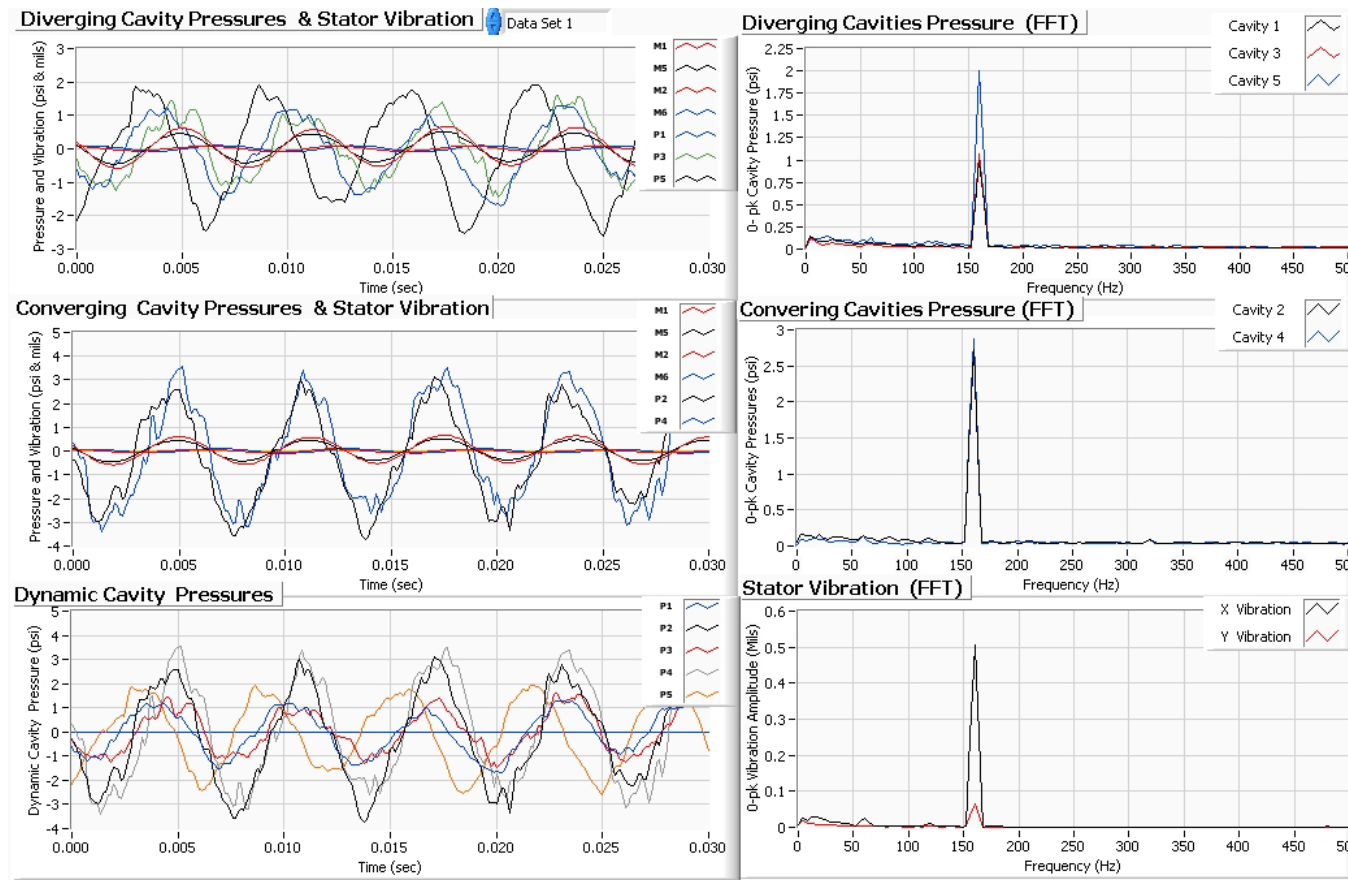

$160 \mathrm{~Hz} 0$ Degree Position X Direction Excitation

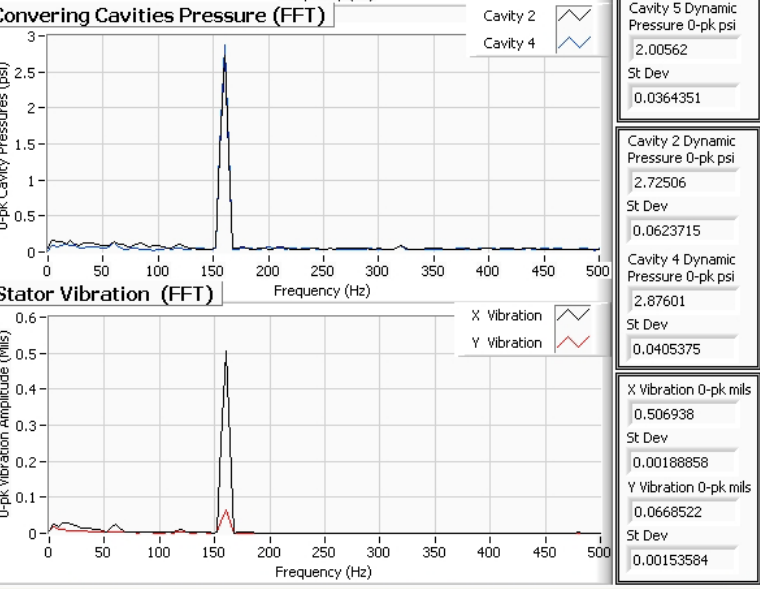



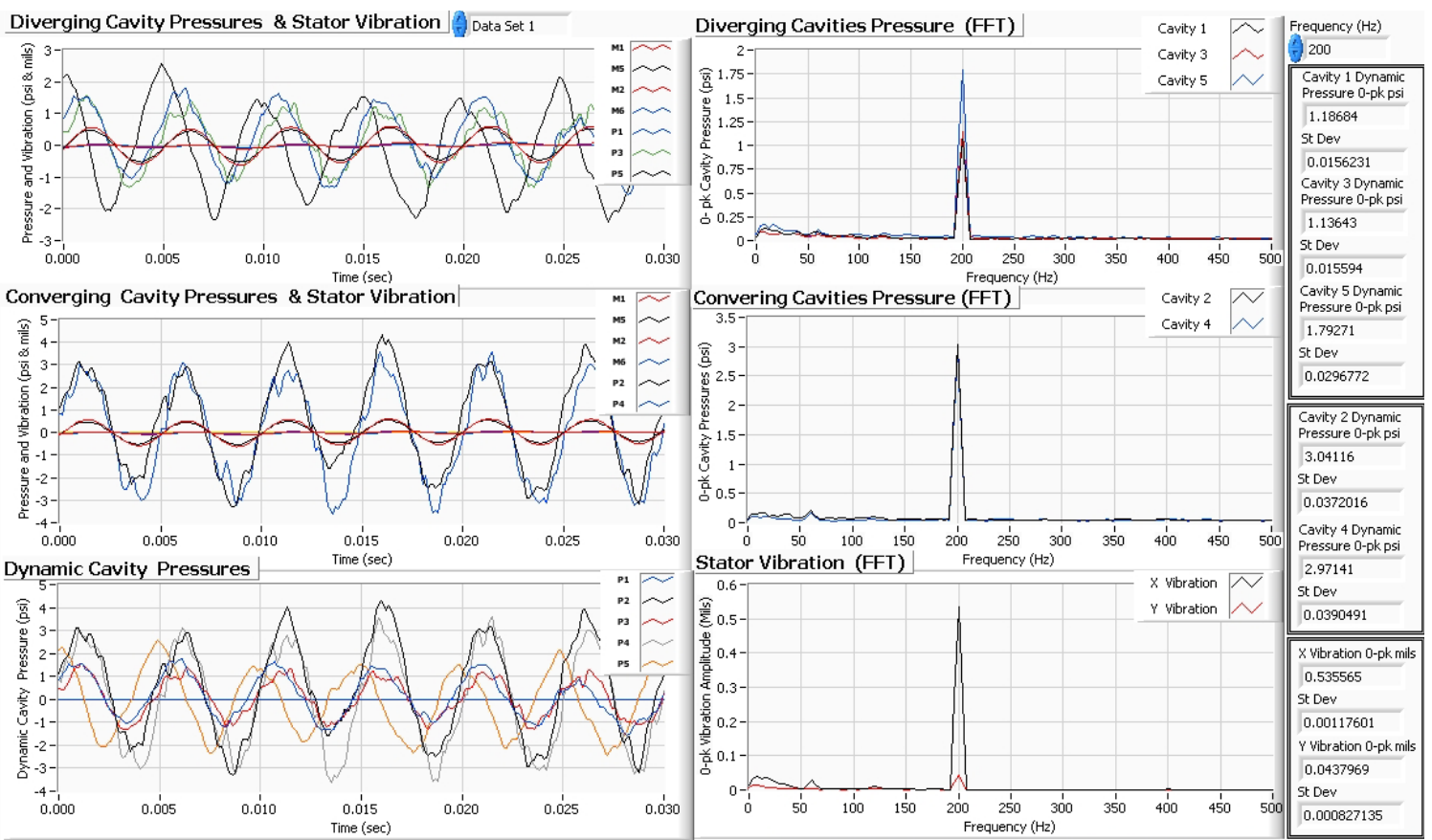

$200 \mathrm{~Hz} 0$ Degree Position X Direction Excitation
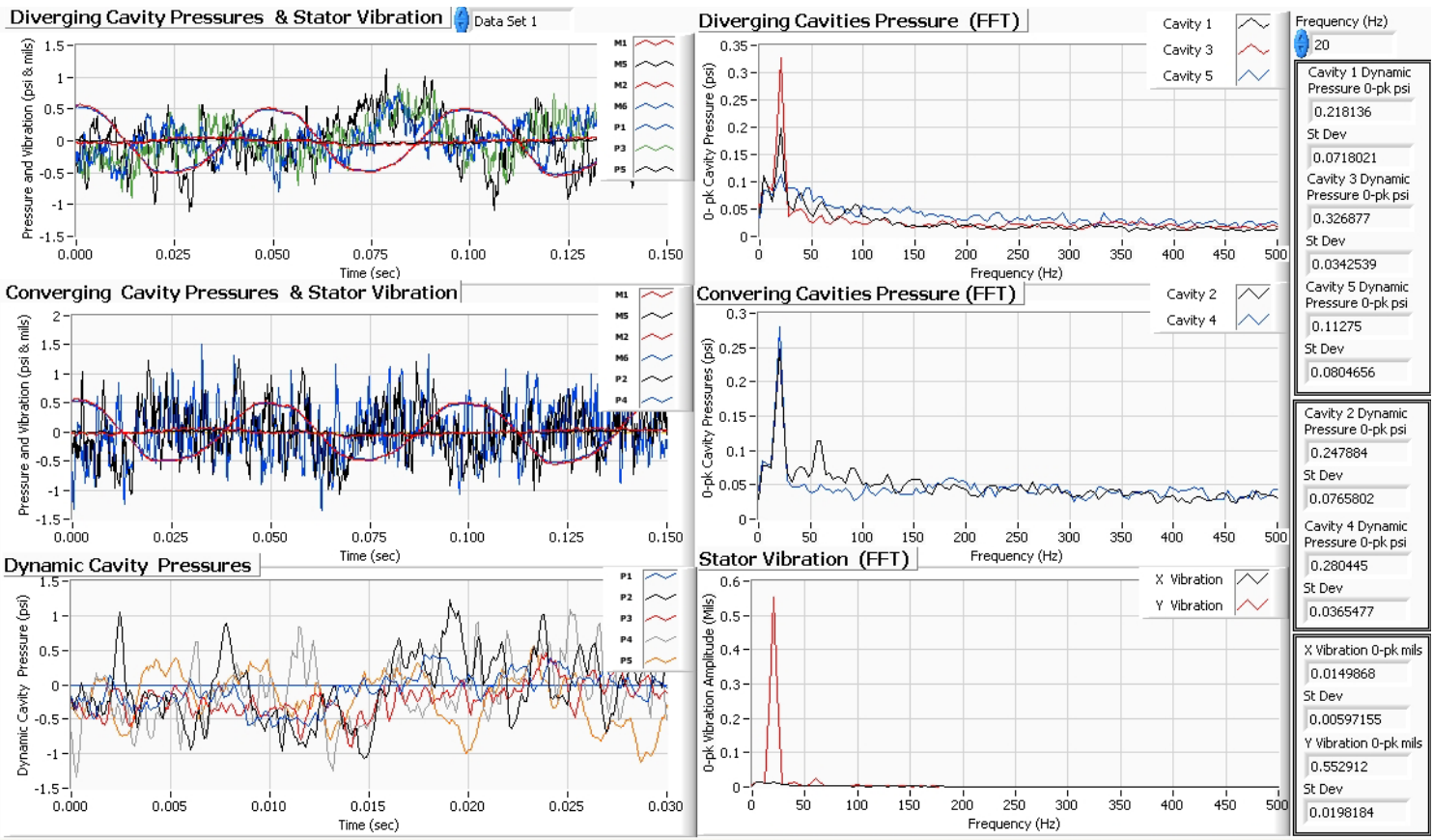

$20 \mathrm{~Hz} 0$ Degree Position Y Direction Excitation 


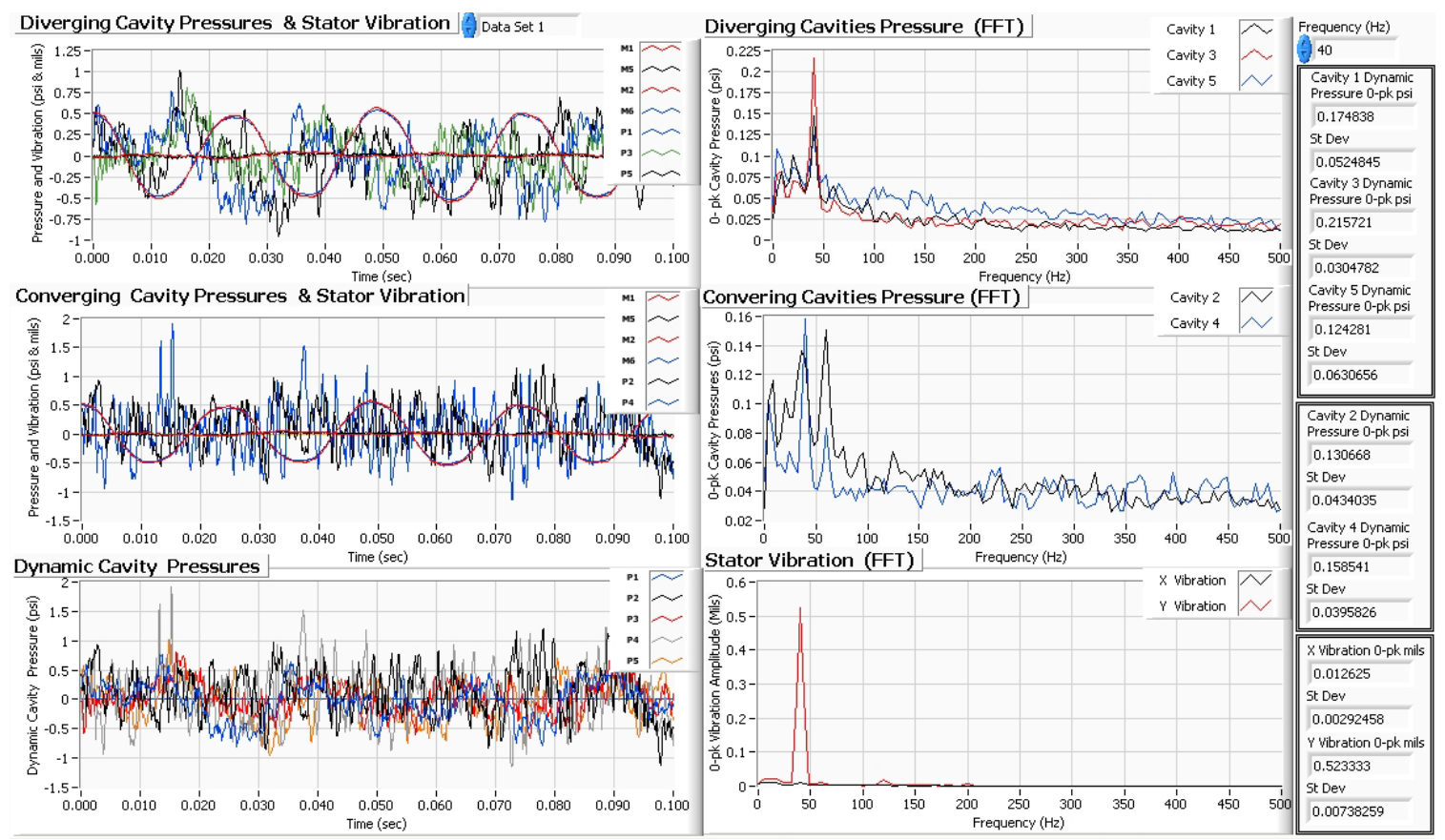

$40 \mathrm{~Hz} 0$ Degree Position Y Direction Excitation
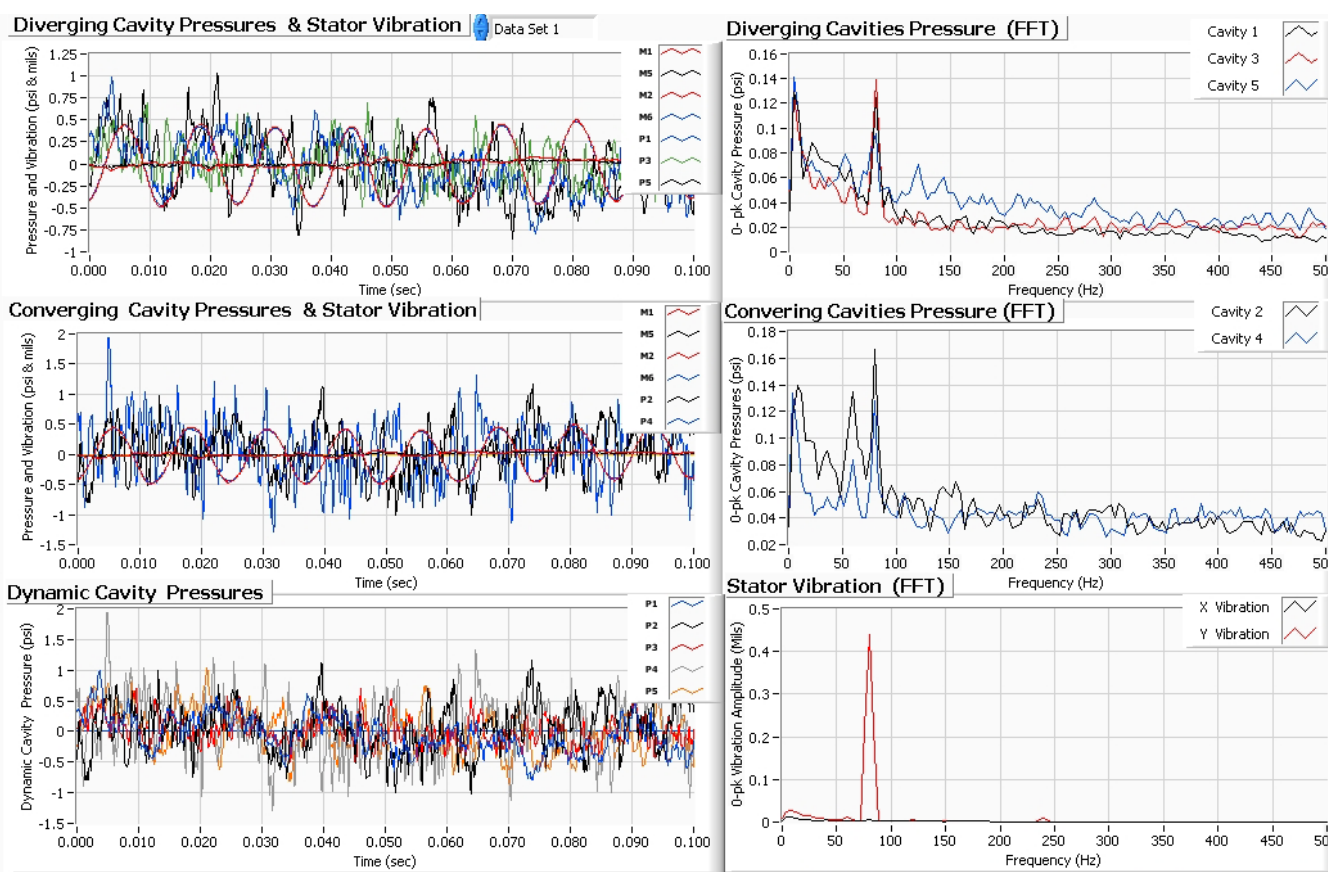

80 Hz 0 Degree Position Y Direction Excitation

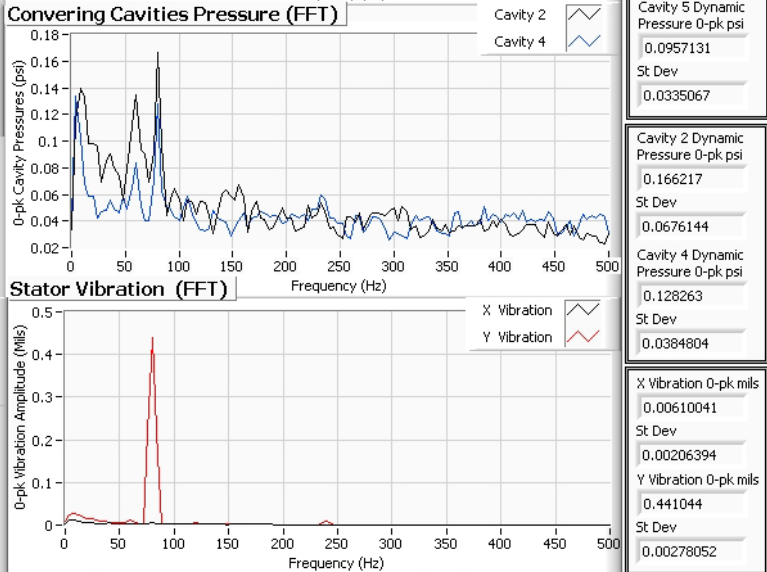




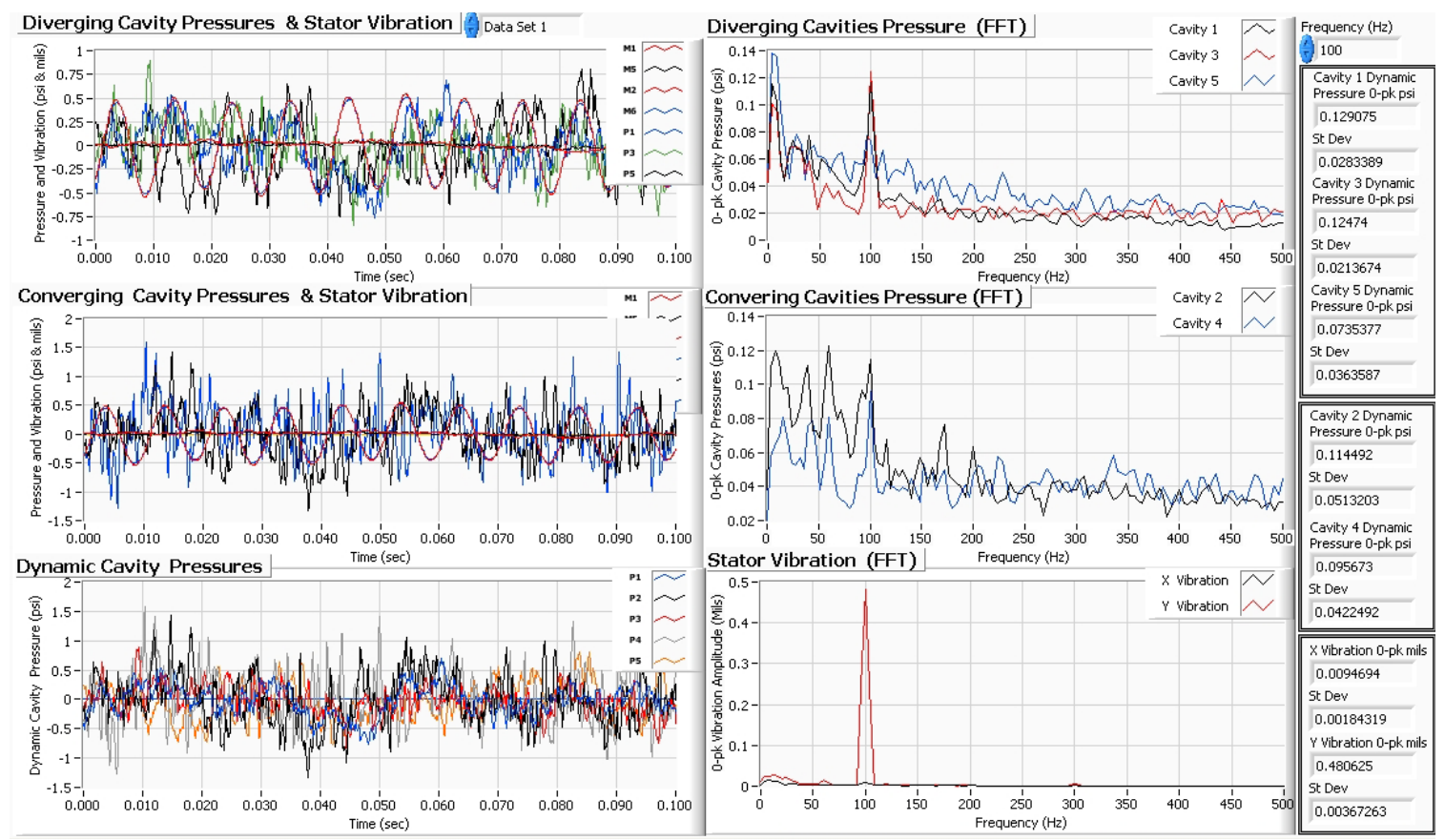

$100 \mathrm{~Hz} 0$ Degree Position Y Direction Excitation
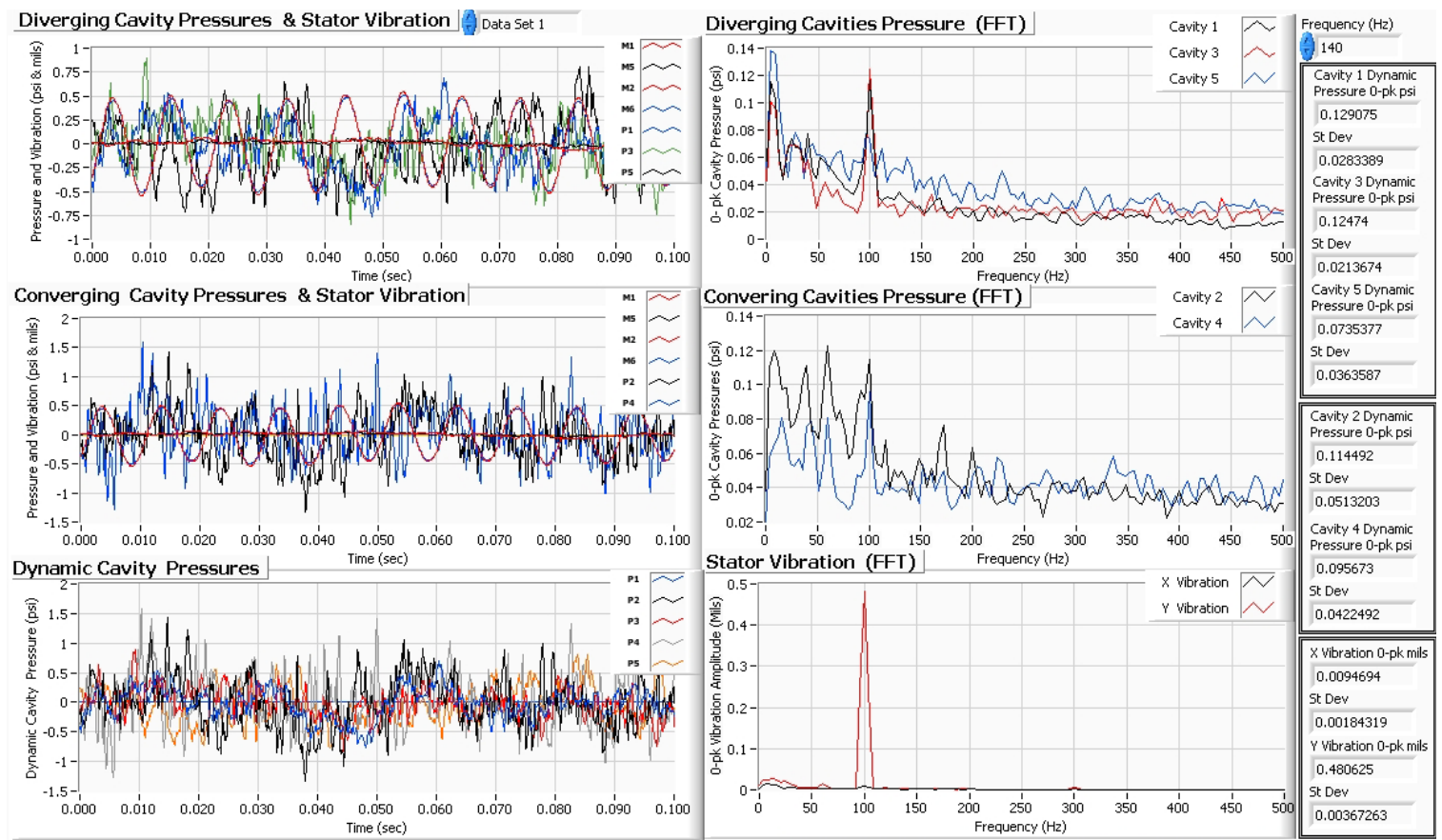

$140 \mathrm{~Hz} 0$ Degree Position Y Direction Excitation 

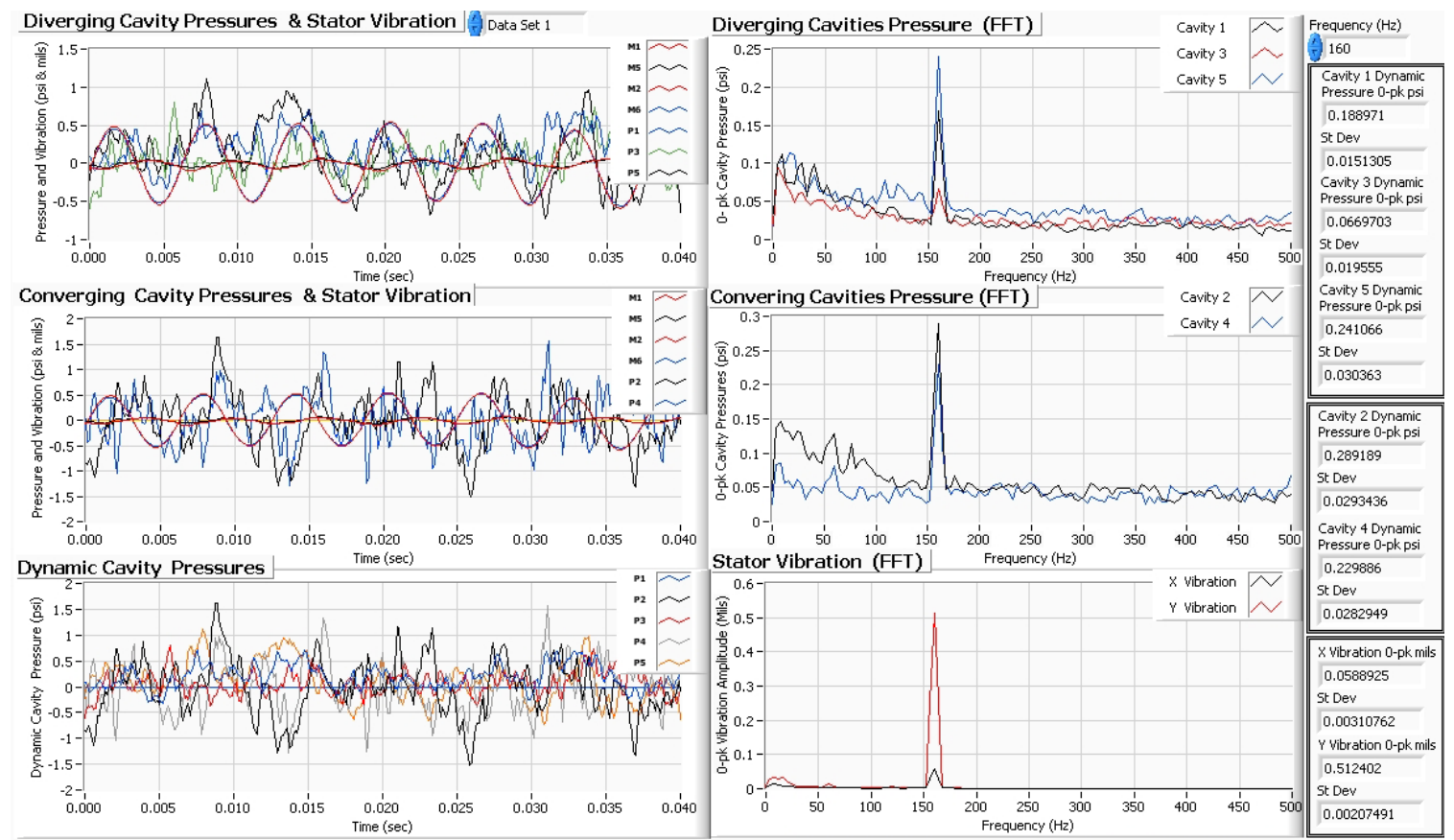

$160 \mathrm{~Hz} 0$ Degree Position Y Direction Excitation
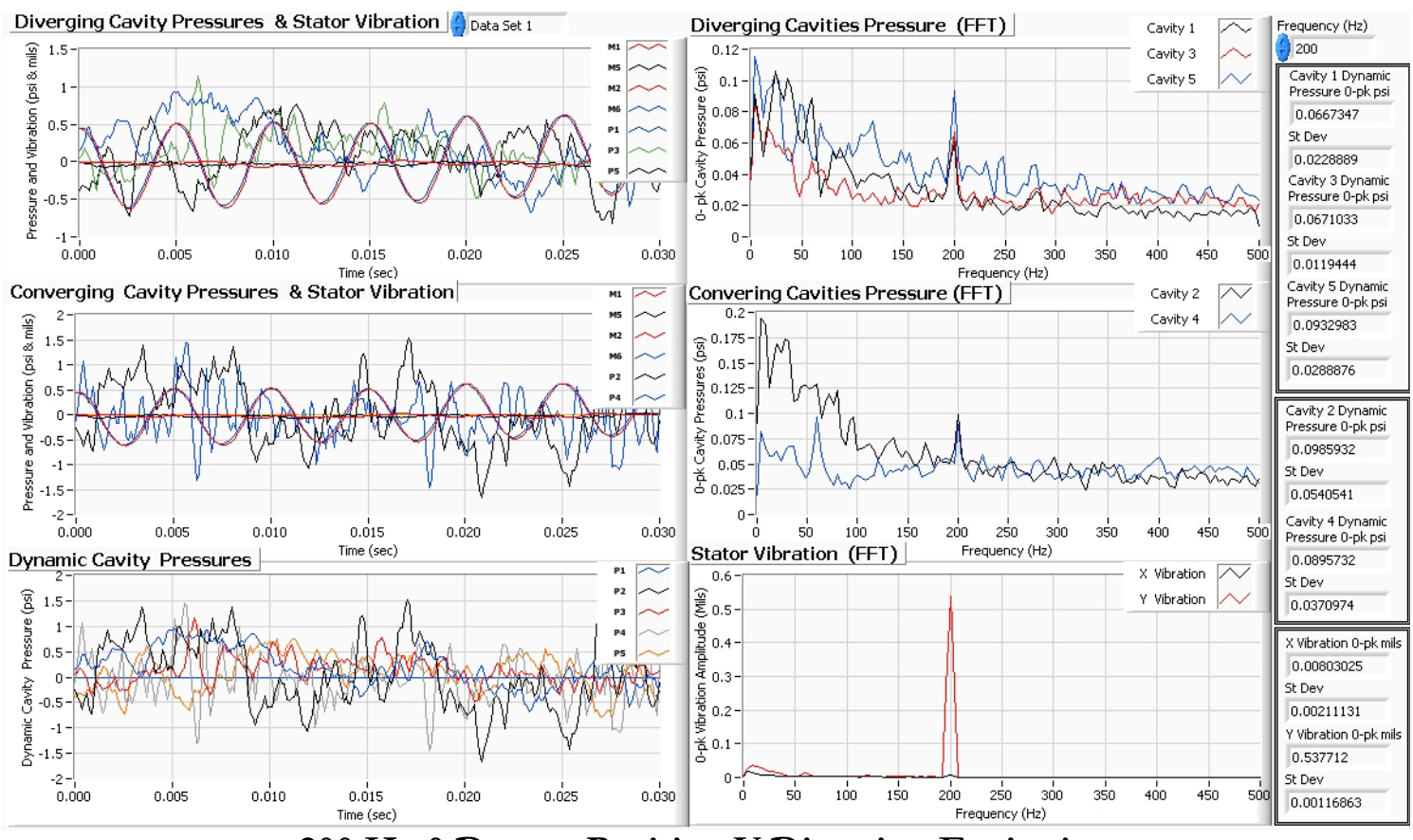

$200 \mathrm{~Hz} 0$ Degree Position Y Direction Excitation 
Dynamic Cavity Pressure Tests

6 Bladed Fully Partitioned PDS

45 DEGREE POSITION 

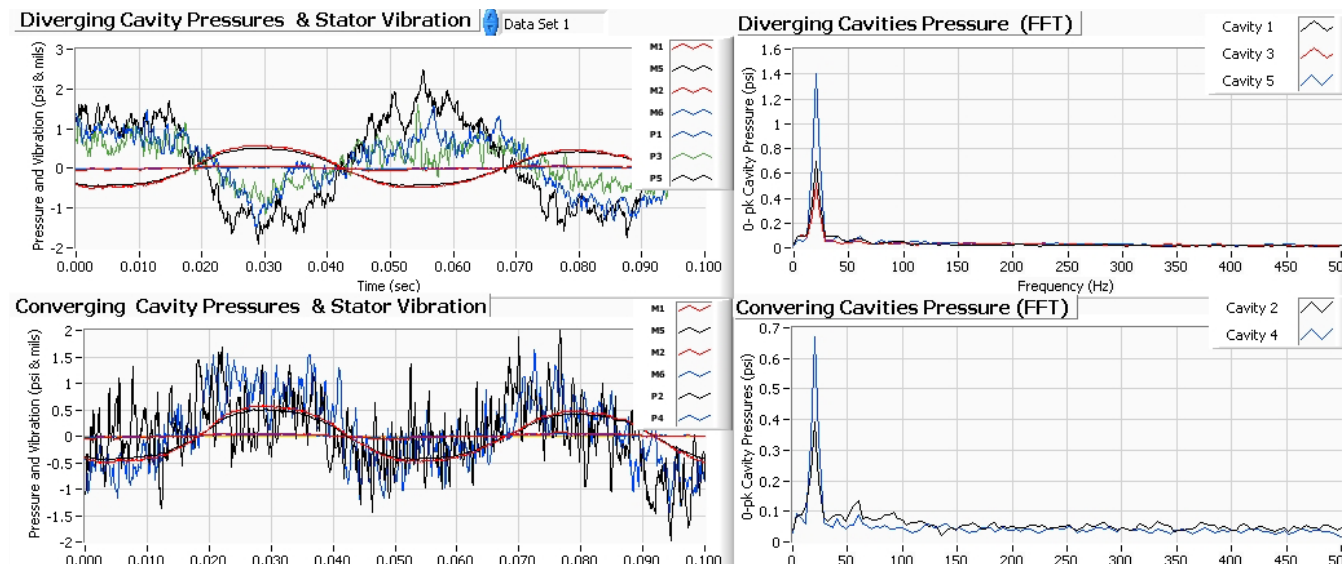

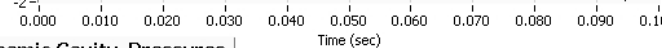

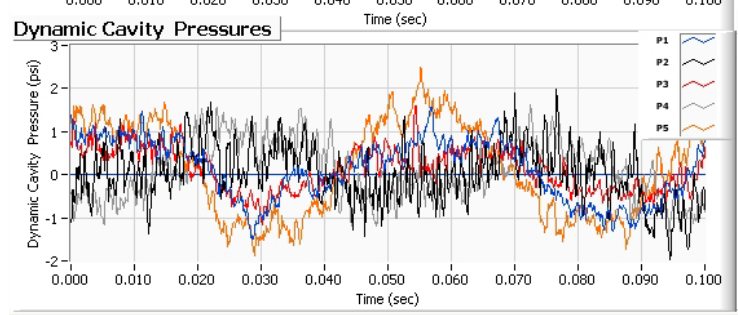

20 Hz 45 Degree Position X Direction Excitation
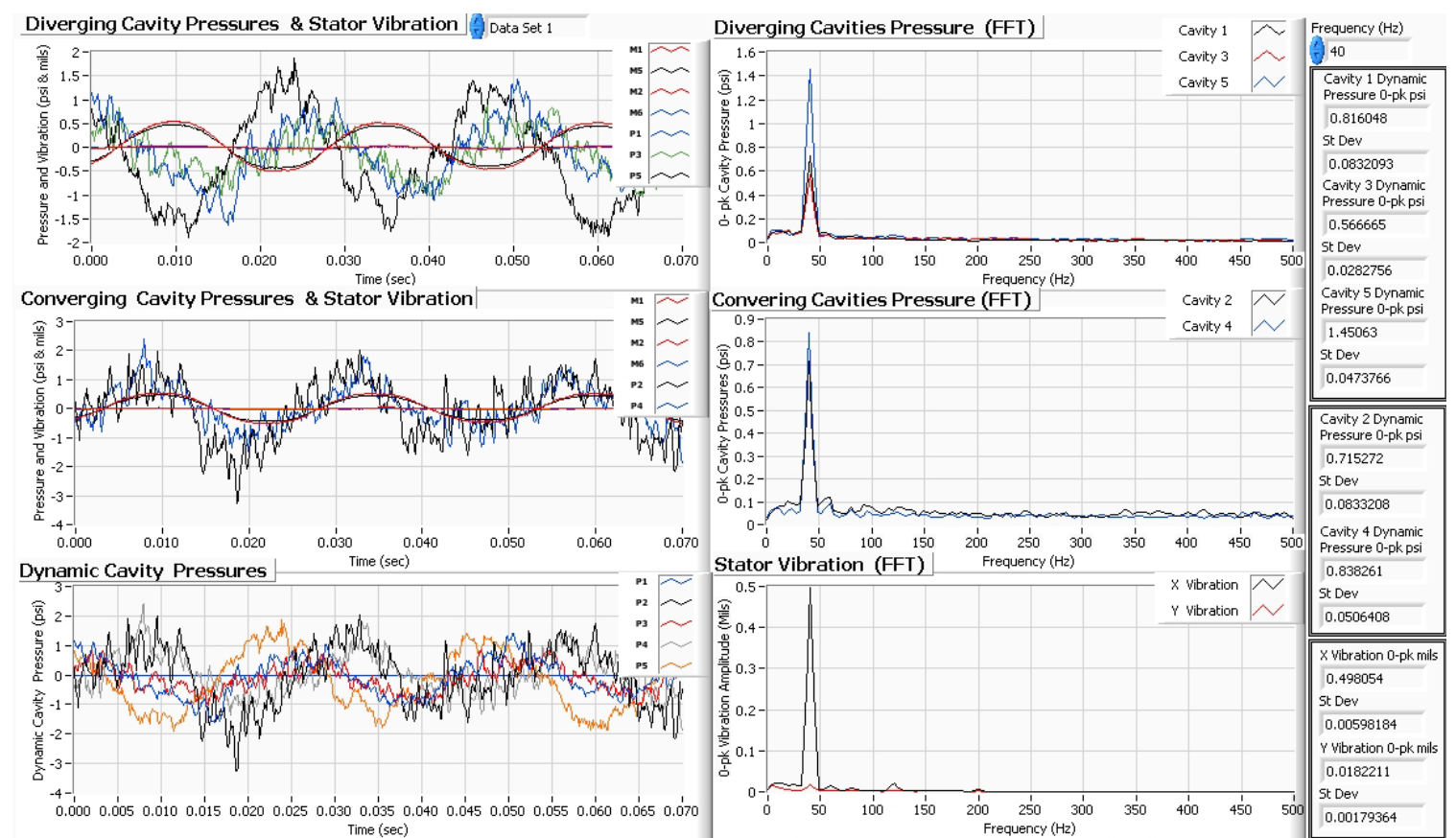

$40 \mathrm{~Hz} 45$ Degree Position X Direction Excitation 

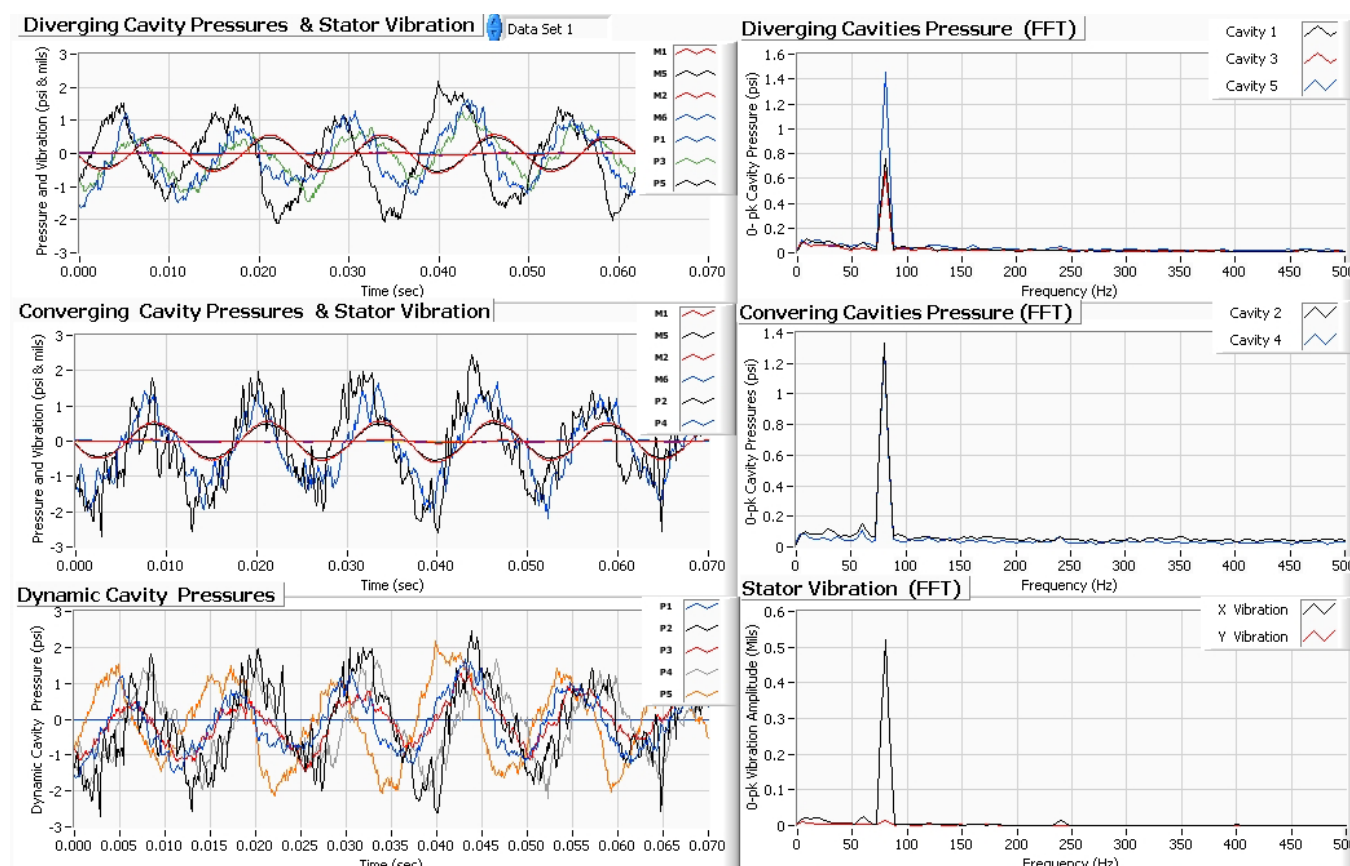

$80 \mathrm{~Hz} 45$ Degree Position X Direction Excitation
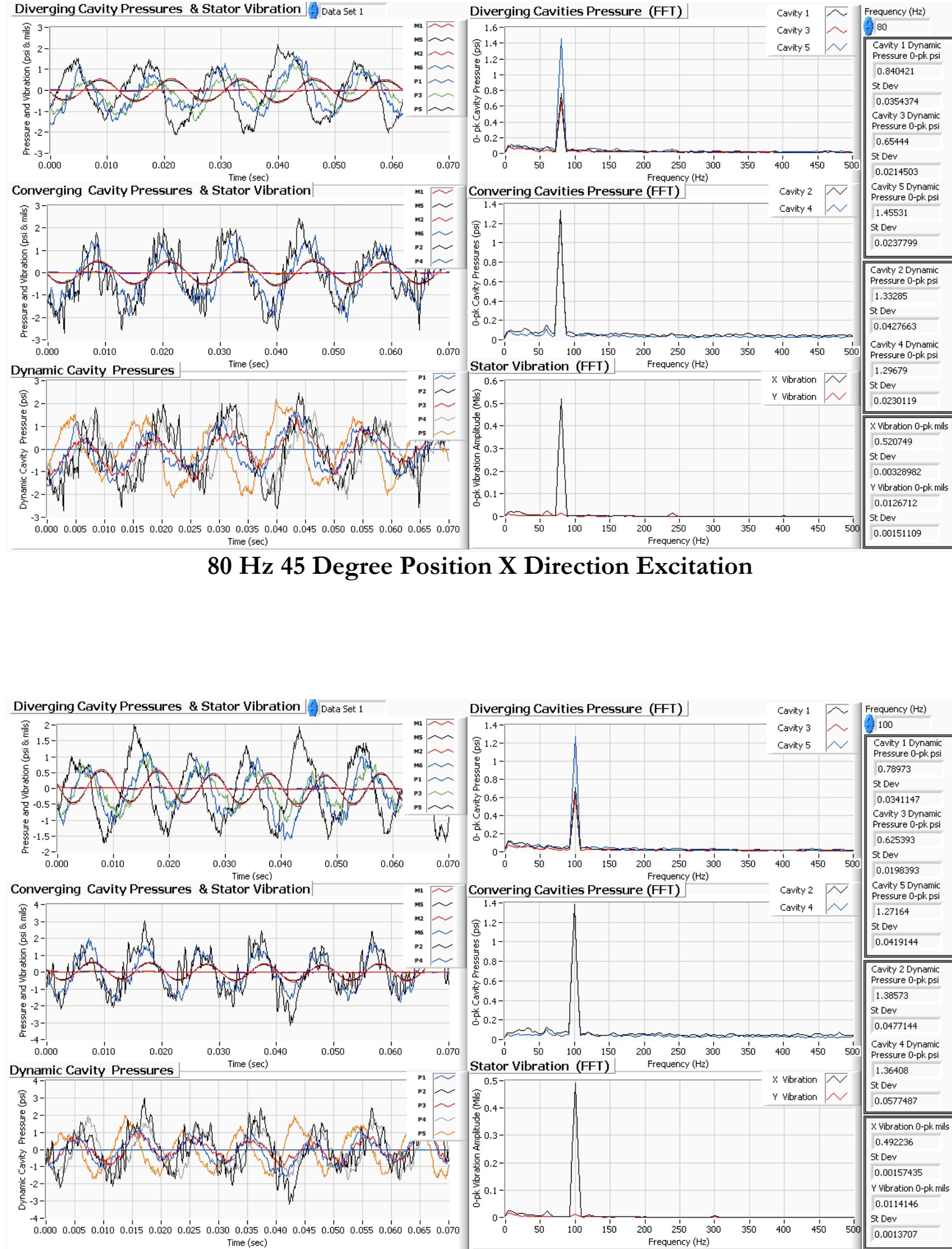

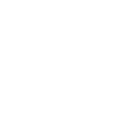



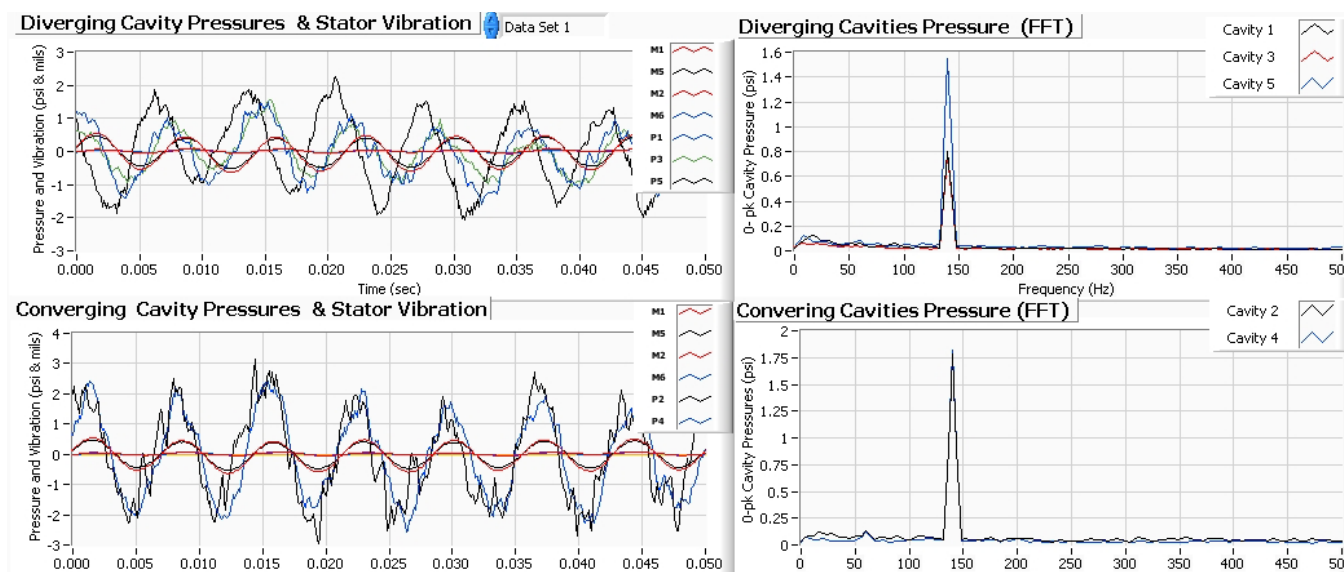

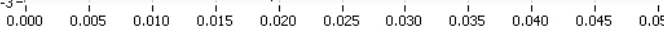
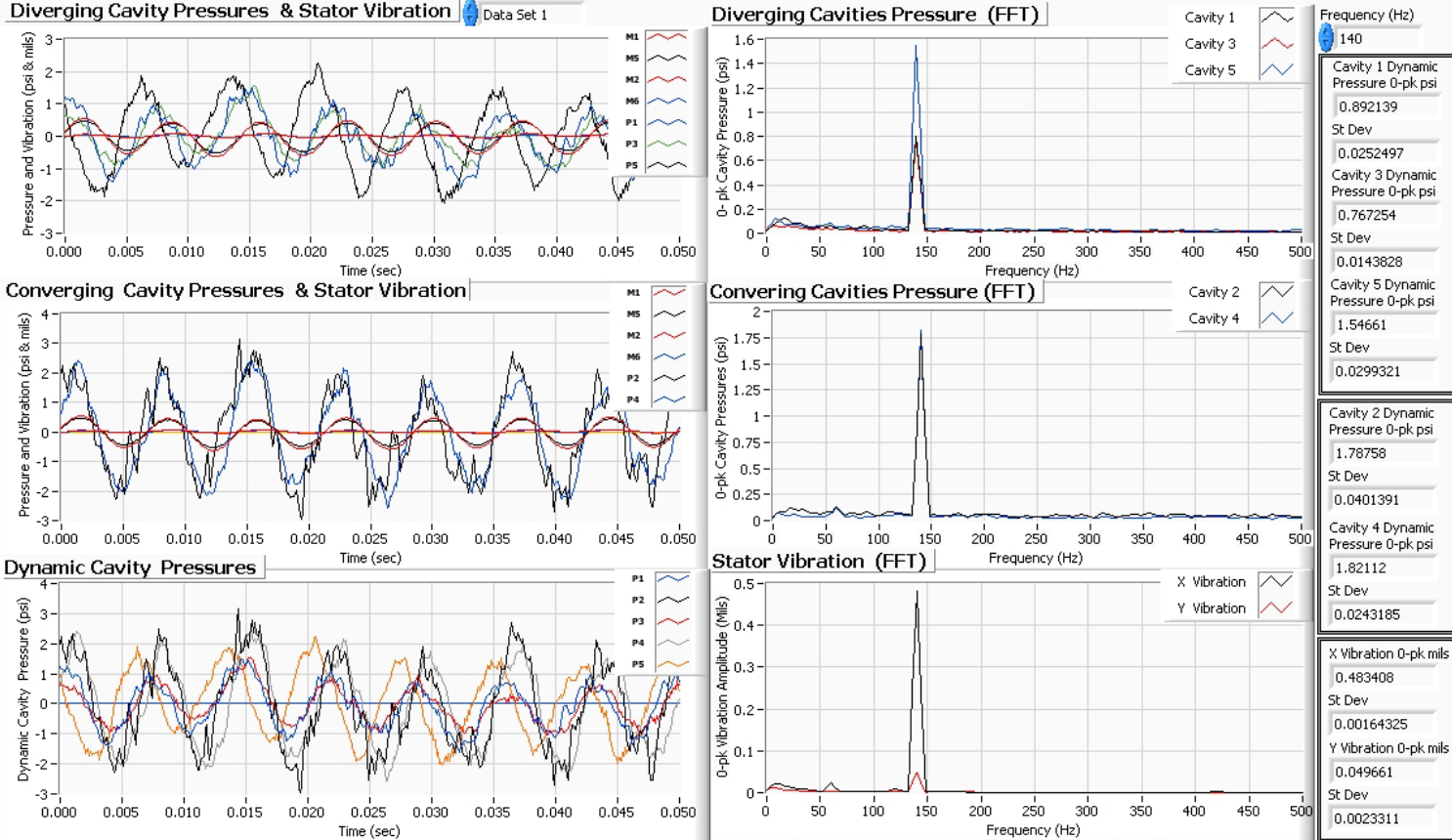

$140 \mathrm{~Hz} 45$ Degree Position X Direction Excitation
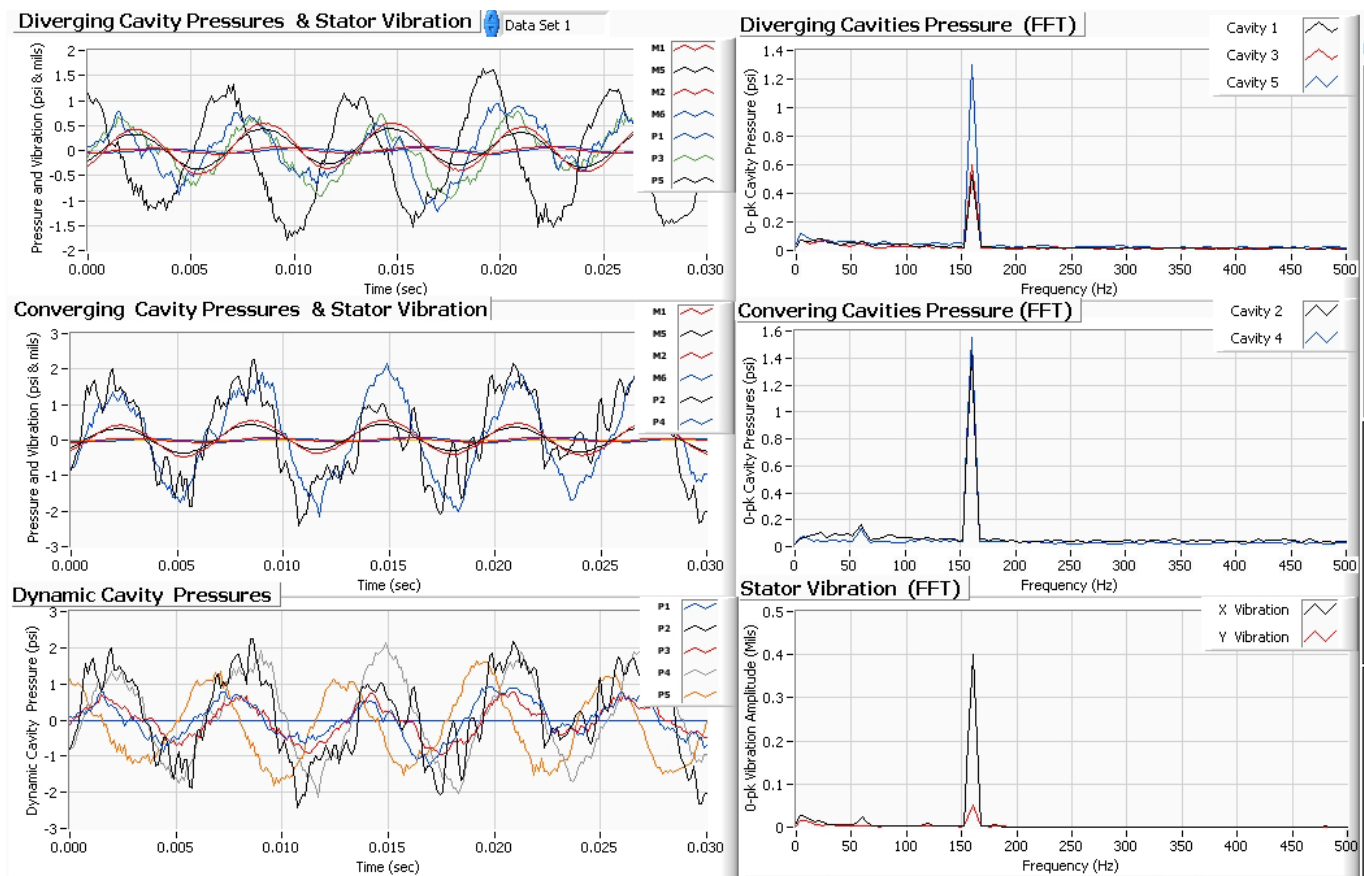

$160 \mathrm{~Hz} 45$ Degree Position X Direction Excitation 

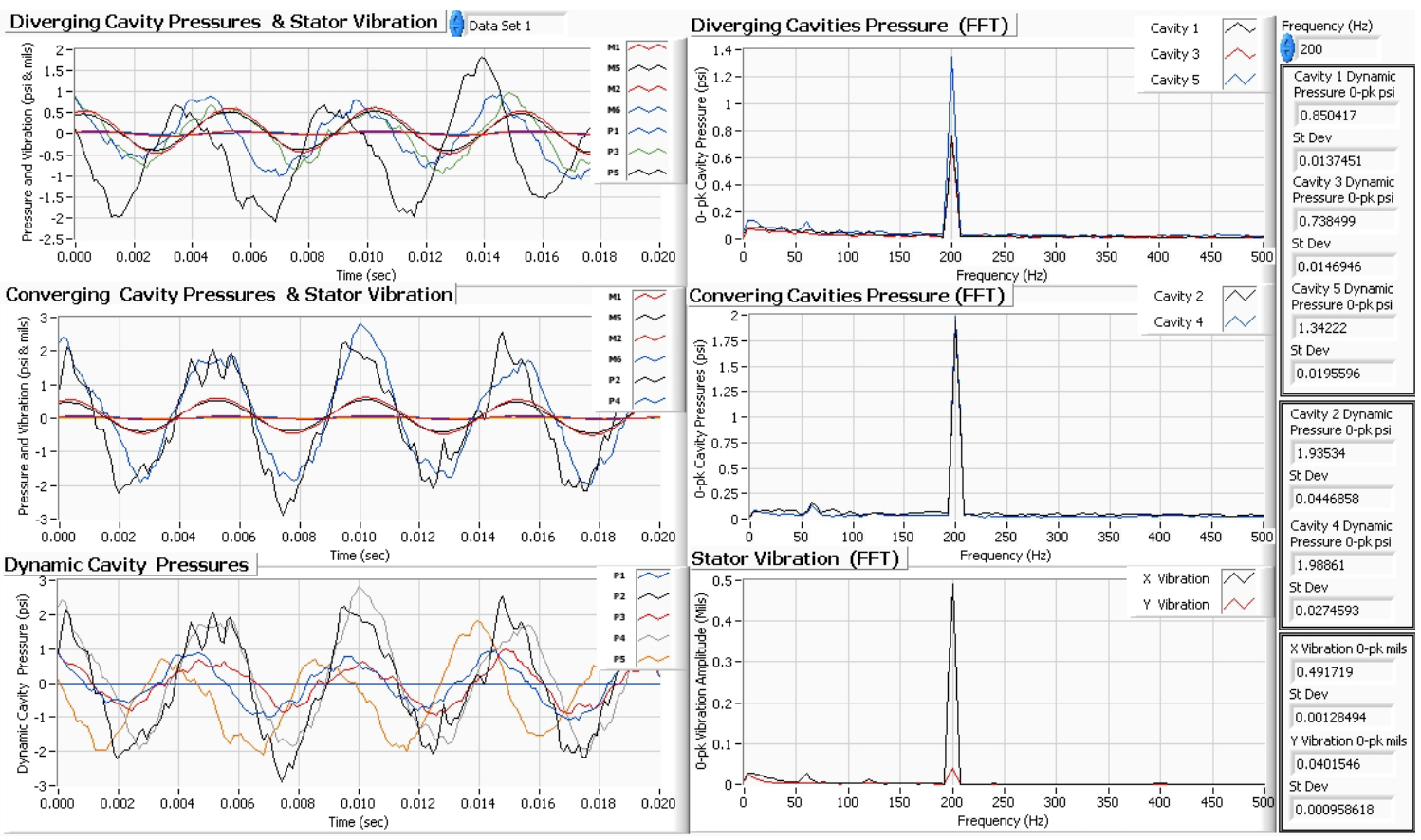

$200 \mathrm{~Hz} 45$ Degree Position X Direction Excitation
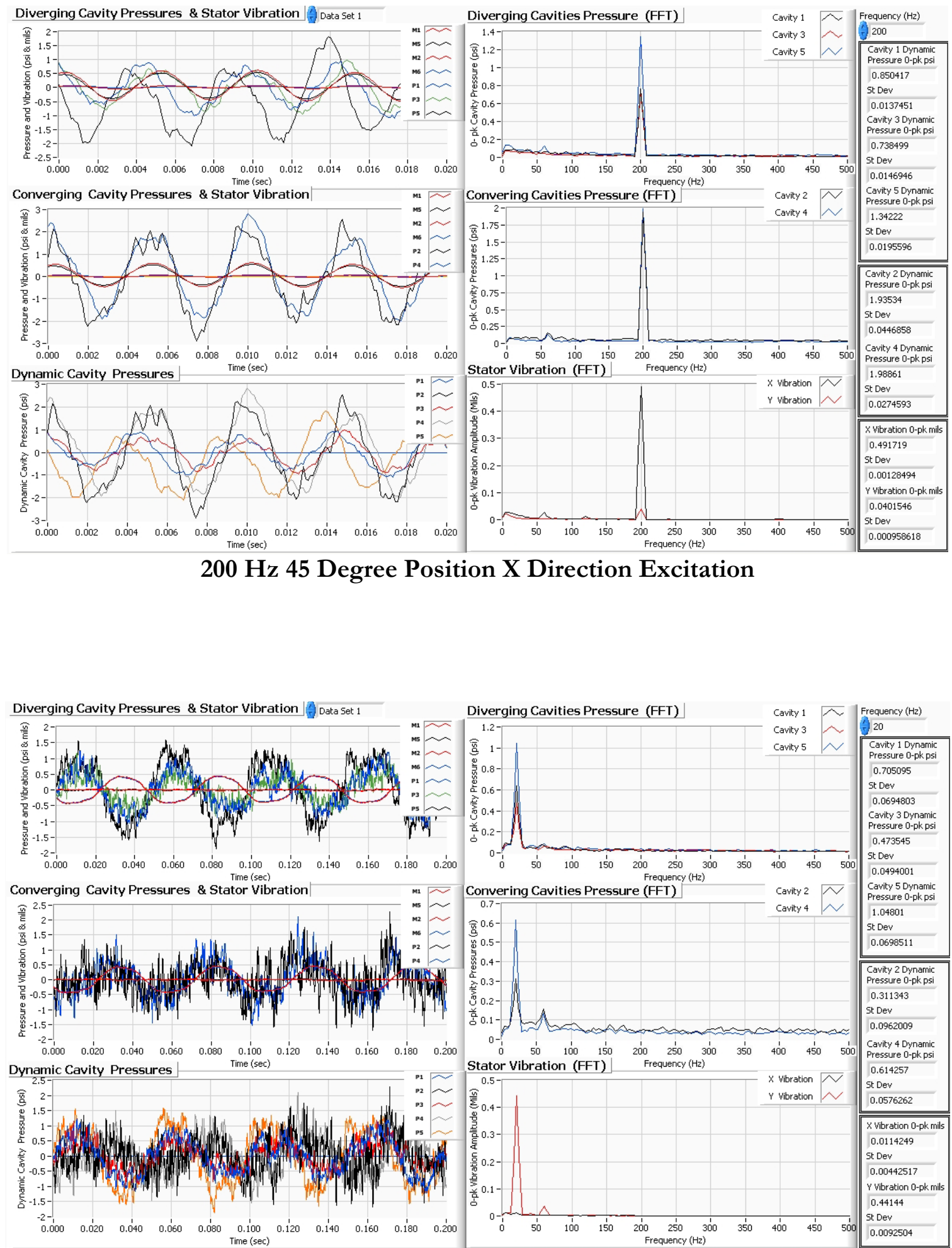

\section{0 $z$ 45 Degree Position X Direction Excitation}

\section{$20 \mathrm{~Hz} 45$ Degree Position Y Direction Excitation}



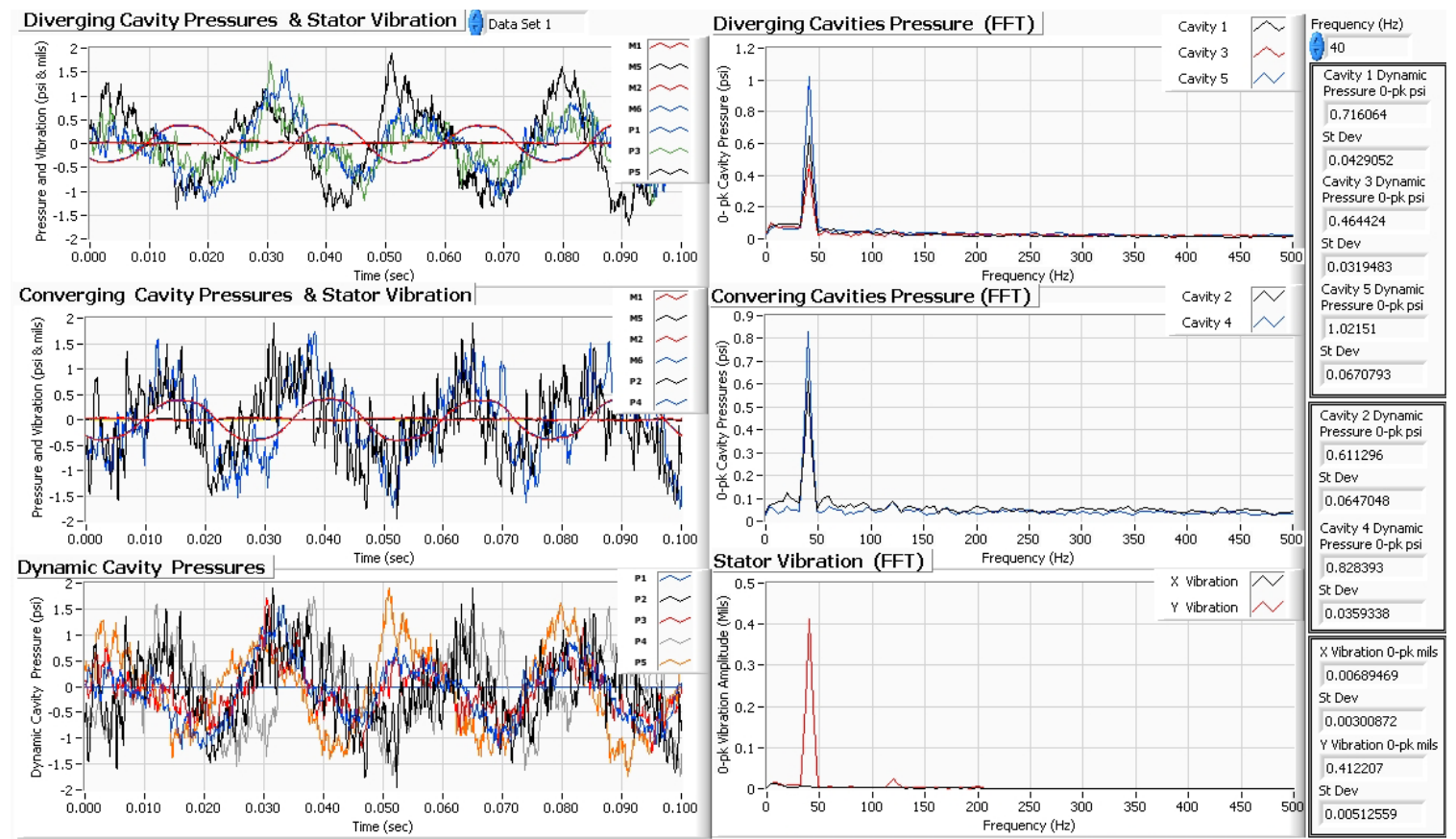

$40 \mathrm{~Hz} 45$ Degree Position Y Direction Excitation
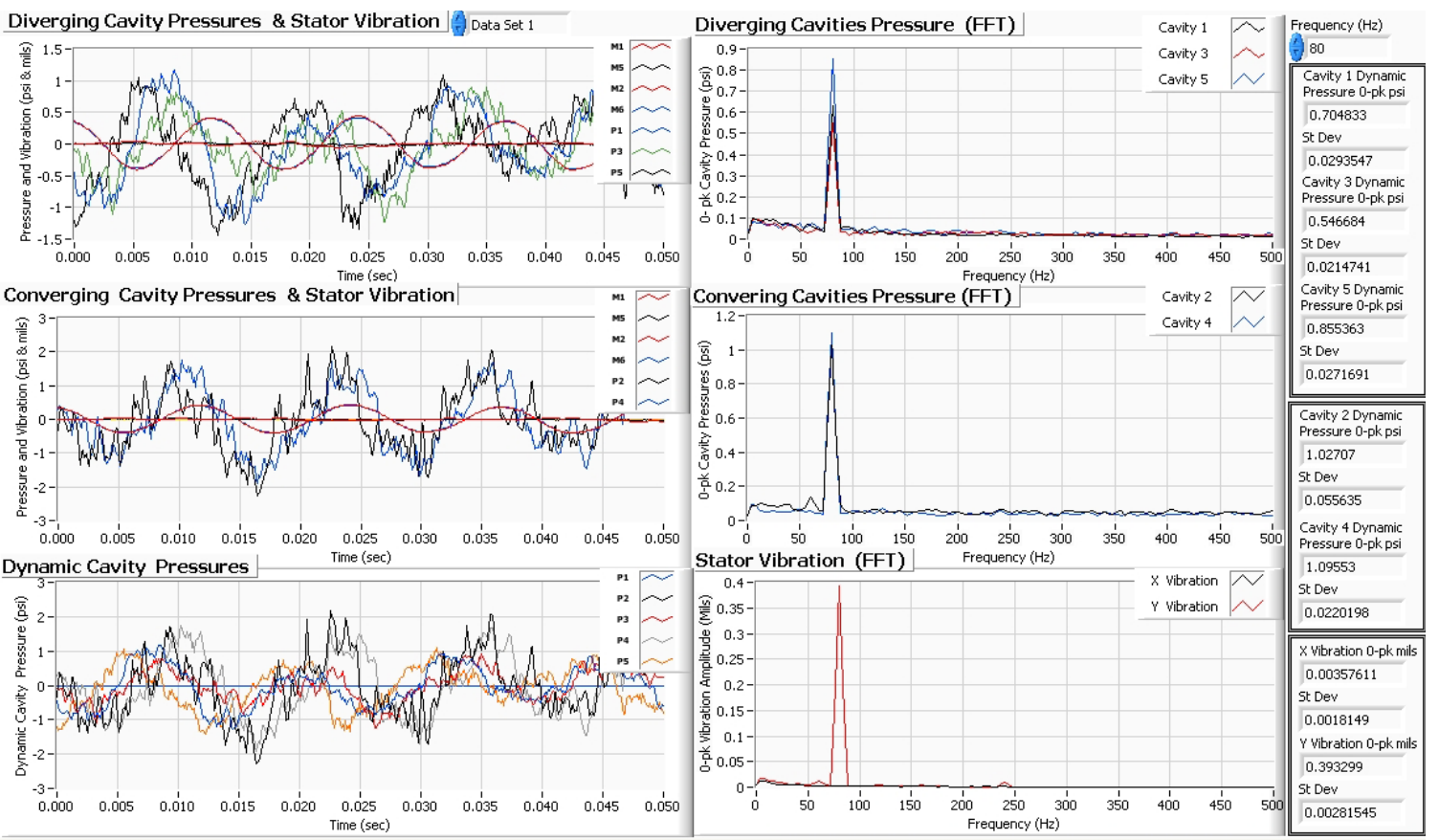

$80 \mathrm{~Hz} 45$ Degree Position Y Direction Excitation 

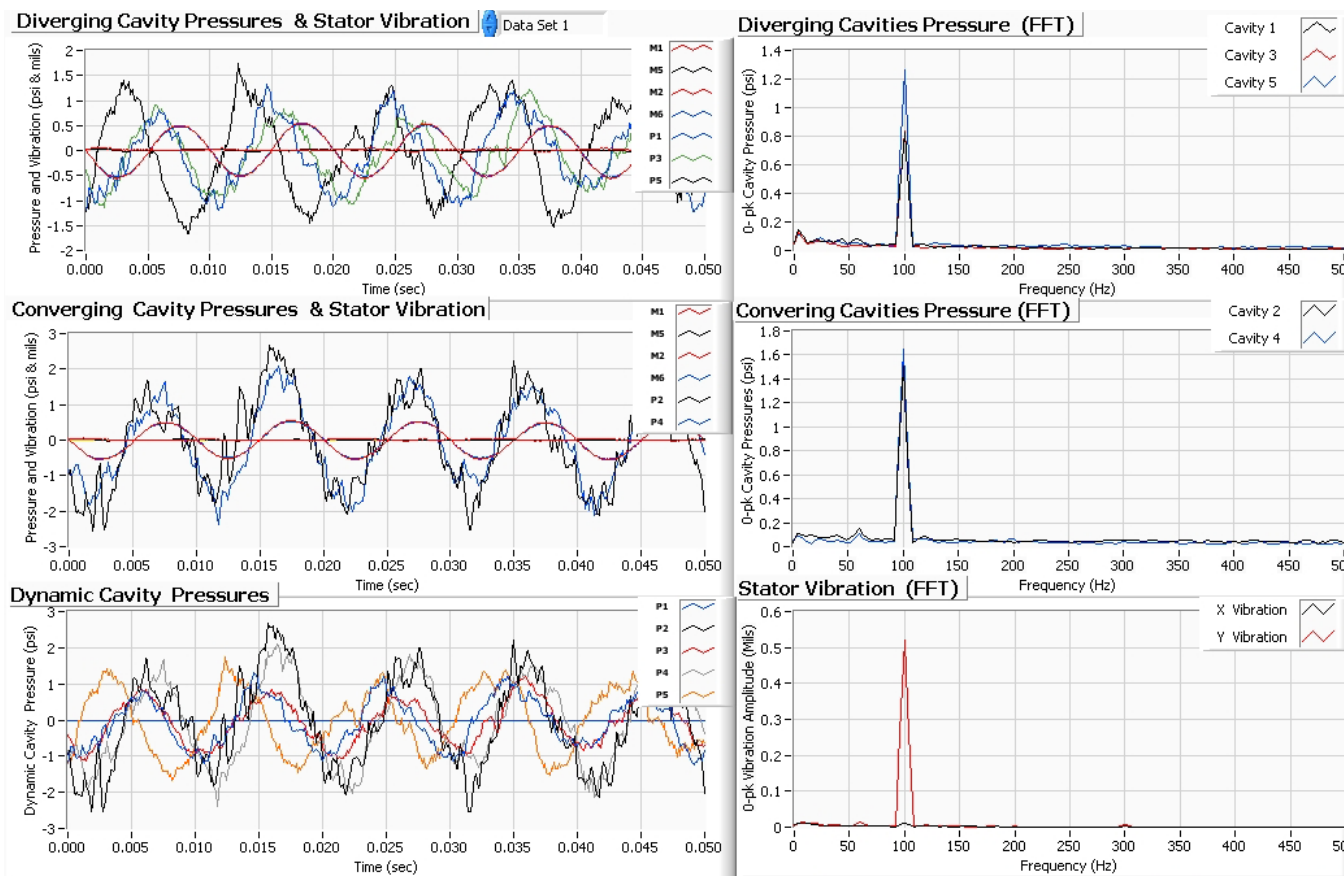

$100 \mathrm{~Hz} 45$ Degree Position Y Direction Excitation
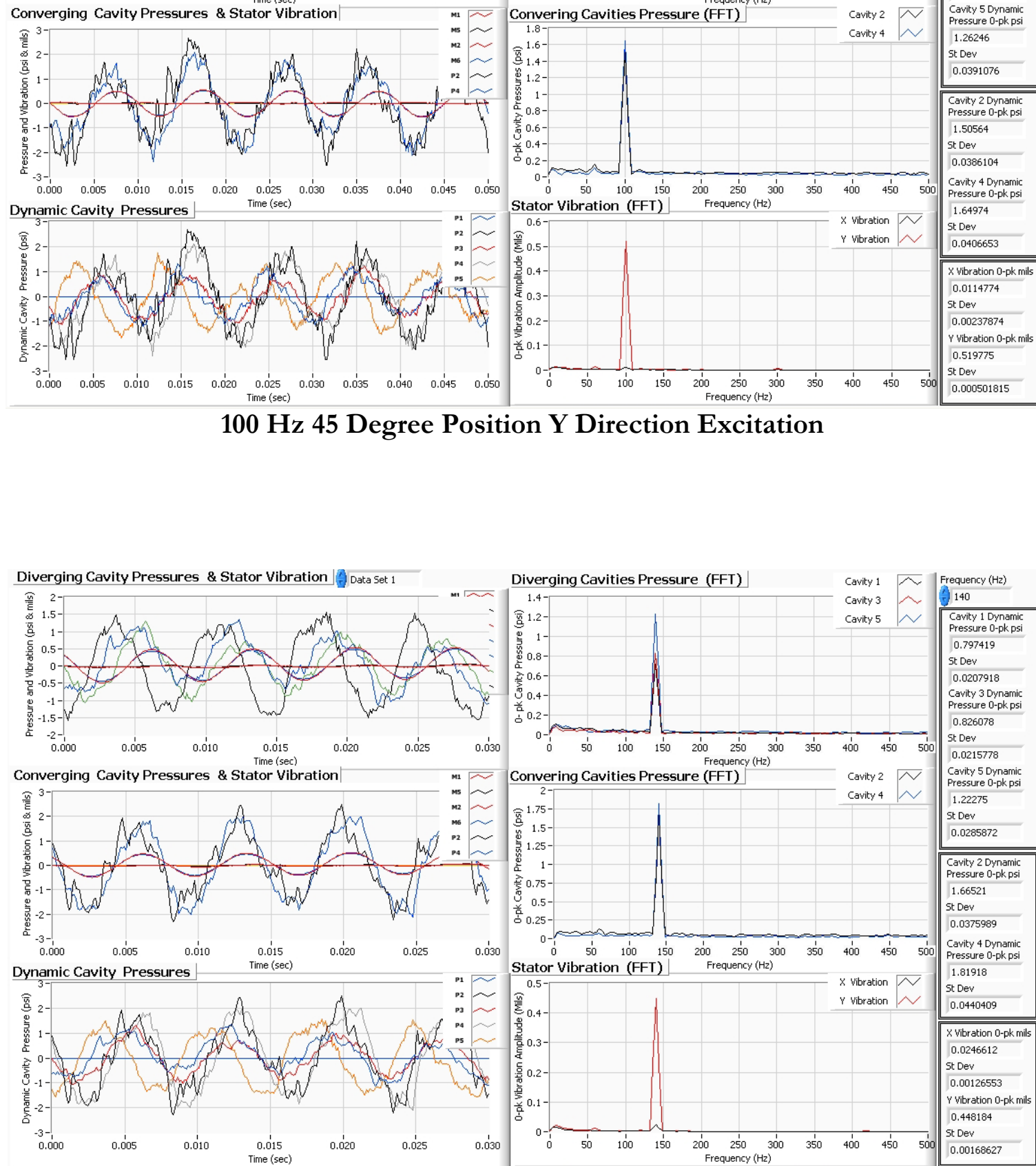

\begin{tabular}{l} 
equency (Hz) \\
100 \\
\hline Cavity 1 Dynamic \\
Pressure 0-pk psi \\
\hline 0.920773 \\
St Dev \\
\hline 0.0308513 \\
Cavity 3 Dynamic \\
Pressure 0 -pk psi \\
\hline 0.835553 \\
St Dev \\
\hline 0.0210465 \\
Cavity 5 Dynamic \\
Pressure 0-pk psi \\
1.26246 \\
St Dev \\
\hline 0.0391076 \\
\hline Cavity 2 Dynamic \\
Pressure 0-pk psi \\
1.50564 \\
St Dev \\
0.0386104 \\
Cavity 4 Dynamic \\
Pressure 0-pk psi \\
1.64974 \\
St Dev \\
\hline 0.0406653 \\
\hline X vibration 0-pk mils \\
0.0114774 \\
St Dev \\
\hline 0.00237874 \\
Y vibration 0-pk mils \\
0.519775 \\
$5 t$ Dev \\
\hline 0.000501815 \\
\hline
\end{tabular}

100 

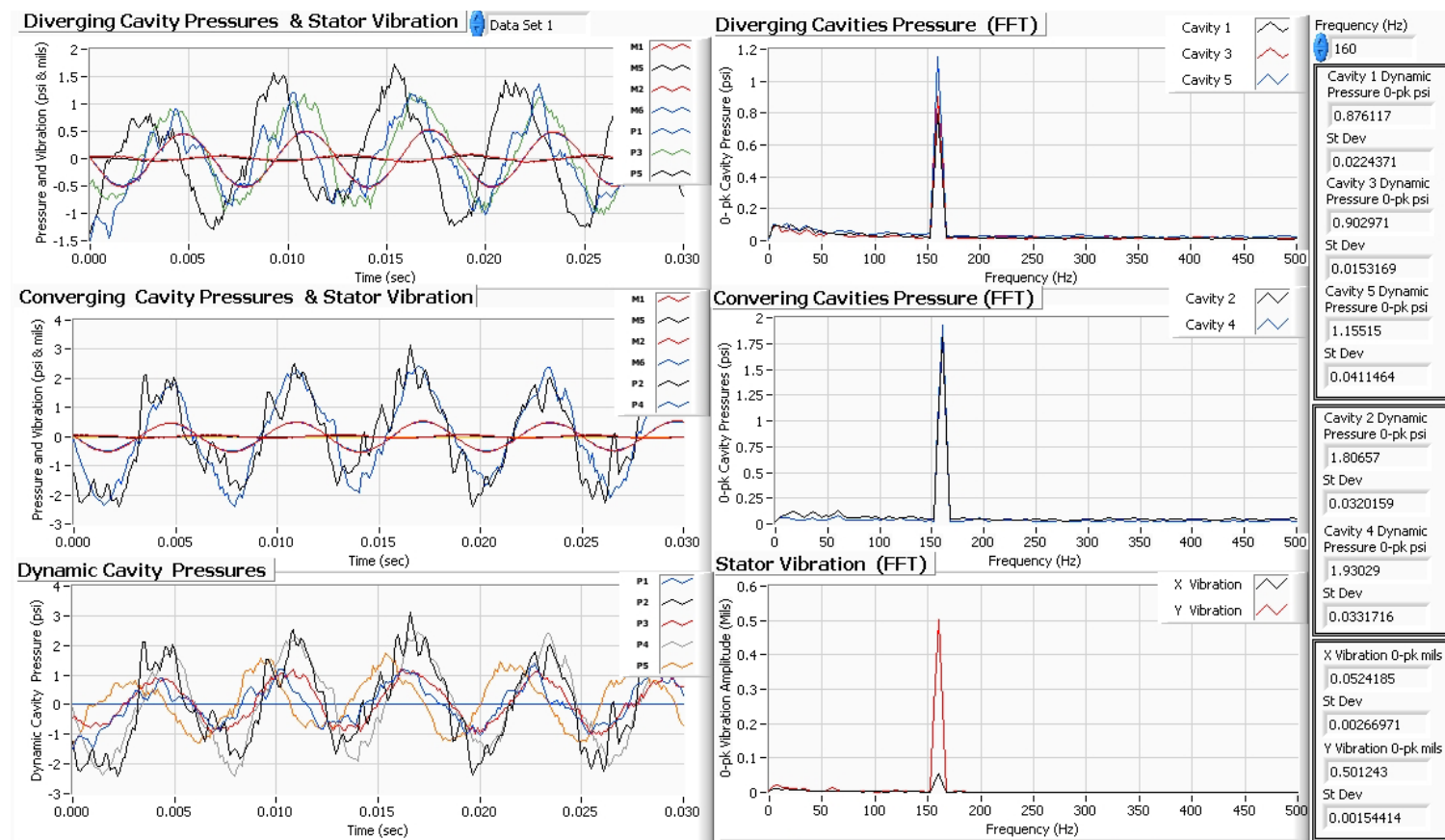

$160 \mathrm{~Hz} 45$ Degree Position Y Direction Excitation
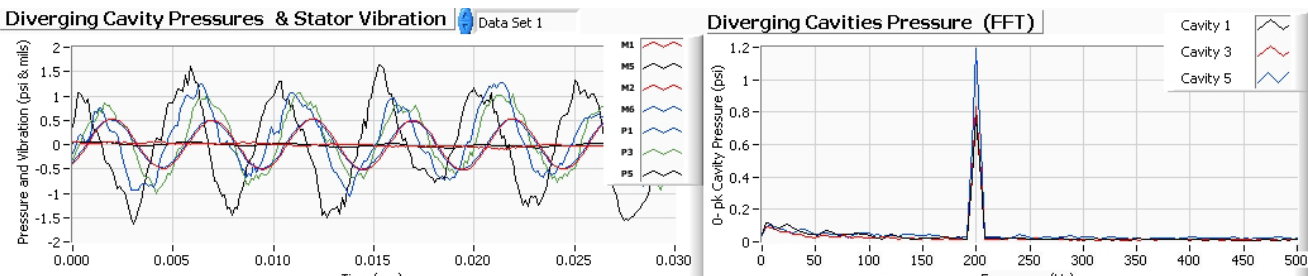

\begin{tabular}{|c|}
\hline $\begin{array}{l}\text { Erequency }(\mathrm{Hz}) \\
200\end{array}$ \\
\hline $\begin{array}{l}\text { Cavity } 1 \text { Dynamic } \\
\text { Pressure 0-pk psi }\end{array}$ \\
\hline 0.864674 \\
\hline St Dev \\
\hline 0.0223555 \\
\hline $\begin{array}{l}\text { Cavity } 3 \text { Dynamic } \\
\text { Pressure 0-pk psi }\end{array}$ \\
\hline 0.831239 \\
\hline St Dev \\
\hline 0.00872258 \\
\hline $\begin{array}{l}\text { Cavity } 5 \text { Dynamic } \\
\text { Pressure 0-pk psi }\end{array}$ \\
\hline 1.19111 \\
\hline St Dev \\
\hline 0.0270992 \\
\hline $\begin{array}{l}\text { Cavity } 2 \text { Dynamic } \\
\text { Pressure 0-pkppsi }\end{array}$ \\
\hline 1.99057 \\
\hline St Dev \\
\hline 0.0472988 \\
\hline $\begin{array}{l}\text { Cavity } 4 \text { Dynamic } \\
\text { Pressure 0-pk psi }\end{array}$ \\
\hline 2.0336 \\
\hline St Dev \\
\hline 0.0302358 \\
\hline X vibration 0-pk mils \\
\hline 0.0106344 \\
\hline St Dev \\
\hline 0.000753544 \\
\hline Y vibration 0-pk mils \\
\hline 0.506207 \\
\hline St Dev \\
\hline 0.000650017 \\
\hline
\end{tabular}
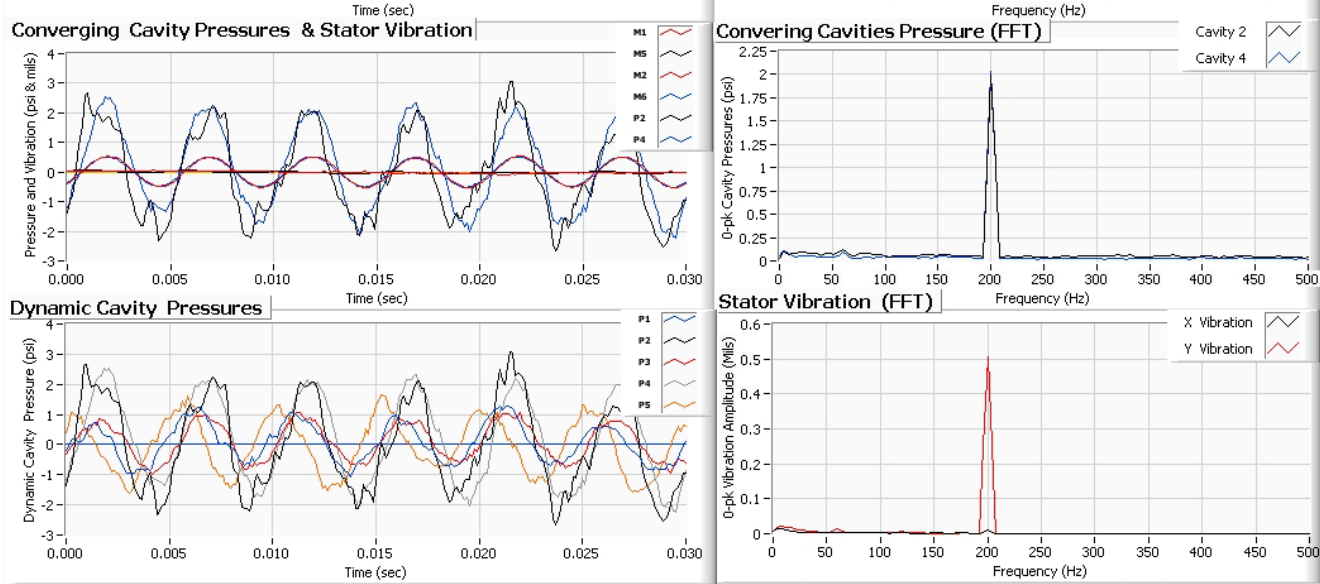

$200 \mathrm{~Hz} 45$ Degree Position Y Direction Excitation 
Dynamic Cavity Pressure Tests

6 Bladed Fully Partitioned PDS

90 DEGREE POSITION 

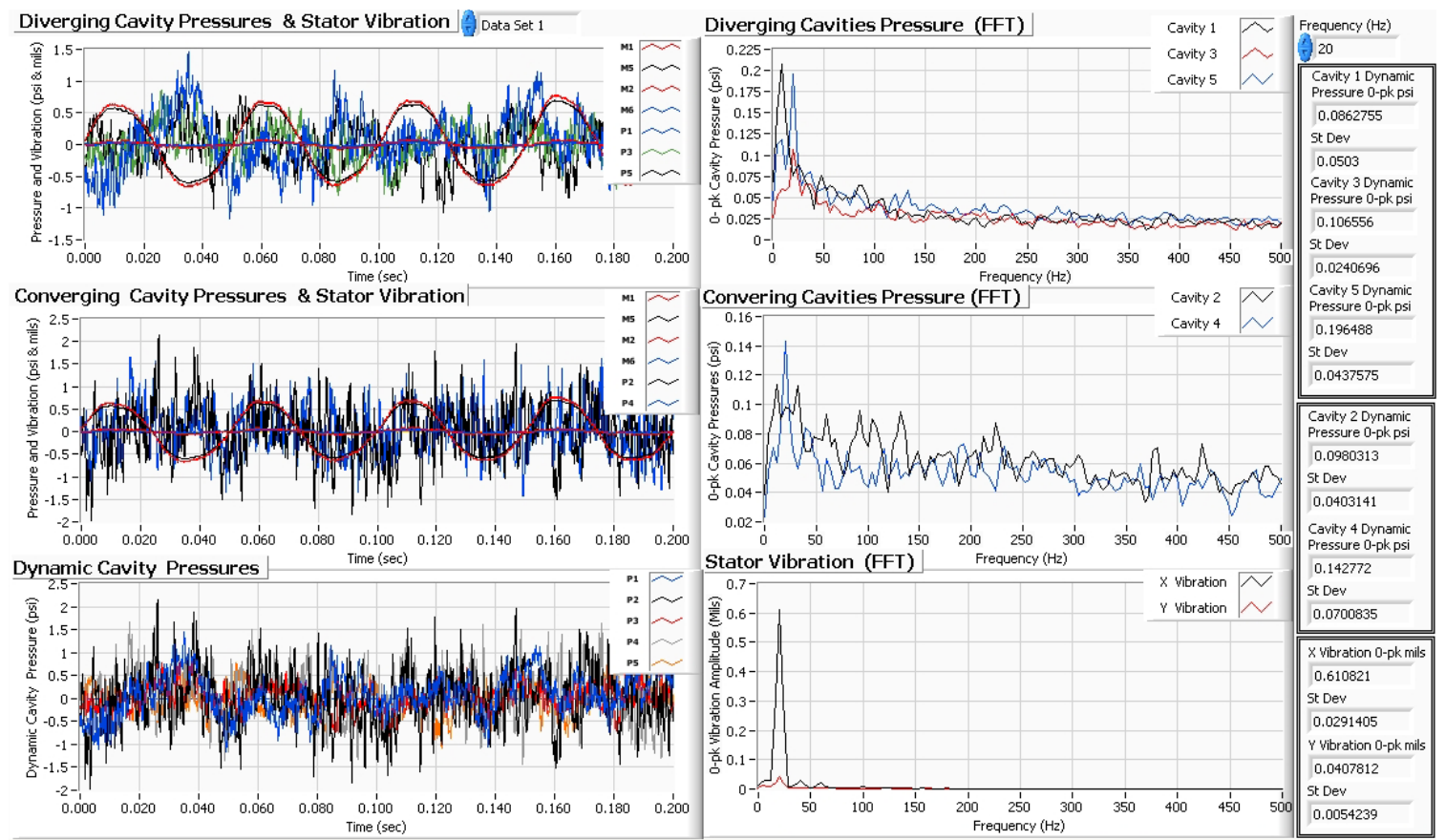

$20 \mathrm{~Hz} 90$ Degree Position X Direction Excitation
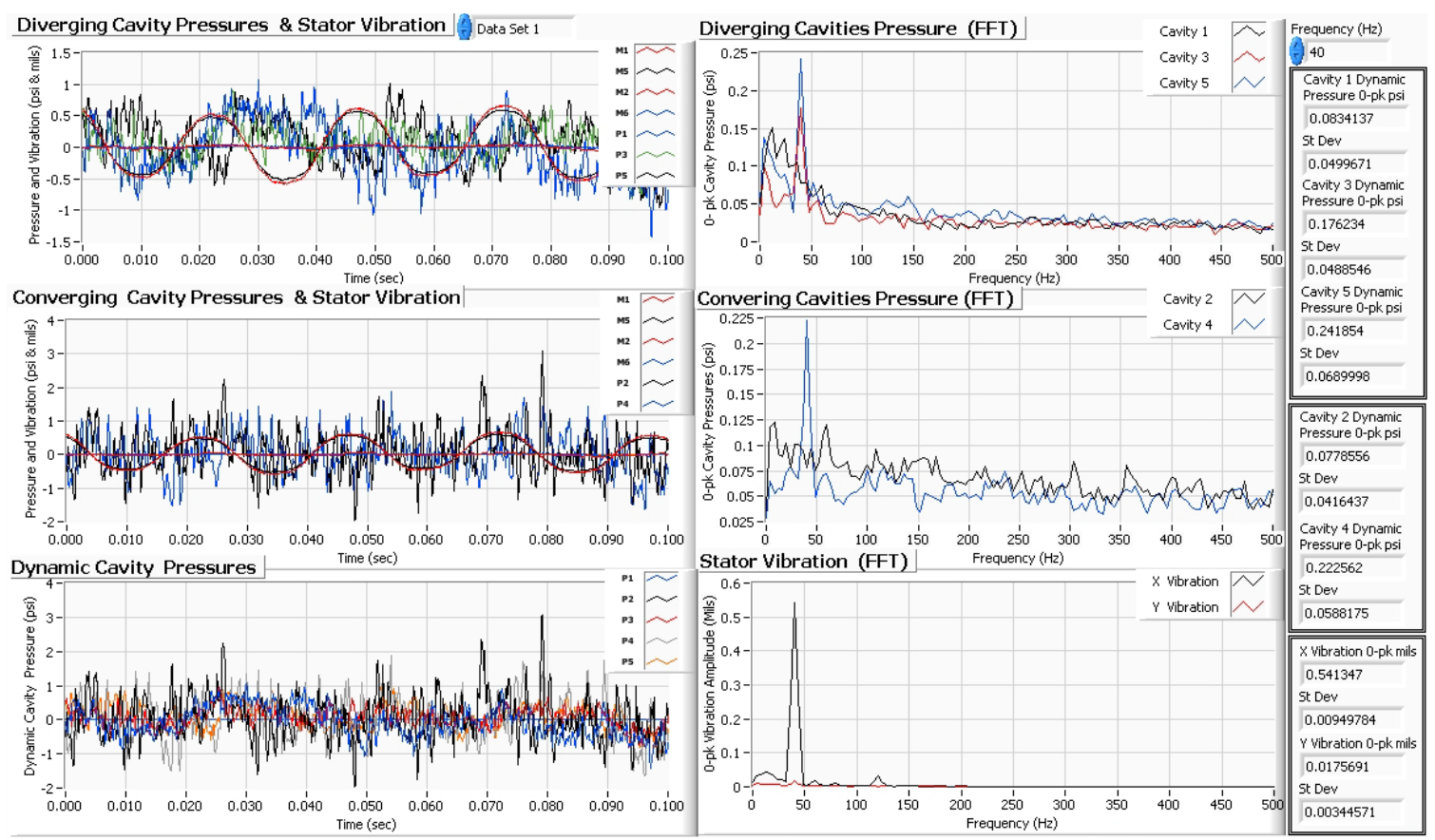

$40 \mathrm{~Hz} 90$ Degree Position X Direction Excitation 

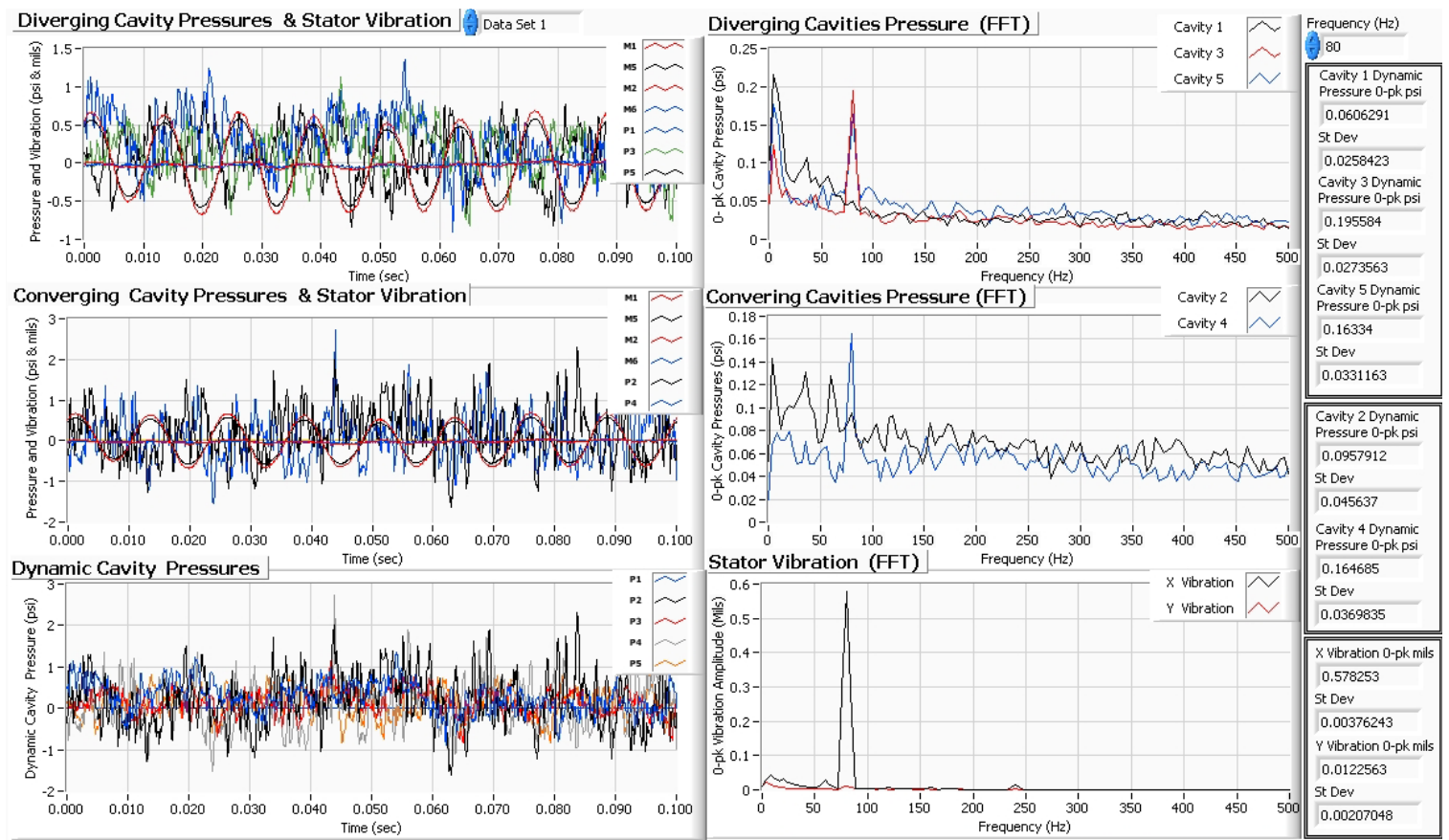

$80 \mathrm{~Hz} 90$ Degree Position X Direction Excitation
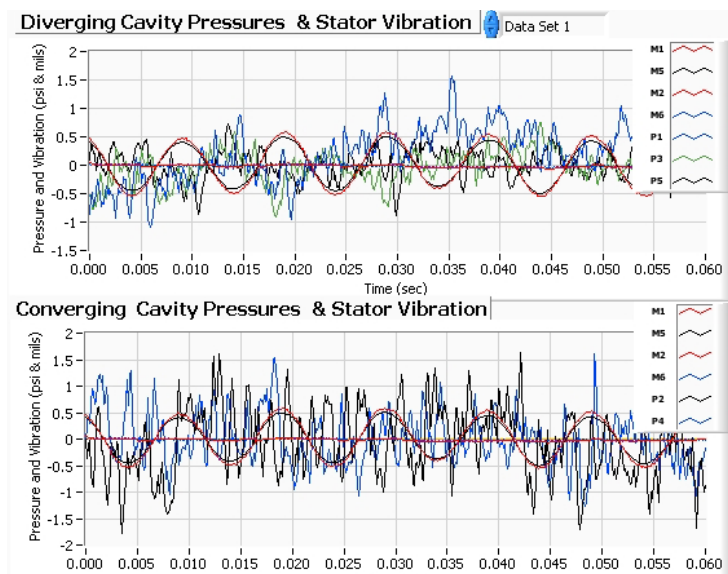

$\begin{array}{lllllllllllll}0.000 & 0.005 & 0.010 & 0.015 & 0.020 & 0.025 & 0.030 & 0.035 & 0.040 & 0.045 & 0.050 & 0.055 & 0.060\end{array}$ Dynamic Cavity Pressures
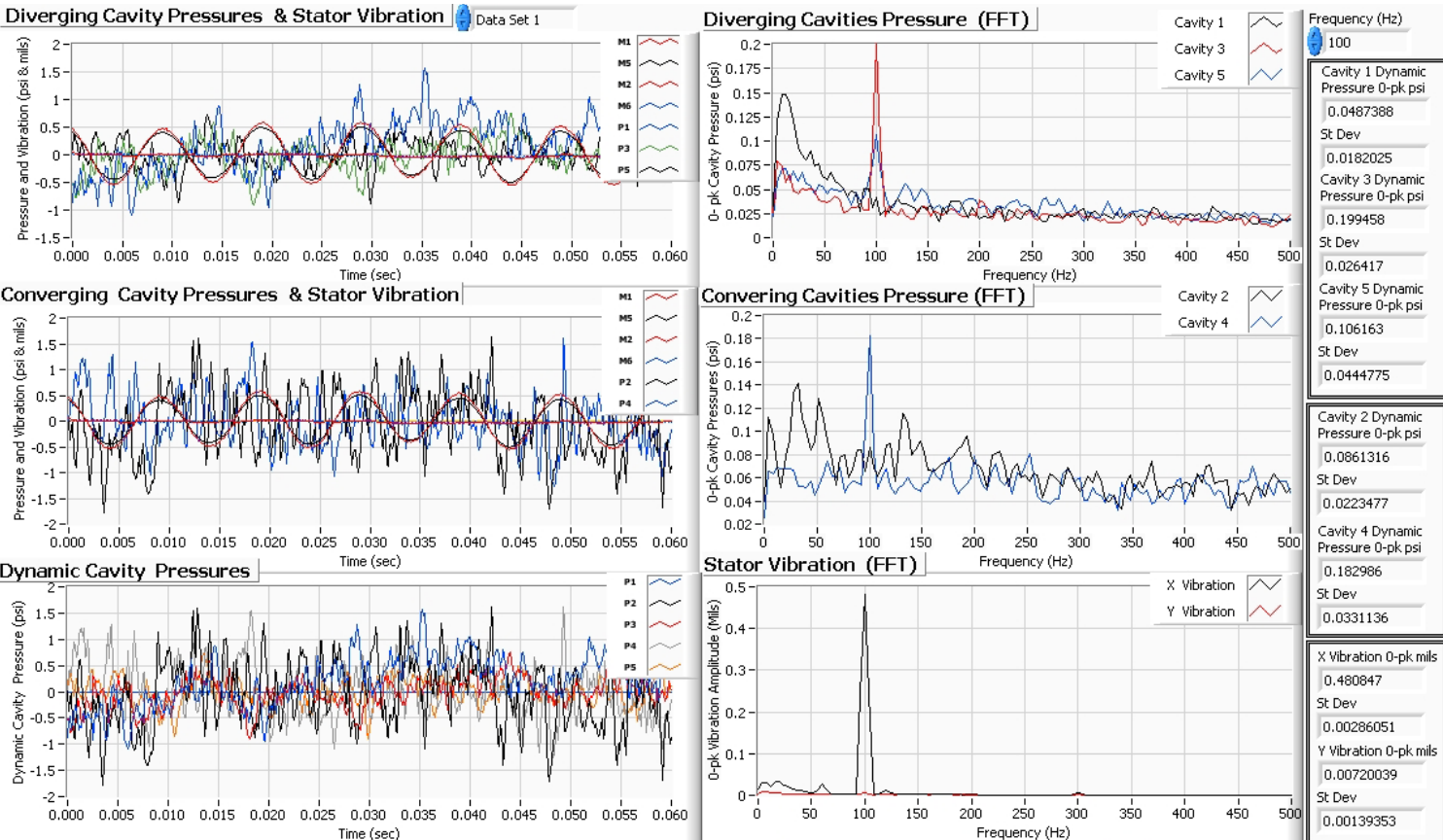

$100 \mathrm{~Hz} 90$ Degree Position X Direction Excitation 

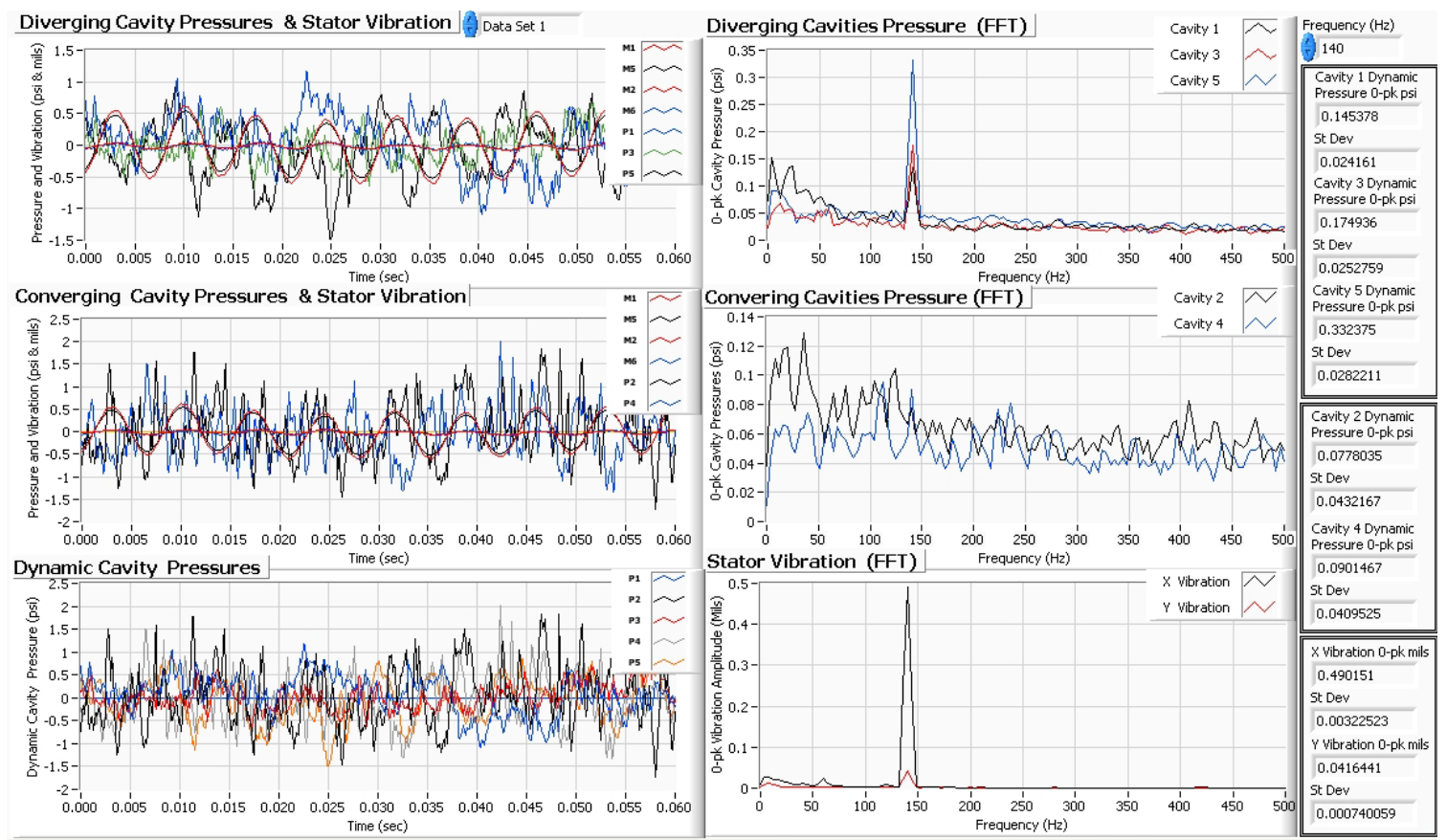

$140 \mathrm{~Hz} 90$ Degree Position X Direction Excitation
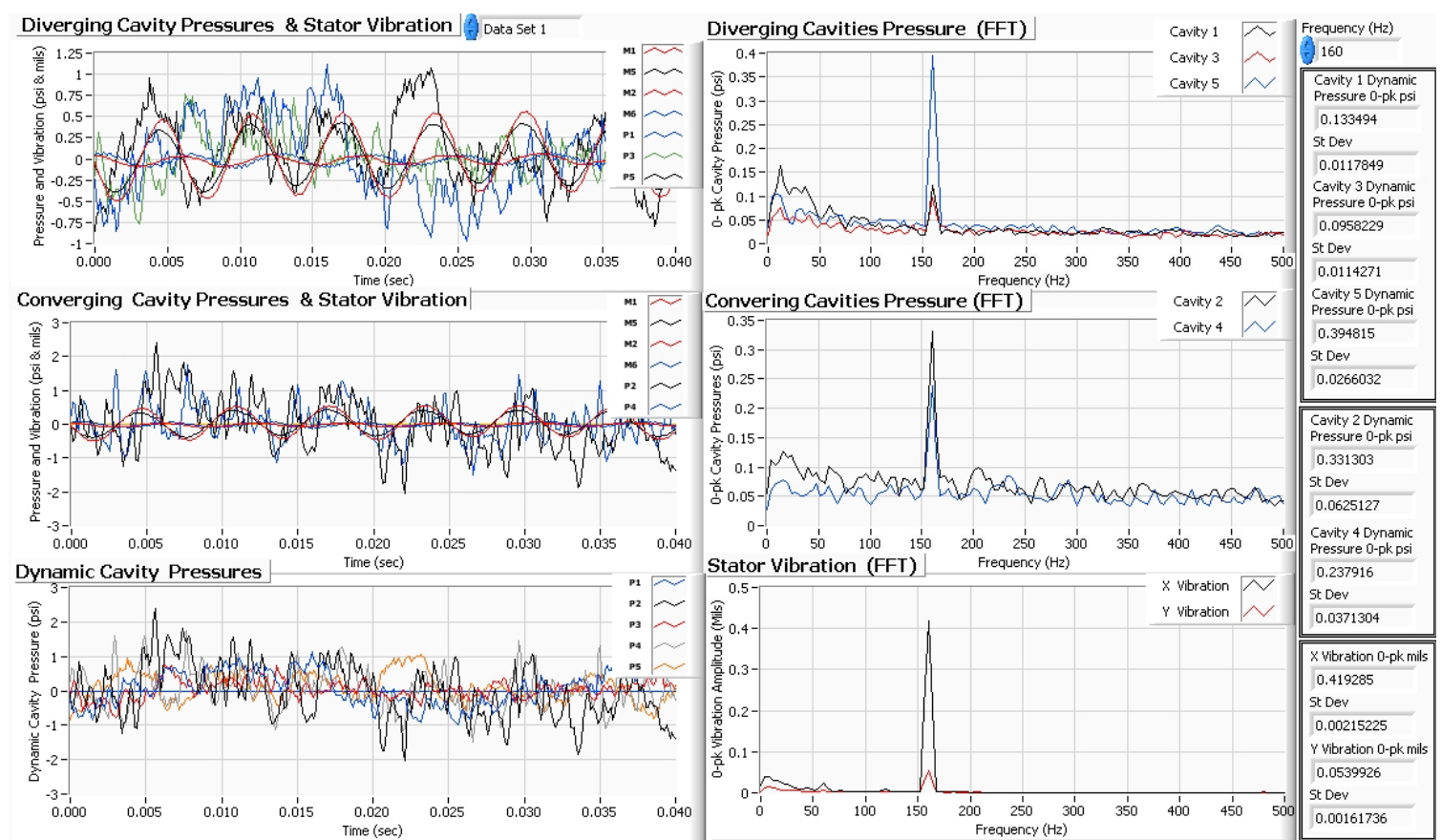

$160 \mathrm{~Hz} 90$ Degree Position X Direction Excitation 


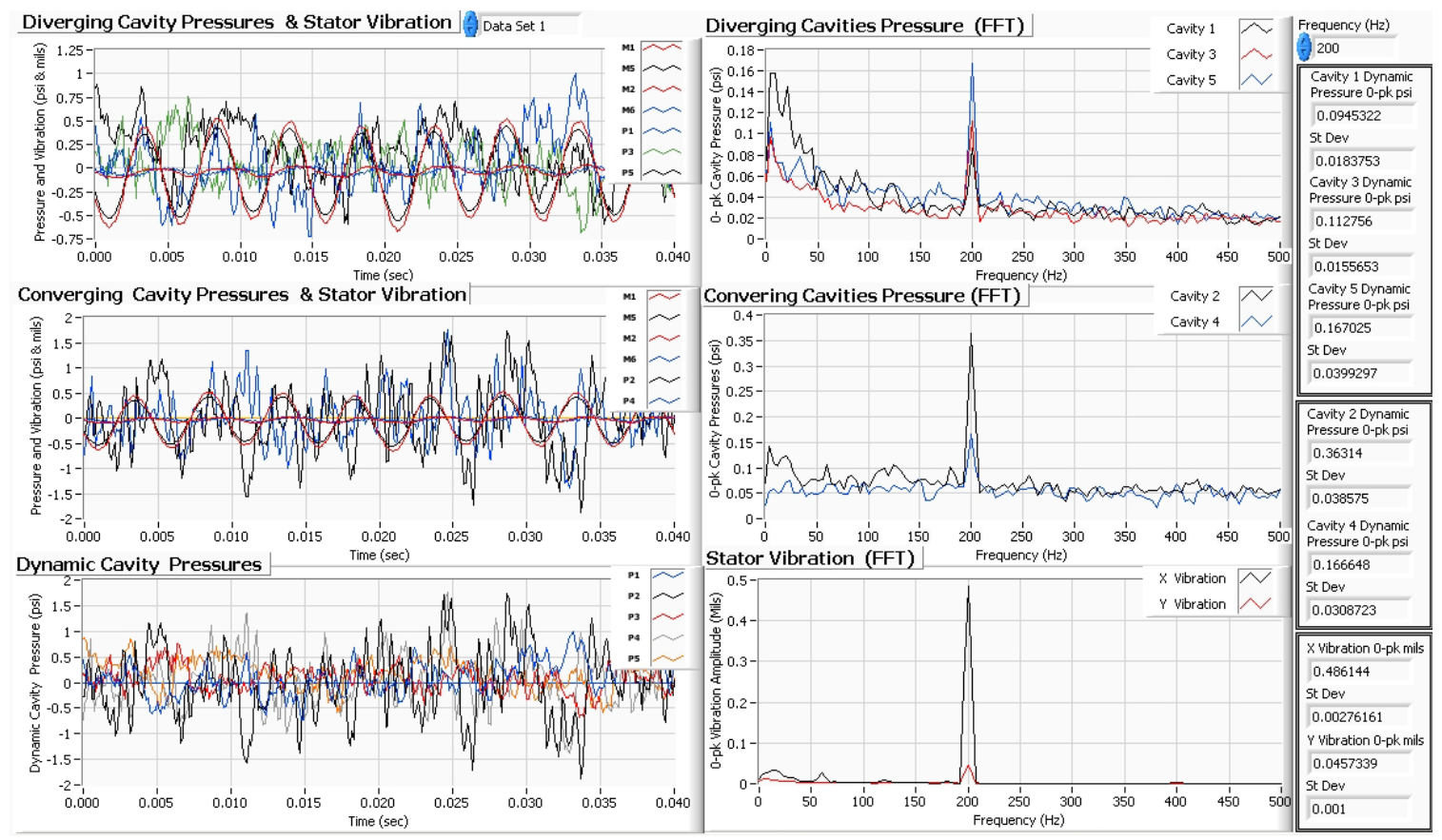

$200 \mathrm{~Hz} 90$ Degree Position X Direction Excitation
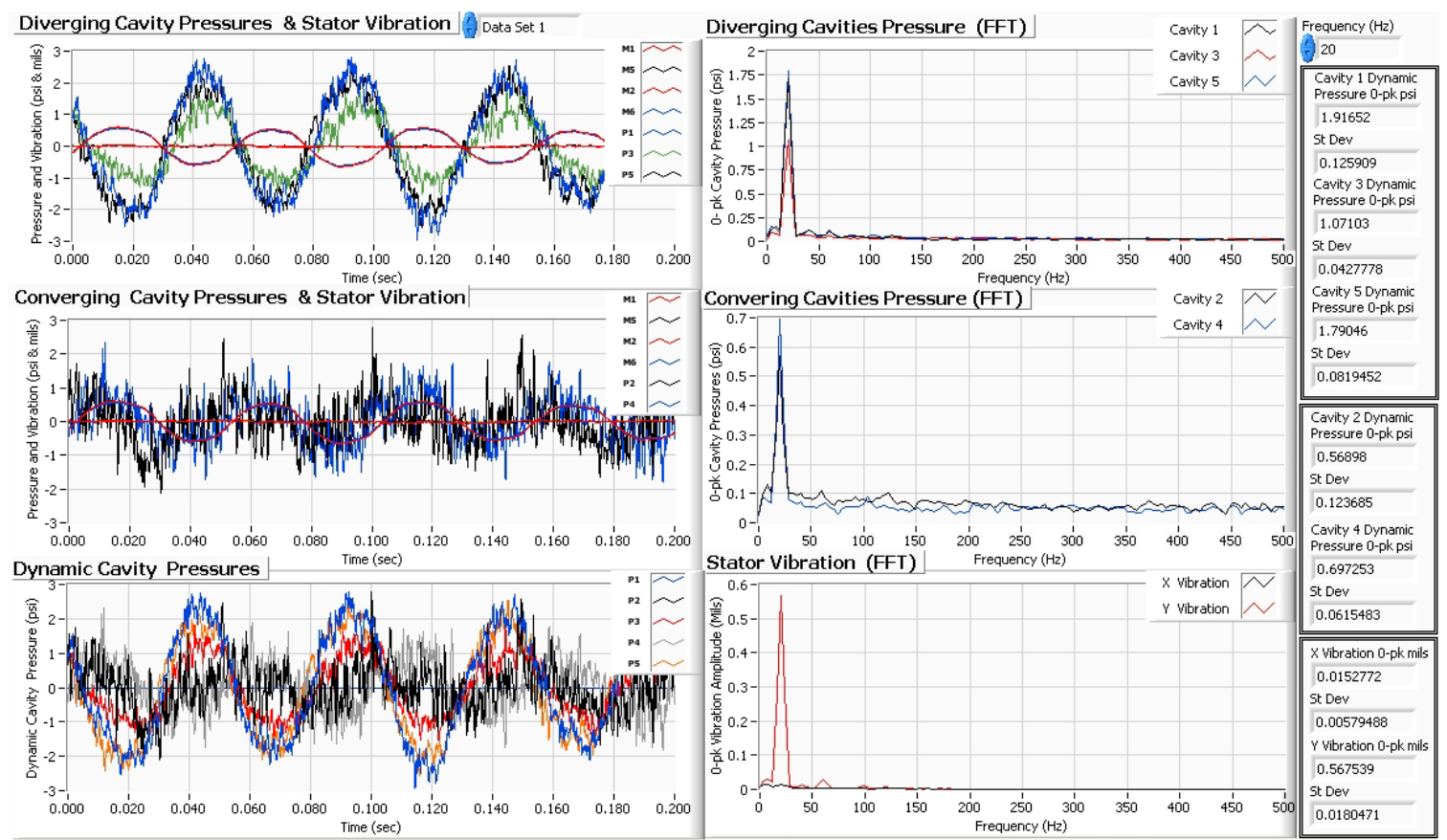

$20 \mathrm{~Hz} 90$ Degree Position Y Direction Excitation 

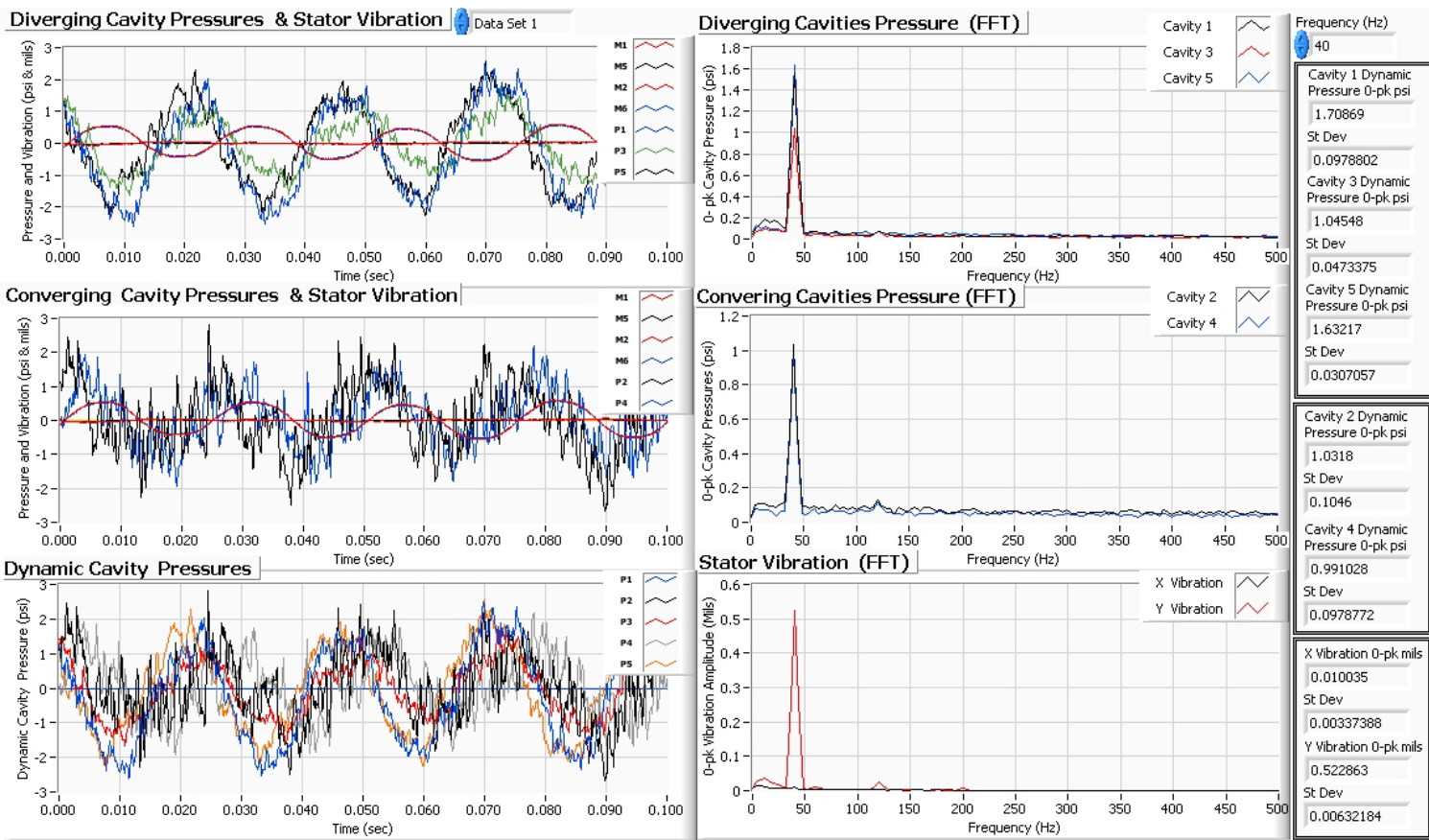

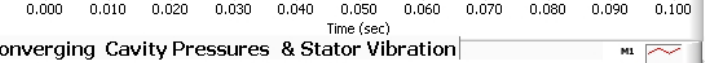
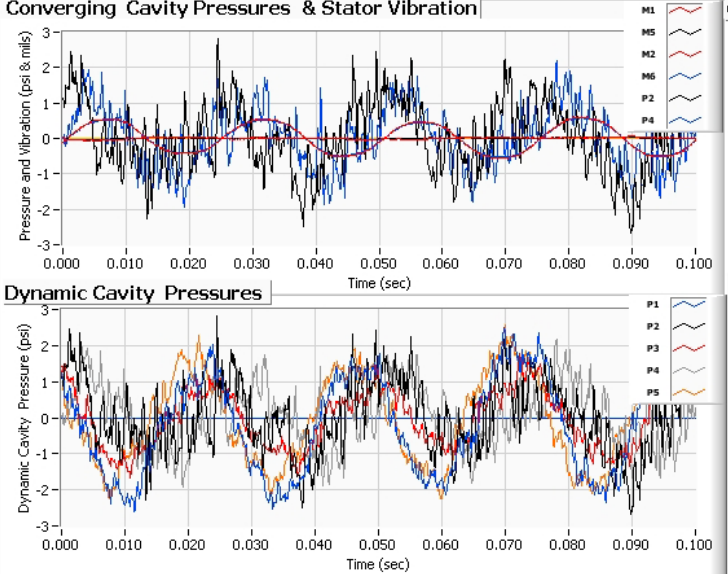

$40 \mathrm{~Hz} 90$ Degree Position Y Direction Excitation
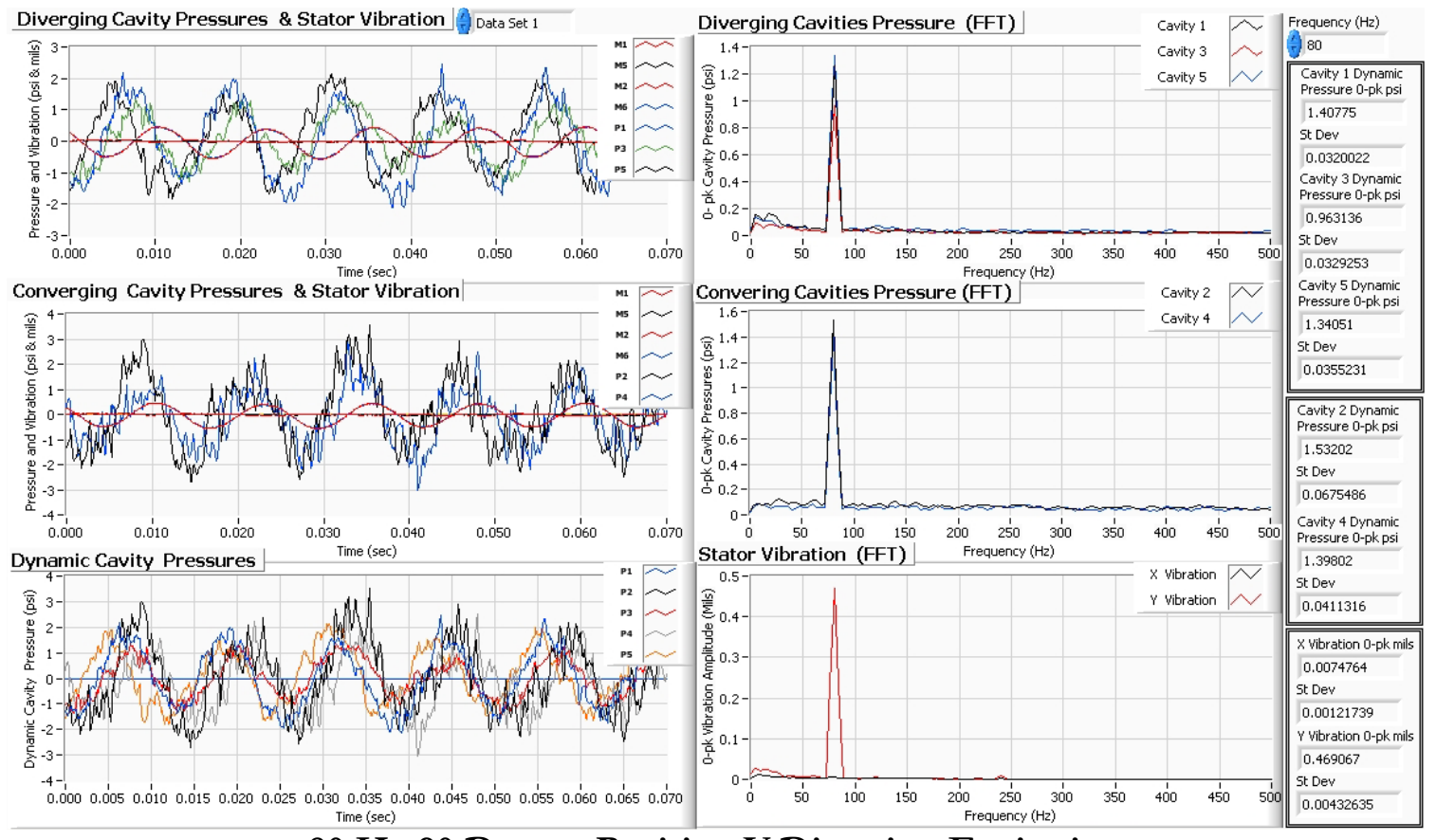

$80 \mathrm{~Hz} 90$ Degree Position Y Direction Excitation 

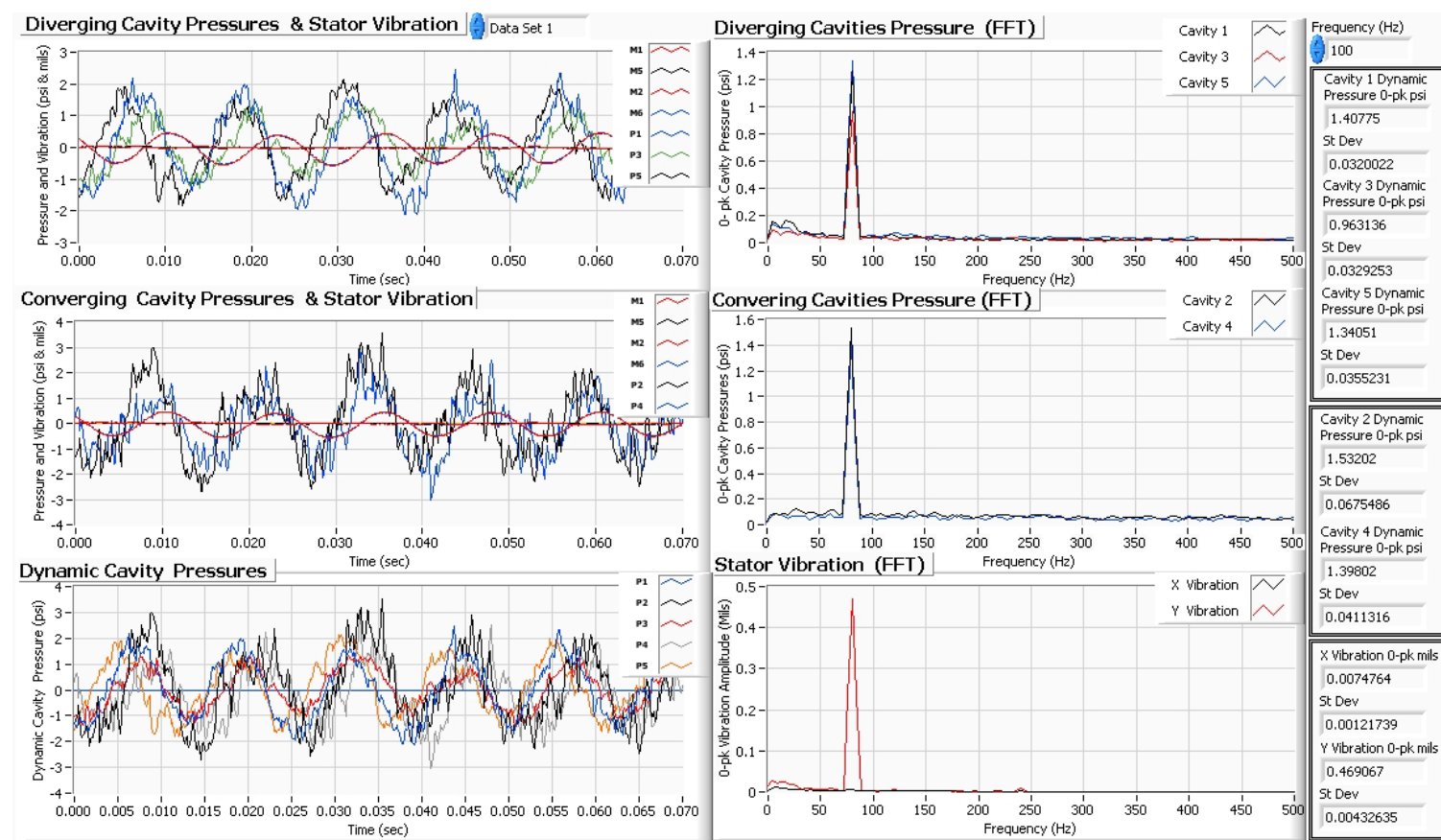

$100 \mathrm{~Hz} 90$ Degree Position Y Direction Excitation
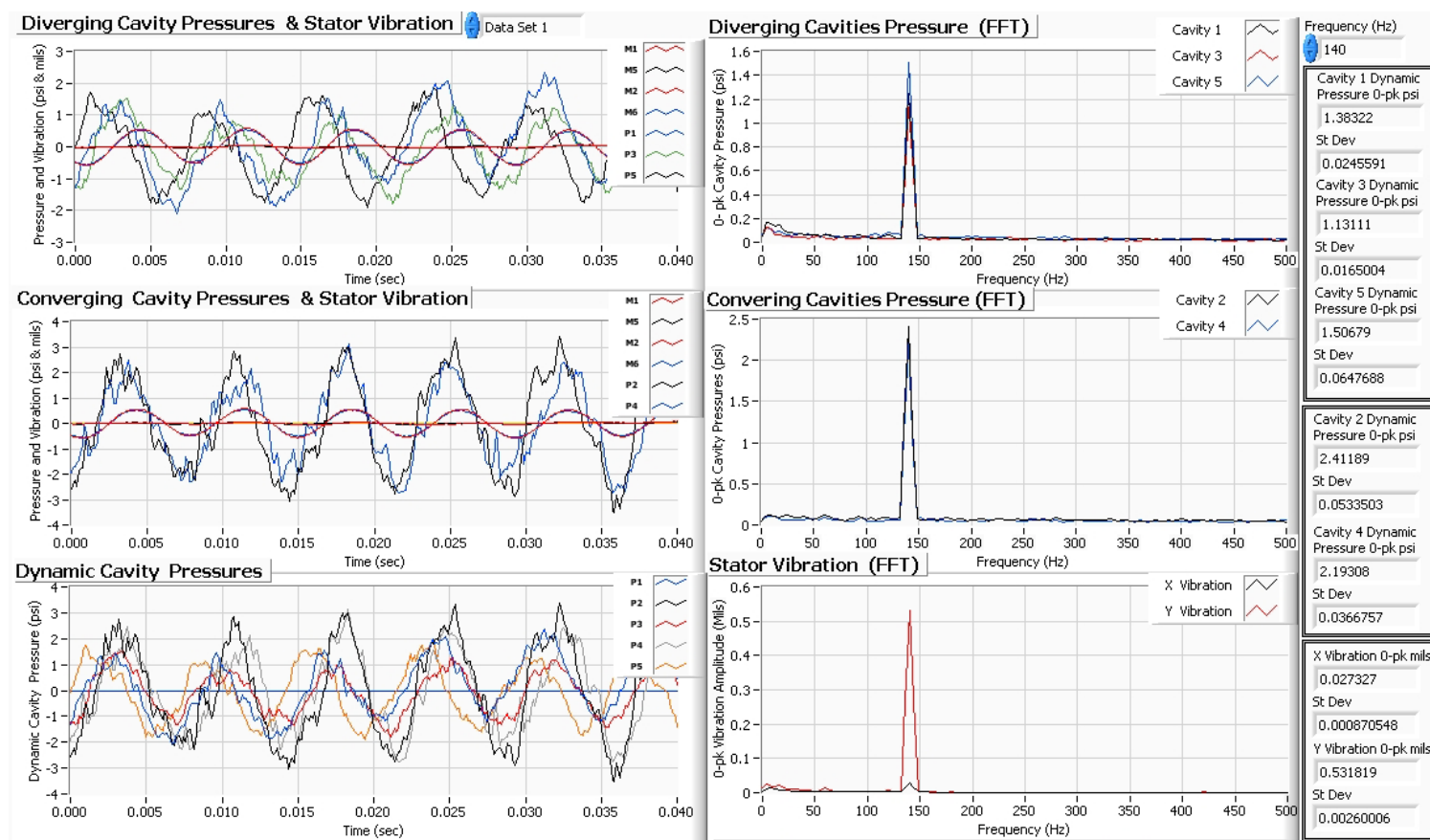

$140 \mathrm{~Hz} 90$ Degree Position Y Direction Excitation 

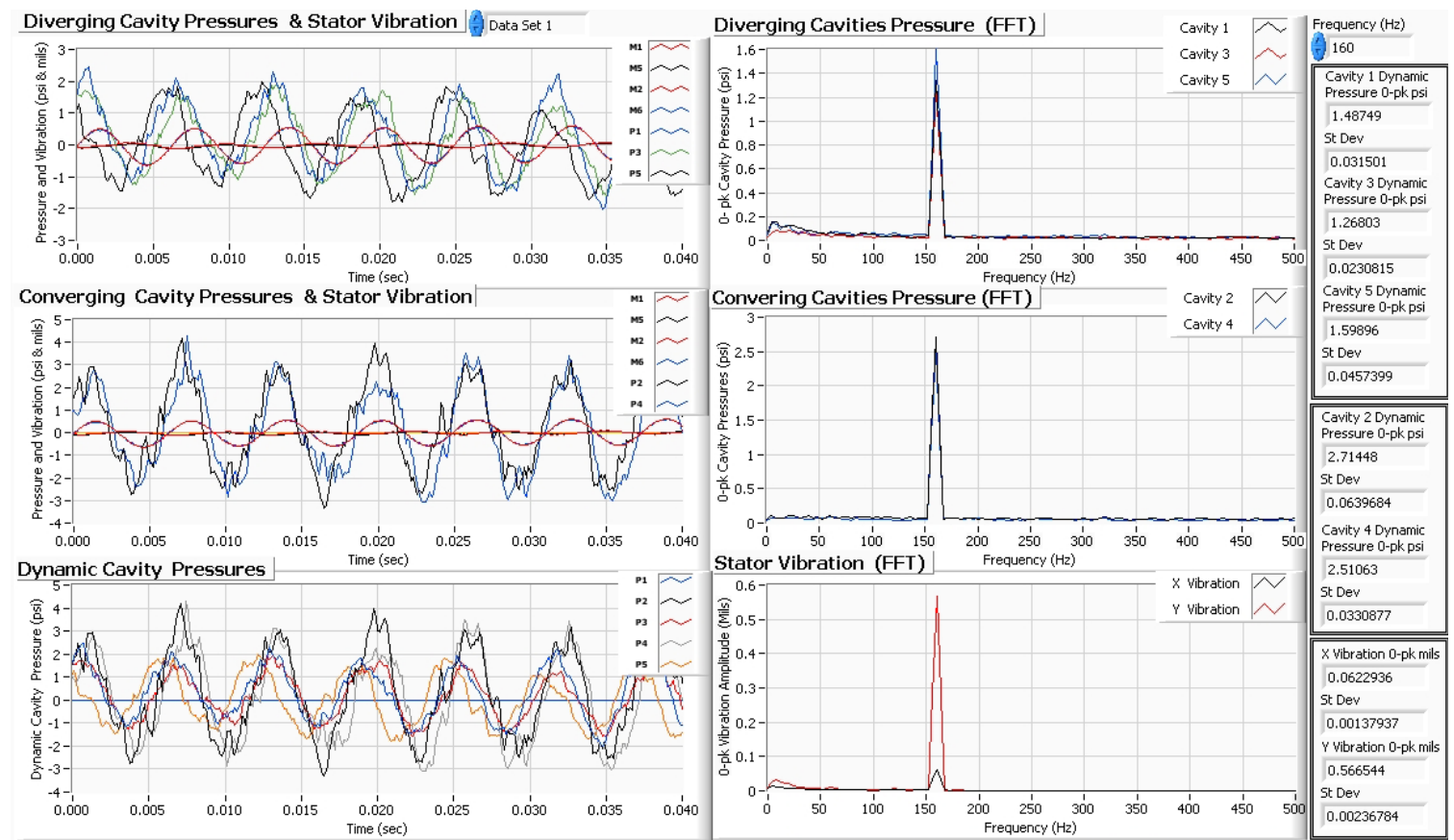

$160 \mathrm{~Hz} 90$ Degree Position Y Direction Excitation
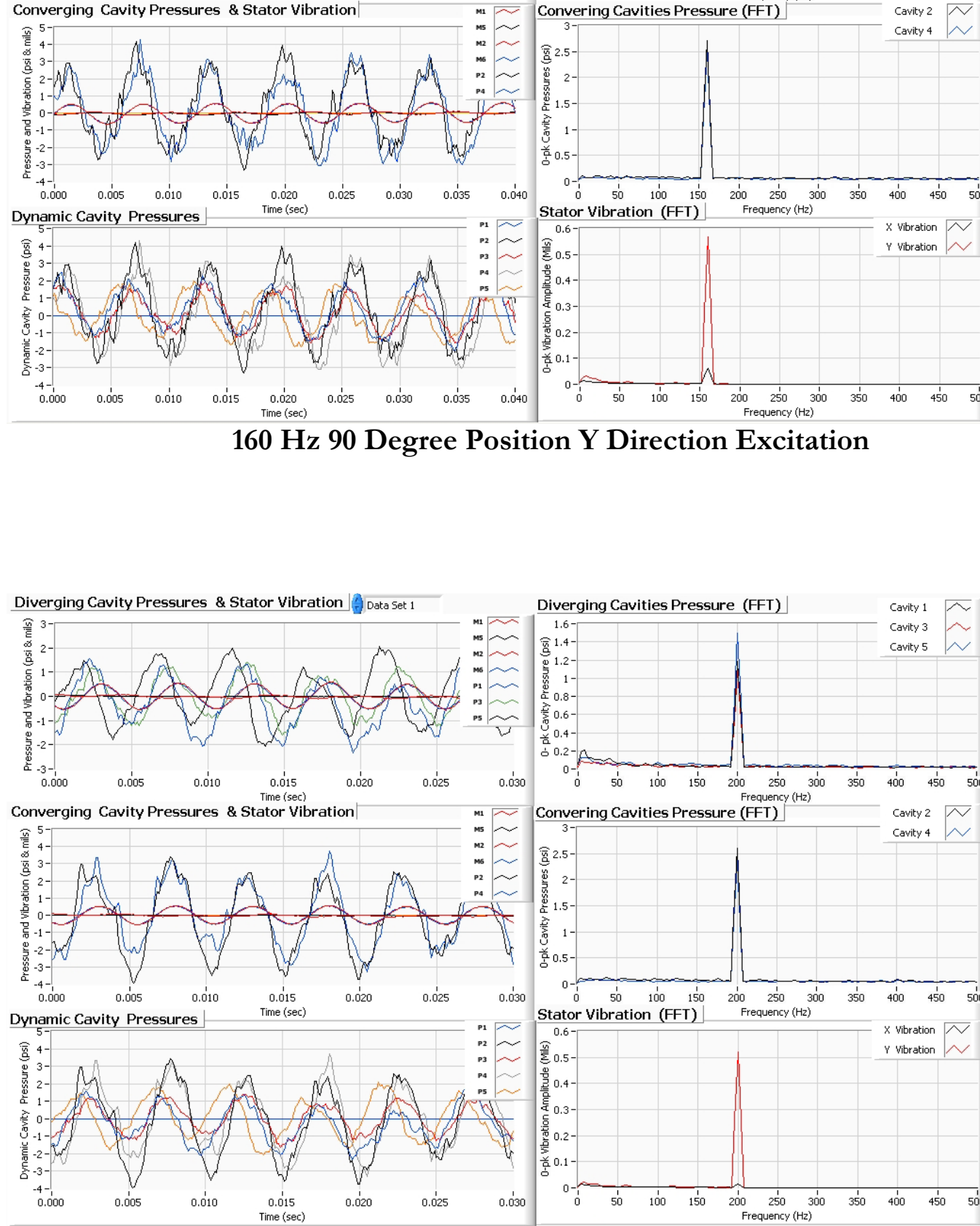

0.0023678

.


APPENDIX E

TEST RIG PICTURES AND DRAWINGS 


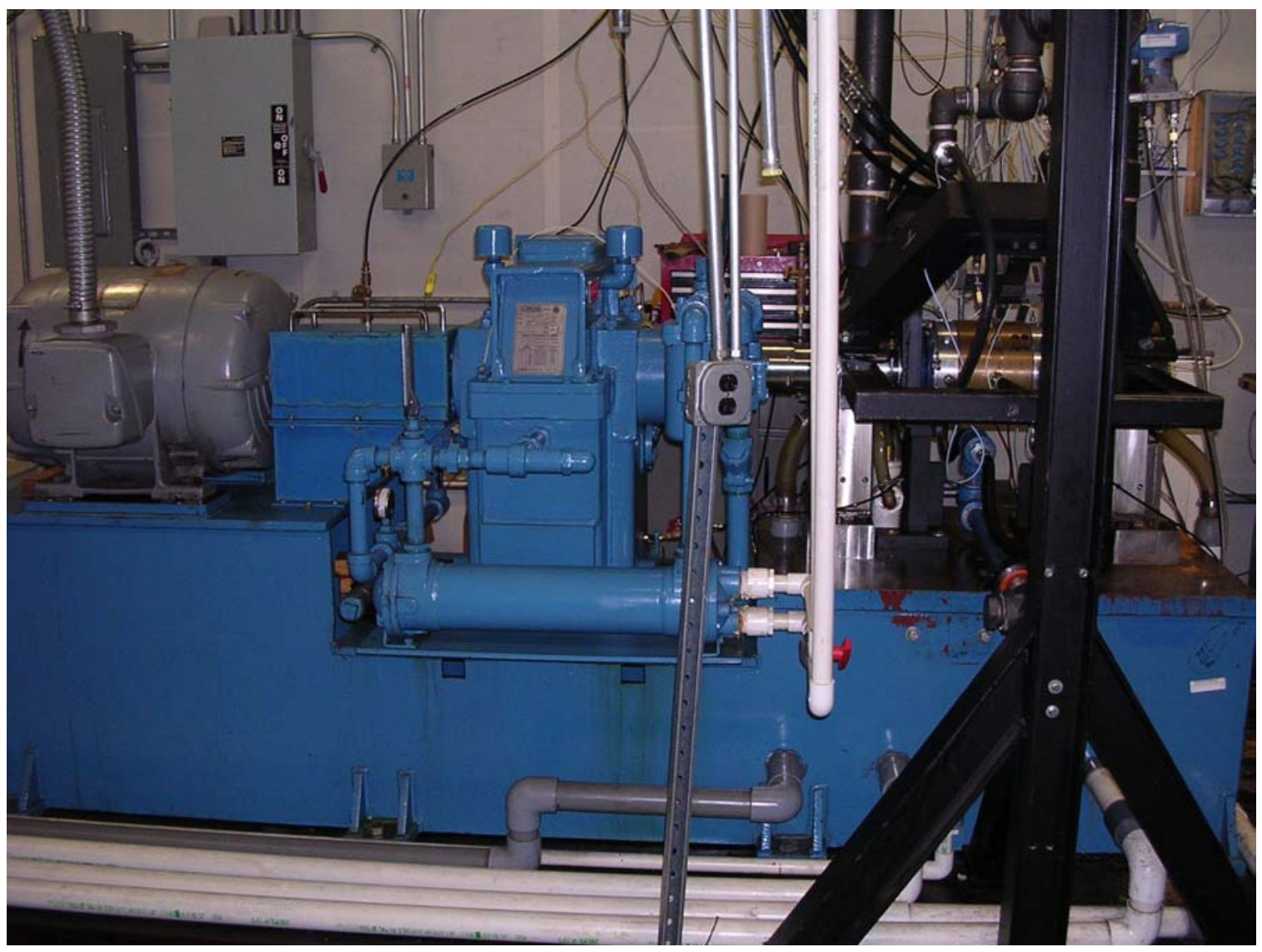

TEST RIG SIDE VIEW

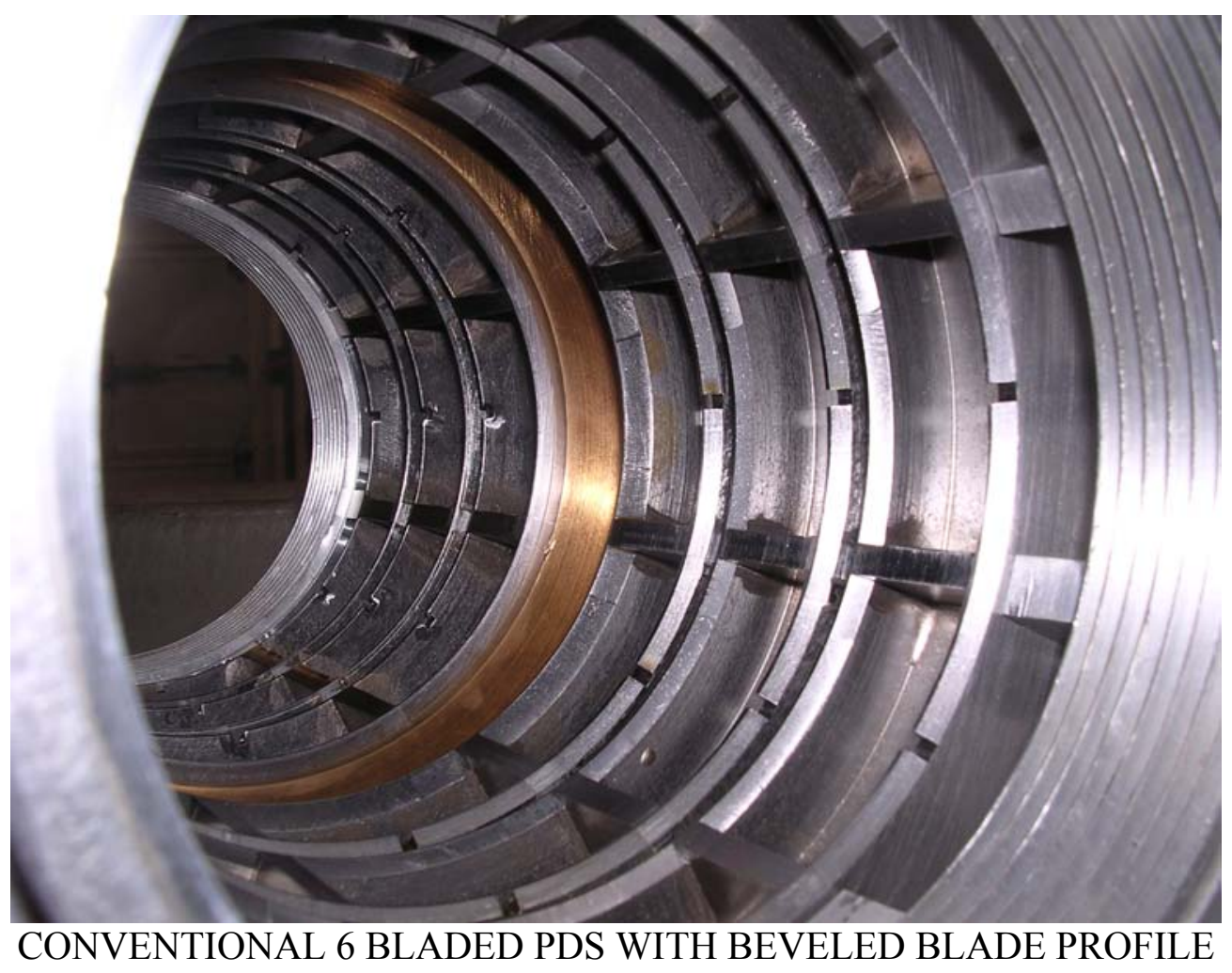




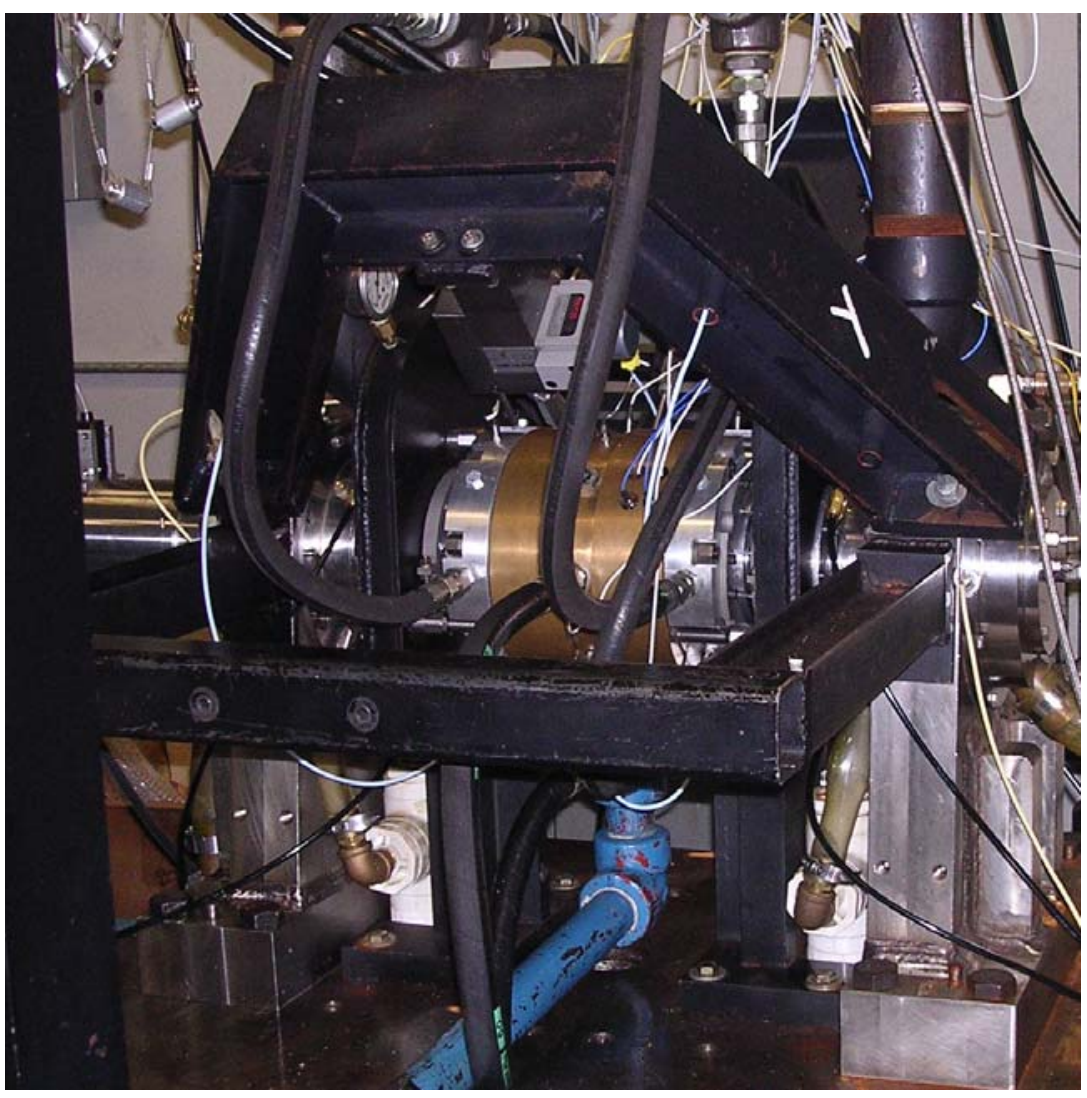

TEST RIG CLOSE-UP

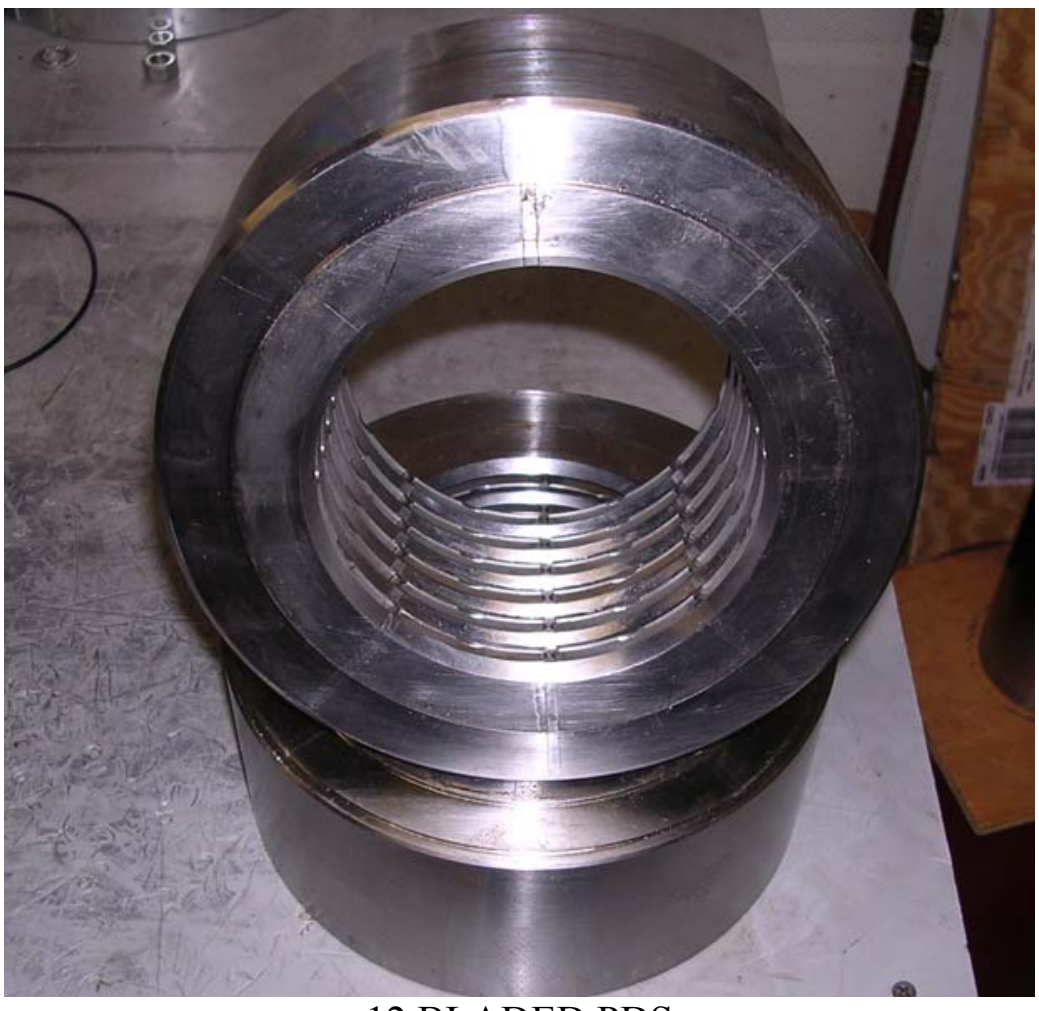

12 BLADED PDS 


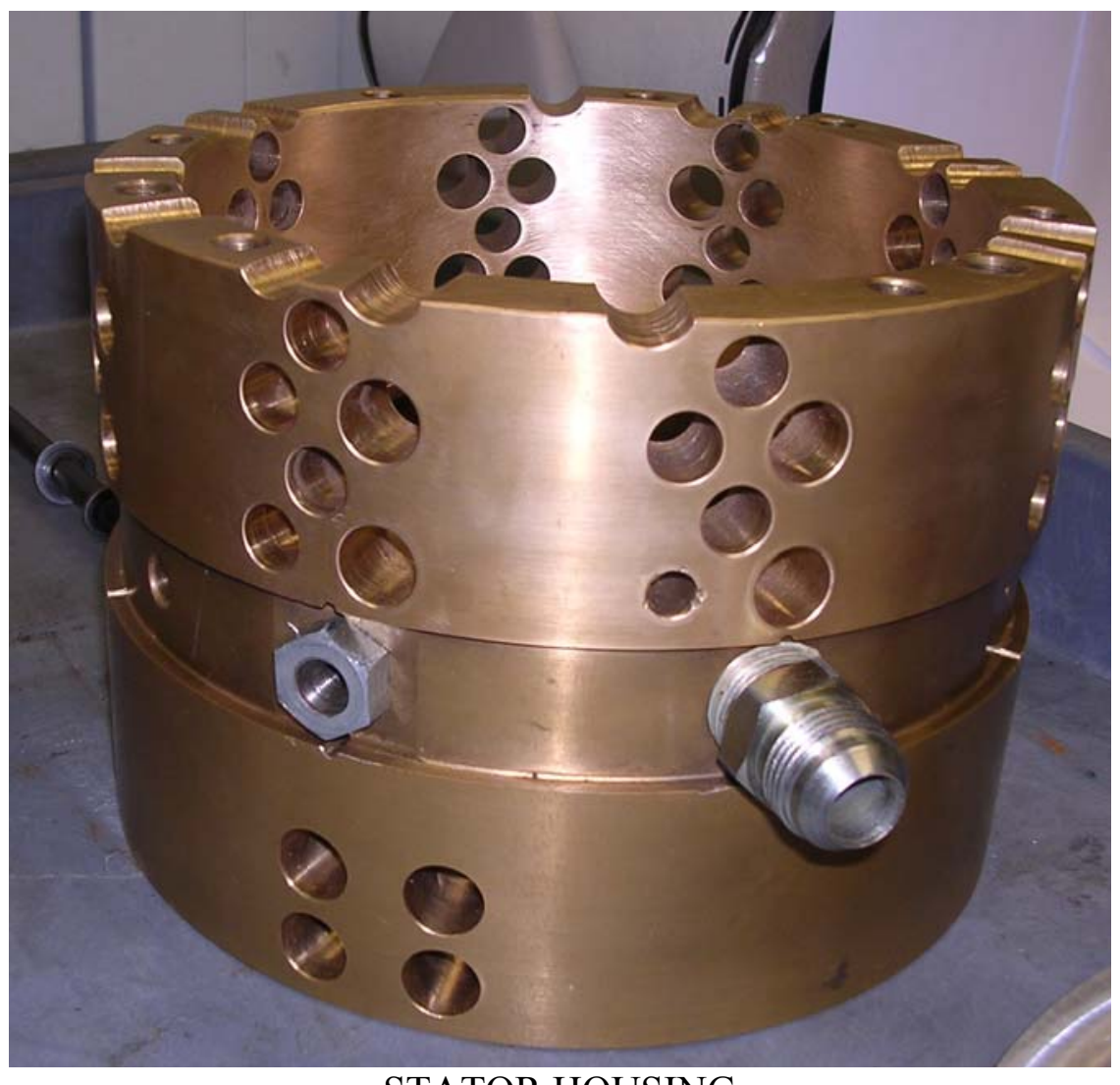

STATOR HOUSING

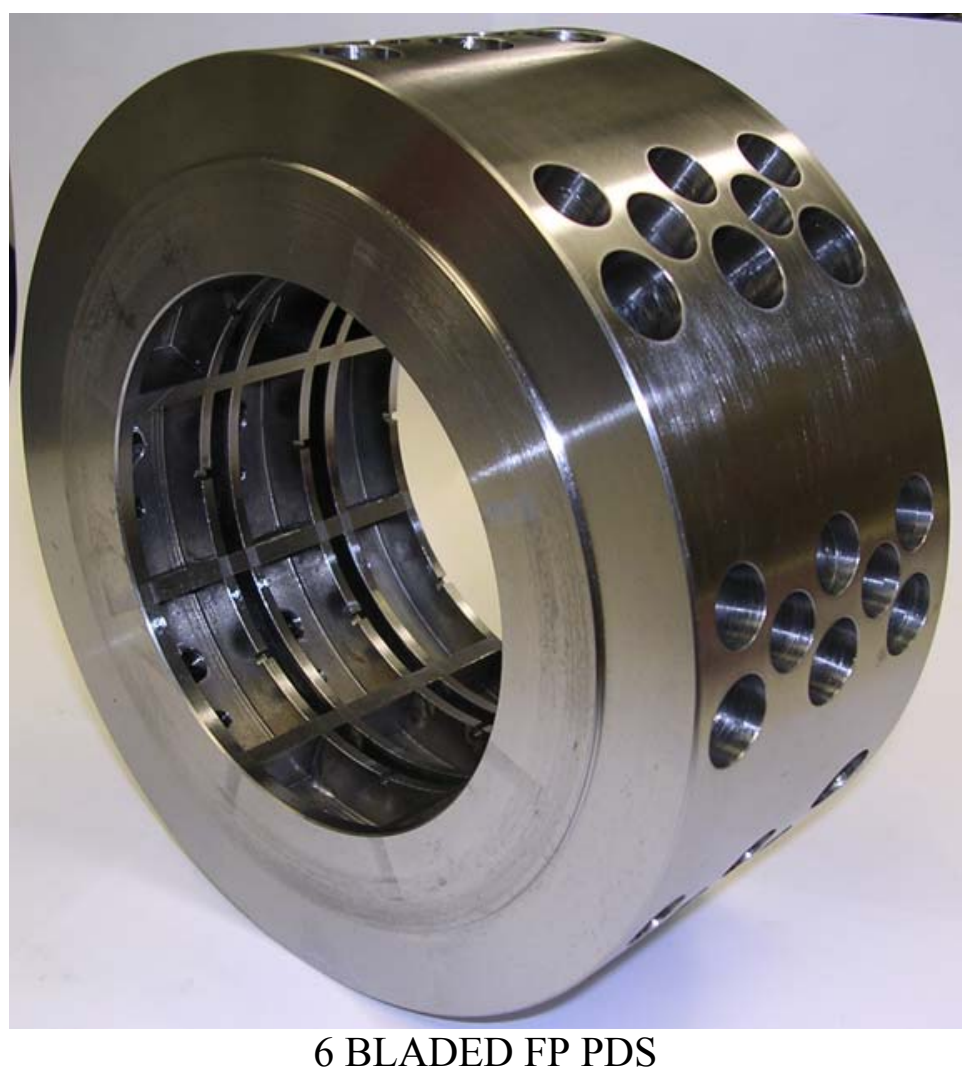




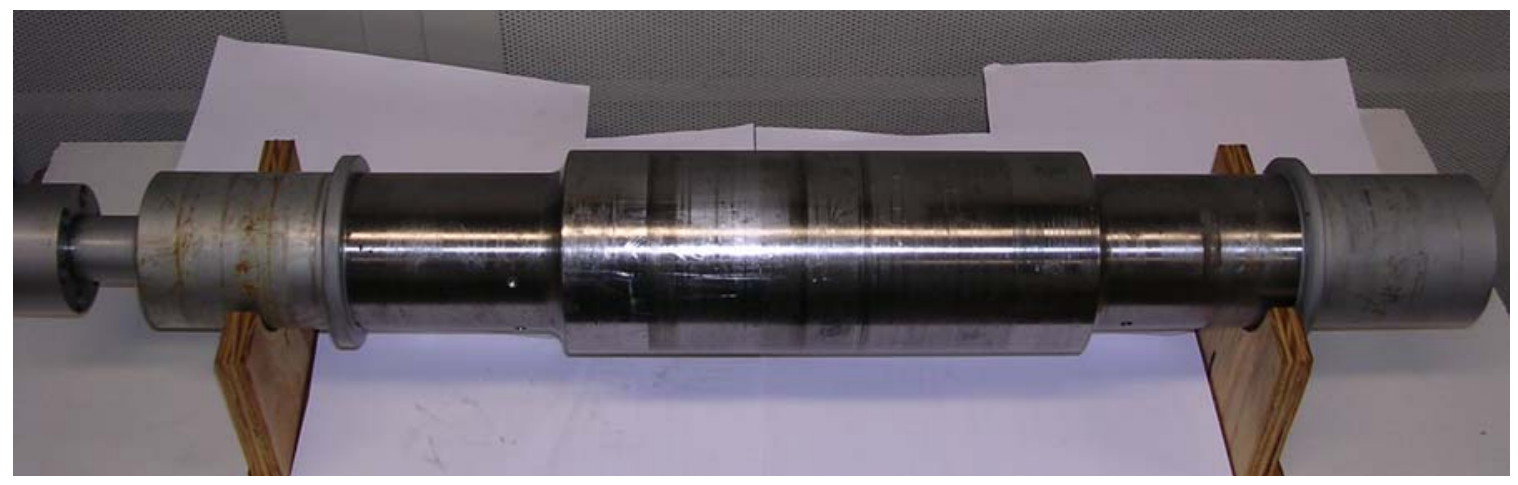

TEST ROTOR

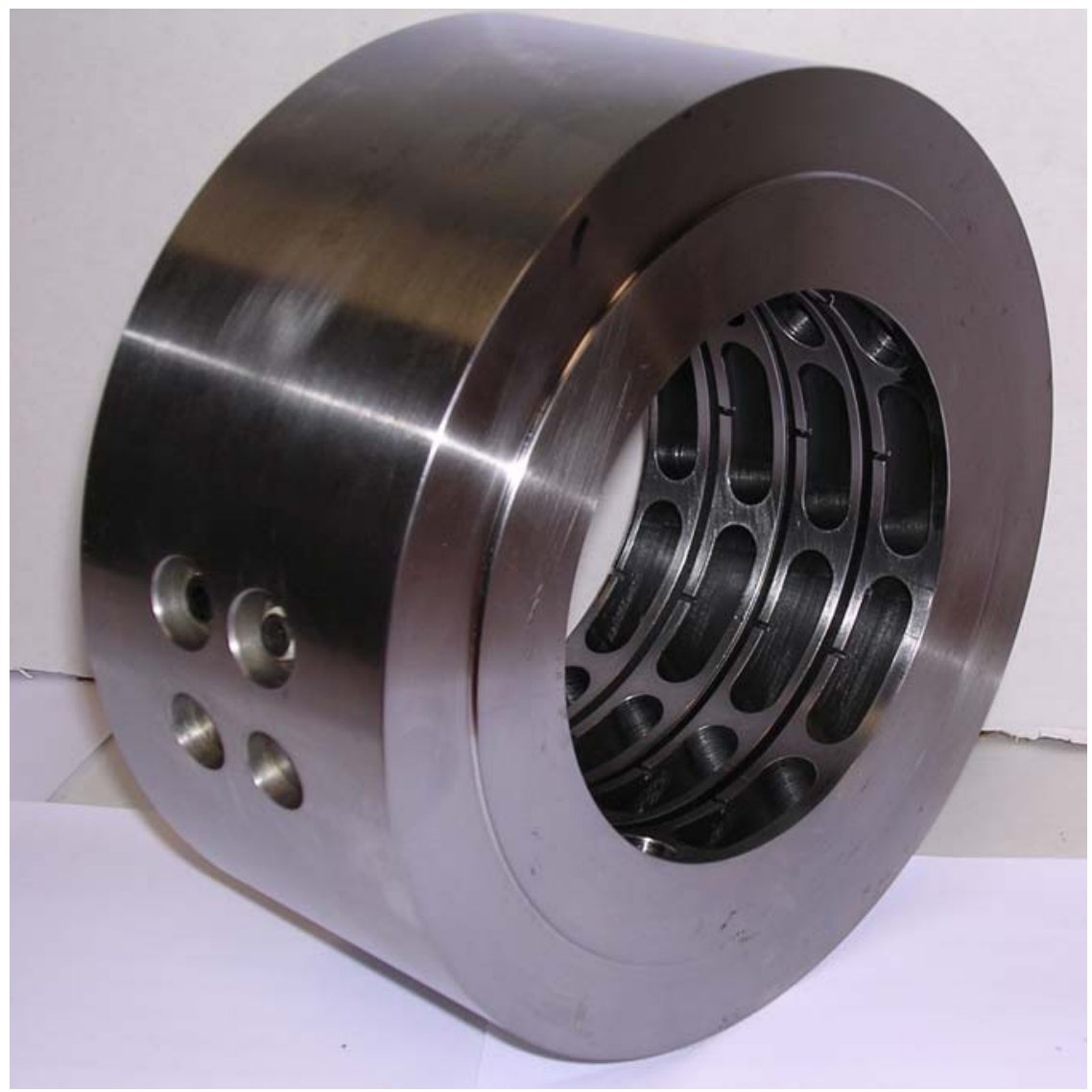

8 BLADED PDS 


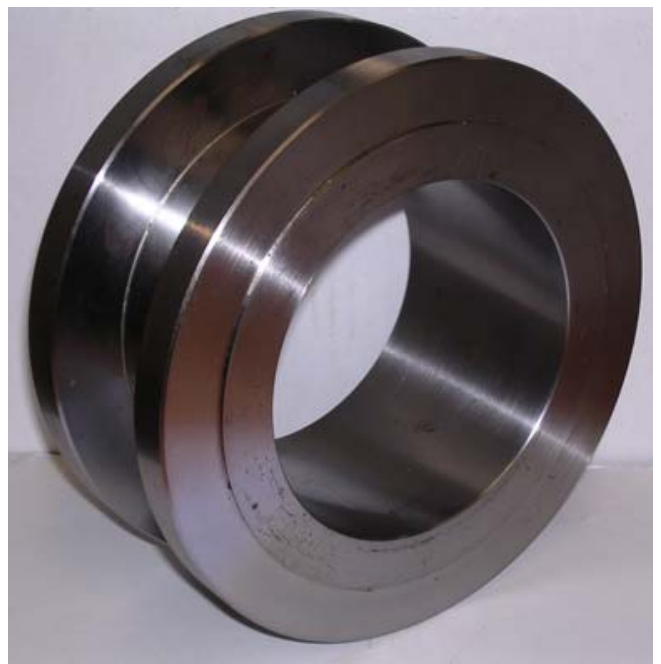

BASELINE INSERT

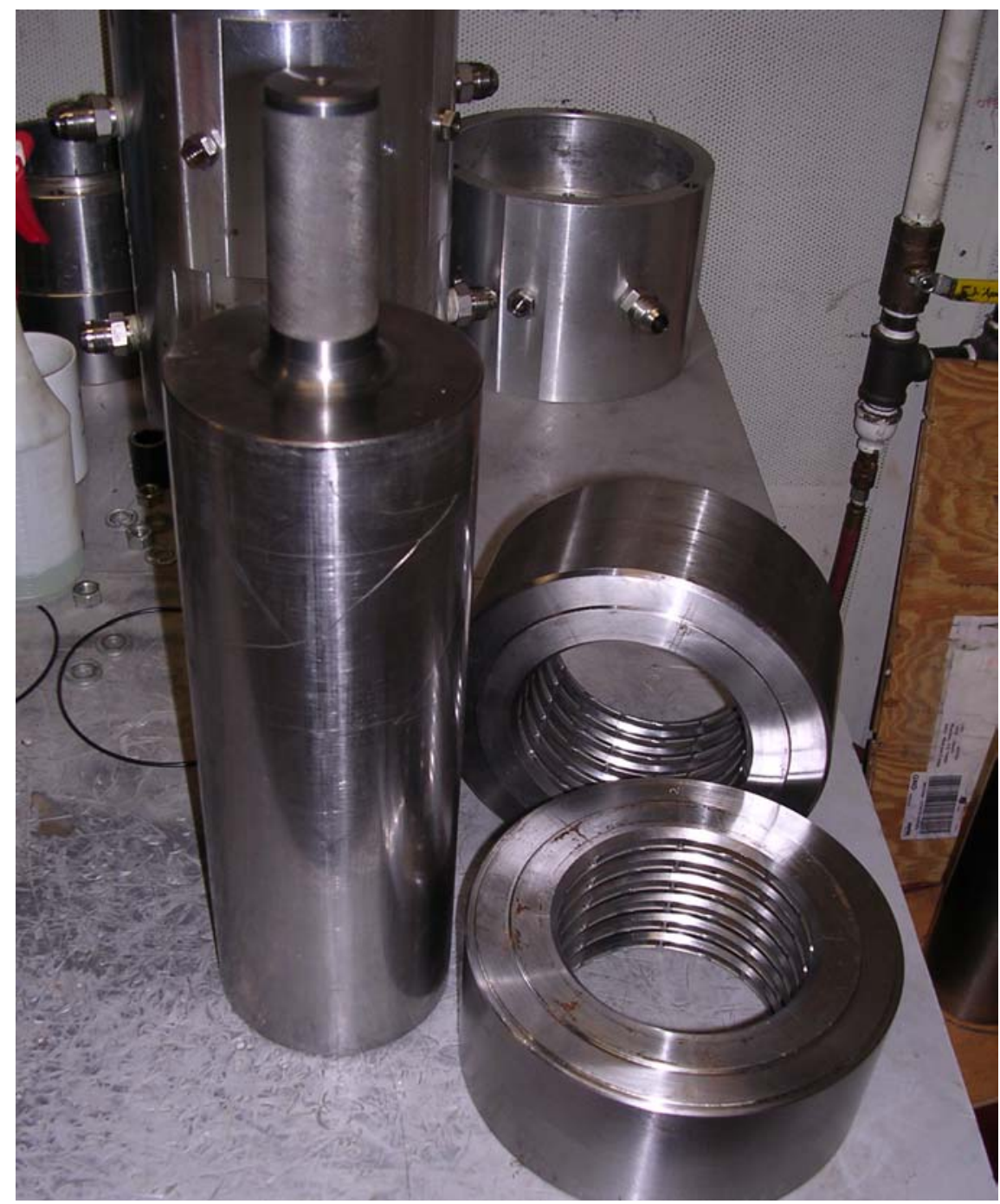

12 BLADED PDS WITH ASSEMBLY MANDREL 


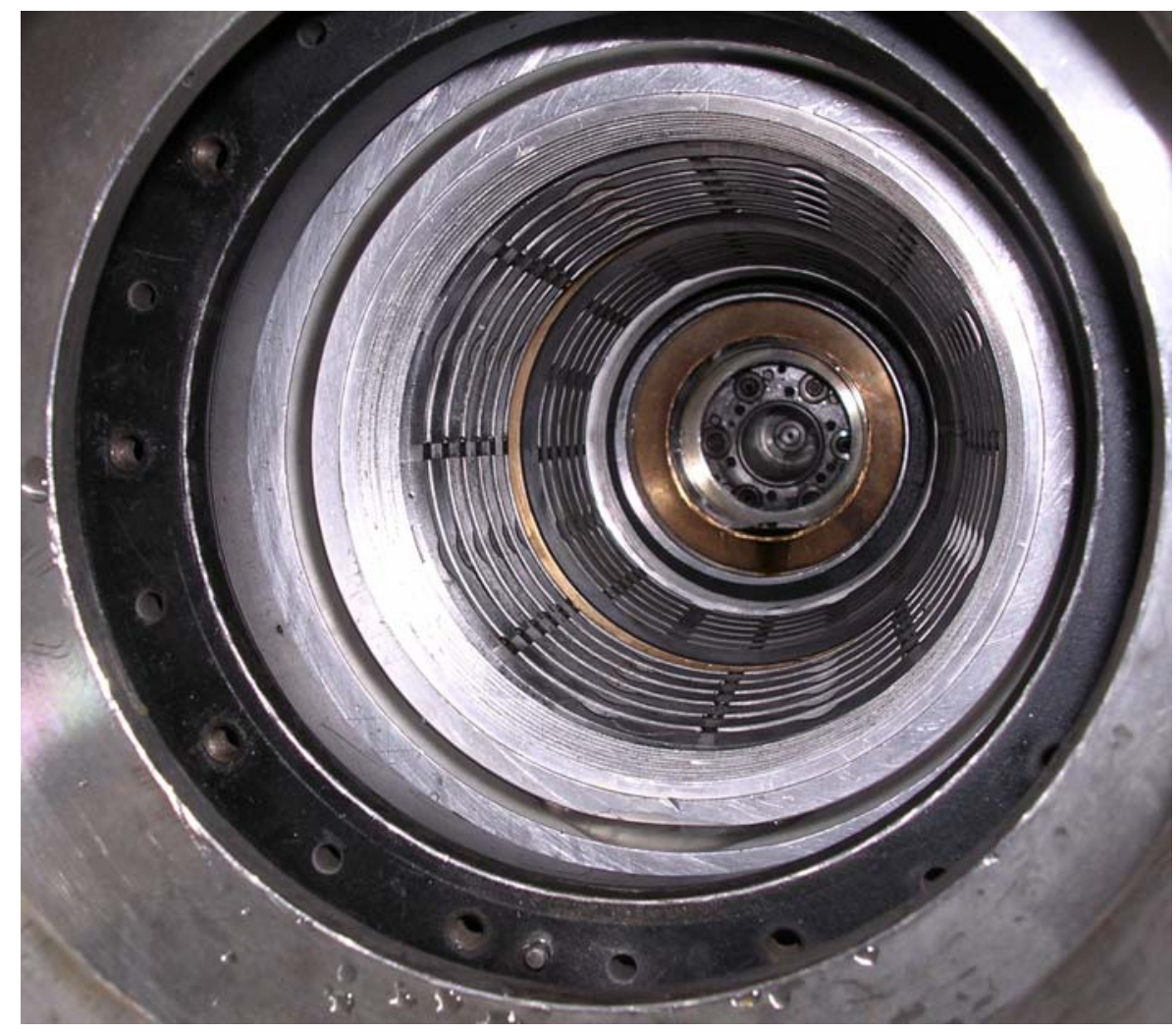

ROTOR REMOVED: 12 BLADED ASSEMBLY

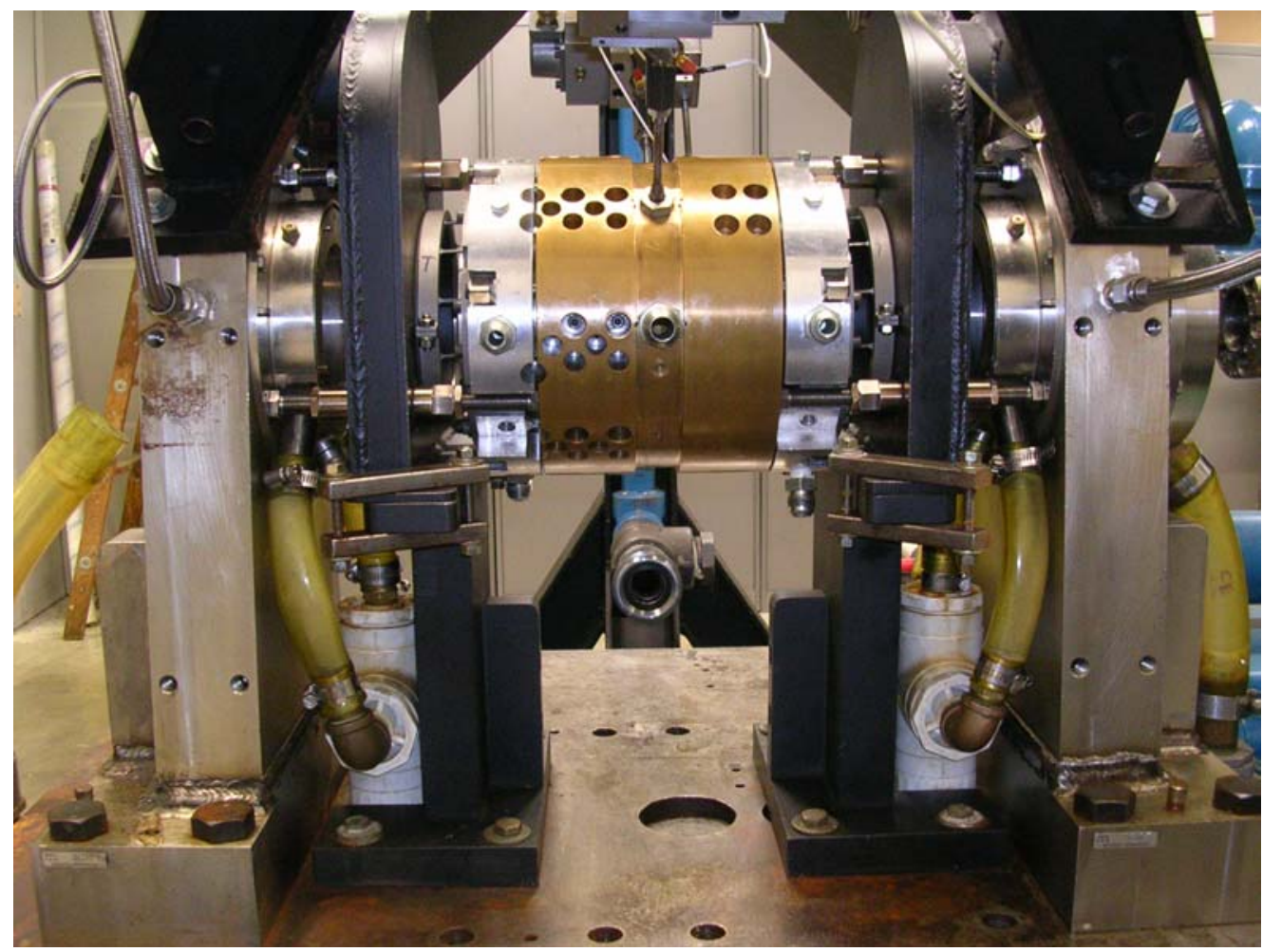

ROTOR REMOVED: TES RIG SIDE VIEW 


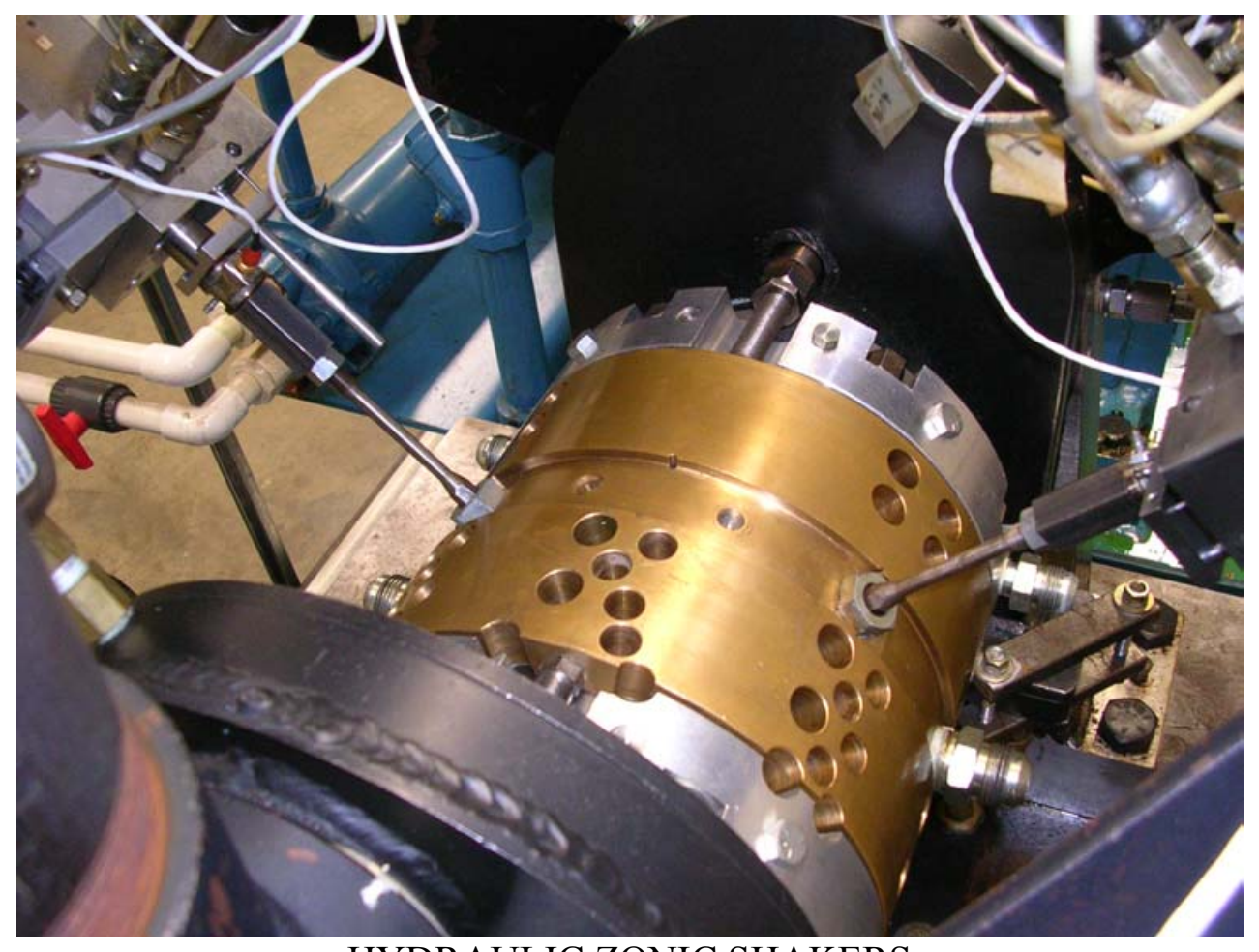

HYDRAULIC ZONIC SHAKERS

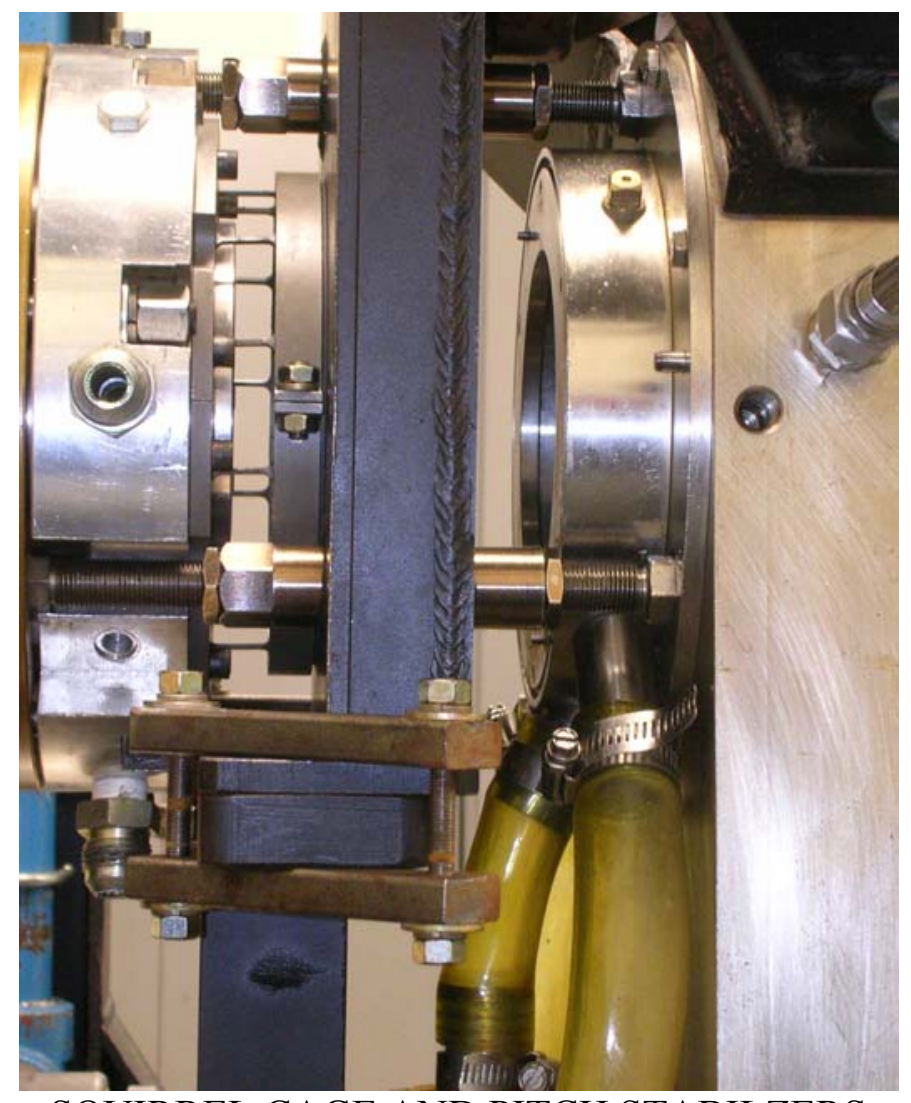

SQUIRREL CAGE AND PITCH STABILZERS 


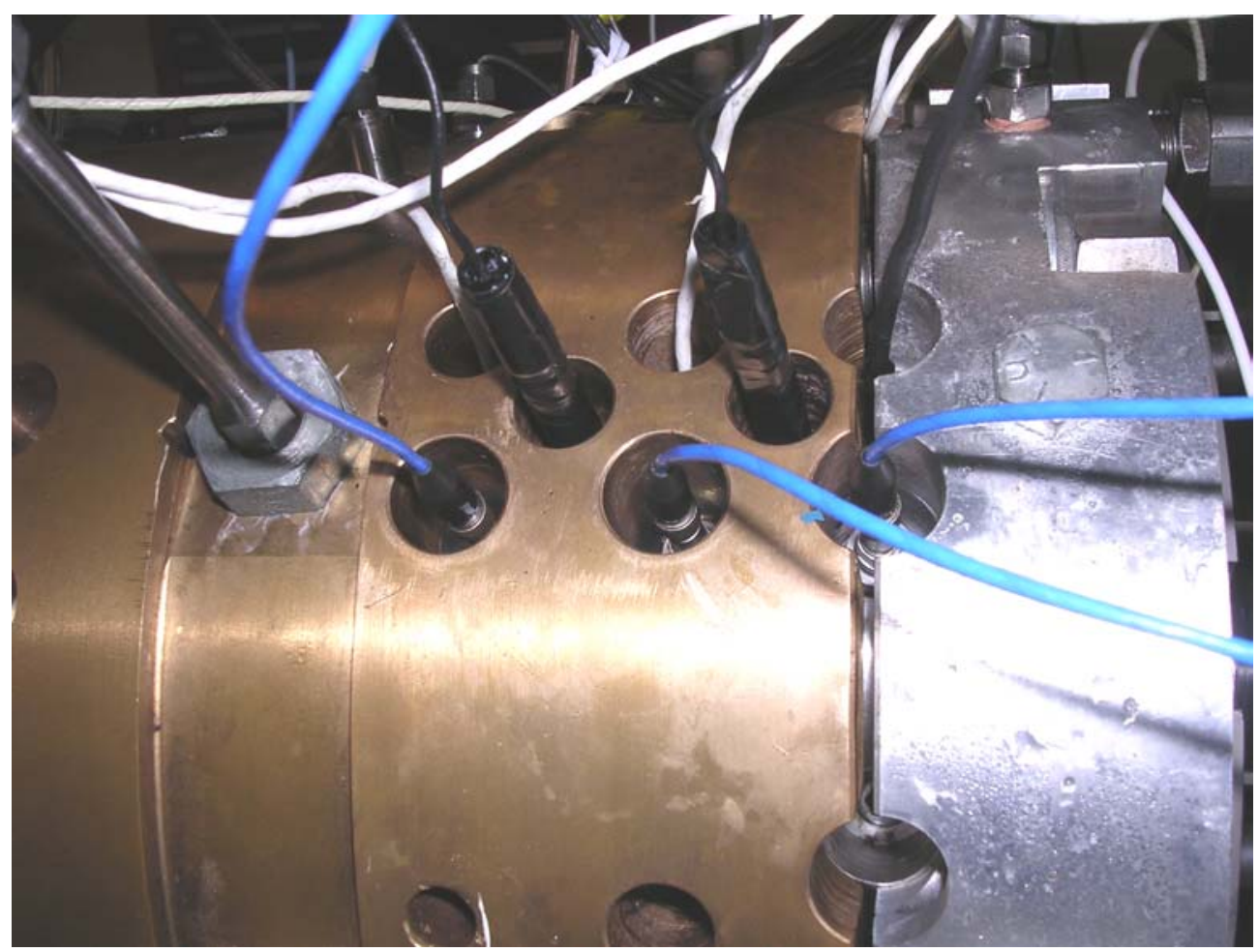

PRESSURE TRANSDUCERS: INSTALLED AT 90 DEGREE POSITION

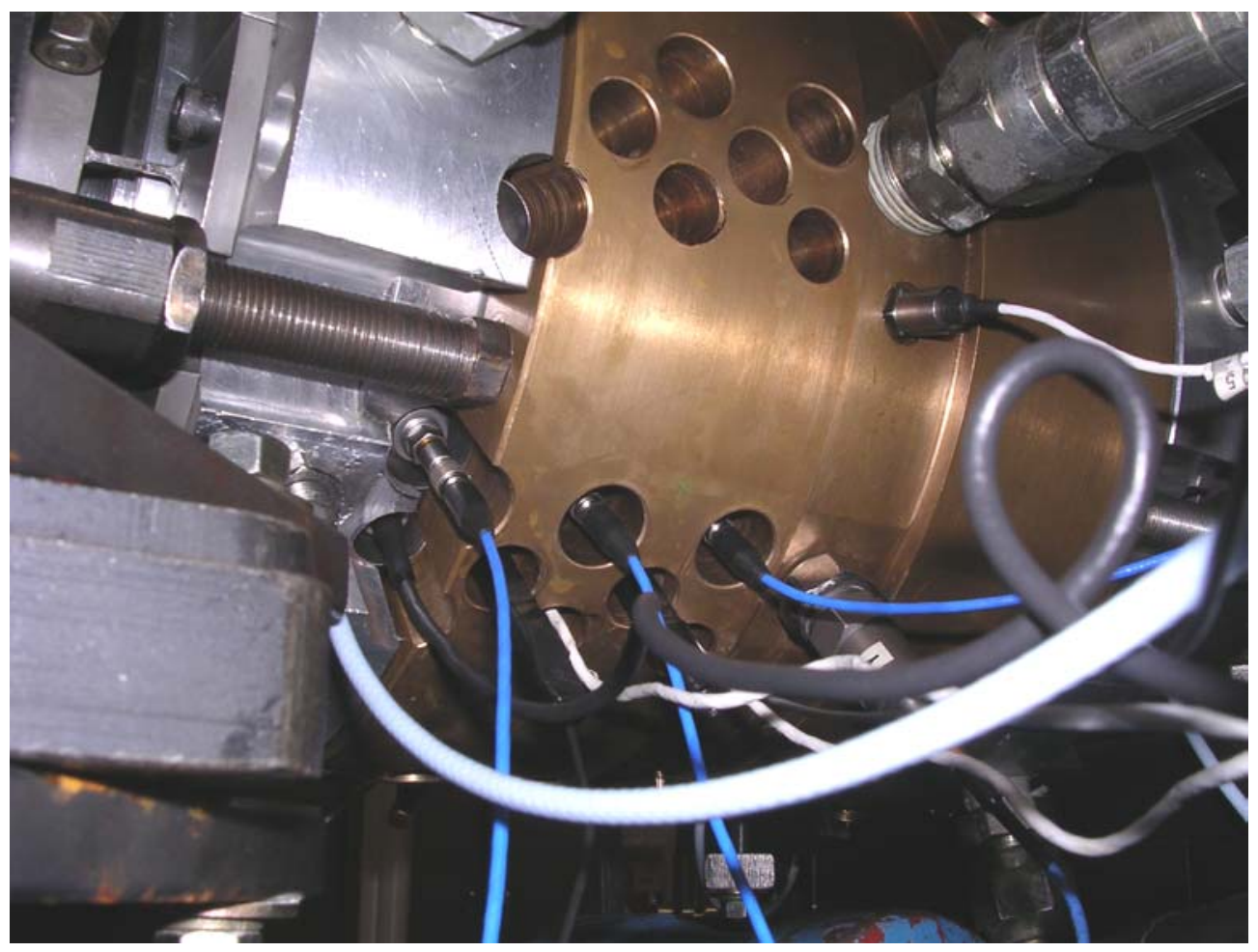

PRESSURE TRANSDUCERS: INSTALLED AT 270 DEGREE POSITION 


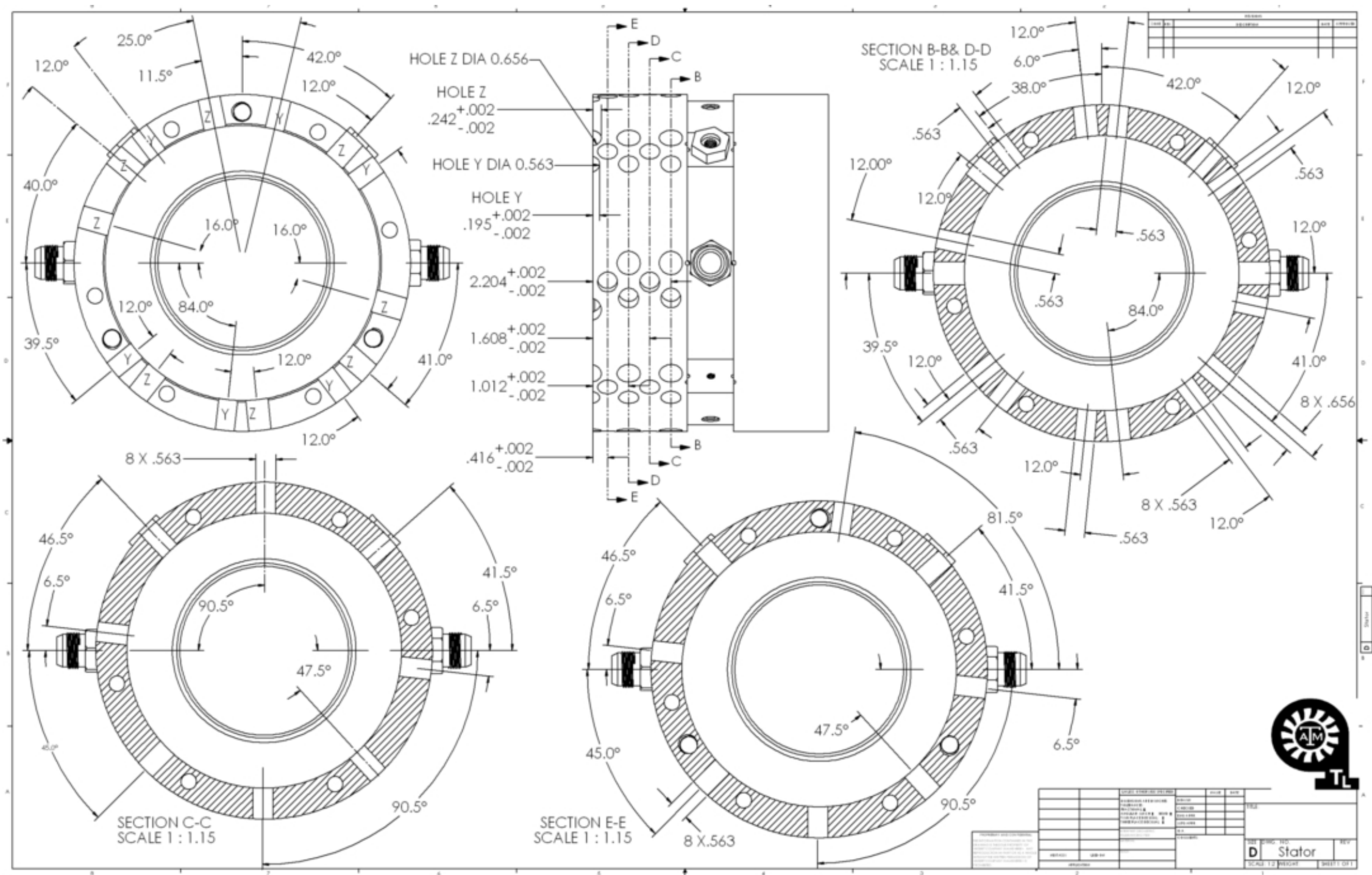

STATOR HOUSING: PRESSURE TRANSUDCER HOLE DETAIL 


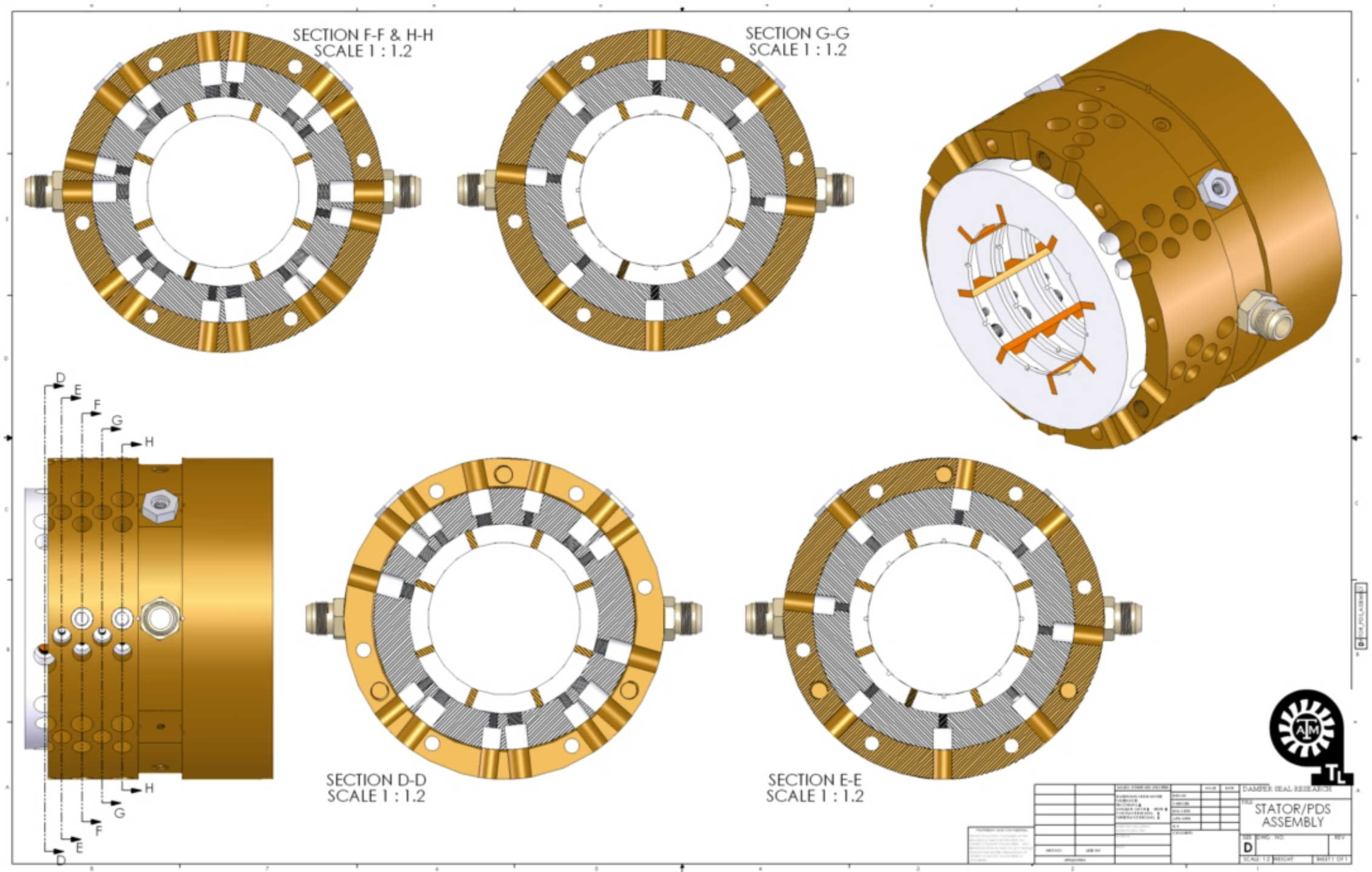

STATOR HOUSING AND 6 BLADED FP PDS ASSEMBLY 


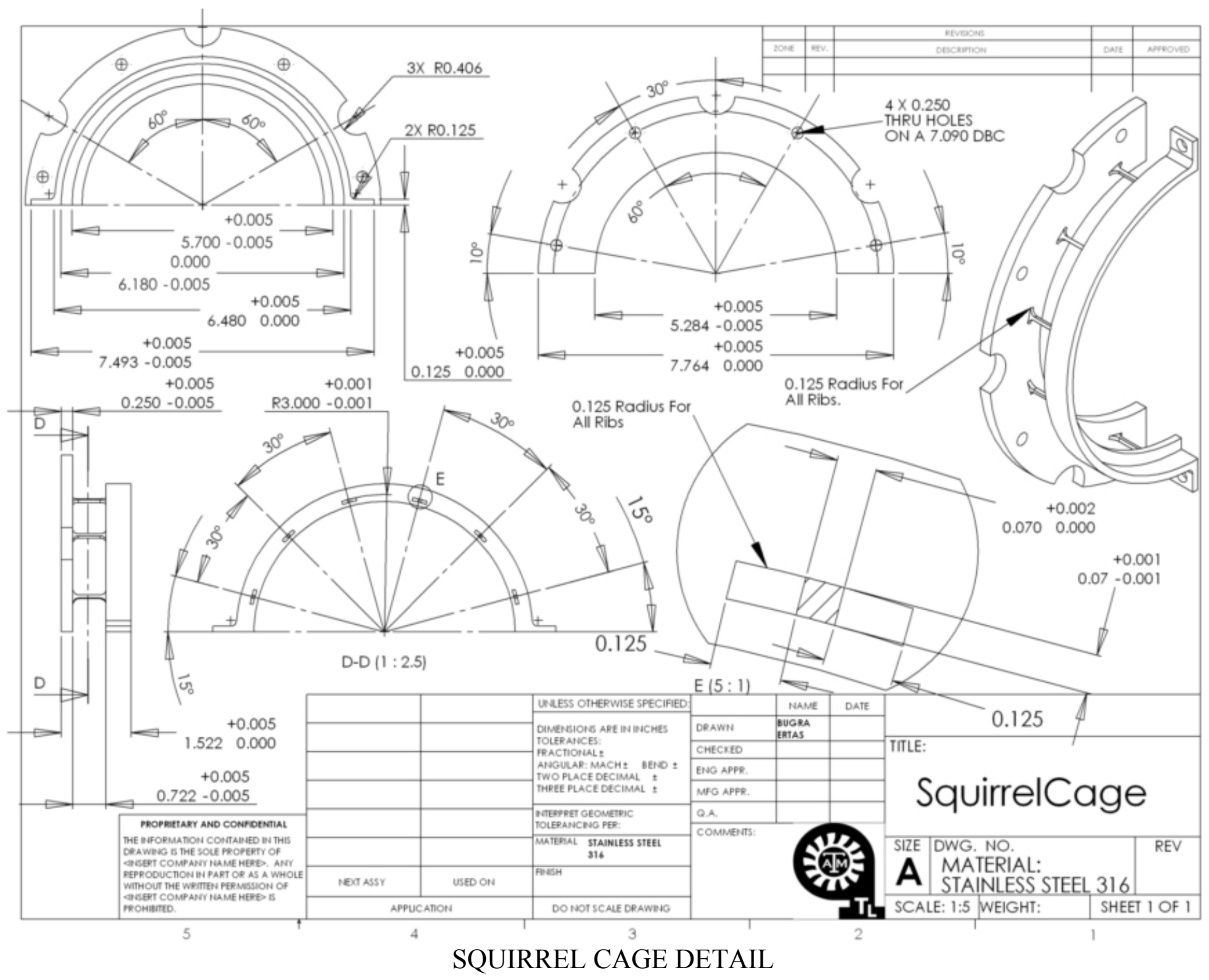




\section{VITA}

Bugra Han Ertas

75 Willett Street Apt 5-i

Albany, NY 12210

\section{EDUCATION:}

B.S. Mechanical Engineering Texas Tech University 1998

M.S. Mechanical Engineering Texas A\&M University 2001

\section{EXPERIENCE:}

Research Assistant

Texas A\&M University

Turbomachinery Laboratory

1999-2005

Project/Research Engineer

Bearing Plus Inc.

Houston, TX

2000-2004

Design/Stress Engineer

Weber Aircraft

Gainesville, TX

1997 